


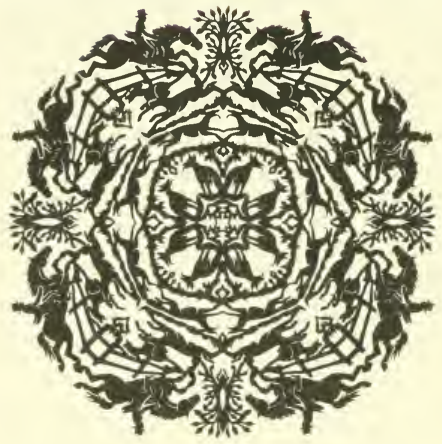

JOHN A.SEAVERNS 


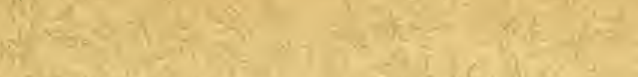





PRINCIPLES AND PRACTICE OF VETERINARY SURGERY. 




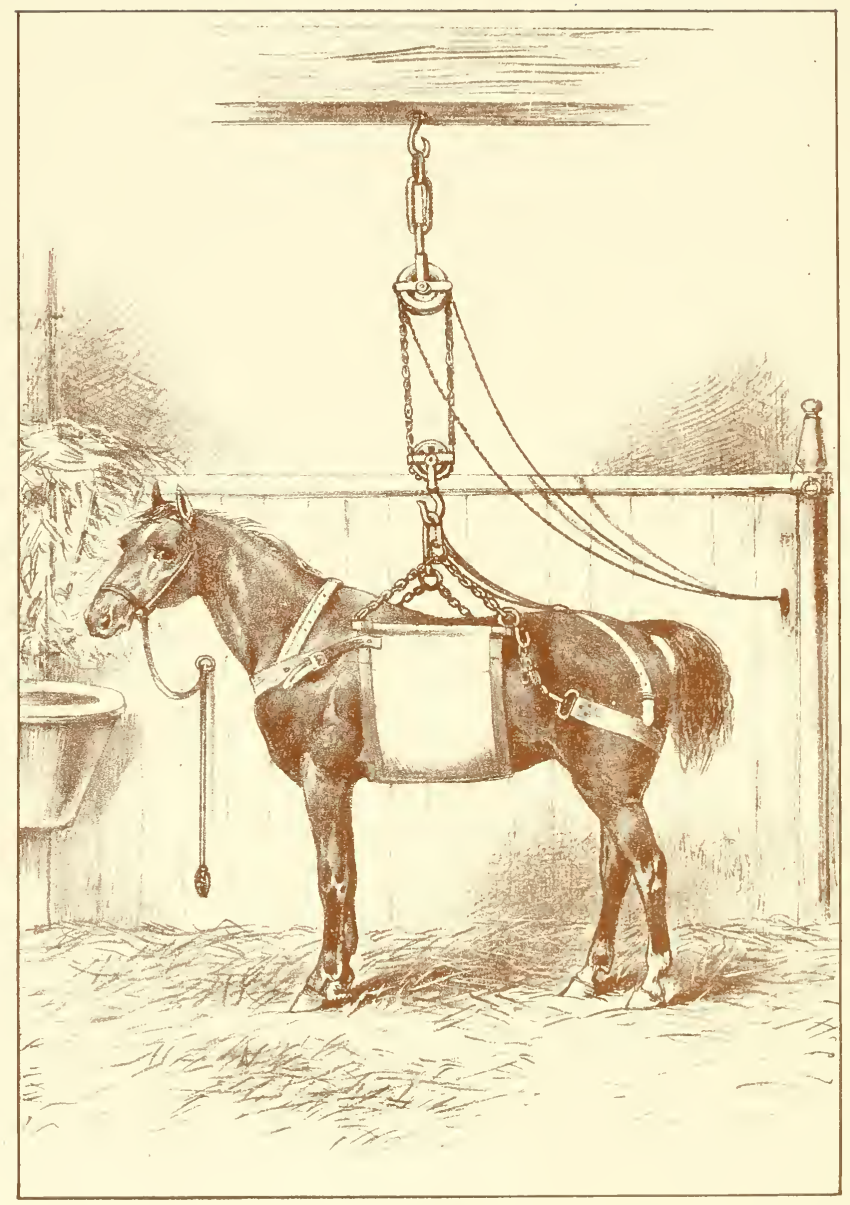

HORSE IN SLINGS 


\section{PRINCIPLES AND PRACTICE}

OF

\section{VETERINARY SURGERY.}

BY

\section{WILLIAM WILLIAMS,}

F.K.C.V.S., F.R.S.E., ETC.

EX-PRESIDENT OF THE ROYAL COLLEGE OF VETERINARY SURGEONS; PRINCIPAL, AND PROFESSOR OF YETERINARY MEDICINE AND SURGERY AT THE NEW VETERINARY COLLEGE, EDINBURGH; EXAMINER IN VETERINARY HYGIENE FOR B.SC. (AGRICULTURE), EDINBURGH UNIVERSITY; PROFESSOR OF VETERINARY SURGERV AND EXAMINER IN AGRICULTURE FOR THE HIGHLAND AND AGRICULTURAL SOCIETV OF SCOTLAND; FOREIGN CORRESPONDENT OF THE SOCIÉTÉ CENTRALE DE MÉDICINE VÉTÉRINAIRE; AND AUTHOR OF "THE PRINCIPLES AND PRACTICE OF VETERINARY MEDICINE.'

EIGHTH EDITION.

REVISED BY THE AUTHOR, ASSISTED BY HIS SON, W. OWEN WILLIAMS, F.R.C.V.S., F.R.P.S.

JOHN MENZIES \& CO., EDINBURGH AND GLASGOW.

J. \& A. CHURCHILL, LONDON. 
PRINTED FOR THE AUTHOR BY J. SKINNER \& COMPANY, AND PUBLISHED BY JOHN MENZIES \& CO., EDINBURGH AND GLASGOW. London, . . . . . . . J. \& A. Churchill. New York, . . . . . . Sabiston \& Murray. Toronto, . . . . . . . . Williamson \& Co. 


\section{CONTENTS.}

\section{CHAPTER I.}

INFLAMMATION.

Definition-Pathology-Phenomena-Causes-Varieties due to Causation -Local Symptoms-Chronic Inflammation-Terminations-Fesolution-Effusion,

\section{CILAPTER II.}

Terminations of Inflammation-continued.

Suppuration-Acute Abscess-Diffuse Suppuration-Superficial Suppuration-Comparison between Pus, Mucus, and Epithelium-Pyogenic Fever or Strangles-Pyæmia-Re-absorption of Pus-Formation of Sinuses--Ulceration-Varieties and Treatnent of Clcers-Mortitication.

\section{CHAPTER III.}

\section{Sruptous of Ivflamiation.}

General Symptoms of Inflammation-Inflammatory Appearances of the Blood-Treatment of Inflammation-Treatment of Chronic Inflammation,

\section{CHAP'TER IV.}

Fractures and Diseases of Bones.

Causes of Fracture-Symptoms-Modes of Union-Treatment of Fractures-Compound Fractures-Separation of Epiphyses-False Joints and Non-Union,

\section{CHAPTER V.}

\section{Particular Fractures.}

Fractures of Inferior Maxilla-Fractures of Anterior Maxilla and of other Facial Bones-Fractures of Cranial Bones-Fracture by "Counter Stroke"-Concussion of the Brain-Hernia Cerebri, 


\section{CHAPTER VI.}

\section{Particular Fractures-continued.}

Fractures of the Vertebræ-On Broken Back-Fracture of the Sacral and Coccygeal Bones-Fracture of the Pelvic Bones-Fracture of the Femur-Fracture of Tilia-Fracture of the Tarsal and Metatarsal Bones,

\section{CHAPTER VII.}

\section{Particular Fractures-continucd.}

Fractures of Scapula-Humerus-Condyles-Olecranon-Radius-Ulna -Trapezium, Carpal, Metacarpal, and Sesamoid Bones-"Spiit Pasterns"-Navicular and Pedal Bones-Broken Ribs-Luxations-

Case of Dislocation of Third Cervical Vertebra, . .

\section{CHAPTER VIII.}

Diseases of the Bones and Articulations.

Classification of the Bones-Ostitis-Sore Shins-Splints-Peculiarity of Splint Lameness-Scrofulous Ostitis,

\section{CHAPTER IX.}

Diseases of the Bones and Articulations-continucd.

Ulceration-Caries-Necrosis-Central and Subperiostal Suppuration -Abscess in the Substance of a Bone, . . .

\section{CHAPTER $X$.}

Non-Inflamatory Diseases of Bones.

Rickets-Mollities Ossium-Osteo-Porosis-Fragilitus Ossium-OsteoSarcoma-Effects of Mercurial Poisoning on Bones,

\section{CHAPTER XI.}

Dislases of the JoINTs.

Classification of the Joints-Anchylosis of the Vertebræ-Acute Synovitis-Chronic Scrofulous Synovitis-Calcification of the Synovial Fringes-Theumatoid Arthritis-Kennel Lameness-Morbid Condition of Cartilage-Traumatic Inflammation of Toints-Hydrops Articulorum-Anchylosis, . . . . .

\section{CHAPTER XII.}

\section{LAMENESS.}

Diagnosis-Definition-Signs-Causes-Congenital Muscular Atony- 
Methods of Examination-Sprains-Strains of Psoæ Muscles-

Chorea-Stringhalt-Shivering-Myositis-Rheumatic Tameness-

Embcli,

\section{CHAPTER XIII.}

Particular Lamenesses.

Shoulder Lameness-Shoulder-Slip-Elhow Lameness-Sprain of Radial or Superior Carpal Ligament-Carpitis,

253-268

\section{CHAPTER XIV.}

Particular Lamenesses-continucd.

Speedy Cut-Broken Knees-Sprain of Flexor Tendons-Sprains of Suspensory Ligament-Sprain of Fetlock-J oint-Sesamoiditis-Ring-

Bones,

\section{CHAPTER XV.}

Particular Lamenesses-continucd.

Hip-Joint Lameness-Sprain of Gluteal Tendons, or Trochanteric Lameness-Sprain and Atrophy of Crural Muscles-Stifle-Joint Lameness -Laceration of Flexor Metatarsi Muscle, . .

\section{CIIAPTER XVI.}

\section{Particular Lamenesses-continucd.}

Diseases of the Hock-Bog-Spavin-Bone-Spavin-Conformation of Hock most subject to Spavin-Thorough-Pin-Capped Hocks-Injuries to Gastrocnemii Muscles-Curb,

\section{CHAPTER XVII.}

\section{Treatment of Lameness.}

Rest-Position of the Limb-Removal of Shoes- -High-Heeled ShoeThin-Heeled Shoe-Hot and Cold Fomentations-Local BlecdingPurgatives-Counter-Irritants-Blisters-Setons-Actual Cautery Theory of their Action,

\section{CHAPTER XVIII.}

\section{Diseases of the Feet.}

Preliminary Observations on Shoeing-Foot Lameness, divided into three kinds : (1.) Diseases of the Bones and Cartilages; (2.) Diseases of the Horn-Secreting Structures; (3.) Accidental Injuries-Disease of the Pyramid of Os Ted s-Side-Bones-Navicular Disease, Theories of -Causes, Pathology, and Treatment-Neurotomy, its favourable and unfavourable Effects-Gelatinous Degeneration of the Navicular Bursa-Pathological Anatomy-Comparison to White Swelling, 


\section{CHAPTER XIX.}

Diseases of the Feet-continued.

Inflammation of the Feet-Divisions of Laminitis-Pathological Changes -Causes-Symptoms-Treatment-Mr. Broad's Treatmeut, .

\section{CHAPTER $\mathrm{XX}$.}

\section{Diseases of the FeET-eontinued.}

Diseases of the IIorn-Secreting Structures-Villitis-Carbuncle of Coronary Band-False-Quarter-Sand-Crack-Horn Tumours-SeedyToe-Corns-Thrush-Canker,

\section{CHAPTER XXI.}

Diseases of the FeET-continuci.

Punctures of the Feet by "Gathered Nails" and by "Pricks" in Shoeing-Importance of thoroughly examining Wounded Feet and of giving Exit to all Discharges-Weak Feet liable to Injuries-Quittor-Treatment-Foul in the Font of Horned Cattle-FootRot in Sheer, . . . . . . . 387-106

\section{CHAPTER XXII.}

Tumours.

Classification,

\section{CHAPTER XXIII.}

Simple Tumours.

Fibroma or Fibrous Tumour-Papilloma-Lipoma-Neuroma-Enchondroma-Crusta-Petrosa Tumours-Fsammoma-Osseous TumoursOsteoma - Parasitic Cysts - Cystic Tumours - Teeth TumoursSarcomata - Melanosis - Actino-Mycosis - Carcinımata - Hard Cancer-Soft Cancer-Epithelial Cancer, . . . 412-447

\section{CमAPTER XXIV.}

\section{WOUNDS.}

Definition-Classification-Incised-Punctured - Lacerated-Contused Gun-Shot-Yoisoned-'I'reatmeut of Wounds-Sutures-CollodionStyptic-Colloid-Shellac Paste-Non-Interference, 


\section{CHAPTER XXV.}

Wounds-continued.

Gun-Shot-Cannou Balls-Wind Contusions-Musket-Shot-Grape-Shot -Sinall-Shot-Consequences of Gun-Shot Wounds-Wounds of the Abdominal Parieties-Wounds and Bruises having Special Names -Burns, Sealds, and the Effects of Cold-Poisoned WoundsSpeedy-Cut-Tread-Cutting or Brushing,

\section{CHAPTER XXVI.}

Wounds-continued.

Methods of Repair-Inmediate Union-Primary Adhesion-Granulation - Secondary Adhesion-Healing under a Scab-Formation of the Cicatrix, and Completion of the Reparative Process, .

\section{CHAPTER XXVII.}

REsUlts of WOUNDS.

Erysipelas, Simple and Phlegmonous-Symptoms and TreatmentTetanus, Idiopathic and Traumatic-Various Forms of-Symptoms -Pathology-Treatment

\section{CHAPTER XXVIII.}

\section{Diseases and Injuries of the Facial Region.}

Wounds of the Lips-Tumours-Bruises of the Mouth-Affections of the Buccal Membrane-Sporadic Aphthæ-Paralysis of the Lips-Optn Parotid Duct-Salivary Calculi--Ptyalisin-Ranula-Epulis-Glossitis-Ulcer and Induration of the Tongue in Ilorned Cattle-Paralysis of the Tongue, . . . . . .

\section{CHAPTER XXIX.}

Diseases and Injuries of the Facial Region-continued.

Parrot-Mouth-Irregularities of the Teeth-Diseases of the Alveolar Processes-Caries of the Teeth-Diseases of Dentition-Dental Irritation in Young Cattle-Dental Cough in Young Horses-Dentition Fever-Supernumerary Teeth-Diseases of the Facial Sinuses-Ozœena, or Nasal Gleet,

\section{CHAPTER XXX.}

Diseases of the Pharixx, Esophages, \&c.

Post-Pharyngeal Abscess-Pus in the Guttural Pouches-Pharyngeal Polypi-Choking, Clinical Records of-Esophagotomy-Dilatation and Stricture of the Esophagus-Wind-Sucking and Crib-Biting, 


\section{CHAPTER XXXI.}

Disgases of the Eyes.

Conjunctivitis - Nebulæ-Albugo-Staphyloma - Ulcers - Glaucoma A maurosis-Strabismus-Ectropium-Entropium-Trichiasis - Distichiasis-Warts-Wounds-Haws-Iachrymal Fistula-StrictureParasites-Fungus Hæmatodes-Periodic Ophthalmia-CataractRemoval of Eyeball-Dislocation of Eyeball-Melanosis of Humours - Examination of Eye by Catoptric Test and by the Ophthalmoscope,

PAGR

\section{CHAPTER XXXII.}

Disfases of the Head, Neck, Veins, \&c.

Otorrhœa-External Canker of the Ear-Poll-Evil-Fistulous WithersCircumscribed and Diffuse Phlebitis-Thrombus-Varicose VeinsPhlebolites-Entrance of Air into the Veins,

\section{CHAPTER XXXIII.}

Disfases of the Arteries and Lymphatics.

Diseases of the Arteries-Arteritis-Embolism-Aneurism-True Aneurism-The Dissecting Aneurism-False Aneurism-Aneurismal Varix -Varicose Aneurism-Degeneration of the Arterial Coats-Calcareous, Cartilaginous, and Fatty Degeneration-Injuries to Arteries -Inflammation of the Absorbents, . . . .

\section{CHAPTER XXXIV.}

Hernie, \&c.

Reducible-Irreducible and Strangulated Herniæ-Unbilical or Exomphalos-Ventral, Inguinal, and Scrotal Hernix-Gut-Tie in Cattle, 596-622

\section{CHAPTER XXXV.}

Diseases of the Bladder, External Organs of Unination, \&o.

Diseases of the Bladder, Vagina, Vesiculie Seminalis, Mammary Gland, \&c.-Cystic Calculi in the Horse, Ox, Sheep, and Dog-Lithotomy -Lithotripsy-Lithotrity-Urethral and Preputial Calculi--Sabulous Matter-Urethritis-Phymosis-Paraphymosis-Auputation of the Penis-Vaginitis-Lencorrhoa-Mammitis, . .

\section{CHAPTER XXXVI.}

\section{Castiation.}

Castration-Precautions to be observed before the Operation is performed - Methods of Operating-Consequences of Castration-SwellingHernia-Scirrhous Cord-Fistula of the Scrotum-Peritonitis-Gangrene-Tetanus-Paralysis.-Amaurosis-Glanders and Farcy, 
CHAPTER XXXVII.

CHAPTER XXXVIII.

AnæSTHESIA

\section{CHAPTER XXXIX.}

Diseases of the Skin.

Classifications : Willan and Bateman's, Bennett's, and Buchanan's-Ery. thematous Inflamıntions-Clipping Horzes-Herpes-Urticaria,

\section{CHAPTER XL.}

DISEASES OF THE SKIN--continued.

Definition-Division-Various forms of Eczema-Simplex-RubrumLichen-Impetiginodes-Acute and Chronic Grease-Elephantiasis - Crusta Labialis-Treatment of Eczematous Eruptions-Effects of Carbolic Acid on the Dog, . . . . .

CHAPTER XLI.

Diseases oF THE SKIN-continued.

Urinous Eczema,

\section{CHAPTER XLII.}

DISEaSeS of the SKIN-continued.

I. Squamous Inflammation of the Skin-Psoriasis-Pityriasis-II. Disordered Sensibility of the Derma-Prurigo-III. Inflammation of the Sebaceous Follicles-Acne-Sitfasts-Treatment, . .

\section{CHAPTER XLIII.}

\section{Diseases of THE SKin-continued.}

Scabies-Different Varieties of Acari--Sarcoptes--Dermatodectes-Symbiotes-Classification-Experiments of Hering, Hertwig, and others as to the Transmissibility of the Mites to Man-Mange in the Horse and $\mathrm{Ox}$-Scab in Sheep-Mange in the Dog and Cat-Treatment of Mange-Diseases due to Epizoa-Poultry Lonsiness-Fleas-Ticks -Bots-Maggots,

\section{CHAPTER XLIV.}

DISEASES OF THE SKIN-continued.

Favus, or Honeycomb Ring-Worm-Experiments of Gruby-The Achorion Schönleinii-The Yellow Crust due to the Exudation-Transmissibility of the Disease from Animals to Man, and vice versaTinea Circinatus-The Tricophyton discovered by MalmstenIdentity of Favus and Tinea Circinatus - Letter from Mr. M'Gillivray of Banff-Treatment of Ring-Worm, . . 


\section{LIST OF ILLUSTRATIONS.}

\section{PHOTO-LITHOGRAPHS.}

Discases of the Bones and Articulations,

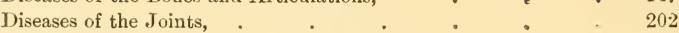

Anchylosis, or Stiffening of the Joints, . . . 230

Tumours, . . . . . . . . 407

\section{LITHOGRAPHS.}

Horse in Slings, .

Three Views of Mr. Walker's Face Cradle,

Actino-Mycosis, . . . . . . 441

Casting by means of Side Lines, . . , , , 655

Do. by means of Hobbles, . . $\quad$. . 656

FIG.

\section{WOODCUTS.}

1. Web of Frog's Foot, showing First Stage of Inflammation, . 7

2. Irregular Contraction of Small Vessels in the Web of a Frog's Foot after the application of Stimuli, $\quad . \quad$. $\quad . \quad 8$

3. Parenchymatous Keratitis, . . . . . 13

4. Inflammation and Gelatinous Degeneration succeeding Neurotomy, 20

5. Purulent Granulations, . . . . . . 32

6. Examples of Cells, . . . . . . . 32

7. Cells from Fresh Catarrhal Sputa, . . . . . 33

8. Pus-Corpuscles, . . . . . . . . 33

9. Oblique Fracture of the Tibia, with External (Ensheathing) Callus, 80

10. Fracture of Metatarsus, showing the Plug within the Medullary Canal, 81

11. Indirect Comminuted Fracture of Basilar Process of Occipital and Sphenoidal Bones, . . . . . . . 104

12. Fracture of Neck of Femur, . . . . . 126

13, 14. Split Pasterns, . . . . . . . . . 136

15, 16. Fracture of Navicular Bone, . $\quad$. $\quad$. $\quad$. 138

17. Trochlea of the Femur, showing the effects of Friction from Luxation of Patella, . . . . . . . . . 143

18. Dislocated Neck, . . . . . . . 145

19. Inferior Extremity of Tibia in a Healthy Condition, . . 152

20. Inferior Extremity of Tibia in a State of Ulceration, . $\quad 152$

21. Sore Shin, . . . . . . . 154

22. Compound Splint, . . . . . . 156

23. Ordinary Splint, . . . . . . . . 158

24. Instrument for Pyro-Puncture, . $\quad$. $\quad$. 159 
25. Necrosis Totalis, .

26. Section of the Bone represented in Fig. 25, $\quad$. $\quad$. $\quad$. 171

27, 28. Abscess in Bone, .

29, 30. Rickets,

31, 32. Osteo-Porosis of Cranio-Facial Bones,

33-35. Effects of Mercury on Bones of Dog,

36. Healthy Cartilage,

37, 39. Diseased Articular Cartilage,

39-41. Diseased Cartilage,

42, 43. Repair of Cartilage, .

44. False Ring-Bone,

45. High Ring-Bone,

46. Low Ring-Bone, .

47. Ring-Bone involving the Os Suffraginis, .

48. Disease of Trochanter Major,

49. Rupture of Flexor Metatarsi,

50. Bone-Spavin,

51. Astragali united to show the Screw Lines,

52, 53. Bone-Spavin,

53A. Occult Bone-Suavin.

201

215

216

217

221

285

286

287

287

292

297

302

305

308

310

54. Spring Truss for Thorough-Pin and Bog-Spavin, . . 311

55, 56. Horse-Shoes,

57. The Foot prepared for the Shoe, .

58. Ossification of the Lateral Cartilages; Fracture of the altered Structure upon one Side,

59. Phalangeal Bones in their naturally Oblique Position,

60. Position of Phalangeal Bones altered by a Thick-Heeled Shue, .

61. Longitudinal Sections of Four Navicular Bones, .

62. External Appearance of Inferior Articulating Surfaces of four Navicular Bones,

3-65. Ulceration of Cartilage, . . . . . . . . 346

66. Effects of Neurotomy, . . . . . . 352

67. Section of the Foot with its various Parts in a healthy Condition, 358

68. Formation of a Horny Exudate at the Toe, . . . 358

68A. Perforation of the Horny Sole by the Toe of the Pedal Bone, . 358

69. Ribbed Appearance of Foot after Laminitis, _ $\quad$ • $\quad 359$

70. Absorption of Inferior Edge of Os Perlis, . . . $\quad 359$

71. Advanced Atrophied Condition of $\mathrm{O}_{s}$ Pedis, . . $\quad 360$

72. Mr. Broad's Shoe, • . • . . . 367

73. Horny Foot, showing Horn Tumour, . . . 378

74. Pedal Bone of same Foot, showing a Cavity at its Toe corresponding to the Horn Tumour in Fig. 73, . . . 378

75. Bony Spiculæ on Plantar Surface of Os Pedis, . . . 381

76. Bistouri Caché, . . . . . . . 390

77. Confirmed Stage of the First Form of Foot-Rot, . . . 400

78. Condition of Internal Wall of Digit in an Early Stage of FootRot, . . . . . . - . 400

79. Condition of a Foot four weeks after the Febrile Stage of Footand-Mouth Disease, . . . . . 402

80. Perpendicular Section of an Accuminated Condyloma, . $\quad 415$

81. Fat Cells, . . • . . . . . 416

82.81. Enchondroma, . $\quad$. $\quad$. $\quad$ - $\quad$. 4418,419 
FIQ.

85. Crusta-Petrosa Tumour, . . . . . 420

86. Development of Teeth, . . . . . . . . 431

87. Diagram of Development of Dentigerous Tumour, . . . 432

89-90. Lymphadenoma Cells, . . . . . . . 441

91-92. Actino-Mycosis, . . . . . . . 441

93. Epithelial Cancer from Palate of $\mathrm{Ox}, \quad$. . . . 447

94-107. Microscopic Anatomy of Cancer, . . . . 447

108. Vertical Section of a Granulating Sore, . . . . . 479

109. Speculum Oris, . $\quad$. $\quad$. $\quad . \quad$. 511

110. Skull cut open to show the situation of Bony Partition of

Superior Maxillary Sinus, $\quad$. $\quad$. $\quad$. $\quad$. $\quad$. 519

111. Aneurism of the Posterior Anrta, . . . 582

112. Inguinal Hernia, . . . . 603

113. Scrotal Hernia, . . . . . . . 606

114. Clams for Castration, . . . . . . . 644

115. Torsion Forceps, . . . . . . . 645

116. Carlisle's Muzzle, . . . . . . . $65 \mathrm{~S}$

117. Sarcoptes Hippopodus, . . . . . . 677

118. Urinous Eczema-External Appearance, . . . 684

119-120. " , , Vertico-transverse Sections of Skin, . 685-686

121. " " " Section of Kidney, Cortical part, . . 687

122. ", " Section of Kidney showing Buundary Layer transverse to Tubules, . . . 688

123. , , , Section of Liver, showing Cells increased in granularity, and also Fatty Degeneration, $\quad 689$

121. ", , Section of Liver, showing Lobules undergoing same changes, . . . . 690

125-127. Sarcoptes Hominis (Acarus Scabie), . . . . 702

128. Sarcoptes Equi, . . . . . . . . 703

129. Dermatodectes Equi, . . . . . . 705

130. Symbiotes Equi, . . . . . . . 706

131. Dermatodectes Bovis, . $\quad$. $\quad . \quad$. 709

132. Symbiotes Bovis, . . . . . . 710

133. Dermatodectes Ovis, . . . . . . 711

134. Sarcoptes Ovis, from above, . . . . . . 714

135. Sarcoptes Ovis, from beneath, . . . . . 714

136. Sarcoptes Suis, . . . . . . . . 715

137. Sarcoptes Canis, . $\quad$. $\quad . \quad$. 715

138-140. Three Follicles of the Skin of the Dog, containing Entozoa, • 716

141. Sarcoptes Cati, . . . . . . 720

142. Branches of Achorion Schönleinii, . . . . 729

143. Fragments of the Branches more highly developed, . $\quad$. $\quad 729$

144. Thalli, Mycelia, and Sporidia of the Achorion Schönleinii, showing

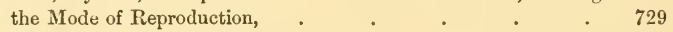

145. Isolated Crusts of Favus, . . . . . . 731

146. Hairs containing Branches of the Achorion Schönleinii, . $\quad 732$

147. Microscopic Appearance of Ring-worm Crust after the addition of

Liquor Potassæ, . . . . . . 736 


\section{PRINCIPLES AND PRACTICE OF VETERINARY SURGERY.}



THE

\section{PRINCIPLES AND PRACTICE}

\section{OF \\ VETERINARY SURGERT.}

\section{CHAPTER I.}

INFLAMMATION.

DEFINITION-PATIOLOGY-PHENONENA-CAUSES-VARIETIES DUE TO CAUSATION-LOCAL SYMPTOMS-CHRONIC INFLAMMATION-TERMINATIONS-RESOLUTION-EFFUSION.

THE process of inflammation is the most important of all morbid actions and conditions.

It has been variously defined by different investigators, according to what they conceived it to be. Thus Hunter says it is simply an increased action of the vessels. In Mr. Syme's opinion, it is "a perverted action of the capillary system, generally attended by heat, redness, pain, and swelling." Dr. Alison describes it as " a peculiar perversion of nutrition or of secretion;" and Dr. Aitken defines it as " a complex morbid process characterised (1.) by a suspension of the concurrent exercise of function among the minute elements of the tissue involved; (2.) by stagnation of the blood, and abnornal adhesiveness of the blood discs in the capillary vessels contiguous to the tissue elements whose functions are suspended; (3.) by contraction of the minute arteries leading to the capillaries of the affected part, with subsequent dilatations and paralysis of the contractile tissue of the affected blood-vessels." Dr. Burdon Sanderson defines it to be "a succession of changes which occur in a 
living tissue when it is injured, provided that the injury is not of such degree as at once to destroy its structure and vitality."

If I were to attempt to give a definition of inflammation, I would say that it is a perverted nutrition of a living part, the effect of irritation or injury.

\section{PATIIOLOGY.}

If a non-vascular structure-the cornea, for example-be excised in the following manner, as recommended by Professor von Recklinghausen, changes which are identical with the earliest of the inflammatory process may be observed.

"The anterior chamber is first punctured so as to let out a drop of aqueous humour, which is placed on the object-glass; the cornea is then excised and placed in the drop with Descemet's membrane upperınost. The preparation thus obtained is examined without a cover-glass, in a closed chamber in which the air is saturated with moisture, so that no evaporation can take place, and consequently no alteration in the density of the liquid in which the cornea is immersed. The healthy cornea is absolutely transparent, and when it is examined under the microscope, in the manner described, no structure can be distinguished. This homogeneity, so essential to the function of the cornea, is a condition inseparable from life; if the observation is continued till the tissue begins to die, its structural elements gradually come into view-first the epithelia, then the lymphoid elements proper to the tissue, then the cornea corpuscles. The explanation of the fact is, that whereas in life the elements of which the cornea is formed affect light exactly in the same degree, their respective refractive powers are slightly altered in the act of dying.

"If a cornea is examined in the same way, which has been irritated a quarter of an hour before by the application of a point of caustic to its surface, the conjunctival epithelial layer can at once be distinguished, along with a few leucocytes, underneath and among the epithelial elements. If an hour or two has elapsed. the proper cornea corpuscles are visible. as dark stellate or spindle-shaped spots on a transparent ground. Of these some are homogeneous, and can be distinguished from the surrounding substance by a slight difference of shade. In 
others, which are finely granular, the processes or rays are subject to slight variations of contour. These amceboid movements of the rays, although very sluggish as compared with those of young protoplasm in general, are rendered much more active by subjecting the preparation to a stream of blood serum; for which purpose Professor Stricker employs the serum of the same animal which has furnished the cornea."

From the above we learn that the application of an irritant produces changes similar to those which take place in a structure when first removed from the influence of vitality, and that in so far it simulates the first post nortem changes. In a vascular structure the alterations of nutrition which result from the application of an irritant are as described in the following pages.

It must be borne in mind that the blood is composed of a solid and a fluid portion. The fluid, liquor sanguinis, composed of albumen, the constituents of the fibrine, water, and various salts in solution; the solid, of two kinds of corpuscles, the white and the red. The red corpuscles move rapidly along in the centre of the stream of blood in a small vessel; while on either side, and close to the walls of the vessel, there is a clear space called the lymph space, containing liquor sanguinis and a few white globules, which move much more slowly than the blood in the centre of the stream.

The fluid part of the blood is that chiefly concerned in nutrition. It contains the nutritive elements in solution, and is absorbed by the various textures. The capillaries consist of a single coat of protoplasm, so that the nutritive material can easily pass out of them, and nutrition is carried on entirely in the tissues which fill up the spaces between the capillaries. Every tissue has an inherent power of attracting and selecting from the blood-which, be it remembered, flows slowly at the side of the vessel-those constituents best suited for its nourishment.

From this it will be seen that the fluid part of the blood is continually transuding through the walls of its vessels, for the purpose of nourishing the tissues. A perversion of this, with a rapid transformation of the transuded material, and an altered mode of the growth of the elements of the inflamed texture, constitute the inflammatory process. When the tissue is irritated, lymph is produced in such abundance that the lymphatics are 
unable to convey it away; it accumulates in the part, forming the inflammatory exudate.

Reasoning upon the foregoing, one is tempted to conclude that inflammation is characterised by an increased nutrition of the part; but such is not the case. During all the processes of inflammation there is no such thing as an increased formation of the natural structures of the inflamed part-they are not even maintained; and it is only after the inflammation has subsided that there is an increased formation of some of the tissues, as the areolar, the bony, and the horny structures.

Whilst the inflamed structure itself suffers deterioration, the process of inflammation is, however, characterised by a production of lowly organized material.

Having now endeavoured to show what I conceive to be the essential nature of inflammation, viz., a perverted nutrition, consequent on injurious irritation of tissues, I shall call attention to the series of experiments that have been made to demonstrate the

\section{PHENOMENA OF INFL.MMMATION.}

The latest observers state that the microscopically visible changes that occur in a transparent vascular membrane of an animal, such as the web of the frog's foot, differ considerably, according to the irritant employed. A weak solution of caustic soda, dilute sulphuric acid, \&c., produce dilatation, first of the arteries, and subsequently of the capillaries, with marked acceleration of the circulation, followed by arterial contraction and eapillary anæmia. But liquor ammoniæ, and carbonate of ammonia in substance, appear always to occasion a certain degree of primary arterial contraction, which begins in one or two minutes after excitation, and is attended with retarded flow of blood through the capillaries; distension of the branches given off by the artery nearer the heart, and increased activity of circulation in the neighbourhood of the irritated part. This state of things lasts for an hour or two, and is succeeded by dilatation and acceleration. Croton oil appears to occupy an intermediate position between the first-named stimulants and ammonia; for while it always gives rise to acceleration of the flow of blood as a primary result, this change is some- 
times associated with widening, sometimes with narrowing, of the arteries.

When the mesentery of the frog is spread out and examined microscopically, the first change which is observed in the circulation, as a result of the exposure to air, consists in dilatation and increase in the length-manifested by more or less contortion-of the arteries. The dilatation begins immediately, and is preceded by no contraction. It is, however, progressive; the diameter of the artery gradually increases for ten or twelve hours, at the end of which period it is often twice as great as it was before: having thus attained its maximum, its size remains unaltered for many hours. This dilatation of the arteries is followed by a similar change in the veins; but inasmuch as there is a considerable interval between the two events, a time occurs at which the arteries, instead of being sensibly smaller than the veins which correspond to them, far exceed them in diameter.

Along with these changes, the rate of movement of the blool is also altered. At the beginning of the process the circulation is quicker than natural. Although the two changes go on at the same time, the acceleration cannot be regarded as a result of the increase of calibre; for the inevitable consequence of dilatation would be diminution, not increase, of the rate of movement, supposing the activity of the heart and the resistance opposed by the capillaries of distribution to be the same. The absence of any relation between the two is still more clearly shown by what is olserved at a later period; for whereas, on the one hand, as has been already stated, the dilatation lasts for many hours, the acceleration is confined to the first stage of the process. The rate of movement soon becomes normal, and this is shortly followed by a change in the opposite direction; so that by the time the arteries are fully dilated, the circulation is much slower than it was originally.

Many experiments have been made with the view of accounting for the dilatation and contraction of the arteries and capillary blood-vessels, and the most important results of these are-that division of a vasa motor nerve produces congestion of all the tissues to which it is distributed; that excitation by the interrupted electrical current, or by mechanical means, prodnces constriction of the minute arteries presided over by the irritated nerve, and consequent anæmia ; that excitation of a sensory nerve 
produces increased activity of the capillary circulation in the part in which the nerve originates; and finally, that all arteries manifest alternating states of contraction and dilatation-movements independent of those of the heart, and of breathing-and ceasing when the vessels are paralyzed by division of their nerves.

Reference to the effect of croton oil shows that acceleration of the flow of the blood through the vessels of the irritated part is sometimes associated with widening, sometimes with narrowing of the arteries; and in order to judge whether the acceleration in the one condition is of a similar or of an opposite nature to what it is in the other, investigators have observed both conditions simultaneously in the same part. If, for example, in the web of the frog's foot the acceleration due to electrical excitation of the central end of the sciatic nerve is of the same nature as inflammation, we should expect it to be increased by local irritation; and, conversely, the effect of irritation, if already existing, to be heightened by exciting the nerve. The very careful experiments of Dr. Riegel show that it is so. Having found that atter section of the sciatic nerve the effects of irritation were slightly retarded, but otherwise unmodified, he repeated the observation in another animal, excited the central end, and then applied croton oil to both webs. On the injured side the accelerating effect of the croton oil lasted much longer than on the other, so that at the time stasis had already set in on the sound side, the circulation was going on more briskly than natural on the injured side.-(Burdon SANderson.)

From the foregoing observations, the reader will gather that acceleration of the flow of blood through the part is the only constant fact observable, whether the cause be the reflex electrical stimulation of a nerve, or the direct irritation of the part by agents which induce either contraction or dilatation.

In all forms of inflammation of sufficient intensity, the circulation, after a varying period of excitation, becomes retarded. The current then ceases, the vessels at the same time become greatly distended, and the blood globules adherent to certain parts of the sides of the vessels, and to each other, so that their iudividual forms can no longer be distinguished. In some cases the vessels have fusiform dilatations of their whole circumference at certain points of their course, or at short intervals pouches sprout from the sides of their walls. These 
pouches are not, however, peculiar to the inflammatory state, and they have been ascribed to structural derangement of the walls of the vessels, in consequence of which they cannot present an equable resistance to the column of blood, and consequently the blood beconies extravasated into the surrounding textures.

Some have affirmed that constriction of the vessels never takes place prior to their dilatation. This, as already explained, has arieen from the kind and strength of the irritant used by these observers; whilst others state that, during contraction, the blood is seen to flow more rapidly through them, and that this is explained by the well-known fact that

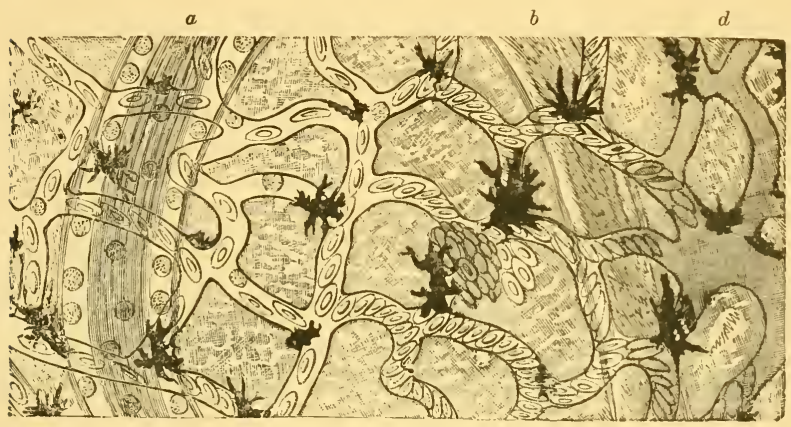

$c$

FIG. 1.-An exact copy of a portion of the web in the foot of a young frog, after a drop of strong alcohol had been placed upon it. The view exhibits a deep-seated artery and vein, somewhat out of focus ; the intermediate or capillary plexus running over them, and pigment cells of various sizes scattered over the whole. On the left of the figure the circulation is still active and natural. About the middle it is more slow, the column of blood is oscillating, and the corpuscles crowded together. On the right, congestion, followed by exudation, has taken place.

a. A deep-seated vein, partially out of focus. The current of blood is of a deeper colour, and not so rapid as that in the artery. It is running in the opposite direction. The lymph space on each side, filled with slightly yellowish blood plasma, is very apparent, containing a number of colourless corpuscles, clinging to, or slowly moving along, the sides of the vessel.

b. A deep-seated artery, out of focus, the rapid current of blood allowing nothing to be perceived but a reddish-yellow broad streak, with lighted spaces at the sides.

Opposite $c$, a laceration of a capillary vessel has produced extravasation of blood, which resembles a brown spot.

At $d$, congestion has occurred, and the blood corpuscles are apparently merged in one semi-transparent reddish mass, entirely filling the vessels. The spaces of the web between the capillaries are rendered thicker and less transparent, partly by the action of the alcohol, partly by the exulation. This latter entirely fills up the spaces, or only coats the vessels. 200 diameters. - (BENNETT.)

liquids flow more rapidly through a narrowed tube. This is not 
the case, for, as Paget observes,."As the vessels are contracting, the blood flows in them more slowly, or begins to oscillate; nay, sometimes, I think, even before the vessels begin visibly to contract, one may observe that the blood moves more slowly in them, as if this were an earlier effect of the stimulus; nor have I seen (what has been commonly described) the acceleration of the flow of blool in the contracting vessels." He again remarks- "It has been commonly said, that as the vessels contract, therefore the movement of blood becomes more rapid in them, as when a river entering a narrow course moves through it with a faster stream, and that then, as the vessels widen, so the stream becomes in the same proportion slower. But this is far from true; the stream becomes slower as the artery or vein becomes narrowed by contraction, and then, as the tube dilates, the stream grows faster, and then, without any appreciable change of size, it may become slower again, till complete stagnation ensues in, at least, some part of the blood-vessels." Now, the reader must remember that arteries are supplied with muscular or contractile power, and when they are acter upon by any influence which causes a contraction of their muscular coat, they must, of course, become narrower, inasmuch as the muscnlar cells lie in rings around them. The consequence then is, that less blood penetrates the part of the body supplied by such arteries. The more muscular an artery is, the more lasting and

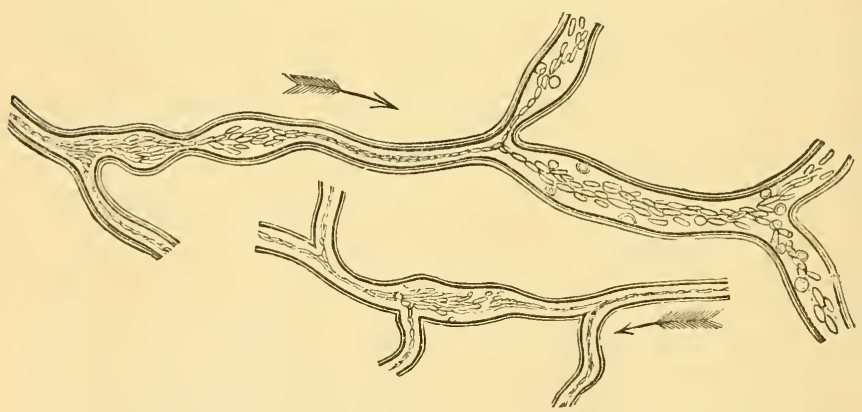

Fig. 2. - Irregular contraction of small vessels in the web of a frog's foot after the application of stimuli. - (WharTon JONEs.)

forcible is the contraction, and the greater the obstruction ex- 
perienced by the current of blood. In the smaller vessels the contraction is rapidly succeeded by dilatation, which continues for a longer or shorter period. When an artery is really in action it gives rise to no hyperæmia; on the contrary, the more active the vessel, the less blood will pass through it. The contraction of the vessels is very irregular, as will be seen from the preceding engraving (Fig. 2).

If contraction of the vessel be the first change observed, it is succeeded by dilatation, and, as already stated, the stream of blood may become faster. Then, without any change being observed in the size of the vessels, it becomes slower, oscillates, and finally completely stagnant, constituting stasis or passive congestion.

The contraction of the vessels can be produced by the application of a stimulus to the nerves supplying them, and the relaxation by cutting these nerves. From this we concludeand the fact is an important one-that relaxation of the muscular fibres of the vessels can be produced by paralysis of the nerves that supply them, or by an interruption of the nervous influence, from whatever cause it may proceed. That the capillaries have the power of contraction has been demonstrated by Professor Lister, who has shown that this contractility is dependent on fusiform (spindle-shaped) cells, which have the power of shortening themselves, and which run transversely round the delicate membrane that forms the walls of the capillaries.

The stoppage of the blood (or stasis) has now to be accounted for, and this is difficult of explanation, as no mechanical obstruction has ever been seen to present itself. If there is no obstruction, why should there be a stoppage?

Some pathologists have asserted that the vessels become mechanically plugged up by the red corpuscles adhering to the sides of them (BoErhave); by multiplication of the colourless corpuscles, and by change in the specific gravity or viscidity of the blood in a part (WHARTON JoNEs), or of the corpuscles in particular (BRUECKE); obstruction of the venous circulation ( $G$. Robinson); or adhesiveness of the blood corpuscles (Lister). These opinions have been combated by Professor Bennett, who says, "That the blood corpuscles indeed have a tendency to aggregate together is certain, and I have frequently seen what Mr. Lister has described, namely, a roll of them projecting from 
a side vessel into a large one, and oscillating in the current without separating; but that this is produced by a viscous condition of these bodies, which causes their surfaces to stick together, as he appears to think, is negatived by the fact that not unfrequently I have seen one or more of them approach rapidly a vessel in the inflamed tissue not yet obstructed, then proceed slowly, oscillate for a while, until at length, getting beyond the diseased parts, it has again darted off with the same velocity as it came. Again, it may frequently be observed, when a vessel is so full of coloured corpuscles that they can no longer be distinguished, and seem to have melted together, that, on rupturing it, these bodies, when extravasated, at once assume their original form, and arrange themselves just the same as they do coming from a healthy structure. I cannot suppose, therefore, that the cause of stasis is connected with adhesiveness of the blood corpuscles; indeed, many of the valuable observations of Mr. Lister himself only confirm what I have long maintained, namely, that they simply are drawn together and brought more closely into contact by some external force, which is excited by irritation of the surrounding tissue."

Later experiments have to some extent confirmed the conclusions of Bennett. Dr. A. Ryneck has made some experiments in the Physiological Laboratory at Grätz, upon the production of stasis, and has shown that all its phenomena can be produced by irritation in the webs of frogs, in which milk or defibrinated blood of mammalia has been substituted for the circulating fluid. If a web so treated be touched with a rod moistened with ammonia, the phenomena of stasis occur in the irritated part; the capillaries become crowded with milk globules, exhibiting the appearance of grev cords. When defibrinated blood is used, the results are even more striking, for in this case the choked vessels soon exhibit in every respect the same appearance as in ordinary inflammation.

These results seem to make it perfectly clear that the local changes which lead to the production of stasis must have their seat either in the walls of the vessels, or in the tissues which immediately surround them. To determine this more precisely, Dr. Ryneck varied this experiment by filling the vessels with an indifferent liquid, such as a solution of common salt of proper strength, so as to remove the blood; then subjecting their 
internal surface for a few moments to an agent, which, by virtue of its chemical action, might be expected to modify or destroy its vitality, such as solution of chromic acid, chloride of gold, or sulphate of copper. The results were decisive: no stasis was produced by irritation in webs which had been thus treated.

It has been observed that there is an apparent increase of the white corpuscles in the inflamed part, and a remarkable disposition in them to adhere to the walls of the vessel; but as no pores are visible in the capillaries under the highest magnifying powers, the fact that the white corpuscles passed through the vessels into the tissues, which was pointed out by Dr. Addison, and which has now been proved, could not be accepted; but in 1846 the statements of Dr. Addison were confirmed by Dr. Augustus Waller, who says (Philosophical Magazine, vol. xxix., p. 397,1846$)$, "In some instances the manner in which the corpuscle escaped from the interior of the tube could be distinctly followed; that part of the tube in contact with the external side of the corpuscle gradually disappeared, and at nearly the same time might be seen the formation of a distinct line of demarcation between the inner segment of the corpuscle and the fluid parts of the blood in contact with it. Any slight agitation then was capable of disengaging the corpuscle from the vessel to which it was now external." The escape of the corpuscle was supposed by Dr. Waller to be due to an exudate being given off from the corpuscle possessing some solvent power over the vessel, or that the solution of the vessel took place in virtue of some of those molecular actions which arise from the contact of two bodies; actions which are known as exerting such extensive influence in digestion, and referable to what is termed the catalytic power. But it is now proved that the capillaries consist of protoplasm, hence the penetration of their contractile walls by the amøboid corpuscles, and the subsequent closure of the openings when the passage is completed, can be readily understood.

In order to do this it must be further borne in mind, as pointed out by Prof. Cohnheim, that a white corpuscle-leucocyte, sarcophyte-is not a cell in the sense in which the term was formerly used, but is a mass of contractile material endowed with the faculty of movement. This movement, from its similarity to that possessed by the amœbæ, has been termed 
amoboid, and is as follows:-The mass constantly clianges its form. But as this goes on in all parts of the hyaline substance, of which it consists, simultaneously, the only way in which it can be understood is by confining the attention to one point at a time; it will then be seen that each act of movement begins by the budding of a ray, or process of contractile substance in a centrifugal direction. What next happens varies in different cases. Sometimes the projection subsides just in the same way as it was formed; at others, the finely granular fluid, which occupies the more central parts of the corpuscle, streams into the offshoot, gradually widening it out until it grows into a mass greater than the remainder, which it finally draws into itself. It is evident that the process last described must always be attended with locomotion, for each time it is repeated the whole mass rallies round a new centre, the position of which corresponds to the extremity of the offshoot.

As already stated, these white corpuscles accumulate in large numbers in the vessels of the inflamed part in close contact with the walls. They then sink into the substance of the walls, and pass through them into the surrounding tissue. During this process it is seen that the corpuscle pierces the walls of the vessels by means of prongs of a delicate homogeneous material, similar at first to a shining thread. Some of these prongs project, and as a prong increases in length it thickens at its base; the substance of the corpuscle now tends towards it, becoming smaller as the prong gets larger; in fact, the contents of the corpuscle are drawn into the substance of the prong, which becomes rounded off' at its tip, and finally assumes the contour of the corpuscle. Having escaped from the vessels into the surrounding tissue, the corpuscles continue their active movements, and increase rapidly in number.

Virchow describes two forms of inflammation, namely-(1st.) The parcnchymatous inflammation, where the process runs its course in the interior of the tissue elements (c.g., connective tissue cells, or germ masses, hepatic and cartilage cells), without one being able to detect the presence of any free fluid which has escaped from the blood; and ( $2 d$.) The secretory (exudative) inflammation of superficial tissue elements, where an increased escape of fluid takes place from the blood, and conveys the new products of growth and altered secretion with it to the surface. 
Virchow is also of opinion that there is no inflammatory exudation at all, in the sense in which it has usually been assumed to exist, but that the exudation is essentially composed of the material which has been generated in the inflamed tissue itself, through the change in its condition, and of the transuded fluid derived from the vessels. If a part possesses a great number of vessels, particularly if they are superficial, it will be able to furnish an exudation. If this is not the case, there will be no exudation, but the whole process will be limited to the occurrence in the real substance of the tissue of the special changes which have been induced by the inflammatory stimulus; and he conclurles by stating that every parenchymatous inflammation has, from its outset, a tendency to alter the histological and functional character of an organ. Every inflammation with free exudation in general affords a certain degree of relief to the part; it conveys away from it a great mass of the noxious matters with which it is clogged, and the part therefore appears comparatively to suffer much less than that which is the seat of a parenchymatous disease.

Of the former (the parenchymatous) he gives an example in

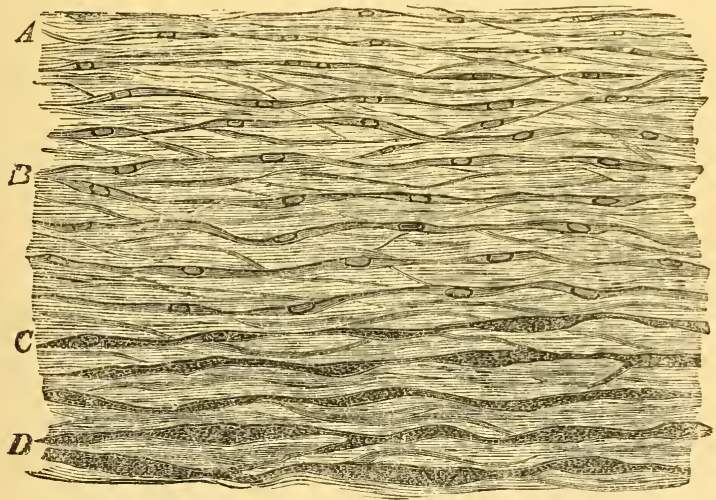

FIg. 3.-Parenchymatous Keratitis. At $A$ the cornea corpuscles are seen in a nearly normal condition, at $B$ enlarged, at $C$ and $D$ still more enlarged, and at the same time clouded. 350 diameters.-(VIRchow.)

inflammation of the cornea; and of the latter form of inflammation we have an example as it occurs in mucous membranes, 
where generally no fibrine is formed, but mucus, which does not exist in the blood. An inflamed mucous membrane produces large masses of epithelium in a short time; they are merely products of the membrane, which is not infiltrated with epithelium or mucus coming from the blood, but the peculiar product of the membrane, and is conveyed to the surface by means of the fluid transuding from the blood. In the same manner, fibrine, instead of being a real exudate from the vessels, is a local product of the tissues on and in which it is found, and is conveyed to the surface, as in pleuritis, in the same way as the mucus of the mucous membrane. We can thus explain that the fibrinous condition of the blood observer in inflammations is a result of the absorption of the increased quantity of fibrine produced by the local metamorphosis of transuded serum.

We may conclude that the effect of inflammation on all living tissues is a modification of the action of individual cells, resulting from some alteration in the properties of the walls of the capillaries nearest to the seat of irritation or injury. Most of these modifications are accompanied by transudation of liquor sanguinis-exudative inflammations; whilst in others, and these are the most destructive, the process is limited to textural changes in the substance of the part-parenchymatous inflainmation. The latter may, however, in some instances be changed into the former, by increasing the stimulus, or by elevating the vitality of the part; for, as already observed, the first change noticeable in a non-vascular structure when irritated is similar to that seen in the same structure when removed from the influences of vital action, i.e., exciserl, and that those processes, peculiar to the inflammatory state, are the natural changes occurring in an injured living structure. Now, if the living structure be much debilitated by any cause previous to the occurrence of the irritation, the natural response will be feeble, delayed, or the part may even die without presenting any signs of exudative reaction.

\section{CAUSES OF INFLAMMATION.}

It will be sufficient here merely to notice the remote causes of inflammation. These may be divided into predisposing 
and exciting causes:-1st. Predisposing causes are influences not of themselves essentially irritant, yet often co-operate with others in inducing the inflammatory process. They may be summarised as follows-debility either of an organ or of the whole body arising from old age; previous disease or a natural weakness; hereditary taint or predisposition; obstruction of the blood-vessels ; plethora; climatic influences; dietetic errors ; insufficient ventilation, and ill-treatment of all kinds.

2d. Exciting or determining Causes.-These are agents which, if powerful, will of themselves be sufficient to determine the inflammatory process even in healthy tissues, but which will do so with more certainty in parts predisposed. They may be arranged as follows:-Direct violence; the application of irritants; exposure to heat and cold; the presence of foreign bodies, or of parts deprived of life; retained concretions or excretions; abnormal conditions of the blood, as in rheumatism and anæmia, or when containing some irritating ingredient, morbid or specific virus, or poisonous drug. I think Dr. Burdon Sanderson simplifies the causes and origin of inflammation very much. He says - "With reference to their origin, all inflammations may be comprised in two classes-extriusic and intrinsic. Of these two terms, the former is applicable to all those cases in which an injury, either sustained by the affected part or inflicted elsewhere, is the obvious cause of the morbid process ; the latter to those inflammations which, from the concealment of their cause, are commonly called idiopathic. If, however, we desire to speak accurately, we must discard this word altogether; for there is no case in which it can be reasonably doubted that an injury must have preceded the earliest sign of local disorder, however little we may know either of the nature of the agent or of the mode of its action. We might advantageously substitute for idiopathic either of the words intrinsic or secondary; but inasmuch as there is no channel by which an agent from within, i.e., from some other part of the body, could penetrate into a tissue, excepting by the blood-vessels or lymphatics, we are entitled to use the only word which fully expresses this view of the mode of introduction of the material cause, and to designate all so-called idiopathic inflammations infective.

"From what has been said it may be readily understood that the primary inflammations naturally affect those parts princi- 
pally which are exposed to external influences, while those of the other class occur by preference in parts and organs to which there is no access excepting through the circulation. These distinctions, however, are not constant, for there are many instances in which secondary inflammations affect external parts, and many others in which internal organs are the seat of primary inflammations, as, for example, when nepliritis arises from exposure to cold. Much more important distinctions, however, may be based on a comparison of the structural changes which the two processes determine in the tissues affected; or, in other words, on their pathological anatomy. In making this comparison, there is one important principle to be borne in mind: In all inflammations, the form of the lesion is dependent on that of the area of influence of the injury. Thus, in those cases of primary inflammation in which it may be supposed that an impression received by afferent nerves distributed to mucous or cutaneous surfaces, is reflected to internal organs (as in the case of nephritis from cold, already referred to), the area of influence of the injury is wide enough to comprise whole organs, and the resulting lesions are of corresponding extent. In the strictly local inflammations, the correspondence in form between cause and effect is, of course, closer and more obvious, the area of a traumatic inflammation being larger than that of the injury which produces it, but of exactly similar form. As regards infective inflammations, the correspondence is not so plain, but the consideration of their pathological anatomy is sufficient to satisfy us that it is equally complete. It is the anatomical character of all infective inflammations that the lesions to which they give rise are disseminated rather than diffused. Particles of matter, of the nature of which we can assert nothing, excepting that they are of extreme minuteness, are conveyed from a primarily inflamed part to other parts previously healthy, and become foci of infective induration or suppuration (miliary tubercles, pyæmic abscesses), each of which is the product-if one may be allowed the expression—of a single seed."

VARIETIES OF INFLAMMATION DUE TO CAUSATION.

And to quote from the same author on this subject:"Although if we be careful to distinguish what is essential to 
the process of inflammation, viz., the altered state of the vessels, from the phenomena which accompany it, and the textural germination which it produces, its characters will appear to us to present very slight variation, yet the visible results by which it manifests itself differ widely in different cases. It is therefore necessary, in order to complete the present subject, to consider in what degree these differences correspond to differences in the causes which produce them.

"Vesication.-If a hot iron is applied to the skin at a sufficient temperature, it at once destroys its vitality. If the temperature be a little below that which is necessary to produce this resuit, the blood contained in the vessels coagulates, and the tissue eventually dies. At a still lower temperature the skin retains its vitality, but blisters are formed at or around the injured part.

"If the mesentery of a guinea-pig is touched with a heated surface, and the effect observed under the microscope, it is found that stasis is produced which is co-extensive with the surface of contact. It is tolerably certain that in like manner, in vesication of the skin by heat, the circulation of the heated part is abruptly brought to a standstill. As, outside of the area of stagnation, it goes on at first with unabated then with increased vigour, while the walls of the capillaries are probably acted upon by the heat in such a manner as to render them more permeable, we can readily understand how it happens that liquor sanguinis is exuded more rapidly and more abundantly than in ordinary inflammations. From the researches of Dr. Samuel of Königsberg it seems probable that the effects of liquid vesicants agree with those of heat in all the respects which have been referred to; so that the peculiarity of the mode of action of vesicant agents in general, would seem to lie in its suddenness, and in the faculty which they possess of at once producing those changes in the capillary wall which in ordinary inflammation require a longer time and a more gradual process for their production. In this way the exudation of liquor sanguinis, instead of being deferred until the slowing of the circulation has commenced, begins immediately, and, favoured by the primary arterial afflux, and the increased intravascular pressure consequent on the sudden capillary obstruction, is so abundant that the liquid collects in blisters.

"Relation between inflammation and the reparative process- 
When the local injury is so intense as to destroy the vitality of the affected part at once, that part becomes surrounded with a zone of inflamed tissue, from which it eventually separates, leaving behind it a granulating surface. To understand this process of demarcation and separation, it is in the first place to be borne in mind that the exuded liquid contains the fibrine-producing elements of the blood, and that contact with dead substance at once determines coagulation of all such fibrinogenous liquids. Accordingly, the first step in the process of reparative separation is the formation, in contact with the dead part, of a more or less solid stratum of fibrine, in which stratum the production of new capillaries and granulation tissue commences."

\section{LOCAL SYMPTOMS OF INFLAMMATION.}

Redness.-In the lower animals this symptom can only be seen in the white parts of the body, such as the white skin of the heels of some horses, and upon the visible mucous membranes. It arises from an increased quantity of blood in the part, both in the capillaries and in the large vessels; and when the congestion is excessive, particularly if it be due to some mechanical cause or obstruction, the redness is increased by the passage of the red corpuscles through the walls of the capillaries without rupture. The corpuscles, in passing through the vessel, become constricted in their centre, so as to assume an hour-glass shape. This passage of the red corpuscles can be seen in the frog's web after ligature of the femoral vein. In some cases the redness may be caused by extravasation of blood, but generally it depends on engorgement. The redness of inflammation is more or less vivid; deepest in the centre, gradually shading off towards the edges of the inflamed part, and partly removable by pressure; but if extravasation be present, its margin will be more defined, and the colour is not removable by pressure.

The aspect of the redness may differ according to various circumstances; if the capillary networks of the part be uniformly distended, the injection will appear as an uniform deep blush; if: on the other hand, they are moulded in the form of villi or folds of mucous membrane, the surface will have the appearance of a pile of red velvet. In fibrous structures the redness has a streaky appearance. The redness does not depend upon the 
formation of new blood-vessels, a process which does not take place till the inflammation is much advanced. Staining of the tissues with transuded hæmatine may occur soon after stasis has been established.

The veterinary practitioner should always carefully distinguish between genuine inflammatory redness and that which closely resembles it in the dead body, namely, hypostatic redness, which depends on mechanical causes or the mode in which death has been produced.

Redness existing only in a depending part of the body, such as the side upon which an animal has lain since its death, without thickening of the part, must never be looked upon as evidence of the inflammatory condition.

The presence or absence of redness is not of itself a proof of the presence or absence of inflammation. Redness exists without inflammation: thus we find the visible mucous membranes red and injected in many diseases, but no one for a moment supposes that these membranes are inflamed. On the other hand, absence of redness is no proof that inflammation does not exist, for some inflammations, as those of the cornea, of the arachnoid membrane, and of articular cartilage, are atteuded with no redness, but rather with opacity, and it is only in conjunction with other indications that redness can be regarded as a symptom of inflammation.

Pain.-The pain of inflammation varies much in degree and in kind, according to its cause, intensity, and seat. The pain of laminitis, of punctured foot, of open joint, or of inflammation of any unyielding fibrous or bony texture, amounts very often to extreme agony. The pain of inflamed serous membrane, more especially of inflamed pleura, is of a sharp, darting kind, giving rise to colicky symptoms. In traumatic peritonitis, on the contrary, although the pain may be excessive, the animal does not always exhibit it, owing to the prostration which is present. The pain of inflamed mucous membrane is dull, or simply an uneasiness not amounting to actual pain. But pain is not a constant symptom of inflammation, and of inflammation without it the following may be enumerated:- Insidious and indolent forms of scrofulous inflammation, especially in horned cattle, in which extensive disorganizations are often produced without the animal ever having manifested any signs of pain; inflammation of a para- 
lyzed part, with sloughing, as in the posterior extremities, after parturient apoplexy; or that inflammation occurring in the foot, pastern, and fetlock of the horse, after neurotomy has been performed; typhoid inflammation of the lungs, where little indi-

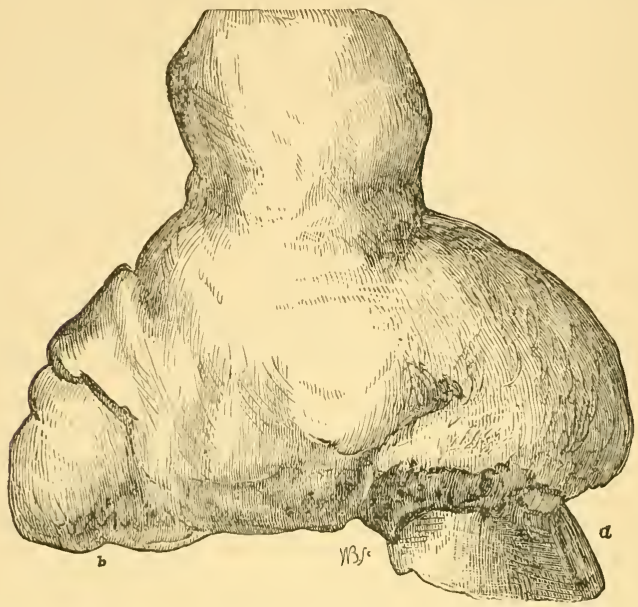

FIG. 4 shows the immensely enlarged condition of the coronet and pastern, from inflammation and gelatinous degeneration, succeeding neurotomy. $a$, Toe of foot ; $b$, Fetlock pad.

cation has been shown during life, and it must also be presumed that the formation of pulmonary abscesses in glanders is unattended by pain. We therefore conclude that the absence of pain is no indication of the absence of inflammation, and that the presence of pain is not impossible without inflammation. Of this we have many examples, as in spasmodic colic, where pain is intense; in tetanus, and in cramp of the voluntary muscles. One peculiar case came under my immediate knowledge, where pain was present to a most exquisite degree, without either spasm or inflammation, and where the external iliac artery of the near (left) side was plugged by a fibrinous clot (embolus). The animal while at rest exhibited nothing unusual, but if put to work in the carriage he would not proceed 500 yards without the limb becoming icy cold; he would 
then groan, break out in sweats upon various parts of the skin, would attempt to lie down; indeed, when taken to his stable, he would lie down, roll, and paw, as if suffering from spasmodic colic. This occurred again and again; and upon the animal being killed, the condition of the artery was discovered.

The sudden cessation of pain in violent inflammation is much to be dreaded, as it gives the practitioner good reason to suspect that the vitality of the part has been lost, from the inflammation having gone on to gangrene.

The situation of pain is not always the seat of the inflammatory action, as in inflammation of the liver the pain is sometimes in the off (right) shoulder, as exhibited by distinct shoulder lameness. This is termed sympathetic pain, and is the only example within my knowledge where this sympathetic or refiected pain can be demonstrated, although the practitioner may surmise that this occurs in inflammation of other organs. In periodic ophthalmia it may be seen to some extent.

The Cause of Pain.-This has been ascribed to compression of the nerves of the part by congestion of the vessels and effusion; an exaltation of nervous function; a painful stretching of the nerves, arising from distension of their small nutritious vessels; or to impression produced on the nervi vasorum by the slight dilatation and elongation of the arteries during each impulse of the blood.

In general, the intensity of the pain depends upon the firmness and inelasticity of the part affected. Thus the pain of laminitis is of a most excruciating character, from the inflamed tissues being confined within the horny foot. The pain of open joint is also of this nature, from the firmness and hardness of the tissues involved; namely, bone, ligaments, synovial membrane, \&c. Such structures as these, along with tendons and faschiæ, possessing little sensibility during health, in disease become extremely sensitive, and the pain in them is often of an agonizing character. Dr. Lionel Beale has demonstrated that in textures which in health exhibit but slight sensitiveness, and become eminently so when inflamed, there is a very great increase in the germinal matter which they contain, and that this often proceeds to such an extent during inflammation that the ramifications of the nerves appear as lines of masses of germinal matter; so that in tissues 
which thus become highly sensitive during inflammation the feeling of pain must be due to the increase of germinal matter of the nerves, as well as that of other tissues. Muscuiar and other softer structures, though endowed with much sensitiveness during health, are not nearly so painful in inflammation. This arises from their yielding nature, giving way and allowing free swelling of the part, which in most cases seems to relieve pain. The veterinarian has a good example of this in lymphangitis, the pain and lameness of which seem to subside as the swelling of the parts appears. In the treatment this should be borne in mind, as remedies that promote swelling (fomentations) afford very marked relief.

Swelling.-The swelling of inflammation depends upon-1st. The congestion of the vessels; and $2 d$. The exudation of liquor sanguinis and the diapedesis of corpuscles into the tissues.

Swelling of an external inflamed part may be looked uponexcept in the case of the articulations, where it is, when very great, indicative of ulceration-as a favourable sign, its occurrence often affording relief. But when it occurs in the organs essential to life, such as the parenchyma of the lungs, or upon the glottis, its presence may soon put an end to the life of the animal. Swelling is not always an indication of inflammatory action; the swellings of dropsy, anasarca, and of purpura and scarlatina, are very different from those of inflammation. The swelling of purpura is very characteristic, terminating abruptly, superiorly, as if a cord had been drawn around the limb; the swelling of anasarca will be found to be most bulky at its most depending part. Tumours are also examples of swelling without inflammation; cartilage, which is non-vascular, swells by its cells taking up more matter, and assuming the form of large round corpuscles. In proportion as they take up this matter, they enlarge in all directions, often forming spots or protuberances on the surface of the articulation, as may be seen in navicular lameness.

Heat.-The temperature of the inflamed part seems to be considerably increased, affecting the sensations of the observer as well as of the sufferer; hence the name "inflammatio," a burning. But this increase of heat is not so decided as one would imagine, and the experiments of Hunter go to prove that the difference, in most cases, is not more than one degree; and, 
according to some observers, it seems doubtful whether the heat of the inflamed part is even higher than that of the other parts of the body. The greatest rise in the temperature is found where the inflamed part is far removed from the centre of circulation, and where the natural temperature is several degrees below that of the blood at the heart, as in the feet of our patients; and it is also found that in parts remote from the centre of circulation the heat is most distressing.

The sense of heat which the patient experiences must be partly due to the increased sensibility of the inflamed part, and also to the fact that the functions of the sensory nerves are increased and perverted. The greatest degree of heat has been found to exist in rinderpest, and this was not due to any inflammation of the part, but to the rapidity of the textural changes that took place in that plague. In this disease the highest record made by me was $109^{\circ}$. The highest temperature recorded by writers on human medicine is $110 \frac{3}{4}^{\circ}$; this was in tetanus, which is not an inflammatory disease.

Some late experiments upon the production of increased heat in a part inflamed may be interesting to the reader.

The experiments of Mr. Simon, corroborated by those of M. O. Weber, go to prove that inflammation does actually cause a local production of heat, for the application of a thermoelectric needle showed (1st.) that the blood passing to an inflamed part is less warm than that part itself; (2d.) that the venous blood returning from an inflamed part is warmer than the arterial blood supplying it, though less warm than the focus of inflammation; and ( $3 d$.) that the venous blood returning from an inflamed limb is warmer than the corresponding current on the opposite side of the body.

The subject has been taken up by MM. Jacobson and Bernhardt; and the results of their investigations are opposed to the above, and corroborative of those of Hunter. They excited pleurisy of one side, or general inflammation of the peritoneal cavity in rabbits, by injecting dilute acetic acid or caustic ammonia; and when inflammation was established, they compared the temperature of the inflamed serous sacs with that of others, or with the blood in the right or left ventricles of the heart. In a preliminary set of experiments they discovered that great exactness was obtainable in ascertaining the temperature of 
internal parts by the insertion of the thermo-electric needle, and that, as a general rule, the pleural cavities were from about one-tenth to one-fifth of a degree per cent. cooler than the peritoneal cavity, and from one-fifth to one-half of a degree cooler than the left side of the heart. It was, therefore, clear that, if a temperature of more than one-half a degree per cent. was present after inflammation had been set up, it could not be due to the blood current, but must have arisen from some local and independent source of heat. But the results of experiments showed that, so far from any augmentation, there was a distinct depression of temperature on the inflamed side, amounting in one case, when the pleural sac was filled with fibrinous fluid, to as much as one-half of a degree per cent. less than that of the peritoneum, and about one-third of a degree below that of the opposite pleura, and more than one-half of a degree cooler than that of the left heart. Again, in peritonitis the temperature was less than that of the left heart by as much as two-thirds of a degree per cent, and in one case it was $1.1^{\circ}$ per cent. (equal to $2^{\circ}$ Fahr.) below that of the blood in the left ventricle.

To settle this uncertainty, experiments have been instituted, which have led to the following conclusions:-1st. That arterial blood supplied to an inflamed limb is less warm than the seat of inflammation; $2 d$. That the venous blood returning from an inflamed limb, though less warm than the focus of inflammation, is warmer than the arterial blood supplied to it; and $3 d$. That the venous blood returning from an inflamed limb is warmer than the corresponding current on the opposite side of the body. On the evidence of these experiments, which were made on three dogs with compound fracture of the leg, Mr. Simon ventures to say that the inflamed part is no mere recipient of heat, but is itself actively calorific; and that in proportion to its heated venous outflow the temperature of the common mass of circulating blood is necessarily raised; and that the local disorder represents an influence which tends to diffusion throughout the body, and thus be an important cause of inflammatory fever.

Impairment of Funetion.-Perversion or impairment of the functional properties of a part uuder inflammation is a very common, indeed almost a constant, accompaniment of its various stayes. In the first stage it may be increased, as may be 
witnessed in the delirium of the first stage of phrenitis, and almost suspended during the comatose or later stage. In enteritis it is often found that the bowels will act excitedly, small quantities of fæces being passed very frequently at the commencement of that disease, but at a later stage their action will be entirely suspended.

In inflammation of the muscles, again, we find that there is almost total loss of their proper contractile power, and that what remains of it is brought into action with difficulty and pain; showing that though their functional activity is lost, their sensibility is highly exalted.

\section{CHRONIC INFLAMMATION.}

Inflammations, according to the severity of their causes, and duration of their action, manifest certain alterations in their progress and termination, and the terms acute, sub-acute, and chronic have reference to the periods of the duration of the inflammation, or the rapidity or slowness of its course.

In chronic inflammation the action of the irritant, though less immediate and severe, is much more prolonged, and has a greater tendency to excite the formation of an abundance of tissue, which, though less highly organized than the normal, yet is not so prone to undergo those retrogressive changes which characterise the exudates of acute inflammation.

Whilst the more highly vascular organs are by no means exempt from chronic inflammation, it is found that the less or non-vascular tissues more commonly undergo those changes which characterise it; changes due either to the nature of the irritant, the vital tone of the tissue irritated, or to the strength of the cause being insufficient to excite the more acute and rapid inflammation.

Chronic inflammation may run its course independently of the acute; it may also supervene or precede it; but generally the milder it is at the outset, and the more prolonged in its course, the more highly organized and more permanent will be its products. From this it will be gathered that chronic inflammation - unimportant perhaps when not located in vital organsbecomes exceedingly grave when involving organs or tissues essential to life, inasmuch as the inflammatory new formation 
may so press upon or even destroy the structure of an organ as to render it incapable of performing its function.

Of this we have examples in consolidation of the lungs, cirrhosis of the liver, \&c.

There are some chronic inflammations which are essentially destructive to the tissue which they involve, and in which there is no tendency to the formation of new tissue in the inflamed part itself-as, for example, in inflammation of articular cartilage; but even in these, with but few exceptions, there is an increased formation of fibrous, and even more highly organized tissue, i.e., bone, in close proximity to the focus of inflammation.

\section{TERMINATIONS OF INFLAMMATION.}

Many pathologists are of opinion that there are great objections to the expression used here, and that what are regarded as terminations are conditions co-existent with the various stages of inflammation. Some have even gone so far as to say that resolution is not a termination; but we must at least retain that term, as it seems the only termination. The words results, or events, are now used instead of terminations; and, retaining resolution as a termination, these are-Effusion of Serum, Exudation of Coagulable Lymph, Suppuration, Ulceration, Gangrene, and Sphacelus.

\section{RESOLUTION.}

This is said to occur when the symptoms gradually subside, and when there is a cessation of the transudation of the fluid part of the blood from the vessels; or, in other words, a restoration of the nutritive functions of the part into their normal condition, and the absorption of the substance contained in the tissue. This subsidence of inflammation may be sudden, when it is called delitescence; or it may be gradual, when it is called resolution; or it may be sudden, and the inflammation may as suddenly appear in another part of the body, and then metastasis is said to have taken place.

Resolution occurs in two ways:-1st. The sudden cessation of the irritation, delitescence, and removal of the exuded materials before coagulation or solidification has been effected. In this process the veins and lymphatics take up the exurlate in its 
normal fluid condition, as it occurs in ordinary serum when blood is drawn from the body before the fibrine has coagulated.

$2 d$. The exudation is more complete, the exuded lymph is consolidated, or even transformed into a low form of fibrous tissue, surrounded by and soaked in much serosity. At this point its organization is arrested, the contents of the cells converted into fatty granules, the cell walls break down, the granules escape into the surrounding fluid (serosity), forming a fluid resembling chyle-called pathological milk by Virchow. In this condition it is absorbed into the circulation, and after undergoing other transformations whilst mixed with the blood-converted into urea, hippurates, ammonia, carbonic acid, \&c.-is finally ejected from the system by the excretory organs, leaving the inflamed part in its original condition, or perhaps slightly altered.

The termination which has been called "Adhesion" is another method by which the exudate is disposed of, and presents a more complete and higher organization of the lymph than the former. In this process the cells, instead of undergoing fatty degeneration, become developed into a form of fibrous tissue, which in the course of time becomes vascular by the formation of new blood-vessels within its substance; and whilst organization is going on in the tissue, the surrounding serum is absorbed, leaving the new formation as part of the economy, remaining so, as in the adhesions of pleuritis, \&c., throughout the animal's life.

The formation of the new blood-vessels is very interesting, and is supposed to be effected as follows:-Coincident with the structural development of the cells and intermediate substance into connective tissue, new blood-vessels are formed by outgrowths from the walls of the original vessels of the surrounding parts. These outgrowths first appear as slight pouches on several original vessels ; these pouches or dilatations first present themselves on one point of a vessel, then on another, as if its walls yielded a little; they gradually extend themselves as blind canals from the original vessels, directing their course towards the edge or surface of the new material, and are crnwded with blood globules, which are pushed into them from the main stream. Still extending, they converge and meet; the partition wall that is at first formed by the meeting of their closed ends clears away, and a perfect arched tube is thus made, 
through which the blood, diverging from the main stream, ant theo rejoining it, may be continuously propelled: or a delicate threadlike process shoots from a vessel, and becomes connected with corresponding shoots from other vessels. These fine processes widen out, become tubular, and their cavities form canals continuous with those of the parent vessels. When the new blood-vessel has begun to project it sometimes bursts; the bloodglobules that issue from the ruptured pouch collect in an uncertain mass within the tissue like a mere ecchymosis, but before long they manifest a definite direction; and the cluster bends towards the line in which the new blood-vessel might have formed, and opens into a portion of the arch, or into some adjacent vessel. For this mode of formation from vessels the name of channelling seems appropriate, for it appears certain that the blood-globules here make their way in the parenchyma of the tissue unconfined by membranous walls. The new vessels possess a very simple structure, their walls being a thin membrane with imbedded nuclei.-(Virchow, Paget.)

Effusion differs from the so-called exudation of lymph in the fact that it occurs from the surface of serous membranes, blisters on the skin, or in a very loose areolar tissue, where the process of perverted nutrition is of the most rapid kind.

The fluid found in serous cavities and in serous abscesses partakes of the general characters of the serum of the blood slightly modified, being of a higher specific gravity and containing more albumen, with more or less fibrine. Many specimens of the effusion found in pleuritis, for example, contain fibrine in an imperfectly developed condition-fibrine which does not coagulate until it is exposed to the atmosphere.

Now, if the fibrine were transuded through the walls of the vessels, it would in all cases coagulate within the body; but when, owing to the inflammation being near or upon the surface, or in very loose areolar tissue, the transformation of the transuded material takes place rapidly, and hence imperfectly-that is to say, when the fluid sweats through the walls of the cells in an imperfectly developed condition, or, when occurring upon a serous membrane (it is also the case in a mucous membrane), the epithelial scales are rapidly thrown off, along with the fluid they secrete.

lu many effusions we find completely developed fibrine 
floating in the liquid, and often forming bands of adhesion between the opposing surfaces of the inflamed membrane.

In every instance of undoubted pleuritis, effusion of serum takes place; and it is ridiculous to hear some speak of the differences that are observable in the symptoms when this occurs, and of the great danger to be apprehended. The effusion is part and parcel of the process of inflammation from almost the outset, and the only danger is owing either to the constitution of the animal being bad, or the treatment irrational, or the attack of more than ordinary severity, that the effusion may become excessive, and cause death by suffocation.

Serous effusion is sometimes the result of mechanical congestion, as witnessed in cedema of the extremities, in ascites, from disease of the liver, spleen, or heart, but this is totally unconnected with inflammation; in such instances the fluid is clear, and generally contains but little fibrine, or any substance that coagulates upon exposure to the air.

The formation of a non coagulating effusion in the various cavities is very propitious; for so long as it remains liquid, absorption may still ensue without its undergoing any ulterior changes when the inflammation subsides. The subsidence of the inflammatory action, however, is necessary, for, from what has been already said, there is impairment of function, and alsorption does not readily take place.

It has been shown by Beale, Simon, and others, that there are two essential characteristics of inflammatory effusion:1st. It contains certain ingredients in larger proportion than that in which they exist in the blood-excess of chloride of sodium, of phosphates, and albumen; and $2 d$., organic germs find in it $\mathbf{a}$ suitable place for growth.

\section{BLOOD EFFUSION, OR EXTRAVASATION.}

This occurs chiefly from rupture of new blood-vessels developed in the newly-formed material which has just become vascular.-(Rokitansky.) But we find it when there has been no time for the formation of such new vessels, namely, at the very commencement of an acute inflammation of the substance of the lungs; but this is rare, and betokens either a very bad constitution, or that the inflammation is due to a very malignant 
epizootic influence, or that it arises from that condition of blood observable in purpura. These hæmorrhages must not, however, be confounded with blood-staining of the part through the oozing of some of the colouring matter of the blood. The natural colour of the inflammatory new formations is greyish or yellowish-white (straw colour very often); and even when they contain blood-vessels, this opacity prevents their having any uniform tinge of redness when they are recent. (ExampleSurface of lung in pleuro-pneumonia.) When they present a tinge of redness, it is either because of hæmorrhage into them, or because they have imbibed the dissolved colouring matter of the blood (hæmatodine); and when this imbibition happens during life, or soon after death, it is important, as indicating an ill-conditioned state of the blood, in which the colouring matter of the corpuscles becomes unnaturally soluble. 


\section{CHAPTER II.}

TERMINATIONS OF INFLAMMATION-continued.

SUPPURATION-ACUTE ABSCESS-DIFFUSE SUPPURATION-SUPERFICIAL SUPPURATION-COMPARISON BETWEEN MUCUS, EPITHELIUM, AND PUS-PYOGENIC FEVER, OR STRANGLES-PYEMIA-RE-ABSORPTION OF PUS-FORMATION OF SINUSES-ULCERATION-VARIETIES AND TREATMENT OF ULCERS-MORTIFICATION.

The formation of pus is termed suppuraition, and it takes place in three distinct ways-(1.) circumseribed; (2.) diffused; and (3.) superficial suppurations.

As an example of the first or circumscribed form, I shall take what is called an abscess or phlegmon, in which the suppuration is enclosed in a cavity (as in the abscess of strangles) whose walls are composed of areolar tissue. In the first stage, the cells of the connective or areolar tissue are charged with the material (lympli) formed by them during the first stage of the inflammation; there is an enlargement of the cells, their nuclei divide, and for some time multiply excessively. This is soon followed by division of the cells themselves, and round about the irritated or inflamed parts, where single cells formerly lay, pairs or groups of cells are subsequently found, out of which a new formation (connective tissue) grows. In the interior of this growth, where the cells were at an early period abundantly filled with nuclei, numberless little cells soon appear, which at first still preserve the direction and forms of the previous connective tissue corpuscles.

These accumulations of little cells occur somewhat later, as diffuse infiltrations of roundish masses encircled by the inter- 
mediate tissue, which continually liquefies and becomes more

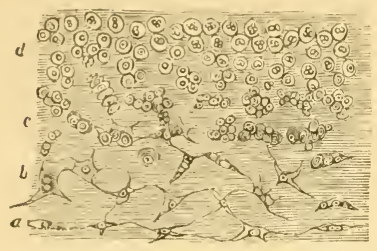

Fig. 5.-Puruient granulations from the subcutaneous tissue of a rabbit round about a ligature. a. Connective tissue corpuscles. $b$. Enlargement of the corpuscles, with division of the nuclei. $c$. Division of the cells (granulations). $d$. levelopment of the pus corpuscles. (VIrchow.)

process, new connective tissue

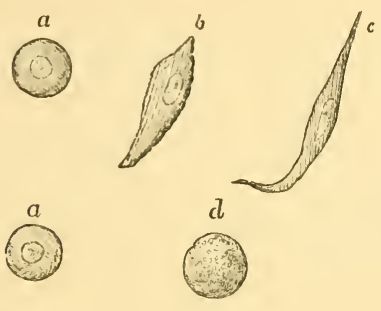

Examples of Cells.

Fic. 6.- $a, a$. Young cells. $b$ and $c$. Connecting tissue cells; and $d$. Pus cell, all derived from $a$. power it was enabled to find its way to the surface, but experiments have proved this to be incorrect. Bones have been placed in cavities full of pus, and left there for weeks, and when they were afterwards weighed, they have, if anything, become heavier, through the absorption of fluid matters, but no softening has been produced, except that caused by decomposition. How far the tissue is destroyed by solution chiefly depends upon the question whether the substance that surrounds the young cells becomes completely fluid. If it retains a certain degree of consistence, the process is confined to the production of granulations, and these may as well proceed from a 
surface whose continuity is perfect, as from one in which there is a breach. In every case granulations arise out of the tissue, and it is not essential there should be loss of substance; they are found upon bone without any loss of substance having preceded them. They are found also in direct contact with the cutis under intact epidermis, and with mucous membranes; and only in proportion as they become developed do the mucous nembranes lose their normal character.

Well-formed, perfectly elaborated, healthy pus is a smooth, rather greasy, sometimes viscid, yellowish-white, or cream-coloured substance, of a higher specific gravity than water, averaging generally about 1.030 having little or no smell, and of an alkaline reaction. Microscopically it is found to consist of certain essential constituents, namely, pus corpuscles; which measure about $\frac{1}{2500}$ to $\frac{1}{3000}$ of an inch in diameter, are pellucid, filled with semi-fluid alluminous contents, and sometimes containing a few minute oil globules. Along with the pus cells floating in the clear liquor puris are often seen minute clear particles, which seem to have some relation, as nuclei or rudiments of the cells. These minute particles are not more than $\frac{1}{1} \frac{1}{0,000}$ of an inch in size. These two solid constituents float in a fluid or serum, called the liquor puris, and the shape of the cells depends on the density of this liquor puris. Sometimes a distinct, circular, dark-edged nucleus may be seen in the paler corpuscles, and sometimes two or even three particles, like a divided nucleus. 
These, then, are the component parts of good, healthy, or laudable pus, showing a benign form of inflarnmation, and that the disease is going on regularly and promises a fortunate issue. When, however, the process deviates from the usual course in an animal otherwise healthy, variations are found in the cells, with multiform mixtures of withered cells; molecular and fatty matter; escaped and shrivelled nuclei, blood corpuscles, and fragments of fibrogenous material; the liquor puris is thin, liquid, or watery, and the pus is then said to be ichorous. When the colouring matter of the blood is mixed with it, it is called sanies, or sanious pus.

Many chemical and vital changes are found to bring about a decomposition in pus while yet in contact with living parts, although it is probable that germs in the atmosphere, or gases formed within the body, may have to do with the change; but phosphuretted and sulphuretted hydrogen and ammonia will be found frequently developed in abscesses, especially if the pus be in contact with bone, or near the alimentary canal, or in the foot: the smell is then most offensive; it is then called foetid pus. Pus may also contain certain specific properties; that is to say, it may be impregnated with certain specific poisons, as that of glanders, variola, \&c.

Healthy or landable pus has no smell, except that peculiar to the animal in which it may exist; it has an alkaline reaction when freshly drawn from an abscess, but it readily becomes acid from the generation of what is supposed to be lactic acid.

It will be gathered from the foregoing remarks that the boundary or wall of an abscess consists of newly formed areolar tissue, which has maintained the firmness and solidity of the part by activity of nuclear growth. There is sometimes found to be a thin, opaque, yellowish-white layer, easily detached, separating the suppuration from the denser part. This has been called pyogenic membranc, from the supposition that its function is to secrete the pus; whereas the cells of the denser part are-by premature and continuous development-growing into pus cells; that is to say, there is no secrction of pus by any membrane, but a continual formation of it by proliferation of the cells proper to the part.

When suppuration takes place in the cavities of the body, it 
is still circunscribed; however, it is not called an abscess, but "purulent effusion."

Alscesses are occasionally found to exist without the usual signs of inflammation; they are then called "cold abscesses;" and when slowly formed, chronic or old abscesses.

Abscesses are divided into acute and chronic.

\section{ACUTE ABSCESS.}

Symptoms.-Symptoms of inflammation precede the surpuration; there is heat, pain, and swelling in the part, with more or less symptomatic fever; for a time the pain increases, and changes to a throbbing character. The swelling becomes harder from increased exudation, and when the abscess is deeply situated, there is some œdema of the surrounding areolar tissue. Concomitant with these symptoms, a tendency to rigor or shivering may be observed by the staring coat, or actual rigors of a severe character may occur.

In the centre of the swelling pus is formed, and around this a layer of condensed plastic lymph, being the boundary or cyst of the abscess. The quantity of pus increases, and by its presence causes the absorption of the boundary of the abscess, more particularly of that portion nearest to the free surfaces of the body, generally the skin. The swelling becomes soft in the mildle, but continues hard at the base, and œedematous at a greater distance. The next change is the elevation of the centre into a prominent hairless spot. This is called the pointing of the abscess.

In general, some time before the abscess points, fluctuation is discoverable, particularly if it is superficially situated; but if deep-seated, and where the superimposed structures are still tense and thick, the quantity of pus but scanty, the perception of fluid is obscure; it becomes, however, more and more distinct as the tissues external to it become thinner.

A noticeable feature in the pointing of an abscess in the lower animals is the removal of the hairs, these falling off and leaving a bare spot on the most prominent part of the swelling. This is accounted for by the absorption of the skin and destruction of the hair follicles, which take place before the abscess finally bursts. When the collection of matter is small, or 
thickly covered, a very nice-practised sense of touch, the tactus eruditus, is requisite for detecting its presence; but if the abscess be large or superficial, simple palpation at one point is sufficient to detect it.

In feeling for fluctuation, the points of the fingers of one hand are placed on the dependent part of the abscess, whilst with the fingers of the other hand the upper part of the swelling is gently pressed or tapped, when the undulations of the pus will be distinctly felt.

In the treatment of acute abscess soothing remedies are the most useful, and of these heat and moisture are the best, as they not only soothe, but promote the formation and growth of pus. They should consist of warm fomentations and poultices, and if the pain be excessive, anodynes may be given internally and applied locally. As soon as pus is formed, a free incision should be made into the abscess, to allow its ready escape; then a warm poultice is to be applied for a few days, but a long-continued application of poultices is apt to give rise to weak action in the cavity of the abscess. There is no necessity for violently squeezing the abscess after it is opened, nor to introduce pledgets of tow into it, as practised by some. After the removal of the poultice all that is necessary is to keep the parts clean, and apply a weak solution of carbolic acid to the lips of the wound. Generally, abscesses should be opened; they should always be opened when too deep-seated to point; but abscesses in the neighbourhood of a joint should be treated with great caution, and, as a rule, allowed to burst spontaneously. The abscess of simple strangles should not, in my opinion, be opened, but allowed to run its natural course.

The best instrument for opening an abscess is Syme's abscess knife, the point to be inserted, and the opening so made enlarged as the knife is withdrawn.

\section{DIFFUSE SUPPURATION.}

We have examples of this in purulent infiltration of an organ, as in the lungs in acute glanders. The inflammation extends through a wide extent of tissue, and the boundaries of the disease are ill defined. The development and growth of the pus cells is exceedingly rapid, the tissue being as if soaked in pus, 
and should the animal not die quickly, large sloughs or death of portions of structure may take place.

Formation of pus, occurring in loose texture-the popliteal space, poll, withers-may cause infiltration by gravity, thus leading to a secondary destruction of tissue, and the formation of sinuses.

The progress of purulent effusion is probably not dissimilar to that of the phlegmonous abscess, but the inflammation is of a different type, and all the processes less complete. Thus we find no barrier of condensed tissue to circumscribe the abscess, or limit the pus. The process of suppuration is less perfect, so that the pus contains shreds or even large portions of mortified and loose connective tissue. There is no vital transformation into a fluid albuminous substance here, as in a healthy abscess. The pus is also thinner, containing a large portion of liquor puris, and also sometimes flakes of lymph. There is no pointing, as in true abscess, for the pus passes readily from its original seat by gravitation towards the most depending position, presenting a soft, broad surface, without any indication of pointing. I have often seen these collections of pus not only in the lungs of glandered horses, but in the lungs of horned cattle, that have survived and apparently recovered from pleuro-pneumonia.

Wounds on the gluteal region, more especially near the tuberosity of the ischium, are apt to cause very extensive infiltration of pus throughout the inter-muscular areolar tissue of the thigh. Very often, after an apparent recovery from a wound in this region, the lower part of the thigh will begin to swell, and on examination the enlargement will be found to contain a large quantity of pus, extending, perhaps, from immediately above the hock to the groin, the pus having burrowed from the wound above, and by gravitation forced its way down among the tendons of the various muscles. Sometimes there are direct signs of pointing, sometimes not. All such collections, wherever situated, should be removed by puncturing the lowest part of the swelling.

\section{SUPERFICIAL SUPPURATION}

May be witnessed in inflammation of mucous membranes and the skin, and the growth of pus can be clearly traced where 
columnar and stratified epithelium exists. I shall here quote from Virchow, who says:- "If you follow the development of pus upon the skin, when the process is unaccompanied by ulceration, you will constantly see that the suppuration proceeds from the rete malphigi. It consists in a growth and development of new cells in this part of the cuticle. In proportion as these cells proliferate, a separation of the harder layers of the epidermis ensues, and they are lifted up in the form of a vesicle or pustule. The place where the suppuration chiefly occurs corresponds to the superficial layers of the rete, which is already in process of conversion into epithelium; if the membrane of the vesicle be stripped of, this layer usually adheres to the epidermis, and is stripped off with it. In the deeper layers we may watch how the cellular elements, which originally have only single nuclei, divide, and how their nuclei become more abundant, and single cells have their places taken by several, which in their turn again provide themselves with dividing nuclei. Here, too, people have generally helped themselves out of the difficulty by assuming that, in the first instance, an exudation was poured out, which produced the pus in itself, and this is the reason why most investigators into the development of pus especially selected fluids which were secreter from injured surfaces. It was very conceivable that, as long as no doubts were entertained with regard to discontinuous formation of cells, the young cells should, without more inquiry, be looked upon as independent new formations; and that the notion should be entertained that germs arose in the exuded fluids, and gradually becoming more numerous, supplied the pus. But the matter stands really thus:-The longer the suppuration lasts, the more certainly is one series of cells after the other in the rete involved in the process of proliferation, and whilst the vesicle is rising up, the quantity of the cells which grow into its cavity is constantly becoming greater. When a variolous pustule forms, there is at first only a drop of clear fluid present, but nothing arises in it; it only loosens the neighbouring parts of the rete malphigi.

"Precisely the same is the ease with mucous membranes. There is not a single mucous membrane which may not, under certain circumstances, furnish puriform elements. But here, too, a certain difference always presents itself. A nucous 
membrane is all the more in a condition to produce pus without ulceration the more completely the epithelium which it possesses is stratified. All mucous membranes with a single layer of cylindrical epithelium (intestines) are much less adapted to the production of pus; that which is produced on them, even though it has quite the appearance of pus, frequently turns out, upon close examination, to be only epithelium. The intestinal mucous membrane, especially that of the small intestines, scarcely ever produces pus without ulceration. The mucous membrane of the uterus, and of the fallopian tubes, though it is frequently covered with a thick mass of quite a puriform appearance, almost always secretes epithelial cells only; whilst on other mucous membranes, on that of the urethra, for example, we see enormous quantities of pus secreted, as in gonorrhœea, without even the slightest ulceration being present on the surface. This depends essentially upon the presence of several strata of cells, the upper forming a kind of protection to the deeper ones, of which the proliferation is thus for a time secured. The pus is at last either borne away by the production of new masses of pus beneath it, or there occurs simultaneously a transudation of fluid, which removes the pus cells from the surface, just as in the secretion of semen the epithelial elements of the seminal tubes furnish the spermatozoa, and, in addition, a fluid which sweeps them away. But the spermatozoa do not arise in this fluid; this is only the vehicle for their onward movement. In this manner we frequently see fluid exude on the surface of the body without our being able to regard it as a cystoblastema. If a proliferation of epithelium simultaneously takes place upon the surface, the elements detached by the transuded fluid will also be found to consist of nothing but proliferating epithelium."

COMPARISON BETWEEN MUCUS, EPITHELIUM, AND PUS.

If now pus, mucus, and epithelial cells be compared with one .mother, it appears that there certainly does exist a series of transitional forms or intermediate stages between pus corpuscles and the ordinary epithelial structures. By the side of perfectly formed pus corpuscles provided with several nuclei are very commonly found somewhat larger, round, granular cells with single 
nuclei, the so-called mucus corpuscle. A little further on we see perhaps still larger cells of a typical form, and with single large nuclei, and these we call epithelial cells. But the epithelial cells are flat, angular, or cylindrical; whilst mucus and pus corpuscles, under all circumstances, remain round. Fven from this circumstance may be derived an explanation of the fact that, whilst the epithelial cells which cover and are in close opposition to one another, acquire a certain firmness of cohesion, mucus and pus corpuscles, which lie but loosely one against the other, and are of a spherical shape, retain a great degree of mobility, and are easily displaced. It has been said that mucus corpuscies are nothing more than young epithelium. Another step, and pus corpuscles would be nothing more than young mucus corpuscles. This is a somewhat erroneous notion. It cannot be maintained that a cell which, up to the point when it becomes a so-called mucus corpuscle has preserved its form as a spherical body, is still in a condition to assume the typical form of the epithelium, which ought to exist in the part; and just as little can it be said that a pus corpuscle, after it has developed itself in the regular manner, is capable of again entering upon a course of development calculated to produce a relatively permanent element of the body. The cells in which the development of epithelial, mucus, and pus cells originate are young forms, but they are not pus corpuscles. In pus, every new cell at a very early period sets about dividing its nucleus. After a short time the division of the nucleus reaches a ligh pitch without any further growth on the part of the cell. In mucus, the cells are wont merely to grow, and in some instances to become very large; but they do not pass certain limits, and, above all, they do not assume any typical form. In epithelium, on the contrary, the elements begin even at a very early period to assume their peculiar form; for "what is to become a hook right early gets a crook." The very youngest elements, however, which are found in pathological conditions, cannot be called epithelial cells, or at least they have as yet nothing typical about them, but are indifferent formative cells which might also become mucus or pus corpuscles.

Pus, mucus, and epithelial cells are therefore pathologically equivalent parts, which may indeed replace one another, but cannot perform each other's functions. 
Observations and experiments made known since the publieation of the first edition of this work compel me to modify the view I then expressed, that the formation of pus was not due to wandering leucocytes; for Cohnheim, Von Recklinghausen, Stricker, Sanderson, and others have demonstrated more or less clearly that pus corpuscles, at the commencement of every acute inflammation, may be derived from the blood-i.e., that they may be emigrant white corpuscles which have escaped through the walls of the capillaries. But even Cohnheim sees that there is nothing in the facts which contradicts the previously accepted belief, supported as it is by an overwhelming mass of evidence, that the later generations are the offspring of the inflamed tissues by proliferation of their cell elements.

The experiments which most strongly demonstrate that the white corpuscles have the power of locating themselves in inflamed tissues, in virtue of their own inherent power of movement, are as follows. Stricker irritated one eye of a frog by cauterizing the cornea through, then excised the cornea of the opposite eye, and inserted it beneath the membrana nictitans of the irritated eye, and finally united the edge of that membrane with the opposite margin of the cutis by ligatures. After twenty-four hours the transplanted cornea was removed and examined, and found to exhibit inflammatory changes, which, although on the whole less advanced than those found in an unexcised cornea at the same period after irritation, were equally characteristic.

These results scarcely admit of misrepresentation; they are, however, rendered much more decisive and satisfactory by varying the conditions of the experiments in such a way as to show that the changes observed are not due to the penetration of leucocytes from the liquid in which the cornea is immersed, and secondly, that they are not a mere result of its transplantation into an unnatural position. The first of these objects is readily attained by dividing the cornea immediately after excision, plunging one-half in water so as to kill it instantly, and then placing the dead and the living portion together underneath the membrana nictitans of the opposite eye. It is then found that whereas the same inflammatory changes as before go on in the living half, the other half remains inactive. 
The second result is attained by the observation of what happens when, instead of first cauterizing the eye which is destined to be the recipient of the transplanted cornea, it is left uninjured. At the end of twenty-four hours the corpuscles of the transplanted cornea are found quite unaltered, and so distinct that the plan is strongly recommended as a method of demonstrating their normal character-(KLEIN, SANDERSON.)

These varied results seem therefore to show, beyond the possibility of dispute, that the structural changes in the cornea of the frog cannot be dependent either upon any influence exercised by the nervous system, or by transmission of the irritative effects from one structural element to another, so that we have good ground for concluding with Professor Stricker that they result exclusively from the stimulating influence of the exuded liquid. The precise physical or chemical conditions are as yet unknown, and are at the present moment subjects of further investigation. Whilst these experiments prove that the formation of pus is sometimes due to the power of the white corpuscles of penetrating living tissue, they do not destroy the facts that the formation of pus may be independent of their presence. In the case of pus formation in cartilage-where the cartilage cells are isolated in cavities in the matrix, having no communication with each other, but entirely closed-it is seen when the surface of the cartilage is irritated, that the cells in the neighbourhood of the irritation enlarge, and expand their capsules. The protoplasm of which each cell consists becomes more granular, and soon contains two corpuscles in its interior instead of one, and has a gathering of protoplasmic matter around itself. This process of division is repeated in each segment until every cavity contains a mass of nucleated cells, which at length assume characters corresponding with those of newly formed pus corpuscles, while at the same time the original interstitial substance gradually wastes away and is finally represented by a sponge-like stroma, in the holes of which groups of young cells are contained.

In this process we have a typical example of germination; the permanent cells which have for their function the maintenance of the unchanging life of the tissue, are replaced by a more numerous progeny of transitory mobile cells, which live 
at the expense of what remains of the tissue, and eventually destroy it.

\section{FEBRA PYOGENICA, OR STRANGLES.}

As an example of suppurative disease, associated with a varying degree of febrile disturbance, we have in the horse what from time immemorial has been termed Strangles. The origin of the term strangles arises from the fact that in some cases it is associated with symptoms of choking or strangulation, and it was divided by the old writers into simple and bastard strangles; the first form being that which ran a regular course, and the second consisting in the formation of multiple or successive abscesses.

Simple strangles may be defined to be a febrile disease, generally attacking young horses, and terminating in the formation of an abscess or abscesses in the areolar tissue of the submaxillary space.

Irregular or bastard strangles, on the other hand, is a very grave affection, in which the connective tissue of the lymphatics of the submaxillary region, and sometimes the salivary glands, are the seat of acute suppurative inflammation, associated with a low febrile state of the system, a tendency to suppurative action in various parts of the animal body, more especially in the mesenteric, bronchial, axillary, and inguinal glands, and to purulent infiltrations in the parenchyma of organs or into the subcutaneous areolar tissue.

It is thought by some that strangles is contagious, from the circumstance that when one case occurs in a stud others very often soon follow. This, however, is not sufficient proof of its being contagious. It is a debateable point whether strangles is contagious or not. Many proofs can be brought forward in support of either view, some observers stating that they have induced it in inoculation-Gohier, Reynal, Toggia. In some instances-like typhoid fever of man-it seems contagious to some extent, while in others it shows no tendency to spread by this mode. My own experience leads me to conclude that it is a non-contagious disease, but whilst there is still any doubt about the matter, it is better to take precautions against its diffusion by separating the affected from the healthy.

Although it is generally found to attack young horses, it is 
by no means uncommon to find aged animals take the disease. In some localities, and during some years, it assumes the form of an enzootic, attacking animals of all ages.

It is an unsettled question whether horses take strangles more than once in their lives, and the point is not easily solved, as the history of animals is liard to trace. I think it may be laid down as a rule that it rarely attacks the same horse more than once, after which the predisposition to it disappears.

The causes of strangles are very obscure. It is thought that dentition has something to do with it; and this may be accepted as a tangible reason, as the majority of cases occur during the active period of dentition. But it by no means follows that this is the only predisposing cause, for animals with full mouths are very often attacked with strangles. Domestication has also been looked upon as the cause of strangles; but this cannot be, otherwise animals that have never been stabled would not suffer, whereas horses at grass very often have it.

Horses from a few months to six years old are the common subjects of strangles. When they are very young, the disease generally assumes a virulent form, and often proves fatal, either from internal abscesses, purulent infiltrations, or a condition similar to the hectic fever of human beings.

Symptoms.-Strangles manifests itself in three ways.

1st. It commences with the common symptoms of a mild catarrhal affection. The animal is somewhat dull, has a slight cough, some soreness of throat, a disinclination to feed, and more or less inability to swallow. The submaxillary space swells, is hot and tender, the swelling filling up the whole space or confined to one side only; and is either diffused or circumscribed. There is generally some dribbling of saliva from the mouth, and a discharge from the nostrils. In most instances the pulse is somewhat hurried, and the respiratory movements slightly increased.

2 l. For some weeks, or even months, prior to the local manifestation of the disease, the animal is unthrifty, loses flesh, becomes hide-bound, drawn up at the flank; if at grass, stands apart from his fellows, has more or less cough, often stretches himself as if fatigued, shivers on the application of slight cold. his coat stares, his growth is arrested. The horseman says that " he is breeding strangles," and time confirms the correctness 
of this opinion, the local signs of the disease becoming developeu, and very often to a more severe extent than in the first form.

$3 d$. The premonitory signs are those which have given the name to the disease, namely, those simulating strangulation, with great difficulty of respiration, accompanied by a loud trumpetlike sound, emitted more especially during inspiration. This sound may arise from spasm of the muscles that close the glottis, namely, the crico-thyroideus, crico-arytenoideus lateralis, thyro-arytenoideus, \&c. ; or from an odematous condition of it (crlema glottidis). If from the first cause, the inspiratory sound only is heard; but if from the second, both movements may be accompanied by the roaring noise, the inspiratory to a greater extent than the expiratory.

The treatment of regular strangles is very simple, and consists of fomentations and poultices to the part, to promote the growth of the pus; slight febrifuge medicines, as the nitrate of potash, to modify any accompanying fever; keeping the bowels in order by laxative diet or very mild aperients, as oil, or the sulphate of magnesia. If the tumour points and bursts spontaneously, it is advisable not to interfere with its course, either by incision or external irritants; but if it is indolent, "seeming to hang fire," as it were, it is good practice to apply a blister. Some writers and teachers condemn this plan of treatment. I feel quite sure that such are men of no experience, a blister often acting like magic in promoting the suppurative action in the tumour, or in dispersing it altogether without suppuration. The best plan is to apply a good fly blister, and a warm poultice in about twenty-four hours afterwards. When the abscess has burst, all that is necessary is to keep it clean, and to give the animal good food, with tonics, the preparations of iron being the best. After apparent recovery from strangles, care must be taken not to expose the animal to any debilitating influence, as work or cold, too soon, for secondary abscesses may form in the interual organs.

The second form of strangles-namely, that ushered in by a lengthened period of unthriftiness-must be treated locally, in the same manner as the first form; but the constitution will require to be supported by good food and tonics before there are any local manifestations. If the animal is at grass, it must be taken up and protected from the vicissitudes of the weather 
by being lodged in a roomy, airy piace; must have an allowance of corn-crushed oats in preference; and be regularly fed and carefully watched, as the most severe forms of the disease, namely, those associated with internal abscesses, are ushered in by the above premonitory signs. If the tumours suppurate kindly, and the disease runs its course regularly, the animal will soon regain its former condition, will, in fact, thrive better than ever, and will seem as if the system had been rid of some deleterious material.

In the third form, great watchfulness must be used for fear that the patient dies of suffocation. To prevent this the operation of tracheotomy may have to be performed.

As to the necessity of this operation, and the time for its performance, there is a difference of opinion. Some think it ought to be performed early, to enable the glottis to remain in a state of repose, and thus recover more speedily than if kept in activity by the respiratory movements; while others condemn the operation except as a dernier resort. Of the two opinions the former is the more humane and rational, but the latter is supported by a more extensive experience. Without discussing the point, I may state that my own practice consists in hot fomentations to the throat, succeeded by a mustard liniment, making the animal inhale watery vapour for some hours and watching the case carefully. If the dyspnœea increase, I perform tracheotomy; but if it decrease even slightly, I go on with the steaming and fomentations. There is a difficulty in applying poultices to the throat, and I find a good substitute in woollen waste confined to the parts within the hood. A poultice soon gets cold, and is apt to do more harm than good if not carefully applied and continually watched. Hot fomentations and packing with wool are therefore preferable.

In performing tracheotomy, the best plan is to elevate the horse's head, extend the nose, so as to draw the inferior cervical muscles into a state of tension, then to feel along the course of the trachea for the part least covered with muscular and adipose tissue, and to make a bold incision through the skin and subcutaneous structures on to the trachea. The incision must be about four inches long, exposing the trachea clearly to view. When this is done, introduce the point of a sharp-pointed bistoury into the trachea, between the upper exposed rings, 
divide two or three clearly across, and introduce the tube, taking care in doing so that the divided rings are not pressed into the trachea.

The tracheotomy tube must be tied around the animal's neck and to the mane, in order to keep it steadily in position.

There is no need for casting the horse for this operation; if it be quickly done, there is scarcely a necessity for the twitch, as the poor animal is too ill to be very unruly. The tube should be kept in until it is manifest that the animal is able to breathe freely through the nostrils. This is ascertained by putting the hand over the tube for a few minutes, when, if the patient breathes naturally and without distress, it may be removed, and the lips of the wound brought together by metallic sutures.

In a period varying from a day to a week after the first symptoms, the local manifestatious of strangles are developed, and until these are apparent, the disease is classified as laryngitis.

The local lesions are generally much more severe than in the first or even second form. The tumours are multiple, or diffused over the sides of the face, nostrils, submaxillary space, and parotidean region; suppurating at several points, and discharging an unhealthy pus. Horses confined in ill-ventilated stables suffer most commonly from this form, and if not removel to a healthier situation, it is apt to degenerate into a hectic condition, succeeded by pyæmia, or to terminate in glanders.

The hectic condition is that in which the animal continues to lose flesh very rapidly, to have a discharge from one or both nostrils of a tenacious muco-purulent material. Abscesses continue to be formed one after another on the face and the submaxillary space; the patient becomes hide-bound, with a dry, scurfy skin, and drawn up in the flank. The hair of the mane and the tail is easily pulled out; the pulse is soft, compressible, feeble, and quick; the legs, at first fine, begin to swell, and the various serous cavities fill with serum, the patient becoming affected with glanders and farcy, or it may die of exhaustion, diarrhœe, excessive secretion of urine, loaded with hippurates and oxalates, or of dropsy of the serous cavities.

The treatment of this condition consists first in the removal of the horse from an unhealthy to a healthy situation. He should be kept in a box by himself, and have, in addition to 
a liberal allowance of corn, hay, or grass, a gallon of new milk during the day; he will soon become fond of it. He should be made to drink it instead of water, and if kept without the latter for a few hours, will drink the milk with avidity. If the appetite is entirely lost, six eggs ought to be beaten up and mixed with each gallon of milk.

The strength must also be kept up with tonics, iron, quinine, wine, or beer, and acids; and should these fail, nux vomica and arsenic in small doses.

I have seen many cases of this kind of mal-condition, which I have ventured to compare to the hectic fever of the human being, rapidly recover, with very little treatment, after being removed from a crowded stable to an airy, loose box.

Prasina differs from the latter condition by the development of abscesses, or of purulent infiltrations in various internal organs, and from irregular strangles by the abscesses being secondary to, and not concomitant with, those in the submaxillary space. In reality there is but little difference, except that in the one case the internal collections of pus are due to a diathesis, and in the other to the absorption of some deleterious material generated in the pus of the primary abscess.

Mr. Haycock was, I think, the first to point out the occurrence of pyæmia succeeding strangles (see his Contribution to Veterinary Pathology, 1849-50), under the term "Phlebitis." Since then the subject has received considerable attention, but even to this day cases are described as pyæmia which are in reality extensive abscesses, infiltrations, excessive suppurations, or farcy.

Pyæmia may be defined to be a typhoid condition, accompanied by multiple abscesses or purulent infiltrations into one or more organs, resulting generally from strangles, parturition, and from injury or surgical operation.

Four theories have been promulgated with the view of explaining the pathognomy of pyæmia:-

1st. "That this condition is owing to an admixture of the blood with pus (pyohemia of Piorry), and that the pus corpuscles being larger than the coloured ones of blood, are arrested in the minute capillaries, and give rise to secondary abscesses. $2 d$. That it is owing to the presence of some irritant body, 
which, not being able to escape from the economy, produces capillary phlebitis. $3 d$. That it is dependent on a property possessed by pus of coagulating the blood. 4th. That it is caused by the presence of a peculiar poison, which contaminates the system."-(BenNetT.)

The first theory is founded upon the assumption that pus cells differ from the white globules of the blood; whereas in reality no actual difference, either in size, structure, or behaviour, on the addition of re-agents can be detected; therefore, if the capillaries are sufficiently large to admit the passage of the white blood globule, they must consequently be large enough to admit the passage of the pus cell.

The second explanation was advanced by Cruveilhier, who, on injecting mercury, ink, and other substances into the blood of a living animal, found that abscesses were formed wherever these accumulated; hence it seemed to follow that the impaction of certain substances in the tissues may induce local inflammations, and lead to abscesses; but that such is not the necessary result of admixture of pus with the blood is proved not only by the previous observations, but by numerous experiments of Lebert and Sédillot, in which the animals recovered.

The third doctrine was advanced by Mr. Henry Lee, and resulted from observing that when pus was mingled with recently drawn blood, it coagulated more rapidly and more firmly than under ordinary circumstances.

But although this may be true as regards pus when mixed with recently drawn blood, it does not follow that it induces coagulation of living blood in the vessels of an animal; and numerous experiments by Lebert, Sédillot, Bennett, and the late Professor Barlow, show that such does not take place: in some cases death followed, in others the animals lived, and the pus corpuscles were dissolved.

The fourth theory is based upon the opinion that good pus is innocuous, and that the bad effects occasionally produced depend on its becoming putrid, or being otherwise altered. It is now generally believed that pyæmia is due to a poisonous principle associated with the pus; hence it is proposed to substitute the terms ichoræmia (VIRCHOw), or septicæmia (VOGEL), for pyæmia.

The signs of pyæmia may not manifest themselves for some time after the primary abscess has discharged itself; never in 
my experience brfore the pus has come into contact with the atmosphere, and they will depend upon the seat of the secondary purulent collections. In the human patient the symptoms are very characteristic; in addition to others, the breath has a smell of new-made hay, and the skin, especially by the side of the nose, and about the eyebrows, becomes yellow; but in the horse these are not recognisable. In one case of purulent infiltration into the lungs succeeding strangles, I did think that the smell of new hay was present; but it was soon succeeded by a most offensive odour of decomposing animal matter. I think the most prominent and characteristic sign is a total loss of appetite, or an apparent loathing of food, associated with occasional colicky pains when the abscesses are in the mesentery; with symptoms of pneumonia and foetid breath when in the lungs, and yellowness of the mucous membranes when in the liver. The animal's head is held low and drooping, the countenance is sunken and cadaverous looking, and the body generally emaciated.

Haycock describes it as phlebitis, and it appears that in his case inflammation of the veins was present; but it by no means follows that this is always the case. I have made careful examinations of several, and found this condition sometimes present, sometimes absent.

As regards treatment, I know of none that can arrest the progress of the disease when it has set in; all that can be done is to be careful that the surroundings of a case of strangles are healthy, specially avoiding bad drainage and bad ventilation. When I was in practice at Bradford, in Yorkshire, nearly every case of strangles which occurred near the Bradford Beck (brook or rivulet) and Canal proved fatal, pyæmia supervening; this fatal result could only be accounted for by the putridity of the water, both in the canal and the beck, and by the exhalations from them having found their way into the stables in their neighbourhood, and set up a septic fermentation in the pus when exposed to their contaminating influence.

If the secondary abscesses form near the surface of the body, as in the groin or subscapular region, they must be opened, and in all cases the animal must be supported with tonics, good food, milk, eggs, wine, beer, quinine, and small doses of the chlorate of potash; but should the lymphatics become 
corded, and abscesses form in the glands along their course, the disease will have degenerated into farcy, and may ultimately terminate in glanders.

\section{RE-ABSORPTION OF PUS.}

It was imagined at one time that pus might again be taken up from the different points at which it had been formed, and that a favourable turn was thereby effected in a suppurative disease; but pus, as pus, is not absorbed; and it is always its fluid part (liquor puris) which is taken up, and as this disappears the pus becomes thicker, constituting what is termed inspissated pus, which contains the pus cells in a shrivelled condition. Not only is the serum in which the cells float absorbed, but also that contained within them; and in proportion as this is the case, the cells become smaller, more irregular, more angular, more uneven; they assume the most singular forms, lie closely pressed together, forming the cheesy concretions which have been confounded with tubercle.

There is another method by which pus is removed,- - that is, by fatty degeneration of the pus cells. Every cell sets fatty particles free within it-breaks up; and at last nothing remains but fat granules and intervening fluid of a milky appearance, and constituting what is termed pathological milk, which is absorbed: here, again, pus is not re-absorbed as pus, but as water, fat, and salts.-(VIRCHow.)

Inspissated pus, in pieces of varying size, dry and hard, is often found in the guttural pouches of subjects on the dissecting table; these are the results of preceding catarrhal attacks and formation of pus in these cavities. They are also found in old abscesses, and give rise to tumours in various parts of the body. -(See Tumours.)

\section{FORMATION OF SINUSES.}

When pus is seated in parts, the action of which is defective owing to local or general causes, or when it cannot be completely evacuated, the cavity that remains does not contract completely, and the wound is called a sinus or fistula.

In the course of time the surface of the sinus becomes smooth and condensed, so as to resemble mucous membrane, or even 
skin, being, in old confirmed cases, lined with a downy hair, and so thick and callous that its walls resemble cartilage in their hardness. This hardening of the walls was formerly thought to depend upon a morbid disposition of the part, and to require extirpation, as an essential step to the animal's recovery. The applications and operations practised for this purpose were extremely severe, consisting of the actual cautery, arsenic, corrosive sublimate, and other powerful escharoties; but a more enlightened pathology has led to their disuse, and it is now found sufficient simply to remove the cause of the irritation.

"In treating sinuses, the objects are to promote granulating action on their surfaces, and to press their sides together. They are not healed by filling up, any more than the original cavity of the abscess, but contract until they become obliterated, or close more directly by union of the opposite surfaces."-(SYME.)

Great care must be taken to avoid confining the discharge of the sinus, since, if prevented from escaping, it distends the sides of the cavity, and for the same reason it is always proper to afford the matter an opening for its complete escape, either by enlarging the one already existing, or making a new one. The method of doing this will be explained hereafter in connection with the different regions in which sinuses are apt to be seated.

\section{ULCERATION.}

Ulceration is another result of inflammation: it consists essentially in molecular degeneration of a part, and goes on by three simultaneous processes. (1.) An exudation of inflammatory lymph and serum surrounds the mass of young cells, which constantly continue to grow on the surface, and at the margin of the ulcer, causing the destruction of the normal textures. (2.) Cells are thus continually growing on the surface, to be carried off by fresh exudation. (3.) Liquefaction of the gelatinous interstitial material supervenes, and so destruction of tissue takes place continually.-(Goodsir, Aitkex.)

The difference between ulceration and mortification will be described hereafter, but I may here state that I cannot conceive ulceration to be molecular death of a part, but rather the absorption of living degenerate textures, by which gaps or solutions of continuity are formed. 


\section{VARIETIES AND TREATMENT OF ULCERS.}

An ulcer is defined to be a solution of continuity caused by ulceration, and its varieties in Veterinary Surgery may be arranged under six heads. 1st. Healthy ulcer; $2 d$. Weak ulcer; $3 d$. Indolent ulcer; $4 t h$. Inflamed ulcer; $5 t h$. Gangremous or phagedenic, or sloughing ulcer; and 6th. Specific ulcer.

1st. The healthy ulcer is smooth at its edges, which are neither everted nor inverted, adherent to the granulations, and when they rise to a level with the skin, a film or cicatrix is formed like a semi-transparent ring round the edges, and gradually spreads over the wound. The granulations are small, firm, numerous, and of a fine florid colour, pointed at their tops, and discharging a thick laudable pus.

But little treatment is required for this kind of ulcer, beyond rest and cleanliness.

2d. The weak ulcer. The granulations are pale, large, flabby, not pointed, but even bulbous at their tops, less vascular, and less apt to bleed on being touched than those of the healthy ulcer; they are unattended with pain, rise above the level of the skin, so that the margins of the ulcer are hid from view. The discharge is thin, pale, and watery.

This kind of ulcer is caused by some debilitating local or general influence. It is often found in the hind legs of lowbred animals, and arises in them from the venous congestion and dropsical effusion, which are so often met with in roundlegged cart horses. It is associated perhaps with some constitutional weakness, arising from bad food, or other cause of general debility. Healthy ulcers, when improperly treated, are apt to degenerate to this form.

The treatment ought to consist of some mild astringent dressing, as the solution of sulphate of zinc or of copper, the periodical use of the nitrate of silver-say, every three days, if thought requisite-and the careful application of a bandage, both to the ulcer and to its neighbourhood, in order to stimulate the granulations and to correct the tendency to congestion. The general system is to be corrected by a gentle purgative, succeeded by tonics and diuretics; at the same time the strength is to be kept up by good, nourishing, but easily digestible food, dry food being preferable to moist. The patient 
ought to have regular exercise, and great attention must be paid to eleanliness.

$3 d$. Indolent ulcer, found usually about the coronet of old horses. Its edges are thick, prominent, comparatively insensible, smooth, shining, firm, incompressible, and without any appearance of cicatrix; the surrounding parts are swollen, hard, incompressible, and if the skin be white, discoloration will be seen from congestion of the vessels; the surface of the ulcer is nearly devoid of granulations, smooth, glossy, and whitish, grey, or brown in colour; the discharge is thin, watery, and scanty. The treatment of this ulcer is by blister, succeeded by gently stimulating dressings, but bandages do more harm than good. The constitutional treatment must depend upon the condition of the animal, but generally a purgative is useful.

4th. Inflamed ulcer. The edges are red or purple, swollen, hot, tense, tender, and painful; the sore presents no granulations, but has a raw, pulpy, foul, and even livid appearance; the discharge is offensive, profuse, mingled with blood and ulcerative débris; the pain is great, and there is always some attendant fever. This ulcer may be caused by the presence of some foreign body in the part, which must be removed; it may be a piece of dead tissue, skin, ligament, or bone, as when this ulcer is produced by a tread. Whatever it be, the first step in the treatment must be its removal. This being done, the inflamed ulcer must be converted into a healthy one by means calculated to subdue excessive irritability. The most useful local applications for this purpose are warm fomentations and poultices, combined with opiates; the general treatment to consist of a purgative, succeeded by febrifuges, and the diet to be light, and easy of digestion.

5th. The phagedenic, gangrenous, or sloughing ulcer, which may arise from constitutional debility or from local causes, as frost-bites, is that in which the edges are very irregular, and of a dark purple appearance, extending a considerable way into the surrounding parts; they are often inverted, and exceedingly painful. The surface of the ulcer is uneven, of a dark, livid colour, presenting a very irritable appearance and much surrounding swelling. The discharge is thin, ichorous, and mixed with blood. The ulcer enlarges with great rapidity, the destructive process being carried on both by ulceration and 
sloughing. In some cases many points of ulceration form in the neighbourhood of each other, the ulceration taking place around several central sloughs; these by enlargement coalesce, and ultimately form one large ulcer, embracing, as in some cases of "carbuncle of the coronary band," a space of several inches. The treatment of this variety is a matter of great urgency and importance, but not always satisfactory.

Treatment.-Free scarifications of the part, to relieve congestion; the removal of all sources of irritation, and the application of emollient and antiseptic poultices or fomentations, constitute the best local treatment. If the ulceration continues to spread, I have seen benefit arise from the gentle application of nitrate of silver to the edges. The ccnstitutional derangement must be treated by purgatives, anodynes, or sedatives, succeeded by tonics; and the diet regulated according to the stage and variety of the accompanying symptoms.

Specific ulcers will be described with the diseases of which they are symptomatic.

\section{MORTIFICATION, OR DEATH OF A PART.}

This is of two kinds, complete and incomplete. In the soft structures, the first is called sphacelus, and the second gangrene; whilst mortification of the blood is termed necræmia, and that of the hard structures, as the bones, necrosis. When the dear tissue is visible to the naked eye, it is called a slough; when it is soft, the process is called sloughing, which means the progressive death of the part as well as the process by which the slough is separated; the same meaning is applied to exfoliation, or the process of separating a sequestrum or dead piece of bone. But so long as the dead particles of tissue ejected in the ulcerative process are in the form of minute particles, visible only with the microscope, the disease is spoken of as ulceration, not mortification or sloughing.

Degeneration of a part may be distinguished from its death by the fact that the degenerated part never putrifies, and that no process (sloughing) ensues for its separation or isolation, such as can be seen in the case of a dead part. "However degenerate a tissue may be, it either remains in continuity with those around it, or it is absorbed. If the same tissue were dead, 
those around it would separate from it, and it would be ejected from them."-(PAGET.)

Mortification of the soft parts may be white or black in appearance, and it may be humid (moist) or dry. The mortified part has a black appearance when the blood is extravasated into the tissue, giving it a purple or dingy hue, whilst to the touch it is soft and doughy. It has a dull-white appearance in frost-bites, when by the action of cold the blood has been driven from the part.

Moist gangrene occurs when the blood transudes, and after its transudation separates into its constituent parts. The serum being set free, dissolves the red globules, raises up the surface of the cuticle in bladders, forming what are termed " phlyctenæ." Air generated by incipient putrefaction is not unfrequently contained in the phlyctenæ, giving to the finger touching the part a sensation of crepitation.

Dry mortification is very rarely met with, but it has been observed to follow the use of ergotised rye as food on the Continent of Europe, where it not only attacks the lower animals, but human beings, in the form of mortification of the extremities. It may, however, occur in any part of the body which has been deprived of its blood and a further supply cut off. Sloughing of the cornea has been observed in animals fed upon food deficient in nitrogenous elements, and in my experience cases have occurred of sloughing of the tail from dry gangrene in horned cattle that have been starved, ill-treated, or have suffered from debilitating diseases or old age. This form of mortification may also be observed in the course of scarlatina in the horse, "when some extreme part of the organism, such as the ears, will suddenly present a blanched appearance; the skin of these organs will suddenly shrink and become hard and dry, as though frozen, and in the course of a day or two these blanched portions snap of, leaving exposed a raw surface, which speedily suppurates."-(НАусоск.) Mortification of the tails of monkeys is too well known to need comment.

NeCRemia, or death of the blood, is well shown in those dire diseases which affect horned cattle, namely, splenic apoplexy and quarter-ill. In these diseases we have local manifestations of the general blood crasis, by the development of large patches of extravasation of dead and decomposing blood, the death and 
decomposition being apparent in the part by the formation or escape of the gaseous products of putrefaction simultaneously with the appearance of the extravasation, giving the swellings a feeling of crepitation even at the very outset of the disease.

Mortification may arise independently of the inflammatory process from defective quantity of blood in a part. This may be due to obstruction of its artery of supply, owing to injury, the formation of a clot within, or pressure constantly maintained upon it. Portions of tissue may also perish when, by injury or by progressive ulceration or absorption, all their minute blood-vessels are destroyed and their supply of blood cut off. I have seen a case where all the gluteal muscles of one side were mortified, from the small arteries having been crushed and destroyed in a railway accident, but where the larger arteries of supply did not suffer. The horse lived several days.

Necrosis may follow the separation of periosteum from the surface of a bone, when it is either stripped off or raised by effusion, or when there is suppuration beneath it.

Sometimes a tumour will slough in its centre from defective supply of blood. Again, a part will slough from the application of a strong chemical agent, as corrosive sublimate or arsenious acid.

Blood defective in quality also produces mortification, without being accompanied by inflammation, and occasionally mere passive congestion of a part may lead to its death; but this is a rare occurrence.

The simplest example of mortification of a part, not from deficiency of blood in it, is that from strangulation, as in strangulated hernia. Here, if the strangulation is sudden and complete, the stagnation is equally so, and the death of the part follows very quickly, with but little excess of blood in it. But if the strangulation be less in degree, the veins suffer more from the gradual compression than the arteries do; the vessels become engorged with blood admitted to them faster than it can leave them, and so, after intense congestion, mortification ensues. (Example-inversion of the uterus.)

The mortification arising from passive congestion and that from strangulation are said to arise from defect in the flow of the blood. 
The other form of mortification we have to deal with is that which goes under the denomination of Inflammatory Mortification. The death of an inflamed part is a very complex matter, and in certain kinds of it stagnation, degeneration, and pressure may act as causes. Thus, inflammatory congestion may end in perfect stagnation of the blood in the vessels, the blood so stagnated may mortify or die, and this death of the blood may lead to the mortification of the tissues that need it for their support.

Degeneration of the proper tissues of the part is a constant accompaniment of the inflammatory process, and this degeneration may result in death, from absence of the proper conditions of nutrition; and, again, effusion of fluid may so compress the inflamed part, and, by the swelling, so elongate the blood-vessels, as to diminish the influx of fresh blood, even when little of that which is in the part is stagnant.

The intensity of an inflammation is not alone a measure of the probability of mortification ensuing, neither is mere debility, for we see inflammations without mortification in very enfeebled cases. Want of condition in an animal, if put to severe exertion, will often cause mortification from excessive congestion, especially of the lungs, the mortification here seeming to arise from complete stagnation of the blood in the pulmonary vessels, with death of that blood. Again, in enteritis, mortification ensues from severity of the congestive rather tihan the truly inflammatory process. Cases of partial gangrene of the lungs, depending on pneumonia affecting bad-constitutioned animals, more especially if such have been kept in ill-drained or ill-ventilated stables, are not uncommon.

When a mortification has a disposition to spread, its dark colour is gradually lost in the surrounding parts, whereas, when it ceases to spread, a red line, called the line of demarcation, separates the dead from the living parts. This line is always regarded as most important,indicating that sloughing has ceased to spread, and that a process has begun for the removal of the sphacelated part from the system.

In this process, consolidation of the tissues by the formation of organizable lymph precedes the suppuration and ulceration; and thus hæmorrhage from the vessels and infiltration of the decomposing material into loose structures aro both prevented. 
The final act in the separation of dead tissue is that of ulceration of portions of living tissue, which are in immediate contact with the dead. A groove is formed by this ulceration, which circumscribes and entrenches the dead part, and then, gradually deepening and converging, undermines it, till, reaching its centre, the separation is complete, and the slough falls off, or is dislodged by the discharge of the ulcerated living surface. Concomitant with this process of destruction, one of repair is set up; as the ulcerated groove deepens, so do granulation cells rise from its surface, so that, as one might say, that which was yesterday ulcerating is to-day granulating; and thus, very soon after the slough has separated, the whole surface of the living part, from which it was detached, is covered with granulations, and proceeds like an ordinary ulcer towards healing.

There is one peculiarity in mortification, namely, mortification by contact. Thus a spot of mortification of the size of a fiveshilling piece is discovered upon a portion of intestine, surrounded by perfectly healthy structures, until the portion of the intestine in immediate contact with the gangrenous spot is examined. This will be found to be gangrenous also, although, when divested of its convolutions, it may be several feet from the originally mortified spot, the intervening lengtli of gut being free from mortification. 


\section{CHAPTER III.}

\section{SYMPTOMS OF INFLAMMATION.}

GENERAL SYMPTOMS OF INFLAMMATION-INFLAMMATORY APPEARANCES OF THE BLOOD-TREATMENT OF INFLAMMATION-TREATMENT OF CHRONIC INFLAMMATION.

THE symptoms of inflammation are of two kinds, namely, local and constitutional. The first-named have been already described. The constitutional symptoms are those indicative of sympathetic, symptomatic, or inflammatory fever, and are of the greatest importance, indicating the nature of the disease when the inflammation exists in an internal organ removed from sight or touch, and often guiding the method of treatment. There are some inflammations that are unattended by any appreciable signs of fever; others, again, by symptoms that are so insignificant as to pass unobserved; but when an inflammation is sufficiently extensive, the general system is disturbed by fever (pyrexia).

Prominent amongst the symptoms of inflammatory fever are rigors (shiverings), elevation of internal temperature, and debility; followed by, or alternating with, increased heat of skin, increased frequency, perhaps force, and often hardness of the pulse, with disturbance or derangement of the natural functions of the auimal body. The rigors are sometimes severe, or amounting only to mere chilliness, a slight staring of the coat being the only sign; but they are always important, as they mark the outset of the febrile disturbance, and it is worth observing that rigors more commonly attend the commencement of spontaneous inflammation than of inflammation caused by external injuries. They are of very frequent occurrence in horses that present no signs of local disease; they are dull, off 
their feed, seemingly little wrong or amiss, but the coat is harsh and staring, and actual shivering is easily induced. An animal in such a state may be on the eve of an attack of severe inflammation of some important internal organ. Generally speaking, the febrile state succeeds the manifestations of the local symptoms of the inflammation, and therefore it may be considered generally that the fever is the natural effect of the inflammation.

The inflammatory fever is not always proportioned in its degree of violence to either the size or the importance of the part inflamed. It may be modified in the outset, or very early indeed, by the nature of the part upon which the inflammation has seized. In inflammation of the stomach and bowels, and some of the other abdominal organs, and particularly in traumatic peritonitis, the disease has a peculiarly depressive effect upon the action of the heart, and this gives a peculiar character to the fever, lessens the amount of the reaction, or abridges its duration-affects especially the character of the pulse, which is herc small and thready, and carries with it a strong tendency to death, by failure of the heart's action (asthenia).

The small and thready pulse, being chiefly observed in inflammation of the abdominal organs, has been called the abdominal pulse, the artery resembling a hard thrilling thread.

As observed in connection with surgical practice, inflammatory ferer is always a secondary affection. A most minute description of it is given by Professor Miller, which I shall endeavour to condense, and apply to what is observable in Veterinary Surgery; and, like Professor Miller, I shall consider the disorder of the general frame according to its systems.

1st. The Nervous.-There are, if one might judge, dull pains in various parts of the animal body, stiffness in the loins and in the limbs, restlessness, and a variety of postures, especially of the limbs, shifting from one leg to another, pointing of the feet; and when the respiratory organs are not the seat of the inflammation, frequent lying down for a short time; no desire for natural movement, dulness, walking performed sluggishly, and with manifest disinclination; watchfulness, the senses of sight and hearing being evidently exalted; and in aggravated cases as those arising from neglected wounds in the feet, delirium and coma may ensue, the eves red and suffused, the visible 
mucous membrane red and injected, and the skin hot and dry, or partial sweats bedew the body.

2d. The Vascular.-The pulse indicates excitement; it is increased in frequency, ranging from 60 to 90 , or even to 120 per minute, hard, rolling like a cord below the finger, ancl yielding but little to its pressure. The arterial coats are exercising an increased amount of tonicity, and resist the impulse of the blood; or an irregularity of movement in the artery may exist, and thus a thrill or jar is imparted to the finger. There is increased fulness, as if the vessel itself were enlarged and held a larger quantity of blood at each impulse; the heart is acting, not only more rapidly, but more powerfully, than in health, and the circulation is truly accelerated; frequency, hardness, or thrilling is seldom absent, but fulness may be wanting, and the pulse may be small instead of full. This is observable, as already mentioned, in serious abdominal disease. The small thready pulse always exists in connection with great nervous depression, and debilitated through rapid astion of the heart, to which circumstance its smallness is probably due. In affections of the brain, and in non-inflammatory disorders of the stomach producing coma, the pulse is commonly slow and full, the suspension of cerebral influence appearing to diminish the rapidity, without affecting the force of the heart's action. There are peculiarities also to be taken into account. The pulse may be naturally slow, intermittent, or rapid; in the common-bred cart-horse, perhaps not above $30 \mathrm{or}$ 35 ; in the thorough or highly-bred horse, especially if he be of a nervous temperament, 45 or 50 ; and in some animals of both descriptions intcrmitting, from some unknown cause or peculiar idiosyncrasy. Allowance must be made for these when previous inquiry has satisfied us that our patient is the subject of one or other of these peculiarities.

$3 d$. The Respiratory.-Pespiration is quickened, the breath is hotter than usual-in stable phraseology, the horse blows.

4th. The Digestive.-The appearance of the tongue does not vary in our patients as much as in the human being, but the mouth is dry, hot, and injected, soapy, lathery, and sometimes the tongue is contracted, and the secretions of the mouth sour or even fotid. There is sometimes very great thirst, loss of appetite and loathing of food, and the bowels are constipated. 
5th. The Secerning.-The secretions and excretions in general are materially diminished; the bowels are constipated, mainly from want of mucous secretion from their lining membrane; the skin is hot and dry; or, if pain be the characteristic of the local inflammation, there will be partial sweats on the surface of the body; the mouth is parched and dry; the urine is scanty, highcoloured, sparingly aqueous, and holding much saline matter in solution.

6th. The Nutritive.-Digestion and assimilation are interrupted; as the fever advances, so does emaciation; and strength becomes more and more reduced.

As already observed, there is no fixed relation between the degree or intensity of internal inflammations and the constitutional fever attending them; nor is the fever always proportioned in its degree of violence to either the size or importance of the part inflamed. This is insisted on by Drs. Alison and Watson, and their conclusions may be accepted, and shortly stated. Dr. Alison writes:- “In some cases where we are sure that we have had inflammation going on under our inspection to extensive effusion of pus, the pulse has been feeble, the skin cool and damp, and the patient exhausted and faint on the slightest exertion; while in others there is high and more inflammatory fever, and in some of these the organ inflamerl has been so to no extent, and its function comparatively little affected, but yet the patient has become comatose early, as in typhus, and died so." Every veterinarian of experience will be able to bear out the correctness of these observations from the statistics of his own practice. Many inflammations will have come under his notice where the danger has been comparatively slight, and the fever high; and others where the local inflammation has been most imminent, and the accompanying fever has been hardly observable. The situation, the extent, and the degree of the local inflammation being the same, the fever generally runs higher in the young plethoric animal than in the more aged class of patients. Young horses newly bought from the breeder, and brought into large towns, suffer more severely from fever, when the local disease is perhaps very trivial, than those which are used to town work, town diet, and nanagement, and are in what is termed condition. The type of the inflammatory fever is modified by other circum- 
stances, such as the state of the atmosphere, ventilation, drainage, and when any zymotic disease is associated with the local inflammation. Animals which are located in country districts present a much higher type of fever, and bear bloodletting much better than those crowded together in large town establishments. Again, the fever is modified in its expression by the nature of the part that is inflamed. The pulse and fever that accompany acute laminitis are at once characteristic of the disease, and no man of common observing powers would fail to recognise this formidable malady if he once felt the pulse, strong, hard, and full, and continuing so for days even after the vital powers have begun to fail. The type of the fever undergoes a change when suppuration takes place; when it (the fever) continues long, and when mortification or gangrene occurs to any extent. The febrile state generally follows the local disease, but there is good reason to believe that the pyrexial condition and the condition of inflammation in a part may be excited, in some instances, conjointly; or, at all events, their periods of coinmencement may correspond so closely that it is difficult to conceive that one is the effect of the other. There is every reason to believe that, in some instances, the fever precedes the local inflammation; as in strangles, where it may be said to be present in some cases for an indefinite but short period before there is any manifestation of local symptoms. The animal is then said to be "breeding strangles," and in epizootic pneumonia in the horse, I am convinced that, in many instances, the local inflammation succeeds the fever, and that much of the non-success in the treatment of this disease has arisen from ignorance of this fact.

When inflammation proceeds to suppuration a severe fit of shivering is often the first indication of the formation of pus. The nature of the fever then alters, and becomes characterised (except in laminitis, and acute suppurative arthritis, or an open joint) by softness and feebleness of the pulse, and by much nervous prostration and debility. A copious formation of pus may proceed from a mucous membrane without any appreciable degree of suffering, whilst a most trifling amount of pus from a serous or synovial membrane will be often associated with fever of even a fatal character. In any case, the character of the fever depends greatly on the constitution of the patient: if this 
be good, the fever is characterised by much heat, and a strong, full pulse; on the contrary, if the animal's constitution be impaired, or if it be out of condition, the fever is of a low type, called typhoid, asthenic, or adynamic, and is characterised by prostration, sinking of the pulse, great impairment of the heart's action, with a tendency to collapse; and the skin is covered by cold, clammy sweats. Sometimes these adynamic characters pass into that typhoid state in which nervous symptoms, such as delirium, coma, and tremors, prevail. These characters are known as ncrvous or ataxic. There is unconsciousness, grinding of the teeth, the mouth becomes foetid, the tongue dry, and tremors affect the voluntary muscles. This form sets in as a result of some unhealthy tendency of the inflammatory process, or when mortification of the inflamed part occurs; but any cause by which the system becomes vitiated will bring about this kind of fever without death of the inflamed part, such as the poison of rinderpest, acute glanders, and other allied diseases. Putrescence of the products of inflammation, degenerating and decomposing, poison the fluids circulating amongst them, and when absorbed induce the typhoid state. This form of fever tends to death by complete sinking of the circulation, with diminution and loss of animal heat; or deepening stupor, with oppressed respiration, supervenes; or the animal dies by combination of both asthenia and coma.

Be it understood, however, that sympathetic inflammatory fever is especially marked by the absence of certain symptoms which distinguish the idiopathic fever, more especially of petechiæ, and of any special eruption on the skin.

\section{INFLAMMATORY APPEARANCES OF THE BLOOD.}

The chief appreciable change in the constitution of the blood is a great increase in the quantity of its fibrine, which, according to Andral and others, may range from $2 \frac{1}{2}$ to 10 per 1000 parts. It is, however, a very difficult matter to ascertain with any certainty the exact increase, for it is impossible, by any process yet discovered, to separate the fibrine from the white corpuscles of the blood.

Some pathologists state that there is an increase of the 
red globules during the very early stage of the inflammation; but all are agreed that, as the inflammation advances, these diminish in number, falling considerably below their normal quantity.

As already pointed out, the white corpuscles are increased in the vessels and tissues of the inflamed part, and where the lymphatic system is excited by the inflammation, in the circulation generally.

The buffy coat is always found in blood drawn from the healthy horse; consequently, this appearance cannot be depended upon as proving the existence of inflammatory disease, but the fibrine of the blood during the inflammatory process has an unusual tendency to separate from the colouring matter, and to the aggregation of its particles during coagulation; the clot or crassamentum is firmer and denser than that of healthy blood, and the surface is hollowed out into a cuplike form, in which case the blood is said to be both buffed and cupped.

Much difference of opinion exists as to the production of the buffy coat, and I would beg to refer the reader to the observations of Dr. Richardson of London, Professor Lister, London, and others, for further information.

\section{TREATMENT OF INFLAMMATION.}

If the practitioner is called to a case of injury of any kind, his first duty is to prevent inflammation by every means in his power. In the case of a punctured foot, or any other injury where the cause is still remaining, he has to remove that cause, and, by enlarging the wound, allow the escape of any blood or serum that may have collected around the foreign body, and in its immediate vicinity. He will also apply soothing remedies to the part, such as warm or cold applications or poultices, and enjoin strict quietude. I prefer warm to cold applications, and recommend them strongly in the majority of cases. It is also a safe and commendable practice to administer a purgative, consisting of from four to six drachms of aloes in a ball: by pursuing such a line of treatment, every part of the inflammatory process may be averted, or at least modified to a considerable extent.

But if inflammation be fully established, and the exciting 
cause still remain, the duty of the practitioner is to effect its removal as speedily and as effectually as possible. This having been accomplished, the inflammation may be subdued by comparatively slight means, and with little danger to the patient. But if this important preliminary be neglected or imperfectly performed, the most powerful remedial measures may be employed with no avail. If, for example, the practitioner be called to a case of lameness caused by a foreign body in the foot, or by pressure of the shoe upon some one particular spot, he will remove that shoe or that foreign body, give an outlet to the serum or pus which it may have created, and by a gentle purge, a poultice, and rest, dissipate all the formidable symptoms, the local inflammation, and the general fever. But, on the other hand, if he leaves the foreign body in the inflamed part; neglects to give an outlet to the discharge; gives purgatives and sedatives; bleeds, foments, and poultices; blisters, or pursues all the most heroic methods of treatment-what will be the result? Increase of the lameness, of the local inflammation, and of the accompanying fever; and if death of the animal does not ensue, the pus will force its way out at the coronet, disconnecting the hoof from the sensitive foot, and producing such a morbid condition of the part as may take a long time to recover. This is not a fanciful illustration, as the majority of practitioners can bear testimony, and some of them to their cost and annoyance. Let it be remembered that the first step in the treatment of every disease, whether surgical or medical, is to remove the cause: all treatment is of minor importance compared with this. "Remove the cause, and the effect will cease," is an old maxim which should never be forgotten by the veterinary surgeon. When the cause has been as effectually removed as may be in his power, he will be in a position to proceed with remedial measures. Those which are opposed to the advance and persistence of inflammation, are termed antiphlogistics; and one of the most important of these is blood-letting, which is of two kinds, namely, local and general.

Until a recent period, bleeding was considered by almost all as the great thing needful in all acute inflammatory affectionsthat it cut short as well as cured every inflammation. I shall 
now endeavour to explain the views of the old and of the new school of therapeutics, upon this important point.

The Old View.-Of all antiphlogistic remedies, the most important was blood-letting; and the effect of general bloodletting in arresting inflammation was said to be owing principally to its lessening the force of the heart's action, causing derivation of the blood from the part, and facilitating the action of other remedies. "A sedative result on the heart's action is effected by withdrawing from the central organ of circulation a part of its natural stimulus, the blood, by which its action is habitually maintained, and partly by the intervention of an impression produced on the nervous system; it being well known that the sudden diminution of pressure on the brain and medulla oblongata has a remarkable effect in diminishing the frequency and force of the heart's action."

Dr. Alison says:- "The effect of blood-letting in causing derivation from parts actually inflamed to other parts of the body has not been studied with so much care as might have been expected from the pains bestowed upon it by Haller. Whether this effect is, as he thought he had ascertained, inexplicable on merely mechanical principles; or whether, as Magendie and Poisenille assert, it is merely the effect of the contractile power of the vessels, and the forced state of distension in which they exist during life, causing a flow to any point where an opening is made; it is quite certain that a movement in that direction is immediately perceived in all the small vessels that can be seen under the field of the microscope, on a puncture being made in any one of them; and in Haller's observations it distinctly appeared that this movement often inverted the natural course of the circulation, and often extended to blood stagnating in vessels, and caused globules to separate, and become distinct, which had previously combined in irregular masses. This being so, it cannot be doubted that similar changes must be effected, in a greater or a less degree, in the blood stagnating in inflamed parts, when an exit is given to the blood from other parts of the circulatory system, whether by general or local blood-letting. And it does not seem possible to understand on what other principle than this blood-letting can be useful, as it undoubtedly is, in certain cases of inflammation, chiefly abdominal, when the pulse is smaller and even feebler than 
natural, but becomes fuller and stronger; or in others 'chiefly of the head and abdomen likewise), when it is slower than natural, small and sharp, and becomes more frequent and fuller after the evacuation."

The action of other remedies is facilitated by blood-letting. " By lessening that morbid impetus of blood and increased tone of the vascular coats by which, during the state of inflammatory fever, the natural secretions are apparently impeded, and at the same time promoting absorption into the blood, as loss of blood is well known to do, it favours the effect of all other evacuating remedies; and further, by its precedence it renders certain remedies-as mercury and opium-decidedly beneficial, which otherwise would have proved either incperative or absolutely injurious."

To this view is opposed that of the late school of therapeutics; and by the followers of that school it is believed that all bleedings and other antiphlogistic remedies are entirely opposed to a sound pathology. Dr. Bennett says, that when an exudation has once occurred, in order that it may be removed, it must undergo certain changes or transformations, for which an increased supply of blood to the part is absolutely necessary; that blood-letting can in no way lessen the amount of blood in an inflamed part, or assist in the excretion of morbid products from the vital fluid; that the character of the pulse cannot be a safe guide to the propriety of bleeding, as its condition is the result, and not the cause, of the inflammation; and that the increased throbbing and circulation of blood about an inflamed part is a result of the inflammatory process-a wise provision, as he says, of nature, to further vital changes, and one which ought to be assisted rather than opposed. Such are the views of, first, the antiphlogistic school, and, secondly, of those who hold that it is impossible to cut short an inflammation, and make it the rule of practice to further the natural changes necessary for the removal of the products of the inflammatory process.

I must confess that I can neither assent to the views of the extreme antiphlogistians nor to those of the writers who condemn bleeding altogether, being quite confident that I have seen many cases of congestive inflammatory disease cut short by a timely abstraction of blood. I have experience of the method 
of treatment that was in vogue twenty years ago, when repeated and heavy blood-letting was the rule, and from my recollections I cannot but severely censure such an irrational method. Where local bleeding can be effected, it is preferable in all cases to general blood-letting.

General blood-letting is commonly performed by opening the jugular vein in our patients. For this purpose a fleam is preferable to a lancet, for reasons that need not be discussed here. The operation is termed venesection, or phlebotomy. If the blood is drawn from an artery, the operation is called arteriotomy, and is best performed by a lancet. In some cases of cerebral meningitis, the temporal artery has been opened with good effect, and the wound in the skin closed by means of a pin, retained in its position by a small quantity of tow or thread. If the hæmorrhage is not arrested by this method in the case of arteriotomy, a pin or needle can be placed underneath the artery. By acupressure in this way, it will be most effectually arrested, at the cost, however, of the vessel, which will become obliterated at that spot. But this is of no moment, as the collateral circulation will be sufficient to maintain the integrity of the part.

A cow requires a larger fleam than the horse. Of this fact the instrument maker is well aware, and the intending phlebotomist can get fleams suitable for all his patients.

Local blood-letting is performed upon the inflamed part, or as near to it as possible, and the object is speedily to unload the engorged blood-vessels. It may be done by scarification, incision, or puncture. In conjunctivitis, for example, it may be drawn from the vessels of the inflamed part by scarifying the inner surface of the eyelids, or by opening the angular vein. In inflammatory disease of the foot, any quantity can be obtained by puncturing the coronary plexus of veins.

When general blood-letting from the jugular is practised, the animal's head should be elevated, and a full stream of blood allowed to flow from a sufficiently large orifice in the vein. If this be done, a smaller amount of blood drawn will make a much greater impression on the pulse than when the orifice in the vein is small, the blood flowing in a trickling stream, and the head depressed.

The question of a second blood-letting remains to be con- 
sidered. If the blood flows freely from the vein during the first bleeding, and if the animal stands a full blood-letting, say from five to seven quarts, without manifesting symptoms of syncope by sighing and sweating, he may be considered to have borne the operation well; but if, on the contrary, the patient manifests these signs shortly after the vein is opened, it will be well for the practitioner to desist from further depletion. The urgent symptoms of the disease may by the bleeding be relieved, but may return again after a longer or shorter period, and demand a repetition of the remedy; but before this is done the reaction of the system generally, the local symptoms, and the urgency for relief, must be taken into consideration. If the reaction be great, and of a sthenic character, with the pulse full and strong, the operation may be repeated; but if it be asthenic, the pulse rapid, quick, and jerking, the respiration oppressed, with partial sweats on the body, and the extremities cold, the bleeding is not to be repeated. The appearance of the blood after it has coagulated is not of very great service as an indication either for re-bleeding or refraining from it. The firmness of the coagulum has been considered as a mark of the tonic state of the system, and as a warranty for repeating the bleeding when the part is as yet unrelieved, and the reaction continues of the sthenic type. On the contrary, a looseness of the consistence of the clot is a sign of weakness, and that the bleeding should not be repeated.

The proportion of serum to the crassamentum, and also its altered character, are arguments for or against bleeding. If the quantity of serum is large, the bleeding should not be repeated. When the properties of the serum are so altered that it coagulates and forms one mass with the clot, bleeding is always injurious; and when the serum, which has little or no affinity to the red globules in health, readily dissolves them, it is an unerring sign that further bleeding should be avoided.

There are many instances for not esteeming the firmness and dimensions of the buffed coat as an indication for bleeding, even when it has the cupped appearance; for this condition exists in many debilitating affections, more especially in epizootics affecting the fibrous and serous membranes; and no one now thinks of bleeding in these diseases. 
It is well known that the buffy or sthenic appearance of the blood depends greatly upon the manner in which the blood is drawn. If this is done in a full stream, these characters are ever present in the healthy horse; but if the stream be slow, and if the blood runs down the side of the vessel, there will be little or no buffy appearance. The form of the vessel into which the blood is received, and its temperature, will also affect the process of its coagulation. Therefore, as already mentioned, the mere appearance of the blood is not a guide to the repetition of the bleeding. As a rule to be safely followed, one good bleeding from a strong and previously healthy patient is sufficient in nearly all cases.

Epizootic influences are opposed to blood-letting, and in epizootics of all kinds, even if the temperature indicates high fever, above $104^{\circ} \mathrm{F}$., we should not hastily have recourse to the fleam, but should remember that the disease depends on a morbid poison, has a course to run, and is not amenable to the mere abstraction of blood. "When the inflammatory fever has been insidious, so that the first stage has passed over unchecked, or modified by previously existing constitutional disease, or complicated with organic local disease; or when they denote debility, exhaustion, or the so-called typhoid state, they generally prove improper cases for blood-letting, even when seen within the first few days."-(AITKEn.)

The next important class of antiphlogistic agents in the treatment of many inflammations consists of purgatives, more especially the aloetic in the horse, saline in the ox and sheep, and of jalap or castor oil in the dog. (1.) They act by remov ing from and freeing the intestinal canal of accumulated food and frees, or other irritating and acrid matters. (2.) They subdue the inflammatory tendency by causing a discharge of a large quantity of serous fluid charged with albumen. They direct large quantities of blood to the intestinal mucous membrane, and they determine to the same surfaces a large amount of nervous influence, and thus act on the principle of derivation. They diminish effusion, and check the force of the heart's action. Aloes, in virtue of its nauseating properties, is most valuable.

The use of purgatives is indicated in inflammatory fever arising from all external injuries, unless they be of sush gravity 
as to necessitate the use of slings from the outset; if such be the case, the weight of the animal being thrown upon the abdominal viscera, renders the use of purgatives dangerous. In inflammatory fever arising from inflammations of mucous membranes, or when at any time the mucous membranes exhibit signs of irritation, even after an external injury, the administration of purgatives, more especially in the horse, is contra-indicated. In thoracic affections, even when the serous membranes are affected, they should be given with caution, but they are of great service in encephalitis, hepatic congestions, \&c.

Opium.-To subdue pain and soothe the nervous system, there is no remedy to equal opium. Its use is of vital importance in some inflammations, especially in inflammation of the intestines.

Aconite is another valuable remedy. Its beneficial effects are more apparent in cases of inflammation, accompanied by excitement rather than pain; it improves the tone, whilst it diminishes the rapidity, of the heart's action. Belladonna is a favourite remedy with some practitioners, but it is not so useful as either opium or aconite; it, however, seems to exercise a beneficial effect upon inflammations of the larynx.

Antimony in all its forms has no effect upon the heart's action in the horse or ox, but is successfully employed in the treatment of canine inflammations.

Mercury is but seldom employed in the treatment of inflammation; its use in both the horse and the ox has been followed by alarming, and sometimes fatal, symptoms. For many years cases have been treated by me without the use of mercury, and I am of opinion that it may be excluded from the list of antiphlogistic remedies.

Alkaline remedies in some manner retard the formation of fibrogenous elements in the tissues; they also increase the secretion of urine, and particularly the expulsion by this channel of the products derived from destruction of the albuminous compounds of the body. Alkalies, having combined with acids in the system, generally pass out of the body as salts, and tend to leave behind them an excess of alkali in the blood. For this reason, the neutral salts of the alkalies suit the lower animals, more especially the horse and ox (in which 
acidity is not such a normal condition of system as in man and the dog). We therefore find that the nitrate of potash is a most valuable antiphlogistic.

Stimulants.-When, after the subsidence of inflammatory excitement, the process of repair does not progress satisfactorily, when in fact it hangs fire, either from excessive severity of the inflammation, depressive treatment, or other influence, it may be necessary to have recourse to stimulants applied to the part itself if superficially situated, or administered internally, when the seat of the inflammation is beyond our reach. But stimulants, particularly alcoholic stimulants, whether wine, spirits, or beer, must be very carefully administered-particularly in horse practice-and only persisted in when their beneficial effects are perceptible. If they improve the appetite or pulse they may be continued, but not otherwise.

Local stimulants to superficial parts, after the subsidence of active symptoms, will often promote their recovery; thus an ulcer or superficial mucous membrane may be touched with nitrate of silver or other stimulant, or stimulated with cold water. Where joints or synovial membranes are to be treated, the stimulating effects of blisters will extend to deeper structures.

One other remedy in the treatment of inflammation is counter-irritation, and this is supposed to act by producing artificial metastasis, or a removal of the diseased action to less important parts of the body than that originally affected. I need not here enter into a discussion of the value of blisters and other counter-irritants. They are useful when properly applied, and very injurious, especially in the horse, when indiscriminately used. Their beneficial effects are most apparent in inflammations of the joints, superficial fibrous structures, and in inflammation, acute and chronic, of the mucous membranes. Their injurious effects are seen in acute inflammations of the serous sacs, of the pulmonary parenchyma, and in extensive diseases arising from depressing zymotic influences.

"The more intense forms of counter-irritant treatment are so painful that it is well worth while to be critical as to their value; and it deserves more general notice than it has yet received, that some of the most accurate clinical observers of the day are profoundly sceptical on this subject. In various cases 
of thoracic inflammation, for instance, where thousands of practitioners employ blistering as a matter of course, the unsurpassed authority of Professor Skoda pronounces such treatment to be always powerless for good, though sometimes powerful for harm. And probably a large proportion of treatment by long-continued setons and issues has subsisted, less from any sure knowledge of its doing good, than as a remnant of the old belief that morbid humours could thus be set running from the body. Assuredly the whole subject requires careful clinical reconsideration; towards which, in this place, only two suggestions are offered.

"In the first place, particularly with regard to the uses of blistering, there is a source of fallacy against which the student will do well to guard himself. Not every cutaneous inflammation excited for surgical purposes is intended to be counterirritant and derivative. There are cases (presently to be again adverted to) where it acts simply as a further stimulant to the part originally inflamed. When, for instance, we apply strong blistering fluid directly over a knee-joint with chronic inflammatory effusion, the action of the irritant propagates itself, in lessening degrees, through the intervening small thickness of parts, and sensibly affects the synovial surface; where frequently at first it causes some increase of effusion; and where at any rate the desired removal of fluid only begins when the superinduced excitement has begun to subsicle. And it is by reason of this action that, with regard to superficial parts, blistering, if it does not resolve the inflammation, commonly determines them to suppurate; a fact, sometimes illustrated in the treatment of indolent inguinal buboes, where it may happen that blistering is deliberately used in order to force this alternative on the part, and, either by one way or the other, to bring the inflammation to a close.

"Similarly, we may sometimes be proceeding rather too drastically when we blister the walls of the visceral cavities. I have often seen cerebral distress appear to be much aggravated by the application of a blister to the scalp; and it has happened, in making the post mortem examination of a patient to whose abdomen a blister had been applied, to find on the inner surface of the abdominal wall a red patch (probably of more injected muscular substance seen through the peritoneum) corresponding to the area of blistered skin. 
"In the second place, it deserves full trial whether every intense and consequently painful form of counter-irritation might not be superseded by the employment of other means, less intense, but more extensively applied; whether, for instance, ten inches of poultice may not be equivalent to three inches of blister, or to one inch of issue. For probably among the agencies now spoken of there is none to which we can look with better grounded confidence than to the diffuse local action of warmth, as supplied by poultices and fomentations; particularly when it can be so administered as to affect a surface of skin very greatly larger than the quantity of inflamed texture which we desire to relieve.

"In many chronic-probably also in some acute-inflammations, especially in those which arise from catching cold, or are associated with gout or chronic rheumatism, the excitement of the entire skin by baths of hot air or hot vapour is often of the most striking and immediate benefit. And though in most of these cases the result of the exterior heat is probably something more than mere blood-derivation to the skin, yet not the less on that account are they important illustrations of counter-stimulant treatment.

"Pending the better settlement of what is doubtful in the present subject, there are cantions which every one admits to be necessary if the counter-irritant treatment of inflammation is to succeed according to its intention. In proportion, namely, as the counter-irritants which we employ are of severe local action, we must take care-first, that they be not so applied as to involve the inflamed part in their direct irritant operation; and, secondly, that they be not so applied as to aggravate any existing febrile disturbance."-(Smon.)

\section{TREATMENT OF CHRONIC INFLAMMATION.}

All depressing remedies are contra-indicated, and the object must be to rouse and stimulate, not only the part diseased, but the system generally, by good food, iron, quinine (and iodates, if there is any deposit or thickening), and by the direct application of stimulants to the part itself, when superficially situated.

It should not be forgotten, however, that all mere remedies are but of secondary consideration in the treatment of inflant- 
mation. The removal of the cause is the first essential, and after this, absolute repose of the inflamed part. If these considerations are kept in view, and the practitioner is aware that the tendency of most inflammations is to a favourable termination, he will understand that, in many cases, the accidental symptoms of urgency require treatment, rather than the disease itself. 


\section{CHAPTER IV.}

FRACTURES AND DISEASES OF BONES.

CAUSES OF FRACTURE-SYMPTOMS-MODES OF UNION-TREATMENT OF FRAOTURES - COMPOUND FRACTURES - COMPOUND COMMINUTED FRACTURES-SEPARATION OF EPIPHYSES-FALSE JOINTS AND NON-UNION.

A Fracture is said to occur in three ways:-1st. By external violence, operating directly upon the injured part: $2 d$. By external violence, producing such concussion upon the bone as not to break it where the force is applied, but at some other part: $3 d$. By inordinate action of the muscles, as in broken back. But some bones are more liable to fracture than others. The bones of the pelvis, shoulders, thighs, pasterns, legs, vertebræ, and of the face and skull, seem to be more frequently broken than the other bones of the horse. In the dog, the leg and thigh bones. Although bones are fractured in animals of all ages, it is worthy of notice that the bones of the old are more readily broken than those of the young.

Bones are rendered liable to fracture from trivial causes by a previously diseased condition. The navicular bone, by a peculiar atrophy or caries. The os pedis, by atrophy and fragility, induced by ehronic inflammation; and the pelvic bones, by a degenerative disease, partaking of the nature of fragilitus ossium and necrosis.

A solution of continuity of bone (fracture) may be transverse, oblique, or longitudinal, according as it is at a right or an acute angle with, or parallel to, the long axis of the part of the bone in which it is situated. A fracture is said to be simple when a bone is broken at one part, without any injury of soft parts; compound or open, when there is an open wound communicating with the broken bone; comminuted, when the bone is broken into several fragments; complicated, when, together 
with the fracture, there is serious injury of the adjoining structures, as laceration of vessels, open joint, or serious contusion of the tissues; fracture with wiund, when the wound does not communicate with the fracture; impacted, when one fragment is lodged in the other; and partial, when the continuity of only a part of the bone is broken. This last variety has been called by some bending, with partial fracture, and by others green-stick fracture. Bending may take place without fracture, but this, as well as bending with partial fracture, is very rare in our patients, although $I$ have seen it both in dogs and young horses.

\section{THE SYMPTOMS OF FRACTURE.}

When fracture occurs in one or more bones of a limb, the syrnptoms are-great lameness suddenly manifested, obvious deformity (with some exceptions, to be mentioned), preternatural mobility, crepitus, and inability to bear weight upon that limb. These are the general symptoms; the particular ones, as well as the causes of the several fractures, will be hereafter described, and it will therefore be unnecessary to refer to them under this head.

\section{MODES OF UNION.}

The injury inflicted in a fracture is rarely limited to the bone. The two or more fragments, driven in opposite directions, penetrate and wound the adjacent tissues, giving rise to more or less less hæmorrhage. If the skin is broken, suppuration generally follows, and the repair is attended with difficulty; but if the injuries are subcutaneous, and the air has no access to the damaged part, the repair is more easily effected.

The extravasation of blood about fractures is not only uncertain in amount, but unequal in the several tissues. Its presence is useful for diagnosis, particularly in the diagnosis of fracture of the humerus.

A ridiculous idea has prevailed amongst horsemen, that fractured bones in the horse never unite. This is incorrect, and, owing to the same tendency that produces splints, syavins \&c., the process of union and repair is a very rapid and efficient one, provided the solution of continuity does not extend into a 
joint having extensive motion, and that the fractured cnds can be liept at rest.

An early consequence of fracture appears to be an exudation of lymph, which is at first dimly granular, but becomes, at a later period, ruddy, elastic, or moderately firm and succulent. It soon attains firmness, when it is called a callus.

There are two methods according to which the callus may

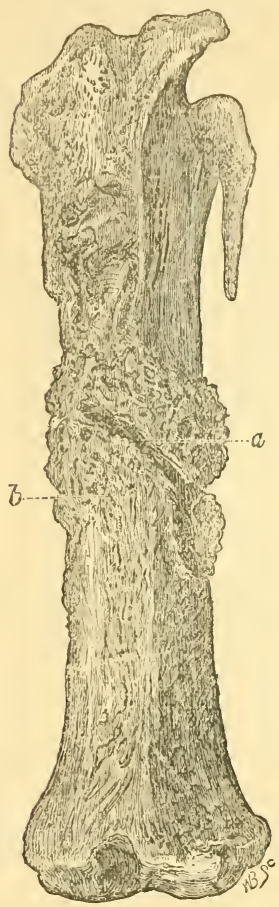

FIG. 9.-Oblique fracture of the tibia, with external (ensheathing) callus, marked $a$ and $b$. The line extending from $a$ indicates the seat of the fracture, the new deposit (callus) being purposely removed.

be placed. In one method the broken ends or smaller fragments of bone are completely enclosed in a new material; they are ensheathed or held together by it, as two portions of a rod might be by a ring fastened round them both.

The new material, in such a case, surrounding the fracture is termed " provisional," “external," or "ensheathing" callus.

In the other method the new material is only placed between those parts of the broken bone whose surfaces are opposed; between these, it is inlaid, filling the space that would else exist between them, and uniting them by being fixed to both (like the process of gluing two pieces of wood). Reparative material thus placed has been called intermediate callus. In either method, there is usually some reparative material deposited in and near the medullary tissue, and this is called interior callus.

In fractures that oceur in the lower animals, with the exception of those found in the fixed bones, the ensheathing callus is usual.

From the researches of Haller, Duhamel, Hunter, Dupuytren, Paget, and Stanley, we find that nature never 
accomplishes the union of a fracture without two successive deposits of callus, and Dupuytren arranged the phenomena, from the time of fracture to the exact and complete reunion, into five different periods.

In tice first stage, comprehending a period of eight or ten days, blood is extravasated into the medullary canal between the fragments, and under the periosteum, raising up the latter from the bone for some distance above and below the fracture; the medullary membrane becomes swollen and separated from the bone, and the periosteum is not only raised from the bone, but also becomes red, soft, swollen, and preternaturally vascular. The fragments of bone may thus be said to be surrounded with blood, which not only fills the medullary canal and space between the fragments, but also separates the latter from the detached periosteum. This blood may be organized or become absorbed, and liquor sanguinis effused into the parts at first occupied by it.

In the sceond stage, comprising the interval between the tenth or twelfth to the twentieth or iwenty-fifth day, the "tumour of callus," as it is called by Dupuytren, is formed. The substance between the periosteum and bone is converted into a structure like fibro-cartilage, and within the medullary canal there is also developed a fibro-cartilage, but the substance between the fragments retains the appearance of coagulable lymph.

In the third stage, extending from the twentieth or twenty-fifth day to the thirtieth, fortieth, or sixtieth day, according to age and strength, the fibro-cartilage between the periosteum

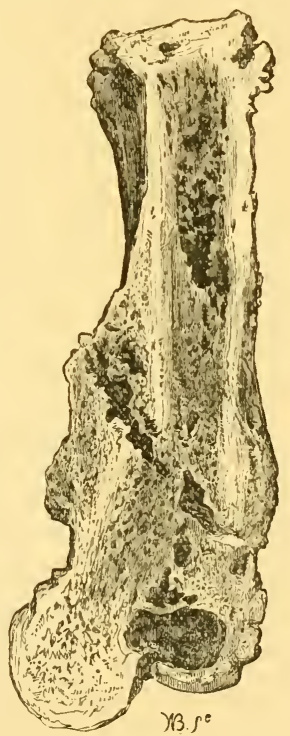

Fig. 10.-Fracture of meta. tarsal, showing the plug within the medullary canal. The plug is ossified. and bone, and that within the medullary canal, are both con. verter into bone, the external forming a ring, ferrule, or clasp; and the internal a plug, or peg, filling up the medullary canal, 
and together constituting the provisional callus. The external ring, embracing both fragments, and the plug, within the medullary canal, constitute nature's provision for keeping the fragments in apposition and at rest. The substance between the fragments is, during this stage, changed into fibro-cartilage.

In the fourth stage, extending to the fifth or sixth month, it is converted into bone, constituting the permanent or definitive eallus.

In the fifth stage, extending from the fifth or sixtl month to the tenth or twelfth, the provisional callus, being no longer necessary, disappears, and the medullary canal is restored. Such are the views of Dupuytren; and such is the method by which fractures are repaired in the lower animals. The only exceptions which I have observed, have been the union of fractures of the lower maxillary bone, when the fracture has been longitudinal, and bound so firmly as to admit of no motion whatever between the fragments. Doubtless, the fractures of our patients would unite, as those of the bones of the human being, by the reparative material being thrown out between the broken ends-that is, by intermediate or permanent callus-provided the parts could be kept in a state of complete repose; but this being impossible, nature has provided a method by which they are held in a state of quietude, until the permanent callus is finally deposited between the broken fragments.

The latter part of the process of repair is that of the shaping and the modelling of the fragments and their bond of union. These consist of-(1.) The removal of sharp projecting points and edges from the fragments; (2.) The closing or covering of the exposed ends of the medullary tissue; (3.) The formation of a compact external wall and cancellous interior for the new bone; and (4.) The making of these continuous with the walls and cancellous tissue of the fragments. The first is effected by the absorption of the offending points and angles, and in the absorption of bone the earthy matters are first removed. The closing or covering of the parts of the broken medullary tube, which are exposed in fractures with much displacement, is slowly accomplished by the formation of a thin layer of compact tissue similar to that which covers the cancellous tissue at the articular ends of bones.

The callus, before ossifying, may become, according to Paget, 
either fibrous or cartilaginous, or may assume a structure intermediate between these, and in any of these cases ossification may ensue. In different specimens, or sometimes in different parts of the same, the reparative material has in one displayed fibrous tissue with a few imbedded corpuscles, like the large, nearly round, nuclei of cartilage cells; in another, a less appearance of fibrous structure, with more abundant nucleated cells, having all the characters of true cartilage cells; and in another, a yet more nearly perfect cartilage. Through any of these structures the reparative new bone may be formed. It may be formed, first, where the reparative material is in contact with the old bone, and thence extending, it may seem as if it grew from the old bone; it may be also formed in the new material in detached centres of ossification, from which it may extend through the intervening tissues, and connect itself with the old bone.

The new bone, by whatever mode it is formed, appears to acquire its proper microscopic characters. Its corpuscles, or lacunæ, at first simple, round, or oval shaped, become jagged at their edges, and subsequently acquire their canals, which appear to be hollowed out in the pre-formed bone as minute channels communicating with one or more of the lacunæ. The laminated canals for blood-vessels are later formed. At first, all the new bone forms a minutely cancellous structure, which is light, spongy, soft, and succulent, with a reddish juice, rather than marrow; and is altogether like the bones of the foetus at their first construction. But it gradually assimilates itself to the structure of the bones that it repairs, its outer portions assuming a compact laminated structure, and its inner acquiring wider cancellous spaces and a more perfect medulla. It acquires a definite periosteum, which is at first thin and lamellar, but gradually assumes toughness and compactness.

Repair of fracture by formation of a false joint is an arrestment of the process before ossification has cornmenced.

TREATMENT OF FRACTURES.

In the treatment of a fracture, it is of great consequence to perform reduction as soon as possible after the injury is sustained, in order to prevent the bad effects of continued irritation, and before the occurrence of swelling and thickening of the 
parts which surround the broken bones can prove an impediment to their proper adjustment. When swelling and tension are actually present, no time should be lost in vain attempts to allay these by means of fomentations, \&c., but the practitioner must at once reduce the fracture-the horse being first placed in slings (see Frontispiece)-and place the limb in a steady and fixed position by means of splints and bandages. The source of irritation being thus removed, the swelling will soon disappear. The best material for splints is strong leather, what is called "bend-leather," the pieces being made sufficiently long to extend to a distance beyond the superior and inferior articulations of the fractured bones, and broad enough to envelop and enclose the whole circumference of the limb. Holes may be cut in the leather where the splints pass over any sharp eminence, as, for example, over the trapezium in the knee. Gutta-percha is recommended by some practitioners, and it answers very well, but I prefer the strong leather. Before it is applied, it should be well soaked in warm water; when thus softened, it may be moulded to the shape of the limb with the greatest ease. The splints are to be retained in their position by bandages. When swelling and tension are present before the fracture is reduced, the splints may be maintained in position by the looped bandage; which consists of strips of calico, about two or three inches broad, and long enough when folded double to pass round the limb, with a few inches of excess; one of the ends is then drawn through the loop, and tied to the other. This bandage is useful when the degree of tightness requires to be altered, but it must be replaced by the common roller as soon as the swelling has subsided, and supplemented by one or two layers of bandages saturated with starch, dextrine-or what answers the purpose equally well, and is much cheaper-flour paste, made by boiling flour in water. When dry, the bandages become immoveable, and support the limb so effectually, that the animal soon puts weight upon it. In using the starch bandage, great care is requisite in its adjustment: it must not press unequally upon any part of the limb, all hollows are to be padded with tow, and it must never project beyond the extremities of the dry bandages, which should always extend below to the foot: or its edges, becoming hardened, may cut and wound the skin, causing irritation and pain; rendering the patient uneasy, feverish, and the limb liable to mortifi- 
cation by the swelling so produced. Another method of bandaging is by the careful application of tarred eord to the whole limb over the leather, the cord commonly used for thatching stacks answering every purpose. The animal, if a horse, is to be kept in a state of quietude in the slings for a period varying from two to three months. In foals and young unbroken horses, the slings must be dispensed with, and it is wonderful to see how carefully an animal,-gay and spirited, perhaps, before the accident,-will nurse the broken limb; for such, in addition to splints, bandages, \&c., a comfortable loose place, bedded with sawdust, chaff, or short straw, is all that is necessary. When the bones of horned cattle are fractured, they must be treated exactly in the same manner as those of the young horse, slings being as a rule inadmissible. The limbs of dogs when broken require nothing but the starch bandage, and in the course of a very few weeks they will be found completely recovered. Such, then, are the general principles whereby fractures are to be treated. I have had experience in the use of the plaster of Paris (sulphate of lime) treatment, and can recommend it. It is also recommended by Mr. Broad of Bath, and Mr. R. Spooner Itart of Calcutta, who state that, if properly adjusted, it forms the best material for retaining fractured bones in proper position. It is applied as follows:Cut thin calico into narrow slips, mix the gypsum with cold water to a consistence thicker than cream; the bandage is then to be soaked in it, rolled up quickly, and bound round the leg, but not too tightly, the animal to be kept still for a few minutes to enable the plaster to set firmly. To prevent dogs from biting it off, Mr. Broad recommends that the bandage be sprinkled, before it sets, with cayenne pepper. Splints made of block-tin form a very convenient apparatus for retaining fractured bones in their proper position. They are easily made to the shape and form of any part of the limb, are light, easily applied, and retained in position by bandages. They should be rounded at their edges, and all spaces between them and the irregularities of the limb padded with tow. Mr. Robinson, V.S., Greenock, was the first to suggest the idea to me. He finds them useful in broken knees, and other injuries where suppression of motion is desirable.

An animal may be lame for some weeks or months after a fracture has united; but if no articulation is involved 
recovery will gradually become complete. Some practitioners fire their patients for the removal of such lameness; such practice cannot be too highly condemned. It is useless, nay, mischievous and cruel. I wonder what would be said of a surgeon who fired for a broken leg! The thing is absurd.

\section{COMPOUND FRACTURES.}

By this term is meant a fracture having communication with the external air by means of a wound, which is produced at once by the same cause as that which gave rise to the fracture: or afterwards by one or more fragments being forced through the skin, or at a more remote period by slunghing and ulceration of the surrounding soft parts. This wound makes a most important difference in respect of the danger and difficulty of cure. There is apt to arise from this source violent inflammation and fever, terminating in profuse suppuration or gangrene. The object in treating such is to obtain immediate union of the wound, and thus convert a compound fracture into a simple one. There are many cases on record of a successful issue being obtained from the treatment of compound fracture in the horse; but in the majority of cases the terminations are very unfavourable. If the bone projects through the wound, and cannot be returned, unless in the case of a valuable stud animal, the patient had better be destroyed; but if treatment be determined upon, the first consideration is the reduction of the fracture; this may be done by extension and counter-extension, aided by proper manipulation. If the fracture be transverse, these means will be successful; but if it be oblique, it may become advisable to enlarge the wound, to admit of the replacement of the fragments. In some cases, replacement cannot be effected without removing the sharp ends of the protruding bone with a saw or bone forceps. When the fracture is reduced, the edges of the wound should be carefully brought together, and kept in close approximation by means of plaster, styptic colloid, or other adhesive substance.

After the use of these means, every endeavour should be made to moderate inflammatory action, prevent suppuration, by carbolic acid, or other antiseptic dressing to the wound, and to lessen febrile disturbance. In our patients means must be 
used to keep the fractured ends in their proper position, and splints must be applied. An aperture must be made in that portion of the splint covering the wound, so as to render its removal unnecessary during dressing, and to allow the escape of any discharge which may occur. By these means a compound fracture may be changed into a simple one-the wound uniting by adhesion. The constitutional disturbance must be mitigated by attention to the bowels (a small dose of physic may be given), by allowing the lightest possible diet, and by allaying the pain, if extreme, by opium; every unnecessary movement being at the same time avoided. When immediate union of the wound is not obtained, the practitioner need not despair, provided the constitutional irritation and pain be not extreme, nor the discharge from the wound excessive. When suppuration from the wound has taken place, the parts are to be bathed with tepid water, and the bandage over the wound kept constantly wet by the process of irrigation, to be described hereafter. No pus should be allowed to remain about the wound. All soiled dressings must be avoided; the weak carbolic acid solution must be applied frequently; and in some cases it will be requisite to syringe any pus-containing cavities with this remedy. The animal's strength must now be kept up by generous food; the stable or box kept sweet and well ventilated. If due attention be paid in all these respects, a recovery may be the reward. But too commonly unpropitious symptoms arise about the third day after the accident; the limb swells considerably; the discharge becomes profuse, thin, sanious, and foetid; there is great pain manifested; the fever runs high, the breathing hurried; the pulse is quick, and the appetite lost. In such a case an unsuccessful termination may be looked for, and it is better to anticipate it, and save the poor animal much suffering, by ordering its destruction. In horned cattle and $\operatorname{dogs}$ amputation has been performed, and the animals have gone about on three legs afterwards. I remember seeing a cow in a drove en route to London from Wales with a wooden leg, amputation having been performed above the knee.

\section{SEPARATION OF THE EPIPHYSES.}

Before the epiphyses are ossified to their shafts, they are apt 
to suffer separation from them by such accidents or vis,lence as would-later in the animal's life, and when the process of ossification had become complete-occasion fracture. The symptoms and treatment are the same as those of fracture.

\section{FALSE JOINTS AND NON-UNION.}

Fractured bones sometimes do not unite firmly together, their extremities remain quitc detached, or are merely connected by a fibro-cartilaginous structure.

The constitutional causes so common in man do not, as a rule, apply to the lower animals, but occasionally it is found that a condition of the osseus system, termed mollities ossium, and another partaking of the nature of necrosis, exist, and effectually prevent reunion of the bones. The most common cause of false joint is the want of, or the impossibility of securing, proper apposition of the fractured ends; and its most frequent situation, the anterior spinous process of the ileum. Indeed, it can be safely said that this fracture always ends in a false joint. When broken, the legs of dogs are sometimes united in this way, even after every precaution has been taken to secure all the essentials of recovery. This termination of the healing process may be looked upon as an example of arrested development of the reparative material ; every part of the process of repair being complete except that of ossification; the fragments being held together by a yielding and pliant band.

In order to secure perfect reunion of the fractured ends of bones, it is necessary-(1st.) That little or no motion should exist between them: $(2 d$.) That there be no excess of inflammatory action: $(3 d$.) That there be no interposition of pieces of muscle, tendon, or necrosed bone between the fragments; and (4th.) That there be a proper supply of blood to the part. Curling, Guerton, and others have paid attention to this subject, and have shown that non-union, as well as atrophy of bone, may be due to defective supply of blood, caused by rupture of the nutrient artery of the broken bone.

It is found that if the supply of blood be cut off by injury to the nutrient artery, so that the periosteum has exclusively to supply the blood, either one or both of the fractured pieces become atrophied, and their cancellated structure and walls thinned. While writing this paper, I had an oppor- 
tunity of seeing a case of non-union of the pelvic bones in a healthy animal. The bones were in apposition, the fracture being an oblique one through the shaft and spine of the ileum. Two months after the accident, the bones were moveable and crepitant; there were no inflammiatory signs nor pain present, the animal being in the best of health and spirits, but lame.

Treatment.-An ingenious method is recommended by Mr. Syme:- "This method was devised by Dr. Plyysick, of New York, in 1804; and it consists in passing a skein of silk or cotton between the extremities of the bone, and allowing it to remain until it appears that new bone begins to be formed, when it may be withdrawn and splints applied." Another plan consists in drilling holes in the fragments with the ordinary Archimedean drill, and fixing them in apposition by ivory pegs driven into the drilled looles. I think this method deserving of trial in compound fractures, even in the earlier stages. The pegs must not project from the surface of the bones, but must be cut off level, so that they do not wound the soft structures.

Blisters over the seat of fracture may be tried. They may, by causing the production of a fibrinous exudate, set up the healing process, which may ultimately end in complete consolidation of the disunited fragments.

One more method may be mentioned, namely, that recommended by Miller. It consists in introducing a long needle through the skin, passing it into the false joint, and cutting up the ligamentous bond of union, as well as the self-investing tissues on the bones; covering the opening with collodion and plaster, and keeping the fragments at rest.

There is great danger in allowing an animal to use his limb too freely soon after the apparent union of a fracture; as gradual yielding of the bone may take place, and deformity occur from the weight thus thrown upon the limb; hence the starch bandage should be retained for a considerable time, and the animal kept in a box for three or four weeks after his removal from the slings. The loose box will be better than the stall, as the animal will be able to take some little exercise, and thus allow, as it were, of passive movements of the limb, which will prevent thickening, or adhesion of the tendons to their thecæ.

When a fracture fails to unite, the causes of such nonunion may be looked for in a variety of circumstances; but the 
most common of these, in the lower animals, is the inability to prevent motion in the limb. Temperament has much to do with this. A calm, good-tempered horse may make a good recovery, whilst an irritable, fretful animal will keep an injured limb in a state of continual motion, will take unkindly to the slings, and thus render union an impossibility. In addition to or independently of this want of repose, even in a simple fracture, a piece of muscle or other soft tissue may be imprisoned between the broken ends of the bones, and if this is not removed, union is not likely to take place. When a fracture is oblique, this complication is not at all uncommon.

When a fracture is comminuted, and a large portion of bone denuded of its periosteum, it can be easily seen why union does not take place. Necrosis of the fragments will be almost sure to occur, leading on to suppuration, loss of sulstance, and the exhaustion of the patient.

Again, if the violence causing the fracture be very great indeed, the vitality of the surrounding textures may be destroyed, or the non-union may proceed from impaired vitality of the bone itself; and this may arise from the nutritious artery of the bone being implicated. In such a case, union of the deep-seated parts of the fracture would be very improbable.

Surrounding circumstances and food have an effect upon the repair of fractures. Where stables are overcrowded and ill ventilated, necrosis and suppuration will most likely occur; and if these be associated with improper food, it will be useless for the practitioner to undertake the treatment.

I have seen cases where union of the pelvis did not commence until the food had been changed. If it be possible to give green food, it should always have a preference over all other; failing this, currots, potatoes, or turnips should be allowed, in addition to hay and corn.

The constitutional causes of non-union are those of a debilitating nature, arising from old age, hard usage, or disease. If a great number of horses are stabled together, no matter how good the ventilation, drainage, food, \&c., may be, a peculiar taint is often produced upon their constitutions by an animal malaria, causing a cachexia, or condition of body in which the reparative powers are in a depressed and languid condition; in such the repair of fractures is effected with dificulty. 


\title{
CHAPTER $V$.
}

\author{
PARTICULAR FRACTCRES.
}

FRACTURES OF INFERIOR MAXILLA-FRACTURES OF ANTERIOR MAXILLA AND OF OTHER FACIAL BONES-FRACTURES OF CRANIAL BONES -FRACTURE BY "COUNTER STROKE"-CONCUSSION OF THE BRAIN - HERNIA CEREBRI.

FRACTURES OF THE INFERIOR MAXILLARY BONE.

1st. Compound fracture through the symphysis maxillaris, resulting from an animal falling upon the mouth. The direction of the fracture is longitudinal, involving the alveolar cavities of one or more incisor teeth, and extending through and terminating in the maxillary space; or obliquely, from the centre outwards, through the neck of one or both rami.

Treatment-Fracture through the Symphysis.-Remove loose teeth and foreign bodies; search for any loose pieces of bone, and remove them. When this is done, it will be found that the fragments can easily be brought into apposition by a little pressure, and so maintained by means of copper wire bound firmly round the incisor teeth. If the animal be a male, the tushes may be included in the wire. A calico bandage may then be applied, for the purpose of further strengthening the parts, and keeping out foreign bodies from the wound. Let the animal be kept upon soft but nutritious food; the wound looked to oceasionally, and washed out with a solution of carbolic acid to destroy the foetor which is sure to be present. In the course of three or four weeks the fracture will be found reunited.

If the practitioner suspects that any necrosed portion of bone exists in the wound, he must search for and remove it. Its presence may be suspected by a foetid, purulent, sanious dis- 
charge taking place from the wound, and by the animal not making that progress towards recovery which he has a right to expect.

2 d. Superficial fractures of the lower jaw occur from severe curbs and bits; anteriorly and within the mouth from the bit, posteriorly and under the jaw from the curb.

Symptoms from Injury of the Bit.-Dribbling of saliva from, or foaming in, the mouth; the animal shy or perhaps vicious if the mouth is touched; inability to bear the introduction of the bit; difficulty in masticating food; perhaps hæmorrhage from the mouth, or saliva streaked with blood. Upon examination, the buccal membrane will be found bruised, inflamed, and swollen, with perhaps a piece of bone sticking through it.

Treatment.-Pemove the small fragments. As the fracture is superficial, no bandaging is required; but the animal must not be bitted until the parts are completely healed and hardened, or he will have a bad or weak mouth ever afterwards; indeed, some horses that I have seen never allow a bit to be put into their mouths again without great struggling and resistance. The animal should be fed upon soft diet for some days after the injury, and the wound examined occasionally, as portions of bran, hay, or corn are apt to lodge in it, causing irritation, and retarding the healing process. If the wound discharge a foetid material, it should be syringed with a weak solution of carbolic acid; in any case, the mouth may be washed with this two or three times a day.

Symptoms from Fracture by the Curb.-Swelling and tenderness of either ramus immediately in front of the curb; sinuses shortly form, and within them loose pieces of necrosed bone may be detected by the probe. The discharge is curdled, fotid, but not very profuse. In some of these cases there is no primary fracture, but necrosis of the superficial layer of the bony tissue, arising from continued and severe pressure, producing periostitis, gangrene of the periosteum, and death of the bone, from the pressure, and non-supply of blood to that part of it covered by the gangrenous periosteum. In other cases a bony tumour forms here as a result of periostitis, and of increaserl thickness of the superficial layer of the bone (hyperostosis). In the first and second forms of injury, it is necessary to remove the fragments of bone, whether they are necrosed or not. It is 
waste of time to allow them to be removed by exfoliation. The method which I recommend, is to slit up the skin, and carefully scrape the diseased surface. If this be done, the cure may be effected in a very short time. If the necrosis is very superficial, one or two applications of dilute hydrochloric acid will effectually remove it, and render unnecessary the performance of an operation. Cornmon sense will convince the reader that the cause, namely, the severe curo, must not be again applied.

In the third form of injury, namely, the hyperostosis, all the the treatment necessary is the removal of the cause, and the application of soothing remedies, succeeded by frictions, with iodine ointment.

$3 d$. Fractures of the inferior maxilla occur at the boundaries of the alveolæ of the incisor teeth by the forcible "punching out" of the temporary incisors-more especially the corner bones. This cruel procedure is resorted to for the purpose of making the animal appear older than he really is. It cannot be too highly censured, and no veterinary surgeon should ever be guilty of doing such a thing. It can in no way benefit the horse, and the idea that the removal of the temporary teeth hastens the development of the permanent ones, is founded on the grossest ignorance. Doubtless, the permanent teeth are sooner brought into view. This, however, does not arise from any increase of their growth, but from the removal of the membrane by which they are covered. Still, when the bones are fractured, the veterinary surgeon must give relief as speedily as possible. All loose fragments of bone must be removed, and the wounds washed out. Occasionally, the gum and membrane of the mouth will be found torn to a considerable extent-the ends hanging loosely in the mouth. These must be brought together, and secured by suture. After this is done, all that is necessary is to cleanse the mouth occasionally with a weak solution of chloride of lime or carbolic acid, and to place the animal on soft diet.

$4 t h$. Fracture of the rami of the lower jaw may occur from other causes than that of the lorse falling upon his mouth-such as kicks from other horses, or any other external violence. The fracture may be in front of, posterior to, or in a line with the tush. If the tush is loosened, it must be removed; but this 
is not to be done unless it is loose, as it may be of great service in securing the bone in its position, as already shown. The presence of such a fracture, if simple, will be indicated by tenderness, and perhaps crepitation; if compound, its condition is detected by exploration of the wound. If the fracture extends through the jaw, with displacement, there will be deformity, in addition to the other symptoms.

I have never seen a simple fracture of this part, but such a thing may occur; the most common form, however, is that of a compound comminuted fracture of the external surface only; the alveolus and contained tooth, by breaking the concussive shock, prevent the fracture from extending to the internal surface. Presuming, however, that the fracture is complete, it may be single or double, with displacement. If simple, replacement, adjustment, and retention of the disunited parts in their proper position are the means to be resorted to; if compound and comminuted, the removal of all loose fragments must be the first proceeding. In order to do this effectually, it will often be necessary to enlarge the wound, and, provided there is no previous disease of the bone, a cure may confidently be looked for.

When the broken ends are bronght together, means calculated to retain them in their proper position must be resorted to. If the injury is near the symphysis, the copper wire and bandages already recommended will be sufficient; but if situated posterior to the tush, something more than this is required. Professor Varnell, in the Veterinarian for 1866, recommends a cradle made as follows:- "The cradle will be from fifteen to eighteen inches long; it should extend as far back as the angle of the lower jaw, and as far forward as to be within an inch of the anterior margin of the lower lip. Its sides should turn up, so as to embrace the outer surfaces of each branch of the bone, and be on a line with the upper margin of the under lip, beyond which they should rise to within a short distance of the zygomatic ridges. Posteriorly, it should incline upwards and backwards; the centre of its underneath surface should be pushed upwards, forming thereby a ridge, which is to fill up the space between the rami as far forward as the symphysis of the jawbone. Its angles must be rounded off, and its borders perforated by holes or slits, so as to enable the operator to apply 

FACE CRADLE DESigned by Mr. WALKER, V.S., Bradford.

FIQ. I.

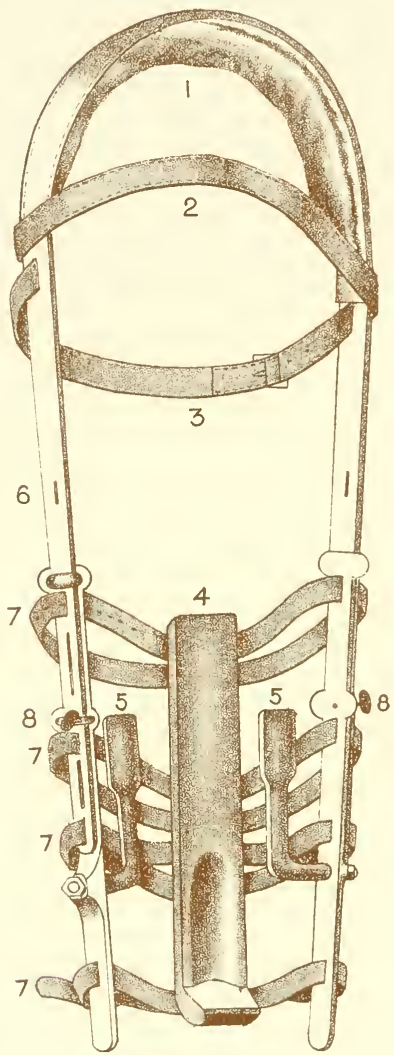

1. Padding to protect the poll.

2. Brow band.

3. Throat lash.

4. Pad for submaxillary space-to be made of wood, and well padded with leather.

5-5. Flanges to rest on first molars.

6. Supplementary strap slits.

7-7-7-7. Straps-which all pass under the lower jaw.

8-8. Thumb-screws for lengthening or shortening the plate, designed by Mr. BroAd, Bath. 
straps, \&c., to secure it in its place. If straps are used, they may be placed as follows:- One may pass from a little below the supero-posterior angles round the back part of the horse's head, behind the ears; a second, from the upper borders of the cradle, a little in front of the supero-posterior angles. This should pass across the brow, in front of the ears. A thirl, a small strap, may pass from one of the two straps to the other, between the ears. A fourth may proceed from the middle of the upper borders of the cradle, across the front of the face, a little below the orbits; and a fifth may pass across the nasal bones, sufficiently above the nostrils, not to interfere with the horse's breathing. The two last-named straps should be partly elastic, the last one more so than the other, so as to allow the horse to open his mouth to a slight extent.

" Should the fracture be compound, some holes will be required in the cradle opposite to the seat of the injury. Perforations of this kind can be made after the cradle is manufactured.

"As horses' heads vary both in size and form, it is not to be expected, even though a practitioner has two or three of these cradles on hand, that any one of them will fit the parts with that exactness he could wish. The inequalities, therefore, must, as before stated, be filled with a padding of tow, or some other agent, in addition to that which should, on all occasions, be used as a lining to the inner surface of a cradle or splint.

"He who is called upon to treat a case of fracture of the lower jaw of a horse must not despair if he does not possess any other splints than those he is obliged to make at the time. The principal object, after the ends of the broken bone are brought in apposition, is to retain them there until they are united; and the practitioner should endeavour to accomplish this with as little inconvenience to the horse as possible."

The method recommended by Professor Varnell seems complicated, but coming from such a source, it deserves every respect. MIr. Walker, V.S., Bradford, has designed a cradle, which has answered the purpose very well in at least two cases, where the fractures were posterior to the tushes, and involving both rami. He.has kindly sent the following drawings of it.-(See Engravings.)

The tendency of the fractured ends to fall inwards is the only real obstacle we have to contend with; so if we can by 
any method fill up the submaxillary space, we shall overcome this difficulty. The pad (a) in Mr. Walker's cradle answers this purpose; and the flanged plates (b) resting on the molar teeth render displacement of the fragments almost an impossibility, whilst every freedom is allowed to the natural movements of the jaw.

It must be borne in mind that the adjustment of the fractured parts must be made with great care, in order that the molar teeth may meet those in the upper jaw as evenly as possible; otherwise the process of mastication will afterwards be imperfect. If any teeth are loosened in their sockets, they must be removed, as they, by acting as foreign bodies, and preventing the proper adjustment of the fractured ends of the bone, retard the process of union. In addition to these means, the uninjured teeth may be fastened together by the copper wire. If the injury has been in existence for a day or two, with swelling, and perhaps systemic disturbance, it will be necessary to remove these symptoms, as well as to adjust the broken bone. Apply fomentations to the part, and give a dose of purgative medicine. Motion of the jaw must, for some days, be prevented as much as possible, supporting the animal during this time by a loose, nourishing diet, such us bran-mashes, boiled linseed, and gruel. If the formation of callus seems retarded from any cause, milk may be allowed-of which the horse will drink freely-and thick oatmeal porridge. In fact the strength of the animal must be kept up without causing the jaw to perform any hard labour. The practitioner will at once see the importance of this. There is one other thing in connection with the treatment of all kinds of fractures of the bones of the mouth that I must insist upon as being most important, namely, that all food be given in a wide-bottomed and shallow manger or tub. If the manger is narrow and deep, displacement of the bones, from the etrorts of the patient to get at the food, is sure to result.

All pieces that are separated from the body of the bone, as well a,s those denuded of periosteum-even if attached by one end-partly split off, as it were-must be removed, either with the common or bone forceps; and the wound must then be covered by fine tow dipped in a weak solution of carbolic acid; a hole, as already described, being left in the mould for the purpose of renewing this dressing, and for cleansing the 


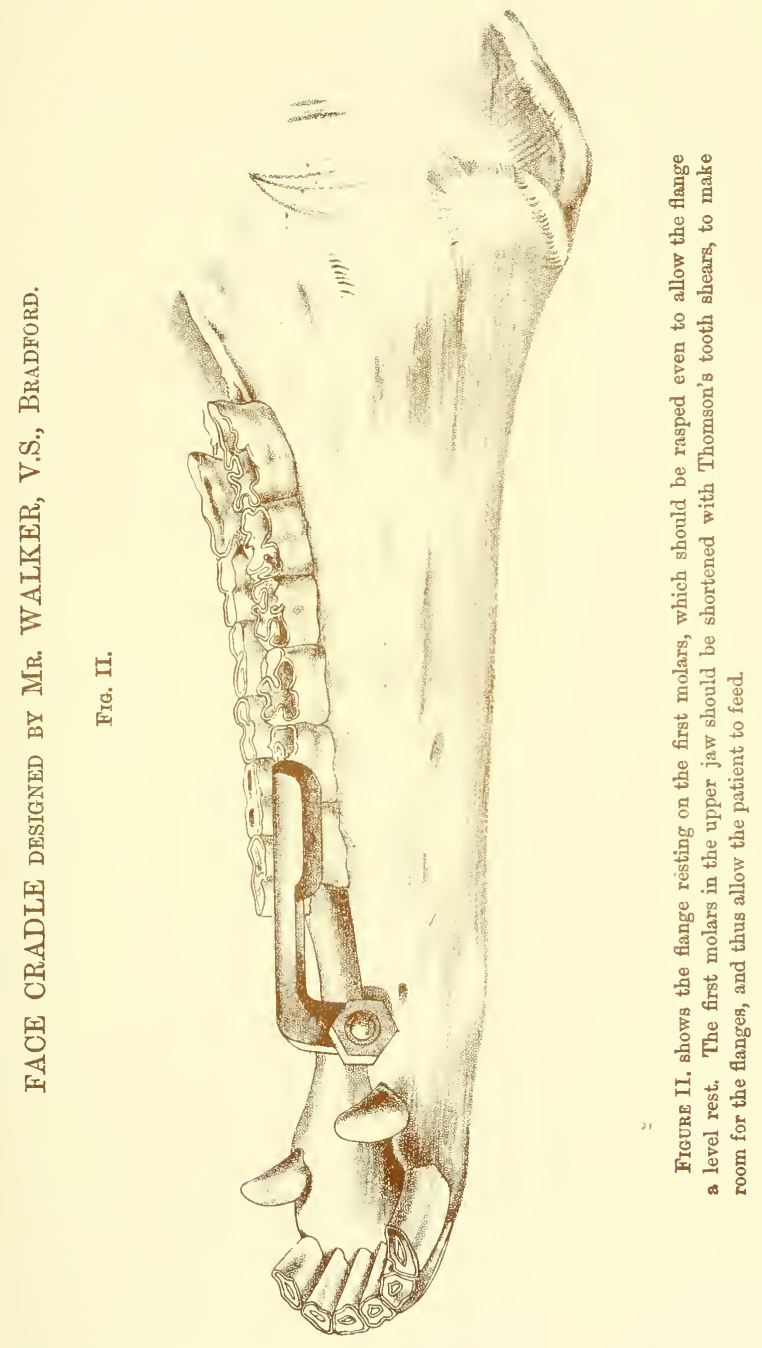



FACE CRADLE DESIGNED BY Mr. WALKER, V.S., BRADFORD.

FIG. III.

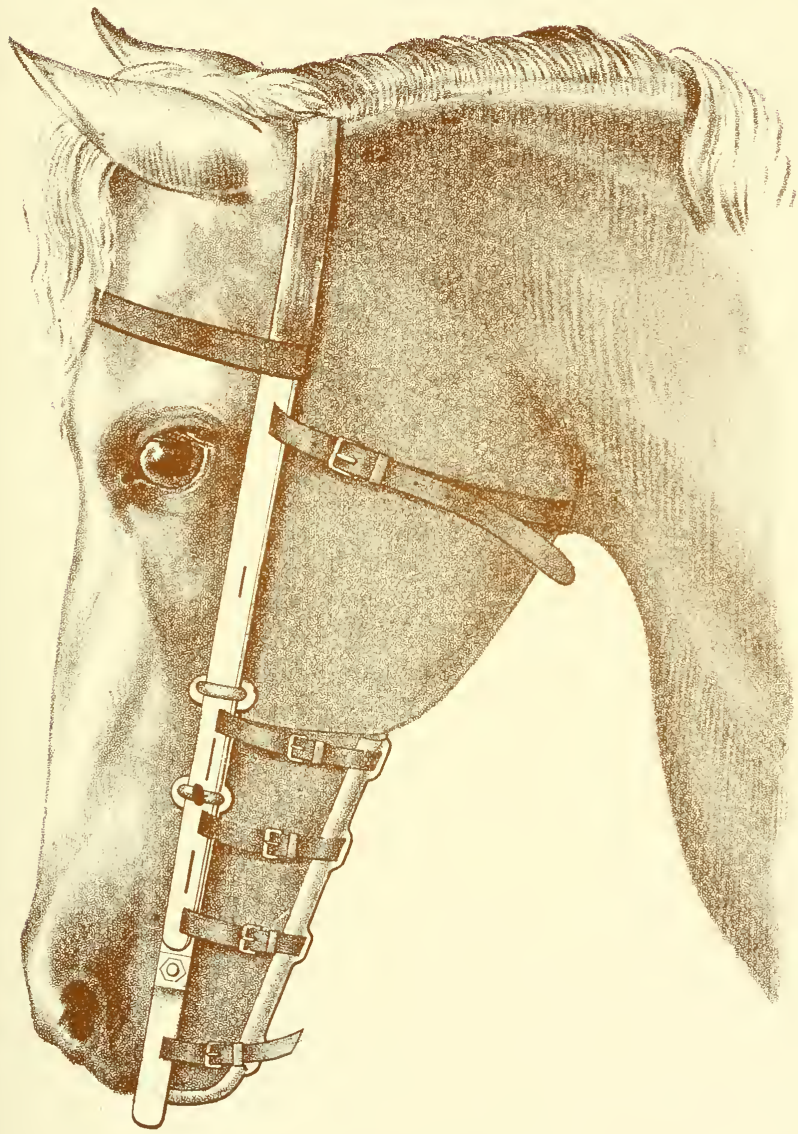

FIGURE III. shows the apparatus fixed on the head. For a smaller head it is necessary to shorten the apparatus by turning the thumb-screws, as, if it extended beyond the muzzle, the patient would be unable to feed. 

wound, if necessary. It is found, since the introduction of antiseptic surgery, that the wound often remains dry, and that it heals by the adhesive inflammation. If such be the case, the dressing must on no account be interfered with, and means must be taken to keep it in its place, either by the application of collodion, styptic-colloid, or gutta-percha paste. When a loose portion of bone remains, its presence may be detected by the continued fœetor of the discharge, and by an ulcer in the skin communicating by means of a sinus with the bone within. In all cases where a loose piece of bone is found in the part some time after the accident, it will be advisable, if its removal is not easily accomplished, to allow some weeks to elapse before forcibly extracting it, in order that the union and consolidation of the fracture may be complete. The opening in the skin may then be enlarged, and the necrosed bone removed by the forceps or tenaculum.

No alarm need be felt at the foetor of the discharge during the first few days after the accident, for as a rule this is always present. The discharge is at first thin, dark-coloured, and fœetid; but if not too profuse, it does not indicate any serious results, for it very shortly becomes lighter in colour, less offensive in odour, and partakes more of the nature of laudable pus. Every facility must be given for the discharge to drain away, and the wound may be injected with a weak solution of hydrochloric acid. I recommend this in preference to all other dressings in such cases, as it dissolves the earthy matters of the necrosed surfaces of the bones, and thus facilitates the formation of a healthy healing surface. I have used it for years, and can speak most highly of it. Much pain is manifested by the animal in some cases of this kind of fracture, from the inferior dental division of the posterior maxillary branch of the fifth nerve being involved in the injury. In such, much relief will be given by dressing the wound with a solution of morphia, or by the adminstration of opium: the local application is the better practice, and as little medicine as possible should be given after the first dose of physic, for fear of causing displacement. If the bowels are constipated, enemas ought to be administered, and the food should be of a laxative nature-linseed, bran, boiled turnips, or carrots; or what Mr. Anderson, V.S., Glasgow, recommends-linseed oil in a warm bran-mash. The patient, 
as a rule, will eat it. Gentle exercise is to be given from day to day; and in order to prevent the patient eating the bedding, it should have a loose box littered with sawdust. In about a fortnight after the accident, if the case does well, chopped hay may be allowed, with boiled oats and bran. I have heard of a case of fracture of the condyle of the lower jaw, with open joint, and that the patient recovered, with perfect motion in the articulation. Such a thing may be possible, but is scarcely credible.

Fracture of Anterior Maxillary Bone must be treated in the way already laid down-that is, by the copper wire and bandage.

Fracture of the Nasal Bones occurs rather frequently, from runaway horses coming in contact with hard substances, such as lamp-posts, \&c. As a rule, one of the bones is fractured, but occasionally both are found broken. In old horses these bones are much more easily fractured than in young ones, for the simple reason that their great elasticity in the young enables them to bear and resist violent shocks of concussion. These fractures may be complete, or partial only,-in the latter case the outer plate of the bone being broken, and the inner bent in without being fractured. When it is complete, it will be found that the Schneiderian membrane is lacerated, with perhaps sharp fragments of the bone sticking through it. This will be more particularly the case in the lower portion of the nose. If the lesion is at the upper part, the fragments will be driven into the facial sinuses. The symptoms of fracture of these bones will be depression; hæmorrhage from the nose; and if the depression is extensive, the breathing will be impaired by the inward bulging of the displaced bones. I remember a case where this impairment was a permanent unsoundness. The fracture was an old one, when seen by me, with considerable depression upon the front of one nostril. When the horse was quiet there was no noise in the breathing, but when put to work, there was a sound emitted during both acts of respiration, accompanied occasionally by hæmorrhage. The case was tried at the Halifax County Court; Mr. Dray, of Leeds, and myself certifying to the unsoundness of the horse; the Judge, Mr. Stansfield, concurring, and giving judgment accordingly.

In the treatment of this fracture, whether it be simple or 
otherwise, an endeavour must be made to readjust the bones in their proper position. Professor Tarnell recommends that " a piece of wood should be prepared, about one and a half inches wide, about half an inch thick, and of sufficient length for the purpose. This piece of wood should have its angles rounded off, and that part of it which is required to be passed up the nasal passage should be covered with a piece of thin wash-leather, to prevent the possibility of any splinter injuring the mucous membrane. The operator being thus provided, and the horse favourably placed and secured, this rude instrument is to be passed up the nostril of the side affected, as far as a little beyond the seat of the fracture, when as much force as may be necessary, or deemed prudent, should be used to raise the depressed fragments of bone. If the fracture is compound, and any portion of the bone projects through the skin (which is not very likely to be the case), it must be dealt with as recommended in compound fracture of the lower jaw; or if it is comminuted, and a fragment is.found to be wholly detached, it should be removed with the forceps.

"The segments of the fractured bone having been adjusted as nearly as possible in their proper position, means should be devised to retain them there. For this purpose two modes suggest themselves. The first is as follows:-To clear the skin as much as possible from dirt and other extraneous matter, and when it is thoroughly dry, to place some straps of strong adhesive plaster across the fractured bones. These should be carried a little beyond their outer boundaries, and in such direction as may be thought best.

"These straps, if well applied, will be found to answer a very good purpose, especially when the bone is much splintered or the skin penetrated, as in compound fracture. In the latter they will close the opening, and thus to some extent reduce it to a simple one; they will also materially support the broken bone, and thus prevent any portion of it from falling into the nasal passage or the nasal sinus.

"The other mode of keeping the fractured bone in its proper position, which may be used with or without the former, and which also tends to arrest hæmorrhage, should it exist to any extent, is to plug the nasal passage with fine tow or cotton wool. This may be done in the following way with perfect safety:- 
"A piece of soft but strong twine may be firmly tied around a small bundle of the above agents, which should be sufficiently large to fill the passage. The free end of the string should be long enough to reach some little distance out of the nostril, whereby the operator may be enabled to hold it firmly while the rest of the passage is being plngged.

"By this arrangement the whole of the plugging can be removed at any time that may be deemed advisable, and there will be no danger of any portion of it passing in a backward direction through the posterior nares into the fauces.

"There need be no apprehension about the horse's breathing being interfered with, if one of the nasal passages only is plugged; but I need not say that both should not be so treated, as the horse cannot breathe through his mouth."(Professor Varnelt, in Veterinarian, November 1866, pp. 875-6.)

If this method should be found impracticable, and more especially if the fracture is a compound one, the broken bones should be raised from the outer surface by means of a strong tenaculum or hook, which is easily introduced into the edge of the fracture; the parts must afterwards be strapped, as recommended above, and in all cases the patient must have rest.

Should the discharge from the nose, which is always sure to succeed the hæmorrhage in this kind of fracture, continue, and become more profuse, foetid, curdled, or sanious, a search must be made for any detached piece of bone which may exist in the part; when found, it must be removed, and the portions of healthy bone in contact with it dressed with the dilute hydrochloric acid, as the mere contact with necrosed bone is apt to cause a necrosed or carious condition of the edges in such contact.

Horses have been condemned for glanders while only suffering from the presence of loose pieces of fractured bone in the nasal region, and it is maintained by some, that fracture of the nasal bones is of itself sufficient to produce glanders; but I cannot conceive how a disease due to a specific virus can arise from a mere accident, although the discharge, ulceration, \&c. may closely resemble the specific disease. To remove fœtor, and to promote a healthy condition of the mucous membrane, the nostrils may be daily syringed with very dilute carbolic acid, solution of ehloride of lime, Condy's fluicl, or by insufflation of iodoform. 
Fractures of the Superior Maxillary Bone are generally caused by blows upon the face with the butt-end of the whip, given by a bad-tempered and savage horseman. As a rule, these fractures are situated anterior to the maxillary spine, and immediately over the superior maxillary sinus, into which the fragments fall. The fracture is usually compound and comminuted, and is easily diagnosed by the egress of the air expelled by the patient during each time of expiration.

Treatment.-Remove all loose portions, bring the edges of the wound together by suture, and apply the collodion dressing.

The Frontal Bones are broken by falls and other severe accidents; the seat of the fracture is usually found in the orbital process. The anatomist knows that the frontal bones enter into the formation of both cranium and face. If the facial portion be fractured, there is but little danger of immediately fatal results; but if the cranial portion is thus injured, death may be instantaneous, particularly if the blow has been sufficient to fracture both plates of the bone. Occasionally, but very rarely, the orbit is pierced by a sharp-pointed instrument, and the orbital plate, which is thinner than any other portion of the cranial bones, is penetrated, and the brain seriously injured. In the most usual form, namely, fracture of the orbital process, it is obvious that many important organs contained within the cavity of the orbit may be seriously hurt, such as the supero-orbital division of the fifth nerve, its accompanying artery, lachrymal glands, and even the eye itself. Sometimes a small piece of the anterior border is, as it were, chipped off, and the tarsal ligament detached from it, which makes the case difficult to manage.

Professor Varnell says that "there is a great tendency in this part of the bone, when injured, to ulcerate and crumble away." I have not observed this; but it may be due to the circumstance that I make it a point in practice never to allow detached fragments of bone to remain in the wound. When the orbital process is completely fractured-say a simple fracture from a severe blow with a blunt instrument-the fractured ends are driven inwards by the force of the concussion. In such a case, the eye will be completely closed; and if there be no swelling or bruise, the closure of the eye is the only symp- 
tom of the injury: there is both paralysis and mechanical displacement of the eyelid.

The treatment of such a case is to elevate the depressed ends of the bone; and to do this it may be necessary to introduce a small lever under the skin, making the incision through which it is to be passed close to the zygomatic ridge, and pushing the instrument subcutaneously until the seat of the fracture is reached, when the elevation may be effected; if the lever is put under the centre of the depression, both ends may be elevated simultaneously. Considerable force is necessary, but it must be applied carefully and steadily. Let there be no jerking, but steady, continued pressure. I have seen the eye recover almost its natural shape when this has been done. Little else is required locally ; the animal is to be kept at rest, the wound made by the operator covered over with collodion, and low diet prescribed; a dose of physic may be given, and the animal carefully watched for a few days by the veterinary surgeon, who will adopt such treatment as may be suggested by the symptoms. If the fifth nerve be damaged, there is found to be a tendency to tetanus; all causes of excitement must therefore be guarded against.

Should the fracture be compound or comminuted, the treatment already laid down must be employed.

The outer plate may be fractured over the centre of the frontal sinus; it may be merely bent inwards, from partial fracture; or it may be completely shattered, and the skin penetrated.

If there be mere depression, there should be no interference, as the indented bone will not press upon any organ, and the only results will be a slight blemish externally, and diminution of the sinus within. A dose of physic should be given-febrifuges if necessary-and a short period of rest allowed.

But if the injury has penetrated the skin, and broken the bone into fragments, it becomes the duty of the veterinarian to remove all such fragments, whether they are attached to the fractured borders, or have been forced into the cavity; to thoroughly cleanse the sinus from all clots of blood and other foreign bodies; for if these remain, their decomposition will be productive of much mischief. When these things are done, the forehead is to be strapped with pitch-plaster, and the constitutional treatment above described adoptod. 
The hæmorrhage may continue for some hours after such an accident, and, of course-from the sinus being continuous with the nasal cavity - the blood will flow through the nostril. This need occasion no alarm; the pouring of cold water, both into the sinus and on the face, will generally arrest it; or two ounces of the tincture of terchloride of iron may be mixed with a bucketful of water, and syringed into the wound by means of an elastic enema syringe.

I do not recommend the application of poultices to injuries of this kind, nor disturbance of the parts after the first dressing, which must be effective and complete; but should the swelling become excessive, or a purulent discharge manifest itself, it becomes a duty to apply fomentations of warm water in which a little carbolic acid has been dissolved, and the general treatment of wounds which will be hereafter laid down must be resorted to. If at any time the wound assume an unhealthy character, with a thin fotid discharge, caries of the bone is indicated, and this will require the hydrochloric acid dressing, care being taken that the diseased bone receives the application. It must never be forgotten by the practitioner thai an injury to any part of the cranium may be much more serious than may at first appear. The effects of the shock, although this is provided for by the double layer of bony plates, may scarcely be apparent; but unexpected symptoms may arise, indicative of severe lesions to the brain and nervous system.

Fracture of the immediate walls of the cranium may be present, involving the vessels of the brain, and when such is the case, the termination is generally fatal.

I have seen the zygomatic ridge broken on its outer edge, the fragments remaining in the wound, and requiring removal.

I have little to add upon fracture of the facial bones. Any of them, where they are exposed to external influences, may be fractured; and should such fractures involve organs or vessels of importance, the case becomes the more complicated. For example, the lachrymal duct may be involved in fracture of the hone of the same name; the fractured bone may heal, but the duct will be spoiled; and the lachrymal secretion, instead of being discharged into the nostril, will flow over the face. Again, the parotid duct may be opened in a fracture of the 
lower jaw, or the inferior maxillary division of the fifth nerve may be injured and pressed open ly a piece of depressed bone, causing excruciating pain, and even fatal results, if not at once removed.

\section{FRACTURES OF TIIE CRANIAL BONES.}

By the arrangement of the cranial bones, an ovate box is formed, which resists external violence after the manner of an arch; yet it is sometimes fractured by the direct application

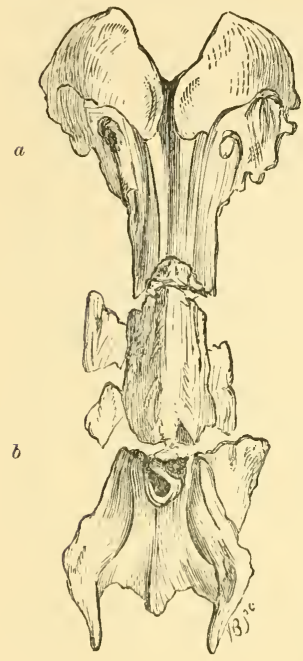

FIG. 11.-Indirect comminuted fracture of basilar process of occipital and sphenoidal bones, from falling on occipital crest. a, Occipital. $b$, Sphenoid. of force to the fractured parts, but more frequently-in the lower animals, especially in the horse - at a part of the skull distant from the place where the violence has been received. This is called "indircct fracture," or fracture by counterstroke, or what is termed by the French, fracture par contrc-coup.

Fractures of the base of the skull are, in all instances, caused by the indirect application of violence, and this is easily explained. If a horse fall, or be struck heavily by a blunt instrument or obtuse body, upon the occipital crest, the force thus applied is resisted by the strength and thickness of this part of the bone, and the shock is conveyed to the basilar process, this being its weakest part.

In all cases this form of fracture is necessarily fatal.

Sometimes the violence of the fall or blow is sufficient to produce fracture of the part to which it is applied, and also to extend to the base of the cranium.

The fractures of the cranium may be conveniently arranged as follows :-

1st. Simple fissure or fracture without depression.

2d. Simple fractures with depression. 
3d. Punctured fractures.

4th. Compound fractures.

5 th. Fracture of external table only.

$6 t h$. Fracture of the internal table only.

1. Simple Fissure.-In this form there is no wound of the soft parts, and the broken pieces preserve their proper level; and if there be no accompanying injury to the parts within the cranium, it is not necessary to do more than guard against the occurrence of inflammation; but the force which breaks the bone may cause separation of the dura mater, laceration of the brain, extravasation of blood within or upon it, or above and below the dura mater; or a mere crack in the outer table, with fracture and depression of the inner table. There may be immediate concussion of the brain; or inflammation of it and its membranes, may subsequently appear to a dangerous or even fatal extent.

2. Simple Fracture, with Depression.-In this form there will be an inequality of the surface of the skull, varying in extent according to the size of the depression, and generally associated with a bruise of the skin. Sometimes the fragments are unyielding, and sometimes they are moveable. A condition of the skin of the head is occasionally seen, which is apt to lead one to suppose that the depression is much greater than it is in reality, or to tlink that the bone is broken, when no fracture exists. This is a swelling caused by extravasation, and it occurs in this manner:-A blow is inflicted by a flat instrument; this blow is sufficient to deaden and paralyze the vessels of the part itself, which remains firmly compressed; but into the surrounding areolar tissue blood becomes extravasated, raising the soft parts to a considerable extent. This swelling is remarkably firm, and this condition should always be kept in mind, or the practitioner may think there is a fracture where none exists, or that the depression of fracture is much greater than it is in reality.

Fracture with depression will be attended by other symptorns than the local ones above described, namely, by compression of the brain; and when a depressed piece of bone occasions the compression, the symptoms present themselves on the occurrence of the injury; but compression may arise from other results of an injury to the head than fracture. For example, an animal has received a blow on the head which may stun him; he re- 
covers from the first shock, and symptoms of compression afterwards appear, and gradually increase; there are then just grounds for supposing that they arise from extravasation of blood within the cranial cavity.

When the compression arises from the formation of pus, the symptoms do not present themselves for several days after the accident, and are preceded by those of inflammation of the brain or its meninges. The history of the case will here be most useful in guiding the practitioner to arrive at a proper conclusion as to the cause of the compression.

There is no certain correspondence between the symptoms of compression and the extent to which a portion of bone may be depressed. In some cases, where depression has been slight, the symptoms have been very marked; whilst in others, the symptoms have been slight and the depression considerable. It is also a remarkable fact that injury to one table of the bone scarcely ever corresponds to that of the other; the inner being nearly always fractured to a greater extent than the outer, and its actual depression much greater than would appear from examination of the parts external to the fracture.

The treatment must be pursued in accordance with the symptoms. In the absence of compression, the indication is to prevent inflammation; and for that purpose purgatives must be given, and low diet with rest and quietude enjoined. It is also necessary to keep the head cool by means of cold wet cloths, and after the period of collapse-which immediately succeeds the injury-has passed off, abstraction of blood may be advisable. The administration of sedative medicines had better be withheld, as they may produce an impression upon the brain, predisposing to inflammatory action.

If compression be present, the practitioner must, without delay, attempt to relieve the brain from pressure. If the bone be not depressed to any great extent, and the symptoms not urgent, bleeding, purging, and the application of cold to the head may be sufficient; for it is sometimes found that the brain becomes accommodated to its new condition, and that the symptoms disappear; but if these means should not succeed, the depressed bone must be elevated. When the symptoms of compression are strongly marked, and the bone depressed to such an extent as to leave no hope from other treatment, the 
fracture must be exposed by a crucial incision, and the bone raised by an elevator. Sometimes it is possible to introduce the elevator under the edge of the fractured bone without using the trephine; in the majority of cases, however, it will be found necessary to remove a part of the bone that is not depressed, so as to admit of the introduction of an elevator, by which the depressed part may be raised to a level with the surrounding parts of the skull.

The best form of trephine is the one with a moveable centrebit. The elevator or lever is a very simple instrument, and can be made by the nearest blacksmith, being merely a chisel with a slight curve or elbow. Should the fracture of the inner table be comminuted, all loose portions must be carefully picked out with the forceps.

Whilst recommending the above treatment, I am bound to admit that the force sufficient to cause fracture of the bones of the skulls of our patients is generally sufficient of itself to produce instant and fatal concussion of the brain; but, on the other hand, it will be found, during a long period of practice, that cases as described occur, especially in a city like Edinburgh, where the streets are hilly, paved with granite, and the horses shod with calkins and toe-pieces, which seem to take from the poor animals the power of falling naturally when they make a slip, and to cause them to tumble head foremost. From these causes, fractures of all kinds are of frequent occurrence.

3. Punctured Fracture.-This is caused by a sharp body, as a pitchfork, or by the animal falling on a pointed instrument of any kind; a mere puncture or cavity being the outward visible effect, but the internal table, from its brittleness, is injured to a greater extent than the outer. This fracture is sometimes called "star-like" or radiated fracture, from the fact that there are numerous fissures or cracks in the bone, radiating from the centre of the fracture.

This is a very dangerous form of fracture, requiring immediate trephining, although symptoms of compression may be absent. The danger arises from the fact that spicula of the inner table are always driven inwards, and if these be not removed, it is certain that inflammation will ensue. If the operation be delayed till then, the animal will most assuredly die.

After the depressed fragments have been removed (and it may 
be possible to do this without trephining or removing any sound portion), the wound must be closed, and the most strict anti. phlogistic treatment pursued, both to prevent inflammation and to subdue it in case of its occurrence.

4. Compound or Open Fracture with Depression, is the most common form met with in our patients; a force strong enough to break the bones being sufficient to drive in the soft parts also.

There is a wide difference of opinion amongst surgical writers as to the necessity of immediately elevating the depressed bone in this form of fracture,-Sir Astley Cooper, Sir B. Brodie, and others laying it down as a law that trephining must be performed as soon as possible, it being useless to do so when inflammation is once established. On the other hand, Professor Samuel Cooper, Sir Philip Crampton, Dease, and Desault maintain that in fractures of the skull with depression, whether it be compound or not, no attempt should be made to elevate the depressed bone, unless very decided symptoms be present of compression or irritation of the brain; and that they have seen many cases terminate favourably without the use of the trephine.

I think that in all such cases, whether there be symptoms of compression or not, the veterinary surgeon should not hesitate to elevate the depressed bone at once; by not doing so, he runs the risk of losing his patient by the inflammatory process setting in, when all chance will be gone. This is imperative where the depression is exposed in consequence of a wound in the soft parts, whether there be any signs of mischief to the brain or not; but if there be depression without wound, and no signs of compression, let him make no such wound by operation.

In ponies that work in coal-pits this is a very frequent form of fracture, arising from the tunnels being made so low that the animals are continually striking their heads against the roof. The force of the blows is here merely strong enough to break the skin and the surface of the bony crest. It is my opinion that in some cases the detached pieces of bone result from the continual blows producing necrosis. It is lamentable to think that these poor animals are worked from week to week, and month to month, without an effort being made to prevent or cure such injuries. I have seen the skin of the forelock detached for several inches-easily raised up as a flap-swollen, inflamed, with an ugly, unhealthy wound underneath it, containing several pieces of necrosed bone, 
causing the poor brute to be nearly mad with pain, and clangerous when any one approached its head. In all such cases the proper treatment is to remove all loose fragments of bone, scrapo the surface of the crest, if in a necrosed condition, dress with the dilute acid already recommended, and bring the flap into its proper position, retaining it there by folds of wet cloth properly adjusted upon the ears and poll. Sutures or plasters should not be used, as they prevent the escape of any pus that may form.

A form of bridle now in use at the pits belonging to the Bowling Iron Company, near Bradford, is the best and most effectual preventive that I have seen. It consists of a plain bridle, having a piece of strong harness leather extending over, and covering the forehead and poll, from about two inches above the eyes to a distance of two or three inches behind the ears, pierced with holes for the ears, and properly adjusted to the peculiarity of each head. After the adoption of this simple contrivance, broken heads did not again occur. It must be kept pliable by frequent oiling, or it is apt to become hardened, and to cause irritation at the base of the ears.

\section{CONCUSSION OF TILE BRAIN.}

This is produced by a blow, or a fall upon the skull itself, or a fall upon the nose, by which a sudden shock is communicated to the brain through the medium of the facial bones. In this way cases have been recorded of fracture of the ethmoid bone. Its symptoms are arranged in three stages-1st. Collapse; $2 d$. Reaction; $3 d$. Inflammation of the brain.

First Stage, or Collapse.-In many instances, the functions of the brain and organs of sense are suspended, so that consciousness is entirely lost, with complete insensibility to external impressions; common sensation and voluntary motion are also lost for the time; this loss is often so great that the animal gives no indication of pain when pricked with a pin or other sharp instrunient; lies motionless upon the ground, or makes a few faint struggles with the limbs; but is altogether without the ability of combining the action of the muscles, so as to perform any particular movement. The extremities, and surface of the body generally, are cold, the respiratory movements feeble, but not usually stertorous; the pulse is weak, 
fluttering, and perhaps intermittent; the pupils are dilated if the concussion be very severe, but they will usually contract on the application of a light, showing that the retina is not perfectly insensible. If the concussion be slight, the pupils will be found contracted, or possibly one pupil may be contracted and the other dilated. These symptoms may change into the second stage, or into those of compression, or they may terminate fatally without any other change.

Second Stage.-The insensibility is diminished, sensation and volition are partly restored, but ordinary impressions produce little effect, and the animal lies in a kind of sleep, although it is possible to rouse lim for a moment by speaking loudly or harshly; pricking by a pin causes him to show feeble signs of pain; there are occasional signs of restlessness; the pulse and circulation are more vigorous; the skin and the extremities become warm by degrees; and the animal will now and then make efforts to rise, which always produces an accelerated condition of the pulse, with loud beating of the heart. These symptoms may subside, or may pass on to the third stage, namely, that of pure inflammation of the brain, with throbbing of the carotids and vessels of the head, injected conjunctivæ, suffusion of tears, intolerance of light and sound, watchfulness, restlessness and delirium, with strong quick pulse, leat and dryness of the skin, diminution of the secretions, and other symptoms of irritative fever. These symptoms may yield to treatment, which must be prompt to be effectual, or they may pass on to those of compression, and prove fatal.

\section{DIFFERENT WAYS IN WHICH CONCUSSION PROVES FATAL.}

By careful experiments, the whole of the brain and spinal cord have been removed, and so long as artificial respiration was kept up, the action of the heart continued for some hours. From these experiments it has been concluded that the action of the heart is independent of the brain and spinal cord. But it has also been discovered that when any sudden injury, such as a violent concussion, is produced on a part of the brain or spinal cord, an immediate and great depression, or complete suspension, of the action of the heart is the result. From this it is concluded that a sudden injury of the nervous centres. 
such as violent and sudden concussion, suspends the action of the heart, and thus proves fatal by syncope or death beginning at the heart. The vital powers of the heart seem to be destroyed, for when the chest is opened immediately after death, it is impossible to excite contraction of that organ.

The difference between the effects of concussion and a wound of the brain is very remarkable. Mayo observes on this subject- "A great part of the brain of an animal may be gently and quietly sliced away with little or no effect; but if ever so small a portion be suddenly crushed, the heart stops directly."

Again, from the experiments of Chossart and others, there appears some variety of opinion as to the part of the circulation chiefly affected by certain injuries of the nervous centres. In certain injuries of the brain and spinal cord, Chossart found that the capillary circulation appeared for a time to be more affected than the heart's action; but still it is by fainure of the circulation that such injuries ultimately prove fatal. It is only by such knowledge as this that the practitioner can be guided to rational and scientific treatment. A peculiar fact may be introduced here, as exemplifying a lesion which occurs very frequently after the operation of pithing (dividing the spinal cord between the occiput and atlas, or atlas and dentata), namely, rupture of the vena azygos.

In some cases, when death occurs from concussion, the heart is found quite empty, a condition that is not easily accounted for. In other cases it is distended; but the distinguishing peculiarity is, that there is no difference in the quantity of blood in its right and left sides.

"We may sum up by stating" (says Pirrie) "that when concussion proves fatal in the first stage, it is by failure of the action of the heart, which is sometimes instantaneous, sometimes gradually increasing to a fatal termination; and sometimes there is a very partial reaction, and then a second failure of the organs of circulation, which proves fatal."-(PIRRIE, Principles and Practice of Surgery.)

Bearing these facts in remembrance, the practitioner will watch the state of the organs of circulation with care and anxiety. Another way in which concussion proves fatal is by compression, arising from extravasation of blood, serous effusion, or the formation of pus. 


\section{POST MORTEM APPEARANCLS.}

These will vary according to the length of time intervening between the injury and the death of the patient. In cases that are almost instantaneously fatal, laceration of the brain is often observed. In other cases the dura mater is separated from the inner plate; and this is a very common condition. Again, the venous sinuses are found lacerated; and, in other cases, the concussion proves fatal without any lesion being visible either in the brain or its membranes.

In the cases where death occurs at a more advanced stage, traces of inflammation will be present, such as increased vascularity of the membranes, small specks of blood in the brain, various kinds of inflammatory products, lymph, serum, pus, or a combination of these conditions.

It has been supposed, where no lesion is apparent, that the sudden shock disturbing the circulation of the brain is the cause of death; and, again, that the structures are injured, and the injury not discoverable by dissection. Others think that the fatal result arises from condensation of the brain. One writer, Mr. Liston, says - "When a blow is inflicted on the skull, only a slight commotion of the brain is induced; the cranial contents are, as it were, slightly jumbled, and a temporary and trifling disturbance of its functions follows. When, however, the stroke is more severe, the brain is separated from its cranial attachments, both at the point struck and at the part directly opposite. It is thrown upon itself towards its centre; its substance is thereby condensed, its diameter in the direction of the impulse diminished, and a separation between the brain and the cranium is formed at each extremity of that diameter:"

By post mortem examinations it has been ascertained that condensation of the substance of the brain does exist in cases of severe concussion. Such condensation may be sufficient to cause instantaneous extinction of life; or the brain may gradually resume its former condition, or only with such incited action as may be required to reunite the dura mater with the inner table of the skull.

Treatment.-In the first stage, when the symptoms of depression are very great, and there is danger of death from failure of the heart's action, stimulants, as ammonia, should be ad- 
ministered, care being first taken to ascertain whether the power of deglutition be present or not. If the power of swallowing be lost, medicine can still be conveniently given in the form of a ball, which should be well oiled, and pushed as far as possible into the pharynx. From the great danger of reaction being excessive, and the tendency of inflammation to succeed, the administration of alcoholic stimulants will be improper, and means must be employed to restore the circulations that do not tend to have a permanent effect. The animal is to be covered with warm clothing, and even heated blankets can be applied to the extremities and surface of the body with great advantage. It is thought by some of the most eminent of surgeons that very little beyond this should be done in the first stage, as the period of depression tends to diminish the danger of hrmorrhage in case the brain should be wounded; and that if the circulation be artificially excited, there is much danger of compression from extravasation of blood. But whilst the indiscriminate administration of stimulants is thus condemned, the abstraction of blood would be also highly improper, and most injudicious, as the vital depression already existing would be increased to such an extent as to destroy any chance that might remain of saving the patient's life. In the majority of cases, however, it would be useless to attempt bleeding, as the blood would not flow until reaction had commenced, and then the loss of the vital fluid would probably kill the patient.

In the second stage, or return of sensibility, the warm clothing, \&c. should be removed, the patient placed in a cool, dark, and quiet loose box, cold applied to the head, a full dose of purgative medicine given, and the diet to be of the simplest kind, such as bran water. If there be a desire on the part of the animal to eat his straw, he should be bedded with sawdust, strict watch being kept upon him; and if symptoms of excitement be observed, it may be advisable to bleed, if the state of the pulse warrants such a proceeding; but if the practitioner thinks bleeding unadvisable, the heart's action may be moderated by aconite, the most valuable of all sedatives in veterinary practice. It is given in small doses, from seven to ten drops of Fleming's tincture, as a larger dose is apt to produce some amount of preliminary excitement. Before administering even this most simple of sedatives, the practitioner must well consider his case; for sedative 
remedies may produce an impression on the nervous system predisposing it to inflammatory action.

In the third stage, or that of inflammation of the brain, there must be a prompt abstraction of blood, the administration of a full cathartic, and, when the delirium of the animal will allow the attendant to handle him, cold must be applied to the head by means of cold water, or even ice.

It is necessary that the abstraction of blood should not be carried too far, for if there be laceration of the brain, the constitution may be so weakened by such bleeding as to prevent the process of repair being set up in the lesion.

The signs of the inflammatory condition may subside, or may be succeeded by others, denoting that compression of the brain by blood, serum, or pus, is present; and in well-marked cases there is insensibility and unconsciousness, as in the first stage; - with this difference, that in mere concussion the symptoms pass off, and even during its continuance there are occasional fits of restlessness; but during compression they are constant whilst the cause is in operation. The retina is perfectly insensible, the iris motionless, the pupil dilated, the respiration slow, difficult, and stertorous; the animal may breathe through the mouth, the tongue hanging out; and the air puffs out the checks. A cow in the comatose stage of milk fever will present a familiar example of this. Stertorous breathing arises from the relaxation of the velum palati, and of the laryngeal and pharyngeal muscles. This relaxation prevents the free passage of the air, and gives rise to the noise in breathing called stertor.

This condition of the animal is that of complete coma, and death is caused by asphyxia resulting from paralysis of the inuscles of respiration; and from the insensibility destroying the feeling of the want of air, which in ordinary circumstances excites the respiratory movements.

The pulse is generally slow, full, and labouring, as the action of the heart does not seem, as in concussion, to be affected directly, but to become impeded, from the obstruction of the circulation through the pulmonary vessels.

There is loss of the power of swallowing, from paralysis of the muscles of deglutition; the action of the bowels is arrested, from their walls being affected by the general paralysis; the sphincter ani is relaxed, and fæces are passed involuntarily; the bladder is 
paralyzed, and cannot expel the urine, which sometimes dribbles off in small quantities. This indicates a state of great distension of the bladder, and that the urine forces its neck to such an extent as to allow the overflow of its contents.

Some cases are characterised by convulsive twitchings, rapid, feeble pulse, and hurried respiration-symptoms believed to denote wound of the brain rather than concussion.

It has been discovered by dissections that hæmorrhage from concussion may occur in any of the five following situations :1st. Between the cranium and dura mater; $2 d$. Under the dura mater into the cavity of the tunica arachnoidea; $3 d$. Between the tunica arachnoidea and pia mater; $4 t h$. Between the pia mater and brain; and 5th. Into the substance of the brain itself.

The method of trephining for injuries of the cranium is described as follows by the great surgeon Syme:- "If the bone be not sufficiently exposed by the original injury, a crucial or triangular incision must be made through the scalp, the flaps of which are to be dissected back. The pericranium is next scraped off sufficiently to prevent it from impeding the teeth of the saw, which is applied at first with its centre pin, to keep it steady, and afterwards, when a groove has been formed, this obstacle to its progress is removed. The sawing must be conducted cautiously, as the skull is not always equally thick, and is often throughout very thin, with hardly any perceptible diploe. A toothpick or probe should be introduced from time to time, to ascertain whether or not the bone be perforated at any part of the circle, and when the whole seems to be nearly cut through, an elevator or forceps may be employed to raise the detached piece. If circumstances appear to require the removal of more bone, the same means are to be repeated, or the process may be accelerated, if the portion be extensive, by Hey's saw, as it is usually called, which proves convenient for connecting the circular apertures together, so as to separate at once a large portion of cranium. After the operation the wound is to be lightly dressed, and the general treatment conducted with the view of checking any tendency to inflammatory action."

After the operation, well-regulated pressure should be applied to the part by means of pads of tow and bandages; if this is not done, a tumour is apt to make its appearance, which consists of a protrusion of the brain, and is termed- 
HERNIA CEREBRI, OR FUNGUS OF TIIE BRAIN.

This is very similar in appearance to a mushroom, the expanded portion overhanging the skull, while the narrow part projects through the opening in the bone, and is connected with the brain. It is elastic and compressible, destitute of sensibility, of very rapid growth, and when cut into, is found to consist of cerebral matter and clots of blood.

Treatment.-All that can be done is to cut the tumour clean off, and apply pressure by wet pads, and prevent all excitement. The tumour has a great tendency to grow afresh, and, a: a rule, a fatal termination may be looked for. 


\section{CHAPTER VI.}

\section{PARTICULAR FRACTURES-continued.}

FRACTURES OF THE VERTEBRE-ON BROKEN BACK-FRACTURE OF THE SACRAL AND COCCYGEAL BONES-FRACTURE OF THE PELVIC BONES-FRACTURE OF THE FEMUR-FRACTURE OF TIBIAFRACTURE OF THE TARSAL AND METATARSAL BONES.

\section{FRACTURES OF THE VERTEBRA.}

A FRACTURED vertebra, above the origin of the phrenic nerve, with displacement of the fragments, produces death, and is not discovered until a post mortem examination be made. This nerve is formed by the union of branches from the fourth, fifth, and sixth cervical nerves, and conveys motor power to the diaphragm. When this power is cut off, death results from paralysis of this great respiratory muscle. It is very true, as can be seen after an animal is "pithed," that respiration goes on for a short time, but it is performed with great difficulty, and seemingly without the aid of the diaphragm, and becomes slower and slower, until it finally ceases altogether.

The transverse processes of the cervical vertebræ are sometimes fractured, and by pressing upon the cervical nerve in the immediate neighbourhood, cause more or less paralysis of the cervical muscles supplied by that nerve, producing what is commonly termed wry neck-that is, a twisted neck, the head being turned from the seat of the injury. I had once under my care a case of this kind, where the fractured bone had become carious, communicating with the surface by means of fistulous openings. The horse had fallen, months before he was seen by me; the neck had been persistently bent from the beginning, and attempts had been made to straighten it, but without success; the soft parts commenced to swell, and eventually abscesses formed successively, and were discharging the offensive pus peculiar to dizeased bone. The use of the probe did not enable me to form 
any definite conclusion; but feeling sure there was detached bone, the wounds were opened up, a piece of fractured bone in a necrosed condition removed, and the wound brought together by suture. The process of healing was rapid, and no more abscesses formed, but the neck never became quite straight. There was certainly a great improvement, and the animal worked for years afterwards. The spinous processes of the dorsal vertebræ are the seat of fracture, and when this occurs, the detached fragments cause what is known as "fistulous withers;" the sinuses must be explored, and the fragments removed. Occasionally it is found that the tuberous ends of the spines are merely bruised, and are in a state of caries or necrosis from the violence. The treatment proper for this kind of injury is the careful scraping away of the diseased surface.

\section{ON BROKEN BACK.}

Fracture of the spine is of two kinds :-

1. Fracture without displacement.

2. Fracture with displacement.

Both of these fractures may be exactly similar in situation and extent, and they may involve either the body of a vertebra, or its arch, or both.

1st. Fracture without displacement generally occurs in the dorsal vertebra. In our dissecting room it is revealed that broken backs are no uncommon occurrences, and that the fractures have united without the animal having apparently shown any symptoms during life; the bones having been kept in the proper position by their ligamentous connections, and the spinal cord never having been interfered with.

In practice, however, cases are sometimes seen where, after a severe fall, there is partial paralysis, showing clearly that the medulla spinalis is more or less involved in the injury; and as illustrations of this, and indicative of the proper treatment of such injuries, I will briefly relate two cases that occurred in my practice while resident in Bradford.

The first case, a truck horse belonging to the Lancashire and Yorkshire Railway Cumpany, was knocked down with great force by a passing truck. The force was so great as to cause the horse to roll over two or three times on the ground; he got up 
with difficulty, and was, when examined a very short time afterwards, found to present symptoms of paralysis of both posterior extremities. There was knuckling over at the fetlocks when standing, difficulty in moving the legs forward, the peculiar plaiting or crossing of the legs which is observable in partial paralysis, and the rolling, uncertain gait. Nothing could be found upon examination.

The treatment consisted of quietude, rest, dose of physic, and fomentations to the loins. In about six weeks the paralytic symptoms diminished, and in about ten weeks the horse was at work again, and continued to work as a truck horse for four years afterwards, when, in consequence of a lameness in one of his feet, he was turned out to grass. Upon being let loose in the field, he commenced galloping, and going down a hill, his hind feet slipped from under him; he fell heavily, was unable to rise, and had to be destroyed.

The post mortem examination revealed the seat of the old fracture, the third lumbar vertebra; the process of anchylosis having extended to the rest of the lumbar vertebræ, the whole being united as one bone; and there was a recent fracture of the fourth, with displacement and pressure on the cord.

The second case was a high-spirited saddle horse, which was suddenly thrown upon his haunches by a strong check being given him by his rider, who was using a dreadfully severe bit. After he was thrown on his haunches, he recovered with some difficulty, and walked, partially paralyzed, a distance of seven miles into Bradford, when he was seen presenting symptoms similar to case No. 1. The same treatment was adopted; but unfortunately the owner listened to the voice of a farrier in the town, who asked to be allowed to put the lorse in slings. When the poor animal felt the support of the slings, he lay, as horses will do while on the slings, with the legs in a semi-flexed position, and his weight thrown upon the trunk. As a natural result, the back arched upwards, and immediately there was displacement of the fractured vertebra, and total paralysis of both hind limbs. When seen again by me, he was greatly excited and very restless, and was ordered out of the slings. When the slings were removed, the hind limbs were quite unable to support him; he therefore went down, and, being a high-mettled horse, commenced to fight and struggle with the fore legs, to knock his 
head against the wall, manger, \&c., bruising himseif in a fearful manner. Seeing that he was in great agony, and the chance of recovery now hopeless, he was destroyed. The post mortem examination revealed fracture through the body of the last dorsal vertebra. I am satisfied that this case would have eventually recovered if he had not been interfered with, as there was no fever; the appetite was good, and the animal cheerful before the slings were used.

Fractures of the spine occur during the performance of a surgical operation when the animal is cast.

Let it be here clearly understood that such fractures rarely if ever occur during the fall, but when the horse is down on the ground, and struggling from the pain of the operation, or from the inconvenience of his position. Broken backs during the performance of operations are much more common in Scotland than in England; this arises from the different methods in which horses are cast. The late Professor Dick used to say:"Fracture of the body of the bone (a vertebra), while the horse is under operation, sometimes takes place; this is to be prevented by placing a back rope on the horse before he is cast, and then it is to be tied round the legs and back, to prevent the animal struggling while down." 1

Now, with all due deference to the teaching of the late Professor, I must here say that the immense number of broken backs which we hear of in Scotland arises from the circumstance that the animal is fastened so that he cannot struggle; whereas he should be allowed more or less freedom of motion while he is confined in the hobbles.

By using the "back rope," as recommended by Professor Dick, the great muscles of the loins, quarters, and thighs are made to act upon two fixed points, namely, the spinal column on the one hand, and the extremities on the other; and when muscular force is exerted to such an extent as we see when an animal is down, one of the points is likely to give way; whereas, if the extremities are allowed a little freedom, the force is expended in moving them, and there will be but little or no danger of a broken back.

If it were possible, by so fixing the animal, to destroy muscular action, it would be a great convenience to the operator;

1 From Notes of Professor Dick's Lectures, delivered during Session 1855-6. 
but the muscular force remains in spite of all the tying down that can be done with ropes, and bones will as surely be broken.

I have always held the opinion now laid down; and although it might have been shaken during my first college days, it returned to me with greater force from seeing a horse break his back while tied down with the back rope. When I first promulgated this idea, during my first course of lectures at the Edinburgh Veterinary College, the majority of the senior students thought my teaching absurd; and to prove it so one of them purchased a subject for dissection, and before destroying him, had him cast for the instruction of his fellow-students, and secured so firmly that he could not break his back, as he said; but, wonderful to relate, the animal tried to struggle, the back was broken, and he rose no more.

It is a general belief among veterinarians that a horse with a broken back cannot move his tail; but this is liable to exceptions, and it will be found that complete paralysis from fracture will be accompanied at first by movements of the tail. To take this symptom as being diagnostic of fracture, and to conclude that when movement of the tail remains, the injury is a sprain of the psoæ muscles, may lead to error and wrong prognosis. Absence of sensibility and the history of the case are much more to be depended upon.

As a rule, but not without exception, vertebræ are fractured through their bodies, the arches remaining intact.

\section{FRACTURES OF THE OS SACRUM.}

The anterior portion of the border of this bone is sometimes fractured by violent falls, or by the animal becoming "cast in the stall." It will be remembered that the sides of the os sacrum are attached to, and situated below, the posterior spinous process of the ilium, giving support and attachment to the pelvis, and connecting it with the vertebra. When it is fractured, the iliac spine, losing its support, immediately falls, and the highest part of the quarter becomes flattened, which can be easily seen by comparison with its fellow of the other side. Examination per rectum will enable the practitioner easily to diagnose the exact seat of the injury. I'rofessor Dick used to 
say that in this fracture the legs bent under the horse, and that this was its diagnostic sign.

Treatment.-Slinging and rest; and as a rule cases terminate very satisfactorily, all that remains being the flatness of the quarter.

The Coccygeal Bones are also liable to fracture, which may be detected by inability on the part of the patient to raise the tail, difficulty in defreation, and by crepitation.

Treatment.-Strong leather binding laced around the tail, extending from its upper to its lower part, proper padding being at the same time used to prevent excoriation.

The rudimentary spines of the false vertebræ of the tail are sometimes fractured, giving rise to troublesome sinuses.

The wounds to be laid open, and the detached bones removed.

A comminuted fracture of the bones of the tail, with violent bruising of the soft parts, succeeded by inflammation, and even gangrene, is occasionally seen in practice. In such, amputation of the tail and removal of all the fractured bones must at once be resorted to, or serious consequences may result, such as irritative fever or tetanus.

\section{FRACTURE OF THE PELVIC BONES}

May be arranged under seven heads :-

1st. Fracture of anterior iliac spine,

$2 d$. " of posterior iliac spine.

$3 d$. " $\quad$ of the shaft of the ilium.

4th. " through the acetabulum.

$5 t h$. " into the foramen ovale.

6th. " through the symphysis pubis.

$7 t h$. " of the tuberosity of the ischium.

Fracture of Anterior Iliac Spine.-This is the most common form of fracture to which the horse and the cow are liable; it is generally caused by falls, or by the animal striking this part against a wall or side of a doorway, most commonly the latter, and chipping off, as it were, more or less of this prominent part of the quarter. It is not always attended by lameness, and is distinguishable by lateral flatness of that quarter.

The broken piece of bone is drawn inwards and downwards 
by the action of the abdominal muscles attached to it. In this fracture we find the method of repair is by false joint, the fractured ends being connected by a fibrous or a fibro-cartilaginous tissue. This is due to the action of the muscles attached to the fragments keeping them in a state of continual motion, and is an example of arrested development of the reparative material; every other part of the process of repair may be complete but that of ossification; and the fragments are held together by a yielding and pliant bond.

There is very little treatment necessary in a case of this kind. If the soft parts are injured, physic, fomentation, and a short rest will be sufficient.

Now and then, however, we meet with a case where no attempt at repair is made, and the broken fragment or fragments die, or become necrosed, giving rise to the formation of sinuses both near to and below the seat of injury. In such a case it will be necessary to remove the irritating fragments, and treat according to the rules already laid down.

Although a horse that is flat in the quarter may not be unsound, yet he is of less value in the market than if he were all right. It is, therefore, of importance that the practitioner should compare the two quarters of every horse he examines as to soundness; and this should be done by standing behind the horse examined.

The next four forms of fracture of the pelvis present externally symptoms similar to each other-namely, a flatness of the surface of the quarter, and lameness, with great difficulty in extending the foot, particularly if the toe strikes the ground.

The exact seat of the fracture is to be determined by an examination per rectum, when movement of the limb will indicate this by crepitation and motion in the part. As a rule, if the patient be laid, he will have great difficulty in rising; if through the acetabulum, he will scarcely be able to rise even with assistance; and when he is up, the limb is unable to bear any weight, and it will be shorter than its fellow, from the head of the femur being drawn upwards on to the dorsum of the ilium by the action of the gluteal muscles. The trochanter will be felt farther back and higher up than usual; the toe will be turned inwards, and rest upon the coronet of the opposite foot. Fracture through the acetabulum is the only one where re- 
covery is hopeless; all the others being curable, provided the fracture is not due to previous disease of the bones, namelyfragilitus or mollities ossium.

Fracture of the shaft, or into the foramen ovale, may occur without immediate displacement, and the animal show no sign of lameness until such displacement occurs.

In a case which once came under my observation, it was found that the fracture was of several weeks' standing, although the horse had worked perfectly sound in a carriage up to the time of the displacement. ${ }^{1}$

The fracture arose from mollities ossium, which was present also in the tarsal and other bones; and when displacement occurred the animal was at work upon a level road, and going at ordinary speed. He did not fall, but suddenly became very lame.

It has been already stated that fracture through the acetabulum is hopeless, therefore no time should be lost in putting the animal out of pain; but in the other forms, the patient should be slung, and in order to do something to keep the parts at rest, a stiff pitch-plaster ought to be applied to the whole quarter. Apparent recovery will take place in from two to three months, but care should be taken not to put the animal to work too soon.

If the horse has to be moved any distance after the occurrence of any of the curable fractures mentioned, his movements will be made very much easier by the attendants tying a cord round the foot of the lame limb, to assist in its extension, and to prevent the toe coming to the ground first; for it will be seen that if the toe strike the ground first, the patient will knuckle over at the fetlock to a most alarming degree; indeed, this knuckling over will sometimes be so great as to cause the animal to fall to the ground. Cases of this kind have repeatedly occurred in my experience, and by the simple expedient here mentioned-the attendant taking care to pull the foot forwards, and rather upwards before it touches the ground, so as to ensure its falling flat-the animal has walked with comparative ease.

1 I had diagnosed this to be a fracture of long standing before he was destroyed, and arrived at this conclusion from the fact that there was much thickening and indolent swelling to be felt per rectum. 
Fracture through the Symphysis Pulis.-This form of fracture is caused by the posterior extremities suddenly slipping outwards. Its symptoms are-a wide straddling gait behind, the legs being dragged forwards in progression; the feet wide apart, the toes turned outwards; the lameness being sometimes excessive. Examination per rectum will enable the practitioner to determine the extent and exact nature of the lesion.

Very little can be done in the way of active treatment. If the animal is not of much value, he had better be destroyed; but if young, or worth the cost of keep and treatment, he may be slung, the limbs pulled as near to each other as possible, and kept in that position by being fastened with a strap above the hocks. In two or three months a fair recovery may be expected.

Fractures of Tuberosity of Ischium arise from the patient falling backwards upon the buttocks, either from a slip whilst backing, or by being pulled over whilst rearing. The symptom is flatness of the most rounded part of the quarter, observed when standing to one side of the patient. An alteration in the form and shape of the quarters from any of the other kinds of fracture is observable when the practitioner stands at a short distance behind the patient; but this is to be seen to perfection when he stands at a short distance on one side, and on a level with this part of the body. Manipulation will detect crepitus, and cause pain. In some cases there will be considerable swelling of the soft parts, which will partly hide the flatness of the buttock. There is some degree of lameness in these cases; but if rest be enjoined, repair of the fracture will soon take place. No special surgical treatment will be needed, beyond the application of a good stiff charge or plaster after the subsidence of the swelling.

\section{FRACTURES OF THE FEMUR.}

The femur may be fractured through its neck or through its shaft, and both forms are incurable. Fracture through the neck commonly occurs along with fracture through the acetabulum, and is to be detected in the same manner. Both in this form, and when occurring through the shaft, there will be shortening of 
the limb, the toe being turned inwards, except when the head of the femur is forced into the foramen ovale, when the limb will be found longer than its fellow, and the toe will be turned

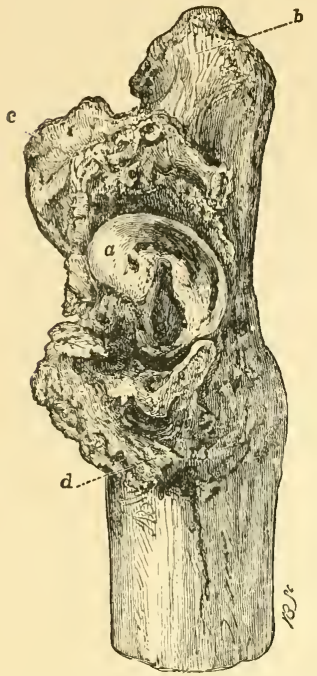
outwards. Fracture of the femur sometimes results from muscular contraction, when the animal is cast and bound for operation, displacement generally being the immediate result; and from the numerous muscles which clothe and are attached to this bone, reduction of the fracture is an impossibility.

Fracture of the Troehanters. I have seen two cases of fracture of trochanter minor externus, from the patient having fallen whilst in the cart, this part of the thigh coming in contact with the shafts. Any one who has seen a horse down, and half turned round in the shafts, will easily understand this. The diagnosis is difficult, and the lameness is peculiar. The elevation of the foot from femur, showing displacement of the ground, and the commence-
head; reunion, with considerable formation of temporary callus. $a$, ment of the act of extension of head; $b$, trochanter major; $c$ and the limb, seem to be performed $d$, surrounding deposit.

with very little difficulty; but the concluding part of the elevation of the limb, and extension of the femur on the pelvis, give the patient much pain, and cause considerable lameness. The foot is let down with a jerk; but after descending a short distance, the pain seems to abate, and the final implanting on the ground is performed with comparative ease and freedom. When the foot is implanted on the ground, another peculiarity may be observed, namely, that the extension of the pelvis on the femur is performed with difficulty. In order to see all these peculiarities, the practitioner must be a close observer. But to assist the young practitioner, let him remember-1st. The first act of raising the foot is done easily; 
$2 d$. The latter part of this, and the abduction of the thigh, cause pain and lameness; $3 d$. The pain caused by the last act produces a jerk when the limb is suddenly dropped, but when it has descended a very short way the pain is relieved; $4 t h$. When the foot is on the ground, and before the first act of elevation, the trunk is carried forward in the act of progression in a painful and peculiar manner. These peculiarities are explained by the fact that the gluteus-externus is attached to this trochanter, and that this muscle is an abductor of the thigh and extensor of the pelvis.

The treatment is rest, and slinging, if the lameness is great, or sufficient to prevent the animal from lying down; with soothing applications to the part.

Fractures of the condyles sometimes occur. One case happened in the practice of a friend of mine during operation. The animal was immediately killed, and an examination made, when it was found that the fracture was of some duration, and it came out afterwards that the animal had been cast in the stall some few weeks previously; that he had shown a little stiffness for a day or two, but that this had passed off: The bone is in my possession.

The patella is fractured longitudinally, or into several fragments, by direct violence; and transversely, by muscular contraction, causing great lameness, more or less inability to extend the limb, swelling in front of stifle joint; these symptoms being common to both kinds, with distinct separation of the two portions when the fracture is transverse. Reunion of this form is an impossibility, owing to the difficulty of bringing the fragments together, and from the presence of the synovia in increased quantity; on the other hand, a longitudinal fracture is sometimes curable, especially if it be a mere crack in the bone. In order to secure as much repose as possible, the part must be thrown into a state of relaxation, and for this purpose a shoe with a high toe-piece, and heels must be put on the foot, a veritable "patten shoe," the animal placed in slings, and cooling applications employed to reduce inflammation and swelling. After a time a blister may be applied, as a considerable effusion is apt to take place into the bursa, the absorption of which is necessary before reunion can take place. 


\section{FRACTURE OF THE TIBIA.}

Perhaps this is the most common form of fracture met with in the extremities, arising from kicks, and occurring most frequently when a number of horses are turned out to grass. The kick is delivered from the side opposite to the fractured limb, and descends upon the internal surface of the tibia, where it is covered entirely by skin, fascia, and strong periosteum. No other symptom of this fracture than a small punctured wound may be present, the animal exhibiting little or no lameness, although the fracture may be extensive. The fracture is generally very oblique, and the periosteum being strong, the ends are not separated. Many cases are on record where horses have worked at all kinds of labour for days after the receipt of injury without manifesting any signs of lameness, until by a sudden twist of the limb separation has taken place. Another cause of displacement is the animal's lying down; when he attempts to rise, the broken ends are forced asunder, perhaps a sharp-pointed extremity of the broken bone driven outwards through the skin, rendering the case almost beyond remedial measures. All cases of punctured wounds of this part, from the violence of kicks, should be treated as if the bone were fractured, until the practitioner is perfectly satisfied upon the matter. The patient should be put in the slings, and kept there until the wound is perfectly healed, and the horse quite free from lameness. If there be no fracture, but little thickening will be left after the subsidence of the inflammation; but if there be a fracture, the veterinarian will be able to detect the presence of the provisional callus. Non-attention to these practical points has caused the destruction of many valuable horses. There is little danger of displacement if the patient be kept in the slings; but if quite sure that fractures exist, the tarred cord should be applied round the leg from the foot upwards, as high up on the thigh as possible. Some portion of the fragments may thus be included in the cord, for it must be rernembered that the cleavage, being oblique, will extend for some distance both above and below the actual wound.

If displacement has occurred before measures can be applied, it will be found almost an impossibility, unless the patient be very young, to perform reduction; and the best course will be 
to destroy the suffering animal; but in the young, and in cases where treatment is insisted upon, the first step nust be the removal of any projecting bone by the bone forceps, and afterwards traction, applied by means of cords to the hock and leg, to produce extension; in this way, and by proper manipulation, the fragments may be approximated and retained in their position by the means already described.

\section{FRACTURE OF THE TARSAL AND METATARSAL BONES.}

The Os Calcis.-Transverse fracture of this bone is caused by muscular contraction, and by the slipping forward of the limb, thus throwing the gastroc-nemius externus, which is attached to the upper extremity, into an unduly extended condition, and the bone suddenly giving way in preference to the tendon of attachment, or the fracture may occur spontaneously when the bone is diseased. This fracture is mostly seen in young horses, where the apophysis is not completely solidified to the body of the bone. This accident arises from the same causes as those which produce "curb;" in the one case, however, the bone is the structure to give way, while in the other the calcaneo-cuboid ligament receives the injury.

Inspection of the limb will demonstrate a flatness of the point of the hock, flaccidity of the tendo achilles, with an apparent shortening of the limb; there is no shortening in reality, but great difficulty in bringing the foot to the ground. The lameness is very considerable; manipulation will enable the practitioner to discover the detached portion of bone resting in front of the os calcis, in close contact with the flexor pedis perforans, on the postero-internal aspect of the hock. It is easily moved by the hand, and can be elevated almost into its natural position.

Treatment.-Apply a high-heeled shoe, then force the fragment into its proper position, if practicable, or as nearly as possible, retaining it there by pads of tow and bandages. The tow must be made into firm pads or rolls, and pressed down in front, and on both sides of the fragment, to prevent it from falling forwards. Over the dry bandage the starch bandage must be carefully laid, extending from the foot over the hock as high as possible, in order to keep the limb in a state of rigidity. 
The patient is now to be placed in slings; in about two months consolidation may be expected; the horse may now be placed in a loose box or sent to grass. I have seen very good recoveries from this accident; but if the bone be in a diseased condition, mollities or fragilitus ossium, treatment will be of no avail.

The tarsal bones are all liable to be fractured by direct violence (see Photo-lithograph, Plate II., Fig. 6-Fracture of the Astragalus), and, if associated with a wound, it is necessary to destroy the animal ; but cases of very severe injuries without wound occur, where the patients completely recover; and examination after the death of the animal, perhaps years afterwards, reveals the fact that the bones had been broken. It will be understood by the anatomist that this applies to the cuneiform and cuboid bones only; for where the astragalus has been involved, only one method of repair can take place, and that is anchylosis of the true hock-joint. It is most difficult to diagnose this form of injury correctly, from the great tendency to immediate swelling of the part, and from the absence of crepitation; the reason being that the bones are firmly bound to each other, and to the metatarsals, by very numerous ligaments.

If the patient be worth treatment, it will be advisable to apply the high-heeled shoe, to place him in the slings, and to abate irritation and fever by fomentations lightly applied, by sedatives, or even anodynes, as aconite and opium, regulating the action of the bowels by laxative food and gentle aperients. After the subsidence of the fever and of the local heat, when two or three weeks have elapsed, a blister will be of great service in removing pain, and in hastening the process of ossification. Fractures occurring below the hock, except from kicks or blows, are extremely rare; they may result from concussion, or the sudden twisting of the limb; but they are much more commonly found to occur from these causes in the anterior extremities. I shall therefore consider them along with those of the fore limbs. 


\section{CHAPTER VII.}

\section{PARTICULAR FRACTURES-continued.}

FRACTURES OF SCAPULA-HUMERUS-CONDFLES-OLECRANON-RADIUS

-ULNA - TRAPEZIUM, CARPAL, METACARPAL, AND SESAMOID BONES

— "SPLIT PASTERNS"-NAVICULAR AND PEDAL BONES-BROKEN RIBS-LUXATIONS-CASE OF DISLOCATION OF THIRD CERVICAL VERTEBRA.

THE College Museum contains many cases of recovered fractured scapulæ; and this bone is liable to be broken through its neck or body by muscular contraction, and through its spine by direct violence, caused by blows or falls,

Compound fracture of the spine will sometimes be found to exist with the fragments completely detached; in such a case, they must be removed.

When the body or neck is the seat of the lesion, if there is not much displacement, speedy reunion will take place, and the animal completely recover, provided the articulation be not involved.

But little can be done in these cases beyond enjoining quietude, placing the animals in slings, and watching for untoward complications.

When the humerus is broken, I am of opinion that perfect soundness is impossible, although Professor Dick taught that such might take place. The practitioner well knows that many powerful muscles are attached to this bone, the action of which will render the retention of the broken bone in anything like its proper position an impossibility. Fractures of the tubercles, of course, like those of the trochanters of the femur, will be an exception to this rule.

When the humerus is broken, there is an immediate shortening of the limb. from the inferior portion of the bone being drawn upwards by the muscles attached to it; great lameness; and there is very often a rupture of the humeral artery, or of one of its 
large branches, with subcutaneous hæmorrhage, causing great engorgement of this part of the limb, and rendering the detection of crepitus an impossibility. But the practitioner need not hesitate in arriving at a correct diagnosis, as the pendulous leg, inability to place any weight upon it, and the sudden engorgement are quite sufficient to prove the existence of the fracture.

This fracture may arise from muscular action; but commonly it is caused by falls. One case, however, has corne under my notice, where it arose from fragility of a portion of the shaft of the bone, from its being continually brought into contact with the carriage pole.

The animal was a very fine carriage horse, one of a pair, with splendid and fast action. Shortly before the final catastrophe he showed signs of a slight lameness, attributable to the shoulder; with short rest and treatment he recovered, and was put to work, at which he continued for some little time. Whilst out one day, and upon a very level road, that between Bingley and Keighley, in Yorkshire, and going at a good pace, he suddenly fell-the leg completely giving way-bringing the other horse, carriage and all, to the ground. He could not rise, the humerus was fractured, and, upon examination, it was found to be in a state of degenerative disease just at the part which was on a level with the carriage pole.

The condyles of the humerus are occasionally fractured, and, as a rule, they never reunite, the reason being the impossibility of keeping the limb in a state of complete rest.

The radius and ulna are fractured by direct violence, and the olecranon by muscular contraction. In dogs this fracture is not at all uncommon; and, if properly fixed with bandages, generally recover without leaving much lameness. I have noticed one case of dislocation of the elbow-joint, accompanied by rupture of the internal lateral ligament; although treated for some time, it did no good, and was ultimately destroyed. The dislocation was easily reduced, and bound up very firmly; but owing to diseased action having taken place in the joint, it was seen that anchylosis would be the result. In the case of a brood mare, or valuable stallion, cases of this kind might very justly be treated; but in horses required for work it will be better to order the patient to be put out of pain, as a horse with a stiff elbow is of little use. A dislocation is easily distinguished from fracture, as in the one 
case you have displacement and contortion without crepitus, whereas the sound is present in the other. There never need be any difficulty in diagnosing fracture when displacement has resulted; the difficulty is in forming a correct opinion when this has not taken place.

The olecranon is fractured transversely by over-extension; it snaps across about its middle. It will be remembered that the very powerful triceps and scapulo-ulnaris muscles are attached to the olecranon. When the animal slips forward to any great extent, these muscles are thrown into a state of great tension; in fact, they are extended beyond their limit. The results from this are, laceration of their tendons of insertion, fracture of the bone into which they are inserted, rupture of the muscles themselves, or all these lesions combined. Any of these cause immediate and great lameness; the animal will stand with the whole limb in an exceedingly relaxed and semi-flexed condition, with almost total inability to move it, and to support any weight upon it. The detached bone being drawn upwards into the deep bed of muscular tissue posterior to the humerus, and the parts, as a rule, immediately swelling, crepitation eannot be detected, and it becomes a matter of great difficulty to form a correct diagnosis. Mr. Anderson, V.S., Glasgow, has discovered an almost unfailing way, and it is as follows:-Let the practitioner place his knee firmly against the knee of the patient's injured leg, and by firm pressure he is to straighten the semiflexed limb, and to keep it in that position, while an assistant is directed to lift up the horse's opposite fore foot. If the olecranon is fractured, the patient is unable to stand; but if there be merely laceration, he will be able to bear his weight, with the assistance at the knee given by the practitioner.

There are two positions in which the limb is maintained when suffering from this injury, namely, semi-flexed with the foot in advance, with the heel touching the ground; or semi-flexed with the foot behind, knuckling over, the toe only touching the ground, and turned inwards.

Correct diagnosis is of the greatest importance in cases of this kind, as in laceration of the tendons, or of the muscular structure, a recovery may be expected; whereas, if the bone is broken, little good can be done if the fracture is complete, and the fragments are separated by muscular action. 
In this fracture it will be found that union is by false joint. This will interfere more or less with the action of the animal ever afterwards; but by far the most common result is intense inflammation, set up by the irritation of the broken fragments, which become necrosed, abscesses form, commonly communicating with the elbow-joint, and the animal dying (or having to be destroyed) from irritative fever.

Reasoning from these facts, I am of opinion that the most rational method of treatment will be at once to cut down upon and remove the fragment or fragments which may be separated from the bone, bringing the lips of the wound together by suture, applying the slings, and other appropriate remedies.

There is a peculiarity about injuries to the neighbourhood of the elbow which leads to the burrowing of any pus that may form into the articulation, and in this way cause a most distressing complication-that of open joint. Kicks or punctures, although they may be inflicted upon a part of the fore arm, at a distance of four inches from the joint, will often produce this dreaded complication, if great care is not taken in giving free vent to all collections of matter; and the reason for it is to be found in the peculiarly firm nature of the muscular and other structures of this part, the muscles being individually clothed in firm thecæ, and the whole enveloped in the brachial fascia.

Treatment.-Rest in the slings, and the maintenance of the limb in the position most calculated to favour the process of repair by a properly adjusted shoe-high-heeled or otherwiseor by the removal of the shoe, as each individual case presents its own peculiarity of symptoms. These details of practice must be left to the judgment of the practitioner, who alone will be able to carry out what may strike him as essential to the wellbeing of his patient.

Fracture by direct violence is that most commonly met with, and, in the majority of instances, from falls in the cart; the elbow being thrown across the shaft, and both radius and ulna broken through into the articulation.

This lesion is easily diagnosed by distortion and crepitus. No treatment can be recommended.

If the ulna only is broken, it will be found that the fracture is through "the beak of the olecranon," iuvolving the surface, which articulates with the humerus between and behind the condyles. 
No treatment, as the whole joint is sure to become involved in the inflammatory actiun.

The radius is broken at its upper third, the ulna sometimes being involved; if it be transverse, or not sufficiently oblique to involve the elbow-joint, it is, as a rule, amenable to treatment. I have seen many cases do very well. It is much easier to treat than when the ulna only has given way, for in the latter instance it is very difficult to bring the displaced fragments into proper position, as the muscles act upon it, and draw the fragments apart.

But when both radius and ulna have given way, the displacement is less, and we have greater command over the limb than over the ulna, which is fixed to the radius by interosseous ligamentous tissue. In the treatment of fractured radius, or of both bones combined, the reduction must be effected by extension and counter-extension, and the fragments kept in apposition by bandages, tar-cord, and by the long splint.

The radius is broken at its lower third from direct violence, as kicks and blows; the nearer it is to the knee, the greater the difficulty in managing the treatment.

Fractures of the bones of the knee arise from direct violence, as kicks and falls. As a rule, there is no treatment. The upper and middle row of carpal bones are most commonly the bones broken. If from a fall, the tendon of the extensor metacarpi magnus is lacerated and torn, and the wound will be upon the front of the knee.

One case of fracture of the trapezium has been mentioned to me by Mr. Anderson, Glasgow. The case was treated for a long time, but, I believe, with no good result.

The metacarpal bones, as well as the suffragines, more especially the last-named bones, are broken into many fragments, sometimes into scores of pieces, most mysteriously, while the animal is galloped upon sandy or soft ground. The sandy beach of Portobello is noted for this.

Transverse fractures of the sesamoids sometimes occur spontaneously when the bones are in a fragile condition from organic disease, and are incurable. The symptoms are descent of the fetlock pad, elevation of the toe, with great lameness, and the presence of a depression, marking the seat of the fracture in the bone or bones. 
Simple fractures of the pastern bones, even when the joint is involved, make good recoveries when properly treated by bandages, splints, and rest in the slings.

Split pastern is of common occurrence.

The diagnostic signs of this are very sudden and severe lameness, occurring when the animal is upon a hard road; the lesion arising from concussion.

Sometimes crepitus can be detected, but very often this is absent, from the fact that the bone is bound most securely by unyielding ligaments. The suffragines are most commonly broken, but it is not a rare thing to find the coronæ fractured also.

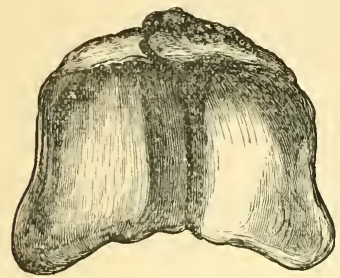

Fig. 13.--Perpendicular split through the central groove on the upper extremity of os suffraginis.

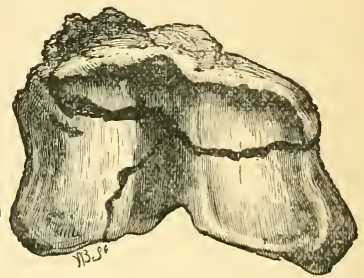

Fig. 14. - Irregular fracture of upper extremity of os suffraginis.

When one bone only is broken, recovery is the rule, although the articulation may be involved.

In the course of a day or two after the accident, the pastern will swell, and the swelling is of a hard, unyielding character. This is a favourable sign, as it indicates the formation of plastic lymph in the part, and the commencement of the process of repair.

There is no necessity for very firm bandaging, especially if crepitus be absent. The shoe should be carefully removed, and the horse allowed to stand with the lame foot upon sawdust, or some material which will adapt itself to the form and peculiarities of the position of the foot. The toe of the foot will only touch the level ground, and it will be a great relief to the horse if the material he stands upon will allow the heel also to be supported. In about two or three months, depending upon the progress of the case, if the injury be to a fore extremity, the slings may be removed; but if it be a hind leg, the slings must be made use of for a longer period. 
I have seen one case of transverse fracture of the sesamoid bones, both bones being involved. The diagnosis was difficult, as there was much swelling around the fetlock; the toe was elevated; the fetlock pad came to the ground; and there was very great lameness; and the symptoms were exactly like those of rupture of the superior suspensory ligament. The horse, being an old one, was destroyed. I do not see that there can be a possibility of rendering an animal serviceable after an accident of this kind. In the first place, it would be almost impossible to keep the fragments in contact, owing to the tendency of the fetlock to fall downwards and the toe to be drawn upwards by the extensor pedis; and, secondly, the sesamoids, articulating by one surface with the metacarpal, the fetlock joint would become anchylosed, and by the other surface forming a synovial bursa, over which the perforans glides, the tendon would become diseased to a considerable extent, constituting a permanent lameness.

Fracture of the navicular bone occurs in two ways; first, by direct puncture, as when a nail penetrates the frog. These cases are rare, but they do happen; the symptoms are excessive lameness, presence of the foreign body, or of the wound from which it has been removed, synovial discharge, abundant, often bloody, and after a time mixed with flaky pus; abscesses commonly form around the coronet; the animal suffers from a high degree of irritative fever, and if not put out of his suffering, dies from pain and exhaustion. It is of the greatest importance to know if the bone be broken or not, as many cases of open navicular joint recover when uncomplicated with fracture.

In open joint without fracture, the pain and fever may be as great at first as when fracture is present, but the discharge from the wound will be synovial, straw-coloured, or mixed with a small quantity of odourless pus; whereas, if the bone be broken, there will be more or less continual oozing of blood from its cancellated tissue, tinging the discharge with a dirty-red hue, and giving it a foetid odour. When these symptoms are present, say in two days after the injury, the animal had better be destroyed. It is as well to wait, say for two days, as the colour may be due to the oozing of blood from the soft structures, and not from the bone.

The other way in which the navicular bone is fractured, is 
when it has been nearly worn through by the caries of chronic navicular disease. Very often this fracture is accompanied by rupture of the diseased portion of the tendo perforans, and in such a case the toe is immediately turned up, making the diagnosis certain; when this complication is not present, it is more difficult to arrive at a sure conclusion, and the history of the case must be taken into account. The horse has been lame, but serviceable, for a long time, and all at once he becomes helplessly lame and unfit for further use. The practitioner must draw his own conclusions, and reason upon the case, as no set rule can be laid down.

This form of fracture occurs very often after the operation of neurotomy, from the increased freedom of motion which results upon the removal of pain; both tendon and bone giving way, the fetlock coming to the ground, \&c. I have seen one case that goes to prove that mere fracture of the bone after this operation does not always cause inconvenience to the animal; and subjoined are two woodcuts, of the navicular bone from a horse
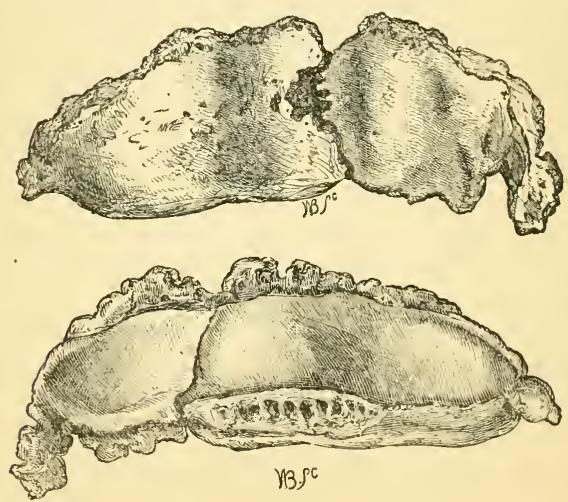

Figs. 15 and 16 show both surfaces of the Navicular bone, described in the text.

unnerved by me at the Veterinary College more than three years before he was destroyed. During the whole of that time he performed his work well and soundly, and ultimately had to be put away, owing to the occurrence of necrosis of the os pedis. 
Upon examination, the navicular bone was found fractured, and the fracture reunited by false joint. I am of opinion that the fracture had occurred immediately after the operation, and that union had taken place very shortly afterwards. The case was under very favourable circumstances, being driven in the cart by a very careful old man, and never pushed beyond a walking pace.

The os pedis is fractured in various ways:-1st. By violence, as when the calkins of the shoe are entangled in a railway point, the horse falling, and a large portion of the crust becoming detached, and at the same time the bone being lacerated. $2 d$. From the falling of heavy weights upon the feet. $3 d$. By concussion; but before this form of fracture can occur the bone must be in a fragile condition, resulting from chronic laminitis. $4 t h$. From the prick of a nail in shoeing. This fracture may be immediate, or a piece of bone may become detached from the pressure of the nail, thus constituting a fracture by partial necrosis.

The first kind may be considered beyond treatment, unless, indeed, the fracture be very slight, and easily removed. The proper treatment will be the removal of all detached horn and fragments of bone, the application of poultices, and the administration of febrifuges, with rest in the slings until the fever subsides.

It is curious to observe how soon a portion of foot stripped of its crust is sheathed and coated over by the horny secretion of the sensitive laminæ; in a very few days, if the laceration of the soft tissues has not been great-where the horn is merely stripped off-it will be found that the soft structures are covered over by a layer of protecting horn, which prevents further irritation, and enables the patient to move the foot with comparative ease. Had the advocates of the hypothesis, that the horny laminæ are secreted by the coronary substance, only remembered what they must have observed in the course of their practice, they never would have propounded such an absurdity. ${ }^{1}$ After the pain and fever have subsided, the practitioner must determine if a shoe can be applied with advantage or not. If it be

1 Notwithstanding the severe strictures of one critic, who states that the laminæ do not secrete horn, I maintain my view that a horny secretion is formed by them independently of the coronary band. 
thought necessary, it must be a bar-shoe, made as light as possible, and nailed to the uninjured part; the foot is then to be enveloped in a soft dressing, consisting of fine tow, tar, and bandages. But if the bone be broken into several fragments, or when it is cleft through its centre, and into the pedal articulation, it will be advisable to destroy the patient.

The second and third forms are, as a rule, incurable. The last form is partial, but is always to be considered of great importance, although by careful and timely treatment the results may be satisfactory. The symptoms are those of pricked foot, with or without suppuration. At first it is impossible to say whether there is a fragment or not. But let the practitioner observe that, when the foot is pricked, the removal of the offending body, the paring out of the foot, and giving exit to the imprisoned pus, will, as a rule, give relief. But if a portion of the bone be detached, the animal will continue as lame as he was prior to the treatment; or it will be found that the pain and fever increase from hour to hour, abscesses form around the coronet, and the animal will die in great agony in the course of a very short time.

The treatment must be prompt. If it be found that relief does not follow the treatment appropriate for punctured font, the practitioner must cut down on the pedal bone at the seat of the pain or suppuration, and explore. He will find a loose fragment; this he must remove as speedily as he can. It may be no larger than a small pea, or it may be the size of a bean, but out it must come. When this is effected, relief is generally obtained; the after treatment being poultices, cathartics, anodynes, and febrifuges.

\section{BROKEN RIBS.}

The attention of the veterinarian is but seldom called to this injury, yet post mortem examinations reveal in many instances that the ribs have been fractured at some time or other of the animal's existence; the method of repair being always by the ensheathing callus. In none of these cases has there been any record of the accident, nor indeed would there be any symptoms present to indicate such, if the fracture had not been a compound one.

The causes are direct violence, such as kicks from other horses, and blows. 
In compound fracture-the only kind that the veterinarian will be most likely called upon to attend, as in simple fracture it is rarely necessary to do anything-it may be found that the lung is wounded, and that the patient will be suffering from pneumonia. It might be supposed that collapse of the lungs would immediately occur upon the admission of air into the cavity of the thorax; but this does not take place to such an extent as one might imagine. The air will be found to rush in and out of the thorax through the wound at each respiratory movement; that is, it will rush in during expiration, and out during inspiration.

Treatment.-To find out if the lung is punctured, and if the fragment or fragments of the bone are displaced, and to replace them in their proper position. All this may be done by introducing the finger into the wound, and by converting the compound into simple fracture; closing the external opening by a good stiff plaster-the Burgundy or the common pitch will do very well-to limit the movernents of the chest by a broad belt, and to watch carefully for signs of pleurisy and pneumonia.

If called upon immediately after the accident, purgatives may be prescribed with advantage; but should the case have been neglected until inflammation of the pulmonary organs begins to manifest itself, purgatives must be withheld, and sedatives, as aconite, with the nitrate of potash, administered.

The ribs may be fractured, and the skin not broken, but when the subcutaneous tissues are bruised to a considerable extent, it will be almost impossible to diagnose the fracture. It may only be supposed to exist by the severity of the injury, and by the animal perhaps manifesting symptoms of pleurisy. It is a remarkable fact that broken ribs, when broken inwards, although it must be at the risk of wounding the lungs, are more readily repaired than when an opening is made externally; this is due to the spores, or organic particles contained in the atmosphere-being the germs of low forms of life discovered by the microscope, causing putrescence and suppuration. This fact will point out the importance of closing the wound of compound fracture, and also of arresting suppuration, if it has already commenced, by carbolic acid dressings. 


\section{LUXATIONS, OR DISLOCATIONS WITHOUT FRACTURE,}

Are very rare in our patients; the most common forms are those of the patella of the horse, the head of femur and carpus of the dog, and that partial form which may be said to exist in what is termed "knuckling over" at the fetlock in the horse. It is said by some that the shoulder-joint is dislocated without fracture in the horse, and I am inclined to think that such a thing may occur, but it can only be very rarely met with; the anatomy of the articulation, the broad and extensive head of the humerus, allowing the comparatively small glenoid cavity of the scapula a freedom and extent of motion which renders dislocation almost an impossibility. The following case, I think, proves that the injury can take place. A horse slipped and fell; when he rose, one fore leg was found powerless, pendulous, shorter than its fellow, and bulging outwards at the shoulderjoint. He was taken to a stable, where he immediately lay down, groaned in agony, was very restless, and unable to rise to his feet. A practitioner, who was sent for, pronounced the shoulder to be out; he secured the animal's other legs by means of hobbles, applied ropes to the lame limb above the knee and at the pastern, and had several men to pull steadily at the ropes to cause extension. When all was ready, he slipped his boots off, and suddenly jumped with force on the damaged joint, which immediately gave a kind of crack, and the bulging suddenly disappeared. The ropes and hobbles were now removed, the horse assisted to his feet, when he was found to be nearly well, and walked home, a distance of about three miles, that night. I was a boy at the time, but I remember the whole thing very vividly. The late Mr. Barlow was of opinion that this dislocation was an impossibility; but when I mentioned the above case to him, he was much struck, and confessed that it was sufficient to stagger him.

Luxation of the Patella occurs in young horses that are grazed on very hilly pastures, from the mal-position in which the limbs have to be constantly kept, owing to the inequality of the ground. The patella is forced outwards; the internal lateral ligament becomes stretched across the internal prominence of the trochlea of the femur, and is thus torn or chafed. At first, the luxation is only partial, the patella slips in and out of its 
position with a clucking noise at every step the animal takes; very commonly both patellæ are in the same condition. When the luxation is complete, the internal lateral ligament must be ruptured through its whole thickness, and the patella slips outwards. The limb now becomes stretched backwards; there is total inability on the part of the animal to flex it, owing to the action of the vasti, rectus femoris, crurcus, \&c., being interfered with.

Trcatment.-Forcible extension and keeping the limb in a forward position by means of a strong cord round the

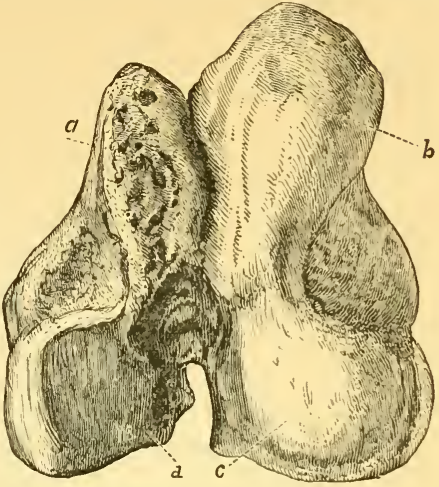

Fig. 17.-Trochlea of the femur, showing the effects of friction from luxation of patella, $a, \mathrm{Ex}$ ternal, and $b$, internal trochlea. $d$ and $c$, Condyles of the femur.

foot. The leg must be kept in this position by the cord being fastened round the neck of the animal for some hours; a shoe higher at the toe than at the heels should be put on, with a projecting piece of iron at the toe, and a smart blister applied to the stifle. Professor Dick used to say that this would perform a cure, even after the ligament had entirely given way, provided the animal were kept at rest.

If the dislocation has been existent for any time, the articular cartilage on the surfaces of the trochlea and patella becomes worn by attrition; the articular laminal layer of bone is then rubbed off, the cancellated tisane exposed, and there is a deposition of porcellaneous material (porcellaneous deposit) in the opened-up Haversian canals, giving the articulating surfaces a shining or glistening appearance. A restoration of the animal to usefulness will be now a matter beyond possibility; indeed it is hardly conceivable that a complete dislocation with rupture of the ligament can at any time be curable. 
Partial disolocation of the Fetlock Joints, arising from diseases of the feet, over-work, or a natural tendency to "knuckle over," is an occurrence of which little need be said, and with the exception of rest and perhaps a blister, no treatment can be recommended. ${ }^{1}$

This form of unsoundness may also arise from relaxation of the binding ligaments, and this may be the result of chronic inflammation of them, leading to a degeneration of their proper structure, when they will be found pulpy, more or less thickened, and the areolar tissue which is amongst their fibres highly vascular.

The hear of the Femur may be dislocated in the ox, dog, and eat; but in the horse this is an impossibility without fracture, owing to the fact that in the horse the "pubio fcmoral ligament" is found. This ligament arises from the head of the femur, passes from the acetabulum through the cotyloid notch, then runs along a grove on the under side of the pubis to the median line, where it crosses its fellow from the opposite side, forming a cross, $X$, and becomes finally lost in the faschia of the abdominal muscles. In this manner the head of each femoral bone is kept in position by the muscles of the opposite side of the abdomen. Reduction may be easily effected in the dog and cat by extension and manipulation, and the parts maintained in position by a stift Burgundy pitch plaster; but in the cow any treatment may be considered questionable, and if the animal is at all fat, she should be made into beef.

The symptoms of this dislocation are similar to those of fracture, but there will be no crepitation. As a rule, in the dog and cat the dislocated bone will be found on the dorsum of the ilium. There will be shortening of the limb, and total inability on the part of the patient to perform the ordinary movements; but should the dislocation be into the foramen ovale, the limb will appear longer than its fellow, and the foot turned outwards.

Dislocation of the Carpus, or of one of the phalanges, is of com-

1 Note.-Since the above has been in type, I have had the opportunity of dissecting a limb, where habitual "knuckling over" at the fetlock was found to be due to an arrested development of the extensor suffraginis. The tendon of this muscle terminated at the upper head of the metacarpus parvus externus, the action of the extensor muscle thus terminating at the knee, and the fetlock knuckled over from the want of the support of its tendon. 
mon occurrence in running dogs, especially greyhounds. It is easily reduced, but is very apt to return.

DISLOCATION OF THE CERVICAL VERTEBR.E WITHOUT FRACTURE.

An animal, in August 1871, was found unable to rise one morning, but with some assistance was got on to its feet, when the neck, which had been previously all right, presented the appearance shown in the annexed woodcut (Fig. 18). Four days afterwards it was sent to the College, a distance of about two miles, for my opinion. The animal (an old mare) walked with a slight staggering gait, knuckled over occasionally at the fetlocks, could eat well, and move the head up and down with a moderate degree of freedom. There was no swelling of the soft parts from bruising or inflammation, the prominence of the curve being

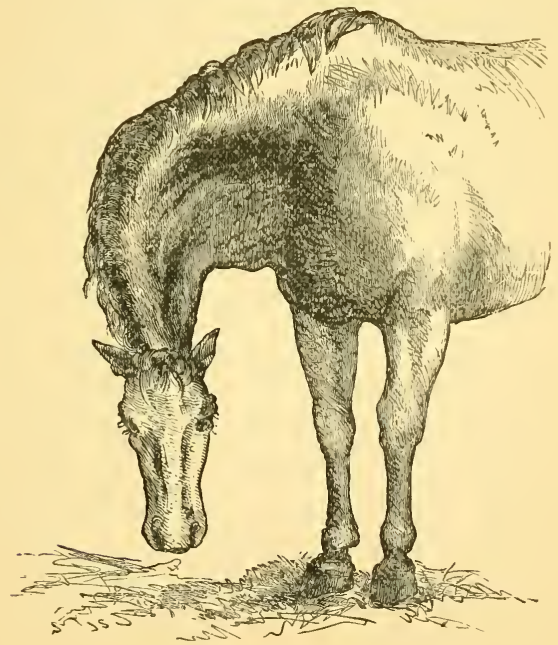

Fig. 18.

hard bone. Upon attempting to straighten the neck, the animal would become paralyzed in the limbs, this paralysis passing away when the pressure was removed from the neck. Being very old, she was ordered to be destroyed. When the neck was stretched 
by a cord to the wall, previous to the division of the carotid artery, she fell paralyzed, and died before she was bled.

The post mortem revealed that the luxation had been reduced by the stretching; that the fibro-cartilaginous disc, between the third and fourth cervical vertebræ, was in a pulpy condition; that the spinal cord and its surroundings were but slightly affected; and that there was no fracture. This case proves that there can be luxation without fracture; that such a lesion does not prove fatal if the spinal cord is not pressed upon; and that reduction-causing pressure upon the cord-may be followed by immediate death. I have heard of similar cases, but never saw one before. I have, however, seen another case since the publication of the first edition of this book.

Twisted or wry neck may occur from a variety of causes other than dislocation, such as over-stretching or bruising of the muscles of one side of the neck, causing inflammation; or from rheumatism affecting them, inducing loss of function of the muscles of one side of the neck. It is also a symptom of hemiplegia. These various conditions may be distinguished from luxation by the absence of the diagnostic symptom of the latter, namely, the sudden paralysis of the limbs when attempts are made to straighten the neck. 


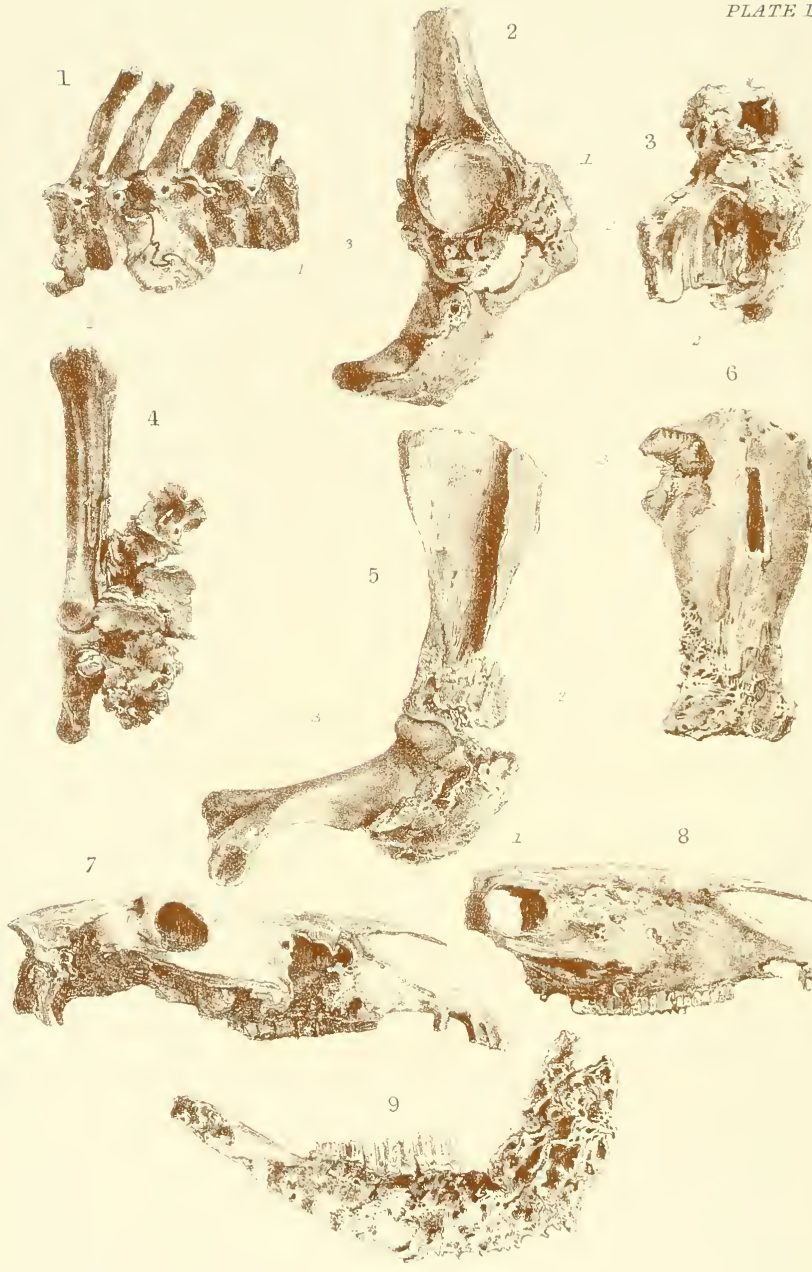


\section{DISEASES OF THE BONES AND ARTICULATIONS.}

\section{EXPLANATION OF PHOTO-LITHOGRAPH, PLATE I.}

1. Fragiditus Ossium, with fracture through the body of the fourteenth dorsal vertebra. (1.) Large fragile osseous tumour. (2.) Line of fracture.

2. Rheumatoid Disease of Hip-Jornt. (1.) Cotyloid cavity, showing porcellaneous deposit. (2.) and (3.) Addimentary bones, partially filling up the foramen ovale.

3. Diseased Condition of the Os Calcis and Astragalus, produced by repeated bruising, the animal from which it was obtained being a vicious kicker.

4. Osteophytes. Through the centre of the mass a canal is left for the passage of the flexor tendons, blood-vessels, and nerves.

5. Ossification of the Flexor Brachin in chronic shoulder lameness.

6. Necrosis Totalis of the Scapula. (1.) and (3.) show large cloacæ or foramina grandia. (2.) represents the sequestral capsule. The specimen from which this figure was taken was highly prized by Professor Dick.

7. Caries of Superior Maxillary Bone and alveolar processes, arising from disease of the two anterior molar teeth.

8. True Mollities Ossium, or softening of bone, by absorption of the earthy salts, without alteration in animal basis. Case described in the text.

9. Actinomycosis (Osteo-Sarcoma). Lower jaw of ox, the hollow spaces in the specimen, when fresh, being filled by sarcous material. 


\section{CHAPTER VIII.}

\section{DISEASES OF THE BONES AND ARTICULATIONS.}

\section{CLASSIFICATION OF THE BONES-OSTITIS-SORE SHINS-SPLINTS-}

PECULIARITY OF SPLINT LAMENESS-SCROFULOUS OSTITIS.

THe diseases affecting the bones of the lower animals may, for the convenience of description, be classified under two heads, namely, inflammatory and non-inflammatory. The line of demarcation between the two is not very well defined, as what may be essentially a non-inflammatory disease in itself might be productive of inflammation. Inflammation of bone (ostitis) can scarcely be considered independently of inflammation of the periosteuin (periostitis), as both structures are so closely related that we camnot have inflammation of the one, without the other participating in the diseased action.

A correct knowledge of the pathology of bone being of the greatest importance to the veterinarian, and the study of this particular branch of veterinary pathology having hitherto been neglected by the profession, I feel it necessary to give a slight outline of the general anatomy of bone, in order that its pathology may be better understood.

Bones are divided into long, short, flat, and irregular. With the exception of the cranial bones, the short, irregular, and flat bones are composed chiefly of cancellated tissue enclosed in a thin shell; and it is found that, owing to their structure, they, as well as the extremities of the long bones, undergo pathological changes somewhat similar to those of the softer structures.

The bones of the cranium are composed of two bony platesa very dense one internally, another less dense externally, and an intermediate vascular and medullary structure. 
The long bones consist of a shaft and two extremities, or epiphyses, which are much looser in structure than the shaft, are developed separately from it, and are supplied by numerous blood-vessels passing directly into them. The shaft is composed of very hard tissue, encloses a cavity called the medullary canal, and on its outside a number of small lines or grooves are to be seen, which are the oblique openings, through which vessels pass from the periosteum into the dense structure of the bone.

The bones of race-horses contain more compact tissue in their shafts than those of lower-bred animals. The dense structure-compact tissue-contains the Haversian canals, conveying blood-vessels; and the canaliculi, which are smaller canals conveying blood plasma to the lacunæ. In this manner this portion of the bone receives its supply of nutritious material from the blood, and without this arrangement the bony structure could not receive nutrition. Each Haversian canal is about $\frac{1}{600}$ of an inch in diameter, and collectively they run in a longitudinal direction, but have many transverse branches of communication. The canaliculi average $\frac{\pi, \frac{1}{15}, 0 \gamma}{0}$ of an inch in diameter, and appear as dark radiating lines, decreasing in diameter as they recede from the lacunæ; they are, along with the lacunæ, filled with the fluid, colourless portion of the blood.

The lacunæ are irregularly oval, stellate, dark-looking bodies, lying with their long diameters, which are about $\frac{1}{1800}$ of an inch, parallel to the bony lamellæ. Each long bone is, in addition to the periosteal vessels, supplied by a nutrient artery, which passes directly into the bone, and breaks up into branches in the interior of the medullary canal.

It will be seen that the compact bone is abundantly provided with vessels, entering from numerous points, covered by periosteum and endosteum; that these nutrient vessels are exceedingly minute, and surrounded by a dense structure; and that, in consequence of this peculiarity, the effects of inflammatory action will be very distinctive, and the symptoms most acute. Fortunately, however, inflammation of the shafts of the long bones is exceedingly rare in the lower animals. The extremities or epiphyses of the shaft are, as already stated, developed separately from the shaft, and they exceed it in circumference, are irregular in outline, expanded, roughened externally, and 
composed of cancelli, with a very thin layer of compact tissue. Whilst they are of greater circumference, their weight is not relatively greater than the shaft, their increase being due to an expansion of substance. This arrangement lightens and strengthens the bone, and modifies inflammatory action, which consequently partakes more of the nature of that of the soft tissues.

The hardest part of the bone is a thin laminal layer, lying next to the articular cartilage. It is non-vascular, insensible, and destitute of Haversian canals, and, in inflammatory disease of the articulations, it is affected in a remarkable way, which will be referred to hereafter.

Each long bone has a canal in the centre of its shaft, called the medullary canal, which is not continued to the extremities. It contains medulla or marrow, and is lined by a delicate vascular membrane, the endosteum, which is prolonged into the cancellated structure and Haversian canals. It is supplied with blood by the nutrient arteries, which anastomose with those supplied by the periosteum.

The periosteum invests the whole bone, except its articular extremities. It is a complex structure, consisting of a fibrous membrane, and a lining of germinal or nucleated membrane; both of which are continued into the Haversian canals. This inner lining assists in the formation of new bone, but the fibrous portion has no formative power.

Many experiments have been made to prove that the periosteum possessed this formative power, but they are still inconclusive; and the only practical deduction obtained is, that when it is destroyed, there is a want of nutrition and reproductive power, in proportion to the destruction of the formative membrane and the vessels passing along with it to the substance of the bone.

The presence of this formative membrane, internal to the fibrous coat, will at once explain how subperiosteal exudation, as in the case of splints and other exostoses, is converted into bony structure.

Having in view all the peculiarities of structure found in the various bones, we will now consider the diseases affecting them. 


\section{OSTITIS, OR INFLAMMATION OF BONE,}

May be acute or chronic. It may involve the whole substance and extent of a bone, or it may be confined to a portion of it (circumscribed inflammation). The causes are external injury, concussion, and hereditary tendency.

Acute inflammation of bone, involving the shaft, is found to affect young race-horses in the disease termed "sore shins." This disease usually involves the periosteum and external layer of the bone only; and such cases terminate by a deposition being thrown out between the periosteum and the bone, which, becoming organized, forms a permanent thickening, depending more or less upon the degree of the diseased action; but in rare cases the whole of the bone is affected, and the inflammation is of such an acute nature that the vitality of the bone is destroyed; the exudation blocking up the Haversian canals and canaliculi, and thus arresting the nutritive functions. The dense structure of the bone does not permit the blood-vessels to relieve themselves by pouring out their liquid contents, as in the softer tissues, and the part dies by the pressure on its vessels, even when the diseased action is not sufficiently active to produce this death of the bone. According to Goodsir, the first changes that occur in the bone are to be distinguished within the Haversian canals. These dilate or become opened up; and the result of this is the conversion of the contiguous canals into one cavity, and the consequent removal or absorption of all the osseous texture of the part.

Concurrent with this softening and opening up of the bony texture, an external swelling makes its appearance; the vessels of the periosteum and contiguous soft parts, becoming involved, throw out a deposit upon the surface of the bone. This exudate, as a rule, becomes converted into bone, leaving the parts permanently altered in shape and appearance; or it may become absorbed before it is ossified, and the parts regain their former condition.

The results of inflammation of bone, where resolution does not take place, are either an increased condensation or an abnormal rarefaction. Of both these forms we have good instances in most cases of ostitis, whether occurring in the navicular or other bones. (See figures of Navicular Bones.) 
In the first form, the Haversian canals and the cancellated spaces are blocked up by earthy matters, which give to the bone

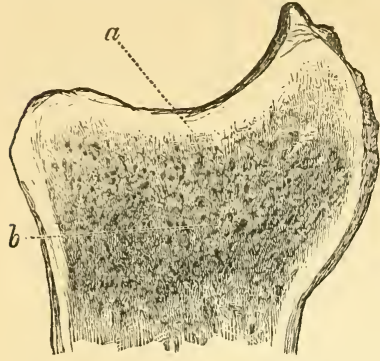

Fig. 19.--Inferior extremity of tibia in a healthy condition. $a$, Laminal layer. $b$, Cancellated tissue.

an ivory-like appearance when sawn acrosis; at the same time the bone is heavier and denser. In the latter, or rarefaction of bone, there is a diminution of its density and weight, owing to its tissues being expanded, and to its canals and cells being enlarged, with thinning of its osseons layers, and the formation of communications between its various interspaces. Whilst this is going on, the outer surface of the bone may become thickened by a deposition of new bone, or it may be removed by absorption; or both conditions may be co-existent upon the

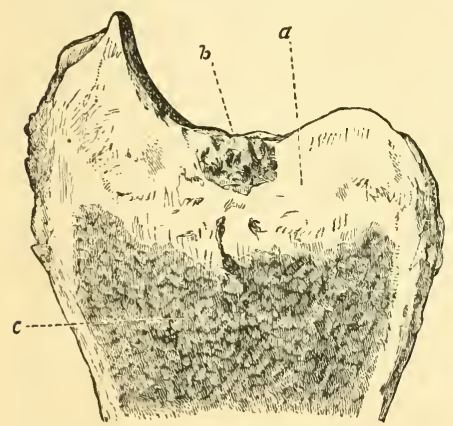

FIG. 20.-Interior extremity of tibia in a state of ulceration. $a$, Thickened laminal layer. $b$, Carious spot. $c$, Cancellated tissue opened up.

as a process of repair contemporary with that of destruction. During the early stages of the process of rarefaction, the bony texture is found softened, and has a porous appearance; but later on the effect is to render the whole substance of the inflamed part more brittle and liable to fracture, as in the case of "fractured spavin," fractured navicular bone, \&c. 
The outer surface of the inflamed bone presents a spongy appearance, from a deposition of new bone; the periosteum is thickened, and in the more acute cases it is detached from the bone, which, when exposed, has a white and smooth appearance, with hardly any change in its structure, the diseased action having been too rapid to allow it to open out.

When the inflammation is of the chronic form, the pathological changes occur more slowly, and when different parts of a bone are affected, new osseous material is deposited here and there, giving the bone an irregularity of shape, as in multiple splints.

A very moderate degree of inflammation in the outer lamella of bone, produces a gelatinous, dark red exudation, which gradually changes its colour to bluish-red and reddish-white, and at length becomes quite white; at the same time, passing from its original gelatinous condition, it forms a coagulum, like the white of an egg, then becomes a soft flexible cartilage, and finally reddish-white succulent bone. In this state it invests the bone, and constitutes, according to its quantity, either a white, porous, and scarcely perceptible film, or a thicker layer, that resembles felt or velvet. The periosteum appears at first injected, bluishred, infiltrated, and decidedly swollen, and generally has but a loose connection with the exudation. The ossified exudation unites with the surface of the bone, and either forms uninterruptedly an addition to the compact wall, or is connected with it by a spongy layer (diploetic). The inflammation may recur in the exudation at any period of its existence, leading to a corresponding increase in its size.

Such, then, are the general outlines of the various changes that occur consequent upon inflammation of bone.

OSTITIS AND PERIOSTITIS OF THE METACARPAL BONES, OR "SORE SHINS."

This is a form of disease affecting the metacarpal bones of young horses, particularly race-horses under four years old, and is due to the fact that they are called upon to perform an amount of work at an age when the condition of their bones is totally unfit to bear it.

The bones of the young, being in a state of development 
and growth, are much more vascuiar, and contain a greater quantity of animal matter than the bones of those of mature age; they are consequently unable to stand the shocks of concussion, to which they are subjected in their training.

Sore shins may affect the whole shaft of the bone (see Fig. 21), and may be so excessive as to cause necrosis to supervene; but usually the inflammation is circumscribed and confined to the lower extremity and anterior portion of the bone. The leg with

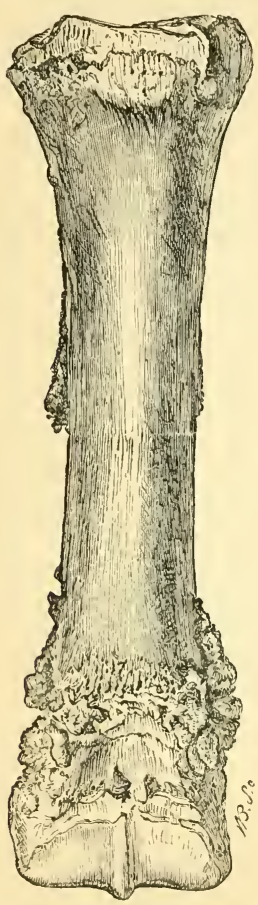

FIG. 21.-Sore shin.

which the animal leads in the gallop is more liable to be affected than its fellow.

Symptoms.-Lameness occurring after a gallop, insidious at first; the horse restless, shifting his weight from one leg to the other if both legs are affected, or, if only one, standing with his foot pointed. If the inflammation is acute, there will be fever, with acceleration of the pulse and respiratory movements, and the bones are sensitive when pressed upon. Swelling is an early symptom; at first it is elastic, tense, and doughy to the touch; it depends on the thickening of the periosteum; and on the presence of a subperiosteal exudate; afterwards, the swelling may become odematous, from effusion into the areolar tissue external to the periosteum, but it always maintains the elastic feel underneath the odema.

If the subperiosteal exudation is very great, and the swelling involves a large extent of the surface of the bone, the most active treatment must be employed in order to prevent necrosis. In some rare cases, the exudate becomes converted into a thin, sanious matter, which corrodes the surrounding tissues, causing great febrile disturbance, and may cause the death of the patient, from its absorption into the general circulation.

In the less acute cases, where the cause has been less severe, 
the disease is more circumscribed and more limited in its effects, or the limbs of the animal have been more able to bear it; it becomes ultimately quite hard, from the organization of the exudate into bony matter, and when this is effected, the lameness and pain generally disappear.

In the acute and extensive form, when the exudate does net break down into sanies, or when suppuration does not immediately take place, it becomes after a time hardened, and finally converted into an ensheathing ring or case of new bone around the old bone, forming a support for the limb, as will be described under Necrosis.

The treatment of the acute form is by free subcutaneous incisions through the periosteum; this will relieve the pain, cut short the disease, and by allowing the exudate to escape, will prevent the separation of the periosteum from the bone, a result to be dreaded in every acute case, as leading to the necrosis of the bone by the removal of its blood supply. In addition to this, warm and soothing fomentations are to be used at first, succeeded by cold, and afterwards by blisters. The constitutional treatment to consist of a smart purgative, followed by alteratives or sedatives, with low diet, and the animal to be kept as quiet as possible. In the less severe form, incisions are not called for, and the cold application may be employed from the outset, succeeded by blisters, as in the acute form.

\section{SPLINTS}

Are another form of ostitis and periostitis, affecting the metacarpal bones, and, in rare instances, the metatarsals. They have a great analogy to the last-named disease, differing only in situation; splints being usually found upon the inner surface of the leg, involving the inner small and large metacarpal bones, whereas the other form is usually found upon the surfaces of the lower third of the large bone only. The reason for this may be found in the pace of the race-horse being the gallop, in which the weight of the animal's body is thrown upon the anterior portion of the bone with fearful velocity at each successive bound, causing the inflammation of the bone in that part by which the shock is received. Splints, on the other hand, are caused by a moderate pace, the trot or gentle 
gallop, where the animal is more kept up to the bridle, and the weight thus thrown more directly upon the upper extremity of the bone, and is thence transinitted to the seat of splint, in the same way as a blow will cause an indirect fracture, as already

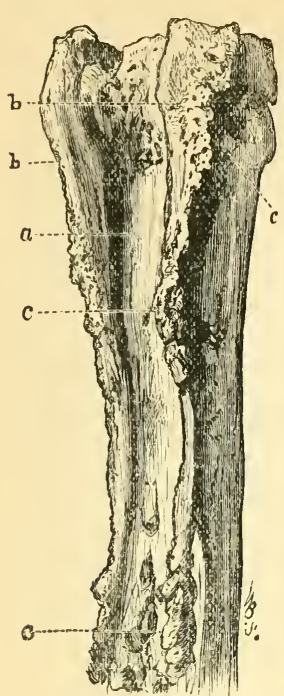

Fig. 22. - Compound splint, involving the three metacarpal bones, $a$, Inter-metacarpal groove, presenting a smooth healthy surface. $b, b$, Osseous deposit, involving the extremities of the lameness, and in a position removed metacarpal and superior sesa- from either articulation or tendon, is lar ossifications, involving the not looked upon as an unsolundness, small metacarpals, lower end of large metacarpal, and forming points of attachment to the supe- fied as causes of unsoundness, as they rior sesamoidean ligament above its bifureation.

NoTe-The tions of this form of important por- ness; and are inclicative of nore tions of this form of splint, namely, disease than is apparent either to the
those involving the ligamentous structures, are hidden from view in the living subject.

eye or touch of the examiner; disease involving articulating surfaces, ligamentous structures, or interfering with the movement of a tendon. 
Professor Dick taught that a splint never interfered with the action of the flexor-pedis tendon; that the soft parts adapted themselves to the hard; and that, although a splint might press upon the tendon, a corresponding hollow was made for it by absorption of the softer structure. I cannot agree with this. Experience has proved to me that it is wrong, and that the lameness, depending upon the interference of the splint, can be completely cured by the removal of the deposit.

A simple splint on the outer side of the leg is more apt to cause lameness than one on the inner side.

The causes of splints are concussion and hereditary predisposition, more especially that arising from shape and form of leg, which descends from parent to offspring.

Splints are most generally found in horses that are newly put to work, or they may arise in the unbroken colt when he is allowed to gallop and play in the fields. The class of horse most subject is the lighter-bred horse, or that which is called upon to go beyond a walking pace. Heavy cart-horses seldom have splints; but I see no reason why they should not, provided their legs were subjected to the same amount of concussion.

Splints are exostoses due to a circumscribed superficial inflammation of the bone and periosteum, and not inflammation of the interosseous ligament, as described by some writers. Numerous specimens in the College Museum prove the correctness of this view. The effects of the concussion which produces this form of ostitis may be due to the immature age of the bone, to peculiarity of shape in the leg, to the method by which the animal is shod, or to work at an early age. old horses throw out splints occasionally.

Splints do not always produce lameness. If of the simple kind, when the horse is very young, and before he is broken in, lameness is but seldom seen; and when over six or seven years of age, he seems exempt from lameness, although the deposit might be of considerable size.

When simple splints cause lameness, it is during their formation, that is, during the inflammatory stage, and before the periosteum has adapted itself to the pressure. The young horse of good action is the one most likely to suffer, as the effects of shock or concussion are greater and more destructive to his limbs than to the limbs of a horse with lower action. 


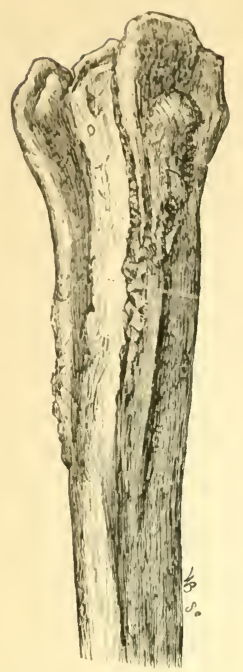

FIG. 23. - Ordinary form of splint on the inner side of near fore leg, involving the large and small metacarpals. In the original specimen the intercoseuus ligament is not ossified.

\section{PECULIARITY OF SPLINT LAMENESS.}

The lameness may precede the appearance of any swelling or deposit, and in such a case it is apt to be confounded with that arising from other diseases. Jut if the following observations are kept in remembrance, no mistake need be made:-

1st. The age of the animal. The young lrorse is most liable to splint lameness, the older horse to navicular disease.

2d. The peculiarity of action. A horse lame from splint will walk apparently or nearly sound, but will trot very lame, the drop of the head and body upon the sound side being very great, and out of all proportion to the apparent soundness of the walk.

3 d. A want of flexion may be observed at the knee.

$4 t h$. When the patient first comes out, and is made to trot, he may go moderately sound, but after a time the lameness increases, the concussion being a cause of pain. In navicular disease the lameness generally decreases with exercise.

$5 t h$. I'ressure upon the part of the leg where splint is likely to be will cause pain; some heat is present; and, by a careful manipulation, a hard swelling perhaps smaller than a pea may be felt. In some cases the exostoses soon develop themselves, and then there can be no further difficulty; but in others, this does not wceur for several weeks, and these are most unsatisfactory to the surgeon. In some rare cases the lameness is very excessive, the horse being scarcely able to put any weight upon the affected limb; standing with the toe only touching the ground, with great heat and swelling of the part affected, at the same time suffering from constitutional disturbance to a considerable degree. In such a case, the treatment must be prompt, to 
relieve the animal from its sufferings, and the best is "subcutaneous periostiotomy," as recommended by the late Professor Sewell.

The operation is performed by making a transverse incision with the rowelling scissors, immediately below the enlargement, introducing the "periostiotomy knife" flatwise under the skin, as far as the upper end of the splint, turning the cutting edge inwards on to the bone, and cutting through the periosteum into the new formation. It may be necessary to cast the horse, but, as a rule, the application of cocaine and the twitch keeps him quiet enough.

Some practitioners pass a seton over the deposit after the operation, and I recommend it as good practice.

In all cases a purgative must be administered; and in the milder forms, fomentations and the application of a cooling lotion are quite sufficient to remove the inflammation and lameness; but if they fail to do so, a blister should be applied, and in obstinate cases it may be found necessary to fire the part, the method by pyro-puncture being preferable, as it leaves but little blemish; the instrument to be made hot, and applied

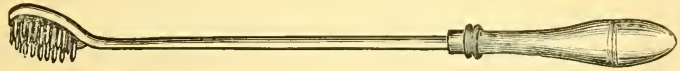

Fig. 24.-Instrument for pyro-puncture.

with sufficient pressure to pierce the skin, and to enter the new deposit.

If there be a return of lameness when the patient is put to work after an apparent cure, it will be advisable to take the shoes off, turn him into a loose place, and allow a long rest. When the deposit is sufficiently prominent to interfere with the action of the flexor tendon, the treatment is to cut down upon it and remove it by the bone forceps or Volkmann's spoon.

Sometimes the splint is large enough to interfere with the action of the opposite fore foot, that is to say, it is liable to be struck by the other foot during rapid action. In such a case a boot must be used, and the absorption of the deposit excited by the application of the ointment of the biniodide of mercury; or if it be a sharp, prominent splint, it is to be removed by the bone forceps. This striking of the enlargement with the opposite foot is apt to cause the horse to fall, 
from the suddenness and violence of the pain, or to induce inflammation in the splint, and very severe lameness.

Splints in the lind legs seldom cause lameness; they are usually upon the outer side of the metatarsal bone. There are many examples of ostitis that will be described more appropriately under the head of lameness. Sore shins and splints are perhaps the only ones, usually met with in practice, of inflammation of the compact shaft of the bone; the other forms being found attacking the cancellated structure composing the shori and irregular bones, and the extremities of the long ones. But before passing on to these, I shall describe scrofulous inflamma. tion of bones, caries, necrosis, and the non-inflammatory diseases.

\section{SCROFULOUS OSTITIS IN YOUNG ANIMALS.}

Tubercular or scrofulous arthritis and ostitis is not an uncommon disease, and is known as joint-ill. It attacks animals of all ages, but particularly the young. In cattle it sometimes assumes the form of malignant "foul in the foot" (paronychia ungularis maligna), and the following is the pathological anatomy of a case of "foul in the foot" arising from scrofula, occurring in my own practice.

The animal was a two-year-old heifer, which had been suffering for about five months from foul of the foot; having defied all the ordinary remedies to effect a cure, and the fact that several animals on the same farm, and of the same breed, were similarly effected, induced the veterinary surgeon in attendance to send one of the affected feet of this malignant case to the College for examination.

The part sent was a fore foot, having been cut through about the lower third of the metacarpus, showing the fetlock joint and the two complete digits attached.-(See Photo-lithograph, Plate II., Figure 9.)

In external appearance it was very mueh swollen, and studded round with ulcers of various shapes and sizes, from that of a sixpence to that of a halfpenny. The fetlock joint was completely open on the right side, and deep nleerations of the articular surfices of the bones had taken place, more particularly of the trochlea of the metacarpus.

On attempting to remove the skin, it was found closely ad- 
herent to the subcutaneous tissue, and intimately blended with it. The subcutaneous substance consisted of an inflammatory exudate of considerable thickness, having a pale bluish appearance, a vitreous character, and quite structureless; it was so intimately infiltrated within the textures of the tendons and ligaments beneath, that it was with difficulty detached from them.

Throughout this exudate were a number of sinuses of various sizes and figures, containing a yellow semi-fluid, granular-looking substance, which, on examination with the microscope, presented all the characteristic appearances of tubercular pus, and a very few tubercle corpuscles mixed with it.

The cavities in which the substance was contained varied in size from a pin-head to that of a bean, the smaller ones being for the most part circular in figure, while the larger ones varied very much, their form and outline making it evident that their enlargement was due to the confluence of two or more smaller ones. Notwithstanding the number of these little cavities, there was very little of that apparent inflammation in the immediately surrounding textures which we invariably find in the formation of an ordinary abscess.

On making a longitudinal section of the bones, I found in the first or upper phalanges not only the medullary canal filled with lymphoid material, but the whole of the cancellated tissue entirely replaced by that substance. The bones of the second or middle phalanx also contained a considerable quantity of it, but there was no ulceration of the articular extremities. There was a slight calcareous-looking deposit on the bones of the first phalanx, but on examination it had no bony structure.

The old writers described this disease as "joint-ill," and ascribed it to rheumatism. I am of opinion that they were wrong, as in its progress it differs most materially from rheumatism. If the patient be under three or even four weeks old, the first noticeable sign of the disease is a dribbling of urine from the umbilical cord. In fact, the urachus has again become pervious, and allows the urine to escape, although the urethra is quite in a normal condition, the animal having been seen to urinate in a proper manner. Concomitant with this unnatural flow of urine, the patient will be found stiff and lame in one, two, or more of its limbs or joints; the affected parts are swollen, 
hot, and tender; the respirations hurried; but the appetite remains tolerably good for some time; the swollen joints suppurate; and the disease is now complicated with open joint; abscesses form on various parts of the body, the patient loses flesh, becomes unable to rise, and dies, a miserable object.

The causes are extrinsic and intrinsic.

The extrinsic are exposure to cold, a bad mother-that is to say, a mother that is without sufficient milk for the foal-long fasting, as in cases where the mother is put to work shortly after the birth of the foal, and only allowed to suckle her young two or three times a day. I have seen the disease produced in pet lambs when brought up on cow's milk; these circumstances produce debility of the constitution, indigestion, and mal-assimilation.

The intrinsic cause is the scrofulous diathesis, and this may arise independently of any external circumstances.

Pathology.-To understand this correctly, it must be remembered that the growth of the bones is very rapid in young animals, and that a large quantity of blood is required in the part, in order to supply the nutritive plasma for such growth; in fact, the bones, more particularly their extremities, are in a state of healthy congestion. The blood supply must be abundant in quantity, and healthy in quality; but in this disease the latter condition is absent; and instead of supplying healthy material for the growth and nourishment of the bones, it conveys a degraded form of an albuminoid matter, which is deposited in the structure of the bones, and there excites a form of strumous inflammation leading on to caries, the removal of the articular cartilage, and the formation of abscesses in and around the joints, which make their way through the ligaments and synovial membrane, and burst externally by many openings, which communicate with the joint by circuitous sinuses.

The probabilities of effecting a cure will much depend upon the severity and situation of the arthrodial inflammation. If the larger joints, or those having extensive motion, as the true hock-joint or elbow, be already opened by the suppuration, the case may be looked upon as hopeless.

If treatment is to be adopted, it must in the first place be directed to the removal of all extrinsic causes; secondly, to promote a better condition of the system generally; and thirdly, to modify the local manifestations of the disease. 
In order to promote a better tone of the system, attention must be paid to the state of the digestive organs. Diarrhœa may be present, the fæces containing clots of curdled milk, mixed with much mucus, fœetid in odour, and irritating to the anus; or the bowels may be constipated. In both conditions, a very gentle laxative, as four ounces of castor oil, with two drams of the bicarbonate of soda, nay be given; and when the bowels are restored to their proper state, a pint of lime water in a little milk two or three times daily, in addition to half-ounce doses of the syrup of the compound phosphates or "chemical food" twice per day. I can speak with great confidence of the phosphates as being most beneficial in all debilitating diseases of young animals. Great care must be taken to see that the patient suckles its mother sufficiently often, and if he be unable to stand to do so, he must be lifted and held up, If the mother has not sufficient milk, the deficiency must be supplied with cow's milk, care being taken that this does not constipate the bowels; the addition of a small quantity of sugar and water to it will be useful. The mother mnust rest from work, and be supplied with good food; a mixture of beans, oats, bran, and grass if in season, will be most suitable.

The lime water is recommended as an antacid. At one time it was supposed to supply lime to the bones; it is now held that the bone diseases of the young are not due to the want of lime. but to its elimination from the system by the kidneys. Authorities, however, are divided upon this subject.

Simulating this disease, both in its symptoms during life and to some extent in the lesions found after death, is another affection of the articulations and bones, arising from the absorption of septic matter, viz-

\section{OMPHALITIS,}

Or inflammation of the umbilical cord, seen in very young animals, particularly foals, a few days or a week or more old.

In many instances the first noticeable symptom of this condition is a dribbling of urine from the umbilical cord. The urachus, at first impervious, has again become pervious, allowing the urine to escape, although the urethra is quite in a normal condition, the animal having been seen to urinate in the natural manner. In some instances the above symptom may be preceded by great restlessness, colicky pains, a tendency to lie upon 
the back, pain evinced when the umbilicus is pressed upon, some swelling of the parts and a doughy feeling, with constipation of the bowels, accelerated breathing, and rapid prostration. In other instances the first noticeable sign may be lameness in one or more limbs, with swelling of one or more joints, considerable fever, accelerated respiratory movements, \&c., whilst, again, all these symptoms may be almost simultaneously manifested.

The nature of the disease was first described by Bollinger, who found that the arthritis is due to the condition of the urachus, and the treatment should be directed to that condition, and the experience of veterinarians in this country has confirmed this view.

The causes are sometimes very obscure, but any ordinary cause, such as cold-particularly damp-cold-or being kept in ill-drained places, may induce it, and it has been observed that where mares and foals are pastured in fields that have been lately dressed with bone manure, the foals are very apt to suffer from this disease.

The application of a ligature to the umbilical cord-a reprehensible practice, particularly if applied too close to the abdomen - is another cause of the disease.

Any and all of these causes induce inflammation in the remains of the umbilical vessels, artery, vein, and urachus, which soon assumes a septic character; the septic products are absorbed into the circulation, cause the inflammation of the articulations and bones, and it is now found that the most successful method of treating it is to excise the remains of the umbilicus close to the floor of the abdomen, remove all débris of blood, \&c. contained in the surrounding tissue, foment well, and dress antiseptically, and if there be colicky pain, freely foment the parts.

The excision of the cord is necessary whether the urachus remain patent or not, and is to be performed in all cases where the abdominal pain or joint affection are associated with heat, swelling, or tenderness of the umbilical region. If there be constipation of the bowels, enemas, containing a teaspoonful of glycerine, are advisable.

In conclusion, it must be always remembered that the health of the mother plays a most important part in the production and removal of this disease, and must upon all occasions demand the veterinary surgeon's care and attention. 


\section{CHAPTER IX.}

\section{DISEASES OF THE BONES AND ARTICULATIONS-continued.}

\section{ULCERATION - CARIES - NECROSIS - CENTRAL AND SUBPERIOSTEAL SUPPURATION-ABSCESS IN THE SUBSTANCE OF A BONE.}

WRITERS upon human surgery make a distinction between ulceration of bone and earies, both conditions being associated with the formation of pus. Thus, Professor Syme says- "By ulceration we mean that condition of bone in which there is loss of substance, together with suppuration, but in which the ulcer has a tendency to heal. In caries, on the contrary, while there is a loss of substance, together with suppuration, there is so far from being any tendency to heal, that healing is very difficult to accomplish." The same authority, quoting from Liston, says:- "It may tend to prevent confusion of the two morbid states, if we confine the term ulceration to suppuration in and absorption of bone, whilst the vessels retain a considerable power of action, throw out new matter, and procure a reparation of the breach; and this condition of the osseous tissue exists when the disease is situated on the surface of the bone, and when it has been induced by an external cause. On the contrary, the term caries will denote that particular kind of ulceration in which reparation is hardly attempted by nature, and is with difficulty obtained by the most active influence, and this disease will be most generally found to affect the cancellated structure.

To the veterinary pathologist this difference is not at all satisfactory, and it may be laid down as a rule that ulceration of bone with a discharge of pus is the result of external injurythat, in fact, there is necrosis, or actual death of a layer of 
bone, more or less deep, as the case may be, the injury being inflicted upon a part of the bone other than its articular end; whilst caries is confined to the extremities of the long bones and cancellated structure of the short and irregular bones; and when not caused by external injury, is unaccompanied by the formation of pus. Of this we have many examples in navicular disease, bone-spavin, ring-bone, \&c.

Professor Spence says, that "The peculiar obstinacy of a truly carious surface arises from the fact that a large part of it is really dead." This, in my opinion, is necrosis, and when the necrosis occurs in our patients, it is always accompanied by suppuration. I shall, therefore, distinguish these two terminations of ostitis, when not caused by external injury, as-1st. Caries or removal of degenerated bone tissue by absorption without suppuration, commonly found to exist in the articular ends of the bones; and, $2 d$. Necrosis, or death of a bone, or a portion of bone, accompanied by suppuration; the dead bone being removed by expulsion or surgical interference.

Caries may arise from traumatic or idiopathic inflammation of the synovial membrane, or from ostitis commencing in the cancellated structure of the bone, and it may be defined as a slow absorptive process by which the bone becomes eroded and cribriform in appearance, the absorption extending to a distance, the bone converted into a brittle mass, and the surrounding parts more vascular and swollen than in the normal state. Or the destructive process is limited, by a deposition of bony material within the cancellated structure of the bone, rendering it of an ivory-like appearance when cut into, and increasing its density and weight.-(See Fig. 20, page 152.)

Caries commences in the interior of a bone, and makes its way outwards; the bone acquires a red hue; its articular surface becomes soft; its laminal layer and articular cartilage are removed, exposing the cancellated structure, from which vascular processes shoot out in the form of red teat-like granulations; the surface of the opposing bone-forming the articulationbecomes similarly diseased, the granulations from the one bone coalesce with those from the other, and form a vascular connection between the interior of both bones. In this manner the Irocess of anchylosis is commenced.

When caries is situated in a bone over which a tendon plays, 
as the navicular, sesamoid, or bicipital groove of the humerus, the fibres of the tendon become "ruptured by abrasion" upon the roughened surface, or by an inflammatory softening-gelatination-of the tendon, whereby its smooth, cartilaginous surface becomes roughened; string-like processes of its fibres are seen upon it; which finally unite to vascular projections from the bone, and form a bond of union between the two. The progress of caries is thus arrested by the adhesion of the tendon to the bone.

The effect of caries is to remove the bony laminal layer and the cartilage, and thus destroy the articulation which it may invade; but whilst this process of destruction goes on within the bone and upon its extremity, we find that the periosteal surface and compact tissue become the seat of new bony deposit, situated beneath the periosteum, which envelops the diseased bone, as it were, in a ring, and extends to all the bones of an articulation which may be involved in the caries; finally, binding them firmly together; in fact, converting two or more bones into one, and destroying motion. The united bones now perform the functions of one bone; and in virtue of this the pain produced by the attrition of the roughened surfaces of the bones one upon the other is no longer caused.

Within the bone the degenerative process, in the majority of cases that have been carefully examined by me, is limited by consolidation of the cancelli; and in a recent specimen which I have in my possession, I found that the first alteration to be observed, in addition to the increased vascularity, was the removal of the fatty material-which is found abundantly in bone during health-and the formation of an organizable lymph, which is finally converted into bone, giving to the original trabeculated structure an ivory-like appearance.

The external signs of caries vary according to its seat. If it be in the bones of an articulation having a distensible capsular ligament, as the true hock, there will be swelling of the joint by distension of its synovial membrane and capsular ligament, with heat, pain, and lameness. But if it be limited to the mere gliding articulations, their synovial membranes are too limited to allow of this distension; and before the appearance of the external subperiosteal deposition just referred to, the speciality of the lameness and heat of the part are the only signs. 
Treatment.-When caries is firmly established, the only method by which it can be repaired is by assisting the process of adhesion between the opposing surfaces. This is to be effected by rest; and by the actual cautery, which has a wonderful effect in allaying the diseased action and removing the pain. It also promotes the formation of reparative material, and hastens the process of repair. It must be understood that when anchylosis occurs in articulations with extensive motion, such as the true hock, elbow, \&c., the animal is of little use, and no treatment is of any avail; but when caries is commencing in these situations, its progress may be arrested by rest and the cautery, or other severe external irritant. But when the mere gliding joints are its seat, then anchylosis may be looked upon as a radical cure.

Necrosis, or death of a bone, corresponds to mortification of the soft structures, and is as distinct from caries as mortification is from ulceration. Necrosis is divided into four varieties, namely, $1 s t$, the scrofulous; $2 d$, the superficial, or that which involves the outer lamella, and presents itself in the flat and long bones; $3 d$, that form which destroys the internal part of a bone, and in which the outer shell is not affected; and $4 t h$, that in which the whole thickness of the bone dies. The last three forms are respectively named external, "superficialis or partialis," central or internal, and general necrosis, or necrosis totalis.

Necrosis superficialis depends upon very acute superficial ostitis, periostitis, or any injury which destroys the periosteum, as well as injuring the bone itself. It was thought at one time that the mere removal of the periosteum was sufficient to produce this form of necrosis, but it is now ascertained that such is not the case, and that when necrosis and exfoliation occur, they do so from the violence which separated the periosteum having destroyed the vitality of the bone itself.

I am not aware that this form occurs except from direct violence to the part, such as blows, kicks, \&c., which expose the surface of the bone, and excite inflammation in the adjoining tissues. The necrosed portion presents a white, waxy appearance, as if it had been carefully macerated, sonorous when struck by a probe, and if exposed to the atmosphere before separation, it becomes changed in colour, passing through various degrees of green, brown, and black. These changes are attributed to the 
action of the atmosphere and the character of the discharge. The limits of the dead portion are not always easily defined, and at all times its outlines are very irregular. If the skin should be uncut, or be healed, over the seat of the injury, an abscess soon forms, which, if left alone, discharges itself; and if the ulcer is examined by a probe, the bone will be found bare, and perhaps loose. The abscess does not heal until the loose portion, called an exfoliation, is removed; in fact, there is now a fistulous communication between the dead bone and the atmosphere. The separation of the dead bone is effected by the absorption of the layer of living bone immediately contiguous to it, by a process analogous to sloughing of the soft parts.

When the dead bone is removed-naturally or by surgical interference-the surface beneath it granulates, and bony matter is formed in the gap, which, however, is not always sufficient to fill it, but to round off its edges, and the part is left permanently deficient.

Free vent must be given to the pus, which is curdy in consistence, and foetid in odour-the dead bone examined, and when found to be loose removed by the forceps. If the necrosed portion be a mere thin pellicle, the dilute hydrochloric acid will often dissolve it, and thus save a prolonged process of exfoliation.

This form of necrosis often attacks the inferior maxillary bone from the pressure of the curb, and the os pedis, from pressure of a foreign body, as the shoe nail.

Necrosis totalis affects the shafts of the long bones, more especially the metacarpals and the metatarsals, and also the scapulæ. One specimen of the latter is now in the Veterinary College Museum.-(See Photo-lithograph, Plate I., Fig. 6.) The cause of this form is violent concussion, as from galloping or jumping, producing violent ostitis, which from its rapidity does not permit the dense texture of the bone to open out, so as to allow the Haversian canals to accommodate the congested vessels, or permit them to relieve themselves sufficiently by effusion. In addition to this, the small amount of exudation which ensues blocks up the canaliculi and lacunæ of the bone, arresting its nutrition, and finally destroying its vitality.

When the vitality of the bone is destroyed, a large deposit 
of osseous matter takes place under the periosteum, and in

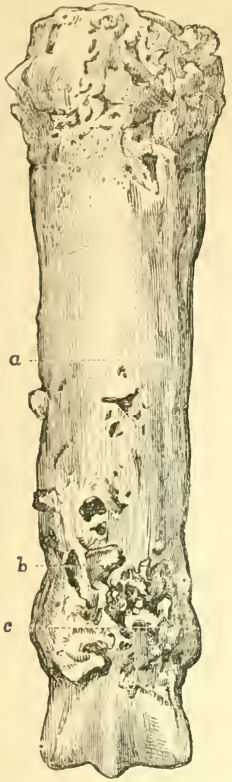

FIG. 25.-Necrosis totalis of metatarsal bone. $a$, Se questral capsule. $b$ and $c$, pansion or rarefaction of its tissue, by Cloacæ, or foramina grandia. the enlargement of its Haversian canals, assumes a rosy colour, and becomes succulent. It gradually acquires an areolar structure, and is thus more rarefied; at length it disappears altogether, and a red, soft, spongy substance, or layer of granulations, oceupies its place. This change is produced by the inflammatory process, which gives rise to suppuration and granulation; the bony tissue beginning with the Haversian canals is dissolved by the matter secreted within them; while the granulations which shoot forth at the same time fill up the enlarged canals. The immediate result of this process is the formation of a furrow of demareation, which encircles the maryin of the dead bone, and is filled up with granula- 
tions; and so far as the process is completed on that surface, of the living bone which faces the dead, so far is the sequestrum separated." The necrosed portion is very irregular in its outline, and the luxuriant granulations from the living parts shoot into these irregularities, causing a dove-tailing closely resembling actual union. From various specimens in my possession, I find that the dead bone, when of any considerable magnitude, is removed in numerous portions, that is to say, it becomes broken into several fragments, which find their way to the surface through the cloacr time after time. In the annexed illustration five sequestra were found within the capsule.

The cloacæ, or foramina grandia, serving as outlets for the pus and sequestra, present themselves about the middle or lower third of the new bone as ovalshaped openings; and a remarkable fact has been pointed out by Professor Goodsir, connected with these, namely, that " they are almost invariably opposite to a smooth or unaltered surface of the dead shaft, and that they result from the pus, thrown out from the granulating internal surface of the new shaft, making its way to the exterior, by the parts not yet closed, in consequence of having been opposite portions of the old shaft, which had not afforded separate osseous centres."

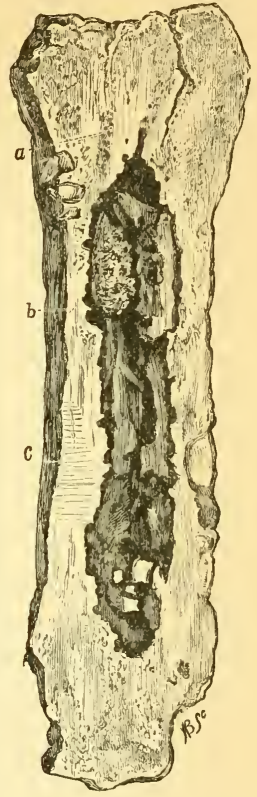

FIG. 26.- Section of the bone represented in Fig. 25, showing five sequestra, or dead pieces of the original bone. $a$, Capsule; $b$ and $c$, Sequestra.

By this, Goodsir means that the smooth surface of that part of the old shaft had not afforded spiculæ of bone adherent to the periosteum, when that membrane became separated from the old shaft, to act as centres of ossification; for he founded the doctrine that the formation of new bone depended not so much on the periosteum, as upon the spiculæ of living bone which were attached to it. He says:- "When the entire shaft of a bone is attacked with violent inflammation, there is generally 
time, before the death of the bone takes place, for the separation of more or less numerous portions of its surface. When the entire periosteum has separated from the shaft, it carries with it minute portions of the surface of the bone. Each of these is covered on its external surface by the periosteum, on its internal by a layer of granulations, the result of the organized matter which originally filled the Haversian canals; the gradual enlargement and subsequent blending of which ultimately allowed their vascular contents to combine, with the layer of granulations just described, to form the separating medium between the dead shaft and its minute living remnants. These minute separated portions, after having advanced somewhat in development, appear, when carelessly examined, particularly in dried specimens, to be situated in the substance of the periosteum, and have been adduced, by the advocates of the agency of that membrane in forming new bone, as evidences of the truth of their opinions."

The causes of necrosis totalis are ostitis and periostitis of a violent form, brought about by galloping, jumping, or any other cause of violent concussion; it is situated in the long bones, or in the os pedis in some cases of very acute laminitis, with sloughing; and a partial or total necrosis of this bone sometimes results after neurotomy.

The symptoms are violent pains, manifested by lameness, if in the extremities, with swelling, at first doughy and elastic, by reason of the effusion between the periosteum and bone, and of the thickening of the periosteum itself; it presents no distinct boundary when involving the whole length of the shaft. The swelling gradually becomes harder as the process of ossification goes on; but at various parts, more especially towards the lower portion of the bone, soft points can be detected in it, attended eventually by fluctuation. These finally burst, and discharge pus, which may at first be odourless; but after the abscesses have been open a short time, it emits that peculiar fotor characteristic of diseased or dead bone. If the abscesses be examined with the probe, the bone will be found bare, and perhaps loose.

Treatment.-If a considerable length of a shaft is dead, some time must be allowed for the consolidation of the capsule before any attempt is made for the removal of the sequestrum, taking 
care that the animal is properly cared for, by having good food, comfort, and cleanliness; but if the bone be not a weight-bearing one, as the inferior maxilla, no time should be lost in removing all portions of the dead bone. The following case will illustrate the necessary treatment.

A bay mare was brought to the College last winter with a fistulous opening in the lower jaw, from which flowed a large quantity of very fœtid pus. Upon examination, about five inches of the original bone, extending from the incisors backwards, was found enclosed in a capsule of new bone, in which were several cloacæ connected with the fistula. When the probe was introduced, loose pieces of bone could be detected within the capsule. The animal was cast, the skin dissected from the largest fistula, and the cloacæ enlarged with the trephine. It was found necessary to make two openings in the capsule, before the sequestræ could be removed by the forceps; the parts were now washed out by syringing with dilute carbolic acid (the foetor being very offensive), and the animal allowed to rise. Three days afterwards the discharge had entirely ceased; the wound healed up rapidly, and the mare continues well to this day.

The same principle of treatment will apply to necrosis, wherever situated, care always being taken that every portion is removed, and that strong and proper instruments are used. The symptoms of partial necrosis of the os pedis will be exactly like those of partial fracture of it (described hereafter); and the symptoms of total necrosis in acute laminitis will be swelling round the coronet, with great lameness, separation of the crust from the sensitive foot, discharge of a dark-coloured sanious matter from the upper part of the foot, and finally, if the animal live, sloughing of the whole hoof, accompanied by great constitutional excitement and irritative fever; the pulse, at first hard, full, and rapid, finally becomes small, thready, and quick; with foetor of the breath in some cases, and death in a very short space of time. The fotor of the breath and the very rapid sinking are supposed to be caused by absorption of the sanious discharge.

I think I need scarcely remind the reader that in compound fractures, when the fragments are completely detached and separated from the bone, they die, constituting necrosis by 
fracture; that in all cases their presence is made manifest by the formation of fistulous abscesses; and that their removal is imperative.

In the ox tribe, the digital bones suffer from necrosis, owing to an inflammation of the inter-digital fibrous tissue.

It was supposed at one time that a sequestrum was reduced in size by the action of the absorbents; but it is now placed beyond all doubt that dead bone is in every respect a foreign body, and cannot be acted upon by the absorbents more than any other insoluble substance. But at the same time it must be admitted that a sequestrum is much smaller than the original bone; this can only he accomited for by the fact that its animal basis is dissolved by the purulent discharge, and that it is thos rendered brittle, and liable to be broken into fragments by the movements of the animal.

Internal necrosis is very rare in the lower animals, one specimen only in the Museum presenting any appearance of it. The following are its symptoms in the human being, namely, most excruciating pains, supposed to arise from the resistance of the outer shell to the swelling of the inflamed part. The fever is often so high as to prevent the patient obtaining repose. The enlargement, exceedingly hard and diffuse, depends on the swollen condition of the bone, and in time becomes very vascular and softened. The medullary membrane is completely destroyed. The swelling continues for a long time, but abscesses gradually form in the soft parts; the outer layer of the old bone expands and grows, during the continuance of the suppuration, by the dilation of the Haversian canals; whilst new bone is formed externally, so that the sequestral capsule is partly of old bone and partly of new. When the sequestrum is removed, and the process of regeneration complete, the bone consists from without inwards of the outer shell of new bone, the layer of old bone which is not necrosed, and the central portion of new bone formed by the granulations within, and which for a long time occupies the place of the medullary canal.

The interior of the bone, at first solid, becomes opened up by the gradual enlargement of the Haversian canals of the new bone, whereby its structure becomes cellular, and in this way it incompletely supplies the place of the medullary eanal.

Many experiments have been made which show that the 
destruction of the medulla is sufficient to cause the death of a bone, and it has also been carefully noted that the power of reproduction varies in the different bones of the body, being great in some, and less in others, and in some absent altogether. Thus the long bones, the lower jaw, and scapula possess the power of reproduction to a great extent; the cuboid and other short bones cannot be reproduced.

Portions of the cranium, under particular circumstances, says Pirrie, may to a certain extent be reproduced; but if both tables be destroyed, together with the pericranium, there will be very slight reproduction; for the dura mater has very little tendency to produce new bone, and for this reason reproduction does not take place after the operation of trephining the skull. The bones of the face, however, seem to have the property, for in several instances $I$ have witnessed complete reproduction after trephining the maxillary sinuses.

\section{SUBPERIOSTEAL SUPPURATION.}

Suppuration beneath the periosteum is not uncommon, and may result from blows, such as "speedy-cut," or from such operations as "punching" for bone-spavin. The symptoms of this form of suppuration are, diffuse swelling, extending in every direction from the seat of injury. The swelling is tense and drum-like when felt; it may be large, whilst it contains but little pus; after a time, however, abscesses form on various parts of it, and discharge a foetid pus. The animal is very lame, and a high state of irritative fever supervenes, which may run on to a fatal termination. The treatment for cases of subperiosteal suppuration must be prompt, and should consist in making free incisions through the periosteum, and thus give vent to the pus.

Punctured wounds, more especially in the neighbourhood of the elbow-joint, are very apt to be complicated by a collection of pus under the periostum, which, if not allowed to escape by free incision, is apt to burrow upwards into the articulation, thus causing that dreaded complication, open joint. I have seen wounds that were several inches from the articulation terminate in this way. 


\section{ABSCESS IN THE SUBSTANCE OF A BONE.}

The formation of abscess in the substance of a bone is of rare occurrence, and 1 have only witnessed one marked case of it. The bone is now in my possession, and the two figures represent its external and internal appearances.

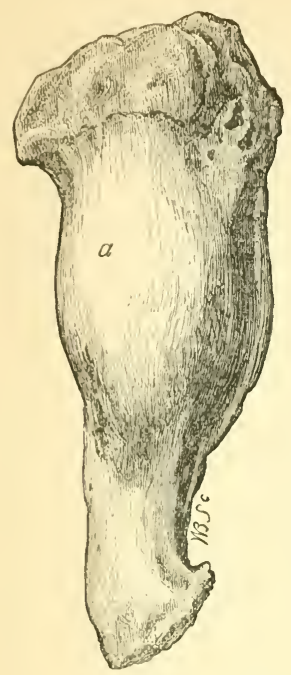

FIG. 27.

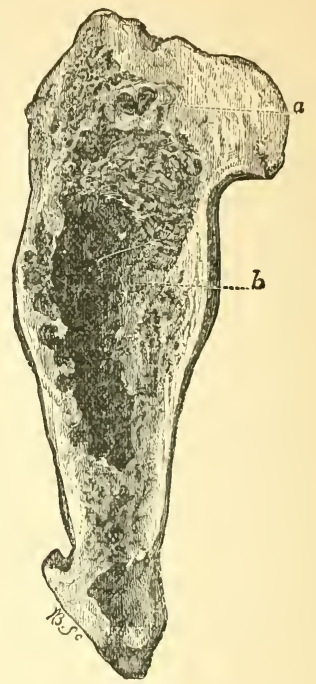

Fig. 28.

FIG. 27.- External view of humerus of a horse, with central abscess.

Fig. 28. - Internal view of same bone. At $a$ is a well-defined abscess, containing inspissated pus; $b$ shows the dilated condition of the medullary canal, the cavity being originally filled with pus.

An abscess may form within the cancellated structure, which is hallowerl out, as in the illustration, and is generally situated near the articular extremity of a long bone. The same amount of inflammation necessary to produce pus in the cancelli, would, if situated in the compact tissue, cause necrosis. It is accompanied by thickening of the outer shell of the bone, as in necrosis. The diagnosis of internal abscess will be very difficult, on account of the depth of its seat. The enlarged bone, with pain, will be our only guides until the pus has pierced 
the bone, and found its way to the surface of the skin. In long-continued cases of lameness, with enlargement of a bone, as in the specimen, the practitioner should open the enlarged portion by the trephine, the probabilities being that he will find either pus, or a sequestrum, or both, imprisoned, and that their removal will bring on a favourable termination. In the case from which the specimen was obtained, no other reason could be given for the abscess than that the pole of the carriage, by continually striking this part, brought on inflammation and suppuration of the medullary membrane, as well as periostitis.

When the trephine has to be used, it is nearly always necessary to cast the patient; making a careful incision on to the diseased bone; avoiding injury to important blood-vessels and nerves; dividing the muscular structures in the direction of their long axes; separating all the soft tissues from the bone before the trephine is applied, or its teeth will become clogged; and, finally, washing out the abscess thoroughly with water in which a small quantity of the pure carbolic acid has been dissolved; and leaving the lower portion of the wound in the soft structures open, to allow the escape of the discharges.

Mr. Stanley says that "circumscribed abscess is, in some cases, attributable to the softening of tubercular matter, analogous to a pulmonary vomica, and that the contents may be discharged, leaving a cavity resembling a tuberculous cavity in the lungs." The circumscribed abscess, according to the same writer, "usually remains of a small size, but in some cases it has enlarged much beyond the natural limits of the bone. Such an enlargement is not the effect of a simple expansion of the walls of the bone; for in some of these cases the osseous walls of the abscess have increased in thickness, with enlargement of the cavity. The process consists of the combined action of absorption on the inside of the abscess, and of osseous deposit on its outside, whereby its osseous walls may acquire any degree of thickness, according to the predominance of absorption in the one direction, or of deposit in the other." 


\section{CHAPTER X.}

NON-INFLAMMATORY DISEASES OF BONES.

RICKETS-MOLLITIES OSSIUM-OSTEO-POROSIS-FRAGILITUS OSSIUMOSTEO-SARCOMA-EFFECTS OF MERCURIAL POISONING ON BONES.

\section{RICKETS.}

TIIE terms "rickets" and "rachitis" are used to denote an unnatural softness of the osseous system in young animals, and is referable to disordered nutrition of the osseous tissue; in fact, to the mal-assimilation and non-development of the

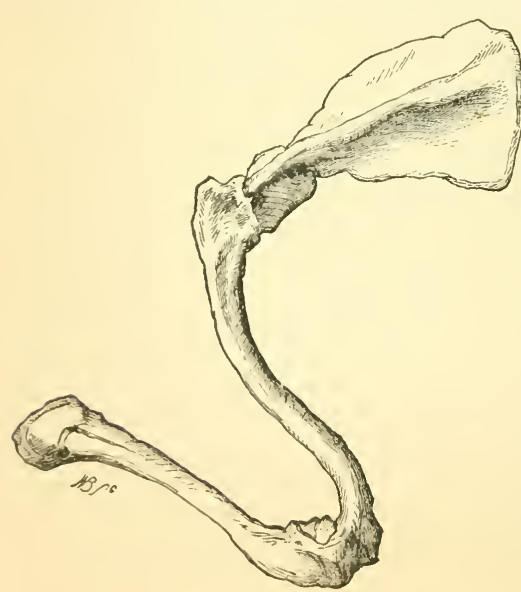

Fig. 29. materials necessary to the formation of bone.

Rickets may be witnessed in foals, calves, and young dogs, more especially youngspaniels and pointers. In foalsand calves the metacarpal bones are those which soonest bend; in dogs, the lower third of the humerus; giving to the dog a dwarf-like appearance : he stands with his fore legs wide apart, and walks with a peculiar rolling gait, throwing the weight of his body from one limb to another in 
a peculiarly ludicrous manner, the sternum almost touching the ground. In foals and calves, the shafts of the metacarpals are the first to give way, the curvatures being from within outwards; thus the knees are thrown outwards, and the toes drawn inwards. The articulations and extremities of the bones sometimes become enlarged, hot, and painful; causing so much lameness that the little animal merely touches the ground with the toes of the affected limbs, and as the animal increases in size the spinal column becomes too feeble to bear the weight of the body, and symptoms of paralysis are observable.

When the bones of the posterior extremities are affected, the toes are turned outwards, the hocks inwards: the points of the calci almost touching each other; the animal being what is termed "cowhocked," the metatarsals at their middle being

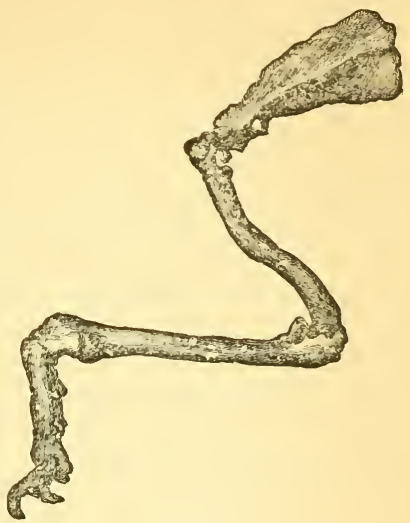

FIG. 30.

FIGS. 29 and 30 illustrate the bent condition of the humerus in rickety dogs. bent inwards and rather backwards. In the dog the humeri-the bones most commonly involved-are bent laterally by the action of the muscles, their inferior extremities being pulled upwards and outwards as $i$, specimens 29 and 30 , and the lower third of the shafts curved inwards, the curvatures being so great as almost to bend the bones completely upon themselves.

In rickets, not only is there a deficiency of the inorganic elements of the bones originally-namely, the phosphate and carbonate of lime-but there is a want of power to assimilate these salts; for during the progress of the disease there is inordinate excretion of them from the system, as shown by deposits in the urine. The structure of the bones is soft, cartilaginous, and open: but if the animal be allowed to 
live, earthy matter is deposited in the bones, which eventually become firm; the curvatures, however, remain. It is a noticeable fact that the centre of the curve in a rickety bone becomes strengthened by a deposition of additional bony matter; thus we always see that a horse with bent legs has depositions in the very centre of the arch, strengthening the weakest part, and making the bones bulkier than natural.

In hydrocephalus the rickety condition of the bones is the result of the pressure of the cranial fluid.

Rickets appears when the patient is a few weeks or months old, and is caused by constitutional debility, the scrofulous diathesis, or by external and preventible causes. Thus we find it in calves which are not allowed to suckle their mothers; in foals, when the mothers are taken to work during the day, and their offspring allowed to suckle perhaps every morning and night, or at most three times a day. Young animals fed upon artificial foods in lieu of milk, and kept without exercise, or when placed under any condition which interferes with those natural functions peculiar to youth, may become rickety.

In all cases there is a tendency to irregularity in the bowels, the milk passing through them in an ill-digested, curdy condition, white in colour and sour in odour, "the white skit," as it is vulgarly called. In a high-bred calf that I once observed, this kind of purging had existed for several weeks. At the time I saw him he was about three months old; had remained healthy until he was six weeks old, at which time he manifested symptoms of rickets; and when examined by me was found to have bending of the bones of the extremities, enlargement of the cranium, with separation of the bones at their sutures, and that he was blind from the pressure of the fluid contained within the cranial cavity.

The treatment of rickets must be directed to the removal of all influences deleterious to the constitution; and to the support of the weak and bent limbs. To fulfil the first purpose, the most appropriate remedies are dry and pure air; a large, roomy, loose box, or, if the weather be warm and dry, a nice field or paddock, and the constant companionship of the mother. If the patient be a calf which has not been allowed to suckle from its birth, 
great care must be taken that it be fed repeatedly, though moderately, during the day.

The constitutional treatment must be directed to the condition of the digestive apparatus. A very gentle aperient might at first be given with advantage, such as four ounces of castor oil-or a dose corresponding to the age of the patientmade into an emulsion, with two drachms of the bicarbonate of potash or soda, and a sufficiency of water. This should be succeeded by small quantities of lime.water, say half a pint, two or three times a day, in the same quantity of milk; and when the acid condition of the bowels has passed away, which will be indicated by absence of the sour or acid smell and better colour of the fæces, the preparations of iron will be found of great service, more especially the syrup of the phosphates, great care being taken that the digestive organs are not again thrown out of order. As a rule, the mother possesses an abundant quantity of milk; but should it be otherwise, the patient's food must be made sufficient for its wants by an additional supply of milk or of linseed tea,-a very good substitute, and often a desirable variety. Cod-liver oil may be given with advantage; and if the animal be valuable, the expense will be amply repaid by a successful issue.

The curvatures in the limbs are to be supported by slight but firm splints of wood, applied to the concave aspect of the curves, taking care that they are of sufficient length to reach the unbent portions of the limbs, both above and below the curves. A moderately firm bandage round both splint and leg will cause the latter to approach the straight line, the bones at this age being elastic and pliable. The splints must be so padded as not to bruise the prominent parts of the legs, and when properly and skilfully applied, may remain on for some weeks. If there be much pain and lameness, fomentations and frictions must be repeatedly made use of, and the splints removed from time to time.

The pathological anatomy of rachitis is thus given by Rokitansky:- "The texture of the bones is affected in two ways, of which sometimes one predominates, sometimes the other. In the first case the bone is rarefied and increased in size-expanded, in fact. A pale yellowish-red jelly is effused into its enlarged canals and cells, into the medullary cavities, 
and even under the periosteum. The bone itself is abundantly supplied with vessels, and full of blood, and its colour is therefore darker than is natural, and red. Occasionally this change reaches to such a degree that the cells of the spongy bones, and those in the interior of the medullary tubes, become excessively distended, and, as their walls disappear, are merged into larger cavities; several cavities become single spacious chambers, and the bones uncommonly soft and fragile. In the sccond case the bone is, in addition, deprived of more or less of its mineral constituents; and sometimes it is completely reduced to its cartilaginous element, and appears like a bone that has been steeped in acid. The bony corpuscles are empty, and their rays have disappeared; and when this is the case, the lamellar structure is here and there obliterated. At other parts the lamellæ appear, as it were, to have fallen asunder, and the corpuscles are seen quite distinctly interspersed between them. It is upon this condition that the flexibility of rickety bones depends." " It is remarkable," says Rokitansky, " that in cases of general rickets the reduction of a bone to its cartilaginous element so preponderates in some bones as to go on, even to completion, without any trace of rarefaction."

The late Professor Barlow taught that in rickets the cartilage yielded neither chondrine nor gelatine. This theory was based upon one given in Simon's Animal Chemistry. However, it is now ascertained that the analysed bones were not rickety, but affected by mollities ossium.

To conclude the subject of rickets, I must say that the swelling of the articulations seems to indicate that the disease does not consist in simple absence of the due quantity of phosphate of lime, and analysis has proved that the cartilage itself is altered in its constitution, the fat increased, and the fluoride of calcium, present in healthy bone, is absent in rickets. Analyses of some rickety bones give-animal matter 81.12, earthy matter 18.88 in 100 parts; against animal matter $31 \cdot 10$, earthy matter 68.90 in healthy bones.

Allied to rickets is what I may describe as true atrophy of the bones, or

\section{MOLLITIES OSSIUM,}

A remarkable case of which I have had the opportunity 
of seeing, and in which there was removal of the earthy constituents of the cranial and facial bones, more especially at their borders, without any visible external alteration of structure. There was neither swelling nor abscesses; in fact, nothing to indicate the pathological change. In this case examination after death revealed softening, thinning, and perforation at the sutures and in the bodies of the bones, giving them a pitted and worm-eaten appearance. The perforations were filled, upon the cranium, by the pericranium and dura mater, which were adherent to one another. The palatine bones and alveolar processes were soft, porous, and spongy.-(See Photo-lithograph, Plate I., Fig. 8.) An examination of the plate will show that the borders of the various bones are separated, in some places, to the extent of nearly half an inch. The ethmoidal bone was completely absorbed, and the brain lodged in the frontal sinuses, filling them, as well as its proper cavity.

The ease is so interesting, instructive, and unique, that I cannot do better than describe it. The patient was a grey Clydesdale gelding, nine years old. He had always been in good health until within about three months of the time when I saw him. At this time he was suffering from the effects of delirium-the third attack within three months-and now, having a discharge from the nostril, was supposed to be glandered. I found him blind (amaurotic), but not quite unconscious; was very somnolent, or semi-comatose; but would wake up with a frightened start when anything disturbed him. He was in fair condition; the nasal discharge was of the nature of a thin reddish serosity, and more abundant from one nostril than from the other. He was unable to masticate hay and corn, or did so very imperfectly, and was becoming worse in this respect. His powers of deglutition were also imperfect, and on examination I found the teeth were loose, and that he could not close his mouth firmly; but the lips were not pendulous. Upon inquiry, I found that he had been supported by thick gruel, oatmeal balls forced into his mouth, bread and milk, boiled potatoes, \&c. In fact, being a great favourite, he had been well taken care of.

Being anxious to know the source of the nasal discharge, about which my opinion was sought by the attending practitioner, I explored the facial sinuses by means of a gimlet, and discovered that the bones were soft, thin, and easily perforated, and that the sinuses were not filled with the red serosity 
above mentioned. Externally, there was nothing to denote this alteration in the bones, and the only observable change of structure was wasting of the masseter muscles, and, as a consequence, an apparent undue prominence of the zygomatic ridge. My prognosis was unfavourable, and in a few days afterwards he died comatose. On making an examination, the brain was found very white, and greatly enlarged, the enlargement partaking of the character of a fibrous development; the dura mater was much thickened, in some places upwards of an inch in thickness. The lateral ventricles were nearly obliterated by growth of fibrous tissue in the brain substance. The cranial nerves, with the exception of the fifth pair, were normal in appearance; but this (the fifth) pair were greatly enlarged, not only in their general bulk, but in each of their fibres, which were coarse, stringy, and distinctly separated by an abundance of fibrous tissue. As already stated, the ethmoidal bone was absorbed, and the brain and thickened dura mater filled the frontal sinuses.

How can this strange disease be accounted for? If the patient had been human, syphilis or mercury would have been blamed; but here was a patient that had never been mercurialized, never syphilitic. It might with reason be said that the attack of phrenitis had led to the brain enlargement, and that the absorption of the cranial bones had resulted from internal pressure from the growth of the cerebral mass. But how about the facial bones? Was the altered structure of the fifth pair of nerves sufficient to account for this? My colleague, Dr. Young, is of opinion that the disease was primarily in the bones, and that the pathological alterations found in the brain and nerves were a result of the osseous change.

\section{OSTEO-POROSIS.}

This disease was first described in this country by Professor Varnell, in the Veterinarian of 1860 . It appears from his careful and elaborate report that at one farm near Reading, no fewer than six horses had been affected with this strange malady when he reported upon it. Since then, however, many practitioners have witnessed it, and all agree that Mr. Varnell's report is a most faithful one. Mr. Anderson of Glasgow, and 
Mr. Robinson of Greenock, have seen it frequently; and I am indebted to them for many valuable hints upon the peculiarity of the symptoms, and for specimens of the bones of the head suffering from the disease.

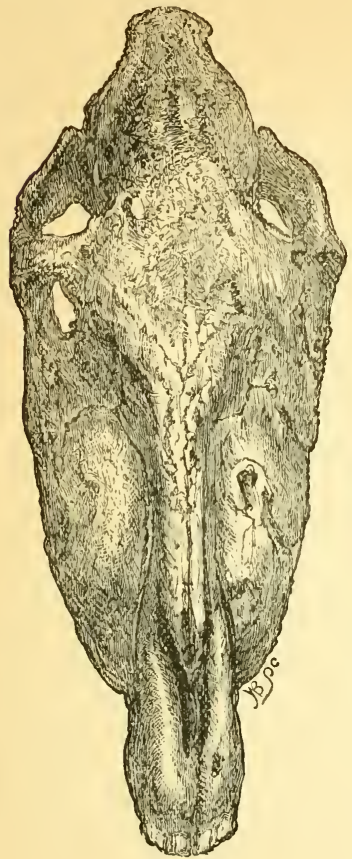

FIG. 31.

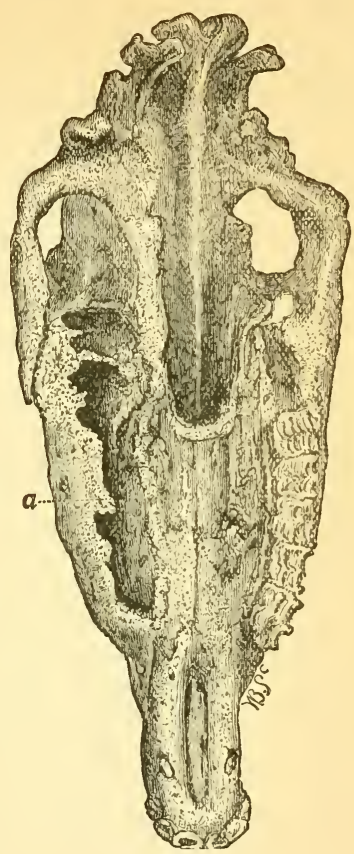

FIG. 32.

FIGs. 31 and 32.-Cranio-facial bones affected with osteo-porosis; the front view, Fig. 31 , representing the enlarged condition of the face; and Fig. 32 showing the disease extended into the alveolar cavities.

As a rule, the bones of the face are the first to suffer; but this is liable to exceptions, as the following extracts from the Veterinarian of 1860 will show. The symptoms were described to Mr. Varnell by a Mr. Wallin :- "My attention," says Mr. Wallin, "was in most instances first directed to defective action, perhaps in one joint or limb only, in which, upon exami- 
nation, tenderness would be evinced, with inordinate heat, and in a few days visible enlargement would be observed to exist. In the course of a week, or thereabouts, another of the limbs would become affected in the same way, when the acute symptoms of the first attacked would perhaps have partially passed off. In this way all the four extremities, one after the other, eventually became diseased. The appetite was generally good, and for a time the animals did not lose flesh very fast; but after a while, from the great constitutional disturbance set up, they fell off in their appetites, and began to waste very rapidly. The pulse seldom rose much above the natural standard, and the excretions appeared to be natural."

It appears that these horses had been, all but one, bred on the same farm, and were the offspring of different mares and different sires. The facts gained by Professor Varnell are very interesting, and may be briefly stated as follows:-

They had been fed on the same food as other horses on the farm which had never shown any symptoms of the disease. The owner had been in the habit of feeding his horses in the same way for years without any previous ill effects. Bran or pollard, along with the ordinary produce of the farm, formed the greater part of their diet. They all drank the same kind of water as the other stock. Professor Varnell examined the food they were getting, and pronounced it good. It appears that male animals only were attacked; none of the fillies had ever been affected with the disease. Another fact that Professor Varnell mentions is most interesting, namely, that the gentleman had another farm a short distance only from when the disease existed. On that farm not a single instance occurred, although the horses were bred from the same parents, and partook of the same kind of food. I will detail the symptoms in the two animals that were suffering at the time of his visit in Professor Varnell's own words. He says:- "I was first shown a brown cart-horse, five years old. He was standing in the cart-horse stable with other horses; was in very fair condition, and at first sight appeared as if nothing were the matter with him; the pulse was only a little above the ordinary standard, and the excretions, as far as I could see or learn, were natural. I ordered him to be led out of the stable, when I observed that lie stepped short, flexed his limbs with difficulty, and apparently 
with much pain, particularly the near fore and the off hind legs, the knee and hock joints of which were hotter than natural, slightly swollen, and tender when pressed upon. These symptoms were also present in the other two limbs, but in a much less degree of intensity. On causing the horse to be turned round, or moved in a backward direction, a decidedly rigid state of the loins was observed, and by pressing upon any part of the back considerable pain was evinced. On examining the animal's head, I was particularly struck with the enlarged and roundish appearance of the facial region. Each ramus of the lower jaw, the upper maxillary, and the nasal bones were evidently enlarged, and pressure on them caused some amount of pain. On looking into the mouth, I observed that the mucous membrane was of a purplish tint, except at the margin of the gums, where it was of a pinkish colour, crossed here and there by bluish lines (veins). The lining membrane of the nasal passages and the conjunctival membrane also were paler than natural. This condition of the membranes, I was assured by Mr. Wallin, could not have been caused by any medicine that had been given."

In the other case, a chesnut horse, which Professor Varnell examined, the joints were swollen, hot, and tender; there was the same rigidity of the back, and the peculiar colour of the mucous membranes; but the enlargement of the bones did not exist. This case had been ill for about six months.

The post mortem examination revealed the following condition of parts :- The internal organs, with the exception of a peculiar pallor, showed no marks of disease. The chesnut horse was sent to London, and on his arrival at Paddington Station he was found unable to walk from the liorse-box to the van that was sent to take him to the Royal Veterinary College. On his attempting to walk, his fore legs gave way at the elbow-joint on one side, and the shoulder-joint on the other, in consequence of the articular and capsular ligaments, and the tendons of the muscles, which are attached near to these joints, becoming detached (as was found to be the case afterwards) from their bony connections, by the tearing away of portions of diseased bones. The horse was destroyed upon the spot, and the carcase taken to the College for dissection. "The soft parts," says Professor Varnell, "generally were paler than is natural, especially the muscles of those limbs which had been thrown out of 
use from the pain produced whenever any movement was attempted to be made. But in these structures I did not detect the slightest traces of fatty degeneration. The fat generally had a mottled, watery appearance, which is very common in animals that are rapidly losing flesh, and this had been the case in a very marked degree for a short time before the horse was destroyed. In disarticulating the limbs from the trunk, and also the several bones of each limb one from the other, the appearance of the interior of each joint was remarkable. In most instances, although not in all, the articular cartilage was of a dark slate colour, much thinner than is natural, and in many places it was entirely lost. This was especially the case round the margin of the articulations, leaving the bone at that part quite exposed. The synovial membrane was considerably thickened, especially in those parts where it is most vascular. The quantity of synovia in each joint was small, of a dark colour, and in some cases mixed with blood. The character of some of the articulating surfaces, however, was quite different. In such, the articular cartilage was pale-coloured, and in some places of a palish yellow, velvety to the feel, and evidently containing fat; thereby indicating that the cartilage cells had disappeared, and fat had become deposited in their place. The ends of the bones were so much softened, that by applying a slight degree of force to the capsular or articular ligaments, small portions of the bone could easily be detached. The periosteal covering of the flat and irregular, and also some parts of the long bones, was very vascular, and could easily be stripped off. The bones generally were likewise so very soft that they could be cut with a knife in any direction with the greatest ease; and if pressure were applied to the cut surfaces, or where the periosteum had been removed, blood would ooze from numerous points. In the interior of the bones the cancelli were filled with a red gelatinous substance. The ribs, the vertebræ, and indeed all the irregular and flat bones, were in the same condition. The shafts of the long bones of the extremities were not visibly increased in size, nor was the shell or compact structure much altered. The ends of these bones, however, were enlarged and soft; and on making a section through them, in their long diameter, the medullary canal, and especially the cancelli near to the extremities, had a singular, although not a uniform, appearance. In 
some of them, the whole of the interior was of a dark red colour, from congestion of the vessels and effusion of blood into the areolar interspaces; in others, one-half only of the interior was in this state, the other part being filled with a peculiar fat, and consequently very pale in colour. It was at the end of the bone affected in this way that the articular cartilage was of a palish yellow colour, velvety to the feel, and also slightly greasy. Fven the teeth did not escape the malady, one of their constituents being evidently affected, which was evinced by the crusta petrosa being much thicker and more spongy than natural."

The pathological anatomy of the bones was investigated by Dr. G. Harley, and communicated to Professor Varnell in a letter, dated 21st March 1860:- “The horse's bones which I examined for you presented two well-marked varieties of morbid change, one of which was most characteristically seen in the bones of the head, the other in the long bones of the extremities.

" 1 st. As regards those of the head. The disease, although it affected all the bones, was most advanced in the lower jaw. I shall therefore limit my remarks to a description of it. The bone was considerably hypertrophied in its tranverse diameter; the periosteum readily detached; the osseous tissue was of a pink colour, and on pressure a quantity of blood oozed from its surface, as if from a sponge. The osseous tissue was elastic to the touch, and so soft that it could with facility be cut with a knife. The surface of the section had a somewhat fleshy appearance, but to the nail it conveyed rather the impression of cartilage. When examined microscopically the osseous tissue was seen to be expanded into a network of fine fibres. The Haversian canals, on the other hand, have become so enlarged that at first sight they might be mistaken for bony tissue, and the bony tissue mistaken for the Haversian canals. On examination with a higher power, not only is the osseous tissue seen to be rarefied, but the canaliculi proceeding from the lacunæ or bone corpuscles, as they were formerly called, are in many cases obliterated. The lacunæ themselves are also not so distinct as in healthy bone. The enlarged Haversian spaces are filled with a gelatinous matter, mingled with a small portion of fat, and here and there pervaded with fine, scarcely visible fibres.

" $2 d$. Long Bones of the Extremities.-The periosteum, although 
readily detached, does not come away with so much facility as from the bones of the head, except towards the ends of the bones, where, strange to say, the ligaments had become detached by the mere movements of the animal during life. The outer surface of the shaft of the bone is firm enough, but on section it is found to be greatly thinned, in consequence of an enlargement of the medullary canal having taken place at the expense of the osseous structure.

"The dilatation of the medullary cavity is most marked towards the extremities of the bone, and there the marrow is deeply tinged with blood. The osseous tissue, which in some parts is reduced to one-half, or even one-third of its normal thickness, does not present the spongy appearance of the cranial bones, but, on the contrary, is dense, and so hard that it cannot be cut with a knife.

"After the calcareous matter is removed by acid, the section of the animal matter has a somewhat glistening cartilaginous appearance, here and there dotted with opaque white spots. Under the microscope, a thin section reveals a curious condition of things; the Haversian canals, as well as the concentric bony lamellæ surrounding them, appear perfectly normal. The lacunæ and canaliculi, as far as can be ascertained in the decalcified bone, are equally healthy; but, on close inspection, the opaque white spots observed by the naked eye are seen to be irregularly shaped cavities in the osseous tissue, filled with fat cells. These cavities vary in size, from that of a pin's point to a milletseed. They are not limited to any particular portion of the osseous structure, although they seem to have commenced, for the most part, in the lacunæ. Some, however, may, with equal truth, be said to have originated in the Haversian canals, the walls of which have been gradually broken down and eaten away, as the morbid condition advanced; the place of the earthy and animal matter being gradually taken up by fat corpuscles."

Professor Varnell says, in continuation of his most admirable description, that " the bones, after being macerated and dried, exhibited many peculiarities which were not apparent in their fresh state; for instance, the ulceration of the articular surfaces, which seemed to be altogether confined to the cartilages of incrustation, was found on their removal to affect the bone 
beneath; and the loss of structure, however small it might be in the cartilage, extended more or less deeply into the bone; the size of the cavity increasing with its depth. This fact, I think, proves beyond disputation that the loss of the cartilage depended primarily upon the diseased condition of the bone, and that the general thinning of the cartilage arose from the same cause. Scarcely an articulation of the whole body was free from this ' worm-eaten' condition of both bone and cartilage.

"It was found likewise, in cleaning the bones after maceration, that, from their extreme softness, great care was necessary to prevent their different processes (epiphyses) from being detached, and the application of the slightest force to a portion of ligament or teindon, that was not sufficiently separated by decomposition, would be certain to effect their removal.

"The external surfaces also of the bones, as contrasted with those in a normal condition, had a very singular aspect, especially in the flat and irregular bones. The ends of the long bones were similarly affected, but the shafts of most of them deviated but little in external appearance from a healthy state. A transverse section, however, of the middle of the shaft showed very distinctly that the osseous laminæ surrounding the Haversian canals were very much thinner than is natural, thus necessarily leading to a considerable enlargement of the latter. In the firstnamed, however, and in the flat bones, instead of the surface being smooth and dense, it had a character not very unlike a fine sponge. The foramina for the passage of the blood-vessels, and especially those belonging to the periosteal membrane, were much dilated, thus giving the bone a kind of honeycomb appearance." (Figs. 30 and 31 illustrate this peculiar appearance very well.)

It is found that the weight of the bones is much diminished in osteo-porosis. The articular surfaces undergo changes that are peculiar; thus, in Professor Varnell's cases, the articular cartilage upon one end of the femur was much thinner than is natural, of a dark slate colour in some places, and altogether lost in others; the bone beneath it being very soft, and almost black from congestion of its vessels and extravasation of blood into its interstices. On the other end of the bone, the cartilage was in a different condition, pale in colour, and in some places of a yellowish hue, soft and velvety to the touch, 
and containing a small quantity of fat. Beneath the cartilage the bone was found soft, the lamellæ very thin, with the interspaces filled with a greasy white-looking substance, fat, and the extremity of the bone generally non-vascular. The synovial membrane was much thickened in some joints, but the quantity of synovia in all was small, dark in colour, and in some cases mixed with clots of blood. The periosteum of all the bones was easily stripped off, and the bones themselves were soft and easily cut with the knife. Within the bones the cancelli were filled with a red gelatinous substance. The ribs, the vertebræ, and all the irregular and flat bones were in the same condition. The shafts of the long bones were externally but little different from healthy ones; but on making a longitudinal section of them, the medullary canal and the cancellated spaces near their extremities were dark red in colour, from congestion of the vessels and extravasation of blood into the areolar spaces. In other parts a peculiar fat was found, giving a paleness to the colour; and the bones were near their ends so filled that the cartilage appeared to be of a palish yellow colour, and was velvety and slightly greasy to the touch.

In some cases the teeth do not escape; the crusta petrosa being evidently increased in thickness, and more spongy than natural. No class or breed of horses seems exempt from this disease, as I have records of cases of it in carriage horses, in ponies, and in thorough-bred ones, whilst Professor Varnell's cases belong to the cart breed. It seldom affects horses above six years old; frequently, indeed, the animals have ranged from two up to four years old; but my later observation teaches me that very old horses are not exempt from the disease. Professor Varnell enters into a very lucid examination of the cause, and leaves one with the impression that it is due to food or water deficient in the salts of lime. Experience, however, seems to prove that the absence of lime is not a constant factor so far as the life-history of the animal can be traced, nor can it be conclusively proved to be due to any peculiar geological formation or particular locality. One of Mr. Robinson of Greenock's cases was attacked while in London, during the summer season, with symptoms analogous to those of rheumatism, and all his cases were well-cared-for animals. It has been witnessed in Shetland ponies. In America the disease is called Big-head, from the peculiar swollen state of the jaws; it is there supposed to be due to the feeding upon maize, and it has 
latterly appeared in thorough-bred stock in Australia, where it is erroneously ascribed by some to forcing the growth of the young by over-feeding.

Osteo-porosis is not confined to the horse and sheep; cattle reared on bad ill-drained land, poorly kept in the winter, grazed on sterile upland pastures during dry summers, ol otherwise illused, being liable to be affected with it. They present symptoms of rheumatic lameness, terminating in spontaneous fracture of the bones involved.

The disease in sheep, as explained by the following history, kindly supplied by Mr. Robertson of Kelso, is not uncommon.

"Sheep of all breeds and of every age are, under certain conditions of soil and cultivation, externally liable to a peculiar form of non-inflammatory disease of bone, more nearly resembling osteo-porosis, as here described, than aught else I am aware of.

"Every appearance of this disease with which I am acquainted has been in stock pastured on what is known as weak moorland, recently reclaimed and limed, or fed with roots grown on scil of this character undergoing reclamation. It is deserving of notice that so long as this class of soil is left undisturbed, the sheep, although grazing sparsely, are in good health; but no sooner is it drained and limed, and put under such rotation as is deemed best fitted to accelerate its being laid down again to grass, than a strange class of diseases make their appearance amongst the sheep confined to this land; amongst the most conspicuous of these affections is this peculiar bone disease.

"There is nothing either in the general health of the sheep, their powers or manner of locomotion, or external appearance of the bones, which afterwards show so much alteration of structure, to attract attention, or even raise the suspicion that the animals are not in good health and doing well.

"The first indication that there is aught seriously wrong is in all probability the finding some morning of one or more of the flock helpless from a fractured limb; or this condition may be observed actually to occur while the sheep are being turned by a dog in the usual course of telling and inspection.

"The fractures, as a rule; occur in the long bones of the limbs. On carefully examining these bones, there is frequently little to be observed of an abnormal nature on their exterior surface. 
Oecasionally the periosteum will be thicker, more vaseular and more easily stripped off; the fractured surfaces, however, or such as are exposed, on making a section through the shaft, disclose great general vascuiarity, and eonsequent heightening in colour.

"The medulla appears not only to contain an extra amount of blood-vessels loaded with blood, but at many parts these seem to have ruptured, and extravasation to have occurred. This heightening in colour and extravasation of blood is not only present in the large nutrient canal, but distributed throughout the entire mutrient system of the bone. Obvious alteration of structure in all these cases is found to exist both in the compact tissue of the shaft, and in the entire cancellated and compact tissue of the extremities. In the former, the bony laminæ surrounding the Haversian eanals are expanded, opened out, and thinner, resulting in a eonsequent dilatation of these latter ; the same conditions exist to an equal extent in the cancellated tissue of the extremities.

"Although this rarefaction of the true bone tissue may have proceeded to a great extent, leaving merely a thin shell of eompact bone around the great medullary eanal, in no ease is there an entire want of bony matter, or a replacement of this by cartilaginous or fatty tissue. The bone will not cut with a knife, but may be fragile enough to admit of being erushed on the applieation of even moderate force.

"It is no doubt from this apparent eondition of the extra amount of blood contained in the vessels and nutrient eanals, its effusion, and the very dark colour imparted to the bones, which has led ordinary observers to speak of them as being in a state of rottenness and decay.

"Although all breeds and ages of sheep, when circumstanced as stated, seem liable to be thus affected, I have met with it most frequently amongst eross-bred sheep, and in these when under two years of age, most probably because they are the elass of sheep most frequently subjected to the adverse influenees mentioned.

"In regard to the treatment of this disease, we ean, from experiments which have been carried out, speak with tolerable certainty as respects the efficacy of preventive measures. When sheep are placed either on pasture or on roots, with such con- 
ditions of soil and cultivation as indicated, the addition to this natural diet of from one quarter to one half pound daily of some nutritious material, as linseed cake, or a mixture of linseed cake, oats, and bran, serves effectually to prevent the occurrence of this diseased condition; while, when such has declared itself, a change to a better soil and a different geological formation will, in combination with supplemented food, materially hasten a restoration to robust health.

"At one time it was believed, and many still hold to the opinion, that the immediately operating cause in the production of this diseased condition of bone was the presence of lime salts in the soil, the result of the added alkali, and their transference to and appropriation by the animal system, thus producing a fragility of bone from excess of earthy constituents. Chemical analysis of these bones has, however, disallowed this theory, even if it were not untenable on other grounds.

" It seems most probable that this condition of mal-nutrition is rather to be regarded as resulting from certain changes which the treatment of the soil has brought about, in the material produced therefrom, and their unfitness when received into the animal body for the elaboration of healthy tissue, than from the passage in an unchanged form of any material already existing in the soil into the component structures of the animal body."

In concluding this subject, I must give it as my own opinion that the disease is due to development of the vascular and fibrous structures of the bones, without a corresponding growth of the true osseous and cartilaginous elements; and in support of this view I here quote from Rokitansky, who says- " Osteo-porosis consists of the excessive development of the tissues which occupy the canals and cells of the bones; whilst at the same time the actual quantity of bony matter remains unaltered." The growth of the contents of the canals and cells must naturally cause a rarefaction of their walls, and the bone becomes increased in volume, expanded, as the walls of the expanding cavities become thinner and thinner, till at length apertures are formed, and cavities which communicate one with the other, containing a dark red medulla, traversed by dilated vessels, and which sometimes become ruptured, and the cavities become filled by loose or firm clots of blood. The fact that 
osteo-porosis has not been recorded in aged horses points ont most strongly that it is a disease of growth; and the circumstance of the bones being fragile and easily broken, confirms the view that the cartilaginous basis, as well as the earthy constituents, is not developed; for were the disease due to nondevelopment of earthy matter only, the bones would bend, being more elastic than is natural; but in this disease they are brittle, owing to the absence of the tough animal basis in sufficient quantity.

Osteo-porosis is confounded with fatty degeneration of bone, but it is quite different, both physically and chemically. In the one case, the bone is pink or dark red, and hydrochloric acid produces but little change; but in fatty disease the bone is yellowish; and all that is left of a piece subjected to the action of the acid is a small quantity of an amorphous greasy substance. In fatty degeneration the cartilage degenerates into fat; in osteoporosis it is not developed sufficiently, the bulk of the bone being composed of vascular and fibrous structures mixed with a portion of spongy bone; hence they are frail, and liable to fracture. In one of Mr. Pobinson's cases both fore legs were broken before the animal was destroyed.

It would be hazardous in our present state of knowledge to condescend upon the cause or causes of osteo-porosis, for it will be gathered from the foregoing observations that animals of every class, no matter how treated, are subject to it; the race-horse, from supposed over-feeding and over-forcing; cattle from the want of proper food; and sheep when placed upon pastures which have been improved by draining and liming.

Continental writers recommend the curative treatment of the disease. In this country everything that has been done to arrest its progress, such as good food, tonics, alteratives, the best of attention, ventilation, \&c., have all proved futile when it had once been established. We must therefore conclude with Mr. Robertson that its prevention is to be aimed at, and measures taken for that purpose in every case where animals are placed under conditions or pastured upon land favourable to its development, by making such additions to their natural diet as to blend the whole food into a perfect pabulum, fit for the nourishment of all the tissues of the body; for in all probability it will yet be discovered that the disease is due to 
the absence of some essential ingredient of the food, or its presence in such small quantities as to be insufficient for the necessities of the animal; or the presence of some other constituent of the food in such super-abundance as to destroy the perfect composition of the aliment as a whole, and thus render it unfit for assimilation by, or so change it as to cause it to become even an irritant to, the osseous system of the animal.

\section{FRAGILITUS OSSIUM.}

Fragility of bones results from two pathological conditions, namely-1st. Fatty degeneration of the animal basis; and $2 d$. The presence of an undue quantity of earthy materials, due to old age or to ostitis.

The first form is a constitutional disease, with no symptoms indicative of its presence during life, discovered only after a fracture of such a nature as to be incurable, and when the animal is examined after death.-(See Photo-lithograph, Plate I., Fig. 1.) I have, however, observed that narrow-loined horses are liable to this disease-bad thrivers, as they are called-with small articulations, badly-formed hocks and knees, round pasterns, \&c., and having a tendency to fall lame from bone-spavins, ringbones, or other osseous diseases. As a rule, they are middleaged horses, and the post mortem examination shows the bones - more especially the pelvic and vertebral-in a peculiar greasy condition, yellowish in colour throughout their substance, and with their cancelli filled with a dirty yellowish fat. When microscopically examined, the minute structure of the bone is found to be filled with fat globules; in fact, their structure is not only infiltrated, but partly composed of fat, traversed, as it were, by the natural bony material.

Writers upon surgery describe fatty degeneration of bone under the head of mollities ossium, or softening of the bones; indeed, there seems to be a confusion amongst them on this subject, some giving the pathological anatomy of osteoporosis, others of fatty degeneration. Paget, however, says that two diseases are described as mollitics ossium, namely fatty degeneration, and the simpler softening of bone, or osteomalacia rhachitismus adultorum, in which the bones are flexible rather than brittle. 
The case related at page 183 goes to prove that the two conditions are quite distinct in the horse, as in that case there was simply a removal of the earthy constituents, rendering the bones flexible and easily cut with the knife. I contend this is the true mollities ossium; whereas in the disease now under consideration fatty material is substituted for the cartilaginous basis, without removal of the earthy matter, and in this way the bones are rendered brittle, and liable to fracture from unappreciable causes.

The second form of fragilitus ossium, resulting from old age, can scarcely be looked upon as a disease, since it is as much the natural state of the senile skeleton as the soft pliable condition of the bones is that of adolescence.

Bones, when affected by chronic inflammation, however, are rendered exceedingly brittle and liable to fracture; and it is interesting to note the progress of ostitis leading to such a condition. First of all, if the extremity of a bone be examined, by making a section of it, when in the early stages of inflammation, it will be discovered that the first change has been the removal of the fat from its cancelli; that a fibrinous exudate has taken its place; and that this deposition is being gradually replaced by osseous material. After a time, the open, areolar appearance of the interior of the bone assumes that of compact tissue, the bone at the same time increasing in weight. This condition may continue for a very indefinite period, and in some cases no further change takes place, but in others open spaces are formed by absorption of the new bone. These spaces are small at first, but become enlarged by removal of their walls, and by the coalescence of two or more of them. Thus the solid, compact bone again becomes cancellated, but this cancellated structure is very different from the original; for the bony trabeculæ are now deficient in animal material, are mostly made up of the earthy constituents of bone, and are thus brittle and ready to break upon the slightest application of force. The bones most liable to assume this condition are the tarsal, cuneiform, navicular, and pedal bones; and these are all composed of a very thin, compact shell, and much cancellated tissue. This compact layer is frequently involved in the diseased process going on within, and covered with small nodules of bone; at other times it is ulcerated on its surface, or both conditions may 
be co-existent. The pedal bones, however, when chronically inflamed, become absorbed at their inferior borders externally, and undergo the change described within their substance. New formations, as bone-spavins and ring-bones, when of long standing, are also liable to take on this action, becoming brittle and easily broken.

\section{OSTEO-SARCOMA.}

Spina ventosa, or, as it is called by Gamgee, " fibro-plastic degeneration of bone," is a disease very rarely found, except in horned cattle, affecting the lower jaws generally; but in one case which came under my notice the upper jaw was the seat of the disease.-(See Photo-lithograph, Plate IV., Fig. 7.) These tumours consist of an osseous crust, forming the walls of a cavity, divided into several compartments containing a reddish fluid tubercular material, or inspissated pus mixed with pieces of bony or cartilaginous substance, bounded, as it were, by cyst-like walls. These three conditions were all present in the case illustrated, and serve to form three definite stages of the disease:1st. The formation of a cyst containing a jelly-like fluid; $2 d$. The transformation of this fluid into a grey caseous material, which, when microscopically examined, presented a merely granular appearance; and $3 d$. The softening of the tubercular matter into a semi-fluid, pus-like substance, mixed with pieces of cartilage and bone.

The cause of this disease has been attributed to external violence; but I have great reason to doubt the accuracy of such a conclusion, and I am of opinion that the causes are intrinsic, and due to the scrofulous diathesis. It occurs in young animals mostly, and is said to affect steers more than bulls, its seat being the neighbourhood of the second and third molar teeth, where at first a small circumscribed swelling occurs. When felt, the tumour is found to be hot, and that pressure causes some pain to the animal. At first the animal experiences no inconvenience; indeed, it seems to suffer but little throughout the various stages of the disease, provided the teech do not become carious, when of course the sufferings of the animal will be severe, aud it will lose flesh from inability to feed. The bullock from which the drawing was obtained was quite fat, 
and sold for $£ 29$ the day before he was slaughtered, and was as able to masticate his food as if nothing ailed him. It is on record, however, that the teeth drop out of their sockets, and that fistulæ open into the mouth.

Treatment, in any but the early stages, is out of the question, and the only thing to be done is to feed the animal, if possible, for the fat market. If, however, called in before the tumour has attained any considerable magnitude, the practitioner may remove it. To effect this, he must dissect the skin and subcutaneous structures from the tumour; trephine in one or two places, as starting-points; and remove the whole of the diseased structures, including the teeth surrounded by them, by either the bone forceps or saw. The operator must be careful not to leave any portion of the diseased parts, or it will most assuredly become the nucleus of a new growth. When the removal is complete, the skin is to be brought together by metallic or carbolised catgut sutures, and over all collodion or styptic colloid is to be thickly painted. In this manner the wound will often heal without suppuration.

The practitioner will of course understand the necessity of carefully feeding the animal upon soft diet for some days after the operation, and that some attention must be paid to the parts operated upon.

\section{THE EFHECTS OF MERCURY ON BONE.}

The accompanying woodcuts-for the use of which I am indebted to Professor Bennett-illustrate the effects of mercury on the bones of a dog, the skeleton of which is now in the Museum of the University of Edinburgh. The history of the case is given by Professor Bennett in his Principles and Practice of Medicine, and is as follows:- "The dog lived in the shop of Mr. Ballantyne, eighteen years ago, in Carrubber's Close. At that time the work carried on consisted almost exclusively of painting with vermillion and lacquering Japan articles. The dog, who never left the premises, was frequently seen lapping the vermillion oil paint; and there can be no doubt that in this way there was introduced into his system a considerable quantity of mercury. After death, the dog was dissected. Numerous 
cancerous-like masses were found in the lungs and internal viscera, and his skeleton was preserved. It will be seen that the shafts of the long bones, and not their extremities, were

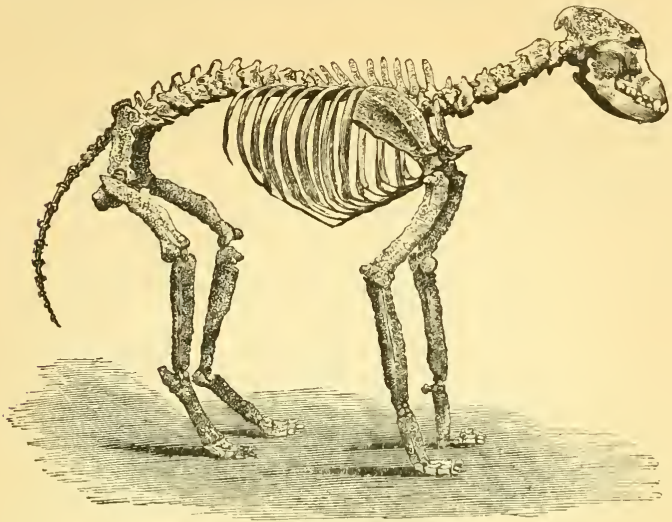

Fig. 33 - Skeleton of Dog.

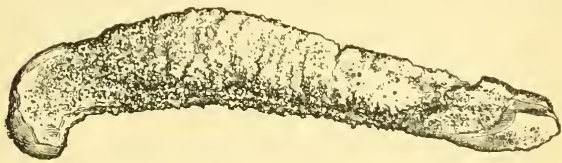

FIG. 34.

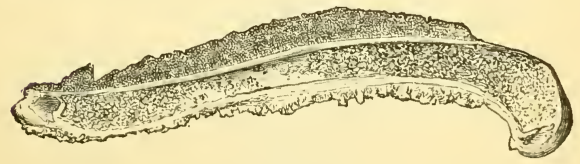

FIG. 35.

Figs. 34 and 35.-Two views of the Femur.

attacked. The disease closely resembles what may be observed in many other specimens of so-called syphilitic disease. Yet in this $\operatorname{dog}$ we have the positive proof that it was caused by mercury, as all attempts to communicate the syphilis to dogs by inoculation have failed." 


\section{DISEASES OF THE JOINTS.}

\section{EXPLANATION OF PHOTO-LITHOGRAPH, PLATE II.}

1. Rheumatoid Inflamation of Shoulder-Joint, showing porcellaneous condition of articular surface, and ossification of synovial fringes. (1.) Extremity of scapula. (2.) Upper end of humerus.

2. Scrofulous Disease of Elbow-Joint of Ox, the lower extremity of humerus being shown. (1.) Tubercular deposits represented by the white spots. (2.) Destructive absorption of the cartilage and bone.

3. Rheumatoid Disease of Stifle-Joint, showing-(1.) Grooving of the bone, and the porcellaneous deposit; and (2.) The formation of the addimentary bones described in the text.

4. Removal of Articular Cartilage and Compact Laminal LAYERS from the surfaces of tibia and astragalus in a case of open joint. (1.) New ossific deposit external to the articulation. (2.) Portion of laminal layer not yet removed, but which in the specimen is " tunnelled under" by absorption. (3.) Lower end of tibia; which at (4.) contains a pit-like ulcer.

5. Hock-Joint in Rheumatoid Bog-Spavin. (1.) Grooving of articular surface into which the porcelain is deposited. (2.) Addimentary bones, formed by ossification of the synovial fringes.

6. Fracture of Astragalus and Bone-Spavin. (1.) Astragalus. (2.) Bone-spavin. (3.) and (4.) Metatarsal bones.

7. Caries of the Point of Os Calcis (Capped Hock). (1.) Superior, and (2.) Inferior extremities of the bone.

8. Scrofulous Inflammation of the Phalangeal Bones of Ox in "Foul in the foot. (1.) and (2.) Show the contrast between an ulcerated and healthy articulation. It will be noticed that although the destructive process is much advanced, there is but little or no attempt at repair by external deposition of bone.

9. Tubercular or Scrofulous Inflammation of the Foot of s Cow, commonly called "foul." At (1.) and (2.) are well-marked collections of "yellow tubercle." 

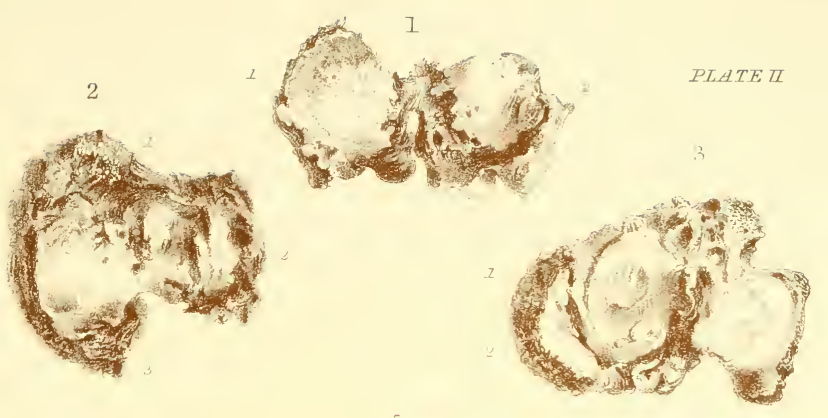

4
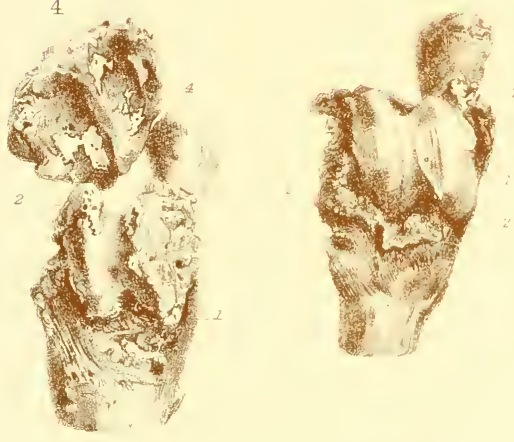

6

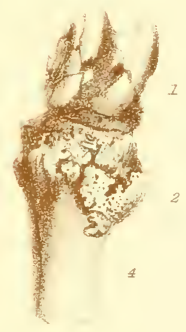

8
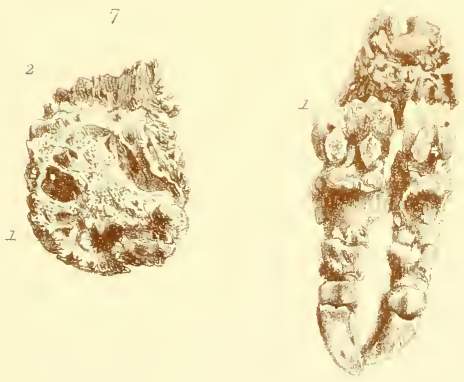

9

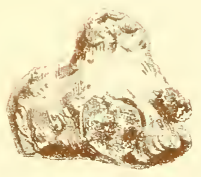





\section{CHAPTER XI.}

\section{DISEASES OF THE JOINTS.}

CLASSIFICATION OF THE JOINTS-ANCHYLOSIS OF THE VERTEBREACUTE SYNOVITIS-CHRONIC SCROFULOUS SYNOVITIS-CALCIFICATION OF THE SYNOVIAL FRINGES-RHEUMATOID ARTHRITISMORBID CONDITION OF CARTILAGE-TRAUMATIC INFLAMMATION OF THE JOINTS-IIYDROPS-ARTICULORUM-ANCHYLOSIS.

JoINTs are complex in their structure, consisting of bones, articular cartilage which covers their extremities, synovial membrane, capsular and binding ligaments, fat, blood-vessels, and nerves. Owing to these various component parts possessing different degrees of organization and vitality, it will be easily understood that the diseases of the joints are both numerous and important, more especially when the extensive motion they are called upon to perform is taken into consideration.

The joints are divided into three classes, namely, the immoveable, the moveable, and the mixed. The immoveable joints, or sutures, are those connecting flat bones together, as in the bones of the head, and are subject to no special diseases; it is therefore with only the two latter classes that we have to deal.

In the mixed joints the ends of the bones are not covered by cartilage of incrustation, nor is there a true articular cavity lubricated by synovia; the bones, joined together by powerful kinding ligaments, are yet separated from each other by a thick broad pad or disc of fibro-cartilage. This simple kind of articulation is subject to but one form of disease, namely, chronic inflammation, causing the gradual conversion of the fibro-cartilaginous pad into bony tissue. In this manner the vertebral column, which is extremely elastic and mobile in its entirety, is converted, more especially in the lumbar and dorsal regions, into a stiff, unyielding structure, rendering the movements of 
the horse uneasy to its rider, and destroying that motion of adaptability which is pre-eminently characteristic of that animal, and which enables it to perform an amount of speed with a weight upon the back that would, without this provision, be sufficient to cause by its concussion such injury to its internal economy as to produce disease, and even death.

Causes.-This disease is generally produced by the animal being compelled to bear a weight disproportionate to its strength. In some cases, however, it proceeds from no external appreciable cause; it must therefore arise from a constitutional diathesis; the bones becoming fragile, fatty, and degenerate in condition, liable to fracture from trivial causes, such as the moderate contraction of the muscles attached to them, or even to be spontaneously broken. (See Photo-lithograph, Plate IV., Fig. 1.)

The specimen from which this plate is taken beautifully illustrates this peculiar pathological condition. When newly prepared, and up to the present time, it is found to resemble, in its external appearance, an old fossil bone of a dirty rusty-brown colour, greasy to the touch, and crumbling under moderate pressure. Microscopically examined, it presented numerous oil globules, its bone corpuscles much altered in shape, and in many of their special characteristics. It will be seen that an old-standing fracture exists, and that a process of repair, represented by the enlargement, with a fissure running through its whole thickness, had gone on to a considerable extent. The animal from which the specimen was taken presented no symptoms of having its back in this condition, nor of the fracture during life. He was a very old pony, and was accidentally bought for dissection. The whole of his spinal column, from the sacrum to the middle of the cervical region, was more or less affected; the anchylosis being complete between all the lumbar and dorsal vertebræ, but only in the rudimentary state in those of the cervical region. Had this animal been cast for an operation, the result would inevitably have been a broken back, no matter how carefully such casting had been performed. Veterinarians will bear this in mind, and are here exhorted to make a careful post mortem examination of every case of such an accident, not only as to the mere presence of the fracture, but into the pathological condition of the bones. 
The symptoms of this disease are very obscure; some slight stiffness about the loins may be observable; disinclination on the part of the horse to lie down in the stable, more or less clonic spasm of the lumbar and gluteal muscles (shivering), or some degree of stringhalt.

Cart-horses are more subject to this disease than any other class of our patients, and this is explained by the fact that they are loaded with heavy weights during the period of their growth, when their age and strength are not calculated to bear them. When hunters and hacks, as age advances, become stilty in their action, unpleasant to ride, the change is often as much due to this increased stiffness in the back as to that in the joints of the extremities.

Sometimes horses suspected to be suffering from this disense present symptoms of its temporary aggravation by a greater degree of immobility in the loins and back, a greater extent of the spasmodic action before referred to, and by signs of partial paralysis; there will be reeling of the gait, crossing and drawing inwards of the hind feet, inability, without danger of falling, to turn round quickly, great difficulty in backing, during which process a peculiarly sudden quivering elevation of the tail may often be observed.

The meninges of the cord are in such cases more or less involved in the inflammatory action.

This aggravation may be produced by a slight cold, indigestion, or other trivial external or internal cause.

For the original disease nothing can be recommended except the removal of its cause, if possible, and a period of rest; but for the exacerbations active treatment is required. The constitutional treatment will depend upon the cause. As a rule, a purgative-if its administration is not contra-indicated by the presence of a catarrhal affection or chest disease-should be given, and belladonna in moderate doses, in preference to any other sedative, because of its special effect upon the cord, and its power of diminishing muscular contrastility. The local treatment to consist of fomentations to the loins, or a sheep-skin, and the application of gently stimulating embrocations.

If these do not succeed, a blister may be applied with advantage. If the hair be clipped for a space four inches broad, and twelve to sixteen inches long, upon both sides of the spine, the 
hair around its borders well saturated with grease, and the blister carefully applied to the clipped patch only, no danger may be apprehended. It should, however, be washed off at the end of twenty-four hours.

The diathrodial or true joints are divided into three varieties, namely, arthrodia or gliding joints, the enarthrodia, and the ginglymoid.

The arthrodia are liable to inflammation, and ulceration of the articular surfaces, arising primarily in the bones entering into their conformation.

The enarthrodia, or ball-and-socket joints, are in our patients seldom diseased, and generally the cause of such disease arises intrinsically, such as from the rheumatoid or the tubercular diathesis. They are clothed by muscular tissue, and but rarely suffer from accidents.

The ginglymoid or hinged joints are exposed to injuries from without, such as sprains and punctures, which are productive of a variety of diseases, namely, inflammation of the synovial membrane (acute and chronic), destruction of the articular cartilage, caries of the extremities of the bones, a deposition of calcareous matter in their structure, called the porcellaneous deposit, loose bodies in the synovial cavity, defective secretion of synovia, increased secretion of it, constituting dropsy of the articulation, and anchylosis; these are also liable to be accidentally opened.

When inflammation of a joint arises from a strain, the ligaments are not only stretched, but are more or less lacerated at their points of attachment. These ligaments, it will be remembered are lined upon their unattached internal surfaces by synovial membrane, which becomes inflamed when they are strained, and from these points the inflammation extends along the whole synovial surface of the articulation.

The true hock-joint, namely, that constituted by the tibia and astragalus, may be taken as a type in our description of these various forms of pathological changes.

INFLAMMATION OF THE SYNOVIAL MEMBRANE, OR ACUTE SYNOVITIS.

The primary effects of inflammation upon this membrane are more or less injection of the vessels, opacity, and dulness of its 
surface. In some cases it will be of an extremely dark red colour, owing to congestion, and may present an appearance of brownish or even crimson spots.

At the outset of the congestion, this, like all other secreting membranes, is preternaturally dry upon its surface, owing to its secreting powers being for a time in abeyance; but very shortly there will be a superabundant secretion of an unhealthy synovia, aqueous in its character, and containing flakes of lymph. The membrane becomes thickened by interstitial deposition; loses its translucency, and becomes rough on its internal surface, to which flakes of lymph are found adherent. This exudation of lymph is confined to the synovial surface, and does not extend to the articular cartilage. In severe cases suppuration may occur, and, if the disease cannot be arrested, ulceration of the cartilage is sure to follow.

The synovial fringes will be found to be in a higher state of inflammation than any other portion of the membrane.

Any true joint in the body may suffer from inflammation of its synovial membrane, but some are more liable than others. This will be afterwards referred to under the head of LAMENESS.

The causes are strains, punctures, the localisation of rheumatic poison, the deposition of tubercular matter into the substance, and upon the surface of the synovial membrane, as in the "grapes" of horned cattle, and from the deposition in and around the joints of mineral poisons, as seen in horses which are employed in and about large smelting works.

The symptoms are lameness and fever, varying in their degree according to the severity of the articular inflammation. If the disease be severe, the pain acute, the fever will be high, the pulse quick and irritable, partial sweats bedew the body, but more especially the affected limb, and the lameness will be so great that the animal cannot put its foot to the ground. The affected joint soon commences to swell; the swelling being tense, yet elastic, in the first stages; but soon becoming hard, firm, and unyielding, from an exudation into the subcutaneous structures. There will be increased heat of the parts, and they will be tender to the touch.

It is of the utmost consequence to arrest the disease before the cartilage becomes involved, or it will be incurable; and 
if it be in one of the posterior articulations, such as the hock, it is often necessary to place the animal in slings. Fomentations of warm water must be applied to the part repeatedly, and some soothing application rubbed on, such as a weak solution of opium or aconite. The shoe must be removed; but if the practitioner thinks that the patient will be able to rest better with a high-heeled shoe, it should be immediately applied.

The constitutional treatment will depend upon the severity of the symptoms. In all cases a moderately strong purgative is beneficial; and if the pain be very great, opium, two or three times a day, will best relieve it; care being taken that the bowels do not become constipated. When the pain is less acute, aconite may be used in preference to the opium.

If these remedies fail to give relief in the course of three or four days, a blister must be applied. No bad results need be feared from such an application. In many cases the pain will subside and the fever abate in the course of a few hours after the blister has commenced to act.

If any lameness and thickening of the joint threaten to remain, it may be necessary to apply the actual cautery.

In conclusion, it must be borne in mind that absolute repose of the part affected, if such a thing were possible in our practice, must be the first and greatest consideration in the treatment of the disease.

\section{CHRONIC SCROFULOUS SYNOVITIS.}

\section{(See Photo-lithograph, Plate II., Fig. 2.)}

This affection is, within my experience, confined to horned cattle, where the process of "in-and-in" breeding has been carried out to too great an extent. The joints most usually affected are the elbow and stifle, and the foot-joints in the disease called "foul in the foot." It attacks animals of all ages, but generally they are three years old and upwards.

At the outset, it is difficult of diagnosis, the symptoms being a lameness arising from no apparent cause, without swelling, or any indication of pain other than the lameness; but after a time a slight swelling may appear about the joint affected, without 
much heat, or pain upon pressure. The swelling gradually enlarges, and is of a doughy, elastic nature; the limb below it seems to waste; the animal begins to lose condition; the lameness and pain increase; and suppuration may take place; but this is by no means a uniform result.

In some cases constitutional symptoms of scrofula may precede the manifestation of the joint disease; when this is the case, the diagnosis will be rendered easy, and the practitioner need not hesitate to condemn the animal to slaughter.

Pathological Anatomy.-The structures around the joint will be found infiltrated in circumscribed spots with a yellowishwhite, caseous material, in which the Bacillus tuberculosis may occasionally be found, and the synovial membrane studded upon its surface with yellowish-white globular bodies, which, when cut into, present the same tubercular character. These bodies vary from the size of a turnip seed to that of a large pea.

The membrane itself is found of a greyish-white colour, and gelatinous in appearance, with its vessels in some parts highly injected; the synovial fluid curdy, and more opaque than natural; the cartilages of incrustation, as well as the ends of the bones removed by ulceration, causing the interior of the joint to present a worm-eaten appearance. In the interior of the bones, round spots of tubercular matter are seen in the cancelli.

In dissecting the various structures, it will be found that the skin, the subcutaneous tissues, and synovial membrane are matted together into an almost inseparable mass.

Hereditary predisposition is an acknowledged fact, and whenever scrofula in any of its forms makes its appearance in a herd, it will be high time to infuse new blood into it. Exceptional cases may, however, be induced by neglect or other debilitating influence.

The treatment can be but palliative; the preparation of the animal for the butcher being the only end to be gained. The application of blisters to the part will often remove the inflammation for a brief period, and thus allow the animal to put on fat.

The patient must be stall-fed, kept as quiet as possible; to have food of the most fattening nature; cod-liver oil may be given night and morning. If the disease has made much progress before the practitioner is called in, or if, in spite of the treatment recommended, it continue to advance, the animal had better be put out of its suffering. 


\section{CALCIFICATION OF THE SYNOVIAL FRINGES.}

In the dissection of old horses which have been stiff in their joints, the synovial fringes are often found in a state of calcification, the ossific or calcareous deposits varying from the size of a millet seed to that of a small bean. These occasionally become detached, and find their way into the interior of the articulation, causing sudden and violent lameness, which disappears as suddenly as it came, by the detached body regaining its original position. A condition similar to this has been termed fimbriated synovial membrane by pathologists. In the horse the stifle joint is that most commonly affected.

\section{RHEUMATOID ARTHRITIS.}

The condition of the synovial membrane described in the last paragraph is probably due to a peculiar inflammation, which has been termed rheumatic gout or chronic rheumatic arthritis-a disease differing from gout and rheumatism in many particulars, although possessing some characteristics common to both. This affection in man has been studied and described by Dr. Haygarth of Bath, by Cruveilhier, and more particularly by Dr. Adams of Dublin. 1 By Dr. Haygarth it was called "nodosity of the joints;" and by Cruveilhier, "usure des cartilages articulaires." To both of these terms Dr. Adams takes exception, and says-" The term suggested by Cruveilhier, it is plain, would localise the disease too much, confining it merely to one of the articular textures; whereas we know that, when fully formed, most, if not all, of these textures are implicated; and as to the term 'nodosity of the joints,' the swellings of the joints which we notice in this disease are by no means hard, as the term nodosity would imply. On the contrary, as in its early stages the swellings are principally constituted by the effusion of much synovial fluid into the interior of the joint, they are soft and fluctuating."

I am sure that every veterinary surgeon of experience, when he has read the following account, abbreviated from Dr. Adams' work, will at once see the great resemblance it bears to many cases he has met with in his practice.

"The disease may be a constitutional or a local disorder; the 1 A Treatise on Rheumatic Gout, or Chronic Rhcumatic Arthritis, all of the Joints. 
constitutional originating in sedentary pursuits, sudden exposure to cold when the body has been over-heated, or as a sequel to rheumatic fever; the local, from habitual over-exertion of a particular joint, or from accident."

Symptoms. - A singular rigidity in the affected joints, which, when first moved, emit a crackling sound. The crackling sensation, felt as well as heard in the human patient, and the rigidity of the limbs, followed by pains, are very considerable at the moment the patient commences to move about, particularly in the morning, after the repose of the night; and in some cases there are painful spasms of the muscles.

The veterinarian frequently finds both these symptoms, namely, the crackling noise and the painful and spasmodic uplifting of the limbs, especially the hind ones, in many of his patients, whose joints present the other symptoms of the disease in man - the swelling, pain, and tenderness to the touch.

The swelling in and around the affected joints, which is noticed in the earlier stages of the disease, arises principally from the increased effusion into the synovial sacs of the articulation, a result of the chronic synovitis which at this period exists. In the later stages of the affection, exostotic growths can be occasionally felt through the integuments.-(See Photolithograph, Plate II., Figs. 1, 3, and 5.) These spring from the margins of the articular surfaces, as well as from the periosteum and bone in the neighbourhood of the diseased joint. In the tibio-femoral articulation (stifle-joint) rims of new bones can be seen to range themselves along the margin of the condyles of the femur and tibia.-(See particularly Fig. 3 in the same plate.)

Articular rigidity, or false anchylosis, is not an unusual consequence of the disease; but true anchylosis, or the complete fusion together of two bones, is exceedingly rare; nor does the disease in its ordinary course proceed to suppuration.

The synovial bursæ in the neighbourhood of the affected joint are occasionally found distended, and synovial cysts become, as it were accidentally, developed in the areolar tissue surrounding the affected articulation, having no communication with the diseased joint. As the disease advances, the fluid contained in these cysts may become absorbed, and the sacs converted into solid tumours. 
Anatomical character of the Disease.-The capsules of the joint are thickened, and at the same time distended with a large quantity of synovial fluid. At a later stage the fluid is removed by absorption, and the capsular membranes acquire a preternatural density. Some of the normal structures, as the round ligament in the hip, and the semi-lunar cartilages in the stifle-joint, are completely absorbed, if the joint has been long and severely affected. The lateral ligaments of the ginglymoid joints become preternaturally elongated, and new growths, varying in consistence-some being cartilaginous throughout, others bony in their centres and cartilaginous at their circumference-are found in and around the affected joints. These are first developed in the synovial membrane, and are almost always attached to it by a slender cord, or by a broad base; and around the rim of the cotyloid and glemoid cavities additional foreign bodies-called by Adams "addimentary bones"-are usually found deepening and enlarging the "cavities of reception" for the heads and condyles of bones composing the articulations.-(See Photo-lithograph, Plate I., Fig. 2.) The articular cartilages are removed, and their place supplied by an ivory-like enamel-the porcellaneous deposit-in the hip and shoulder joints, the surfaces of the bones become as smooth, in whole or in part, as an ivory ball.-(See Photo-lithograph, Plate II., Fig. 1.) In the ginglymoid joints the place of the removed cartilage is supplied by means of patches of ivory or porcelain-like enamel, marked out by parallel grooves, hollowed out in the direction of the movements of extension and flexion.-(See Photolithograph, Plate II., Figs. 3 and 5.)

I think that I have produced sufficient evidence from the symptoms during life, and the appearances of the bones after death, to prove the similarity, if not the identity, of this disease with the rheumatic gont, or more properly the rheumatoid affection of the joints of the human being. What veterinary surgeon can have failed to notice the peculiar stiffness of the limbs, and the crackling noise emitted when the joints are moved in some cases of bog-spavin and navicular lameness? And no one, I am sure, is prepared to deny that the peculiar alterations of structure and the intractable nature of the morbid processes are not due to a diathesis or constitutional condition similar to that which in man predisposes to this malady. 
In support of this view of the pathology of this disease, I take the liberty of quoting some remarks made by Dr. G. W. Balfour of Edinburgh in a clinical lecture published in the Edinburgh Medical Journal, February 1870, page 713. He says-“'You may remember, at the close of my last lecture, that I exhibited to you the bones of a horse affected with rheumatoid arthritis. The case to which I then referred is still under treatment, though improved; and I purpose referring now more at large to the subject. I brought these horse bones the other day for several distinct reasons:-1st. To show you well-marked examples of eburnation on a larger scale than we ever have them in human joints; $2 d$. To show you that this eburnation, in the horse at least, is not confined to the surface of the bone-is not therefore produced, as has been supposed, by mere consolidation and polishing produced by friction, as you would polish a piece of hard wood or dead bone; for you remember, in the one bone, the os naviculare-a sesamoid bone, not exposed to friction or pressure at all-this eburnation had extended throughout the whole substance of the bone, showing that probably the process is an integral part of the disease, and does not depend on friction at all,-as, indeed, in living bones we could scarcely expect would be the case; and $3 d$. To show that horses, who live so very differently from man, both as regards food and drink, and who never have gout, are yet commonly affected by a disease which produces precisely the same pathological results as rheumatoid arthritis in man, thus affording additional proof that rheumatoid arthritis has nothing gouty in its nature; and showing that, to obtain anything like a proper and profitable pathological history of this disease, we must include the lower animals in our survey. As yet very little is known of rheumatoid arthritis, and yet it is of importance to know that little; because it is of the utmost consequence to recognise both the acute and chronic disease, the treatment being so dissimilar from that of the diseases with which they are apt to be confounded-chiefly rheumatism and gout-but which may also in the early stages include locomotor ataxy and Bright's disease, both of which are frequently associated with pains which might be mistaken for those of rheumatoid arthritis, an old disease, long known by those skilled, but little recognised generally by the profession. . . . . Rheumatoid arthritis appears to be a disease arising from exposure to cold and damp, but only in a 
person constitutionally predisposed by debility from some cause or other. But, truly, we know nothing certain in regard to this, and we require to have our knowledge of human pathology collated with that of the horse before we can form any rational theory on the subject. The blood, the urine, and the perspiration have all been analysed with merely negative results, and yet the morbid anatomy of the disease is perhaps the richest and the most extraordinary belonging to any complaint, the disease affecting, in its peculiar way, every bone and every joint in the body. In the early stage of the disease, there is found simply effusion of synovia, with vascular injections, signs of arthritis; at a later stage the fluid becomes absorbed, the capsular membrane thickened, and the cartilage ulcerated. At a very early period this cartilage splits up, and becomes removed apparently by a slow process of absorption, the whole surface of the joint being then denuded, the osseous surface becoming polished and eburnated, as in those specimens."

The treatment can, generally, only be palliative, and is chiefly constitutional. The administration of alkalies-particularly potash-is sometimes followed by amelioration of the symptoms. Salicylate of soda has also been given in some cases with marked beneficial results, but these are inconstant. The bowels should be regulated by an occasional purgative, the animal carefully fed, and put to slow work, kept in a dry stable, and when any sudden increase of lameness, denoting a fresh attack, occurs, fomentations, or perhaps moderate blisters, are to be prescribed.

\section{MORBID CONDITION OF CARTILAGE.}

Articular cartilage becomes diseased in consequence of synovitis, disease of the bone to which it adheres, or independently of any morbid condition of adjacent structures, and such disease may commence upon its free or its attached surface, or in the middle of its substance.

In violent inflammation of joints, as those arising from puncture, the destruction of the cartilage is accompanied, or even preceded, by the destructive absorption of the vascular extremity of the bone immediately contiguous to its articular laminal layer. In this manner we can explain the presence of floating pieces of cartilage and bone in the synovial fluid. 
Cartilage may become destroyed from disease arising within itself, independently of any morbid condition in the bones or synovial membrane; and to account for this, seeing that cartilage is a non-vascular structure, Goodsir, Redfern, and others made many important investigations.

The anatomist will be aware that cartilage in a healthy state, when microscopically examined, is seen to consist of a homogeneous matrix, called the hyaline substance, mixed with fibrous tissue in fibro-cartilage, and nucleated cells, called the cartilage corpuscles, embedded in its substance, arranged in a certain order. Cartilage contains no blood-vessels, but derives its nourishment by imbibition from the surrounding fluids and textures. In the large joints, blood-vessels may occasionally be seen penetrating the cartilage, but these do not seem to form nutrient loops; hence, to all intents and purposes, articular cartilage contaius no blood-

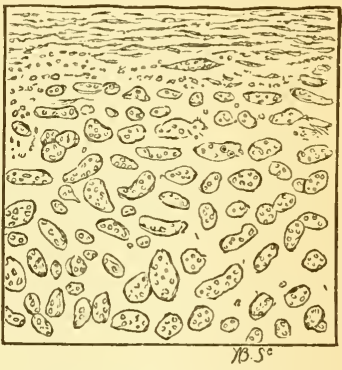

FIG. 36.-Healthy cartilage. vessels, nor have any nerves been discovered in it; consequently, it possesses no sensibility.

It was first demonstrated by Goodsir that ulceration of eartilage is accompanied by enlargement and alteration in the form and arrangement of the cells. "Instead of being of their usual form, they are larger, rounded, or oviform, and instead of two or three nucleated cells in their interior, contain a mass of them. At the very edge of the ulcerated cartilage, the cellular contents communicate with a diseased membrane by openings more or less extended. Some of the ovoidal masses in the enlarged corpuscles may be seen half released from their cavities by the removal of the cartilage." 1 Goodsir describes a false membrane of a gelatinous nature, which covers the cartilage during the progress of the disease, both in scrofulous and simple inflammation. As far as my investigations go, I have only found this membrane in two instances.

The texture of the cartilage does not, during the progress

1 Goodsin's Anatomical and Pathological Observations. 
of the ulceration, exhibit any traces of vascularity, and the membrane, which was supposed by Goodsir to be the cause of the ulceration, is regarded as the result of the disease. The formation of this false membrane, which is sometimes on the bone, sometimes on the attached surface of the cartilage, has conveyed the erroneous impression, entertained by some observers, that in one form of destruction of articular cartilages, the destruction is preceded by the formation of vessels in the substance of the cartilage itself. It is quite certain that in nearly all instances of destruction of the articular cartilages, in the lower animals at least, there have been extensive ulcerations without the presence of any vascular membrane; and in one instance that fell under my notice, a false membrane was

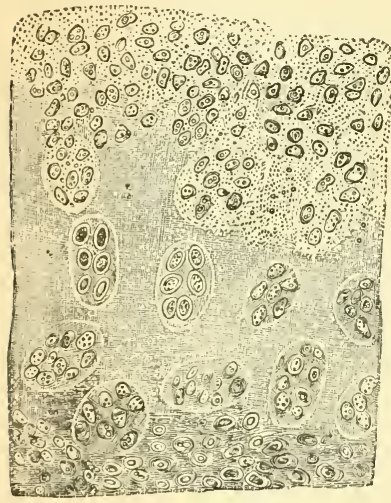

Fig. 37.

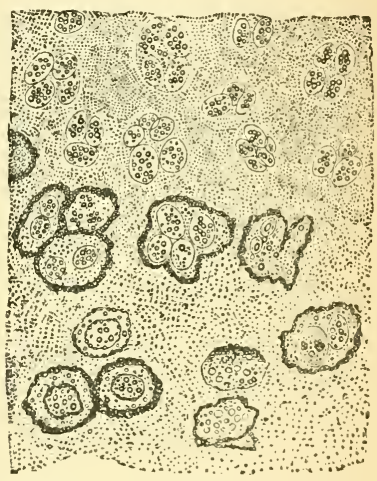

FIG. 33.

FIG. 37.-Diseased articular cartilage, showing the enlargement of the corpuscles, the increase of the nuclei within them, and their escape into the softened matrix.-(REDFERN.)

Fig. 38.-Deepest part of a vertical section from the cuneiform surface of the semi-lunar bone, showing the cells, with thickened walls.-(REDFERN.)

found covering the articular surface of the astragalus, the cartilage of which presented no signs of ulceration; whilst the tibial cartilage of the same joint was ulcerated without being covered by any adventitious membrane.

The further changes are the bursting of the enlarged cells at the surface of the cartilage, which by this change becomes disintegrated. In some cases, the nuclei which have escaped from the cells become elongated, and incorporated with the 
hyaline substance, giving it unusual softness, and causing it to swell, fibrillate, and split into fibrous bands projecting into the joint. In other cases, the nuclei degenerate into fatty material

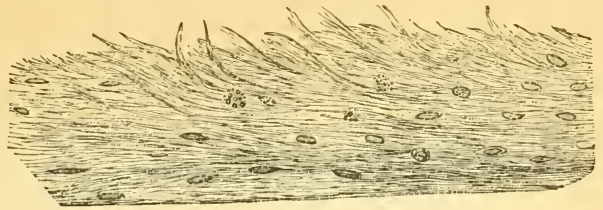

FIG. 39.

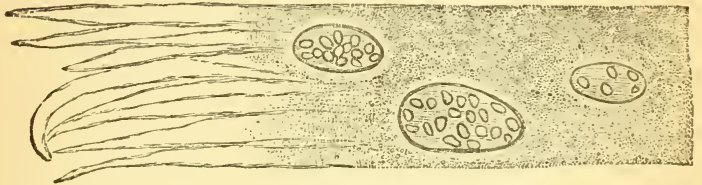

FIG. 40 .

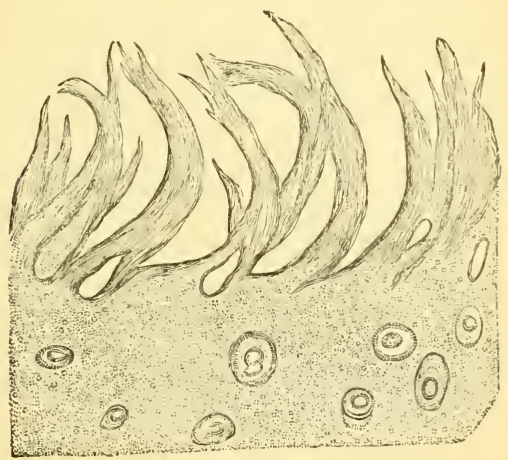

FIG. 41.

Fig. 39. - Cartilage from diseased human patella, showing on the surface fibrous tissue with imbedded cells and nuclei.-(REDFERN.) This figure represents very exactly the commencement of the fibrillation and splitting up of the cartilage, lining the inferior surface of the navicular bone in navicular disease, before adhesion has taken place, between the bone and tendon.

Fig. 40 shows a more complete formation of bands and filres upon the surface of the cartilage. -(REDFEnN.)

Fig. 41.-Vertical section from the cartilage of the central part of the glenoid cavity of the (human) tibia, showing the splitting into fibres on the surface.-(REDFERN.) This appearance of the cartilage represents very fairly the condition of the navicular cartilage when the bone and tendon have become adherent. 
within the cartilage corpuscles. Another change is the infiltration of the cartilage with the salts of lime, this change commencing in the cell wall. This may often be seen in the cartilage of the navicular bone. The alteration in the hyaline substance consists in its losing its glistening appearance, and being split up into shreds of fibres projecting into the joint.

Dr. Redfern has arrived at the following conclusions from his inquiries into this subject:-

1st. That all the known forms of disease in articular cartilage are connected with changes in the texture, which are essentially similar to each other.

$2 d$. That during the progress of these changes the cells of the cartilage become enlarged, rounded, and filled with corpuscles, in lieu of healthy cells; bursting subsequently, and discharging the contents into the texture on the surface; whilst the hyaline substance splits into bands and fibres, and the changed hyaline substance, and the discharged corpuscles of the cells afterwards form, in many cases, a fibro-nucleated membrane on the surface of the diseased cartilage.

$3 d$. That these changes are referable only to an abnormal nutrition as their immediate cause, and in no case to mechanical or chemical actions, such as attrition, or digestion in a diseased secretion.

4th. That most extensive disease may go on in many joints at the same time, and may proceed to destroy the whole thickness of the cartilage in particular parts, without the patient's knowledge, and while he is engaged in active occupation.

$5 t h$. That the disease commences most frequently on the free surface, but may proceed from the bone to affect the attached surface, or may take place in the middle of the thickness of the cartilage.

$6 t h$. That it is at least very doubtful if the symptoms which are believed to indicate the existence of ulceration of articular cartilages are not really dependent on a morbid change in the bone.

$7 t h$. That disease of the whole thickness of an articular cartilage at particular parts admits of a natural cure, by the formation of a fibro-nucleated membrane from the substance of the cartilage, without the occurrence of any new exudation.

When cartilage is destroyed, it is never reproduced. If the 
destruction be superficial, the breach may be repaired by the formation of the fibro-nucleated membrane. In such cases the affected part will present a velvety appearance. If the synovial membrane be involved, the fibro-nucleated membrane may be incorporated with an exudation from the synovial surface.

Dr. Redfern, in his researches On the Healing of Wounds in Articular Cartilages, has satisfactorily demonstrated that the repair of cartilage is always a very slow process. In one experiment, in which he had made three incisions into the cartilage of a patella, and two into that of the trochlear surface of the femur of a dog, he found that no union had taken place in twenty-nine weeks, but a reparative process had just commenced. In another case, similar incisions were firmly united by fibrous tissue in twenty-four weeks and five days.

The appearance of the parts, upon examination by Dr. Redfern at the end of this time, I beg to give in his own words :-

"A slightly increased quantity of synovia exists in the joint; the patella rests by the external half of its articular surface on the inner side of the internal condyle of the femur, and the part of the fibrous capsule of the joint which lies upon the trochlear surface of the femur, and glides over it, presents a dense white and smooth spot, similar in appearance to fibro-cartilage, and of exactly the same size and shape as the trochlea. The edges of the trochlear surface of the femur, and the vertical ridge on the cartilage of the patella, are less prominent than usual, and rounded. The cartilage of the femur appears perfectly healthy, and that of the tibia somewhat softer than natural, and the cartilage of the patella is more transparent, and has a small perforation in its centre leading down to the bone. The position of the incisions is difficult to see on the patella, and is only marked by the slightest curvilinear depressions; on the femur, one incision is recognised with the greatest difficulty, and the position of the other cannot be seen with the naked eye.

" On microscopical examination, the superficial cells over a considerable extent of the surface of the cartilage of the patella, and especially in the neighbourhood of the central depression, have become much enlarged, many measuring $\frac{1}{1000}$ of an inch in diameter; they are nearly spherical, and their contents appear to have divided into three or four masses like nuclei; they are lodged in hyaline substance of greater transparency and much 
greater softness than ordinary, so that the cells can be pressed out of it.

"In the neighbourhood of the incisions into the cartilage of the patella, and upon the trochlear surface of the femur, though not extending upon the condyloid surfaces, the substance of the superficial layers of the cartilage has become converted into a membrane composed of granules, nuclei, and fibres. The formation of this membrane may readily be traced by an examination of it at the line of junction of the trochlear and condyloid surfaces, where it becomes thin before it ceases to be observed. The contents of the cells first become granular, and the hyaline substance soft and more transparent; the walls of the cells disappear gradually, and scarcely anything but cell membranes, granules, and molecules, lying in a hyaline mass, can be seen; fibres are then formed between the nuclei, and the latter either disappear altogether or elongate into nuclear fibres, and thus a dense fibrous mass is produced. If during this process the cells have become larger than natural, they give to the junction of the cartilage and membrane a notched appearance, by bursting and discharging their contents into the latter; but if no enlargement has taken place, the textures run into each other so insensibly that no distinct line of demarcation can be drawn between them.

"The portions of the texture through which incisions were made present similar appearances in every instance. Not the slightest difficulty is experienced in making sections through both the cut surfaces and the substance by which they are firmly united. Such sections show the cut surfaces to be very uneven, and hollowed into small pits of the size of the cartilage cells of these parts. The pits are obviously produced by the half-destroyed cells, the former contents of which are now seen lying on the surface. No evident change has taken place in the texture of the cartilage at a little distance from the cut surfaces, except that here and there the intercellular substance presents a fibrous appearance. There is no obvious enlargement of the cells, or crowding of their interior with corpuscles, as is frequently seen to occur under similar circumstances. The substance uniting the cut surfaces consists of a hyaline, granular, and indistinctly striated mass, in which there are numbers of rounded, oblong, elongating, or irregularly shaped 
corpuscles, varying in size from $\frac{1}{5000}$ to $\frac{1}{2300}$ of an inch in diameter, those in the adjacent cartilage measuring more uniformly about $\frac{1}{3000}$ of an inch. The corpuscles are more numerous in the substance connecting the cut surfaces than in the cartilage itself, and the intercellular substance of the same part is slightly more transparent than that of the adjacent texture, the appearance of fibres being most distinct in those parts which are directly in contact with the cartilaginous surfaces.-(Fig. 42.) In sections obtained from the cartilage of the femoral trochlea the mass between the cut surfaces is less transparent, its fibres are much more perfect, and the corpuscles are smaller and less easily seen.-(Fig. 43.) The fibrous and nucleated membrane formed on the surface of the cartilage is continuous with the uniting medium, and differs from it merely in having its fibres parallel to the surface.

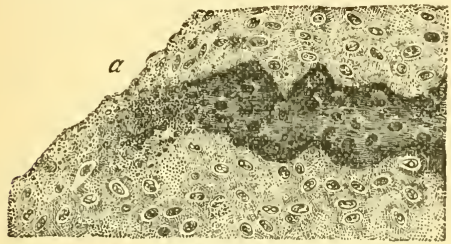

Fig. 42.

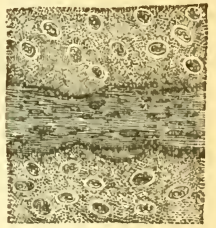

FIG. 43 .

Frg. 42.- Vertical section of the cartilage of the patella of a dog passing through an oblique incision made twenty-four weeks and five days before examination, and showing perfect and firm union of the cut surfaces.(REDFERN.)

Fig. 43. - Section of the cartilage of the femur of a dog passing through an oblique incision made twenty-four weeks and five days before examination, and showing the perfect fibrous tissue developed in the healing of the wound.-(REDFERN. )

"It may be here remarked, that the lameness which occurred in this case is by no means to be viewed as the result of the injury to the cartilages; for except when dislocation, or some other accidental occurrence, takes place, incisions in the articular cartilages of the knee-joints of dogs are attended with no lameness or evidence of suffering whatever, after the first few days, which are required for the healing of the external wound. Neither is it to be supposed that articular cartilages are in a favourable position for the healing of wounds; for, independently of the continued movement, which is no less detri- 
mental here than in the healing of ordinary wounds, many actions which take place in these textures proceed very slowly, and therefore are in greater danger of being interfered with in their progress. In an instance in which I made three incisions into the cartilage of the patella, and two into that of the trochlear surface of the femur, no adhesion had taken place in any of the instances when the parts were examined twentynine weeks afterwards, though no inflammation of the joint, dislocation, lameness, or other apparent cause for the want of union had occurred. Yet some of the cut surfaces were in such close contact, before the parts were examined by means of sections, as to lead to the supposition that union had taken place. On the examination of these wounds, no effused matter of any kind appeared on the perfectly smooth cut surfaces; but as the cells near to them in the substance of the cartilage were obviously enlarged and rounded, having in their interior three or four corpuscles into which their nucleus appeared to have divided, or a number of bright granules, there appears reason to believe that union might still have cccurred had more time been allowed.

"After the foregoing observations, I no longer entertain the slightest doubt that wounds in articular cartilages are capable of perfect union by the formation of fibrous tissue out of the texture of the cut surfaces. The essential parts of the process appear to be the softening of the intercellular substance of the cartilage, the release of the nuclei of its cells, the formation of white fibrous tissue from the softened intercellular substance, and of nuclear fibres, by the elongation of the free nuclei. It does not appear necessary that the cells should become much enlarged or crowded with corpuscles, for the union may take place without any enlargement of the cells, or increase in the number of their corpuscular contents ; these changes, therefore, though they contribute materially in some cases to the rapidity of completion of the process under consideration, are clearly not essential in any of its stages."

When the destructive process has removed the whole depth of the cartilage to the bone, and when the laminal layer has been removed, the breach is repaired by an exudation from the vessels of the bone, which, becoming converted into bony matter, occupies the affected part. 
When none of these results have taken place, the process of destruction is arrested and limited by a deposition into the bone of a peculiarly hard, calcareous material-the porcellaneous deposit-which fills up the cavity, and blocks the cancelli and canals of the bone, and by its smooth and polished surface makes up to some extent for the want of the cartilage.

In old horses the cartilage of incrustation is exceedingly thin, and in some cases it will be found converted by ossification into the above-mentioned ivory or porcellaneous deposit. This deposit is exceedingly dense, and derives its hardness from the Haversian canals being filled up by additional laminæ.

The depressions or cavities termed sulci, which are found in many close-fitting joints, as the elbow and true hock joints, must not be mistaken for a diseased condition. They are merely cavities for storing synovia.

TRAUMATIC INFLAMMATION OF JOINTS.

An open joint, when occasioned by puncture or incision, is not at first (if unassociated with fracture) attended by severe local or constitutional disturbance; but at the end of a period varying from two to ten days pain comes on, and spreads over the joint, which soon presents a considerable amount of swelling and tension. The swelling is at first tense, but elastic; however, it soon becomes hard and unyielding, and accompanied by great constitutional disturbance, the pulse rising in frequency, becoming hard and wiry in its character, and the animal evincing acute and agonising pain by partial tremors and sweats upon his body. In fact, all the constitutional symptoms indicate a state of great irritability. The lameness is excessive. The animal is scarcely able to put its foot to the ground, whilst at the same time the pain causes it to keep it in an almost continual state of motion. An injury not at first penetrating the joint may do so in the course of three or four days, by sloughing of the tissues around it, these having been destroyed but not removed by the violence of the injury.

The discharge of synovia may be very trifling for some days after the accident; but it gradually increases as the inflammation advances, is thin in its consistency, and mixed with flakes 
of lymph; coagulates upon the lips of the wound, and oozing through this there will be a thin watery discharge. There is exudation of a large quantity of lymph into the tissues surrounding the joint, which becomes partly organized, forming a hard. firm swelling. The secretion from the wound is now unhealthy, purulent, or tinged with blood, whilst abscesses begin to form around the articulation.

The fever and debility increase from day to day if the local symptoms be not arrested; and finally the animal dies from the irritation.

Condition of the Part.-From the admission of air into the synovial cavity, inflammation of the synovial membrane is excited; this extends into the extremities of the bones constituting the articulation; their cancelli become turgid, the Haversian canals enlarged; the tissues contained within them form a disorganized mass, infiltrated with ichor; absorption of the termination of the vascular part of the bone immediately contiguous to the non-vascular laminal layer ensues, by which it is detached (along with the cartilage covering it) from the extremity of the bone, and may be found floating in thin masses in the synovia within the joint. This process goes on simultaneously at the extremities of both the bones of the articulation, thus exposing their vascular interiors, from which loops or spongy granulations spring up luxuriantly. These unite with each other, and form vascular communications from the cancellated structure of one bone to that of the other, this being the first step in the process of internal anchylosis. From the blood-vessels of these new formations an exudate is formed, which is finally converted into bony matter.

These granulations easily bleed, and when blood is seen in the synovial discharge, the veterinarian will understand that the laminal extremities of the bones have been removed, that their vascular interior is exposed, and that, if the joint affected be one of extensive motion, it will be useless to keep the animal longer in its misery.

Treatment.-The treatment of open joint, to be successful, must have two objects in view:-1st. To promote the healing of the wound by the first intention, or by the adhesive process. $2 d$. To prevent inflammation. For these purposes, both local and general measures are required, the local being the 
more important. It includes the apposition of the lips of the wound by pinning, or by suture, which must be either metallic or catgut. If there are any foreign bodies, such as dirt, gravel, or portions of disintegrated tissue in the wound, they must be carefully removed before its lips are brought together. To support the pin or suture, and to prevent the admission of air and germs into the wound, a thin paste made of spirit varnish and iodoform must be applied, by being painted on in successive layers with a camel's-hair pencil. The next thing to be done is to place the animal in slings as soon as possible. I consider this essential to the successful treatment of open joint, as it places the patient in the most favourable position for repose, and by preventing him from making even the attempt to lie down, does away with the danger of reopening the wound. All other local applications, by interfering with the healing process in the wound, are at this stage calculated to do harm.

Wounds upon or near articulations should never be meddled with, by any probing, for the purpose of discovering if there be fracture of the bones. If fracture exist, the lameness will be excessive from the first. A meddlesome interference with the probe has often caused open joint, when the original injury had not penetrated the synovial membrane.

The constitutional treatment must be that calculated to lessen pain and irritation; a small purgative, combined with opium, to be followed at intervals of four to six hours by doses of opium or of aconite; and enemas of warm water, two or three times a day, will be beneficial, unloading the rectum, and enabling the animal to pass fæces without straining.

If after the wound heal by these measures the inflammation of the joint continue, as in all probability it will, cold must be applied; and the best method of doing this is by irrigationthat is, by allowing a continual stream of cold water to trickle over the surface of the joint. This is easily done by attaching a gutta-percha pipe to a tap, fastening the pipe to a convenient part of the slings, and carrying its free extremity on to the lame limb, and fixing it above the inflamed joint by means of a bandage.

If this plan of treatment does not seem to succeed, a blister must be used. 
Such, then, is the treatment when the lips of the wound admit of being brought into apposition, and when the practitioner has been called in before the advent of suppuration. If it be a lacerated wound, its lips must be brought together as well as possible, and retained in that position by the application of the styptic colloid.

If these means are ineffective, if the injury has been inflicted for a longer period than a few hours, when inflammation is already established, and pus has commenced to be formed to plug up the wound at this stage would only cause the fluid accumlated within the capsule of the joint to burst out at some other spot, but even in this stage much can be done by irrigating the joint and surrounding structures with a solution of corrosive sublimate-1 to 500 parts of water-in order to destroy all germs which may have gained entrance, and then by covering the external wound with several layers of iodoform and varnish paste. If the discharges be profuse they must be allowed to escape, but much can be done to prevent the admission of atmospheric germs by the careful adjustment of boracic lint and light antiseptic bandages, through which the discharges will escape. If the discharges diminish and the bandages cause no inconvenience or irritation, they should not be removed until the cure has been completed. Every wound which has commenced to suppurate must heal by granulations; and the more perfect formation of these goes on in the deeper-seated parts of the wound-that is to say, that the healing is from within outwards. The plugging of the outer orifice while pus is being formed causes it to accumulate in the joint and surrounding structures, adds to the suffering of the animal, and in too many cases causes its death.

An extended experience warrants me in recommending the application of a blister to the whole surface of the joint, as the most successful treatment that can be adopted if the wound has failed to heal by the primary or adhesive process. The blister acts by removing pain, limiting motion, exciting the formation of healthy granulations, and (as a result of the swelling it produces) causing the approximation of the surfaces of the wound.

The coagulum of synovia which accumulates upon the wound should never be removed, as it prevents the admission of air and of germs into the joint, and thus limits the formation of pus. 
Many cases when so treated make good recoveries; but if ulceration of the cartilage and removal of the laminal ends of the bones occur (and this change will be indicated by hæmorrhage, or by increased pain and twitching movements of the limb), and if the articulation be one of extensive motion, the attendant will understand that the repair can only be by anchylosis, and that anchylosis in such a joint will render the animal unfit for further use; but if the joint be one of limited motion, the animal may become fit for slow work, even after the joint has been destroyed. The animal's shoes should be removed, provided this be done carefully, and before great lameness has manifested itself. If one of the lower articulations, particularly of the fore extremities, be the seat of the lesion, the animal can be made to stand in a tub of cold water, and the trouble of affixing the pipe for the purpose of irrigation be avoided.

All cases of open joint require a long period of rest after the wound has healed; and it is generally necessary to blister repeatedly, or even to fire, before the remains of the inflammation excited in the bones and synovial membrane are finally removed.

Several abscesses, some mere points of pus, others of a greater size, form in the exudate which has been formed outside of the cavity of the articulation during the progress of the disease. It may be considered necessary to open them surgically, if they cause increased pain. It may be well to do so; but if they are mere accumulations without pain, the cautious surgeon will not interfere, rather allowing them to burst spontaneously.

The horse should be kept in the slings until he is able to bear a moderate amount of weight upon the affected limb.

During the early period of the disease, the food must be spare, light, and cooling; but when the fever has abated, it must be of the most nourishing kind, in order to compensate for the great waste of tissue and emaciation which are so characteristic of open joint.

\section{DROPSY OF THE JOINTS, OR HYDROPS ARTICULORUM,}

Is a morbid condition, with or without inflammation, as in bogspavin, and its analogues termed wind-galls. In this affection the synovia is more abundant and serous than is natural, and 
distends whole joints or bursæ. There is seldom much pain or lameness, because the ligamentous and other textures have been yielding gradually, and are but passively distended by the fluid. The quantity of the secretion is liable to variations, disappearing more or less upon exercise, and accumulating after a short rest. During long periods of rest, however, the accumulations may disappear, and appear again when the animal is put to work.

Young growing cart-horses are very subject to the formation of these fluid enlargements, more especially about the hocks. When they are full grown, however, their joints may become quite fine.

The treatment most appropriate is the application of blisters and pressure; but this will be again referred to under the head of LAMENESS.

ANCHYLOSIS, OR THE STIFFENING OF THE JOINTS.

There are several forms of anchylosis:-1st. Osseous, or true; $2 d$. Fibrous; $3 d$. Ligamentous ; $4 t h$. Spurious.

1st. The true anchylosis may be general and complete at every part of the joint, as in some bone-spavins and true ring-bones, where all trace of the articulation has been lost, and the opposing bones so firmly united as to appear as one bone. Before this can happen, the articular cartilages and laminal layers of the opposing bones must be removed by an ulcerative absorption, and an exudate thrown out from their cancellated structure; which, becoming organized into bony matter, cements the bones together. This is the natural termination and cure of such causes of lameness, and it is usually accompanied by a deposition of bone upon the outside of the affected joint, which aids in the completion of the anchylosis.

$2 d$. The fibrous.-The opposing surface of the bones may be united by fibrous tissue. As a rule, however, this is only found during the earlier stages of true anchylosis.

$3 d$. The ligaments become rigid to such an extent as to make a joint more or less stiff and immoveable, whilst the articular surfaces of the bones remain healthy. Stiff joints of old horses are examples of this.

4th. The spurious is that which depends on deposition in textures external to the joint, uniting the various structures to each 
other. This form is always present to some extent after acute inflammation of a joint; and appears around the lower joints of a limb which is mal-formed by contraction of the flexor tendon, or rupture of the suspensory ligament, as shown in Photo-lithograph, Plate III., Fig. 6. This form can be removed by forcible extension of the articulation, after division of the contracted tendon. It is also present to some extent after open joint successfully treated, and disappears gradually by absorption when the joint is brought into use. Its removal may be accelerated by repeated applications of iodine to the skin. 
ANCHYLOSIS, OR THE STIFFENING OF THE JOLNTS.

EXPLANATION OF PHOTO-LITHOGRAPH, PLATE III.

1. Anchylosis of Shoulder-Joint.

2. Incipient Anchylosis of Elbow-Joint, showing removal of cartilage and laminal layer of the bone, on extremity of humerus.

3. 4. and 5. show Anchylosis of the Carpal, and First and Second Phalangeal Articulations.

6. Anchylosis and Destruction of all the Phalangeal ArticulaTIONs in an old-standing case of "Break-down." 

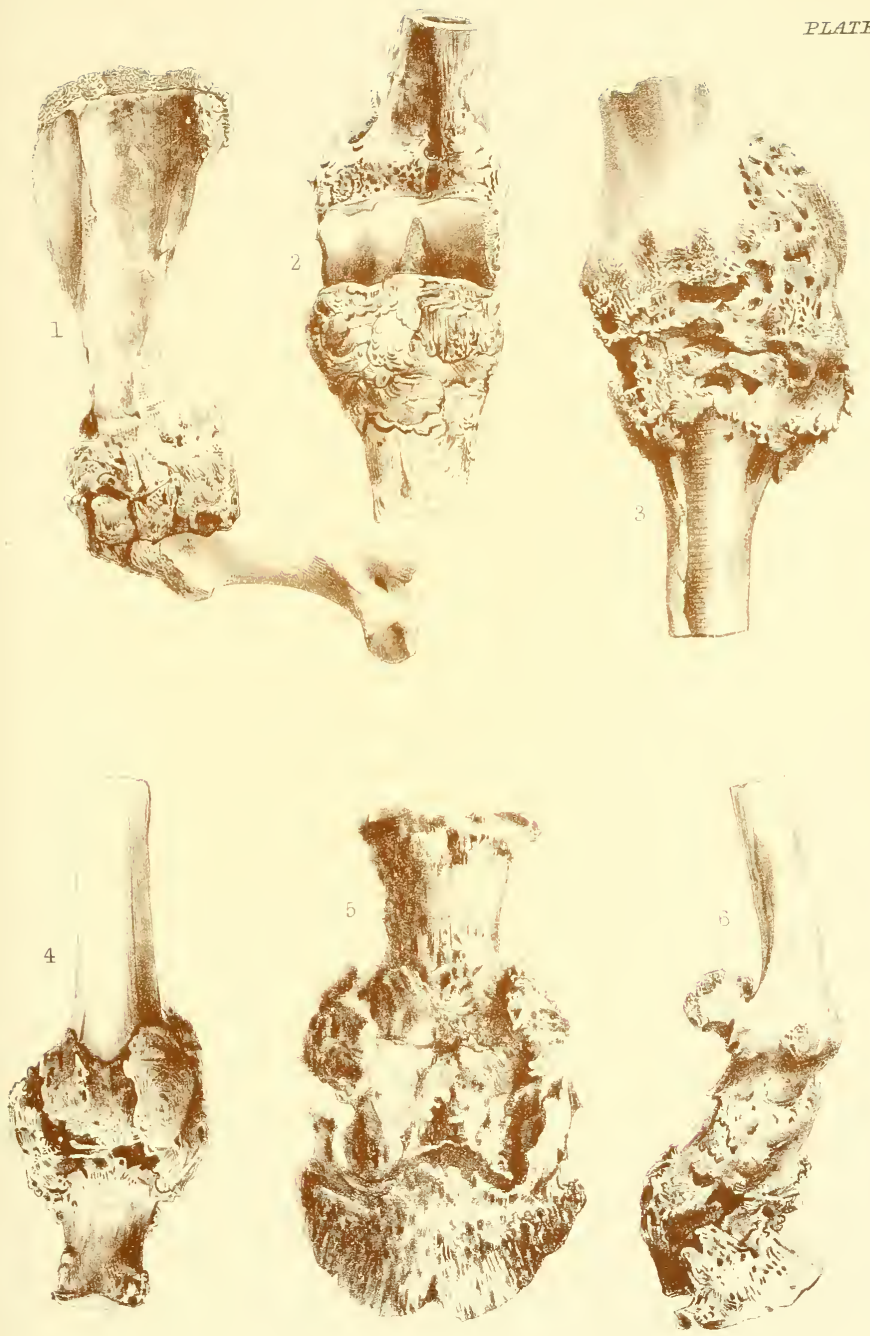



\title{
CHAPTER XII.
}

\author{
LAMENESS.
}

DIAGNOSIS - DEFINITION - SIGNS - CAUSES - CONGENITAL MUSCULAR ATONY - EMBOLI-METHODS OF EXAMINATION-SPRAINS-STRAINS OF PSOE MUSCLES-CHOREA-STRINGHALT-SHIVERING.

Having entered into the pathology of the Diseases of Bones and Articulations, I shall now consider the question which may be looked upon as being the one to which the greatest importance may be attached, namely, that of Lameness.

Diagnosis of Lameness. - The readiness with which some men are able to detect lameness seems to be an instinctive gift. Of such was Professor Dick, who could, at a glance, even when a horse had been moved but a few yards, tell the seat and the cause from which he was lame. But whilst this is so easy a matter to some, to others it is a task of great difficulty, requiring long-continued practical study and observation. There are, however, cases where the most experienced are at a loss, and where even the most skilful differ in opinion. To the young man entering upon the duties of his profession, this matter is apt to cause many anxious thoughts and uneasy moments, as a mistake at this period may interfere very materially with his success in life. To such I would say, Never express a decided opinion until you are thoroughly satisfied as to its correctness.

The first point to be determined is the limb in which the patient is lame. This may seem an easy matter, but in reality it is attended with no little difficulty. Thus, a mistake may be made by expressing an opinion that the lameness is in the hind leg, when in reality it is in the fore, and vice versa. This 
error has been so often committed, that the peculiarity of gait which has led to it has been called "cross-lameness." For example, a horse lame in the off fore leg is trotted from the observer; he seems as if he were lame in the near hind, for the quarter seems to ascend and descend. But when the animal is trotted towards the observer, it will be seen that the irregular motion of the hind quarters depends upon the elevation and dropping of the head and body; and that the lameness in reality is in the fore, and not the hind limb. An opinion must not be given before the horse has been trotted from and towards the observer. Of course there are many cases where lameness is so apparent that such an examination is quite uncalled for; at the samo time, there are many other cases so slight in degree as to require the most rigid scrutiny.

The next difficulty is the detection of lameness when it is situated in both fore feet. When such is the case, an animal may seem to go as if sound. Advantage has been taken of this by low horse-dealers, who, when they have a horse lame in one fore foot, make him lame in the other also. This is by them technically termed "Beaning," and consists in placing a small piece of iron tightly under the shoe of the sound foot, which produces so much pain as to cause lameness. A more refined method has latterly come into vogue, that of paring the toe of the sound foot nearly to the quick, and so adapting the shoe as to press upon the weakened spot.

A horse lame in both feet, although he may not drop in his gait, will be short in action; will go, as it has been more forcibly than elegantly expressed, "like a cat on hot bricks." Each foot is carefully put to the ground, and quickly lifted up again, while at the same time there is a rolling motion of the body. In other cases he may exhibit the lameness in one foot as he goes from, and in the other as he approaches the observer. Such cases are very confusing, and require all the veterinary surgeon's discriminative powers. Care must be taken not to confound peculiarity of action with lameness. For example, a horse, especially a young one, may appear lame in the near fore foot, if led with a short rein, and his head pulled to one side, or when he is first bitted. This is called " bridle lameness ;" it disappears when the animal is run in a slack rein. Again, horses which are habitually exercised in a ring, or 
round a circle, invariably seem lame upon the fore limb nearest the centre of the ring. When the lameness is in both hind limbs, the difficulty of diagnosis is not so great, as the animal is both stiff and lame; but mere stiffness must be distinguished from lameness, although it is often confounded with it. No doubt a stiff horse is an unsound horse; but he may be very useful for slow work, and at a suitable price. Mere stiffness may indicate age or fatigue, and often passes away after a little exercise; but lameness is indicative of actual pain or disease, and although it may pass off with exereise, still it must not be confounded with stiffness.

There are some forms of lameness which are apparent in the stable only, the movements caused by bringing the patients out of the stable being sufficient to produce the total disappearance of the lameness. Such being the case, the examiner should see the horse in the stable as well as out of it.

The manifestation of lameness by the animal is shown in two ways:-1st. During repose; $2 d$. During movement. In some cases, the appearance, or expression, is much more palpable while the animal is standing still, as in many foot lamenesses. For example, a horse will continually point, or even elevate, the foot which is suffering pain; if both feet, each foot alternately will be pointed or elevated. But if he is made to move, the extent of the lameness does not seem equivalent to the amount of pain so expressed. In other cases, the patient will stand perfectly firm, although in the great majority of cases the pastern of the lame limb is more upright than that of the sound one, as if he feared to put as much weight upon it; but when made to move, he will immediately exhibit the lameness. Again, many cases are seen in practice where the patients come out of the stable sound, but when they have performed some work or exercise, lameness becomes manifest. Others, again, leave the stable very lame, and become freer in their action when they have been warmed with exercise. Such cases are apt to deceive; therefore the veterinary surgeon should take every precaution against being taken unawares. Some horses exhibit their lameness when they "turn round." They may go well enough if led straight to or from the observer; but when sharply turned round, they at once manifest their unsoundness; and when an examiner as to soundness finds a man turn his 
horse " pel him to be turned quickly. Slight chorea or stringhalt is seldom detected except during the turn, and I have seen cases where it was manifested when the animal was turned one way only.

In other cases lameness may exist in two or more limbs, but not equally; and when so complicated, the animal may endeavour to save the lame limbs by throwing his weight from them in a manner so peculiar that it requires great care to distinguish the true nature of the case, and to form a correct opinion.

Again, there are some horses which walk down hill in so peculiar a manner, that they may be supposed to be lame. This kind of walk has been termed a "three-cornered walk." The animal sways from side to side most awkwardly; his hind quarters being turned to the one side or the other, going forwards broadside-on, similar to an animal going down hill with a heavy load behind him. Young horses, when being broken-in, should be corrected of this fault, as it is most unsightly; and to see a rider on a horse of this kind reminds one very forcibly of Don Quixote and Rosinante.

A touch of the whip or spur will cause the horse to improve lis paces, and at once show that it is not lameness, but laziness, from which he is suffering.

The signs of lameness manifested during repose are very important, and sometimes diagnostic. A horse suffering acute pain in one of his legs will, if it be a fore limb, point the foot: by pointing is meant the extension of one limb in advance of its fellow. This is done for the purpose of relieving pain, and is performed by throwing the flexors into a state of relaxation, and removing any tension or pressure from the painfu] part. This pointing does not apply to every lameness in a fore limb, nor particularly to any cause of it, for in some rare instances a horse may be lame in both fore feet from chronic navicular disease, yet never point.

The pointing of elbow lameness is characteristic, the fore arm being extended, the knee in a state of flexion, and the foot perhaps upon a level with or posterior to its fellow. In severe shoulder-lameness, the pointing, if it can be called such, is backwards, the limb relaxed, the knee bent, and the foot pos- 
terior to its fellow ; sometimes the toe only touching the ground; the whole of the limb semi-pendulous, consequent upon the inability of the muscles to elevate and bring it forward without pain; as a man fails to lift his arm when his shoulder is injured or diseased.

If the lameness be in a hind limb, the patient may stand with it either flexed, "knuckling over" at the fetlock, or with the foot off the ground altogether. When he stands with his lame (hind) leg in advance of the sound one, the position generally indicates disease in or below the hock.

A horse with acute pain in the fore feet will stand with his hind ones advanced beneath the body, resting first one fore foot and then the other, and every time he moves will rear up his head, and stretch out his neck in expression of the pain he suffers; but should the pain be in both hind feet, he will stand with his fore feet beneath his chest and towards the central line of gravity; his body pushed forwards, and head hung down, in order to remove the weight as far as possible from the seat of pain. He will first ease one hind foot, and then the other, breathing heavily, and showing other signs of acute pain. Pain in both hind feet often interferes with the act of urination, by preventing that stretching of the body which is so characteristic of that act in the horse. In such cases the poor patient will endeavour to stretch himself, will elevate the tail, but with a groan quickly assume his former posture, and suddenly "pick up" the feet alternately. From this fact it is often supposed that a horse suffering acute pain in the hind feet has some disease of the urinary organs.

The practitioner having satisfied himself which leg an animal is lame in, he must now endeavour to find out the seat of its cause. The late Professor Dick taught us to cause the shoe to be removed, and the foot examined in every case of lameness. This is a good rule to be followed, especially by young practitioners. He used to relate a case (showing the importance of this rule) where the os suffraginis was fractured. His great skill in lameness led him to think, upon examination, that the fracture was not the only cause of the lameness; he had the shoe removed, and discovered a wound from a nail in the foot, containing purulent matter. The fact of the horse being injured in the foot explained the cause of the fracture, - the animal, on 
account of the pain, having trod unevenly, prevented the equal distribution of his weight, and caused the fracture. The Professor had the foot attended to, as well as the fracture, and the patient made a good recovery. Now, had he been satisfied, as the majority of men would have been, that the fracture was the only cause, the purulent matter confined in the foot would have forced its way out at the coronet, a high degree of fever would have been excited, the case much complicated, and the Professor accused of mal-treatment.

After duly examining the foot, and being satisfied that the lameness is not there, the practitioner must endeavour to find out where it is ; but before considering the various lamenesses in detail, we must refer to the definition of the word and the variety of its causes.

The word lame, according to Percivall, is from the AngloSaxon lam, weak; the terms lame and weak are synonymous in some parts of England; thus it is a common expression to hear, "that is a lame story," for a weak story; or a "lame sermon," for a poor, weak sermon; and more commonly, a "lame excuse."

The same writer defines it to be- "The manifestation in the act of progression, by one or more of the limbs, of pain, weakness, inability, or impediment."

This definition is very near the mark, but I think it would read better as follows:-A manifestation by one or more of the limbs, of pain, weakness, inability, or impediment; the act of progression not being necessary to the manifestation of such. Percivall, however, must always be looked upon as a great authority, as he seems to have been a practical man in most matters, as well as an elegant writer.

Lameness is not of itself a disease, but a sign of it. It is the expression of pain or inability, the result of disease, accident, or malformation in the limb or limbs by which it is manifested. It may, however, arise from disease apart from the limbs, as from injury to or diseases of the spinal cord or nerves, from cerebral disease, and occasionally disease of the liver. It may exist for a short time independent of disease-a mere expression of pain without disease, as from a stone in the foot, or a badly-fitting shoe; but if these causes of pain exist for any length of time, inflammation is sure to follow. Disease much oftener exists 
in a limb without lameness than lameness without disease. Thus, a horse may have a wound, ulcer, bony deposit, or a tumour, without evincing lameness. From these particulars we may argue that lameness is never present without pain, although Percivall asserts that inability, in the absence of pain, will be found as a cause of lameness, and he says- "Dislocation of the patella occasions no pain, and yet the horse is too lame even to move. The partial or complete anchylosis of a joint may cease to be attended with pain, and yet there may be permanent and irremoveable lameness." I think we may safely take exception to these conclusions, as they are not borne out by every-day experience.

Complete anchylosis may exist without pain, and yet the patient is lame; but it will be found that such anchylosis exists in some joint of extensive motion, and prevents flexion and extension in the whole limb.

Pain, then, may be generally said to be the common cause of lameness. The patient feels the pain either when it moves the limb, or when it bears weight or presses upon it. During motion the patient endeavours to avoid throwing pressure upon the lame lim.b, by treading lightly or stepping short, and by removing wejght as far from the seat of pain as it possibly can, not only by using the lame limb in a manner best calculated for this puipose-as by treading on the heels when the pain is in the antirior part of a limb or foot, and upon the toes when in the postierior part-but also by throwing the weight from the lame limb as much as it possibly can.

Wreakness of the limbs may cause lameness and inability to perform the function of progression properly. A characteristic example of this has been described by Mr. George Armatage, under the title of "Congenital Muscular Atony," or a want of devilopment of muscular fibre in the extensor muscles of the fore arm of foals. In this form of lameness the animal stands almost upon the front part of the fetlock-joints; the flexor musicles healthy, fully developed, and having no antagonistic pow'er opposed to them, in consequence of the arrested development of the extensors, draw up the limbs posteriorly; the heel of the foot and the fetlock pad being in close contact, the little animal being at the same time almost unable to move. I have found this kind of lameness occurring at any time during the 
first year of the animal's life, and it may not always be viewed as "congenital." Grazing on very bare pastures is apt to cause it, more especially if the foal be short in the neck, or when so formed that he has to bend over on his fore limbs considerably before he is able to obtain his bite of grass. Continuance in this position for a long time each day causes a weakening and arrestment of development of the extensor muscles, whilst at the same time the flexors called into action are excited to increased development. We have thus, concomitantly, atrophy of the extensors and hypertrophy of the flexors.

Mr. Armatage recommends the continued application of mild blisters to the anterior region of the arm, to excite the exudation of plastic material and the development of the exudate into muscular fibre, and, by bringing more blood to the part, to increase its tone and power, and elevate its functional activity.

A horse may be lame from excess of tonicity in the musıles of a limb, accompanied by much pain, as in cramp, which rend.ers him for the time being dead lame.

In other cases lameness may be due to disease in the bloodvessels of the limb or their parent trunks. For example, m.ost extreme lameness is manifested in limbs when their "arteries of supply" become plugged by thrombi. Cases of this kind have occurred in my own practice, where the horse became dreadfully lame, and exhibited symptoms of great agony whenever put to work, while in the stable no signs of pain riere present; but when he had worked in the carriage for about 500 yards, he would show signs of weakness in one hind limb, commence to sweat, and finally become immoveable with pain. After a short period of repose, these symptoms would disappear, but appear again if any attempt was made to rork him.

In addition to pain and lameness, the limb was deathly cold to the touch, and upon a post mortem examination being made, the external iliac artery of that side was found nearly obliterated by a deposition of fibrine.

In the detection of the lame limb, the following rules may be laid down for the guidance of the young practitioner:-When the lame limb comes to the ground during progression the animal suddenly elevates that side of his body and drops the 
other side. If the lameness be in a fore limb the head as well as the fore part of the trunk is raised from the lame and dropped upon the sound limb. This is called "nodding." If the lameness be in a hind limb, the quarter of the same side will be elevated and that of the sound side thrown forwards and downwards by a jerking motion; the head being moderately steady if the pain be not great, and jerked if there be acute agony. It is only by carefully noting these facts that we are able to detect the limb in which an animal may be lame.

The signs indicative of the seat of lameness are of two kinds:-(1.) Those manifested by action; and (2.) Those discoverable by examination, while the animal is in a state of rest. In some instances the latter are alone sufficient to indicate the seat and nature of the disease, but the lameness must be of a severe character, manifested by "pointing," standing with the lame limb flexed, or even completely elevated from the ground; or, as in laminitis, with the unaffected feet and limbs placed as much under the body as possible.

In the majority of cases, however, it is necessary to cause the patient to perform some movement; and it is agreed by all practical men that the slow trot is the best pace. A horse may walk lame, but if such be the case, he must be very lame. There are cases, however, as in "slight splint lameness," where it is necessary to urge the animal to a sharp trot before any deviation from the normal gait can be distinguished.

The following rules may be useful for the guidance of young practitioners:-The horse should be led out of the stable in a snaftle bridle, with the rein over the head. Let the man who leads him be ordered to hold the rein at about eighteen inches from the mouth, and let there be no holding up of the animal's head by a tight curb or rein, to prevent "nodding;" but, at the same time, the rein must not be too long, for if this be the case, a spirited animal may turn round so far as to kick the man with his hind foot. The horse should be trotted immediately after he is taken out; and for this reason, that any very slight lameness may disappear if he be walked any distance. Many low dealers will knock a horse about in the stall to remove such lameness. This should be looked to in every suspicious case. If the horse be frisky, he must be cooled down and very carefully led, in order that the examiner may 
have a fair chance. It has been already stated that it is necessary to make the horse run both from and to the observer, and it may be necessary to do this repeatedly; but if an examiner cannot determine the existence in two or three minutes, it is better to put the horse up again, as the exercise has a tendency to decrease, or even to remove the lameness. In some very slight cases, it may be necessary to place a rider on the horse, as the weight upon the back will cause the manifestation of the lameness. As a rule, however; it is better not to do this, especially if the animal be a spirited one.

There are some lamenesses which are only manifested after sharp work, and in such cases it is necessary to give the horse half-an-hour's trot or gallop, tying him afterwards in a stall until he becomes cool. When taken out of the stable and trotted after such a test, lameness, if in existence, will most assuredly be detectable. Some veterinary surgeons do this with every horse they examine; but it is quite needless in ordinary cases, and it is only when some suspicion exists that such a test is necessary, such as badly-formed hocks, splints near the knee, or some alteration of structure in any part of the limb, or in cases where there is "pointing" while at rest, or where the disease is very slight.

The gait only is sometimes sufficient to determine the seat of lameness, and in some cases it is the only guide; but it is a good rule, and one that should never be neglected, to examine the lame limb while the animal is in a state of rest.

By the latter method we discover lameness by positive and negative signs. For example, if there be heat, pain, or swelling in any part of the limb, discoverable by manipulation, the evidence is positive that the cause is in such a part; but if, on the other hand, there be neither pain, heat, nor swelling in the limb, nothing in the superficial parts of the foot to account for it, we must conclude that it is deep-seated in the foot, or in a part thickly clothed by healthy tissues, and we must arrive at a conclusion by negative evidence, assisted by peculiarities of gait.

Lameness may be caused by a strain of a ligament, muscular tissue, tendon, by fractures, diseased bones, cartilage, or fibrocartilage, morbid conditions of the skin; neuromatous and other tumours; plugging of arteries; accidents, as "pricks" in shoe. 
ing, "treads," wounds, ulcers, rheumatism, and reflex nervous action, as in diseased liver.

\section{A SPRAIN, OR STRAIN,}

Is violence inflicted upon any soft structure, with extension, and often rupture of its fibres. Professor Dick was of opinion that there was always rupture of the fibres in a sprained tendon or ligament. But I think that the condition which we term strain may arise from repeated extension or slight stretching, without the fibres being at first ruptured at all; and that an altered nutrition is so produced, which leads on to inflammation of the part, with interfibrillar exuciation, and finally to the softening of some portion of the fibres, by which they lose their toughness, and become broken across. A very slight strain may be a mere bruise, with ecchymosis; whereas violent and great extension may rupture the whole structure of a part.

Extension is not always the cause of a strain, as a muscle may be injured by the opposite condition, namely, violent contractions; its fibres and their thecæ broken across their long axes; or its tendinous fibres torn from their attachments at either or both of its extremities.

Strains may be confined to the thecæ or sheaths only, but these are of but little importance compared with injury to the ligaments or tendons themselves. It has been already stater that synovitis may arise from strain of a ligament, by the inflam. mation extending to the small synovial surface which is found on most "binding ligaments."

Muscular strains are found in various parts of the trunk and limbs. Thus, a horse may be strained in the neck, as a result of a fall upon the head. If the fall be very severe, the strain may be complicated with severe injury to the spinal cord, or with fracture of the vertebræ, causing perhaps the sudden death of the animal.

The muscles of the dorsal region may be sprained by the hind feet slipping backwards. When a muscle is strained the injury is succeeded by pain, swelling, heat, and loss of functivis. An inflamed muscle can no longer contract; hence, in some strains, the symptoms resemble those of paralysis.

This swelling of an inflamed muscle is very often succeeded 
by loss of substance (atrophy), and sometimes by fatty degeneration of its fibres, whereby they lose their red fleshy appearance, and assume that of whitish threads of fat. When microscopically examined, the sarcous elements-the real contractile tissue within the sarcolemma-is replaced by glistening oil particles, so that the functional power is completely destroyed. If the whole muscle be involved, its contractile - power no longer exists; and this loss of power will vary according to the extent of the muscular structure involved in the primary lesion.

Atrophy of the fibrillæ, and consequent fatty degeneration of their contents, is often due to pressure by an inflammatory exudate formed in the spaces of their connecting areolar tissue; and it is important, practically, to remember this, for the reason that the sooner an exudate can be removed, the less chance there is of degenerative changes taking place in the true muscular elements.

The changes that occur in inflammation of muscular tissue, whether arising from strain or other causes, may be briefly described thus:-1st. Swelling from congestion and exudation; $2 d$. Atrophy, from the pressure of the exudate upon the muscular fibrillæ, and from loss of function; $3 d$. Fatty degeneration of the sarcous elements, and permanent loss of contractility.

Treatment.-Repose; soothing applications, succeeded by slight, and afterwards stronger irritants. Purgatives and cooling diet at first, followed by good nursing.

\section{STRAIN OF THE PSOAE MUSCLES.}

Much confusion prevails among veterinary surgeons as to the proper diagnosis of this injury, some classifying all cases of inability, or paralysis of the hind limbs, unless broken back can be detected, as sprain of the psoæ muscles; whilst others deny the existence of such an injury at all.

Sprain of the psoæ muscles simulates paralysis, broken back, and that mysterious disease which has been erroneously termed hysteria by Mr. Haycock,-a disease which, in reality, is due to the presence of a large amount of effete materials, especially urea, in the circulation, and which may be termed Azoturis.

The psoæ muscles-magnus and parvus-are, along with the iliacus, sartorius, \&c., situated within the pelvic and sub-lumbar 
regions, originating at the heads of the last ribs and last dorsal vertebra, extending in a backward direction under the bodies of the lumbar vertebræ to the ilio-pectineal eminence on the brim of the pelvis, and the internal trochanter of the femur. Their action is to bend the haunch upon the pelvis, to draw it forward in progression, or while the hind quarters are stationary and fixed points, their action produces that appearance called "roached back."

It is important to remember the action of these muscles, for when injured they cease to act, and the haunch will be thrown into the opposite condition of "roached back;" and from this circumstance the injury may be readily distinguished from azoturia.

Strain of these muscles is caused by injury, such as " being cast in the stall," or by any other accident capable of producing violent extension of them; hence arises the difficulty of determining between it and "broken back." It may, however, be distinguished from the paralysis of broken back by the power of flexing and extending the limbs being still retained by the animal whilst it is lying down; there may be inability to rise from the ground, as the psoæ muscles assist very materially in that operation; but when raised by means of slings from the recumbent position, and when the hind feet are firmly placed upon the ground, the horse is able to stand moderately well, and command the movements of the limbs to some extent, although there is always a tendency to knuckling over at the fetlockjoints.

The muscles of one side may be injured; in such a ease the loss of power is limited to one side only.

If the injury be not sufficiently severe to destroy the power of rising, it will be seen that, when the horse is made to walk, he drags his limbs or trails them to some extent. There is scarcely any elevation of the feet or flexion of the joints, and a great tendency is shown to knuckle over at every step.

Examination per rectum will reveal heat, tenderness, and swelling under the spine; and in the majority of cases that have come under my observation, external swelling around the rectum and perinæum, or vagina, if the patient be a mare, will make its appearance in the course of a few hours after the accident. 
The acute inflammation is generally succeeded by a condition of atrophy, and for some time afterwards the animal will show signs of weakness and inability; but these will pass away as the muscles regain their power and tone.

Trcatment.-Rest in the slings; enemas, to act as internal fomentations; aperients, and febrifuges if fever be present. In cases where the appetite is not much impaired, and but little or no fever supervenes upon the injury, the administration of medicine should be withheld. Fomentations must be applied to the loins and perinæal region; and after the first few days, mild external stimulants, as weak ammonia liniment, also good food and careful tending. It may be laid down as a general principle that slings do harm when the animal is unable to stand in them, and if both fetlocks knuckle forwards, and the whole weight of the patient be thrown upon the abdomen, slings should not be used; but should he be able to stand when the feet are implanted on the ground, the slings are very useful. After some time has elapsed, and when the patient appears not to make the desired progress towards recovery, a good blister to the loins and quarters will be very beneficial, and will hasten the development of muscular tissue. If the season be favourable, a run at grass in some quiet place will complete the cure.

Muscles are liable to become deranged in their function either by an exaltation or depression of their contractile power, and these conditions are generally exhibited in the muscles of the lumbar, gluteal, and femoral regions.

The causes of these aberrations of function are often very obscure, and seldom demonstrable. It has been already pointed out that diseases of the spinal bones are present during life without any very decided external manifestations; and from this it may be inferred that such diseases as "stringhalt," shivering, or that form of partial paralysis vulgarily termed "jinked-back," as well as that peculiarly obscure nervo-muscular disease called by French veterinary writers "immobilité," and by English horsemen "German horses," are all probably due to some disease of the sensory track of the spinal cord, similar to the "loco-motor ataxia" of the human patient, which is thus described by Aitken in his Science and Practice of Medicine, 1866:- "In the erect posture the muscles may sustain a heavy 
weight, and general paralysis does not supervene for months or even years. There is then a gradual and progressive loss of the power of co-ordination in the acts of volition. An awkward unsteady gait is the earliest indication of such progressive paralysis. At first the feet are moved in a slatternly manner, the heels lounging on the ground, and then, as the disease advances, they are thrown involuntarily to the right or left without purpose, and without the power of restraining their irregular movements. The act of turning round is performed with great difficulty. ... If the patient is put on his legs with his eyes shut, and his feet close together, it is seen that, although he has the muscular power he has not the muscular sensibility to preserve his body from falling, or to guide him in taking even a few steps forward with his eyes closed. He will reel and tumble about like a drunken man."

Functional irregularity of muscular action may arise from fatty degeneration of the muscles, without any disease being found in the nerves or nervous centres.

\section{CHOREA.}

Definition.-An irregular convulsive clonic action of the voluntary muscles, confined generally in the horse to the posterior extremities-constituting "stringhalt," and in the dog as a sequel to distemper-to the anterior ones, neck, and face, by which they are withdrawn from the control of volition, more especially during sleep.

Many pathological views are entertained regarding this disease. By some it is regarded as entirely functional, and independent of organic change. By others it is held that it is due, at least in some cases, to some disease of the blood, the precise nature of which is as yet unknown, and that it may be associated with some diseases, as rheumatism and diseases of the heart.

Professor Dick was of opinion that it was due to the presence of tumours in the lateral ventricles of the brain, and supported his views by a post mortem proof. But tumours in the ventricles may be present without chorea, and chorea is very often present without such tumours. Other writers have traced its origin to a hypertrophied condition of the nerves given off from the lumbar plexus, to the pressure of some 
exostoses on a nerve, and to paralysis of the muscles antagonistic to those affected with the spasm. In one case which fell under my notice, melanosis within the spinal canal was the cause of chorea; but the spasm (clonic, or rapidly alternating contractions and relaxations) of chorea is not a phenomenon of persistent spinal irritation, while tonic spasm is a mark of such a condition.

Chorea may be divided into partial, as in stringhalt in the horse, and general, as seen occasionally in dogs.

Stringhalt may be defined to be an involuntary convulsive motion of the muscles, generally those of one or both hind legs, but occasionally it is seen in the fore legs also.

The limb or limbs affected are convulsively elevated from the ground, and brought down again with more than natural force. It is not always to be noticed at every step the horse takes. He may go several paces, as many as twenty, without exhibiting any signs of stringhalt; then, all at once, the limb or limbs will be suddenly elevated from the ground with a peculiarly sharp sudden jerk. It is necessary sometimes to turn the animal round from right to left, and from left to right, in order to make him show any signs of stringhalt, the symptoms of the disease being exhibited as he turns one way only. It is generally developed slowly, but I have seen very aggravater cases come on in one night, and as age advances it always becomes worse. It should be viewed as an unsoundness, and as a cause of depreciation of the animal's value. In two horses which had suffered from very violent stringhalt, the post mortcm examination revealed exostoses on the shaft of the ilium, involving the great sciatic nerves. I think its cause is sometimes peripheral, as when a bone-spavin presses upon the nerves of the hock. The stringhalt then is due to reflex nervous action.

In grey horses, stringhalt is occasionally due to a deposition of melanotic material in the sheath of the great crural nerves. I am inclined to the opinion that ehronic stringhalt, or that form of it not dependent on rheumatism, arises from a congested condition of the nerves and their sheaths, and that there is always a tendency to the occurrence of neuritis from causes that would otherwise have no effect upon the nervous system. This view is supported by the fact that injuries to the feet, or any part of the limbs affected with stringhalt, are very prone to be succeeded by increase of the spasm, by much nervous excitement, and by tetanus. 
Some horses affected with stringhalt, when injured in the feet, become almost unmanageable from the extent of the spasm; they are soon exhausted by the expenditure of nervous and muscular force, and the mortality from such injuries is much greater than in horses free from stringhalt.

There is no treatment in chronic stringhalt. I have divided the tibial nerves when it has been associated with bone-spavin. In this case it was confined to one leg only, and to a fearful extent, the foot being caught up with extreme rapidity, and brought down with great violence, insomuch that the shoe was repeatedly broken by the violence of the concussion. There was a bonespavin on the hock, with considerable heat and tenderness. After trying various remedies upon the hock without good result, I divided the tibial nerves; but the animal derived no benefit from the operation. In aggravated cases the limbs are adducted, the foot thrown outwards, as well as elevated, during the act of progression. But where the symptoms are aggravated from any cause-such as an injury, common cold, or other source of febrile disturbance-the severity of the spasmodic action may be much modified by removal of the cause of excitement, and by a cathartic, belladonna, or the bromide of potassium, in suitable doses. Hard work will very often increase stringhalt to such an extent as to call for medical treatment. In the rheumatic form the treatment for rheumatism is to be prescribed. In Australia "stringhalt" is becoming serious, but its cause does not seem to be yet discovered.

IMMOBILITÉ, SHIVERING, SPRAINED BACK, ETC.

Immobilite is a term applied by French veterinarians to those cases of muscular irregularity manifested by the inability of the horse to turn round quickly without falling. He may be able to trot in a straight line well enough, but when turned round sharply, immediately falls. A modified form of this disease is very often encountered when the animal, although able to turn without falling, does so with great difficulty, throwing the hind legs about in an awkward, unsteady manner, and seemingly without power to regulate their movements; the hind quarters reeling from side to side, clearly showing that the muscular movements are imperfectly controlled by the power of volition.

This is commonly called broken, sprained, or jinked back by 
horsemen. It is not due to any fracture, nor always to any external injury, but is a progressive disease, arising from some alteration of structure in the spinal cord from disease of the vertebra, or from granular degenerative disease of the muscles themselves.

"Shivering" is another peculiar nervo-muscular affection of the posterior extremities, resembling stringhalt, and manifested more particularly during the acts of "backing" or "turning round."

In a case of this kind the animal, when made to back, will perform that act with some difficulty; the muscles of the gluteal and femoral regions are thrown into a state of "clonic spasm," contracting and relaxing in a very irregular manner; hence the term "shivering," from the resemblance of the muscular action to trembling or shivering. In many cases the tail is spasmodically elevated and depressed in the manner of a pump-handle, and the limbs elevated from the ground by a peculiar rigid or stiff movement; the foot often suspended for a moment, as if the animal were unable to direct the action of the muscles.

When moved forwards, the necessary actions will be performed tolerably well, but the backward movement is done with more or less difficulty, and sometimes it cannot be performed at all.

Shivering, immobilité, strained back, and their various modifications, must be considered as causes of unsoundness, since their tendency is to increase in severity as the animal becomes older. They often interfere with his condition, and generally give him an aged appearance before he has reached his prime; and they prevent him lying down, particularly if he is confined in a stall. Some horses, while so affected, lie down well enough; the majority, however, scarcely ever do so, but fall down in their sleep occasionally, and being unable to rise again without assistance, often injure themselves by struggling. Animals of this kind should always be slung at night.

Hereditary tendency.-I think there can be no doubt as to hereditary predisposition being one of the causes of the foregoing diseases; indeed, I have had sufficient proof, in my own experience, to convince me that such is the case. Very often one form or other will be found in young animals, two or three 
years old, that have been subjected to no hardship, arising spontaneously, and increasing by slow degrees. I have one instance on record where four young horses, the progeny of a dam that was affected in the back, died from spinal paralysis before they had attained the age of three years. A fifth is now living, and shows signs of aggravated nervo-muscular disease.

\section{MYOSITIS, OR INFLAMMATION OF MUSCULAR STRUCTURE.}

The muscles may be inflamed, either from external or internal causes, those of the lumbar and gluteal regions being most generally so affected. The intrinsic cause of myositis is the presence of the rheumatic poison in the blood; and the extrinsic causes are sprains, the application of direct violence, or of cold and moisture. It is expressed by pain, swelling, heat, and loss of function. Inflammation of the muscles of the dorsal, lumbar, and gluteal regions has often been confounded with "laminitis;" but a careful practitioner need not make such a mistake.

In laminitis, as well as myositis, there is great stiffness. In laminitis there is a tendency, more particularly in the hind feet, to elevate the feet from the ground alternately. In myositis there is no such tendency, the feet being firmly planted on the ground, and there allowed to remain, if the animal be not forcibly moved. In laminitis, when the animal is down, the symptoms of fever and pain are considerably alleviated. In inflammation of the muscles there is no tendency to lie down; and if the animal were forcibly cast, as is sometimes done in laminitis, the symptoms will become aggravated. A horse with fever in his hind feet will generally, immediately on rising, begin to shift them, become very uneasy, much distressed, and at last will lie down with a sigh of relief; the pulse falling, in the course of a few minutes, perhaps thirty to forty beats per minute.

In some cases of laminitis there is a disinclination to lie down; but when such are forcibly laid on a comfortable bed, they generally feel inclined to remain recumbent, and, as a rule, it is only necessary to lay them down once or twice before they find out the benefit, and take advantage of it without assistance.

When the muscles of the loins and quarter are inflamed the symptoms will simulate those of stringhalt. 
I have merely pointed out these essential marks of difference at present; a more detailed account of laminitis will be given hereafter.

The treatment of muscular inflammation, when not caused by such a degree of violence as to destroy the vitality of the parts, is very simple, - a gentle purgative, rest, fomentations. Should atrophy succeed the active stage, a mild blister.

\section{RHEUMATIC LAMENESS.}

The lameness is characterised by what is termed metastasis, or a shifting of the seat of the disease from one part to another. The favourite seats of rheumatism, occurring after influenza, are the sesamoid bursæ; but it may appear in almost any serous or synovial structure. It is not my intention here to enter into the pathology of rheumatism, that being reserved for another volume; but I may lay down a simple rule for its treatment. A strong vesicating blister should be applied to the part and around it without loss of time, all other local applications being in my opinion worse than useless. A blister is supposed to act by attracting the rheumatic poison into its vesicles, and removing it from the system by the bursting of such. It is recommended by Dr. Richardson and others that the blister should be near, but not upon, the diseased spot; but I have found this inconvenient, and a direct application to answer every purpose.

A horse suffering the greatest agony from rheumatism is relieved in the course of one day, provided a numerous crop of vesicles be produced. The cantharides blister is the best; a purgative is useful after the blister. If the lameness be not thus removed, colchicum and nitrate of potash are to be administered; and if these fail, the iodide of potassium, or carbonate of soda or potash, with vegetable tonics.

In dogs, rheumatism is commonly called Kennel Lameness.

It attacks young sporting dogs more frequently than any other class. The cause is usually to be found either in the construction of the kennels or their management; damp or cold situations, bad drainage, a short supply of clean straw, and want of exercise, are each and all liable to produce this intractable disease. The symptoms are general rigidity, accompanied with great pain, as evinced by the anxious expression, hurried breath- 
ing, disinclination to move, and pain on manipulation; the pulse is hard and quick. The tendency of the disease is to localise itself in the joints, attacking one or more at first, disappearing from there, and appearing in others.

Treatment.-Put the patient in a dry warm place, and administer an aperient. Great relief will be obtained by immersion in warm baths; care being taken to prevent chill afterwards. The affected joints are to be stimulated with soap liniment; alkalies, diuretics, and nourishing but easily digested food, are to be given; and when the more acute symptoms have passed off, citrate of quinine and iron.

The terms rheumatism and rheumatic lameness are often misapplied. For example, scrofulous disease of the joints in horned cattle is commonly thought to be rheumatic in its character, and due to external causes, such as cold, damp, \&c.; whereas, in reality, it is an intrinsic disease, and due in the great majority of instances, more especially when it occurs amongst high-bred stock, to a system of in-and-in breeding persisted in for too long a period. Again, a disease-osteo-malacia_often terminating in spontaneous fracture of the bones, which prevails amongst horned cattle pastured on poor land, and occurring mostly during dry summers, is supposed to be of rheumatic origin, whilst in reality the stiffness of the joints and lameness are symptomatic of malcondition of the body generally, and of the osseous system particularly, due to the want of proper food in sufficient abundance, or to something deleterious in it. In Wales this disease is supposed to be caused by the animals eating the purging or mountain flax (Linum Catharticum), which is found growing amongst the pastures in such situations. I cannot endorse the popular idea that this plant is the cause, for cattle refuse to eat, indeed, turn away from it. I look upon it more as an evidence that the land is poor, and the pastures, particularly in very dry seasons, defective in nutritious elements.

The symptoms are depravity of the appetite, rapid emaciation, venous murmurs; stiffness of the limbs, swelling of the joints, and difficulty, or even inability, in rising from the recumbent position.

The post mortem appearances are remarkable. The whole body is wasted, the muscles anæmic and flabby, the blood is thin and watery, and the tissues are odematous and softened. 
Parasites are often present in large numbers in the stomach and intestines. The bones are enlarged and softened, crumble upon the application of slight pressure, and if fractures have occurred some time prior to death, it will be found that the reparative process has been but very imperfectly and feebly established.

In this disease a peculiar pathological process obtains, similar to that which is witnessed in the general emaciation of phthisis pulmonalis, described at page 49 of my Principles and Practice of Veterinary Medicine, namely, the absorption of a tissue, and its redeposition; but in this disease, instead of fat being absorbed, it is found that the calcareous ingredients of some bones are removed by absorption, and deposited in the form of osseous tumours upon various other bones of the skeleton, obliterating cavities of reception, and causing deformity of the bones themselves.

The prevention of this disease is to be accomplished by proper diet; change of pasture if possible, if not, the addition of cake or other nutritious feeding material; whilst the pastures themselves are to be manured with artificial and other dressings - the phosphates of lime, nitrate of soda, or the salts of aminonia, as may be determined upon by a competent analyst.

In the early stages, the disease may be successfully combated by antacids, tonics, occasional but mild aperients, and proper food; but if the osseous system is greatly involved, treatment is useless. 


\section{CHAPTER XIII.}

\section{PARTICULAR LAMENESSES.}

SHOULDER LAMENESS-SHOULDER - SLIP-ELBOW LAMENESS-SPRAIN OF RADIAL OR SUPERIOR CARPAL LIGAMENT-CARPITIS.

\section{SHOULDER LAMENESS.}

There are three forms of shoulder lameness, which may exist independently of each other. One of them is illustrated in Photo-lithograph, Plate II., Fig. 1. These three causes are(1st.) Disease of the shoulder-joint, Photo-lithograph, Plate III., Fig. 1; $(2 d$.$) Sprain of the flexor brachii, Photo-lithograph,$ Plate I., Fig. 5; and (3d.) Sprain of the antea and postea spinati, teres major and minor, muscles, but more particularly of the spinati, as they are mostly concerned in performing the function of binding ligaments, connecting the scapula and the humerus.

Mr. Percivall and others were of opinion that injury to the serratus magnus was a frequent cause of shoulder lameness. Professors Dick and Barlow taught that such could hardly be the case, and my experence leads me to endorse their opinion.

The three above mentioned are the usual causes, but there are others sometimes met with, namely, rheumatism; fracture; liver disease ; formation of abscesses in the brachial glands, as a sequence to, or sometimes a primary manifestation of strangles; open joint and necrosis of scapula.-(See Photo-lithograph, Plate I., Fig. 6.)

Disease of the Shoulder-Joint may arise from a variety of causes, as sprains or rheumatism. Inflammation having been set up in the structures of the joint; the capsular ligament 
becomes thickened, in other parts diminished, in structure; distended by synovia and exudation; the articular cartilage is removed by ulceration, or, in rheumatoid disease, converterl into the porcellaneous deposit; the capsular ligament and synovial membrane, with its fringes, becoming at the same time loaded with calcareous matter.-(See Photo-lithograph, Plate II., Fig. 1.) When the cartilage is ulcerated, there is caries of the heads of both scapula and humerus, and afterwards anchylosis of the articulation.-(See Photo-lithograph, Plate III., Fig. 1.)

At one time all obscure lamenesses in the fore extremity were attributed to the shoulder, and one heard of nothing but shoulder lameness. A reaction, however, took place, after Turner re-discovered navicular disease, in favour of the latter, which for many years bore the palm; but now these extreme views have been much modified, although there are still some who attribute everything to the foot, and others to the shoulder. It must be understood, however, that there are shoulder as well as foot lamenesses, and a proper diagnosis must be made of each case by the practitioner, who, if enlightened, never weds limself to the one theory or the other, but is guided by such facts as present themselves to his notice.

Diagnosis.-The gait of shoulder lameness is indicative of the seat of disease. The patient does not carry his limb straight forward, but with a rotatory motion, the limb being thrown outwards, and the toe made to form the segment of a circle; in other words, he brings the leg forward with a sort of sweep, and in some cases the toe of the foot is dragged or trailed along the ground. In shoulder and knee lameness more particularly, the signs of pain are more discernible during the elevation of the foot from the ground; whereas in foot and other diseases below the knee, the converse is the rule.

Upon manipulation, it will be discovered that pain, heat, and swelling are present. In order to discover the swelling, it will be necessary to push the levator humeri muscle to one side. It is but loosely attached to the shoulder joint, which it covers. The next test is to take hold of the limb, and move it backward and forward, to perform flexion and extension, when the animal will shrink, and evince considerable pain. In some well-marked cases the extension of the fore arm by the examiner will cause 
the animal to rise bodily from the ground. It must, however, be always understood that uneasiness when these movements are performed, or when the shoulder is pressed upon, is of itself insufficient to determine the seat of lameness, as many horses are fidgety, and will not bear such handling. But if there be a drag in the gait, along with the other signs, it may fairly be concluded that the ease is one of shoulder laineness.

Sprain of the Flexor Brachii.-This muscle contains a large amount of tendinous fibres, and is entirely tendinous as it passes over the bicipital groove in front of the superior extremity of the humerus, over which it plays like a rope over a pulley, becoming inserted finally into the inner head of the radius. Both it and the groove are lined with fibro-cartilage, and enclosed within a synovial sac. It extends from the scapula to the head of the radius, and its function is to flex the fore arm upon the shoulder, - an important function, rendering the structures which perform it liable to injury and disease.

In sprain of the flexor brachii, the muscle, along nearly all its course, will be found swollen and inflamed, standing out prominently from the surrounding structures. This swelling, along with that of the bursa in front of the shoulder, pain upon pressure, and the gait peculiar to shoulder lameness, namely, a difficulty in elevating the foot from the ground, are the diagnostic symptoms.

It night be supposed that disease of the bicipital groove would, from contiguity of situation, be always accompanied by disease of the shoulder-joint itself; but such is not the case, as may be seen from an examination of Photo-lithograph, Plate I., Fig. 5. In many cases, however, the joint becomes affected.

The result of sprain of the flexor brachii is inflammation of the tendon and the structures of the bursa, which, if not arrested, will run on to ulceration of the cartilage upon the tuberosities of the humerus, to caries of the bone, degeneration and rupture of the tendinous fibres, and finally to ossification of the whole substance of the muscle and tendon. The reason why ossification, and not atrophy, as in inflammation of other muscles, must be looked for, is the fact that the flexor brachii is mostly 
composed of tendinous fibres, and long-continued inflammation of tendons usually ends in their ossification.

The causes, like those of the next variety, are generally due to the horse being worked at the plough, and on the cff side, by which his limbs and shoulders are thrown into an irregularity of position; that is to say, one foot is placed in the furrow, and the other on the land. One shoulder is thus in a lower position than its fellow, an extra amount of weight being at the same time thrown upon it, so that in the course of time its structures become diseased.

Sprain of the flexor brachii from the above cause is confined generally to the off side, and this can be easily understood from the extra work it has to perform in elevating the foot which is in the furrow to the level of that which is on the land. Ploughing is not the only cause of this form of lameness; but a horse that has been sprained in this tendon is seldom fit for the plough again. He may work on the road well enough; but as soon as he is put in the plough the lameness returns. In some instances rheumatism becomes localised in the flexor brachii, and is a cause of intermitting lameness.

In the treatment of both shoulder-joint and flexor brachii lameness, great advantage is derived from the application of the high-heeled shoe. This enables the horse, while standing, to have the diseased structures in a state of relaxation and repose.

\section{"SHOULDER-SLIP."}

By this is meant that peculiar outward slipping movement of the shoulder-joint at each step the animal takes when the foot of the lame limb is upon the ground, the opposite one elevated from it, and when the weight of the horse is thus thrown upon the lame side.

It would appear almost as if the shoulder were out of joint, hence the term "shoulder-slip;" but such is not the case, as the following observations will explain.

The scapula and humerus are not bound together by lateral or binding ligaments, as is the case with other joints, the movernents between them being so varied and extensive, that inelastic ligaments would not allow them to be performed. They are consequently bound together by muscles, which 
perform the function of ligaments, and admit of a variety and extent of motion by their power of contraction and relaxation.

These muscles are the antea and postea spinatus and teres externus. The spinati, which are mostly concerned in this, are lodged in the fossæ of the scapula, covering its whole external surface, and attached inferiorly to the tuberosities and ridge of the humerus, and to the capsular ligament of the shoulderjoint. It will be seen from their position that their action is mainly to prevent the outward bulging of the head of the humerus, to keep the parts firmly and closely in their respective situations, and to assist in the elevation and adduction of the humerus.

It will be remembered that there is great disparity between the articular head of the humerus and the glenoid cavity of the shoulder, the latter being much smaller than the former. This disparity of size allows a liberty of motion without dislocation that otherwise would be impossible, assisted and kept within moderate bounds by the action of the foregoing muscles.

This form of lameness is generally seen in horses that are worked in the plough, and in the horse that works in the furrow. Mr. Barlow said, "always in the limb that is placed in the furrow ;" but I cannot endorse this, having often seen it in both shoulders.

The explanation is, I think, simple, the functions of the muscles being-1st. To keep the parts firmly together; $2 d$. To prevent the outward bulging of the head of the humerus; $3 d$. To draw the head of humerus outwards when the glenoid cavity overlaps its outer border; and $4 t h$. To assist in lifting the limb. Now, when the limb is, as it were, made longer than its fellow, by the foot being placed upon lower ground in the furrow, it necessarily follows that the work to be performed by these muscles is increased to a considerable extent, as the limb seems to hang from the shoulder; and that, in consequence, the demand upon them is beyond their power of endurance. Inflammation is excited in the muscular structure, and often in the tendons; the exudation presses upon the true sarcous fibrillæ, which, along with degradation of their constituent elements consequent upon the injury, leads on to atrophy. 
A muscle, when inflamed, or when atrophied, loses its power of contractility and its tone; and in this form of lameness the shoulder bulges outwards when the weight of the animal is thrown upon the lame side, because the muscles which bind the shoulder, and limit the great extent of motion between the scapula and humerus, are no longer able to perform their functions. So much then for the shoulder of the off side limb; and we must now endeavour to explain how "shoulder-slip" can occur in the near side, or that which is placed upon the land, and higher than its fellow during ploughing.

The second function of these muscles is to prevent the outward bulging of the shoulder-joint, more particularly the head of the humerus, - a condition of the joint which occurs always when a limb is placed upon higher ground than its fellow, as any one might see by watching a horse working in the plough. There is a bulging outward, a contortion in fact, at every step the animal takes; the parts are thrown out of their equilibrium, first into a state of undue relaxation, by the upward pressure of the humerus, then of undue extension, by the outward bulging. Again, both fore feet may be occasionally in the furrow, and the hind ones also. This, with slipping in and out, and the inequality of the land, brings on, particularly in the young horse, a state of irritation which finally terminates in inflammation and atrophy.

It is seldom that any but young horses suffer from this lameness, as older horses become used to their work, and are able to avoid injuring themselves. Great care is therefore required in bringing the young horse gradually to his work,-in fact, " to break him in" with due caution, by placing him at the side of a steady, quiet old horse; one that will not outpace him, nor fatigue him in his work. When an animal becomes fatigued he loses command over his action, becomes unsteady in his gait, and is thus rendered liable to sprain himself.

Symptoms.-In some cases there will be swelling and heat over the course of the muscles, upon the outer surface of the scapula, and in the joint itself; but in the majority of cases actual lameness does not occur until the muscles have become considerably atrophied, and instead of swelling, there will be a wasting of the shoulder. There will be a hollow space upon either side of the scapular spine, extending its whole length. 
The spine is thus rendered prominent, standing out as a sharp ridge from its surrounding structures. By standing in front of the horse, if one shoulder only is affected, and by comparing the two shoulders, the difference can at once be detected. If both are diseased, this comparison will not be of any service, but it is seldom that they are both alike; in one, the antea spinatus, in the other, the postea spinatus, is the more atrophied. This atrophy is different from that wasting of the shoulders seen in chronic foot lameness. In the one case, the muscles of the external surface of the scapula only are wasted; whilst in the other, all the muscles of the shoulder and arm are in that condition.

Shoulder-slip is curable, provided the tendons of the muscles, the rim of the glenoid cavity of the scapula, or the external trochanter of the humerus, be not organically altered in structure. The alterations found in these are abrasion of the tendons, ulceration of the cartilage covering the bones over which they glide, and caries of the bones themselves.

Treatment.-During the inflammatory stage, purgatives, fomentations, removal of shoes, and rest. After the muscles are wasted, repeated applications of moderate stimulating remedies, such as mild blisters, and a long period of rest in a strawyard, or at grass. When recovered, the patient should be put to other work than ploughing.

Some dealers have no objection to horses with wasted shoulders if they go sound, as it is well known that they will perform their work well enough if not put to the plough. Notwithstanding this, I hold that it is an unsoundness in law, and that it always depreciates the animal's value. I have seen many young carriage-horses suffer from this form of unsoundness, resulting from working in the plough; but I cannot recullect one instance where they were rendered permanently unfit for carriage work.

This fact is important, if it become the universal law that mnsoundness means unfitness for work. I mention this because I have heard lawyers argue and judges rule that such is the "law of warranty."

It might be supposed that the atrophy of the muscles of the shoulder, which is so well marked in this form of lameness, only became apparent after a considerable interval had elapsed subse- 
quent to the sprain of the muscles. Such, however, is not the case; and it may be laid down as a fact that well-pronounced atrophy occurs in the course of three weeks after a sound horse has been put to work; and in many instances it seems the alteration has been due to an irritation, not amounting to inflammation, leading directly to mal-nutrition, without the occurrence of swelling or exudation.

When shoulder lameness arises from disease of the liver, it occurs in the off side only, and is associated with loss of appetite and yellowness of the mucous membranes.

Abscesses in the brachial or pre-scapular ganglia of the lymphatic glands may be the cause of lameness in the shoulder. These glands are situated between the scapula and thoracic walls, and are liable to become inflamed, primarily or secondarily, in strangles, phlebitis and pyæmia caused by wounds upon the limb, or even after neurotomy, causing great pain and lameness.

Inflammation of these glands may be detected by swelling, pain, and heat in front and below the point of the shoulder, in the space existing between the sternum, scapula, and humerus. The limb will be pushed outwards by the swelling, there will be much fever, and the inflammation and suppuration are relieved only by the opening of the abscess or abscesses, spontaneously or surgically.

The pus is very deep-seated, and it is necessary to open the abscess in nearly every case of this kind, and to do it before there are any signs of "pointing." In such cases, I always explore with a "pin-director," and invariably find out where the pus is situated. I then make a deep opening with a "Syme's knife."

\section{ELBOW LAMENESS}

Occurs from disease of the joint, sprain of the lateral ligaments, and from laceration of the triceps extensor brachii muscle.

The internal lateral ligament may be sprained, or even ruptured, by the animal's fore leg slipping outwards. The triceps muscle is often injured in the manner already described at page 132, and by the animal's fore feet slipping forwards. These accidents commonly happen in frosty weather. Cattle are also liable to these injuries, particularly to sprain of the internal ligament.

When the ligaments are sprained, or the triceps injured, the 
diagnosis of the seat of lameness is easy, for there will be swelling, pain, and heat, in addition to difficulty in moving the articulation. When the internal ligament is injured, the horse stands with his foot and limb thrown outwards. This he does to prevent, as much as possible, the injured parts being pressed upon by the pectoral muscles; and when the triceps is the seat of the injury, the fore arm is flexed upon the humerus; the action of the flexor brachii being now unopposed, the knee is elevated, the leg flexed from the knee downwards, the toe of the foot touching the ground, and the limb semi-pendulous. When the horse is made to move, he drops considerably, and seems in danger of falling at every step he takes, the limb itself almost bending double when any weight is thrown upon it. This excessive dropping, during progression, is characteristic of elbow-joint lameness, even when there are no external signs, such as heat, swelling, or pain, visible.

Percivall describes a case of elbow-joint lameness in his book on lameness, publisled in 1849, where the symptoms were similar to those of laminitis. I have never witnessed anything similar to what is described by Percivall, namely, an animal putting the heel first to the ground in elbow-joint lameness. Usually the limb is semi-flexed, in order to relax the articulation as much as possible; the toe, during motion, first coming to the ground.

The diagnostic signs of elbow-joint lameness are, first, the semi-flexed position of the limb whilst the horse is standing still; and the excessive dropping of the head and anterior parts of the body during action.

Professor Dick used to say that the capsular ligament, distended with synovia, could be felt through the skin and subcutaneous structures. I have, however, not been able to confirm this. Photo-lithograph, Plate III., Fig. 2, is a good illustration of elbow-joint lameness.

A small wound upon or near to the olecranon gives rise to very peculiar symptoms. The wound itself may be so small as to escape detection without a very careful examination. It may be situated upon the very point of the olecranon, or upon its side, or even in the space between the thoracic wall and elbow. The wound is inflicted commonly whilst hunting; the rider, not knowing that it has been received, rides his horse, 
and as a result of motion, air is, as it were, pumped into the subcutaneous areolar tissue, inflating it, first in the neighbourhood of the wound, and then, if the movements be continued, over the greater part of the animal's body. I have seen the head swollen to a tremendous extent, the eyes closed by the swollen lids, the neck, shoulders, dorsal and posterior regions so blown up, that the classification of the patient in the animal kingdom became a matter of some difficulty. Such a condition need occasion but little alarm; all that will be requisite is to finc: out the wound, clean it well with warm water, plug it up with tow dipped in collodion, and keep the animal still. In a short time the air will be absorbed, and the swelling dissipated. If the nostrils are so greatly swollen as to interfere with the respiratory functions, endangering the animal's life, it will be necessary to puncture the skin surrounding them, and press out the contained air ; but if this is not the case, there is no necessity for making any punctures.

\section{SPRAIN OF THE RADIAL OR SUPERIOR CARPAL LIGAMENT.}

This ligament, sometimes described as a broad band of fibrous tissue, springs from the posterior surface of the radius, and becomes inserted into the flexor perforatus above the knee, and is also continuous with the perforans muscle and antebrachial fascia. It will be noticed, upon dissecting the fore extremity, that the flexor muscles of the radial region are intermixed with a large quantity of white fibrous tissuein fact, they are semi-tendinous in their structure; and that, both above and below the knee, any undue extension of them is ehecked by ligamentous bands, which arise from the radius and metacarpus magnus, and become continuous with their tendons of insertion.

The peculiarity of construction here noticed enables the horse to sleep while standing. The feet are made fixed points by being planted firmly on the ground, and these muscles, acting as extensors of the shoulder, prevent the auimal from falling by keeping the limbs in a state of rigidity. Had they been composed wholly of muscular tissue, this long-continued action would have been an impossibility; but containing as they do a large amount of tendinous structure which is beyond the influ- 
ence of the nervous system, and hence beyond the possibility of fatigue, the mere implanting of the feet upon the ground, ly causing the muscles to be stretched, produces a state of rigidity and firmness in the limbs that enables the animal to stand and sleep for hours together without danger of falling. The muscles are strengthened and assisted by the ligamentous bands: from this circumstance, and from their acting as checks to over-extension, they are liable to sprain or even rupture.

Sprain of the radial ligament is manifested by lameness, a difficulty in the act of flexing the knee, with swelling, heat, and tenderness immediately above the knee, posterior to the radius. The swelling arises from exudation of lymph into the substance of and around the ligament, and from distension of the synovial bursa, through which the tendons pass, behind the knee. In some cases, both perforatus and perforans also are involved in the injury: in such the lameness, pain, and swelling are excessive, and flexion exceedingly difficult, arising from the swollen tendons being too thick to play through their thecæ,-just as a rope too thick for a pulley retards motion.

The distension of this bursa appears as a tense but fluctuating swelling at the back, and slightly above the knee, and is sometimes called thorough-pin of the knee.

The tendons of the various muscles concerned in the flexion and extension of the knee, pastern, and foot, passing through thecæ upon the surfaces of the carpus, are liable to injury, with distension of their various synovial sheaths from hypersecretion of synovia. We have thus the theca of the flexor metacarpi externus made visible on the outer surface of the os trapezium; the sheath of the flexor metacarpi internus on the inner side of the knee; that of the extensor metacarpi magnus in front of the knee as a swelling on either side of the tendon; and those of the other extensors upon the outer surface of the carpal articulations.

These enlargements, when arising from injury, and consequent thickening of the tendons themselves, or inflammation of the synovial membrane, cause lameness; whilst at other times they are mere bursal distensions giving rise to no inconvenience. 
In the neighbourhood of copper and iron smelting works, bursal enlargements are due to the impregnation of the animal system with mineral poisonous materials. The poison, finding ingress by means of the atmosphere into the lungs and circulation, becomes deposited in and around the articulations, more especially of the knees, and finally produces anchylosis.

All cases where the tendons are thickened must be looked upon as unsound, since they are tied down by the enveloping thecæ and annular ligaments, through which they play; and any thickening of their substance must destroy that facility of motion which is so essential to soundness of action.

The bursa of the extensor metacarpi magnus sometimes becomes greatly distended-constituting what is termed "capped knee,'-from blows and bruises, or the entrance of thorns whilst hunting and jumping thorn fences. These thorns may remain in for years withont causing any apparent inconvenience to the animal beyond some swelling.

When capped knee is first observed it is generally accompanied by some stiffness and pain, showing that more or less inflammation is present. These symptoms should be combated by purgatives, fomentations, and quietude. After a time all signs of inflammation pass away, leaving a fluctuating swelling. This swelling may either be punctured at once, or its reduction attempted by the application of blisters. Of course, if a thorn can be detected it must be at once removed. Blisters, when sufficiently strong, often cause the reduction of these swellings by producing an exudation of lymph into the distended sac, converting the soft, fluctuating swelling into a hardish, indurated mass, which is gradually removed by absorption. But if blisters -and I prefer the cantharidine one to any other, with moderately firm pressure after the soreness of the blister has passerl away-have no effect in reducing the swelling, the practitioner need not hesitate to puncture and allow the contained fluid to escape. The puncture should be made at the lowest margin of the swelling, and upon its inner side, by a transverse incision, in order to blemish as little as possible. After the puncture is made and the fluid pressed out, the walls of the sac must be kept in apposition by means of an antiseptic bandage, rollerl round the knee from above downwards, until they become united by the adhesive inflammation. The bandage should not be dis- 
turbed for five or six days if no inconvenience is caused to the patient; but if any signs of pain are manifested, it should be removed and readjusted. The puncture in the skin must be kept open (and the best plan to do this is to insert a small piece of lint or tow into its orifice, allowing it to remain in for a few hours), in order to allow the escape of any fluid which might collect in the sac. The bandage is placed so as not to cover the wound. There is no danger to be apprehended from opening this bursa. I have done it repeatedly, and always with success; and sufficient inflammation is usually excited without injecting iodine or any other irritant.

Horned cattle, especially milking cows, kept in-doors, are liable to have enormously enlarged knees from distension of these bursæ, caused by bruising while lying upon hard floors. They may be opened with safety; and the best plan of doing so is to insert a seton right through the substance of the swelling, and allow it to remain in for three or four weeks; the knee to to be protected from further injury by a good thick bed, or by a thick flannel bandage wrapped round it.

\section{CARPITIS, OR INFLAMIIATION OF THE KNEE.}

This cause of lameness was investigated and laid before the profession by Mr. Arthur Cherry (Veterinarian, 1845). He gives a very elaborate account of it, which may be read with advantage; but in his over-zeal for what seems a pet theory, he has confounded diseases of other parts of the limb with those of the knee. For example, he says that " heat in the foot is sometimes felt in carpitis," and that what the old farriers called chest-founder arose from this inflammation of the knee. These statements must not be received without great caution, as they are apt to mislead the young practitioner. Mr. Cherry says"Under the term Carpitis I propose to describe a disease of the knee-joint which, in its commonly existing form, has never, as far as I am aware, been specifically described.

" The knee-joint itself has been considered to be exempt from disease, unless from the infliction of direct injury ; indeed, so far did the late Professor Coleman carry his opinion on this subject, that he used to assert, in the most positive manner, that the knee was never the seat of lameness. 
Professor Dick, as far as I can remember, dicl not mention the existence of this form of lameness; and on referring to my notes of his lectures, I can find nothing in them to lead me to think that he did so, although the Museum contains many specimens of the disease.

"The gait in chronic carpitis," says Mr. Cherry, "affecting both knee-joints, gives to a rider the sensation of the chest being displaced from its right position-a sinking or ' foundering,' and which feels as if it would increase at every step of the liorse. When attention is drawn to this peculiar gait, it is easy to be distinguished from the short cat-like step in navicular arthritis, which gives to a rider a sensation of the chest being raised up, or an attempt at doing so ; further, the feet in navicular arthritis are brought to the ground with the toe first, and in consequence the step is short and stilty, from the weight being as much as possible thrown on the column of bones, to relieve the affected tendon, or surface over which it plays; and from the same cause there is great inclination to canter, a pace which, when slow, brings into play but slightly the flexor tendons or navicular joint. In carpitis, on the contrary, these symptoms are reversed, from the cause of lameness being seated on, or amongst, the carpal bones. The effort is not now to throw the weight on the bony column, but on the tendons and ligaments, and by this means to avoid concussion; hence the limb is carried forward as nearly straight as possible; and still further to effect this object, it is thrown with a circumductive motion outwards, and brought to the ground with the heels first, as is shown by the wearing away of the points of the heels of the shoe. This mode of progression produces a long step, and at the same time the fore quarters are dropped or lowered, which has been, I doubt not, the origin of the term 'chest-founder.' There is also a disinclination to canter; and if this pace is attempted, it gives increased pain, and a rocking motion exceedingly unpleasant to the rider.

"The diagnostic characters of the disease affecting one leg only are the same as exist in both, but from the contrast which is afforded by the sound limb, these are much more recognisable than when both are affected; to these, however, one other characteristic must be added-the step of the lame leg being rather longer than that of the sound limb." 
The characters are very much akin to those of shoulder lameness, from which, however, it may be readily distinguishedfirst, by the circumductive manner in which the leg is thrown forwarls, and the straight knee; secondly, by the absence of the dragging of the whole limb; thirdly, by the stepping on the lieels, and the greater firmness on standing; but one of the best guides perlaps in diagnosis is the expression of pain when the affected joint is forcibly flexed or extended, and noting which joint is attempted to be least brought into use during action.

Mr. Cherry says- " I am disposed to a belief that there is in some horses a hereditary idiosyncrasy to this disease; be this as it may, half-bred are much more prone to it than thoroughbred horses."

The inflammation and its consequences may be confined to one of the articulations of the carpus, more particularly to that between the trapezoid and inner small metacarpal; or the disease may involve several, or all of the carpal articulations.

The morbid condition of the joint may consist of simple inflammation, terminating, by proper treatment, in restoration, or inflammation associated witl uleeration of the articular cartilage, earies of the bones, and a deposition of osseous matter external to and around the diseased portion, constituting auchylosis.-(See Photo-lithograph, I'late III., Fig. 3).

The inflammation being deep-seated in the bones, or synovial membranes, is not accompanied by much external heat; and the articulations of the knee, with their synovial membranes and capsular ligaments, being small and incapable of containing much synovia, account for the absence of external swelling until there is a deposition of osseous matter.

In many young horses, especially when they are first put to work, we meet with an occult lameness which is most puzzling. There is no heat, swelling, nor pain upon pressure in any part of the limb or foot. The animal is moderately sound when it first comes out of the stable in the morning, but shows signs of increased lameness after being at work for a short time. In such cases, the practitioner cannot be far wrong in pronouncing the lameness to be in the bones of, or adjacent to, the knee-joint.

The osseous deposits arising from carpitis, when not caused by the fumes of metals, are generally situated on the inner side of the joint. This is due to the bones being here more under 
the centre of gravity, receiving a larger share of the weight, and bearing more concussion than those on the outer side; therefore the head of the inner metacarpal, the trapezoid, and os magnum in the lower, and scaphoid and lunar in the upper row, are most commonly diseased; but it is very rare indeed to find the disease extending to the bones of the upper row.

Treatment.-Removal of the shoes; loose box in preference to the field or stall; with other remedies appropriate to lameness, to be described hereafter. 


\section{CHAPTER XIY.}

\section{PARTICULAR LAMENESSES-continued.}

SPEEDY-CUT-BROKEN KNEES-SPRAIN OF FLEXOR TENDONS-SPRAIYS OF SUSPENSORY LIGAMENT-SPRAIN OF FETLOCK-JOINT-SESAMOIDITIS-RING-BONES.

"SPEEDY-CUT" is the name of an injury to the region of the carpus, which often causes lameness. This injury is inflicted by the horse striking his leg with the opposite foot. Sometimes the bruise is above the knee, upon the skin covering the prominent process upon the internal surface of the radius, which gives attachment to the lateral ligament of the joint. It is only highstepping horses that are liable to inflict this injury in the trot, but in the gallop almost any horse may do it.

When a horse strikes himself, as in speedy-cut, he is liable to fall from the violence of the pain, thus endangering the life of the rider, and breaking his own knees.

In the examination of horses as to soundness, care must be taken to detect any marks of former bruises upon the inner side of the knee arising from this cause. The marks may be on the lower extremity of the radius, or upon any prominent part of the inner surface of the knee or head of the metacarpal bone. Wherever situated, I am of opinion that they should be considered as causes of unsoundness, since they indicate a fault, which at any time may interfere with the usefulness of the animal.

The symptoms of speedy-cut are-inflammation and swelling of the skin, collection of fluid in the subcutaneous areolar tissue, constituting a serous abscess, or the formation of pus in the part, with lameness, accompanied occasionally by a good deal of fever. 
Prevention is better than cure, and many horses will do well enough if properly shod with either the three-quarter or Charlier shoes. The foot must be kept narrow on the inner side, and the shoes removed about every three weeks, otherwise, by growth of the foot, the animal is apt to strike. I have known many cases of this kind where it was essential to remove and readjust the shoes at the end of three weeks, otherwise, mischief was sure to follow. If due attention to shoeing does not prevent it, a boot must be made, extending as high as the knee, with a rim on its upper extremity to keep off, as it were, the interference of the other foot. I do not think that narrow-chested are more liable to "speedy-cut" than wide-chested animals; round high action being generally the cause.

The treatment of the active symptoms arising from the injury consists in fomentations to the part, purgatives, the opening of the abscesses, whether serous or purulent, and the removal of any thickening which may remain, after the active symptoms have passed away, by the application of iodine or biniodide of mercury.

\section{BROKEN KNEES.}

A wound upon the anterior part of the knee, although of itself a mere scratch, is of such importance that it lowers the value of the animal to a very great extent. Many are the excuses for a broken knee. It is very true that many knees are broken through carelessness on the part of the rider or driver; and in judging such the veterinarian must draw a distinction between a good and a bad goer, between a well-made horse and the reverse. Marks of speedy-cut and cutting or brushing must also be looked for, as the infliction of these is apt to cause the animal to fall.

If a horse, well made, with very fine action, has broken knees, the cause may be purely accidental-bad shoeing, carelessness, or to the animal being subject to fits of vertigo.

Broken knees are of five kinds:-1st. When the skin is bruised, but not cut; $2 d$. When the skin is cut; $3 d$. When the skin is divided, more or less lacerated, the tendon of the extensor magnus exposed, and its bursa opened; $4 t r$. When the wound penetrates through the tendon, exposing the carpal articulations; $5 t h$. The last form, with fracture of one or more bones. 
1st. Whien the skin is simply bruised, the hair being, as it were, shaved off, with a little oozing of blood. This need cause no uneasiness as to after-blemish, if properly treated; but if treated in the common way-by hot fomentations, flannel bandages, and stimulating ointments or liniments-a blemish is the sure result.

The proper method of treatment is as follows:-1st. Tie up the animal's head, so that it cannot lie down. This I hold to be important, for the skin may be so injured that but little additional pressure is required to cause it to slough, and sloughing, however trifling, will leave a blemish. In addition to tying the head, all that is required is to sponge the knee lightly with some cooling application, such as the white lotion, repeatedly, for two or three days, or until the inflammation has subsided. If much swelling should arise, a purgative should be given.

$2 d$. When the skin is cut. The edges should be brought together as well as possible, and so kept by plaster, or styptic colloid, or shellac paste dipped in lint or tow. A wound on the knee must never be piuned or stitched, for flexion of the part will most assuredly tear out the pin or suture, with a piece of skin as well, and thus increase the blemish. In addition to the colloid or plaster, a light calico bandage may be applied, the horse's head tied up, and the dressing not interfered with for four or five days, if the case progresses favourably. But if the leg should swell, or show signs of pain and inflammation, the bandages must be removed, and the wounds examined; if found to be suppurating, they are to be lightly washed, and afterwards dressed with mild astringents, or very dilute carbolic acid-one to 200 parts of water is strong enough - or the white lotion, with a small quantity of the carbolic acid, will do very well.

It must be understood that before the edges of the wound are brought together, all foreign bodies, such as dirt, hay-seeds, \&c., are to be removed. Very often a small piece of dead tissue will be seen, presenting a deadened white appearance in the wound. This should be removed by the curved scissors, otherwise it will act as a foreign body.

$3 d$. When the skin is divided, more or less lacerated, exposing the magnus tendon, it does not nccessarily follow that, 
although there is a discharge of synovia from the wound, there is true open joint, for the bursa of the extensor metacarpi magnus is separated from those of the articulations.

Cases of this kind look very formidable, the resulting inflammation being sometimes great, the swelling very considerable, extending as high as the elbow-joint, and as low as the foot; the whole limb being infiltrated with effusion and exudation. The carpal-joint becomes greatly enlarged by a fibrinous deposit, which surrounds it, and the synovial discharge very profuse. Generally, with proper treatment, such cases recover, if the tendon be not crushed.

$4 t h$. If the tendon has been crushed, although neither lacerated nor divided in any way, it may slough in the course of four or five days, its vitality having been destroyed. This sloughing of the tendon is attended with severe symptoms, and is a source of great danger to the animal's life. The sympathetic fever becomes very high ; the respirations and pulse quickened; the bowels constipated; the urinary and other secretions arrested; both the wound and tendon assume a dusky livid or leaden hue; the discharge becomes fotid, sanious, mixed with blood, and the lameness excessive. When the slough is removed, the carpal articulations are exposed to view; the bones are inflamed, and of a red hue. The power of extension is now lost by the separation of the tendon from its attachment, and the limb is persistently flexed. If an attempt be made to extend it forcibly, great pain is inflicted.

The treatment of such cases, whether the division of the tendon be immediate or not, is a matter of anxiety to the veterinary surgeon, and, except when the patient is a valuable stud animal, it is better to destroy it, for even if a cure is effected, the articulation will be anchylosed; and a horse with an anchylosed knee is of little use. But if an attempt is to be made to effect a cure, the limb must be fixed in the straight position by means of the tin splint recommended for fractures, placed behind the knee, and fastened by proper straps and bandages, leaving the wound uncovered.

In the treatment of open bursa, or even when the skin only is divided, it is always advisable to apply the splint for the purpose of preventing motion, and to prevent a horse from accidentally striking the wounded knee against the nuanger, to turn him 
round in the stable, and supply him with food by means of a bag suspended from the stall-posts; the slings in the graver lesious playing a most essential part.

In some rare instances the wound in the skin and tendon is situated at the inferior part of the knee, over the articulations of the lower row of carpal bones and the metacarpals. A wound in this part, although penetrating deeply and opening into the joint, is not nearly so dangerous as one over the articulation between the two rows: for there is but little motion, the succeeding inflammation is not nearly so great, nor the supervening anchylosis so important.

$5 t h$. When the accident has been sufficiently severe to fracture one or more bones of the knee, the animal should be destroyed.

The causes of lameness now to be described, extending to the foot, are mostly common to both anterior and posterior extremities. When they are not common to both, special reference will be made to them. They may be enumerated as follows :-

1. Sprain of the flexor tendons.

2. " of suspensory ligament.

3. Inflammation of the sesamoid bursa.

4. " of the fetlock-joint.

5. Rupture of lateral ligaments of fetlock-joint.

6. Knuckling over.

7. Sprain of inferior suspensory ligaments.

8. Wind-galls.

9. Ring-bones.

10. Sprained pasterns.

\section{SPRAIN OF THE FLEXOR TENDONS.}

The so-called sprain of the back sinews is not primarily in reality a lesion of the flexor tendons proper, nor of their thecæ, but consists of a diseased condition, arising from over-extension of the metacarpal or metatarsal check ligaments, situated below the knee and hock, performing the same functions as the radial ligament which has been already described.

These ligaments arise from below the upper extremities of the 
metacarpal and metatarsal bones, and run down the legs, lodged in the fossæ upon their posterior aspect, bounded on either side by the small canon bones. They are external and posterior to the suspensory ligament, become attached to the great perforans tendons half way down the leg, and are there placed to prevent over-extension of the tendons.

Cart-horses are much more liable to this lameness than any other breed, in consequence of the great labour they perform in drawing heavy loads. This can easily be understood by watching a horse start a heavy weight-how he digs his toes into the ground, making his feet fixed points, upon which the muscles, the flexors particularly, are made to act. The muscles acting upon two fixed points, namely, the elbow and foot, render their tendons tense and stretched. When the impetus is given by which the load is started, great strain is thrown upon them and their check ligaments, by which they are extended; and this extension, acting upon inelastic structures, causes some of the fibres to give way-to become ruptured. Repeated stretching produces a degenerated condition of the fibres, by which they are rendered easily lacerable.

I do not hold that it is always necessary to have actual rupture of the fibres to constitute a sprain of a tendon or ligament. Inflammation can occur in them, non-vascular as they are, without this lesion; and extension, although not producing rupture, is sufficient to produce the inflammatory condition, more especially at the points of insertion into the bones.

An additional cause is found in the kind of shoe that carthorses wear in some parts of the country, shoes with heavy toepieces welded across them. These very materially increase the resistance to the action of the flexors, by adding to the obstacle to be overcome, that obstacle being the foot placed on the ground with the weight of the animal upon it. The foot is elevated upwards and backwards, the last part to leave the ground being the toe; and as it does so it is made to describe the segment of a circle: we therefore find that the toe of the shoe is rounded off as it wears. It will easily be understood how any additional resistance to this last act in its elevation becomes a source of disease in the structures which perform it. No one can deny that toe-picces enable the horse to have a stronger 
hold on the ground, and thus prove an advantage when very heavy loads are to be moved, more especially in hilly districts, but this very advantage of grip becomes a cause of lameness, from the fact that it has to be overcome by the active organs of locomotion.

When a ligament or tendon is inflamed, an exudation takes place both within and external to its substance. That within the structure, or the inter-fibrous exudate, separates the fibres from each other, causes them to diverge from the straight line, and thus the ligament or tendon becomes shortened; hence we find that in this strain a horse is unable to bring the heel of the foot to the ground. This shortening, particularly during the early stages, is increased by the muscles being kept in a contracted condition, to relieve the inflamed part from tension; but after a time descent of the heel is rendered an impossibility by adhesions and actual shortening from organic change in the non-active structures (the tendons and ligaments) of locomotion.

Symptoms.-There will be heat and swelling in the part; very often, during the earlier stages, the ligament can be felt swollen, prominent, and bulging, the tendons themselves being quite normal; when pressed upon, the horse evinces pain; stands with the leg upright, and moves it stiffly, digging his toe into the ground. When in the hind leg, flexion is very imperfectly performed; he seems to throw the limb behind him as he lifts it from the ground, and the fetlock and hock are not flexed as in sound action. When the tendons themselves are involved they will be found swollen upwards and downwards from the seat of the original injury; and this extension of the swelling prevents their gliding through their thecæ, particularly the thecæ situated in the carpal or tarsal fossæ.

To detect very slight sprains, especially if situated in a hind leg, it will often be necessary to compare the thickness of both legs by careful manipulation, as the swelling can only be detected in that way, on account of the coarse hair and thick skin of cart-horses' legs. In the better bred animal, a difference in the thickness can be easily seen as well as felt.

The special treatment required for this lameness is the application of the high-heeled shoe, in order to throw the tendons into a state of relaxation, and a long period of rest. In chronic 
cases, where shortening has permanently taken place, the operation of tenotomy must be performed.

Many horses with contraction of the tendons will perform very slow work moderately well if a piece of iron is attached to the toe of the shoe, projecting an inch or two in front of it, and slightly turned up at its anterior part. This acts as a lever upon the toe, forcing the heel downwards, and prevents "knuckling over" on the front of the fetlock-joint.

Tenotomy, or division of the tendons, is performed as follows:The animal must be cast with the lame limb lying undermost, which is to be detached from the hobble, and kept in a semi-flexed position by a cord placed round the fetlock. The operator must feel for the anterior edge of the tendon, a little below the middle of the leg. He then must introduce a small sharp-pointed scalpel, flatwise, through the skin and subcutaneous tissue, which occupies the space between the tendons and suspensory ligament, until its point touches the skin of the opposite side of the leg, keeping close to the tendon to avoid dividing the artery. The sharp-pointed scalpel is now withdrawn, and a probe-pointed tenotomy knife introduced in the same marner, until the probe point can be felt by the operator under the skin of the opposite side, when its edge must be turned towards the tendon, and the limb forcibly extended by an assistant. This extension will cause the tendon to press firmly on the edge of the knife, the operator at the same time cutting slowly towards the wound in the skin. The movements of the knife must be slow and cautious (or the skin may be wounded), until both perforans and perforatus are cleanly cut. In many cases it is found that the heel cannot be brought to the ground owing to the presence of old adhesions about the fetlock and pastern joints. If these adhesions do not break from the weight of the animal in two or three days, they must be forcibly extended by the operator placing his knee against the front of the horse's leg, and forcibly drawing the leg and foot forward until the adhesions give way.

In performing tenotomy, a small wound is essential; for if a large one be made, a fungous growth springs up from it, which is difficult to remove, and which sets $u p$ the suppurative action, causes irritative fever, delays the healing process, and leaves an ugly blemish.

The incision through the skin is always made in the fore leg 
on the inner side, and for the reason that the operator can better avoid dividing the artery. In the hind leg, it does not matter from which side the knife is introduced, for the artery is there, unless the operation be very low down, protected by the external small metatarsal bone. In all cases the skin must not be wounded on the side opposite to that by which the scalpel is introduced.

The after treatment is very simple, if the operation has been properly performed; the wound may be pinned, the leg lightly bandaged, and occasionally moistened with tepid water; the horse's head tied to the rack for two or three days; at the end of which time the wound will have healed.

As a rule, I have all the shoes removed before I perform an operation of this kind, and the feet rasped to as near their natural proportions as possible. This is important, as the lame foot is apt to be allowed to grow at the heel to an enormous extent. All this overgrowth must be removed, or deformity of the leg will arise from it.

In a few days after the operation, or in some cases immediately, if no adhesions exist, it will be seen that the horse brings the heel of the foot to the ground, and that the toe is turued up when he is made to move. This need not cause any apprehension; indeed, the only drawback to the success of the operation is the contraction of the new formation uniting the divided ends of the teudons, and the re-assumption of the form, to overcome which the operation has been performed. I always feel sure of a successful operation if the animal continues to walk upon the heel for some weeks afterwards, and I never apply a high-heeled shoe to such a case.

The operation, although promising in itself, and successful in restoring the limb to its natural position, very frequently proves a source of disappointment, from the fact that the reparative material, which is thrown out between the ends of the divided tendons, gradually contracts, whereby the tendons are made as short as before it has been performed. In the hind leg this contraction is the usual consequence, and the tendency to it has to be overcome by a lever at the toe of the shoe. This often arrests, but seldom finally prevents it. In the fore extremity the operation is much more successful, the limb maintaining its natural condition for years afterwards. 
There is generally an amount of thickening left in the leg; but since this does not prevent the free use of the limb, it, need not be interfered with, as it gradually becomes absorbed, and in the course of time the leg will look very respectable.

A horse should not be put to hard work for at least six months after the operation has been performed on a fore leg; if on a hind leg he will require a much longer period.

\section{SPRAIN OF THE SUSPENSORY LIGAMENT.}

The superior sesamoidean or suspensory ligament is a broad strong band of white fibrous tissue, arising from the supra-posterior part of the canon bone, lying in the hollow, bounded on either side by the small canon bones; it extends downwards, bifurcating above the fetlock, becoming attached to the lateral parts of the sesamoids, thence it is continued downwards and corwards to the tendon of the extensor pedis at the anteroinferior part of the os suffraginis, where its divisions become united, and are generally lost in the substance of that tendon; but in the young subject a careful dissection generally shows that it again becomes divided, and that its two divisions travel along the lateral part of the os coronæ, terminating there, and on either side of the pyramid of the os pedis.

Much difference of opinion exists amongst veterinarians as to whether this ligament is elastic or not,-Percivall, Dick, and others maintaining that it is so; whilst the late Professor Barlow, from a careful investigation into its structure, argued to the contrary.

I am quite convinced that the latter view is the correct one, and that the sesamoid bones do not descend and ascend during action in the manner described by Percivall, who says- "In proportion as the pastern is upright in position, less weight is imposed upon the sesamoid bones, more upon the pastern, and vice versa. What pressure or weight the pastern bone receives descends to the coronet, and then to the os pedis. But what becomes of the weight upon the sesamoids? they have no bones below to transmit it to. They are in a somewhat similar situation to the splint bones; they call upon their attaching bands (their ligaments) to support them under their load, and their ligaments do so by yielding, they being elastic, so long 
as force is operating; and the instant it is not, they, through their elasticity, again recover their short lengths, and so raise the sesamoid bones into their places. This descent and ascent of the sesamoids is not to be compared with that imperceptible and disputed motion of the splint bones; on the contrary, it is a demonstrable and beautiful descending and ascending motion; a playing up and down, after the manner of a spring of most elastic and exquisite workmanship, imparting at every step the horse takes its anti-concussive influence to every part of his frame. Watch the long and elastic fetlocks of the Arabian or race-horse as he is cantering upon turf, and at every bound he makes will the tufts of hair from those joints be seen dipping upon the ground, though, while the animal is standing still, they may be some inches removed from the surface; nothing can evince more beautifully and plainly than this the movements of the fetlocks.

" The attaching band or ligament upon which the sesamoids repose in their descent, and depend for their re-ascent, is the suspensory. And in order to show what proportion of the superincumbent weight these bones sustain, or rather how insufficient the pastern is without their aid to support the burden, we have only to cut the suspensory ligament through; that done, the horse is in real truth broken down; the feet slip forward and the toes turn up, while the fetlocks bend down upon the ground. Not so after division of the flexor tendons; we occasionally sever them by way of remedy for knuckling over, and all that results in the enabling or forcing the animal to set his heel upon the ground. Once divide the suspensory ligament, however, and no power left is able to sustain the pasterns erect. The suspensory ligament is therefore one of the main-springs of the machine in action; one of the chief of those beautiful contrivances which, while they save the legbones from being smashed to pieces under the weight and shocks they have to sustain at every bound and leap the animal makes, insures his rider ease and safety to his journey's end."

This quotation is very eloquent, but unfortunately the idea it conveys is incorrect in every particular. In the first place, the sesamoids do not sustain any part of the weight of the animal's body, being placed external to the bony skeleton, having no bones either immediately above or below, being, in 
fact, muscular appendages, like the patelloe and naviculares, by which leverage power is given to the muscles whose tendons are inserted into or play over them. They are thus entirely different from the small splint bones, which bear concussion, being placed beneath other weight-bearing bones, and add to the bulk of the lower part of the knee, without increasing its weight to any great extent, which would be the case if the leg were in one piece; and by being attached by inter-osseous ligaments, not elastic ones, as Percivall maintains, deaden and modify concussion much more effectually than if the union were entirely bony.

$2 d$. The suspensory ligament is not to any extent elastic, and is for the purpose of keeping the sesamoids in their position to prevent their descent in the same way as the inferior suspensory ligament prevents their ascent during the movements of the tendons; and also, by keeping the sesamoids firmly pressed against the fetlock-joint, it forms a brace or stay, which prevents over-extension of the fetlock and elevation of the toe, to which there is always a tendency from the pressure of the animal's weight.

$3 d$. The sesamoids do not descend and ascend, as described by Percivall, and even if the suspensory ligament were elastic, this movement in the bones would be an impossibility, since they are firmly attached to the tuberosities on the lateral aspects of the upper part of the body of the os suffraginis by the internal and external lateral sesamoidean ligaments, and to each other by the inter-osseous. In fact, they are pulleys, over which the tendons play like ropes, enabling them the more easily to lift the feet from the ground.

How is it, then, that the fetlock pad comes nearly to the ground at every stricle of the race-horse? It is because the whole limb is pressed downwards upon the fetlocks, pastern, and cotfin bones, which are placed obliquely under the animal's body; and this seeming descent of the sesamoids is simply an increase of this obliquity.

Dissections, and observations on the limb of the living animal, have convinced me that extension at the fetlock-joint is very limited, and that it is an impossibility to increase the obliquity of the pastern, even by very great pressure upon the fetlockjoint, while the suspensory ligament is uncut, and that the in- 
crease of obliquity which is seen during rapid action is due to the movements of the os coronæ on the pedal bone, the articulation between them admitting of extension to a much greater degree than any of the other phalangeal articulations. If it be carefully noted, the movement between the os suffraginis and metacarpal is almost confined to flexion; that between the suffraginis and coronæ very limited in every way; whilst the formation of the inferior articular extremity of the os coronæ and the articular surface of the coffin bone admit of both flexion and extension, the first movement being more limited than the latter. To prove this by analogy, let the experimentalist press a table with the bulb of his finger, and it will be seen at once that the distal is much more capable of extension than the rest of the phalangeal articulations. The comparison is a good one, as the articulations of the human fingers and those of the pastern of the horse are very much alike. It is a necessity to the well-being of the animal that the greatest adaptability to extension should be at the extremity of the limb, as the greatest pressure is there; in fact, the pastern is a lever, extending from the fetlock to the foot, and the greater the length it has, the more oblique it will be.

The limb not only descends at the pastern during motion, but it also ascends at the shoulder, where the body is suspended upon it by the seratus magnus and other muscles. We have thus a spring below and a spring above,-a wonderful and beautiful provision to destroy the effects of concussion, which would be fatal to the animal when all the weight of his body is thrown upon the fore extremity during galloping or jumping. The hind extremity, unlike the fore one, is attached to the trunk by means of an articulation-the hip-joint-because the forces that produce concussion are not applied to it in the same degree as to the fore one; the impulse of rapid pace throwing the weight from the hind upon the fore extremity; hence it is that one spring, namely, the inferior one, is sufficient.

The suspensory ligament being to all intents and purposes inelastic, and the sesamoid bones firmly bound to it, to the fetlock, and to the pastern, assists in supporting those parts of the limb which are below it, namely, the pastern and the foot, by forming a brace or stay, exactly in the same way as a 
rope fixed in like manner would do. Without this aid, the power of the flexor muscles would be quite insulficient for the purpose, and without the suspensory ligament the horse's toes would be turned up, particularly while the muscles were inactive; but with its assistance they are enabled to obtain that repose which is so essential to the well-being of all muscular tissue.

Nothing proves this staying or bracing function of the suspensory ligament and sesamoids so much as rupture of the ligament or fracture of the sesamoid bones. Immediately after these accidents, the toe of the foot is turned up; even in such a case the sesamoids are not moved from their position, but the fixed points of the stay are destroyed.

Sprain of the suspensory ligament is of two kinds, namely(1.) An inflamed condition arising from slight causes; and (2.) Rupture, partial or complete, from more violent ones.

In the first form there will be lameness; swelling felt along the course of the ligament; the animal standing and walking on his toe as much as he can, to remove all tension from the ligament; but in the latter form the fetlock pads descend more or less, depending upon the gravity of the lesion.

The rupture may be completely through the substance of the ligament, above its bifurcation, or one of the branches only may be lacerated, above its attachment to the sesamoid bone; or it may be detached from the bone without transverse rupture.

This lesion occurs both in the fore and hind extremities; in the fore extremities of race-horses and hunters, and more commonly in the hind ones of horses which draw heavy loads. It may be in one or in both legs at the same time. I have often seen it in both hind legs of 'bus horses in hilly districts.

The symptoms are descent of the fetlock pads, turning up of the toes, with heat and swelling of the leg or legs. The lameness is often very great, the animal being almost unable to move.

The injury is a very serious one; but with a long rest and proper treatment a horse so injured may become sufficiently sound to perform moderate work for many years. Yet there is always a weakness left, which must be considered an unsoundness, as it may at any time cause lameness, particularly if the animal be called upon to do a little extra work. 
The marks of the lesion always remain in the shape of a thickening, which may be felt close to the large canon bone, or upon any part of the course of the ligament.

Special Treatment.-Patten shoe or shoes, if they can be applied and borne; firm pledgets of tow placed in the hollow of the heel to support the fetlock, maintained in their position by firm bandaging. The tow should be made into a firm roll, the fetlock pad elevated by an assistant, the roll of tow placed under it so as to completely fill up the hollow of the heel, and fixed in that position by a bandage. Other bandages should be placed round the leg as high as possible, to keep the parts together, and thus diminish the breach to be healed; cold applications applied until the inflammatory symptoms have subsided, and the parts firmly reunited, when a blister may be rubbed on to remove the thickening which remains. A long rest is essential, and if the patient will not lie down the slings are to be used.

In some cases of injury to the foot, the absorbents of the leg become swollen and inflamed. This must not be confounded with tendinous or ligamentous injuries.

\section{SPRAIN OF THE FETLOCK-JOINT.}

The fetlock-joint is liable to inflammation of its synovial membrane from a variety of causes, the most frequent being sprain of one of its lateral ligaments. In comparison with other forms of lameness, this is rare, though, from the frequency of fracture of the upper extremity of the os suffraginis, entering into the composition of the joint, one would be inclined to conclude that the concussion which so often produces fracture would, when applied in a more limited degree, be a frequent source of inflammation; but such is not the case. It may be here observed that squareness of the fetlock, obtained by well-developed tuberosities on the os suffraginis, is much to be desired, a round fetlock being very objectionable. At the same time, it must be remembered that their very squareness renders them more liable to fracture, because the large upper extremity of the suffraginis in a square fetlock is, as it were, unsupported by the body of the borie.

In inflammation of the fetlock-joint, from whatever cause, 
the lameness is characterised by, on the part of the animal, inability to flex it, by heat, swelling, pain on pressure, and more or less pointing of the foot.

\section{SESAMOIDITIS.-INFLAMMATION OF THE SESAMOID BURSA.}

The posterior part of the sesamoid bones is covered by cartilage, forming a groove, over which the flexor perforans plays. Both the cartilaginous pad and the tendon are liable to injury, which causes severe and obstinate lameness.

Sesamoiditis may also arise from an injury to the inferior part of the suspensory ligament-whether involving it above the bifurcation or confined to one branch only-by extension of the inflammation; and is oftener met with in hunters than in any other class of horses.

Sesamoiditis is not so often found in the fore as in the hind extremity, and is rather difficult to diagnose, owing to the accompanying swelling being very limited in extent.

The pathology is as follows:-

1st. Inflammation of the bursa, owing to sprain or other injury.

$2 d$. Increased secretion into the synovial cavity, causing a bulging of its capsule upon either side of the flexor tendon.

$3 d$. Ulceration of the cartilage of incrustation, softening and laceration of the fibres of the tendon, and, finally, adhesion between the bones and tendon,

$4 t h$. When arising from sprain of the suspensory ligament there will be thickening of the ligament or of one of its branches, associated with that loss of cohesion in its structure which seems so characteristic of inflamed ligament, as manifested by the animal going upon the heel, and descent of the fetlock pad.

Symptoms.-Lameness, the horse, unless the suspensory ligament be involved, going on his toe; heat at the back of the fetlock, with swelling of the bursa. This swelling is different in its character from a mere dropsy of the fetlock joint or sheaths of the tendons; in sesamoiditis, although it fluctuates upon pressure, it feels tense, the capsule being fully distended with fluid; whereas in a common wind-gall it is soft, and easily pressed from one side of the leg to the other. This difference in the character of the two enlargements must be remembered. 
The hard enlargement of sesamoiditis is an unsoundness; the soft yielding swelling constituting wind-galls is never reckoned as such.

This bursa is often the seat of rheumatism.

There is a speciality about this lameness which Professor Dick was very particular in describing, namely, that a horse, after a period of rest, may trot quite sound for a short time; that he is liable to fall lame again with very moderate work; and that the lameness is most obstinate to remove.

An injury to the inferior part of the suspensory ligament, whether involving it above the bifureation, or confined to one branch only, may cause sesamoiditis by extension of the inflammation; but, as a rule, it is due to sprain of the tendon or injury to the cartilage of incrustation.

The special treatment consists in the application of a high-heeled shoe, and if the lameness be in a lind limb and very severe, placing the animal in slings, and the constant application of cold water. After the subsidence of the inflammation, the absorption of the enlargement is best effected by the use of Mr. Broad's truss.

The inferior suspensory ligaments of the sesamoid bones are occasionally sprained, causing great lameness, the animal going on the toe, with swelling in the hollow of the heel.

In some rare instances, the ligamentous structures in this part of the limb have been converted into osseous material (osteophytes), forming complete rings round the tendons.-(See Photo-lithograph, Plate I., Fig. 4.)

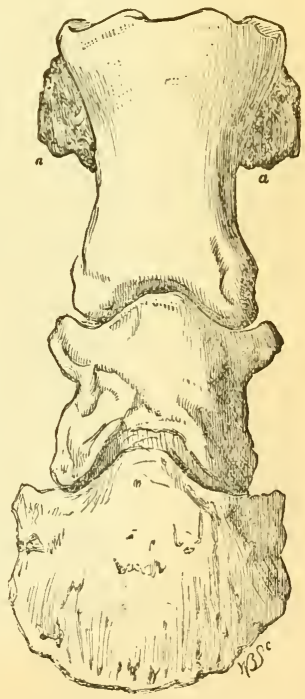

Fig. 44.-False ring-bone, shown at $a, a$.

RING-BONES.

This term is applied to the osseous deposits which are found upon the upper and lower pastern bones, but with 
more propriety to those which take the form of a ring round the bone.

Ring-bone is of two kinds, true and false. The false ringbone may be dismissed in a few words; it is an exostosis, situated about the middle of the os suffraginis, due to an increased development of one or both of the roughened ridges on

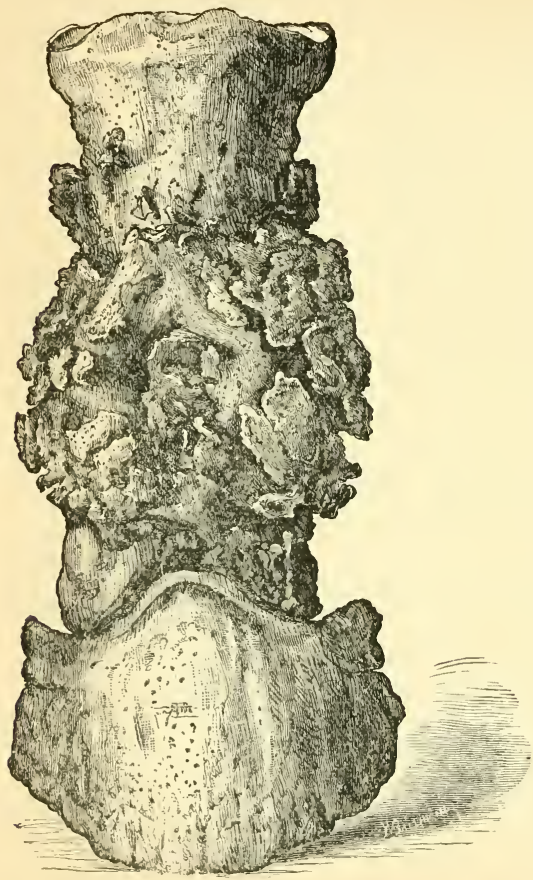

Fig. 45.-High ring-bone.

this bone, which give attachment to the sesamoidean ligaments. When very large, it may cause lameness; as a rule, however, it never gives inconvenience to the animal, and is not always to be looked upon as a cause of unsoundness. It may be compared to a splint thrown out for some beneficial purpose.

The true ringbone is quite another matter, and is an unsoundness in every sense of the word, involving important articulations, and giving rise to obstinate, and often incurable, lameness.

There are two kinds of true ring-bone, the high and the low ; it is called high when it involves the proximal inter-phalangeal articulation or pastern joint; and low, when it involves the distal inter-phalangeal articulation or coffin joint. In many instances both forms exist at the same time. Photo-lithograph, Plate III., Fig. 5, is a good illustration of this. 
Ring-bone must not be confounded with what is termed sidebone; the first is a bone disease, the second a disease of fibrocartilage.

Ring-bones, whether high or low, vary in size; but the degree of lameness does not depend upon the mere size of the new formation. An animal may be very lame, indeed, with but little osseous deposit; and another may show but little lameness, with a very large ring-bone.

Very often the segment of the ring is defective, and the deposit may appear only on one surface of the limb, or on both sides without any prominence in the front. When at the sides, they do not cause the same degree of lameness as when the front is involved.

ling-bones are not the cause, but the result of disease, being the effect of an inflammation originating in the extremities of the bones, or synovial membranes of the articulations which they involve. As a rule, they are the result of ostitis, commencing in the cancellated structure of the bones, the areole of which first became filled with an organizable lymph, convertible into bony material, as already described.-(SeeOstrtis, p.151.) During the progress of the inflammation, the articular cartilage and laminal layer of the end of the bones become removed by absorption, while external to the joint active deposition of bony material is going on, for the purpose of repairing the damage within.

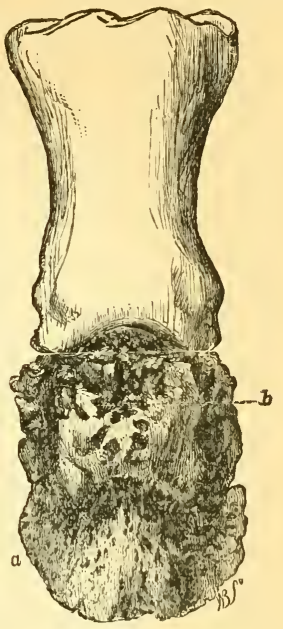

FIG. 46.-Lowering-bone.

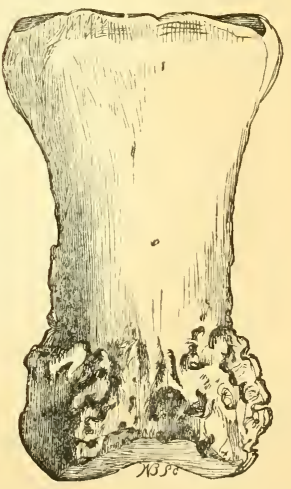

Fig. 47.

In some instances the disease may commence at the inferior extremity of the os suffraginis, and gradually involve the 
articulation and os coronæ. The accompanying figure (Fig. 47) illustrates this. When involving either articulation, ring-bone always causes lameness at its commencement; but when anchylosis is completed, such lameness may almost, if not entirely, disappear.

The lameness of ring-bone precedes the deposition of bony matter, and is due to the presence of inflammation in the bones or synovial membrane.

The gait of a horse lame from this cause is characteristic. If in the fore extremity, except the deposit be on the posterior aspect, the patient puts his heel to the ground first; but when in the hind pastern, the toe touches the ground first always when it is situated in the upper position, except it be in front; when in the lower position, the heel comes down first. From this peculiarity in putting the foot to the ground, it is apt to be confounded with laminitis, seedy-toe, and inflammation of the coronary band. It differs from laminitis by the absence of pain at the toe, freedom from fever, \&c., and by the heat being confined to the upper part of the foot only.

An examination of the foot will determine whether there be a seedy-toe or a sand-crack in its front; and the absence of the striated appearance of the wall of the foot will distinguish it from inflammation of the coronary substance.

Percivall, writing in the year 1823, has thus classified 150 specimens of ring-bone. He, however, includes anchylosis of the fetlock-joint under the term:-

5 complete anchyloses of the fetlock-joint.

18

" $\quad$ " pastern-joint.

The other specimens were simply encrusted, more particularly around their extremities, with layers of new bone, or were variously deformed with exostoses of different shapes, many of which were very large, and several of them confined to one side.

My own observations upon, and examinations of, specimens enable me to agree with Percivall, and to differ from Professor Dick and others, who maintained that the lower form was the most common.

'The causes of ring-bone are hereditary, structural, incidental, and rheumatoid.

Hereditary predisposition is sufficiently proved and acknow- 
iedged; I therefore simply advise breeders of horses never to breed from a sire or dam having ring-bones, unless their origin can be readily traced to some accidental cause.

The structural tendency to ring-bone is manifested in horses with upright pasterns. Percivall explains this in nearly the following words :-

"The pastern and coffin bones constitute the nethermost parts, the pedestals of the columns of bones composing the limbs; and being so, they receive the entire weight and force transmitted from above. The pastern, when long and oblique in position, receives the superincumbent weight in such an indirect line that, bending towards the ground with the fetlock, nothing like jar or concussion follows. The very reverse of this, however, is likely to happen every time the foot of a limb having a short and upright pastern comes to the ground. In it, instead of the weight descending obliquely, it descends directly upon the pastern bones, making them entirely dependent upon the pedal bone for counteractive spring; and should anything occur to destroy or diminish this spring, or to throw more weight, or weight more suddenly, upon it than it can counteract, jar of the whole apparatus ensues; and an effort of nature to strengthen the parts, by investing them with callus and ossification, is likely to be the ultimate result. For we would view ring-bone, disease though it most assuredly must be called, frequently in young horses, as a resource nature seems invariably to adopt whenever the pastern bones and joints are found unequal to the exertions or efforts required of them. And the reason why ring-bone occurs oftener in the hind than in the fore limb will probably be found in the greater stress or strain the hind pasterns undergo in unbacked young horses, particularly in such acts as galloping, jumping, \&c., exercises which they are likely to take of their own accord while running out at pasture."

In the treatment of ring-bone care must be taken to observe how the patient places the foot on the ground. In the fore leg he always puts the heel down first, in order to throw the weight of his body, and the consequent concussion from the column of bones, on to the soft parts. In such a case a thin-heeled barshoe must be put on the foot-the shoe recommended by my friend Mr. Broaa of Bath for laminitis. This gives great relief 
by allowing the animal to throw its weight upon the heels with the least possible effort. I have many cases of ring-bone at the present time, where the horses go moderately sound with such a shoe, that would be unworkably lame if shod in the ordinary way.

If the horse walks on his toe, he must be shod with a highheeled shoe. This only occurs when ring-bone is situated in the hind limbs, and on the sides or back of the pastern; when in front, the horse will go on his heel, and is to be shod with the low.heeled shoe.

Very long pasterns, from the fact that they act too powerfully as levers upon the articulations, are subject to ring-bones; in such cases, I think that they arise from sprain of the ligaments. This occurs, as a rule, in the hind legs, and for this reason, the horse lame from this cause throws his weight upon the column of bones by walking on the toe. Sprain of the flexor tendons, as well as that of the suspensory ligament, both in the fore and hind limbs, are often succeeded by ring-bones. 


\section{CHAPTER XV.}

PARTICULAR LAMENESSES-continued.

HIP-JOINT LANENESS-SPRAIN OF GLUTEAL TENDONS, OR TROCHANTERIC LAMENESS-SPRAIN AND ATROPHY OF CRURAL MUSCLESSTIFLE-JOINT LAMENESS - LACERATION OF FLEXOR METATARSI MUSCLE.

Hip-Joint lameness is of very rare occurrence, except as a result of the scrofulous diathesis in young animals, and of rheumatism in those of mature age. Sprain of the hip-joint is very rare indeed, but it is possible; and when inflammation of the joint occurs from this cause, the same pathological changes are observed as in other joints, namely, redness of the synovial membrane, exudation into the cavity, and if not arrested, ulceration of the articular cartilage and laminal layer of the bones. In rheumatoid disease, the tendency is to the formation of the porcellaneous deposit within, and bony vegetations around, the articulations.

Lameness in the hip, however, is not at all an unfrequent occurrence; still its seat is not the joint, but the head of the trochanter major of the temur.

"The trochanter major is a very large eminence which looks outwards and upwards, and presents, posteriorly, a prominent part, termed the summit, which stands a little higher than the articular head (of the femur), and gives attachment to one of the heads of the gluteus maximus; and anteriorly the convexity, which is rounded and covered externally by cartilage of incrustation, which forms a bursa, over which plays the other tendon of the gluteus maximus, which becomes inserted into the ridge just below."-(Strangeways' Anatomy.)

The above quotation will enable the reader to understand that the trochanter major is a very important protuberance; that it 
is a very strong lever, to which the tendons of powerful muscles are attached; that it has to assist extensive movements, and, in accordance with the importance and extent of its functions, it is liable to injury and disease.

Sprain of the tendon of the gluteus maximus gives rise to inflammation of the synovial bursa on the convexity of the trochanter, as well as in the substance of the tendon. The summit is liable to injury from blows or falls, and the inflammation so produced extends to the convexity. From whatever cause the inflammation arises, and whether it originates at the

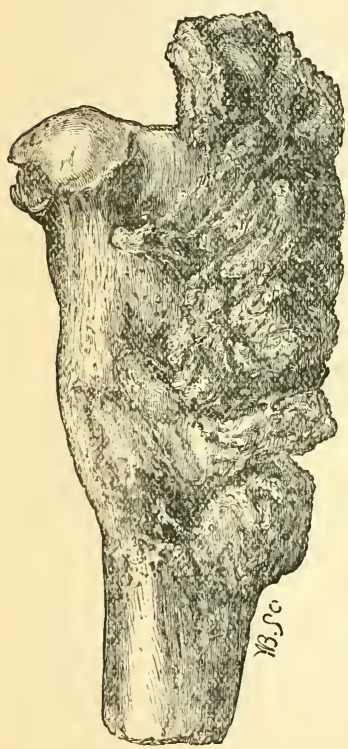

Fig. 48,-Disease of trochanter major, described in the text. summit or the convexity of the trochanter, the exudate which is formed is apt to be converted into osseous material, and the cartilage of incrustation on the convexity to be removed by ulceration.-(See Fig. 48.)

Violent inflammation of the hip-joint is accompanied by very severe synuptoms; and the animal will stand almost immoveable, with the foot raised from the ground, in which position it will be steadily maintained, unless he is forced to move; all movement increasing pain to an agonising extent. There will be fever, loss of appetite and flesh, rapid wasting of the quarter, and inability to lie down. In such a ease the animal must be slung.

The dissection of such cases generally reveals ulceration or laceration of the ligamentum teres, ulceration of the articular cartilage, both on the head of the femur and cotyloid cavity, with suppuration into the cavity of the joint, or partial destruotion of it by a deposition of bony matter. I have never witnessed anchylosis of this joint, but I have a specimen where the acetabulum is almost filled with 
bony material. If the severe symptoms do not soon abate, the practitioner will understand that the above pathological changes are going on, and that the horse will remain incurably lame.

The symptoms of a milder form of hip-joint lameness, and of that arising from disease of the trochanter, do not differ in any peculiarity of gait. There is a hop and a catch in the lame limb, and a want of movement in the quarter, which to a practised eye is quite suggestive. The whole of the quarter on the lame side is elevated with as little motion of the hip as possible; the other articulations being flexed with ease. While standing, the foot of the lame limb in severe cases will often be elevated from the ground; the muscles below the seat of disease exerting themselves, as it were, to support the weight, and thus allowing the gluteals to be in a state of relaxation. In hipjoint lameness heat may be felt, and pain caused, in some cases, by pressure applied per rectum. In trochanteric lameness, a distinct swelling can be detected, both by touch and sight, upon the quarter. In both forms, atrophy of the muscles of the quarter soon manifests itself, and is apt to lead the practitioner to confound the disease with fracture; but in disease the wasting and inequality of the quarter appear after the animal has been lame for some little time, whilst in fracture they are seen immediately after the displacement of the bones.

The swelling of trochanteric lameness is of an irregular roundish shape, on the very point of what is erroneously called the hip-joint; hot to the touch, and painful upon pressure. To see it distinctly, the practitioner should stand both behind and at the side of the patient, as the shades of light sometimes hide it from observation.

In the treatment of either form, the high-heeled shoe is to be put on the foot, to enable the parts to be maintained in a state of repose. The patient requires a long rest, since the lameness is very apt to return; and in this, as well as in other lamenesses, treatment should be resorted to, and rest imposed, before alteration of structure has taken place. Even when all sigus of lameness have disappeared, great care must be taken not to work the animal too soon.

The horses most subject to this lameness are those which draw heavy loads, and care must be taken that they are not forced 
to this too soon after apparent cure, or it will most assuredly cause a return of the lameness. Another fact should be remembered, namely, that a high toe-piece on the shoe, by increasing the resistance to the action of the muscles of the limb, may cause this, as well as many other forms of lameness.

\section{SPRAIN AND ATROPHY OF THE CRURAL MUSCLES.}

That mass of muscular structure known as the crural muscles is liable to sprain. It consists of the rectus femoris, arising from the ilium; the vastus externus and internus, arising from the superior part of the femur; and the crureus, arising from the lower half of the femur. Their muscular structure is very intimately blended, and they finally become inserted into the superior surface of the patella. Their action is to extend and elevate the thigh, and advance it under the body.

The symptoms of injury or sprain of these muscles are inability to extend the stifle and flex the hock, in fact to draw the limb forward; dragging of the toe when the patient moves; when standing still, the patella seems to have slipped down, and there is knuckling over of the fetlock-joint, the whole limb being posterior to its fellow. After a time the muscular structures become atrophied, individual muscles become prominent, and a concavity presents itself between the anterior spine of the ilium and the patella, and this atrophy is seen most markedly after azoturia.

Since the introduction of tramways, this form of injury has become more common, owing to horses slipping on the rails. This form of lameness requires long rest.

\section{STIFLE-JOINT LAMENESS}

Is of two kinds, namely, that within the joint proper, involving the condyles of the femur, the semi-lunar discs, and articular head of the tibia (see Photo-lithograph, Plate II., Fig. 3); and that in the patella articulation. The pathology of both forms is alike-inflammation, ulceration of the articular cartilage and of the semi-lunar dises, when the joint proper is involved, of the deposition of the porcellaneous deposit, both in the cartilage and Haversian canals of the bones, when caused by rheumatoid disease.

In stifle-joint lameness, the limb, when the animal stands, 
is generally bent, the thigh flexed upon the pelvis, and the leg upon the thigh, so that the articular surfaces of the bones are separated, and prevented from pressing against each other; but when made to move, the relative positions of the various bones are altered, and, instead of being flexed, the stifle is maintained in a rigidly extended state. In this manner the joint is locked, its movements suspended, and, as a result, the heel of the foot touches the ground first. This is more especially the case when the posterior part of the joint is diseased.

In lameness from disease of the bursa-patellæ, the horse generally walks with his toe dragging the ground, as if he were afraid to bring the rectus femoris and vasti muscles into action to elevate the patella on the trochlea of the femur; the forward movement of the limb being performed with great difficulty, the toe of the foot describing the segment of a circle at each step. These symptoms are, however, not constant; some cases of well-marked patella lameness put the heel to the ground first. But in all instances the position while standing is that of semi-flexion, this being the best calculated to insure separation of the various articular surfaces, and prevent pressure.

Dissection of stifle-joint lameness reveals a condition of inflammation of the synovial membranes, of the condyles of the femur, and the articular head of the tibia; degeneration into shreddy masses of the semi-lunar dises, laceration of their ligaments, removal of the articular cartilage, or its calcification and removal by friction.

When confined to the bursa-patellæ, the alterations of structure consist in the removal of the articular cartilage from the patella and trochlea of the femur, and thickening of the synovial membrane from interstitial inflammatory exudation.

The bursa-patellæ is situated superficially, and when inflamed the increased secretion poured into it causes a swelling in front of the joint. This swelling must not be confounded with an apparent enlargement of this part, seen in the healthy stifle when the animal stands with the limb in a semi-flexed easy position, when "he stands resting it," as it is commonly called. The swelling arising from disease is persistent, hard, and prominent when the limb is extended; but the enlarge- 
ment arising from the parts being thrown into a state of rest and relaxation is soft, and disappears when the horse is made to stand firmly.

Professor Dick said that stifle-joint lameness could be detected by pressing upon either side of the internal lateral ligament, and that a swelling could be felt caused by the distension of the capsular ligament with synovia. I have never been able to confirm this, and have been compelled to be satisfied with negative symptoms and the gait.

Relaxation of the patella arises from sprain and inflammation of the lateral ligaments, which thus become elongated and allow of partial dislocation of the patella. The symptoms and treatment are similar to those recommended for luxation (described at page 143 , which see).

The muscles of the stifle are subject to cramp, the symptoms being very similar to those of dislocation of the patella, the limb being extended and fixed to the ground. It is also often seen in dogs. It comes on and goes off suddenly. I have known such to arise from indigestion, and the liability to its return to be completely removed by one or two doses of purgative medicine and alteration of diet.

Subcutaneous laceration of the flcxor metatarsi muscle is occasionally a cause of lameness. Irofessor Dick had seen something of it. He used to refer to it in his lectures as presenting symptoms similar to paralysis of the gastrocnemii. He used to say that when the flexor metatarsi muscle, any muscles situated at the anterior part of the stifle or the gastrocnemii were injured, the tendo-achilles fell into a relaxed condition when the limb was elevated from the ground.

Figure 49 is from life, drawn by Professor Vaughan, when a student of the College. The horse was an ill-looking long-legged cart-horse, very subject to rheumatic attacks, for which Mr. Cunningham of Slateford had repeatedly attencled lim. Towards the end of 1871 he was found one morning to be very lame, and when he attempted to move, the near hind leg was thrown upwards and backwards with great violence; at the same time the tendo-achilles was seen to fall into a number of folds. Upon the limb being elevated by the hand, the same seemingly powerless condition of the gastrocnemii was observed. The limb was swollen above and in front of the 
hock, midway between it and the patella. The horse being old and not of much value, the owner, at the suggestion of Mr. Cunningham, sent him to the College for dissection, when it

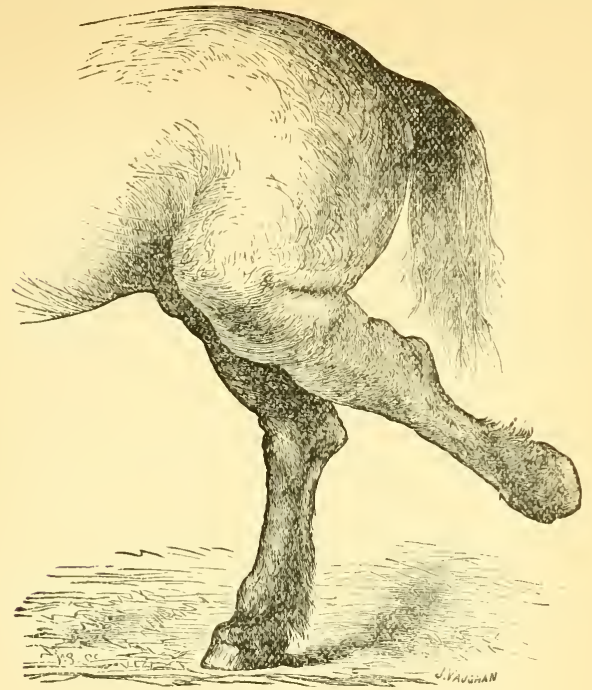

FIG. 49.

was discovered that the flexor metatarsi was lacerated across its whole thickness, at a point corresponding to the extemal swelling; its fibres were pale, and when examined under the microscope their tranverse strix were nearly, and in some places entirely, absent, showing that the sarcous elements were undergoing degeneration. I have met with other instances of the same lesion, but never had the opportunity of making a dissection of the parts prior to this. As to the probability of repair, I think that, provided the animal be not too old, the chances of recovery are very good, and that in the course of time reunion of the divided muscle may be expected; but should the horse be old, or of a weak constitution, the probabilities are that the rupture is a result of degenerative disease of the muscular tissue, and not a mere accident, and 
that the process of repair will be very doubtful and unsatisfactory.

This peculiar lameness is not always aue to rupture of the flexor metatarsi muscle, as in the case just described, for it sometimes happens that its tendons of insertion become detached from the tarsal and metatarsal bones. Such cases are diagnosed by the peculiar gait and the seat of the swelling.

Some few years ago there were three cases of sprain of this muscle in Edinburgh at the same time, two in the College practice and one in Mr. R. Rutherford's. 


\section{CHAPTER XVI.}

\section{PARTICULAR LAMENESSES-continued.}

DISEASES OF THE HOCK-BOG-SPAVIN-BONE-SPAVIN-CONFORMATION OF HOCK MOST SUBJECT TO SPAVIN-THOROUGH-PIN-CAPPED HOCK-INJURIES TO GASTROCNEMII MUSCLES-CURB.

\section{DISEASES OF THE HOCK.}

THE tarsus or hock corresponds to the ankle-joint of man. Its diseases may be divided into three classes, namely-(1.) Diseases of the true hock-joint (that composed of the tibia and astragalus); (2.) Diseases of the gliding articulations formed by the cuneiform bones; and (3.) Diseases of its ligamentous and tendinous structures.

\section{DISEASES OF THE TRUE HOCK-JOINT.}

Bog-Spavin.-A bog-spavin, when caused by inflammation of the joint, is a tense, fluctuating swelling, accompanied by heat and pain. In such instances it is an unsoundness indicating a change within the textures of the joint. There are bog-spavins, however, which may not be looked upon as constituting unsoundness, being mere dropsy of the articulation, arising from some fault of conformation. Professor Dick maintained that such collections were "mere stores of synovia for lubricating the joint, thus proving the best preventatives of disease, by preventing friction." These kinds of bog-spavins are most frequently found in very upright or in very crooked hocks, and in weak hocks of any description; because in all these forms concussion is most severely felt, and exertion is likely to be injurious. In all probability the hyper-secretion of synovia is due to some 
irritation not amounting to actual disease, and may be viewed as a provision of nature to fulfil the purposes ascribed to it by Professor Dick, since it often happens that such hocks remain sound as long as those of a better conformation. They should, however, be looked upon with suspicion, and the practitioner ought at all times to satisfy himself by a severe trial that such loocks are able to bear a reasonable amount of exertion; that the swelling does not increase after such test, and that the animal be neither stiff nor lame in the slightest degree.

Inflammation of the true hock-joint may be acute or chronic. In the acute form the lameness is very great, with fever, loss of condition, and inability to put the foot to the ground. It is apt to terminate in ulceration of the articular cartilage and a partial anchylosis of the joint.

A very constant condition of the joint in the chronic form of bog-spavin, and probably the cause of it, is the gradual conversion of the articular cartilage into calcareous matter-the ivory or porcellaneous deposit.- (See Photo-lithograph, Plate II., Fig. 5.) This condition is associated with the rheumatoid diathesis; and not only is there a deposit in the cartilage, but also in the synovial fringes, which are converted into hard rounded nodules of calcareous matter. Common inflammation of a joint generally tends to ulceration and anchylosis; the rheuinatoid, to calcification of the articular cartilage and synovial fringes.

The sulci of the lock-joint should not be confounded with ulcers.

When articular cartilage has been calcified, or converted into the ivory-like deposit, it no longer prevents the effects of the friction arising from the movements of the bones upon each other. A careful examination of Photo-lithograph, Plate II., Figs. 3 and 5, will at once demonstrate this, for it will be seen that the articular heads of the bones are worn down, leaving well-marked, and, in the hock, deep grooves upon them. The polished appearance given to the diseased part was attributed by Professor Dick to the effect of friction on bone otherwise unchanged; but a more careful examination has shown that it is due to an actual formation of a very lard, earthy matter in the Haversian canals, for the purpose of counteracting and limiting the effects of friction, and that 
its glistening, polished appearance is undoubtedly due to the friction.

What is termed a blood-spavin is simply a distended condition of the vena-saphena, caused by the pressure of a bursal or bony enlargement. It has been described as a disease of the vein, and in some rare instances a varicosity has actually existed.

In rheumatoid diseases of the hock-joint, the crackling sound caused by the first movements of the articulation, already described, has been noticed by some veterinarians. Thus, the late Mr. Farrel of Dublin was able to prognosticate the incurability of hock-joint disease when it was accompanied by that sound.

What is commonly called a "sprung hock" is an enlarged and inflamed condition of the tarsus generally, involving the struc-. tures of the whole articulation, arising from severe sprain, and in some instances associated with fracture of one or more of the smaller bones.

This injury causes extreme lameness, and fever; prevents the animal from lying down and taking its natural rest; and is thus a cause of rapid loss of condition, and sometimes of deatl.

The special treatment is that calculated to allay irritability, fever, and the local pain; and in order that the animal may obtain some degree of rest the slings are essential; and when the acute symptoms have subsided, repeated applications of the biniodide of mercury ointment to stimulate the absorption of the callous enlargements, and perhaps setoning or the actual cautery to remove any remaining lameness.

\section{DISEASES OF THE GLIDING ARTICULATIONS OF THE HOCKS.}

Bone-Spavin.-Bone-spavin is a very common cause of lameness and unsoundness, and it is of importance that a veterinary surgeon be a good judge of a hock; for what may be a spavin in one horse may be a mere peculiarity of conformation in another.

Definition.-The derivation of the word spavin is buried in obscurity; but it is thought by some to be derived from the Italian spavenio, a disease of horses-an unsatisfactory explanation. I find, however, that Rokitansky describes a lamellar bony tumour as "spavined;" possibly the word may have some connection with that form of exostosis. A bone-spavin may be 
defined to be an exostosis on the inner and lower part of the hock, arising from inflammation of the cuneiform and metatarsal bones, terminating generally in anchylosis of one or more of the gliding joints of the hock.

Why spavin should generally appear on the inner, and but rarely on the outer side of the hock, can be explained by satisfactory reasons. 1st. The inner side of the limb is more under the central line of gravity: $2 \boldsymbol{l}$. From the arrangement of the

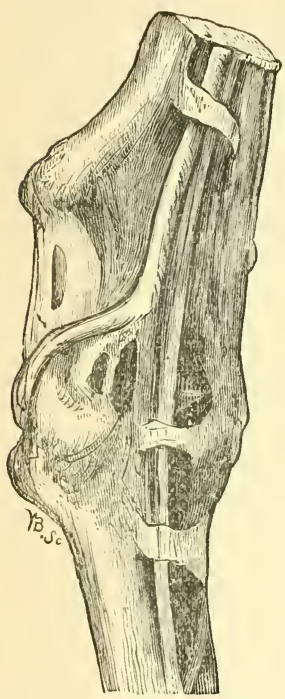

FIG. 50.-Showing situation of bone-spavin, a shallow groove being left in the ossific deposit for the passage of the oblique tendon of the flexor metatarsi, immediately under which the spavin is situated. cuneiforms, and especially the direction of the articular surface of the astragalus, the hock is so constructed that when the limb is flexed the pressure and consequent concussion is thrown on the inner side. Professor Goodsir maintained that a screw-like motion of the true hockjoint, acting unfavourably on the cuneiforms and metatarsals, was the cause of spavin. Professor Barlow said spavin was due to compression of the cuneiforms during extreme flexion. Other pathologists maintain that concussion, produced when the foot comes forcibly to the ground, is the sole cause. It is not my intention to discuss which theory is correct. In all probability they are all more or less so.

Bone-spavin is but very rarely found on the outer side of the hock. Percivall says that he would not be sure "that an exostosis upon the outside would be called a spavin, and that it is commonly the result of injury;" but I have a specimen in my possession, presented by Mr. Stevenson of Newcastle, with a spavin in this unusual position, originating from no apparent cause, and which produced an incurable lameness; but I have seen several cases from which the lameness entirely disappeared.

Spavin arises from causes that are hereditary or constitutional and local. 
The hereditary predisposition to bone-spavin is beyond doubt, all writers of authority being agreed upon the point, which is also well known to breeders of horses. This hereditary predisposition is not always due to peculiarity of conformation, as many breeds or families of horses with well-formed hocks often become unsound from this cause. Peculiarity of conformation is nevertheless not only hereditary, but is of itself a predisposing cause of spavin.

Percivall says-"I am very much disposed to believe in the existence in the system of what I would call an ossific diathesis. I have most assuredly seen unbroke colts so prone in their economy to the production of bone, that, without any assignable outward cause-without recognisable injury of any kind-they have at a very early age exhibited ring-bones, and splints, and spavins. There might have been something peculiar in the construction of their limbs to account for this; at the same time there appeared a more than ordinary propensity in their vascular system to osseous effusion. Growing young horsesand particularly such as are what is called "overgrown" -may be said to be predisposed to spavin, simply from the circumstance of the weakness manifest in their hocks, as well as other joints. When horses whose frames have outgrown their strength, with their long and tender limbs, come to be broketo have weight placed upon their backs at a time when the weight of their own bodies is as much as they are able to bear - then it is that the joints in an especial degree are likely to suffer, and wind-gall and spavin to be the result. Indeed, under such circumstances, spavin, like splint and other transformations of soft and elastic tissue into bone, may be regarded as nature's means of fortification against more serious failures."

These remarks of Percivall are based upon correct observations, and one cannot help admiring the way in which they are put before the reader. There is not one point that I can dispute, unless, indeed, the term osseous effusion, which may not now be looked upon as being consistent with correct pathological knowledge.

The local or exciting causes of spavin are sprains of the ligaments, more particularly the inter-osseous, and concussion of the bones. The old writers ascribed spavins to blows; so unlikely, however, is the seat of them to receive a blow, that there need 
be no hesitation in declaring such an assertion to have been made without foundation. Advantage has nevertheless been taken of it, and one often hears sellers of horses declaring most solemnly that a very prominent spavin is nothing but the result of a kick inflicted before their own eyes, and that it will go away in a few days. But a blow sufficiently heavy to cause such a swelling leaves a mark; and, " not to be done," these gentry often make a small wound on the skin of the enlargement. This is hardly credible, but I speak from actual experience and knowledge of facts that have come under my own observation. Further, I have heard such men confess that such wounds were made for the purpose of accounting for a lameness. The young practitioner must not be misled by such tricks.

One fertile source of spavin is the alteration of the direction of the leg, brought about by the use of high-calkined shoes. These ligh heels alter the relative position of the limb, from the hip downwards, and are a cause of shocks of concussion, which are felt every time the horse puts his foot to the ground during action.

The form of Hock most susceptible to Spavin.-Upon this point there is much difference of opinion amongst veterinary writers; some maintaining that short-pointed, compact hocks are generally spavined; others think sickle or cow-hocks, and hocks "tied in" below; whilst other writers-more especially the advocates of the compression theory-are of opinion that hocks with well-developed calces are most prone to become spavined. These latter base their argument upon the assumption that the point of the hock is the lever of the extremity, and that the parts in front are liable to compression; the extent of such compression depending on the length of the lever. This theory is self-apparently fallacious, as the lever here mentioned acts in a direction contrary to that which would cause compression.

Notwithstanding all these differences, I do not think that there is any kind of hock that can be said to be exempt from spavin, provided a sufficient cause has been applied. Cavalry horses, when the drill and manége exercises were more violent than they are at present, suffered from spavin to a very great exteut. "Since, however, the pace has become moderate, and 
the halts less abrupt, and more time has been given to the cavalry recruit horse for the evolution of his natural powers, the disease has much abated in prevalence."-(PercivalL.)

The hock described as "tied in below" is that form where the head of the metatarsal and cuneiform bones are small compared with the bones above. The cuneiform and metatarsal bones are receivers of weight and distributors of concussion; their capability to the performance of those functions depends upon their size and development, and upon the breadth of surface they present. When not well developed, one cannot fail to see the applicability of the argument that they are predisposed to spavin, and as a rule practice proves its correctness; but there are many exceptions, and one often sees a badlyformed hock remaining sound under very severe tests.

The theory of Goodsir, that the motion of the true hockjoint is like a screw, is well worthy of consideration. I have, by the kindness of Professor Turner, obtained the loan of a cast of several astragali united together, illustrative of the screw-like direction of its articulating surface. The cast was prepared by Mr. Stirling, the curator of the Edinburgh University Anatomical Museum, under Professor Goodsirs immediate superintendence. Being the result of the observations of ono to whom the Edinburgh Veterinary College is so especially indebted, it cannot fail to be interesting.

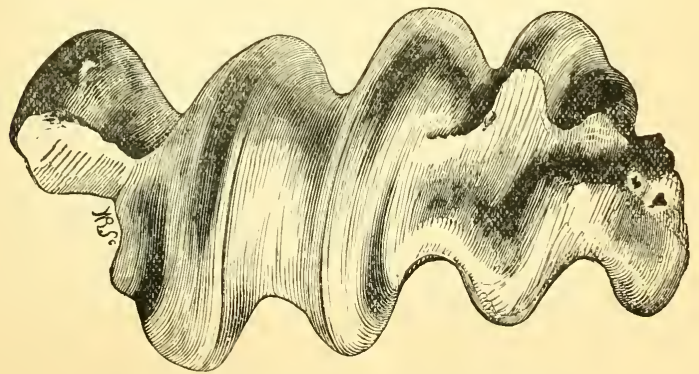

Fig. 51.-Astragali united to show the screw lines.

By carefully examining the oblique pulley-like prominences, and the deep groove between them, on the superior articulating 
surface of an astragalus, the observer will see that the screwlike motion is subject to three deviations. First, the direction is from within outwards for a short distance; then it seems to run perpendicularly; and, finally, the lines of the prominences are seen to curve towards the inmer side and on to the inner third of the cuneiform magnum, the inner prominence of the astragalus terminating abruptly, and leaving a hollow space for the end of the internal prominence (malleolus) of the tibia. This abrupt termination allows the final act of flexion in the lock to be performed with great velocity and suddenness. One can see it in a fast-going, well-actioned horse, giving sharpness and grace to the hock action; but whilst it allows this advantage, it is apt to be a source of mischief, by acting directly upon the cuneiform bones, and proving a source of concussion to them during sharp flexion. This, in addition to the concussion received when the foot comes to the ground, is sufficient to cause inflammation of the cuneiform bones, and spavin.

The relative obliquity and position of the true hock-joint, and the direction of its motion with relation to the production of disease of the lower rows of bones, are subjects well worthy of consideration, and open a fair field of investigation to the veterinary pathologist. Pertinent to this matter, one might naturally ask, Why should there be three rudimentary screws on the surface of the astragalus? The explanation is based upon the fact that the animal must be able to lock the joint whilst asleep on its legs. It has been already explained that the ligaments and the tendinous structure of the muscles of the fore arm enable the fore legs to remain unbent during sleep. In addition to tendinous muscles and ligaments, we find that the posterior extremities (and they are much more liable to bend under the animal's weight, as may be witnessed in cases of debility) are furnished with a lock in the true hock-joint,the lock being the deviation of the screw lines, which enables it to sleep without falling. The remarkable manner in which flexion of the true hock-joint is performed has been remarked by Dr. Graves of Dublin; and a valuable paper by Professor Dick on the subject, and on the conformation of the knee and elbow joints, may be consulted with advantage. It is printed along with his memoirs.

Amongst the variety of hocks that a veterinary surgeon 
examines, what is termed a "coarse hock," is often seen. By this term is meant a hock with well-developed cuneiform bones, giving a prominence to the articulation, very much resembling spavin; and it is generally borne out by experience that such hocks are superior to those of a finer description, standing more wear and tear, and remaining sound. It was laid down as a rule by the late Mr. Barlow, that if both hocks were alike, and the action good, joints coarsely formed should not be condemned as unsound, more particularly if the enlargements were situated towards their posterior aspect.

I have made many dissections, and found that such enlargements were often due to disease, even in horses that were never known to be lame. Why the presence of such deposits did not cause lameness will be better understood after the pathology of spavin has been discussed.

Many horses are foaled with irregular hocks, very often with one hock larger than the other; and such are apt to be condemned as being diseased and unsound. Experience, however, proves that a young horse, rough in his hocks, if put to work before he has arrived at his full growth, is apt to fall lame; and that the same animal, if allowed to rest until he is old enough for work, will remain sound for many years, although a marked disparity may exist between the two hocks. Again, a horse at four years old will look coarse in the hocks, or even spavined; but when he is six, the same horse will appear quite fine and sound, provided he has been properly cared for.

It is almost impossible to lay down a rule as to what constitutes an unsoundness, provided the animal goes sound, and has pretty good hock action. Spavins in front of the hock are generally condemned, and justly so ; but even when so situated, one very often finds they cause no lameness, at least during the middle period of the animal's life.

The lameness of bone-spavin is, as a rule, removeable in the young and middle-aged, but incurable (with few exceptions) in horses past their prime. In the two former it is due to an inflammation, which of itself brings about the reparative process ; in the latter, to a degenerative disease in the bones, partaking of the nature of fragilitus ossium, unaccompanied by a true process of repair.

Pathology.-Bone-spavin consists of inflammation excited in 
the cuneiform bones, or in the cuneiforms and large metatarsal, and sometimes the inner small metatarsal, either from con-

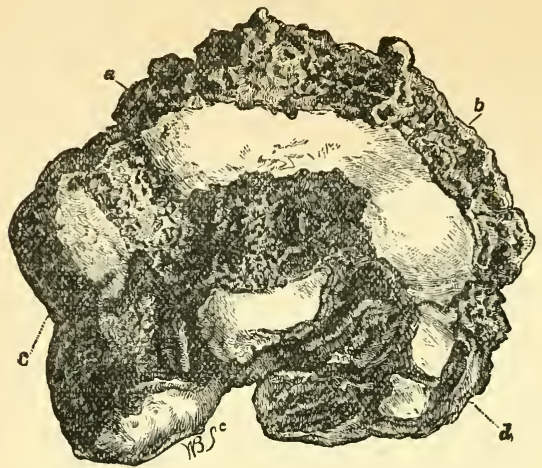

Fig. 52.

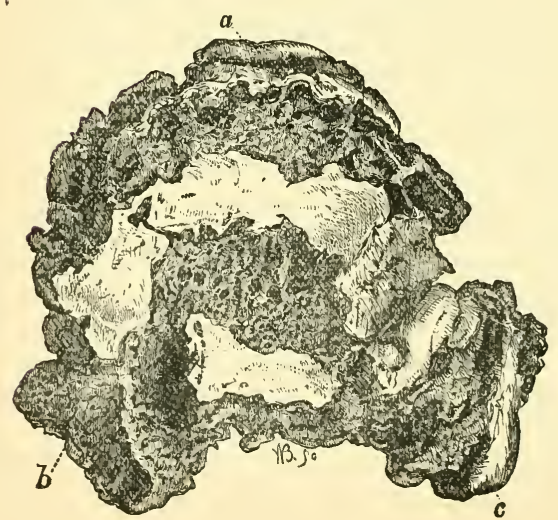

FIG. 53,

FIG. 52 represents the inferior surface of cuneiform medium and parvum and os cuboides in a state of ulceration, with their cancelli exposed. $a, b$, Cuneiform medium ; $c$, Os cuboides ; $d$, Cuneiform
parvum.

FIG. 53. - Articular heads of the three metatarsal bones from same hock. $a$, Large ; $b$, inner small ; $c$, outer small, showing from same responding to that found in the opposing bones. 
cussion applied to the bones themselves, or extension of inflammation of the inter-osseous ligaments, which are, in every case that I have examined, implicated in the morbid process.

The inflammation of the bones (ostitis) originates in the cancellated structure of their interior; an exudation is gradually thrown out between them and their cartilage, perverting the nutrition of the latter, whereby it ulcerates and is removed, leaving the exposed surfaces of the bones in contact with each other, and their cancellated structures in apposition; thus enabling their vessels to communicate with each other, as already described in the chapter on Axchylosis. Concomitant with the destructive process going on in the interior of the bones, an exudate is formed upon their periosteal surface, extending from one diseased bone to another, binding them together by a band of lymph-ultimately converted into bonewhich locks them firmly together, and prevents further motion. In old horses the process of anchylosis is limited to the periosteal surfaces, whilst the destructive action still proceeds upon the articular ones; whereas in the young or middle-aged lymph is thrown out between the ulcerated surfaces of the bones, which is organized into true osseous matter, making the process of anchylosis complete at all points. The incurability of the lameness in the old must be ascribed to this non-termination of the process; for however extensive the deposition of bony matter is upon the external surface of the bones, if their exposed cancelli come into contact with each other, the lameness will most assuredly remain.

We may now understand why the external deposit is not the cause, but the result of the disease, and why its presence is not always accompanied by lameness. So long as the carious surfaces of the bones are unrepaired, so long will the lameness remain; but when the bones are locked togetherare, in fact, converted into one bone, performing the functions of one bone-the lameness disappears, and the reparative material becomes as one of the essential structures of the economy. The destruction of the slight gliding motion of these articulations is of but little consequence, as it does not interfere with the flexile action of the hock.

Reasoning upon these observations, and upon the well-known fact that an old-standing spavin of the young and middle-aged 
horse does not always cause lameness, we are warranted in endorsing the popular and common conclusion, that an old-

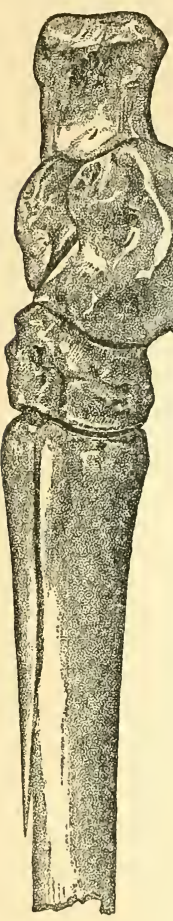

Fig. 53A. standing spavin seldom does harm, and that it is only during its formation it interferes with the usefulness of the animal.

But a spavin, to terminate so favourably, must be limited in its extent; perfect immunity from a recurrrence of lameness may result when it is confined to the cuneiforms and metatarsals; but if the superior surface of the cuneiform magnum and the lower articulating surface of the astragalus are involved, in addition to the others, I do not think that perfect restoration can be effected. In many of the specimens in the College Museum, not only are all the gliding bones involved, but also the bones of the true hock-joint. Such changes must cause permanent lameness.

In conclusion, I may mention that a practice prevails in some parts of the country by which hocks that present a want of symmetry in the seat of spavin, and particularly if one hock is larger than its fellow, are made as nearly alike as possible. The operation is called causticking, and is as follows:-Upon the coarse hock an incision is made a short distance below the spavin, and a piece of caustic inserted subcutaneously to the base of the enlargement. This causes inflammation and a filling up of the hollow beneath the spavin; in fact, it hides the latter by elevating the former.

Occult bone-spavin. Occult Spavin, as shown in Fig. 53A, is a form of hock disease in which inflammation of the cuneiform bones and interosseous ligaments, with anchylosis, is unaccompanied by any apparent external formation of bone ; the spaces between the bones may be filled up with ossific matter as in the illustration, but there is no visible enlargement. 

THE HOCK.

Thorough-pin is a bursal enlargement situated on the inferior lateral aspect of the thigh and upper and posterior part of the hock, arising from disease of the tendon of the flexor pedis perforans muscle, which is enclosed in a synovial sheath, on the inner side of the os calcis, or from dropsy of the sheath, without disease of the tendon. The fluid which fills it may, by pressure, be forced from one side to the other; hence the term thoroughpin, or running through from side to side. This may be described as true thorough-pin, in contradistinction to that associated with very large bog-spavins.

A thorough-pin, however large, does not cause bog-spavin, as there is no real channel of communication between the true hockjoint and the bursa of the perforans tendon; but the capsule of the joint swells upwards and backwards, bulges into the bursa of the tendon, and a large bog-spavin may thus cause the distension of the bursa, and the appearance of thorough-pin.

Thorough-pins are generally found in short, fieshy, upright hocks, where the os calcis is short and ill developed. We can account for such hocks being subject to them by the knowledge that the lever of the limb being a short one, more strain is thrown upon the flexor tendons, the flexors of the foot being extensors of the hock.

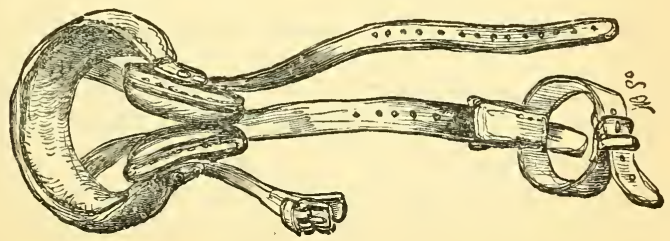

FIG. 54.-Spring truss for thorough-pin and bog-spavin, designed by Mr. Broad, Bath.

Railway shunt horses are very liable to fall unsound from thorough-pin, in consequence of the very heavy truck loads they have to start.

In the treatment of thorough-pins and bog-spavins, rest, the 
high-heeled shoe, and pressure by a spring truss, are the most effective appliances. Some recommend puncturing the sac. This I have performed in some cases with satisfactory results; but as a rule I think the operation should be avoided. The method of puncturing the bursa subcutaneously, by making a very small incision and drawing out the fluid with a syphon, is useless, the sac becoming quite full again in the course of a few hours. If a puncture is to be made at all, it should be at the bottom of the swelling, sufficiently large to allow the complete removal of the fluid; and it should remain open for some days. No fear need be entertained, although signs of pain and fever may ensue, as an open bursa is not so serious as an open joint.

After the sac is emptied, a weak solution of sulphate of zinc or dilute tincture of iodine may be injected; the walls of the sac brought into apposition by firm but careful bandaging; a flannel bandage, owing to its elasticity, being the best of all.

\section{CAPPED HOCK.}

The gastrocnemius internus muscle terminates about half-way down the tibia in a strong tendon; it is at first within the tendon of the gastrocnemius externus; winds round its side; then surmounts it, and, on reaching the point of the os calcis, forms a cap, giving off slips of insertion from each side to the bone. A large synovial bursa exists between the tendons here, and is one of the seats of eapped hock.

There are two forms of capped hock-the synovial and the serous.

Synovial Capped Hock appears as a tense fluctuating swelling, situated upon both sides of the point of the hock; the tendon, being posterior to the bursa, prevents the swelling of the internus from bulging backwards. It is an unsoundness, causing lameness, and sometimes the formation of abscesses from caries or necrosis of the summit of the os calcis.-(See Photo-lithograph, Plate I., Fig. 3.)

The Serous Capped Hock, a serous abscess caused by pressure or violence, is situated in the areolar tissue, between the tendon of the gastrocnemius internus and skin.

It is not an unsoundness, if not causing lameness, and arises generally from the horse striking the point of his hock against 
some hard substance. It very often indicates a kicker, either in harness or in the stable, is unsightly when large, and depreciates the value of the animal.

Displacement of Tendon of Gastrocnemius Internus.-Professor Dick used to relate that he had met with cases of what he called dislocation of the tendon of the gastrocnemius internus, and that the tendon had been torn from its attachment to the os calcis. He said it always fell to the outside, thereby not untwisting itself from the externus; but that it was quite possible for it to fall to the inside. He said that a groove was formed for it on the side of the bone, and that the patient regained his soundness.

This is a rare form of injury. I have recently seen two cases of it, arising from kicking, the animals striking the points of the calces violently, causing inflammation of the bursæ, or synovial capped hock. Although in one case the displacement was evident very shortly after the infliction of the injury, yet in neither instance was there any tearing of the tendons from their attachments, but simply an elongation and loss of cohesion, from inflammation in the ligamentous bands which bind them to the os calcis, as well as from pressure of the increased synovial secretion, allowing the tendons to slip outwards whenever the foot was elevated.

The treatment consists in throwing the part into a state of repose by the application of the high-heeled shoe, cold water to the seat of injury, and when the inflammation is reduced, blister or firing by pyro-puncture.

The tendo-achilles may be torn from its attachment to the os calcis; as a rule this is accompanied by fracture or detachment of the epiphysis, and occurs in young animals before the extremity has become permanently united to the body of the bone.

\section{INJURIES TO GASTROCNEMII MUSCLES.}

Injuries to the gastrocnemii muscles or to their tendons are known by the animal presenting symptoms the reverse of those manifested when the flexor metatarsi is injured, as in the case illustrated at page 297. In injuries to the gastrocnemii, the foot is elevated from the ground, as in stringhalt, the leg being suddenly brought upward and forward at each step. When the animal is standing still there will be knuckling over at the 
fetlock-joint, owing to the loss of power in the gastrocnemii, which act during repose as extensors of the hock. Division of the tendo-achilles is called "ham-strung;" and when such an injury is inflicted, the fetlock is brought to the ground, and the limb is powerless. I have, however, seen such cases do well, when the leg has been fastened on to a long stiff splint, extending from the foot to the stifle in front of the limb, and kept in this position by proper bandages.

\section{CURB.}

This is an injury, sprain, to the calcaneo-cuboid ligament, and not to the cellular tissue, as described by Percivall and others: nor is it a sprain of the broad annular ligament which passes over and binds down the tendons in their passage down the back of the hock, although the annular ligament as well as the tendons may suffer when the injury is very violent. Such cases are commonly called "sprung hock," and are associated with great lameness.

The original seat of the injury in curb may be at the point of attachment of the ligament to the cuboid, or at its ultimate termination on the head of the external small metatarsal bone, or its attachment to the posterior aspect of the os calcis may be lacerated to a considerable extent.

In the first instance, it presents itself as a small hard nodule upon the lower part of the posterior aspect of the hock; so small and so hard that it is sometimes impossible to say whether it is the injured ligament or the bones themselves. In the second, it can easily be recognised as a protuberance upon the back of the hock, from four to five inches below the point of the os calcis.

Curb is apt to cause lameness in young horses, or, when of fresh origin, in horses of any age. Curbs of long standing, being merely the remains of former disease, very seldom cause lameness, and are very often considered by men of experience not to be an unsoundness.

Curby hocks are over-bent or sickle-shaped, and if associated with long calces, are almost sure to become the seat of true curb.

From what has already been said about the leverage power 
of a long os calcis, it will be understood that the ligaments which bind it down are much more liable to sprain when it is long than when it is short. The form of hock the reverse of that liable to thorough-pin is the one predisposed to curb.

An aged horse, when suffering from curb lameness, is generally sound again in a few weeks; but if the patient be a young horse whose bones are not fully consolidated, it takes a much longer time before the parts are restored; and if such an one be put to work before they are thoronghly repaired and strengthened, lameness will in all probability recur. Curb lameness does not depend upon the magnitude of the enlargement, some very large curbs causing little or no lameness, and some small ones proving a source of very severe lameness. Curb lameness is characterised by difficulty in extending the hock, and in some very severe cases by that condition of the tendo-achilles already described, the limb being elevated, and the tendo-achilles remaining in a state of relaxation. This arises from the animal avoiding to exercise muscular force on the os calcis, and endeavouring to mitigate pain.

Horses liable to curb should be shod with a shoe high in the heel, and care should be taken that it be not allowed to wear too low, else lameness is almost sure to recur. Cart-horses with curbs should not be shod with high toe-pieces, or the increased resistance to the action of the muscles upon the calcis will cause lameness.

Whether a curb be an unsoundness or not must be left to the practitioner; legally it is so, but in reality an old curb, unaccompanied by heat of the part, and causing no lameness, does not generally interfere with the usefulness of the animal.

In some parts of the country young animals are fired to strengthen the hocks, and prevent curb and spavin. Such cases, when they come before the veterinary surgeon for examination as to soundness, have a suspicious look about them, but if the hocks are good and the action sound, the marks of the cautery are no indication of unsoundness.

This practice of firing to prevent disease is most cruel and useless. It cannot be too strongly condemned; and Martin's Act should be applied to every one guilty of such barbarity. 
Treatment of Curb.-Rest, a high-heeled shoe, reduction of the inflammation, and afterwards removal of the thickening by means of iodine or its salts. If lameness is persistent, firing.

The tendon of the flexor pedis perforans is liable to injury as it passes through the tarsal groove, succeeded by great lameness, distension of the bursa, the swelling prominent both below and above the hock on its postero-internal aspect. The tendon is bound down at the tarsal groove by the posterior annular ligament, thus preventing the swelling from appearing except above and below.

The superior tarsal ligaments correspond to the radials; the metatarsals to the metacarpals in the fore extremity. Both are liable to sprain, the latter much oftener than the former, characterised by lameness, swelling, and other signs already described. 


\title{
CHAPTER XVII.
}

\author{
TREATMENT OF LAMENESS.
}

\begin{abstract}
REST-POSITION OF THE LIMB-REMOVAL OF SHOES-IIGH-HEELED SHOE-THIN-HEELED SHOE-HOT AND COLD FOMENTATIONS-LOCAL BLEEDING-PURGATIVES-COUNTER-IRRITANTS-BLISTERS-SETONS-ACTUAL CAUTERY-THEORY OF THEIR ACTION.
\end{abstract}

BEFore describing the diseases of the feet, which are so numerous and important as to require special consideration, I will endeavour to give a brief description of the treatment of lameness. The first and most important necessity in this matter is to form a correct diagnosis; without this all is hap-hazard, and calculated to do much harm. The next step is the removal of the cause, if that be possible, and of every circumstance calculated to aggravate the effect. After these things are attended to, the position of the limb and foot demand attention, in order that the patient's efforts to remove pressure and tension from the seat of pain may be assisted. If a lame horse stands with the foot of the lame limb flat upon the ground, that is to say, touches the ground with both heel and toe, and if the feet are strong and good, I am of opinion that all his shoes should be removed, in order that he may stand upon his feet, and be able to poise his body in nature's way. But if his feet are bad and weak, they must be protected by light plain shoes. Trifling cases of lameness, where in all probability recovery will take place in a few days, are exceptions to this method; but in all instances where it is likely the patient will require a period of rest, the plan is to be highly commended.

If, however, the horse is inclined to elevate the heel, to stand on his toe-the posture indicating that parts are thus relieved and pain diminished-he must be encouraged in this by having a patten or high-heeled shoe applied. This will, in many in- 
stances, afford much immediate relief; but if, on the contrary, he is inclined to throw his weight upon the heels, a thin-heeled shoe should be used. The shoe recommended by Mr. Broad, of Bath, for laminitis, which will be described hereafter, answers the purpose in all such cases, except when the posterior part of the limb and heel descend from ruptured sesamoidean ligament, as already mentioned.

Such, then, are the first circumstances to which the practitioner must attend, in order to place the injured structures in a state of repose.

When the parts are put in as complete a state of rest as possible, the effects of the primary lesion will command attention. These are inflammation, with pain, and perhaps swelling.

The inflammation is the result of the injury, and, except in cases where the textures involved have become torn, lacerated, or crushed, it is the only morbid condition present in the early period of the lameness.

It is of the utmost importance to bear this in mind, as by proper attention to the animal, alterations of structure may be prevented; whereas if he be now neglected, worked from day to day, and otherwise improperly treated, organic changes ensue, rendering the lameness incurable, or curable only by a lengthened process of repair.

For the reduction of the inflammation, hot or cold applications to the part are useful. I prefer warm, considering that they are more soothing in the early stages than cold. Notwithstanding my own preference to warm applications in the earlier stages, I must in justice state that the general belief is in the efficacy of cold at first, warm afterwards; and that this belief is based upon the ground that cold moderates excessive vascular action, by causing vital contraction of the vessels, and hence its application immediately after the receipt of blows or injuries restrains inflammation; and that heat acts by soothing the nervous system of the part, relaxes the vessels and tissues, so that the vessels relieve themselves by effusion; in other words, heat promotes the secretory inflammation.

There is, however, no rule to guide the practitioner in prescribing either hot or cold, and the choice is very often a matter of experience. 
In all painful affections, warm fomentations or poultices must, as a rule, be prescribed; in the course of some days, however, if the pain is subsiding, and the parts seemingly relaxed, much benefit will be obtained by making a change to cold, mild astringents, and bandages, to promote absorption of the exudate.

The congested capillaries may be relieved by local bleeding, but the parts upon which such an operation is performed are very few, except about the coronet or the foot. An incision into the coronary plexus will reach the vessels at once; the utility of this is, however, very doubtful, except in rare cases. Bleeding at the toe, although much practised by some, is not to be commended.

Purgatives are very useful during the first stages of lameness, reducing the inflammation; a full dose of aloes may be given with advantage, the diet being properly regulated, and restricted to bran-mashes, a little hay, and the water to be chilled.

After the acute signs of inflammation have subsided, if the lameness still remains, the application of the so-called counterirritants will be rendered necessary.

These consist of rubefacients, blisters, setons, and the actual cautery. The action of these remedies differs only in degree, in rapidity, and in permanence, not in the nature of the exudation which they produce.

The theory of counter-irritation is one of great obscurity. It is all very well to say that counter-irritants act by causing metastasis, or a translation of the disease from one part to another. I need scarcely here discuss the origin of the idea that it is based upon the assumption that no two inflammations can exist in the system at the same time, and that by exciting a manageable superficial inflammation we counteract or remove the deep-seated and unmanageable one. This theory of counterirritation is founded upon a false and irrational basis, and has been the means of destroying the lives of thousands of horses. Without entering into any speculative discussion upon the question, superficial irritants are very beneficial in all cases of chronic lameness, whether it be caused by disease in bone, cartilage, ligament, tendon, or any other structure; and they are often more decidedly beneficial when applied to the diseased structure itself than to the skin covering it. For example, a 
lameness arises from bone-spavin; its eradication is much more certain and rapid when a pointed cautery is applied to the diseased bones than when the hock is fired in the ordinary way. Again, a spavin has been fired and blistered repeatedly without benefit; the bones are "punched"-a barbarous operation, and only to be performed in extreme cases-violent inflammation is excited in the diseased bones, which for a time increases the lameness; but this gradually subsides, and the original lameness is found to be removed.

How, then, are we to account for such results? Certainly not by the theory of metastasis. Dr. Bennett, sceptical as he is in most things, seems to believe in the doctrine of counterirritation, and thus expresses himself:- "Artificial irritations of the skin, to produce internal or distant effects, are caused by what are denominated counter-irritants, including stimulants, frictions, hot applications to parts, sinapisms, blisters, moxas, cauteries, \&c., \&c. These all operate through the nerves by reflex action; some, like warm fomentations, soothe irritation; others, as blisters, create it locally, but remove it where it was primarily seated. How this is accomplished constitutes one of the most vexed questions in therapeutics."-(BennetT's Principles and Practice of Iedicine.)

I am of opinion that the curative action of external irritants is not due to their producing metastasis or counter-irritation, but that they excite within the originally diseased structure a reparative inflammation, partaking in its nature of what is described by Virchow as the "secretory inflammation," which, superseding the original diseased process (whether that be inflammation pure and simple or its effects, ulceration, caries, or the formation of a low form of fibrous tissue), excites the formation of reparative material, by which breaches are united, ulcers healed, and diseased action removed.

To illustrate this view, I will bring forward two familiar examples:-1st. The healing of a sinus or fistula, after the application of a blister, or of the actual cautery to the skin contiguous to it; and $2 d$. The removal of phlebitis by a blister.

In the first instance, we find that a sinus heals after a blister or cautery by the formation of an organizable exudate, which completely fills up the cavity of the sinus; and, in the second, we find that a blister assists in the obliteration of the inflamed 
vein; not by removing the inflammation from it, but by promoting the formation of a large quantity of reparative lymph, and hastening its further development into fibrous tissue, by which the vessel is transformed at the inflamed part into a fibrous cord. Now, if the curative action were due to the removal of inflammation, we should find that in the first case the relief would be only of a temporary nature; the sinus would still remain, being generally the cause, and not the effect, of the morbid action; and in the second, that the inflammation being removed from the coats of the vein, the vessel would, upon removal of the clot, become pervious. But such is not the case. Let the clot be removed ever so often, it is sure to form again, and nothing has the power of overcoming the inflammation of the vessel until it has been transformed into an organized cord -a process most materially hastened by the application of a blister.

I think it may therefore be accepted that external irritantswhether they be simply rubefacients, producing a mere redness of the skin, vesicants or blisters, which cause elevations of the cuticle by fluid underneath it, or eauterization and setons, which promote the suppurative action-remove lameness by assisting nature in a process of repair.

Rubefacients may be employed in the less severe forms of lameness, in sprains of tendons, or in slight affection of joints, along with rest and fomentations, after the more acute symptoms have passed away.

Blisters.-It is usual to apply blisters in all cases of some standing, when organic changes in the parts involved are suspected. Before a blister is applied, the hair should be clipped from the part, which, if dirty, ought to be washed, and when dry, the blister to be applied with smart friction for about ten minutes. To obtain the full effect of a blister, a quantity of ointment is to be thickly laid on after the rubbing-in is completed.

The best agent is cantharides, in the form of acetate, tincture, or ointment; to the limbs, the ointment in preference; one part of cantharides to twelve parts of lard or palm oil. If prepared with a temperature equal to the boiling-point of water $\left(212^{\circ}\right)$, it will be sufficiently strong, and will never blemish. It is a mistake to think that the powdered flies should be mixed 
with the vehicle when it is nearly cold: an ointment so prepared will require three times the quantity of cantharides, The heat melts the cantharidine.

Hints upon blistering generally.-No more than two legs are to be blistered at one time, and three weeks at least must be allowed to elapse before the others are blistered, and between each re-application. It is bad practice to blister extensively in very hot weather; and it is a mistake to suppose that blisters to the loins and back are more apt to irritate the urinary organs than when applied to any other part of the body, provided that it be carefully and properly done.

The evil results of blistering are-

1st. The production of strangury, by the absorbed cantharidine irritating the urinary passes. This is a very rare occurrence, provided the blister has been applied to a moderate extent of surface; but if four legs, or even two, be very extensively blistered at one time, the occurrence of such may be laid down to the indiscretion of the practitioner. In some cases, however, very moderate blistering is followed by strangury, and when it does occur, it is best treated thus:-First wash the blistered surface with warm water, in which a little alkali has been dissolved; dress it with oil; give the animal demulcents to drink, such as cold linseed tea; and administer a few doses of opium and bicarbonate of soda.

$2 d$. The production of a considerable amount of nervous irritability, fidgetiness, quickened pulse, and injected mucous membranes, with loss of appetite. These symptoms are due to a nervous temperament; and if not very severe, had better not be interfered with. Should they become alarming, the animal must be treated as in the first instance; the fomentations being continued for a longer period to the legs. It may be here mentioned that fomentations should not be hot, but soothingly warm.

$3 d$. Sometimes blisters, no matter how carefully applied, produce excessive swellings of the limb or limbs, with a tendency to suppuration and sloughing of the skin. These results are generally due to the animal being in bad health, and in a condition tending to anasarca or to erysipelatous disease. The treatment must consist of purgatives or diuretics, as the case may be, fomentations, astringent lotions, and gentle 
exercise, as soon as the pain is sufficiently subsided to admit of the animal being moved about. In many cases the swellings involve the sheath of the penis and the under surface of the abdomen. Punctures are very useful in such parts, by allowing the escape of the contained fluid. I have seen tetanus arise from a very limited blister to one fore leg.

It is necessary to tie the horse's head to the rack after a blister has been applied, in order that he may not bite it, or lick it with his lips or tongue, and thus blister the mouth and blemish the blistered spot. It is also necessary to tie the head so that the horse cannot lie down, for if he lies upon the blistered limb the vesicant will adhere to that part of the body brought in contact with it whilst the animal is recumbent, and produce an effect upon it as well as upon the part to which it has been purposely applied. If the blistered spot be within reach of the tail, the tail should be tied up, or it is apt to become daubed, and the blister whipped on to the thighs, sheath, or mammary gland.

If the effects are not sufficiently apparent in about thirty hours after the blister has been applied, a very little more, or what is remaining on the skin, which may be sufficient, should be gently rubbed in; and in about forty-eight hours after the application the part is to be washed, and every trace of the blister removed; a little oil being now applied, or, what suits perhaps better, an emulsion of sweet oil, carbonate of potash, and water. It is a mistake to keep the parts soft too long; the eschars should be allowed to accumulate, and to desquamate gradually.

When the head is untied from the rack, a cradle must be put on the animal's neck to prevent him from biting the blistered spot. A cradle is, however, useless when the lower part of the fore legs is blistered, since the animal can elevate his feet from the ground, and thus get at then with his teeth; and when he is lying the cradles are of very little use when a blister is below the knee. In such cases the best method is to keep the head tied up until a thick scab is formed, which will destroy itchiness in the part.

Firing, or the application of the actual cautery, is supposed by some to be beneficial in acting as a suppurant, and by others in forming a permanent bandage round the part; but I appre- 
liend there is not much truth in either of these suppositions. It is a much more severe irritant than a blister, and often removes pain very rapidly when repeated blisters have failed to do so. In bone diseases, and in all causes of chronic lameness, it is of great benefit, and seems to act by powerfully exciting the healing process in the part diseased.

The firing may be in lines, and superficial, the transverse method being the least calculated to blemish; or it may be in points, and deep, by pyro-puncture (see drawing of instrument, Fig. 24, page 159), and into the diseased structure. This latter mothod is the more easily performed, and the more effective.

Nothing is more calculated to dispel the idea of the correctness of the counter-irritation theory than the dissection of a part which has been recently fired (say three days after the operation), when it will be found that the skin, subcutaneous tissue, and the bones-when they are superficially situated, such as those of the hock, pastern, \&c.- - are involved in the inflammatory action so induced. Thus a bone-spavin lameness is removed by the inflammation excited by the cautery in the diseased bones, providing a supply of material for the purpose of uniting them together into one immoveable mass; or, as in caries of a ginglymoid joint, for the repair of destroyed structure, as already explained.

Setons act very satisfactorily in some cases of bone diseases, especially in those accompanied by external heat of the part; they produce a discharge of pus, and their action can be continued for a much longer time than that of blistering or firing. In tendinous or ligamentous lamenesses, with much thickening of the integuments and subcutaneous structures, setons should not be employed, as they leave much additional thickening, and are not so effectual as the actual cautery. 


\section{CHAPTER XVIII.}

\section{DISEASES OF THE FEET.}

PRELIMINARY OBSERVATIONS ON SHOEING-FOOT LAMENESS, DIVIDED INTO TIREE KINDS-(1.) DISEASES OF THE BONES AND C.IRTILAGES; (2.) DISEASES OF THE HORN-SECRETING STRUCTURES; (3.) ACCIDENTAL INJURIES-DISEASE OF THE PYRAMID OF OS PEDIS-SIDE-BONES-NAVICULAR DISEASE, THEORIES OF-CAUSES, PATHOLOGY, AND TREATMENT-NEUROTONY, ITS FAVOURABLE AND UNFAVOURABLE EFFECTS-GELATINOUS DEGENERATION OF THE NAVICULAR BURSA-PATHOLOGICAL ANATONY-COMPARISON TO WHITE SWELLING.

\section{PRELIMINARY REMARKS ON SHOEING.}

There is no subject that calls for more attention than tle consideration of the fuet of the horse. At the present time, so great is the ignorance prevailing amongst owners, shoers, and managers of horses, that the majority of lamenesses are found to arise from mismanagement of these important parts of the animal frame. In the city of Edinburgh above 60 per cent. of the horses engaged for all pur. poses are lame, and above 80 per cent. of such are lame in one or both fore feet. In many English towns, especially in London, the lame horses to be seen in the streets are very few compared with those of Edinburgh; but even in the most favoured parts of the kingdom the number is something enormous compared with other countries; and well might the late Professor Sewell say that he had seen more lame horses between Dover and London than during a sojourn of three months in France and other continental countries.

The writers upon the management of the horse's feet and upon shoeing are very numerous, embracing all classes of men, from peers of the realm down to the groom and shoeing-smith; but 
an analysis of their writings shows that the work of one is often a mere repetition of that of another. Nearly all have run in the same groove, promulgating false ideas from one generation to another, to the incalculable deterioration of the usefulness of the horse. Indeed, such is the prejudice, and so deeply rooted are men's opinions, that it is dangerous for any one to teach a more rational doctrine. If such an one is now and then bold enough to point out the errors of the past, he stands a fair chance of being considered a dreamer, or something worse.

Nearly all writers upon the subject have looked upon the foot as a very wonderful and complex piece of mechanism, and seemingly have forgotten, or have not known, that no matter how complex it may be within, it is enclosed in a simple horny box; that all the efforts of shoeing should be directed to preserve that box in a natural condition; and that its position in relation to the limb should not be altered by the shape or form of the shoe.

Many have maintained, and some still maintain, that the horny foot is an elastic, expanding, and contracting organ, and that its elasticity should be kept intact by paring the sole, peculiar nailing on of the shoe, and by keeping the foot as moist as possible, by stuffing, spongio-piline, \&c. Others, again, suppose that a mechanical advantage can be given to its tendons and ligaments by the form of the shoe; in fact, by improving upon nature.

All these are errors, and have originated with men who have built their conclusions upon mere hypotheses. It is not my intention here to enter minutely into the question of horse-shoeing, but merely to state, in the first place, that it is essential to abolish the drawing-knife; and in the second, that calkins and toe-pieces should be done away with for all kinds of horses except those used for heavy draught in towns where the streets are paved and steep. All horses required to go beyond a walking pace are injured by shoes with turned-up heels and toes. Farm-horses, and those employed upon macadamised roads, are better without than with heel and toe-pieces, although the pace they are required to go is never faster than the walk; in fact, where possible, all horses should be shod with a flat shoe. 
The form of the shoe which I recommend is represented in the following woodcuts; and it ought to be so made and fitted as

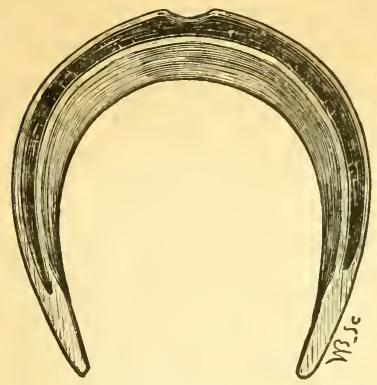

Fig. 55.

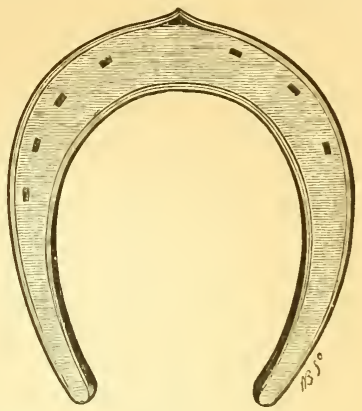

FIG. 56.

Fig. 55. - Inferior (concave) surface of shoe.

FiG. 56.--Superior (flat) surface of shoe, bearing on the sole.

to bear upon all parts of the sole and crust that are calculated to bear pressure. Experience and anatomical investigation point to the conclusion that the sole as well as the crust is intended to perform this weight-bearing function: the sole around the margin of the crust for the distance of about half-an-inch in all parts of the foot except at the heels-i.c., that part of it embraced in the triangle between the wall and bar; in fact, the seat of corn. Here the shoe should rest upon the wall only, being made sufficiently narrow at this part (as seen in Figs. 55, 56). By the application of such a shoe, all parts of the foot calculated to bear weight are called upon to perform their natural

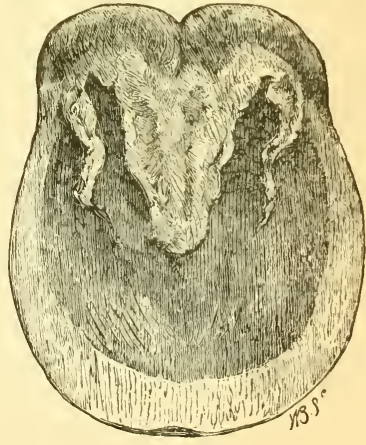

Fig. 57.

The foot prepared for the shoe. The sole, frog, and bars untouched with the knife for two years; the natural length of toe and depth of wall kept in their relative positions by the rasp only. function; the various structures kept in their proper and relative position; the frog allowed to come to the ground to prevent concussion; the weight of the 
animal's body diffused over an extended surface, and not limited to the wall alone, as in the common method of shoeing with a seated shoe; slipping prevented, by the rim of the concave shoe and the wedge-shaped frog grasping the ground.

This method of shoeing has been practised at the forge of the College with the most beneficial results for several years, and even in such a city as Edinburgh, where the streets are so steep and slippery, with the great advantage that horses slip less than when shod with turned-up heels. I feel quite convinced that, if such a shoe were generally adopted, lameness in the feet would be much more rarely met with; but such is the prejudice, even amongst those who have the opportunity of seeing the beneficial results of the system, that it is almost impossible to convince them that it is an improvement. One large cab proprietor says - "All my lame horses go better in your narrow-heeled shoes, especially my ' groggy horses ;' but even he will not have his sound horses so shod.

As to the desirability of pressure upon the sole, my views are borne out by many practical men, especially by Mr. Broad of Bath, a gentleman who has studied the subject very deeply. His essay upon horse-shoeing, which gained the second prize of $£ 30$, given under the auspices of the Scottish Society for the Prevention of Cruelty to Animals, is replete with instructive matter, and ought by all means to be published.

It must, however, be borne in mind that the sole, to bear its due proportion of weight, should be left unmutilated by the knife.

To return to the subject of lameness. The diseases of the feet may be arranged as follows :-

1st. Diseases of the bones and cartilages.

$2 d$. Those originating in the horn-secreting structures; and,

$3 d$. Accidental injuries.

\section{I.-LAMENESS FROM DISEASE OF THE PYRAMIDAL PROCESS OF THE}

OS PEDIS.

This form may exist either in a fore or a hind foot, and results from blows upon the front of the coronet, or from over-extension of the extensor tendon (attached to the point of the pyramid) by the use of high calkins. 
Symptoms.-Swelling on the front of the coronet, varying in size from that of a hazel nut to that of a pigeon's egg, with lameness, which is often very persistent. 'The peculiarity of gait is manifested by the horse putting the heel down first, and often taking the foot up very quickly as soon as the toe comes in contact with the ground. There will be pain on pressure, and some heat; now and then the skin crer the enlargement sloughs, leaving a wouna, which heals with difficulty; or a wound may be present from the first, if the injury has been due to external violence.

Treatment.-Low-heeled bar shoe; fomentations, poultices, and rest; succeeded by blisters, or the application of the actual cautery. In some cases the lameness resists the most active treatment, and upon examination after death, caries is found to have destroyed the pyramid of the bone, and extended into the pedal articulation. Neurotomy, if there be a good foot, might be tried in cases that resist all other treatment.

\section{II.-OSSIFICATION OF THE LATERAL CARTILAGES.}

Side-Bones.-Commonly met with in heavy horses, and in the fore feet. I have seen the lateral cartilages of the hind ones ossified ; but this is very rare, and, so far as I know, never occasions lameness.

The lateral cartilages are two thin plates of fibro-cartilage, of an irregularly quadrangular form, surrounding the wings of the os pedis, which, in virtue of their elasticity, assist the sensitive frog and soft structures of the foot in regaining their natural position after being pressed upwards and outwards by the weight of the animal. An opinion prevails that these fibro-cartilaginous bodies assist in the expansion of the foot. Undoubtedly they expand at their posterior border each time the animal puts his foot to the ground; but in this expansion of the heel they are mere passive agents, being, in fact, pressed outwards by the structures contained in the space between them. They are, however, active agents in causing the contraction of the heel; for when the pressure is removed from their inner surfaces, they then tend to assume their natural position in virtue of their elasticity; and the pressure they exercise upon the sensitive frog forces the heel into its original shape. Briefly, they may 
be said to be forced to expand when the foot is on the ground, and that they actively assist contraction when the weight, which forces the sensitive frog upwards and outwards, is removed from the foot.

It must not be understood that I am advocating the theory that the foot expands upon its inferior surface; that supposition is now entirely disregarded; but no one can deny, what is apparent to the most ordinary observer, that the foot expands at the coronet and heels; not the horny foot, but the soft parts of the heels and coronet. To prevent undue expansion of these, the lateral cartilages are placed as elastic sides.

Ossification of the Latcral Cartilages.-As already stated, side-

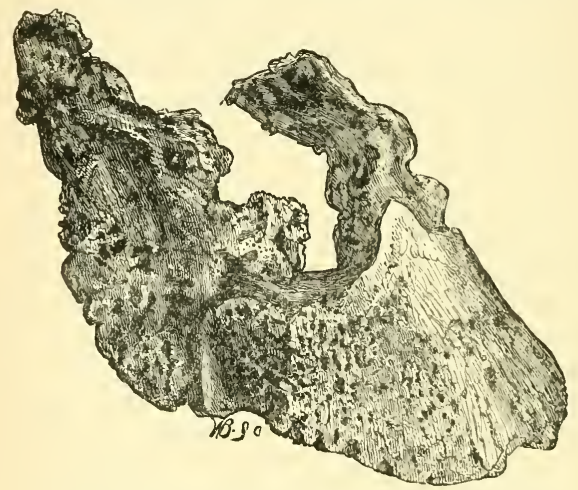

Fig. 58 shows ossifivation of the lateral cartilages, with fracture of the altered structure upon one side, at its junc. tion with the pedal bone.

bones are commonly met with in heavy draught horses; indeed, a great majority of this class is found so affected by the time the animal is six or seven years old; and this seems to arise from the over-expansion of the cartilages caused by the great weight of the animal. The process of ossification is very often a slow one, unaccompanied by any acute inflammatory action, giving the animal no pain, and causing no lameness.

The causes of ossification of these cartilages are-hereditary tendency, and shoeing with high calkins. It is generally admitted that the predisposition to side-bones is hereditary, and 
many breeders of the best class of cart-horses, being aware of the fact, are careful not to breed from an animal with them.

High-heeled shoes prove a cause:-1st. Because the shock received by the heels when the foot comes to the ground is transmitted directly to the cartilages; $2 d$. Because the pressure upon the heels of the wall is unnatural and excessive, the frog being prevented from bearing its proper proportion; and, $3 d$. Because they are pulled inwards and downwards by the sensitive frog being pressed downwards, whilst it horny covering, being removed from the ground, forms no column of support.

Side-bones are a cause of unsoundness, but all horses so affected should not be condemned on this account, and it may be laid down as a general rule, that if the feet are strong, open, and well developed, the horse showing no lameness should not be condemned for side-bones. But if he is stilty in his action, even without actual lameness, or if the feet be contracted, altered in form, weak in the heels, flat or convex in the sole, there should be no hesitation in pronouncing him unsound.

Occasionally the lighter-bred horse is found to have sidebones, which are usually attended with lameness; whether lame or not, such an animal is unsound, since he is unfit to perform his ordinary labour, as trotting upon the roads is sure to set up the inflammatory process in the cartilage, and cause lameness.

To detect these deposits, it is necessary to press upon the cartilages; naturally, these are yielding and elastic, but when ossified they lose this character, becoming hard, unyielding, and enlarged. The deposition of bone may be uniform, involving the whole substance of the fibro-cartilage, or it may be in isolated spots, either at the junction of the cartilage with the pedal bone anteriorly, or involving the posterior borders, forming a hard kernel-like enlargement.

The anterior side-bone more commonly causes lameness than the posterior one.

Side-bones differ from ring-bones, both in the structures they involve and the lameness they occasion. Side-bone lameness is characterised by the toe of the foot being first brought to the ground; when both feet are involved, by a shortness of step and want of elasticity or springiness in action, resembling that of navicular disease.

Treatment.-Bar shoe; rest, blisters, firing; and should these 
fail, neurotomy. The French operation of removing the deposits by excision has not found much favour in this country, nor do I think it likely that it will do so, as the incurability of the lameness is generally due to other structures besides the lateral cartilages being involved in the diseased process. An examination of Fig. 5 in Photo-lithograph, Plate III., will illustrate this, and throw a light upon the circumstance that side-bones are occasionally the cause of incurable lameness.

Neurotomy is very successful in removing this form of lameness, and is attended with more permanently beneficial results than when performed for navicular disease.

It will be useful to bear in mind that when these cartilages are ossified, the horse's gait will lose that elasticity which is so essential to good action. In the cart-horse this is not of much consequence, but in the horse required for other paces than the walk, it is of the greatest importance, not only as a question of soundness or unsoundness, but of the usefulness of the horse and safety of the rider or driver.

\section{III.-NAVICULAR DISEASE.}

This is the most fertile cause of lameness that we know of in the better-bred horse-the bane of horse-flesh. At one time all cases of obscure lameness in the fore extremity were attributed to the shoulder; but after the discovery of James Turner, all were said to arise from navicular-joint disease.

Should the student desire the history of this disease, he will find it treated very fully by Percivall in his book on Lameness (1849). It was called navicular arthritis (PERcivall), and podotrocholitis (BRAUEL); and has been ascribed to various conditions, such as contraction of the foot (CoLEMAN); laceration of the fibres of the perforans tendon, as it passes under the navicular bone (DICK); inflammation of the synovial membrane (Turner, Percivall, and others); inflammation of the synovial membrane only, or of that and the navicular bone (BRAUEL); and inflammation, having its origin in the interior of the navicular bone, leading to exostoses on or caries of the inferior articulating surface, with degeneration of the fibres of the tendon (Broad of Bath), and its causes to a variety of circumstances, as best suited the whim and fancy of the theorist; such as 
non-paring of the foot, destroying its elasticity (CoLEMaN); a shoe thick at the toe, causing increased resistance to the action of the flexors, and rendering the perforans tendon liable to strain (DICK); thin-heeled shoes, by throwing the weight and concussion on the posterior parts of the foot and frog, bruising the synovial membrane; hereditary tendency; bad shoeing; bad nailing; want of exercise ; too much exercise, \&c.

I. Contraction of the Foot--Messrs. Turner and Percivall wrote, that " of contraction there may be two kinds. 1st. A contraction of the heels, called lateral contraction; $2 d$. Contraction of the hoof from below upwards, or vertical contraction;" and these were said by Professor Coleman to be the cause of this lameness. He maintained that "expansion of the hoof is effected by pressure upwards of the frog, and pressure downwards of the navicular bone. By properly thinning of the sole, rasping the quarters, lowering the heels, giving the frog pressure, and keeping the horse in a pond all day, or else tied up with his lame feet in a tub of water, we have no difficulty in removing contracted hoofs. Although difficulty there be none, however, in restoring the original form of the hoof, we too frequently find we have gained nothing by it, because we have not restored the original structures of the parts contained within the hoof. Contraction of the hoof in consequence of the internal parts being squeezed produces inflammation of the laminæ, and ossification of them. This causes the horse, in galloping, to avoid to his utmost coming down upon his heels, or to tread upon hard ground; the concussion at such times being great from loss of elasticity in the laminæ, so that the moment he comes to work he falls lame. In nine cases out of ten of what are termed 'groggy' or 'foundered' horses, these parts, in consequence of chronic inflammation, become altered in structure; effusion of lymph or bony matter taking place." I have thought it expedient to make this extract from Coleman, in order to show the kind of pathology taught by him; and I hope to be able to point ont the mischief brought about by it-conclusions and generalities without one fact to support them.

Contraction of the hoof is not a cause, but an effect, of disease; an atrophy of the structures contained within the horny box consequent upon diminished functional activity and adapta- 
bility of the hoof to the atrophied structures, which it encloses and protects. Professor Dick said there was a kind of contraction of the hoof, in fact a natural tendency to this in the domesticated animal, arising from a want of moisture when he is confined in the stable. This kind of contraction, he maintained, did not cause lameness, "as the soft parts became adapted to the alteration of the hoof." Now, in my opinion, this kind of contraction would be the one most likely to cause lameness; indeed, it would be impossible for an animal not to be so, if the pressure of the drying hoof were sufficient to cause atrophy and absorption of the sensitive tissues within. That horses' feet do become contracted, more especially at the heels, without lameness, I do not deny. I do not think, however, that this is due to any want of moisture, but to the removal of the horn from the heels and sole during the operation of shoeing, for the parts contracted are those situated posterior to the wings of the os pedis, where the space between the two quarters and heels of the hoof is filled by the elastic sensitive frog, a structure possessing but little sensibility. The great barriers to the collapse of the hoof at this part are strong heels, bars, and sole; but if the smith, by the so-called "opening of the heels," remove such a quantity of horn as to weaken the foot, can we wonder that it collapses, and that its sides approximate each other too closely?

Sometimes one foot may be found contracted through its whole extent without lameness. This may be due to some natural peculiarity in the animal, just as we find that a man may have one foot smaller than its fellow; or it may arise from the circumstance of the animal having been lame in that foot or that limb while young. I have often found a small foot to be due to a previous lameness in any part of the limb or the foot, and to accidental circumstances. The explanation is easy. When an animal is lame in any part of the limb, he qvoids putting weight on the foot of that limb; the consequence is loss of function and wasting, and in the young animal, a cessation of growth; whilst the opposite foot, having to bear more than its proper share of weight, becomes enlarged in all directions; in fact, grows rapidly, in order that it may be able to maintain the extra amount of weight thrown upon it. The disparity between the feet will remain through life, 
and the animal may be sound. Again, both fore feet are sometimes apparently contracted without lameness, but if examined closely, they will be found to be small and not contracted; the bones of the limbs will participate in the peculiarity of conformation; they will be narrow from side to sicie, and in all probability the hind feet will be small also. The contraction of the feet due to navicular disease will be found in the foot or feet that are lame, and it is an atrophied condition, resulting from diminished function, disappearing by degrees if the original disease, or the pain resulting therefrom, can be eradicated; for example, if the original freedom of action is restored to the parts by neurotomy, the disease still being present, the foot or feet contracted will regain their original dimensions.

II. Sprain or Laceration of the Fibres of the Perforans Tendon.-This was the theory taught by Professor Dick, and accepted by myself and the great majority of his pupils. He said-" I have endeavoured to demonstrate that primary and permanent disease is established in the synovial capsule, between the tendon and navicular bone, and arises from strain and over. extension of the tendon, where it passes under the navicular bone. It has predisposing causes, such as want of paring, shoeing, and, still more, bad shoeing; hereditary tendency of particular breeds, and high condition, for it is a disease rarely of the agricultural, but of the high-bred horse. In like manner, it has manifest exciting causes, such as strain of the tendon and over-exertion, pressure on the sole, as from travelling with a stone in the foot; and there is the tight shoe, exciting irritation of the foot, which, hot and uneasy in the stable, is aggravated by occasional and violent exercise." Towards the end of Professor Dick's career, the idea that the disease was due to laceration of the tendon became more firmly held by him, and that this was caused by bad shoeing, that is to say, by allowing the toe of the foot to be too long, and applying a shoe, thick and irregular at the toe, which, by increasing the resistance of the foot, when implanted upon the ground, to the action of the flexor muscles, threw an additional strain on the tendon where it passes under the navicular bone.

During the last five years I have made numerous post mortem examinations of navicular disease, and am coivinced that strain 
or laceration of the tendon is never a primary condition, and that the disease commences as an inflammation of the cancellated structure of the navicular bone, or of the cartilage upon its inferior surface. It was very hard for me to believe that a theory which had so much to support it, and which I had advocated ever since my student days, could be erroneous. I am sure that all the students of my predecessor will remember how enthusiastic he was while lecturing upon this subject, and how he showed us specimen after specimen where the tendon was lacerated and adherent to the bone. In order to establish the correctness of this view, it was necessary to have provided a specimen with the tendon diseased or lacerated, and the surface of the bone in its natural, or at least in a slightly altered condition; but this could not be done, and it was taken for granted that the altered structure of the bone arose from the primary lesion being in the tendon.

As a mere speculation, the view that the primary condition arose from laceration of the tendon could do no harm; but as a pathological fact, influencing men's minds upon the question of the non-removal or even non-prevention of this lameness, it has done infinite harm; for it involved the supposition that navicular joint lameness was almost incurable, and as such it is now looked upon by the majority of the profession and the public. Even when most enthusiastic in my views as to the correctness of Professor Dick's theory, I was often startled by seeing many cases of navicular disease, when attended to in the early stages, recover after a short period of rest.

Pathological examinations were made by Professor Dick to an extent surpassed by none; but he contented himself with investigating the condition of the outside of the bone and tendon, and if nothing was apparent there, the lameness was ascribed to some other cause.

If navicular disease were due to sprain and laceration of the tendon, how is it that it so very rarely affects the hind feet ? 1 Is it because the flexors of the hind extremities are more favourably

I I have in my possession one specimen, sent me by Mr. Clark, V.S., CouparAngus, of acute inflammation, with incipient ulceration, of the navicular bone of a hind foot; but even in this case, although the symptoms of lameness were very great and intractable, and were supposed by one veterinary surgeon to be due to fracture of the pelvic bones, there is no appearance whatever of laceration of the fibres of the tendon. 
placed than those of the fore limbs? I think the most devoted advocate of the theory will scarcely venture to answer in the affirmative; but should he feel inclined to think so, let him watch a horse in motion with a load behind him, and he will very soon see that the flexors of the hind limbs are called more powerfully into action than those of the fore ones; for in addition to acting as flexors of the feet and pasterns, they are extensors of the hocks. If strain were the cause, navicular disease, owing to the double function the flexor perforans is called upon to perform, and the more powerful strain thrown upon it in the removal of heavy loads, would be found in the hind, and not in the fore feet.

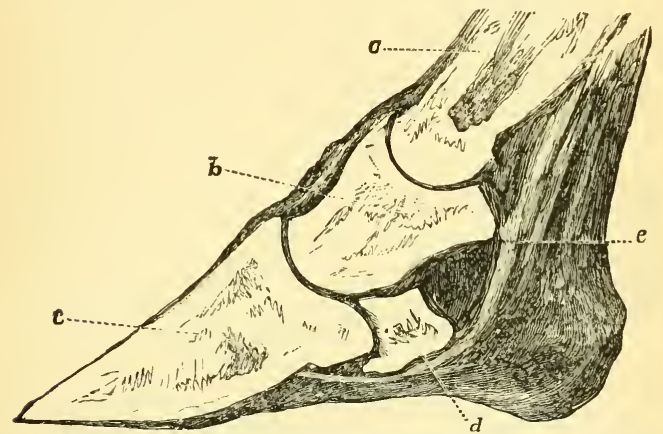

FIG. 59 represents the phalangeal bones in their naturally oblique position, and it will be seen that from the upper part of the long pastern $(a)$ bone, down to the toe of the os pedis $(c)$ is a continuous oblique line. This obliquity of position enables the bone to act as a spring, for the purpose of modifying concussion. Every horseman knows that the more oblique the pastern, the greater the elasticity of step and freedom from jar; that the pace is easy for the horse, and delightful to the rider. The coronary bone $(b)$ rests entirely upon the os pedis, and the navicular bone $(d)$ placed posteriorly bears no weight, but gives increased leverage power to the tendon $(e)$. It is a muscular appendage, like the sesamoids, and is not intended to be a weight-supporting bone.

Navicular disease is due to the rheumatoid diathesis and to concussion, and the liability to suffer from the first-named cause originates in hereditary predisposition and accidental circumstances; and to the second in conformation (as narrow feet with short upright pasterns), and in the unnatural alteration of the relative position of the navicular and weight-bearing 
bones, brought about by the application of shoes which are either turned down, calkined, or made thicker at the heels than at the toes.

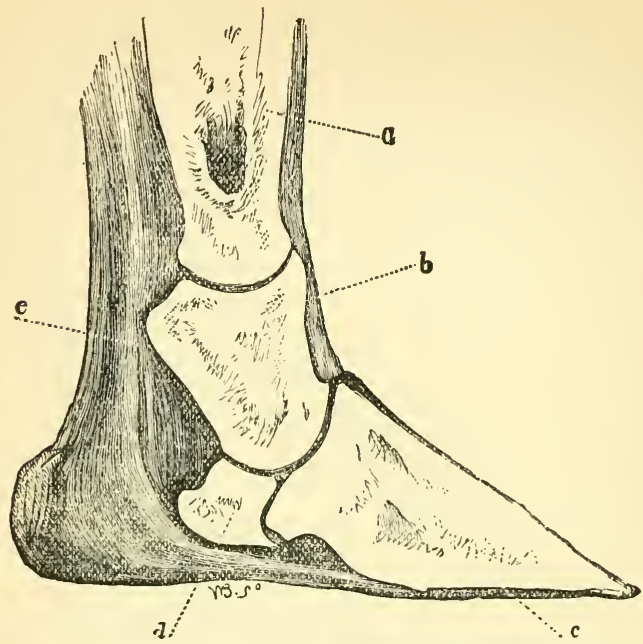

Fig. 60 represents the bones when their position is altered by a thick-heeled shoe; the line is changed from its natural obliquity to a direction approaching the perpendicular. The lower end of the os coronæ $(b)$ is made to rest upon the navicular $(d)$, as well as upon the pedal bone $(c)$. The navicular bone is made to bear weight for which it was not intended, neither is its structure calculated to do so. All weight-bearing bones have their fibres arranged to receive weight upon their extremities, but the fibres of the navicular, and of all the sesamoidean bones or muscular appendages, are not so arranged, and weight thrown upon them induces disease.

The development of navicular lameness from rheumatoid disease is occasionally due to what Professor Dick termed " occasional exercise," which implies long periods of rest, inactivity, and irregular work. Professor Dick maintained that during rest there was a deficiency of synovial secretion, and that when an animal, with his joints and bursæ in this dry condition, was suddenly put to fast work, the effects of the friction upon the surface of tendon, moving under the bone, were laceration of its fibres and navicular disease. Whether 
this dryness of the bursæ be present or not, it is very true that want of exercise is a powerful predisposing cause of the disease, and, doubtless, its effects are due to the localisation of a constitutional tendency to rheumatoid bursitis-(See Rheumatord ARTHRitis.) The other accidental causes are pressure of a stone in the foot, impacted immediately below the bursa, and exciting inflammation; punctures, and allowing the toe of the foot to attain an inordinate length. The most frequent predisposing cause being the abnormally relative position of the phalangeal bones, either arising from congenital formation of limb, or induced by shoeing with thick-heeled shoes; the exciting cause must be looked for in the pace. Race-horses, so long as they are shod with racing shoes, rarely suffer from navicular disease. The pace with them tells upon the column of weight-bearing bones and upon the ligaments and tendons, but when put to harness-work, in carts, omnibuses, \&c., and shod with thick shoes, they soon become unsound.

Hunters, again, are not nearly so prone to become lame from navicular disease as harness-horses; but if strain were the cause, they would be continually falling lame. They are, however, shod with a level shoe, which allows the frog to touch the ground, and the great concussive shocks, which would be otherwise inflicted at every jump the animal is called upon to take, are thus modified or destroyed.

At one time I was of opinion that compression was the cause; that the tendon caused so much pressure upon the bone as to produce irritation, and gave the animal a feeling of uneasiness in the first instance, as evidenced by "pointing of the foot" before lameness was apparent. I am, however, forced to abandon this supposition, and to come to the conclusion that "pointing" indicates a condition of ostitis sufficient to excite a feeling in the horse that something is wrong, which is insufficient to cause actual lameness.

Symptoms.-The lameness is manifested in two ways:-1st. Suddenly, and perhaps without apparent cause, very often immediately after the horse is newly shod. It is then attributed to some fault in the nailing; but on examination, nothing wrong is found in the nailing, or that the shoe has bruised the foot in any part by undue pressure. After a time this lameness may disappear, or it may disappear from one foot, and after an in- 
definite period reappear either in the same foot or its fellow. The rheumatoid form is thus manifested. $2 d$. By a slow and insidious process in one or both feet, and this is the most common form; the first noticeable sign being pointing of the foot, succeeded after a time by "shortness of the step" and lameness, which may be of a transitory character. For example, "a horse may quit the stable apparently sound, and it very often happens that the rider or driver may fancy that the horse now and then goes lame. The foot is examined, and nothing found, and next morning he may seem all right again. The next journey perhaps, or rapid work, brings back the lameness, which after a little rest and a poultice to the foot disappears again; he may perhaps favour it a little, but there is nothing of consequence the matter, but in the end, if the fast work is continued, the case becomes confirmed."-(Percivall.)

The diagnostic signs of navicular lameness are-1st. Negative; 2d. Positive. (1.) The negative signs are the absence of injury to any part of the limb, and of any apparent disease. These are diaguostic of foot lameness, and are of importance, as they limit the seat of the disease to the foot. This region must now be explored, and lameness in the foot may exist from various causes, but in each variety the signs are different. If laminitis be present, there will be heat, tenderness, \&c. If a corn be the cause, its presence is easily detected; in fact, in nearly every other form, except navicular arthritis, there will be some appreciable sign; but in the disease in question-if we except occasional heat and tenderness in the hollow of the heel, or redness of the frog and sole immediately below the navicular bursathere will be no apparent sign. The redness of the frog and sole, now and then met with, is very characteristic when really due to navicular disease; but it is not always to be depended upon, as it may arise from external injury, such as treading upon a stone, and may be superficial only. Pointing of the foot or feet, although a most valuable adjunct to a correct conclusion when taken along with other signs, is of itself quite insufficient to mark the seat of the lameness; for it may depend upon other causes, such as splint, sprain, ring-bone, \&c.; or an animal may point and be perfectly free from any unsoundness. This " pointing" may be a mere habit, or a sign of fatigue. In such instances, however, it differs in its character from the pointing 
of lameness. When a mere habit, or arising from fatigue, the animal stands with one fore foot and then the other in a semiflexed position, in a careless lounging fashion; but he points two limbs simultaneously; that is to say, one fore and the hind limb of the opposite side of his body. But in pointing from disease, one foot only is pointed at a time; and when one limb or foot is diseased, that foot only; when both fore feet, each foot alternately.

(2.) The positive signs. If, along with absence of other disease in the foot or limb, there be the heat and tenderness upon pressure at the hollow of the heel, or the redness of the sole already spoken of, these may be looked upon as positive signs. Many veterinarians, by exercising great pressure upon the heel and violently flexing the pastern, cause the horse to show signs of pain, which they consider sufficient to denote navicular lameness; but the severe way in which some make this examination is sufficient of itself to cause the pain, and even to aggravate any other disease in the limb. I have for a long time ceased to have faith in this method of manipulation, and for the reason that many horses will wince upon pressure, no matter where the lameness may exist, and content myself with the action of the horse, which may be looked upon as furnishing the most positive sign.

A horse suffering from this lameness comes out of the stable after an interval of quietude stiff and lame; he may be scarcely able to put his lame foot to the ground, but after he has been exercised for a short time, particularly if the ground be soft, the great lameness disappears. If he be lame in both feet, his step is short and stilty, and he seems rigid and bound by some stiffness of the muscles of the chest and shoulders. On this account our forefathers called the disease "chest-founder." There is nothing, however, wrong with the shoulders or chest, and the peculiar rigidity of movement and shortness of step are thus explained:-1st. The shortness, because the pain felt in the foot implanted on the ground causes the animal to relieve it as soon as possible by bringing down the other foot, just as a man lame in both feet is compelled to take short steps whilst walking. $2 d$. The stiffness arises from an aversion to all movement on the part of the sufferer, because flexion of the foot excites pain in the bursa. He also naturally 
avoids elevating it to any extent, from an instinctive knowledge that the concussive shock would be materially increased when (after such elevation) it is again brought to the ground. In addition to shortness of step and rigidity of movement, the wear of the shoes is characteristic. They are worn at the toes; the patient avoids bringing his heels down, and he digs his toes into the ground. When the animal is lame in one foot only, this peculiar appearance of the shoe is of great assistance to correct diagnosis.

Professor Dick says that "the symptoms of the early, and, in a practical point of view, the most important stage of the disease, are a peculiar shifting of the feet, and a shortness of the step; while a degree of heat is found in the foot, more especialiy about the heel and coronet. There is a continual pointing or holding of the foot in a relaxed position, dryness of the hoof, throbbing of the plantar arteries (?) and pain on pressure in the hollow of the pastern. The other parts of the limbs are clean and fine; there is general tenderness of the foot on pressure, with tripping and stumbling; and finally, the foot is contracted."

Contraction of the foot always succeeds navicular disease, and in some instances the atrophy is not confined to the foot, but extends to the muscles of the shoulders and fore arm. This wasting has, however, no further connection with the disease than that it results from diminished function, due to limited action, as already explained.

Pathology.-The parts involved are the navicular bone, the articular cartilage, the synovial membrane of the bursa, and the tendon of the flexor pedis perforans. The inflammation is first limited to the cancelli of the bone, or the cartilage, as illustrated by the following plates.

The tendon passes under the bone, as a rope under a pulley, their surfaces being in a more complete co-adaptation from the circumstance of the bone having a process or eminence across its middle, to which the tendon is fitted by a corresponding hollow in its substance. As a rule, it is the crest of the bone that shows the earliest signs of caries; whilst calcification of the cartilage is usually seen upon either side. Inflammation having been set up in the vascular cancellated structure of the bone, the progress of that inflammation may lead to a 
variety of changes, both within its interior and upon its articular surface; in one instance, to the deposition of small calcareous
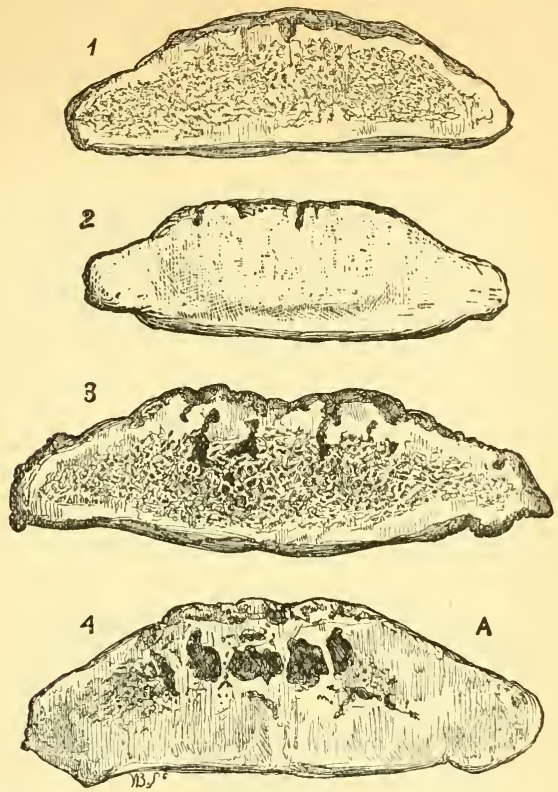

FIG. 61 represents longitudinal sections of four navicular bones, sawn through their middle as near as possible.

1. Healthy bone. Cancelli in their natural condition, with distinct spaces (areolæ) between the bony trabeculæ.

2. Early stage of ostitis. The bone presents almost an ivory-like appearance, from exudation (and ossification of the exudate) into the areolar spaces, rendering the bone almost solid.

3. Second stage. Showing rarefaction of the bony tissue, and expansion of the areolæ; the inflammatory new formation has been absorbed; the cancelli are enlarged, and cavities are formed (indicated by the dark spots) by absorption of the bony trabeculæ. The exterior of this bone shows no signs of disease.

4. In this figure both the processes, viz., consolidation and rarefaction, are going on. In the centre and to the left hand, dark spots (foraminæ) are seen, indicating the destructive (rarefactive) process; whilst to the right, and indeed alinost surrounding the cavities, the bone presents the ivory-like appearance, the natural areolæ being filled with bony matter. 2 and 4 represent changes that are consonant with those of rheumatoid disease.

spots upon and within the cartilage (see No. 2, Fig. 62); 
in another, to the formation of large carious-looking cavities; exposing the vascular interior of the bone (see No. 3, Fig. 62),
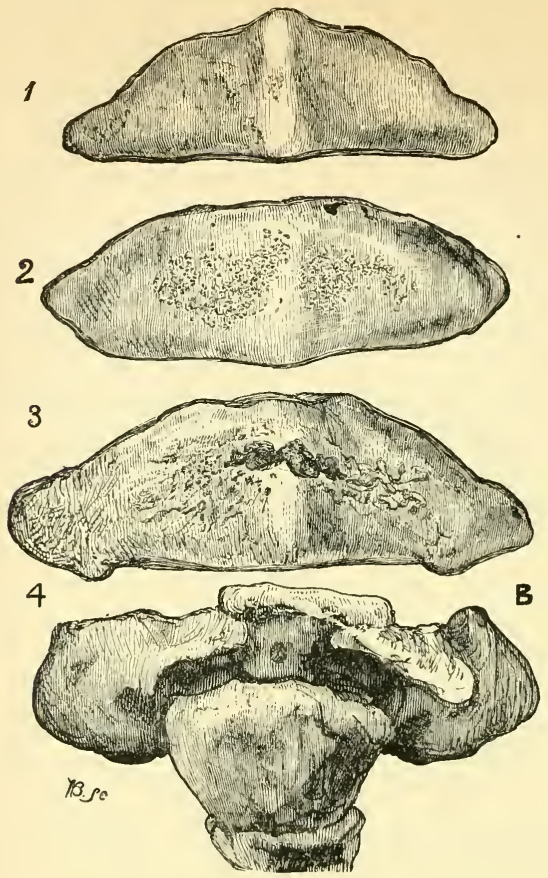

Fic. 62 represents external appearance of inferior articulating surfaces of four navicular bones.

1. Apparently healthy bone; externally it presents no appearance of disease. It is carefully selected as typical of an apparently sound bone, its cartilage of incrustation being intact, and the tendon perfectly healthy. The animal from which it was taken had been lame from navicular disease before its death. Its internal appearance is represented by No. 3 in the preceding illustration.

2. Calcification of the articular cartilage. The calcareous spots indicated by the dark dots; interior of this bone much opened up.

3. Caries of articular surface.

4. Adhesion of tendon to the exposed cancelli of the bone.

with gradual removal of the bone, its texture at same time becoming friable, until at last it may become fractured by some 
trivial accident. In other instances the destructive process is limited (see No. 4, Fig. 61, page 343) by ossification of the cancellated spaces limiting the boundaries of the cavities by a more or less solid osseous wall. Not only are the natural areolæ of the interior structures solidified, but the vascular canals within the trabeculæ are blocked up by osseous material, the more effectually to arrest the process of destruction; and for the same purpose, and to limit motion as much as possible, the tendon becomes adherent to the bone. This adhesion of the tendon occurs in two ways:-1st. Its fibres are lacerated and present loose ends. These are imprisoned by lymph thrown out from the exposed interior of the bone, and are united to it by a new connecting fibrous tissue. $2 d$. By the formation of a false membrane, which is very vascular, similar to that already described (Goodsir's false membrane), extending from the synovial fringes, both on the inferior surface of the bone, and superior surface of the tendon, creeping by slow degrees over the whole articular surfaces, destroying their smoothness, and becoming a bond of union between them. In some specimens all semblance to a bursa has been destroyed by the parts becoming completely united together, and secretion of synovia, being no longer required, has entirely ceased. In the earlier stages the synovial secretion seems to be augmented, and this was considered by Professor Dick to give rise to the fulness which is sometimes observable in the hollow of the heel. In some rare instances the navicular and pedal bones become anchylosed by spiculæ of bone from the inferior border and extremities of the navicular, and from the posterior part of the pedal bone.

The changes in the tendon are indicated by, first, softening and fibrillisation of its superior surface; the splitting up is not altogether due to friction against the roughened surface of the diseased bone, but to degeneration of the structure of the tendon, from the inflammatory action having extended to it from the bone, and increased vascularity of its connecting tissue. This splitting up or rupture of the fibres is manifested by the surface of the tendon being covered by tufts of loose, stringy, whitish fibres, with their free extremities floating in the secretion of the synovial membrane, until an exudate is formed, by which they are at a later stage united to the bone.

The synovial membrane, at a very early stage, is involved in 
the inflammatory action; its vessels are injected, its surface presenting a red streaky appearance; it becomes slightly, but

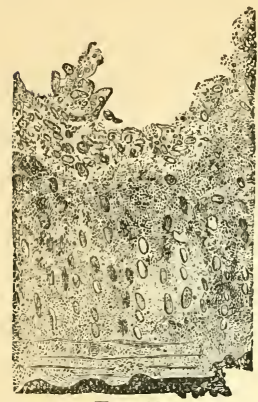

Fig. 63.

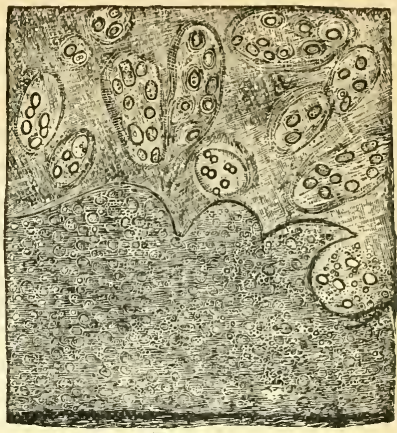

Fig. 64 .

FIG. 63 shows the gradual ejection of the tissues of the cartilage at the ulcerated spots. In the centre of the hollow the superficial parts of the cartilage have disappeared, and the deeper parts are undergoing degeneration, becoming irregular, shrivelled, losing their walls, and discharging their contents.-(After REDFERN.)

Fig. 64.-Vertical section of cartilage, showing the notches produced by bursting of the cells, and the fibrous and granular mass on the free surface.-(REDFERN.)

This figure very fairly illustrates the change in the navicular cartilage at an early stage of its conversion into a finely granular calcareous substance.

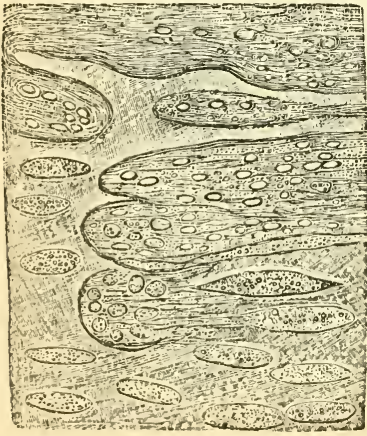

Fif. 65 shows the superficial cells of the cartilage enlarged, and passing into a granular and fibrous mass on the surface. never much thickened; in very chronic cases its free surface becomes involved in the general adhesive process, and united to the tendon and bone.

The changes in the cartilage are the same as when the cartilage of incrustation is inflamed in an articulation (see Chapter XI.) ; it is either removed from the surface of the bone by ulceration, or converted into fibrous tissue or a calcareous material. The supposition that the surface of this cartilage is naturally covered by a membrane is not borne out by microscopic investigation; the supposed membrane being nothing more than the cartilage corpuscles, which are flattened upon the free sur- 
face of all articular cartilages.-(See Fig. 36.) It is true that the navicular bone, like the sesamoids and the bicipital groove of the humerus, is covered by a cartilage with fibrous tissue imbedded in its matrix, but there is no membrane on the surface.

The phenomena observable during the course of the disease here call for remark, more especially the variation in the pointing of the feet. One animal will exhibit this sign before any lameness is manifested, whilst another will have been lame for some time before it begins to point. In the first instance, the pain in the part is due to a very slight degree of inflammatory action, and marks a slow, insidious development of the disease; there is sufficient pain to cause uneasiness whilst the animal is at rest, and when his attention is not called away from it; but when at work, the excitement of exercise, more especially if he be a high-spirited horse, makes him forget his pain, or the pain itself is dissipated by movement. In the other case, sufficient injury has been inflicted to cause immediate lameness, and this may last for some time before the animal becomes habituated to the method by which some degree of relief is secured by pointing. The act of pointing is performed in two ways :- - 1 st. By extension of the limb and of the pastern, the heel of the foot resting upon the ground, the leg stretched forward before its fellow. In this form relief is obtained by the increased obliquity of the pastern, and by an endeavour to approximate the column of bones to their natural position, whereby weight is taken off the navicular bone. The other method is by flexing the limb, touching the ground with the toe only, the heel elevated, the flexor aspect of the limb relaxed, and the inflamed part relieved of tension and pressure as much as possible. This form is indicative of more advanced disease than the former, of denudation of the osseous cancelli, and fibrillisation of the tendon.

The concavity of the sole of the foot is gradually increased, and the frog hardened and elevated from the ground, or softened by a discharge from its cleft and surface. These changes are, however, but a part of the general atrophy of the foot; there is decreased functional activity; the heel is not brought firmly to the ground, and its structure wastes or becomes diseased.

In discussing the causes of the disease, I have stated my opinion that generally the alteration in the direction of the bony column by the shoe, or by a natural upright conformation 
of pastern, is the great cause. In addition to this violent interference with the designs of nature, the elevation of the heels furnishes additional cause of concussion to those already stated, by removing the frog from the ground; the frog being elastic, tough, and strong, intended to touch the ground and diffuse concussion, as well as to prevent slipping at each step the animal takes. When left in its natural condition, it is a large tough, softish body, giving way to the pressure of the fingers when manipulated, expanding each time the foot is put to the ground, the commissures at its sides admitting of this expansion without the other parts of the foot being interfered with. So long as it touches the ground, the sensitive parts within, and particularly the navicular bursa for which it furnishes a soft bed, rests upon a resilient body, free from concussive danger. Besides furnishing this soft bed, it performs another important function; being elastic within and without, it assists the flexors in the first act of elevating the foot from the ground, and it does this in virtue of its inherent power of assuming its original shape when the superincumbent weight is taken away from it. Thus, whilst on the ground, and bearing its proportion of weight, it is flattened from side to side, bulging into the commissures and cleft, ready at every movement, like a piece of india-rubber, to assume its proper form when the least portion of the weight is taken from it. When the flexors are acting, it becomes narrowed from side to side, and deeper from surface to surface, and during this assumption of its natural form, it gives an upward impetus to the foot, which is of essential importance to its easy elevation. The removal of this frog pressure during long periods of rest in the stable is also a fertile source of the disease, because the frog becomes hardened, and does not furnish a soft cushion for the bursa to rest upon. The question may be very properly asked, Why, if concussion be the cause of the disease, the caries does not appear upon both synovial surfaces of the navicular bone? The answer to this may be found in the following remarks, namely-1st. That the lower surface and tendon were intended to rest upon the soft resilient frog, and not upon a hardened body, like what we find in improperly shod horses. $2 d$. That the changes of tissue consequent upon functional activity is greater upon the surface under which the tendon plays. $3 d$. 
Ulcers have always a tendency to open upon parts nearest the surface of the body; and, finally, because this surface, being deprived of its soft cushion (the frog), receives a greater amount of concussion.

Treatment.-Recollecting that the first stage is that of inflammation, all means should be used to arrest this process before any alterations of structure have taken place. Shoes are to be removed; the frogs allowed to touch the ground; blood is to be withdrawn from the toe, or coronary plexus; and the feet placed in a cold water bath for several hours during the day, and in a poultice at night. Whilst in the bath the horse's head must be tied up; but when he is out of it he should be encouraged to lie down, for the purpose of taking weight off the feet. This method of treatment, with an occasional purgative and a cooling diet, has proved most successful. At the end of a fortnight, whether the lameness be removed or not, a mild blister round the coronet is very useful; but should this plan prove of no avail, a seton must be inserted through the frog. If the near foot is to be operated upon, the needle should be introduced from the frog upwards; but if the off one, from the heel downwards, if the operator be not lefthanded. It is seldom necessary to cast the animal. The horny frog must be well pared, and if the needle-a sharp-pointed, short, curved one-be passed quickly, the operation is generally completed before the animal knows much about it. It is necessary that care should be taken not to introduce the needle too deeply, as the tendon may be wounded; the point of the needle should be introduced into the frog about one inch from its toe, and brought out midway between the bulbs of the frog and the anterior boundary of the hollow of the heel. The ends of the tape must be tied together, so as to form a loop, which is to be sufficiently long to admit of a little movement in dressing, but not so long as to extend to the boundary of the wall, or the horse, by treading upon it, will soon destroy it. Before the seton is introduced, it is usual to put on the shoe, for the purpose of removing weight from the irritated frog. About three weeks or a month is the usual time for keeping the seton in the frog, dressing it daily, and keeping the foot clean. After its removal, the frog must be examined, for usually it is "under-run" between the two orifices by 
purulent matter; if such be the case, the detached horn must be removed, and the exposed parts dressed with a little tar. If structural changes have not taken place, this plan of treatment will usually be found effective; but if the lameness continue after a month has elapsed after the removal of the seton, all treatment will be useless, and the animal should be put to slow work, or the pain removed by neurotomy.

This operation (neurotomy) is a very simple one, if care be taken. The incisions ought always to be made in the hollows immediately above the sides of the fetlock-joint, in order that the inner cicatrix be protected from injury from the opposite foot. Two methods of procedure were recommended by Professor Sewell and others, namely, to perform above the fetlock, called the "high operation," or below it, called the "low operation." The high operation includes the division of the trunks of the plantar nerves; and the low, the division of their posterior branches only. Had success attended the latter method, it would have been the more satisfactory plan; but unfortunately it does not entirely remove the lameness.

To operate successfully, the following rules must be followed: -1st. Clip the hair finely over the course of the nerve, that is, immediately anterior to the anterior border of the perforans tendon. $2 d$. Let the animal be made to stand in cold water for an hour before operating; this lessens the tendency to hæmorrhage from the small vessels necessarily cut through. $3 d$. Cast the animal carefully. The leg to be operated upon must be removed from the hobble, but fastened to the hind leg by the side-line extended from above the knee to the thigh; the foot pulled forward by a cord or strap tied round it, and held by an assistant. It is necessary to extend the fetlock. joint, or the incision might be accidentally carried into the sesamoid bursa. 4th. Feel for the edge of the tendon, and cut boldly but carefully down upon the nerve, exposing it without dissecting the areolar tissue. 5th. Divide the nerve as high up as the incision will admit, in order that the skin may overlap its exposed extremity, and dissect out at least an inch of its length. The nerve is always divided at the superior part of the wound, in order that the infliction of pain be not repeated, as would be the case if the first division were made below. 6th. When the nerve is removed, the lips of the wound 
may be brought together by suture, pin, or plaster, the latter being preferable, as leaving less blemish. It is usual, when both legs are to be operated upon, to perform the operation on the one lying undermost, of course dividing the inner nerve, and upon the outer side of the upper leg, before turning the animal. When the operation is completed, the patient is allowed to rise, removed to the stable, and there tied, so that he cannot lie down or interfere with the wounds for three or four days. If pins or sutures have been inserted, a covering of styptic colloid or collodion may be applied after the slight hæmorrhage has ceased; after this is done, if the parts are not interfered with, it will be found that they will unite by the adhesive process.

Unfavourable results of the Operation.-These are many, even in well-selected cases. If the selections are not judicious, the operation is calculated to do much harm, and to bring discredit upon the practitioner.

In determining whether it is judicious to operate or not, the following rules must be borne in mind:-1st. Never operate on a very heavy, thick-legged cart-horse: $2 d$. Never operate where the feet are thin, weak in the heels, full in the sole, or otherwise exhibiting a predisposition to laminitis ; and $3 d$. Operate only where the foot is good and strong, the animial's action not too high, and the lameness otherwise incurable.

The untoward results are fracture of the navicular bone, rupture of the tendon, sloughing of the hoof, and a peculiar gelatinous degeneration of the bursa, tendon, and surrouuding structures, along with the formation of a large quantity of a low form of fibrous tissue.

The first symptom of this degenerative process is a bulging, doughy, or elastic sweiling in the hollow of the heel; the animal at the same time going upon the heel more than usual, and the toe slightly elevated at every step it takes. This peculiarity of gait may at first be so slight as almost to escape notice; but it is a symptom of importance, and should be carefully noted, since it indicates that the tendon and the other structures are losing their cohesion, becoming soft, and more or less extensible. The external swelling increases, until it surrounds the whole of the coronet, and extends upwards to the fetlock-joint. The divided extremities of the 
nerves frequently becoming at the same time very sensitive

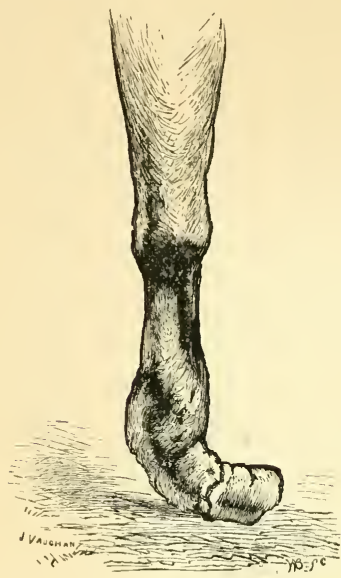

Fig. 66.-Off fore leg after neurotomy, showing external appearance of gelatinous degeneration of its structures, with rupture of the flexor tendon and inferior navicular ligament, and fracture of navicular bone. to the touch, from a deposition between their fibres; lameness may arise from this cause, or it may be absent. As the swelling increases, the coronet and pastern present a tense glistening appearance, and the elevation of the toe becomes greater by degrees, or suddenly, from rupture of the tendon; the leg assumes a form similar to the annexed figure.

The condition illustrated by the woodcut (Fig. 66) is similar to what surgeons denominate gelatinous degeneration, or strumous disease of the joints; and for the purpose of comparison, I give a description of the examination of the leg from which the woorlcut was drawn, and the pathological condi tion of a joint subject to a similar disease in the human subject.

(I.) PATHOLOGICAL APPEARANCES OF THE NAVICULAR-JOINT AND SURROUNDING STRUCTURES OF THE FOOT REPRESENTED IN FIG. 66 .

External Appearances.-In the space usually denominated the pit of the heel, but which now, owing to the altered position of the foot, constituted its posterior part, there was a prominent bulging or elastic doughy swelling. On removing the skin, extensive deposits of a gelatinous or fibrinous material were revealed surrounding the pastern and fetlock joints, involving the extensor and flexor tendons, forming a soft cushion for the lacerated structures to rest upon, and embracing in its interstices a large quantity of a sero-sanguineous fluid.

The flexor perforans had been ruptured at that portion which passes under the navicular bone; but the rupture was not complete, the inferior layer of fibres remaining still unbroken. The 
divided ends of the tendon were some distance apart, and they bore a jagged appearance, owing to the formation of fringe-like processes of a gelatinous material, containing some blood-vessels, which gave to the new formation an appearance similar to that of pale muscular tissue. The inner or anterior surface of the tendon bore evident traces of ulceration and gelatinisation; and it had an appearance of ecchymosis in its structure.

The inferior broad navicular ligament was ruptured, its divided edges presenting the same appearances as those of the tendon, but were considerably more vascular.

Appcarances of the Navicular Bone.-Externally, its posteroinferior surface presented no distinct traces of ulceration; the cartilage was absorbed; and the bone presented a vascular dirtybrown appearance, and its articular cartilage was covered over by a vascular membrane, the processes of which could be distinctly seen penetrating the structure of the bone, and extending over more than one-third of the bursal surface. The bone was fractured at about half an inch from its right extremity.

(ii.) Pathological anatomy of gelatinous degeneration, oR STRUMOUS DISEASE OF THE JOINTS, IN THE HUMAN BFING.

"Gelatinous degeneration may attack any joint in the body, but those most frequently affected are the knee, elbow, and ankle. I shall take the knee-joint as an illustration, as the disease, especially that of the low chronic form, is most frequently there situated.

"In about six weeks after the commencement of the disease, a partial swelling is found on either side of the patella, extending towards the posterior aspect of the lateral ligaments. This increases around the patella, and the markings of the bone are gradually lost in it. The swelling presents either a peculiar elastic or a doughy feel. As the disease goes on, the symptoms become more marked, the colourless swelling increases, the joint becomes more tense, and has a glazed appearance.

"The pathological condition leading to these symptoms consists in a peculiar degeneration of the synovial membrane. This exists under two forms, and these have been described under two separate names-the gelatinous and pulpy degeneration; but they are truly identical in symptoms, and as regarcis 
the essential nature and course of the disease. In the gelatinous degeneration, all the cartilages of incrustation become gradually covered over by masses of new deposition, which extend wherever there is a synovial membrane. In the recent state, this deposit has a gluey or jelly-like character varying in colour from pale yellow to dark brown, and is generally intersected by lines of white membraneous structure. This is the more general character of gelatinous disease; but there is another condition, in which the membrane assumes a sort of granular or fungoid appearance on the surface. This has been described as pulpy degeneration; but all the phenomena are the same as in the former case, and there is no use in making a distinction between the two conditions. The deposit fills up the whole joint, and acts as a kind of soft cushion, protecting the ends of the bones, so that at first, when the swelling is most marked, there is comparatively little pain.

"The cartilage of incrustation is removed by absorption; the absorption is induced by the pressure of the new material on its surface, or by a peculiar action which the deposited substance exerts upon the cartilage. All the textures are ultimately involved; not only the synovial membrane and the cartilage, but also the fibrous textures and the areolar tissue. This gives rise to disorganization, and the formation of abscesses and sinuses in all directions."-(SPENCE's Lectures on Surgery.)

My object in describing this comparison between the white swelling of the human joints and the disorganization that so frequently succeeds neurotomy, is to show how a similar pathological condition may be induced by a circumstance which at first seems to be so very dissimilar; -in the human being, a constitutional taint; in the horse, removal of a portion of the sentient nerve. Further inquiry may discover that the disease from constitutional taint is in reality due to altered innervation consequent upon that taint.

The rupture of the tendon is due to a slow degenerative process, and not to a sudden snapping of its fibres, as was formerly taught.

Now and then, however, sudden rupture of the tendon takes place immediately after the operation; but this is rare in comparison with the degenerative process, which not only involves the tendon, navicular bone and ligaments, but all the surrounding structures of the foot and pastern. 
In the dissection of the foot from which the figure was drawn, the navicular bone was fractured, and the inferior broad ligament ruptured. Neither of these results was due to violence, for the animal had been at rest for several weeks prior to its death, and the various changes had come on gradually.

Another unfavourable result of the operation is loss of the hoof from suppuration induced by accidental injuries to the foot, or from morbid action in the tissues, deprived of their nervous sensibility.

In some cases regeneration of the nerve substance occurs; the divided ends become continuous by growth from their extremities, the nervous current is re-established, and the lameness recurs. These results, however, do not take place for at least two years after the operation is performed. If the lameness is severe, it may be necessary to excise the reunited nerves, and thus again destroy the sentient communication between the diseased part and the nervous centres. It has been already shown that fracture of the navicular bone, when unaccompanied by degenerative disease of the bursa and tendon, may not interfere with the animal's usefulness; the fractures, as in Figs. 15 and 16 , being united by a false joint, and the animal continuing to perform his daily task with apparent ease and comfort.

My experience leads me to the conclusion that the lameness arising from ring-bones or side-bones, when the feet are good and strong, is generally permanently benefited by neurotomy, the animal restored to its full usefulness, and remaining sound for many years. Indeed, in ring-bone lameness I have repeatedly noticed that not only has the lameness been entirely cured, but that there has been a gradual absorption of the osseous new formation, and a restoration of the pastern almost to its natural shape and dimensions. When the operation has been performed for the relief of these lamenesses, there appears to be an absence of any tendency to gelatinous degeneration or other alteration of structure. 


\section{CHAPTER XIX.}

\section{DISEASES OF THE FEET-continued.}

INFLAMMATION OF THE FEET-DIVISIONS OF LAMINITIS-PATHOLOGICAL CHANGES - CAUSES - SYMPTOMS - TREATMENT - MR. BROAD'S TREATMENT.

LAMINITIS, OR INFLAMMATION OF THE FEET.

Inflamition of the feet, independent of its subdivisions into acute, subacute, and chronic, is of two kinds, namely, inflammation primarily limited to the sensitive laminæ and sensitive sole; and ostitis, or inflammation involving the os pedis, laminæ, and sole from the very outset. The causes, course, and tractability of the two forms differ; but the first, if not subdued in a short time, is apt to become developed into the latter.

"Laminitis is one of the most dreadful diseases the horse is liable to, and is not confined to the feet, although its chief seat is there. It is caused by over-exertion, inordinate feeding, drinking cold water when heated, long voyages, from the horse being compelled to stand in a constrained position, or a sudden chill. It is often communicated to the feet from internal organs, as from pneumonia, enteritis or bronchitis; in this case the feet are affected as well as the whole surface of the body, the hair of the mane and tail being often thrown off; and the tendency of laminitis is to throw off the hoof, as well as the common integuments, in consequence of the general irritation."-(Professor Dick.)

Laminitis, arising from over-exertion and from the concussion of hard galloping on the road, as in those reprehensible cases where horses are run against time, is much more intractable than when it appears during diseases of the nucous membranes, when it may pass off as a mere congestive attack, without leaving any structural change; whilst the form caused by concussion leads to ostitis of the most acute kind, sometimes terminating in necrosis of the pedal bone, sloughing of the hoof, and a most agonising death. The symptorns of both varieties are identical, except in their severity, and if the so-called meta- 
static form continues long enough, the pathological changes are the same also.

Pathology.-Inflammation of the sensitive foot, including laminæ, sole, and os pedis. The exudation is greatest at the toe, the foot being most vascular at this point. The pain of laminitis is most agonising and persistent, because the sensitive foot is invested with an unyielding horny box pressing upon the engorged blood-vessels, preventing free exudation and swelling, and thus proving a barrier to the method by which congested blood-vessels are relieved. The exudate in laminitis is found to limit itself to the external surfaces of the sensitive laminæ, but in that form caused by violent concussion, and which with propriety might be termed "peditis," the exudation may be subperiosteal, detaching the periosteum from the pedal bone by the outpouring of an unhealthy, broken-down, dirty reddish coloured fluid; whilst the interstices of the bone, its canals, and lacunæ are filled with the same material, forming a barrier to the free circulation of the blood, and thus assist in hastening the process of necrosis and sloughing.

Mere laminitis may pass off without causing structural change ; there is but a slight exudate, and this is soon absorbed after the subsidence of the inflammatory action. In some cases the removal of the exudate leaves a space between the sensitive and horny laminæ, which has led some observers to think that the disease consists essentially of absorption of the bond of union between the two sets of lamini, the formation of a cavity, and the subsequent filling up of it with imperfect cheesy or seedy horn.

Should the inflammation persist, the exudate accumulates at the toe, increases in thickness, presses upon the. toe of the os pedis in the one direction, and upon the crust in the other, separating the two, forcing the toe of the bone downwards, and, later on, the toe of the crust upwards. The effect of this change in the position of the bone is the formation of a convex sole. The sole, being pressed upon by the point of the bone, is forced downwards, losing its natural concave shape; whilst from the secretory powers of the sensitive sole becoming interfered with, partly arrested or perverted, the horny sole remains thin, weak, cheesy, or spongy, like macerated horn, or even grumous, affording but little protection to the sensitive parts within. The outer horn of the wall, namely, that secreted by the coronary band 


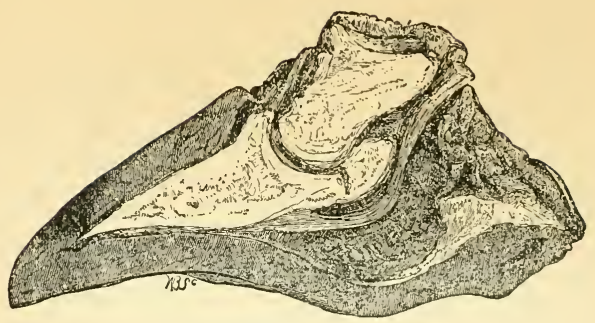

FIG. 67.

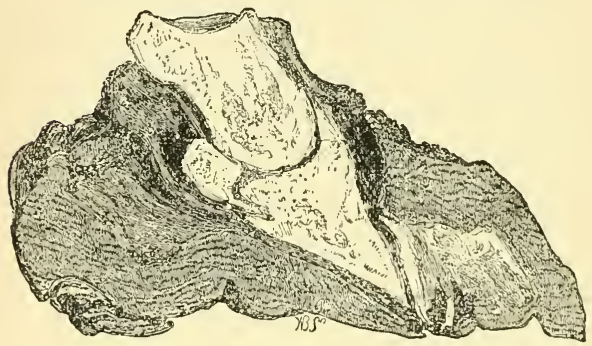

Fig. 68 .

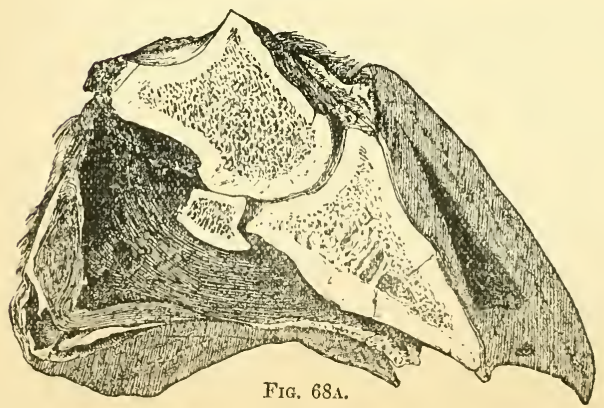

FIG. 67 represents a section of the foot with its various parts in a healthy condition, and in their proper relative positions.

FIG. 68.-Formation of a horny exudate at the toe, causing alteration in the several positions of the os pedis, wall and sole, and the formation of convex or "pumiced" sole. Whilst this process is going on, the extensor pedis is elongated, and the flexors thrown into a passive condition.

Fig, 68 A.-Perforation of the horny sole by the toe of the pedal bone, due to the pressure of the new formation.-(College Nuseum.) 
now presents characteristic appearances; it becomes ribbed, as if there were successive efforts to cast the hoofs. The rings of laminitis are irregular, and run together towards the anterior aspect of the foot, as in the annexed illustration (Fig. 69). This

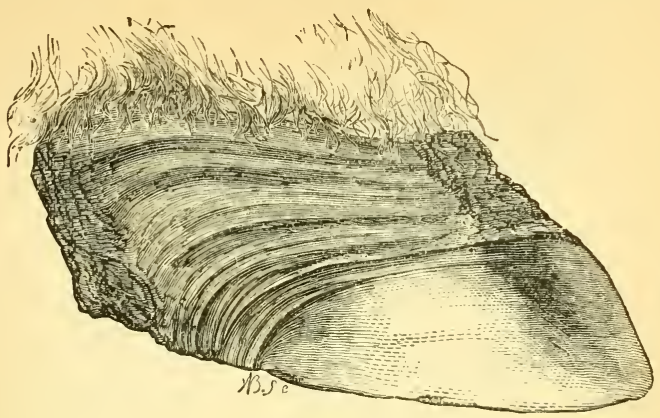

FIG. 69.

distinguishes them from the rings of a healthy unrasped foot, in which they are regular, and have wider interspaces.

The bone, pressed downwards by the exudate, becomes absorbed at its borders, by which it is reduced in bulk, more

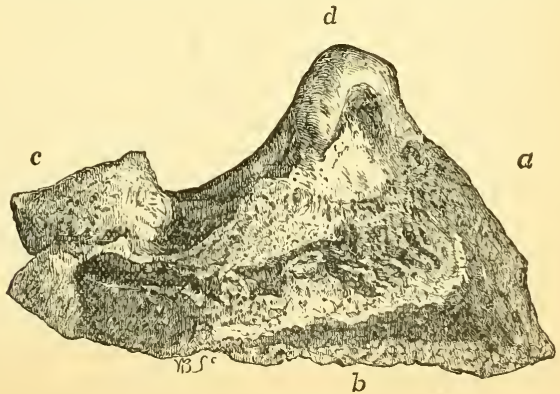

FIG. 70.-Absorption of inferior edge of os pedis. $a$, Anterior aspect. $b$, Plantar edge; the dark shade represents a hollow space from which the bone has been absorbed. $c$, Basilar process. $d$, Pyramidal process.

particularly at its toe and sides, whilst its whole structure uecomes brittle (1st.) by ossification of the exudate within its 
interstices; and (2d.) by removal or absorption, as already described under Navicular Disease.

In addition to the results indicated by the above-described pathological changes, ossification of the sensitive laminxe and

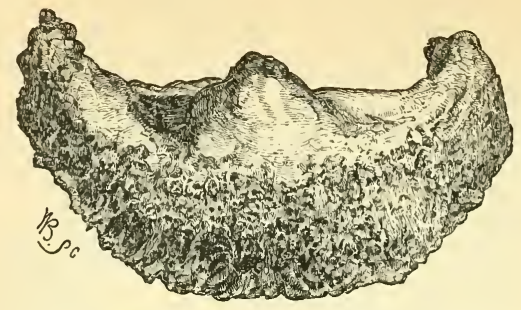

FIG. 71 represents a more advanced atrophied or absorbed condition of the bone, with ossification of the laminæ.

suppuration are occasionally found, the latter occurring more especially at the coronet, detaching a small portion of the upper part of the wall; whilst in other instances a serous fluid collects between the sensitive and horny soles and frogs, and detaches them from each other.

Direct concussion causes laminitis, by its effects upon all the structures of the feet. Usually this form is confined to the two fore feet. But we must look further into the question, to understand the operation of inflammation, or even irritation of an internal organ, in the causation of this disease.

Many writers state that affections of a serous membrane, such as the pleura, terminate, by metastasis, in laminitis. I have carefully studied the question for some years, and have arrived at the conclusion that such is not the case; that when the feet become inflamed during the progress of another clisease, a mucous membrane is involved, and that the inflammation of the lamine is due to an extension of the irritation from its primary seat to the lamine of the feet, as explained by Professor Dick in the words already quoted, namely, "The feet are affected, as well as the whole surface of the body, the hair of the mane and tail being often thrown off." To explain this, it will be necessary to remember that the skin, mucous structures. and laminæe are continuous one with another, and secretory; the nature of their secretions being similar, namely, epithelium 
secreted by the mucous membranes; scales of the same nature, by the skin; and horn cells, similar in every respect to those of the skin and mucous membranes, by the laminæ. It must also be remembered that when a part only is irritated, the effects of such irritation tend to spread, and that when the mucous membranes of the intestinal, uterine, or respiratory passages are inflamed, the skin participates, as may be witnessed in many cases of cutaneous eruption from indigestion, catarrh, \&c. The irritation of the cutaneous surface may be very slight, scarcely observable; but the irritation of its foldings in the feet, surrounded by their unyielding case, becomes a source of great pain; in fact, it may be said that this slight sympathetic irritation becomes the cause of an acute inflammation, because located in unyielding structures. I am convinced there is no real metastatic transference of the inflammation from its original seat, for it is found, when horses die from super-purgation, diarrhœa, or other irritation of the intestinal mucous membrane, that laminitis is often present before death, and post mortem examinations of such reveal the presence of inflammation, both in the feet and in its original seat. It is not necessary to have an inflammatory disease of mucous tissue to induce fever in the feet; a slight irritation, such as that of a moderate cathartic, being sufficient. I have several cases on record where a moderate dose of purgative medicine has caused inflammation of the feet, without seemingly stimulating the intestines to more than moderate purgation. That the skin is in an irritable condition is also proved by the tendency to the non-healing of wounds during laminitis, by the loss of hair, as pointed out by Professor Dick, and by the frequent occurrence of phlebitis, or inflammation of the jugular vein after bleeding from it in this disease. The wound in the skin made for the withdrawal of blood does not heal, but suppurates, and the diseased process extends to the venous coats.

Acute laminitis terminates in resolution of the parts, or in that form which is termed subacute or chronic, in suppuration, and occasionally in gangrene.

Chronic laminitis is that condition of the feet remaining after the subsidence of the febrile symptoms; or it may originate independently of an acute attack. Horses suffering from the chronic form are, however, subject to the acute from 
the most trivial causes; and the acute form, when caused by concussion, if the animal outlive the primary attack, commonly degenerates into the chronic. The pathological changes, namely, the descent of the toe of the pedal bone, the absorption of its borders, formation of convex and weak sole, and the organization of a large mass of imperfect horn at the toe, are identical in both forms, the difference being in the severity of the accompanying febrile disturbance, and in the time in which the changes are accomplished.

The predisposition to inflammation of the feet is often traceable to hereditary tendency and defective conformation, as well as to bad management of the feet. It is said that horses with wide feet are more liable to the disease than others. Such is not my experience. Indeed, it cannot be said that any one shape of foot is more susceptible than another. It is more commonly found iu heavy dray-horses than in those of a lighter breed, this being doubtless due to the great weight the feet of such are compelled to bear, weakened, as they often are, by paring of the sole.

There are two other classes of horses which are very liable to the disease :-(1st.) The horse which has overgrown his breed, that is to say, an animal with the legs and feet of a well-bred and the body of a cart-horse; (2d.) Small ponies that are excessively fat. In both these instances the inflammation can only be attributed to the unnatural weight of the body being greater than the feet are able to bear. I feel satisfied, however, although the weight predisposes, that the removal of the solar horn is the exciting cause. When horse-shoers learn to discard the drawing-knife, laminitis, except from other clearly defined causes, will be much rarer than at present.

The more immediately exciting causes are concussion, excitement, over-exertion, indigestion, more especially when caused by engorgement of the stomach with wheat, or any other cause of intestinal irritation, septic infection, and bad shoeing.

Generally, the disease is confined to the two fore feet, especially when caused by concussion; but it is not very unusual to find all the four feet affected; sometimes the hind feet only, and, in rare instances, one fore or one hind foot. When one foot only is affected, it is often due to an injury to the 
opposite foot or limb, which compels the animal to throw all the weight of that part of his body upon the sound side. The sound foot becomes inflamed, which is indicated by lameness, heat, and pain. Premonitory to the advent of the disease in the sound foot, the patient will sometimes suddenly be seen to bear his weight upon the originally lame side, although the wound, open joint, or other injury from which he may be suffering, continues in all its severity. An ordinary observer will consider the animal to have been unexpectedly relieved of pain; but in a short space of time the hitherto sound foot will be elevated from the ground, the animal at the same time evidencing agonising pain; fever will set in, and the head will be pushed into a corner. These symptoms are manifested to a much severer degree when a hind foot is involved. To prevent this complication to a severe injury or lameness, the shoe is to be removed from the sound foot, which, in very aggravated cases of injury, may be placed in a soft poultice, or allowed to stand upon some soft substance. The leg must be bandaged; and if the horse is unable to lie down, as will generally be the case when a hind limb is involved, he must be placed in slings. I once saw a case where the os pedis of the off hind foot had been forced through the horny sole, in consequence of the animal having received an injury to the near hind leg. The animal was young, and newly taken up to be broken-in. Through some oversight, the horse was shod upon both fore and the off hind feet, the near side foot, owing to the lameness in the leg, being left without the shoe. By this error extra weight was thrown upon the foot of the sound limb-thus elevated above its fellow by the shoe-which caused violent inflammation, and the destructive process above mentioned. The ease is very instructive.

Symptoms of Inflammation of both Fore Feet.-The horse is excessively lame, almost immoveable, especially at starting; he seems as if all his body were cramped; stands with his hind legs drawn under the belly, and the fore feet advanced, in order to relieve them from the weight as much as possible; occasionally he may be seen to sway himself backwards, elevating the toes, throw his weight for a moment upon the heels of the fore feet, and then assume his original position. If compelled to move, he elevates his feet with great difficulty, not 
because the muscles of locomotion are inflamed, as is sometimes supposed, but because he requires all his feet to be upon the ground at the same time to bear the weight of the body. $\mathrm{He}$ will often groan from pain, while sweats bedew the skin.

To diagnose a case quickly, the best method is to push the horse backwards, when it will be seen at once that he will elevate his toes, and throw his weight upon the heeis. The pulse of laminitis is full, strong, and accelerated; and it will maintain the character of strength and fulness even after general debility has manifested itself.

In some instances the animal lies down upon its side, with the legs stretched out, for hours together, evidently feeling great relief from the assumption of this position; whilst in others, particularly during the earlier periods of the disease, it will stand persistently.

When the hind feet only are affected, the patient stands with all his four feet together; the fore ones are pushed under the body, and not extended forwards, as when they are inflamed; the hind ones are extended forwards, in order to throw the weight upon the heels. Many, especially students, suppose that when the hind feet are inflamed, the horse stands with them pushed backwards, to remove them from the centre of gravity as far as possible. Such is not the case, since this position would cause the weight to be borne by the toes, the part of the feet which lie is most careful to relieve, as it is the very spot where the inflammation is most acute. The sufferings of the patient are even greater when the hind feet are affected, and usually the symptoms become greatly aggravated by the standing posture. It is distressing to witness so much pain; the animal standing "all of a heap," with anxious eyes; now nervously elevating one foot from the ground, then its fellow; its respirations hurried and nostrils dilated, in fact, "blowing," as vulgarly expressed; when compelled to move, as soon as the toes of the hind feet are pressed to the ground, he takes a kind of a jump forwards, performing perhaps two or three automatic steps, from the agony of the pain. He rarely stands long when the hind feet are affected, and experiences immediate relief when recumbent. Very often the pulse will fall twenty or thirty beats in the minute in the course of a quarter of an hour after he has assumed this position. 
When all four feet are affected, the symptoms will consist of a combination of the foregoing, with local heat in all the feet, some degree of throbbing of the plantar arteries, and tenderness upon manipulation, or to the touch of the hammer.

Treatment.-Gentle purgatives. On no account must violent cathartics be given, since the operation of purgatives tend to laminitis, and they occasionally cause death when the disease itself has been of a tractable or even mild form. If the bowels are loaded, injections of warm water are very serviceable; but if the constipation is not very urgent, they may be dispensed with.

In addition to a mild aperient, febrifuges are to be administered; and for the fever of laminitis I know of nothing which proves so effectual as the tincture of aconite, in small but repeated doses. In that form arising from irritation of mucous membrane or indigestion, the bicarbonate of soda may be added to the aconite. If the pain be very severe, I think better results are obtained from opium or morphia than from aconite, which seems to act more by reducing fever than by allaying pain. This method of treatment, with poultices to the feet, and the application of Mr. Broad's shoes, has proved of eminent service in the treatment of laminitis.

Practitioners differ in opinion as to whether cold or warm poultices or baths should be applied. Some recommend cold, others prefer warm. For my own part I have found that pain and fever are more speedily removed by warm fomentations and poultices in the early stages, and the resolution of the inflammation is promoted by cold applications after the very urgent symptoms have passed off. There is no necessity to discuss the why and the wherefore of these two methods, suffice it that warmth has a soothing effect upon a part to which it is applied, and that this soothing of pain in laminitis is the first essential step in its treatment.

Mr. Broad strongly recommends exercise, even in the earliest stage of the disease, and very urgently requests me to do so also, but, much as I respect Mr. Broad, I cannot alter the conclusion I have arrived at from much experience of the disease and its treatment. Mr. Broad assures me that he is always successful with exercise. I can only reiterate what I stated in the first edition of this work, that I am of opinion that Mr. Broad would even be more successful than he is, if he were to abandon this 
portion of his treatment. If the disease were a mechanical congestion of the vessels, doubtless such congestion would be relieved by the motion of the limbs ; but we must look for something more than congestion being the origin, namely, irritation, and to the fact that congestion is the result and not the cause of the disease.

The necessity for bleeding in laminitis is a question upon which practitioners differ. Without discussing it, I may state that I consider local bleeding from the coronary plexus advantageous, relieving the congestion and alleviating pain.

The treatment of laminitis has hitherto been often unsatisfactory; but a short time ago a method was recommended by Mr. Broad, V.S., Bath, which at the time secured for him a fair share of abuse and condemnation; one writer going so far as to state that he thought the Society for the Prevention of Cruelty to Animals ought to be informed about it. Fortunately, however, for Mr. Broad, none of his critics had ever tried the plan; whilst others, less prejudiced, and quite as eminent in the profession, gave it a trial, and were satisfied that it was most successful when properly applied.

Mr. Broad's Treatment.-Mr. Broad has kindly written out his method of treatment, and I give it here in his own words :-

\section{" TREATMENT OF ACUTE LAMINITIS.}

"As early as possible get lightly nailed on the feet affected extremely stout, wide-webbed, and long bar shoes, made from iron about twice the ordinary thickness that the particular horses' shoes are made from; make them gradually thin from behind the quarters, so that the heel part of the shoes is wide and thin, and fitted rocker fashion, which enables the horse to throw his weight where he tries to, much better than he can in ordinary shoes or without any; that is, off the pedal bone on to the soft elastic tissues and teudon behind it, which are much less vascular and sensitive.

"The object of putting on very stout shoes is to lessen the jar; as it more commonly occurs that the horses are obliged to be exercised on hard ground, as soft is not obtainable near. (It is a generally acknowledged fact with practical men that stout shoes lessen the jar to the feet of horses working on 
pitching or hard roads much more than light ones do.) If the case is not a severe one, plain shoes, made after the same fashion, will do, although I prefer the bar shoes. I sometimes

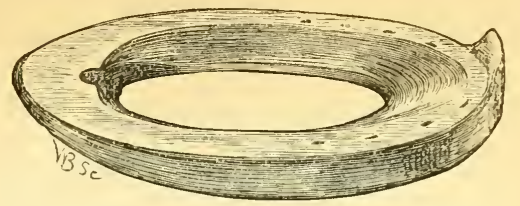

FIG. 72. -Mr. Broad's shoe as described in the text.

use leather soles, but not in all cases. If the heels of the feet are very strong and high, lower them; if not, don't cut or pare the feet in any way. I also give at once an ordinary dose of physic, and as soon as possible get the shoes on, and compel the animal to walk on soft ground, if obtainable; if not, on the road or round the box for a short time, until a little of the soreness passes off; for however difficult it may be for him to move at first, it always lessens from exercise. In severe cases $i^{\downarrow}$. is often necessary for a person to walk behind the animal for a short time to compel him to move on. Regulate the amount of exercise according to circumstances; in a general way, give from half-an-hour to one and a half hours three times per day, allowing the horse to have a loose box when not at exercise. Repeat the physic about the fourth day (depending in a ineasure on the effects of the previous dose, although I never saw any ill effects from the repeated physicking in such cases), until the lameness is removed, and the horse fit to work, which he may do in the same shoes, or lighter ones made somewhat after the same fashion, as it will be better for the horse to do moderate work than to remain idle. In cases arising from metastasis, or excessively long journeys, where there is exhaustion, it is necessary to be very careful with regard to physicking. In country places, when there is a difficulty in getting the special shoes made quickly, the horse may be exercised in a field in his ordinary shoes, or without any, until the special shoes be made and put on. It is very essential that no time be lost before the treatment is commenced, for when the congestive stage has passed, and active inflammation is going on, it is necessary to be very cautious with regard to exercise. If, 
from neglect or improper treatment, separation at the coronet has commenced, or the pedal bone is so far displaced that there is danger of its toe protruding through the sole, or if it is already through, keep the special shoes on, dress daily with hot tar until the opening in the sole has been filled up, and a particle of horny matter thrown out and become dry; after which, as soon as the horse can walk, get him into the wettest pasture that can be found, and if he is not excessively lame, take his shoes off, rasp the heel low, and shorten the toes, so as to bring the sole in contact with the ground. This operation is to be repeated every three or four weeks, which will in time ensure sound and perfect feet as before the attack occurred, notwithstanding that all four of the pedal bones may have protruded through the soles; but it may require from six to twelve months before a new wall has grown down perfect and the sensitive lamina recovered its normal exudative powers. If the feet are not properly and regularly attended to, they will not recover their natural shape, but will be deformed, especially at the toes. A wet pasture is essential, as the horn will grow as much in one month as it would in three months on dry ground.

"Should the horse be suitable for farm work, he may be used on soft land for months before he is fit for fast road work.

"Subacute laminitis is, in the majority of cases, a transitional stage from the acute to the chronic. Very few cases remain subacute which begin as acute; yet you may have cases the symptoms of which may be moderate in intensity, and to be considered as subacute from the beginning.

"The treatment of subacute and chronic laminitis must be a modification of that of the acute disease."

Like Mr. Broad I have treated a great number of horses suffering from laminitis, and very successfully without exercise. If a horse will not lie down of his own accord, he ought to be cast, when very often the relief which is thus obtained by him will cause him to remain recumbent for many hours or even days. If he lie down persistently he ought to be turned over every four hours or so, bedded on clean dry material, and precautions taken against bruising or other injuries. If he be umable to urinate freely, the catheter must be inserted and the urine drawn off at least four times during the twenty-four hours, 
or more frequently if the animal express uneasiness and a desire to micturate.

Every case of laminitis should, if possible, be stabled in a large, airy, well-ventilated loose box, the floor covered with plenty of short straw, chaff, or other material that will not ertangle the animal's feet and legs.

I do not recommend slinging except in cases that will or can not lie down.

If lameness threatens to remain, mild blisters may be applied to the coronets with advantage. 


\section{CHAPTER XX.}

\section{DISEASES OF THE FEET-continued.}

DISEASES OF THE HORN-SECRETING STRUCTURES-VILLITIS-CARBUNCLE OF CORONARY BAND-FALSE-QUARTER - SAND-CRACK - HORN TUMOURS-SEEDY-TOE-CORNS-THRUSH-CANKER.

DISEASES OF THE HORN-SECRETING STRUCTURES OF THE FEET.

LAminitis, if confined to the soft structures of the foot, might with propriety be classified under this head; but as it involves the pedal bones as well as the laminæ, I have arranged it under the first division.

\section{INFLAMMATION OF THE CORONARY SUBSTANCE-VILLITIS (HAYCOCK)-CORONITIS.}

Seen in heavy cart-horses, and occasionally in better-bred ones. Symptoms-harshness and brittleness of the crust; loss of toughness and pliability; increased heat round the coronet and upper part of the crust; often a fulness of the coronary band, and tenderness on pressure; a shuffling gait, if both fore feet are affected; the heels are first put to the ground; but not to the same extent as in laminitis; a desquamation, or a tendency thereto, of the coronary substance, shown by a line of separation between it and the crust; diminished secretion of horn ; and a peculiar striated or striped appearance of the crust, well described by Haycock in his essay on "Villitis." The peculiarity of gait is due to the animal trying to glide his feet along the ground. The duration of this form of lameness is variable, but it generally lasts a few weeks. It is distinguishable from laminitis by the peculiar appearance of the crust, and the bulging round the coronet.

Treatment.-Rest or removal of ordinary shoes; poultices to the feet for a few days, and the application of Mr. Broad's 
shoes, either barred or open; cold applications to the coronets; and when the heat and tenderness have subsided, mild blisters. To reduce any constitutional disturbance, and the local inflammation, purgatives may be given occasionally. If the animal does not become sound with this treatment, he must be allowed a run at grass.

\section{CARBUNCLE OF THE CORONARY BAND, 1}

Called Furunculus by Mr M'Eachran, Montreal, attributed to frost-bites by others, consists of an oval or irregular portion of the coronary substance becoming infiltrated with unhealthy lymph, forming at first a hard swelling of variable size, very painful ; sometimes extending all round the coronet; or appearing at other times as several patches or spots of inflammation; accompanied by great pain, lameness, and a high degree of febrile disturbance. After a few days suppuration sets in, most commonly at several points, with ulceration of the softened portions, forming so many apertures or sinuses, which discharge a thin, unhealthy, ichorous pus. The apertures enlarge by ulceration or even sloughing of their borders, and by confluence constitute a most formidable looking and unhealthy wound. In aggravated and malignant cases, other inflammatory points appear upon the pastern and fetlock, extending in some instances as high as the knee or hock, causing sloughing of great patches of skin and subcutaneous tissues, exposing the tendons, nerves, blood-vessels, and even the cavities of the joints, and producing so high a state of fever and suffering as to destroy the animal's life.

Mr. Robinson of Greenock describes some of his cases to have presented the following symptoms:-Lameness and febrile disturbance. On examining the limb, the integuments, in some part between the knee or hock and the foot, will be found gangrenous, with a dew upon the surface; sinking on pressure; having, in fact, a doughy feel. In a day or two a line of demarcation makes it appearance, and separation of the gangrenous part takes place. When not involving the coronary substance, Mr. Robinson says that the result was always favourable.

1 Carbuncle of the coronary band is a disease similar to malignant onychia of the human heing. 
Treatment. - Poulticing at first, to encourage the separation; afterwards digestives, to stimulate the granulations; and finally mild astringent dressings. Mr. Robinson's cases have all occurred in the winter, and he attributes them to cold and wet. I have seen many cases at times when they could not be attributed to wet or cold, and which seemed to be unmistakeably due to a blood-poison; they were also more malignant and unmanageable than those witnessed by Mr. Robinson.

I believe this disease is hitherto undescribed by any English author; but I know several veterinarians who have met with cases of it as well as myself.

I am inclined to think that it is due to a blood-poison similar to that of glanders and farcy, as it is oftenest seen in unhealthy situations, ill-ventilated stables, and in horses with bad or gross constitutions. I was inclined, when I first saw it, to attribute it to some injury to the parts, and to believe that the unhealthy action was the consequence of such injury, or to the introduction of some deleterious material into the wound; but further experience has convinced me that it often originates from constitutional causes, and that it is an inflammation of the coronary substances, due to the presence of some morbid material in the blood. If the attack be slight, the inflamed point limited, circumscribed, and surrounded by healthy tissue, recovery may be expected; but if there are several points, which by ulceration tend to coalesce, as well as extensive destruction of tissue, an unfavourable termination may be looked for. Even if recovery from the more immediately dangerous symptoms is obtained, such will be the destruction of the horn-secreting structures that the animal will be worthless for the future; therefore the treatment must be directed to the prevention of the spreading of the disease from its original roint; and the best local remedy for this purpose is the nitrate of silver, freely applied in its undiluted state. After the free application of the caustic, the animal ought to be made to stand with the lame foot in cold water, for an hour at a time, repeatedly during the day; poultices are injurious, as they encourage the tendency to sloughing and ulceration. When the foot is removed from the cold water, the parts are to be dressed with a solution of the tincture of terchloride of iron. In all cases of this description, removal of the shoe and ex- 
amination of the foot are imperative. The general febrile disturbances are to be combated by-1st. A brisk cathartic; $2 d$. Sedatives or anodynes, as the case may require-anodynes if the pain be acute, sedatives if the febrile symptoms predominate. The caustic is to be applied every second day, until it is seen that the ulcerations are becoming healthy in appearance. I have tried the carbolic acid dressings, but they are not to be compared with the nitrate of silver, cold water, and astringents. It must be remembered that the caustic is not to be applied for the purpose of destroying healthy tissue, but to induce healthy action in what is already diseased; it must therefore be used with cautious freedom.

The appetite is to be stimulated by tempting food; care must be taken not to let the strength be reduced by the want of proper nourishment; and as soon as the most acute febrile symptoms have passed off, tonics, especially the salts of iron, are to be given. The stable must be well ventilated, clean, and well drained; in fact, the surroundings of the animal are to be of the best kind.

The result of a modified form of this disease may be a falsequarter.

\section{FALSE-QUARTER.}

This consists of one or more clefts or fissures in any part of the crust or wall of the foot, due to the destruction of the secretory coronary band.

The horny wall or crust of the foot being secreted by the coronary substance, it naturally follows that when a part of it is destroyed, the part of the wall below the destroyed portion is no longer supplied with horn from above; and this deficiency causes a chasm or fissure in the wall. It differs from "sandcrack" very materially; is much wider at its base, and contains a modified condition of horn; that is, the horny laminæsecreted by the sensitive ones-denuded of their outer covering. The coronary band also shows a loss of substance corresponding to that in the crust.

A horse having a "false-quarter," although he may not at the time show any signs of lameness, is to be considered unsound, since he is liable to become lame at any time, from injury to the thin horny covering of this part of the foot, which is also 
more or less tender, and requires careful shoeing, in order that no undue weight be thrown upon the weakened portion.

Causes.-Anything that destroys the integrity of the coronary substance, such as treads, quittors, \&c.

The treatment, if there be a wound, must be directed to the diseased coronet; removing all ragged edges, making the wound as level as possible, and moulding the parts into their natural condition, as nearly as can be done, by firm pressure; bringing the edges of the wound together, and inducing it to heal by secondary adhesion. If the case be an old one, the gap in the coronet healed up, and filled with fibrous tissue, but little can be done, except filling the fissure with gutta-percha from time to time as occasion may require, and applying a bar shoe, so as to throw the bearing from the weak part. Blisters to the coronet are sometimes useful.

Tricks are played upon feet of this kind; false-quarters, sandcracks, and seedy-toes are filled with gutta-percha, moulded to the part whilst warm, nicely filed or rasped when cold, and the whole surface of the foot blackened with lamp-black and oil, or covered with tar.

Caution.-Never examine a horse as to soundness if the feet are dirty, without having them washed.

\section{SAND-CRACK.}

A sand-crack consists of a fissure of greater or lesser extent in any part of the foot, commencing at the coronet, and generally found in the inner quarters of the fore and the toes of the hind feet, very rarely in the outer quarters.

Professor Dick taught that " a sand-crack may happen in an instant, from a false step; and hence a horse, though he may spring a sand-crack within an hour after purchase, cannot be returned on that account to the seller." This opinion is held by many other writers and teachers; but it is incorrect, except in very rare instances. A sand-crack proceeds by slow degrees. Prior to its appearance, the horn is imperfectly secreted; it is dry and brittle. Horn is built up of tubes matted together. These tubes are similar to hair, and are secreted by the same kind of cells. And "the same cell which forms the scaly epithelium, epidermis, and hair is utilized in building up the 
horny structures. The special history of the horny appendages of animals consists therefore in the description of the form and disposition of the surfaces from which they spring. Whereas hairs have roots embedded in follicles, the horn tubes spring from papillæ, which stud a surface extended over bony or fibroelastic prominences."-(GAMGEE.)

Horn is often spoken of as "built of hairs firmly matted together" (BARLow). The horn tubes are united together by an inter-tubular substance composed of cells, and produced from the surface between the papillæ. The wall, consisting of horn tubes and agglutinating inter-tubular substance, is secreted by the coronary substance, and is naturally tough, but breaks into fibres if it grows beyond its natural length. This is due to the crumbling of the inter-tubular substance.

In order that normal horn be secreted, it is essential that the papillæ and intermediate spaces in the coronary band, as well as the sensitive laminæ, be in a healthy condition; for it is found that the very tough and natural state of the wall is maintained and preserved by the continual addition to it of horny agglutinating cells, secreted by the sensitive laminæ as it (the wall) descends over the foot.

Having seen, then, that the horn is secreted by the papillæ and the inter-papillary substance, the reader can understand that the brittle condition of the crust leading to sand-crack depends upon a perverted condition of the secreting structures.

Some horses are very liable to these cracks; and when a cure has been apparently effected, they (the cracks) are very apt to return. Such may be said to be naturally predisposed; but the cause must be looked for in the deteriorating effects of bad shoeing, especially in that method which ignores the weight-bearing properties of the sole. When the sole is weakened by the drawing-knife, and shoes applied to the feet, more especially the seated shoes which are put on the fore feet, the weight-bearing surface is limited to the thickness of the wall; and an amount of weight is thus thrown on the crust calculated to overstrain it, and to induce diseased action in the structures to which it is attached, and by which it is secreted. Of itself horn is incapable of diseased action, being a secreted and not a formative material.

A sand-crack commences at the thin upper margin of the 
wall, and is usually small and insignificant at first; but it gradually extends downwards and inwards, and when it has penetrated through the horny structures, lameness becomes apparent; inflammation is set up, both in the laminæ and within the skin above the fissure; the part is very painful, and the lips of the wound, at its upper part, gape as the tissues swell.

In some rare instances, however, lameness may be present before the appearance of the fissure. When such is the case, we may reasonably conclude that the fissure commences on the inner part of the wall, being the converse to the general rule.

When the animal is made to move, the crack is seen to close every time the foot is put to the ground, and to open again when the weight is removed from it; during this closure the borders of the crack grasp some of the sensitive and swollen tissues, causing most excruciating pain and sometimes hæmorrhage. Sand and dirt insinuate themselves into the wound, act as irritants, give rise to the suppurative action, and in some instances to a high degree of irritative fever. It is very painful to witness a horse, especially a heavy cart-horse, attempting to walk with sand-crack at the toe. He keeps the foot elevated from the ground as long as possible; puts down the heel first very carefully; and when the toe is forced to touch the ground, he immediately lifts the foot with a sudden catch, the pain being often so poignant as to cause him to groan.

A sand-crack may occur through the bars, but it is very rare.

Treatment.-If inflamed, remove all sources of irritation, and pare the edges of the crack if they press upon the tissues; in fact, "bottom the crack," and allow the escape of pus, dirt, \&c. ; remove the shoe, and give purgatives; order fomentations and rest. A fungous growth is generally seen filling the fissure; this is the result of the inflammation, depends upon it, and disappears upon its subsidence. It must not be destroyed by caustic, for the application will add to the irritation. When the inflammation and pain have subsided, place a bar shoe (thin heeled, with side clasps, if the crack be in the toe) on the foot, allow sole as well as frog pressure, but remove the pressure from that part of the foot immediately below the crack; pare away the upper part of the crack from its coronary attachment, and let no direct communication remain between the fissured horn and the substance from which new horn is to grow, or the crack will be 
perpetuated. A sand-crack never unites, and a new crust must be cultivated.

There are two operations applicable to sand-crack after the inflammation has subsided, namely, the French plan of "stripping," and the English method of " clasping." The first should be performed only in very aggravated and long-standing cases. It consists in making a groove on either side of the crack; the two grooves originating in a point about the middle of it, extending upwards to the coronet, and gradually diverging from each other, so as to include, at the coronet, nearly an inch of wall on either side of the fissure in the form of the letter $V$. The horn is to be cut completely through, and then the whole of it included in the grooves is to be stripped off from the point to the coronet. ${ }^{1}$ If any remains of the sand-crack be seen in the sensitive laminæ, they are to be cut off with a sharp knife. Cold applications, bandages, and mild astringents, with rest, will constitute all the after treatment necessary; time being allowed for the growth of new horn before the animal is put to work.

The second method-that of "clasping"-is of two kinds, is much milder, and answers the purpose in the majority of cases. First performed as follows:-The horn immediately attached to the coronet above the crack is to be pared away, so as to completely disconnect the diseased horn from the coronary substance ; then a clasp is to be applied, which may now be obtained, with the necessary instruments, from any surgical instrument maker. The second, and the better when the horn is sufficiently thick, as when the crack is in the toe, is to cut a notch with the drawing-knife about half-an-inch from each side of the crack, as near the coronet as possible, and about a quarter of an inch deep, and sufficiently large to allow the imbedding of the head of a small horse-nail. If the crack be an old one and long, other notches are to be cut an inch lower down, and ordinary horse-nails, pointed with a broad point on the reverse side, are carefully driven into the horn through those notches, embracing both sides of the fissure, long clenches being left, and the crack drawn closely together with the pincers, the clenches fastened down, and the whole rasped smooth. The rationale of these methods is to prevent all motion in the crack during the movements of the animal. In this way a horse may be employed for his usual work during the growth of the new horn;

1 It is now found that in many cases the grooves are quite sufficient without stripping the horn from between them. 
whereas, if no clasp or nails be applied, lameness manifests itself, from the pinching of the sensitive laminæ when the edges of the crack approximate each other at the time the foot is on the ground, as already explained. It is always necessary to see that no dirt insinuates itself into the foot through the fissure, and to keep it filled with some firmly adhesive material, such as gutta-percha. If the growth of horn seem inactive, a slight blister to the coronet will prove useful, by promoting a more rapid secretion. At all times, and for sand-cracks in any part of the foot, a bar shoe, if the frog is not diseased and unfit to bear pressure, is useful. In this, as in all other diseases of the feet, the sole is to remain unpared, and allowed to bear its due share of the animal's weight. When the crack is at the quarter a three-quartered bar shoe, to remove weight from it, is to be recommended.

\section{KERATOMA.}

The two following woodeuts, fac-similes of specimens in my possession, will illustrate this condition of the foot:-

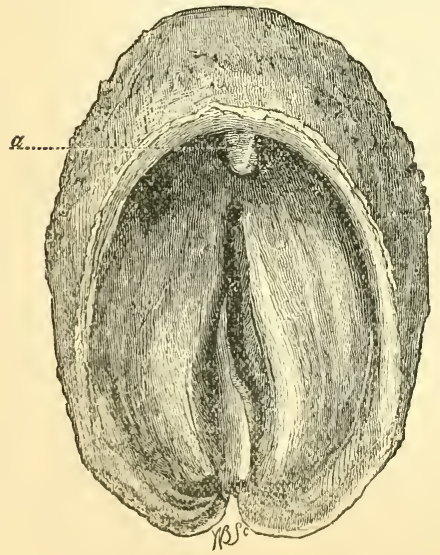

Fig. 73.

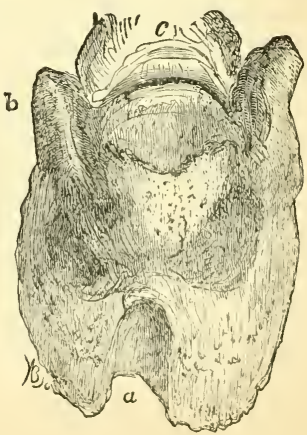

FIG. 74.

Fig. 73.- Horny foot, showing at $a$ horn tumour.

Fig. 74.-Pedal bone of same foot, showing a cavity at its toe corresponding to the horn tumour in Fig. 73. 
The horn tumour seen in Fig. 73 is caused by pressure of the toe-clip of the shoe, this having been hammered too tightly by the smith, or becoming so by the animal striking the toe against the ground. These tumours and their corresponding gaps in the pedal bone are generally found in the toes of the hind feet; but it is by no means an uncommon thing to find them in the fore feet, at the sides where the clips of the shoe are situated. I have a case now under treatment with two in each fore foot. Generally, a kind of fissure is seen in the outer shell of the wall, or a concavity or depression without an actual fissure. There is sometimes lameness; occasionally the animal goes sound, the absorption of the bone accommodating the horn tumour. A horse whose feet are in this state is unsound, as the growth of the tumour is apt to cause lameness. Now and then instances may be seen where there is no apparent cause, no pressure of the clip, the disease being apart from the portion of hoof upon which a clip is usually situated. These tumours are analogous to corns in the human feet, and consist of an increased secretion of horn, generally caused by pressure.

If they cause lameness, a method of treatment is recommended, namely, the removal of the whole of the crust imnıediately over the diseased part, by cutting through it on either side from top to bottom, detaching it from the sole, and tearing it off, leaving the sensitive parts exposed. This operation is called "stripping the wall;" it is a very cruel one, and should never be performed except where the disease has resisted other treatment, as it is sometimes followed by untoward and irremoveable results, which seem to have escaped the notice of those who advocate its performance, not only for horn tumours, but for seedy-toe and sand-crack. These results are-(1.) The formation of successive abscesses and fistulæ in the coronary substance, before and during the growth of the new horn: (2.) Destruction of the sensitive laminæ, non-secretion of the horny duplicates, and the consequent attachment of the horny wall to the os pedis through the medium of a form of fibrous material, easily excited to inflammation and suppuration: (3.) Formation of abscesses at the coronet after the growth of the new wall, arising from the sensitive laminæ, bruised and lacerated by the force of the operation, degenerating in some parts into circular fungoid masses, which by growth separate the wall from its 
attachment, and occasion much lameness. It is, therefore, better to modify any lameness which they may occasion by properly adjusted shoes. Should these prove insufficient, the diseased portion may be isolated by grooving, as in the process of stripping, but the horn left unstripped.

\section{SEEDY-TOE.}

This term is applied to a perverted secretion of horn at the lower margin of the os pedis, by which the crust becomes detached from the horny laminæ.

It is often a result of laminitis, or of the pressure of the clip of the shoe. It consists in the formation, by the surfaces of the sensitive laminæ, of a cheesy or mealy imperfect horn, which is incapable of maintaining the union between the outer wall and laminæ. Being more rapidly secreted than healthy horn, it causes a separation of the crust from the laminæ, and of the sole from the lower margin of the os pedis. Rapidly drying, it shrinks in bulk, causing a vacant space between them, which emits a hollow sound when percussion is applied to the wall. When very extensive, there is usually a bulging of the wall at the part affected.

Although called seedy-toe, $i c$ is not confined to that part of the foot, being often seen in other parts of it, especially the quarters. When not due to laminitis, or to the pressure of the clips, it originates in some inherent cause, such as weak feet, or in the weight-bearing surface of the foot being limited to the wall by bad shoeing.

Lameness is not invariably present; but it must always be considered an unsoundness, as it is easily aggravated, has a tendency to spread, and great lameness is produced by the insinuation of dirt and gravel.

If treatment is to be adopted, it should consist in the removal of all the diseased parts, and in the promotion of the growth of healthy horn by blisters and moisture. Bar shoes, sole pressure, and removal of shoe-clips are the instructions for the farrier.

Some people think that white feet are more liable to suffer from seedy-toe than dark-coloured ones. This is a mistake, seedy-toe being as often found in dark as in white feet, in the fore as well as in the hind feet. 
CORNS.

A corn is the result of a bruise, involving the structures of the sensitive sole, appearing as an ecchymosed spot in the triangular space included between the bars and the wall at the heel; occurring in the fore feet, and almost invariably in the inside heel ; and caused by bad shoeing. Corns are not horn tumours, as tanght by Gamgee and others, but they may become so, if the cause be long applied.

The ordinary seated shoe is the most irrational invention that ever emanated from a man's brain. It is a thing that bears upon no part of the sole except upon the spot that is incapable of bearing such pressure. It is dished out-made concave-all round the foot except at the heels; and the result is corns. A corn consists essentially at first of a bruise and extravasation of blood, from rupture of the small vessels, which insinuates itself

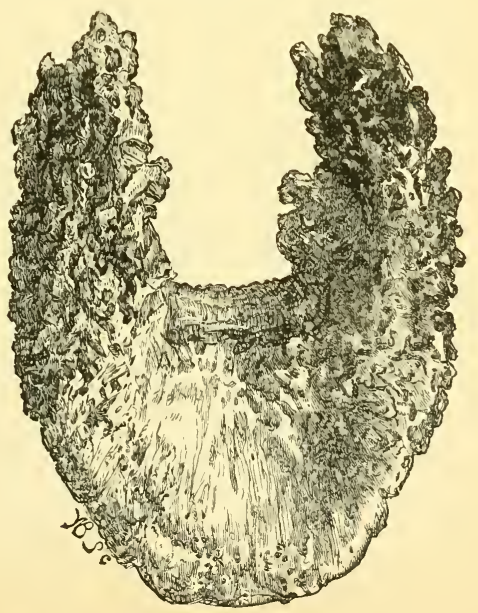

Fig. 75 .

into the horny texture, and gives it the characteristic red appearance; it terminates occasionally in suppuration, partial necrosis, horn tumour, or in the formation of bony spiculæ on the plantar surface of the pedal bone, as represented in the sketch. 
A corn constitutes an unsoundness, although it may not at the time cause lameness.

Treatment.-If inflammation be present, treat accordingly; remove shoes; poultice, \&c. Give exit to pus if the corns have festered; and if there be superficial necrosis of the pedal bone, the sequestrum must be removed. The radical cure, however, is to be eflected by proper shoeing, and the method already laid down is certain, speedy, and recommends itself to all impartial men. Horses that are high steppers, with heel action, are most susceptible to corns, especially if weak in the heels; but corns are seen in the best of feet, and they will continue to be seen while horses are shod with the seated shoe. One other cause of corns is the maceration of the feet by vile combinations called "stoppings," which cannot be too strongly condemned.

\section{THRUSII.}

A discharge of a foetid material from the frog, arising from a diseased condition of the secretory surface of the fibro-fatty frog. The cleft is the part commonly first affected, and when neglected the disease spreads over the whole organ; the horn becoming detached from the bulbs of the heels to the toe of the frog. The causes are extrinsic and intrinsic. The extrinsic causes are filth, the irritating materials generated in the decomposition of the urine and fæces, and contained in stoppings for the feet, such as cow-dung, and maceration of the frog, as when the animal is turned out to a wet pasture : these causes operate by destroying the integrity of the horny frog, and irritating its sensitive counterpart. Thrushes from extrinsic causes are more commonly found in the hind than in the fore feet, because the latter are not in the dirt while the animal is in the stable. The intrinsic causes operate chiefly on the fore feet,-although thrushes may be seen in the fore feet if the animal be at grass, in a wet strawyard, or in a dirty loose box. In the winter time the frog denuded of its horn becomes occasionally frost-bitten, leading to deep sloughing, which may even extend to the navicular bursa, and causing great lameness.

Thrushes are easily cured, when originating from external causes, by cleanliness, and calomel locally applied; and if it be impossible to prevent the animal from standing in his excreta, 
leather soles should be used; these may be moveable, and used only while the horse is in the stable.

When, from the intrinsic cause already referred to, or from some habit of body (cachexia), with swelling of the legs, or grease, the cure becomes a difficult matter, purgatives, dressing with calomel, tonics if necessary, regular exercise, and great cleanliness, will be necessary.

\section{CANKER}

Differs from thrush in the nature of the diseased secretion, in its course and tractability. It usually commences in the frog, and rapidly extends to the sole, and even the sensitive laminæ; but it is not a rare thing to see it commencing in any other part of the plantar surface, or of the laminated structure of the wall, excited by a wound, prick, or other injury. It much resembles grease of the leg, both in its nature and the class of animal it attacks; and very frequently both conditions are coexistent. I have investigated the condition of the parts microscopically, but have failed to detect any malignant characteristic. The papillæ are enlarged by engorgement, or by hypertrophy, but show no trace of eancer or malignant disease; nor of lympinoid or tubercular deposition, as in some cases of foul in the feet of horned cattle. In some instances such bodies, like the sporules of a vegetable parasite, are to be seen under the microscope; perhaps further research will demonstrate that canker is due to the presence of a cryptogam.

The characteristic symptoms of the disease are strongly marked, and consist of an abundant fœetid, colourless discharge from the frog, which is large, spongy, and covered by pallid, stringy prominences of a fungoid nature, intermixed with offensively smelling, semi dried, cheesy masses of matter, composed of imperfect horn cells. It seems as if the various constituents of the horn were in an uncombined state; the sulphur, which is a natural constituent, being secreted as sulphuretted hydrogen, giving the characteristic foetor to the whole secreted mass; the horny matter imperfect, and floating in an abundant liquid material ; the secreting villi enlarged, scantily covered by a thin pellicle of horn, giving them superficially a white appearance, whilst underneath they are turgid, congested, and humid. 
Canker may be confined to one foot. When resulting from an injury it is always so ; but when originating from no ostensible cause, two, three, or even all the feet, may be contemporaneously or successively affected. The sound feet of a horse predisposed to canker very often have an abominable smell, as if the animal suffered from a sulphuretted hydrogen diathesis. I have coined this term, not being able to find another to express my meaning.

Canker is generally confined to heavy cart-horses. Some writers say that neglected thrushes are apt to run on to canker, I am, however, of opinion that such is not the case, unless there be a strong predisposition; and when this is present, thrush, or even an injury, may excite its development. Cauker is a constitutional disease located in the feet, and is due to a cachexia or habit of body, grossness of constitution, as exhibited by thick round legs, large feet, and the lymphatic temperament.

The treatment of canker is early extirpation, not only of the diseased surface, but of the whole horny solar aspect of the foot, and is performed as follows:-With a sharp drawing-knife make a groove at the immediate junction of the sole and wall all round the foot, commencing at the heel, extending round the toe, and to the other heel. Then divide the circle into two halves, by making another groove from the cleft of the frog to the toe of the foot. The patient must now be cast and properly secured. Then with a sharp scalpel cut cleanly through the grooves into the sensitive parts, so as to completely separate the sole from the wall. When this is done, strip off one-half of the sole first, and then the other half, removing every trace of the horny covering of both sole and frog. It must be done thoroughly and effectually. Cut away all fungous growths, and dress the whole of the exposed surface with the nitrate of silver. Pack up with dry tow, bandage, and put on a leather boot. Apply moderate pressure to the fetlock by a tourniquet, to prevent excessive hæmorrhage. I find it unnecessary to do this until after the operation is completed, as bleeding does not begin until the animal is on its feet again. Two days after the operation, the dressings are to be thoroughly soaked in warm water for an hour, and carefully removed. If removed dry, they are apt to tear away pieces of the textures, to which they are firmly agglutinated by the dried blood. Great care must be exercised in doing this, 
as everytliing torn now leaves a raw surface difficult to heal. In some instances the whole of the exposed surface of the foot presents a beautifully healthy appearance, and become gradually covered with natural horn, with very little more treatment than cleanliness, mild astringents, and moderately firm pressure. But the more aggravated ones will continue to present fungoid elevations and morbid secretion, requiring repeated dressings with powerful caustics and astringents. Chromic acid, carefully applied in its undiluted state, is a good remedy, combining with the watery constituents of the fungoid growths, and stimulating the production of healthy horn. It must be sparingly applied, as its affinity for water is so great as to cause a blaze of fire by the intensity of their combination. For at least a week after the operation, the horse should stand without shoes, the soles being padded with tow, bandaged, and booted; but when the feet are able to bear the shoes, they should be put on, since it is much easier to dress the feet when they are on. The after treatment will depend very much on the progress of the disease; remedies seem to lose their effect after two or three applications, and require to be changed. When the chromic acid seems inoperative, a mixture of sulphuric acid and tar might be tried, or the acid carefully put on in its undiluted state. Care, however, must be taken not to continue caustic and escharotic dressings too long, or the whole sensitive sole will be destroyed, necrosis of the bone produced, with great sloughing, and perhaps the death of the horse. Burut alum, sulphate of copper, terchloride of iron, chloride of zinc, sulphate of iron, tannic acid, and other astringents, with an occasional application of carbolic acid, might be tried in their turn. Some cases improve under firm pressure, by means of pledgets of tow and splints; whilst in others, pressure seems to stimulate the diseased action and the production of fungus. There is scarcely a rule which can be laid down. Much depends on the skill of the veterinary attendant. The stripping must not, however, be forgotten, and it may be necessary to repeat the operation. Many horses have recovered that have required it to be done three, or even more times. When the laminæe are involved, the case may be generally looked upon as hopeless; but if treatment is determined upon, the crust must be stripped, so as to expose the whole of the diseased surface.

The constitution, being the source and origin of the ailment, 
must not be forgotten. Purgatives, diuretics, tonics, alteratives, such as arsenic and deobstruents, are to be employed to get it into a good state of health, with regular exercise, good $d r y$ food, great cleanliness, and a dry situation. In all cases of canker the dressings should be removed daily; and when pressure or protection by an artificial sole seems hurtful, the foot should be left uncovered, dressed over with tar, and repeatedly cleansed with cold water every day. If in a fore foot, this is easily done; but a hind foot requires more trouble and care. When a thin pellicle of horn has formed, gentle pressure, by means of pledgets of tow dipped in tar, will prove useful in all cases. Such pressure may be constant or intermitting, as the discretion of the practitioner may lead him to prescribe. 


\section{CHAPTER XXI.}

DISEASES OF THE FEET-continued.

PUNCTURES OF TIIE FEET BY " GATHERED NAILS" AND BY "PRICKS" IN SHOEING-IMPORTANCE OF THOROUGHLY EXAMINING WOUNDED FEET AND OF GIVING EXIT TO ALL DISCHARGES-WEAK FEET LIABLE TO INJURIES-QUITTOR-TREATMENT-FOUL IN THE FOOT OF HORNED CATTLE-FOOT-ROT IN SHEEP.

\section{PRICKS IN SHOEING AND GATHERED NAILS.}

A “ Gathered NaIL" may pierce any part of the sole or frog, but most commonly it is found to have entered one of the commissures of the frog, and about midway between its toe and the bulb of the heel. If the puncture be deep, there is great risk of its penetrating the navicular bursa. The treatment is removal of the nail, paring of the part to allow the escape of pus, poultices, and rest.

Punctures or pricks in shoeing are of two kinds, namely, those actually penetrating the sensitive structures, and those where the nails, not actually penetrating the sensitive parts, are driven so near as to cause bulging of the inner layer of horn, and pressure upon the sensitive interior, leading on to inflammation and great lameness, with or without suppuration.

Many cases of pricks are caused by what are termed "drawn nails;" that is to say, the smith, finding that his nail has gone too near, or even penetrated the sensitive foot, draws it out and drives it in again, taking a more superficial hold of the horn. These cases are more difficult to diagnose than when the nail causing the injury is not removed; but with care it can be seen that, although the nail-hole is pared out, a stellate discoloration remains at the bottom of the cavity made by the knife. This must be followed and thoroughly bottomed, or the suppuration 
imprisoned within the horny foot will cause acute pain, great lameness, violent febrile symptoms, and even death from pain and nervous exhaustion; or the pus may extend upwards along the laminæ, detaching the horny structures from their attachments, finally appearing at the coronet as a soft tumour, which, if pressed upon, will discharge first a sero-sanguineous matter and pus. Sometimes this discharge affords relief, but at others the suppurative action extends round the coronet, breaking out in various sinuses and unhealthy-looking abscesses; the animal at the same time suffering from a continuation of the pain and febrile symptoms, becomes rapidly emaciated, and from continual lying down covered with bruises and sores.

To detect punctured wounds of the feet it is necessary to remove the shoe from the foot affected, then to examine all round the margin of the sole, by pressing it and the crust with the pincers. When the seat of the mischief is pressed upon, the horse will generally evince pain; but the test is not to be depended upon until the sole has been pared out.

"Pricks in shoeing" usually manifest themselves shortly after the horse has been shod; but occasionally there may be no signs for three or four weeks, or even longer ; and in some horses, especially if the puncture be at the toe, the suppuration will appear at the coronet as the first symptom of mischief. This fact has led some writers to suppose that, because the burrowing of the pus does no mischief in these exceptional cases, it is good practice to allow every case to go on unchecked, until the pus has forced its way upwards and discharged itself at the coronet. I am surprised to find that such an idea has gained ground latterly, especially amongst the young members of the profession. It is full of error, founded on a very limited practical experience, and calculated to entail much suffering on the poor patients, and to destroy many a valuable animal's life. The first step in the treatment of these accidents is to pare down to the bottom of the puncture, to allow the escape of the matter, which, by-theby, is generally of a dark colour, and to place the foot in a poultice. Many cases, if so treated, are quite free from lameness in a day afterwards. A purgative is useful, removing sympathetic fever.

Paring of the sole and removal of the horn allow the sensi- 
tive sole to bulge in the form of a fungoid granulation. To this caustic remedies are generally applied. Such applications are, however, calculated to do harm, as the supposed fungus is nothing more than the swollen tissues, which disappear when the irritation has subsided, like any other inflammatory swelling. However, if, after the subsidence of the lameness and inflammation, the bulging still continues, the nitrate of silver or sulphate of copper and tar may be applied; after which the part ought to be exposed to the drying influences of the air, when a scab wili form, affording a natural protection to the tissues, which will now soon heal.

In paring and searching lame feet, great care is to be taken not to make them bleed, as hæmorrhage obscures the operation, and renders it a difficult matter to follow a small spot of discoloration to its termination; and when the structures are wounded with the knife, they are apt to sprout up, inflame, and cause the healing process to be more prolonged than when the parts are carefully pared.

Two or three sizes of "searches" are very useful; the smaller ones, with double-cutting edges, to be employed in the more delicate and concluding part of the operation.

In many cases of punctured foot it is necessary to apply the bar shoe for a few weeks in order to remove the pressure from the seat of injury, and to diffuse it over the frog and healthy part of the foot. Leather soles are also useful.

\section{WEAK FEET.}

Some horses have naturally weak feet, thin heels, and brittle walls, with a tendency to split up into layers where the nails are inserted. These feet require careful shoeing, with stout shoes. and leather soles adapted to their special peculiarities. Horses that show tenderness or slight pain in the feet after work are inuch relieved and benefited by having their feet and legs bathed in water, or by being made to stand in a foot-bath for one or two hours at a time.

\section{QUITTOR,}

A fistulous wound upon the quarters and heels of the coronet, 
generally caused by treads, pricks in shoeing, suppurating corns, or any other injury calculated to excite the suppurative action within the foot, or in the structures of the coronet.

The symptoms of quittor are lameness, swelling upon the coronet, about the centre of which one or more small orifices are seen, discharging either a thin limpid secretion, or a thick, perhaps curdled, pus. From the external orifices, sinuses are found leading generally in a downward direction, beneath the coronary substance, lateral cartilage, and into the foot itself.

In many cases the animal is excessively lame, scarcely able to put the foot to the ground.

A quittor differs from a wound, or a recent abscess on the coronet, by the condition of the parts, which have taken on a peculiar unhealthy action, by the character of the surrounding swelling, which is hard to the touch, and by the presence of sinuses.

The treatment will depend upon the cause. If it be anything within the foot, as a festered corn or a prick, a depending opening must be made at the sole, sufficiently large to allow the free escape of the contained pus. This, along with poultices for a few days, succeeded by a blister to the enlarged coronet, will often be sufficient; the foot being protected in the meantime by a nicely adapted bar shoe, if it be too weak to go without. In all cases of injury to the feet, I think that if it be possible to do without the shoe it is better not to apply it; but if the foot is broken, or if the application of the shoe be imperative, a nicely fitted bar shoe can be put on in such a manner as to remove weight and pressure from the seat of the injury or disease.

In cases of quittor, where no communication exists between the wound and the plantar surface of the foot, it will be necessary for the practitioner to introduce his probe carefully, and to ascertain the depth and direction of the sinuses; when he has satisfied himself on this point, the Bistonri caché (see Fig. $76)$ is to be introduced as deeply into the sinuses as possible,

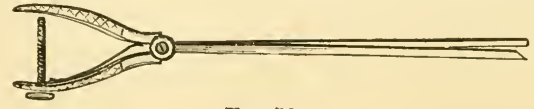

Fra. 76.

the blade opened, and the parts cut as it is drawn outwards. 
The ordinary Bistouri caché will not answer the purpose, as it does not cut to its point. The one delineated in the woodcut is specially adapted for quittors and other fistulous sores, as it cuts to its very point, a matter of great importance when it is necessary to bottom the wound. Very frequently the long continuance of quittor is due to the imprisonment of a piece of necrosed bone, dead cartilage, or other foreign body; and it will be necessary to remove this before a cure can be effected. When the sinus has been opened to its extremity, it is good practice to inject into it a solution of the bichloride of mercury, 3i. ; water, $3 \mathrm{i}$.; a few drops of hydrochloric acid being added to cause the salt to dissolve. One or two injections of this are sufficient to cause the whole exposed surface of the wound to cast off a thin slough, and to leave a healthy granulating surface, requiring no treatment beyond being kept clean. Much harm is done in these cases by the long-continued application of escharotics; but should the wound look unhealthy after the separation of the slough, it may be necessary to apply the solution a second time. A glass syringe is the best instrument, as lead will be acted upon by the salt and the acid.

Poultices assist the separation of the slough, and tend to soothe the irritation. They should therefore be applied for several days.

In quittors of a very intractable nature the whole diseased structures, including the lateral cartilage, have to be removed by surgical operation, which is as follows :- The horse must be cast, a tourniquet applied to the fetlock; an incision is then to be made at the junction of the horny wall and coronary substance, immediately below the seat of disease, the skin is to be carefully dissected from the subcutaneous structures, and folded upwards in the form of a flap. When this is accomplished, the diseased portions are to be carefully removed with the scalpel, taking care not to penetrate the articulation, and the skin brought back to its original position, and retained there by firm but not too tight bandages. If the vessels are enlarged, as sometimes will be the case, they ought to be secured by ligature, but generally this is unnecessary, the hæmorrhage being prevented by the bandage, and it is always better not to introduce a foreign body, as a ligature, into the wound.

There are many unqualified men noted for their success in 
the treatment of quittor. I knew of one, and he certainly managed to cure cases after everybody else had failed. His plan was a simple one, and consisted of the injection of a fluid-the composition of which was a secret-into the sinuses every fourteen days. He never allowed any interference with the foot during the interval, and seldom had to apply his remedy more than twice. I almost think that it was the tincture of the terchloride of iron. I deem it advisable, in cases of quittor, to make the incision into the sinus; but there are cases where these are too small and too numerous to admit of a satisfactory exploration. For such the old plan of "coring" will answer well, and it is as follows:-Powder some corrosive sublimate, or arsenious acid, or a mixture of both, very fine; place a very little of the powder, say five grains, on a small square piece of tissue paper; double this up cornerwise, and then fold into a stiff plug, pointed at one end. Introduce it as carefully and as deeply into the wound as possible, and, if necessary, apply a bandage to keep it in its place; over the whole apply a poultice. In from five to seven days a circular slough will have separated, leaving the whole interior of the wound exposed to view. If this presents a uniform red appearance the sinuses have all been destroyed; but if pale-looking spots or small apertures are seen in it, they indicate that more of the diseased surface requires destruction. This should now be earefully done, or the second slough may extend to important struetures. When a healthy surface has been obtained, and the progress of the case still remains unsatisfactory, a blister must be applied to the coronet. This stimulates the reparative process, and removes pain and lameness.

If any part of the crust shonld at any time press upon the textures involved in the diseased action, it is to be earefully thinned by the rasp. To sum up, the principles of treatment are as follows :-To make a depending orifice by incision, seton, or counter-opening, by seton or cautery remove all foreign bodies, to destroy unhealthy surfaces, allay irritation, and finally to stimulate the reparative process.

The vital powers and vaseularity of the several structures involved vary to a great extent; hence the practitioner sometimes finds that, after doing all he can, the case progresses uusatisfactorily. I have in such instances found it advantageous 
to apply the actual cautery to the diseased structures, making a pointed iron, and pushing it, at a white heat, to the very bottom of the sinuses. The results from this have been satisfactory; the lameness has been relieved, the swelling removed, and lealthy action set up in the wound.

When fistulous openings are situated upon or near to the anterior surface of the coronet, great care and discrimination must be brought to bear upon their treatment, as the pedal articulation is there superficially seated and thinly covered by soft structures, and a free application of an escharotic is sure to cause the slough to extend into the joint.

No horse should work while suffering from quittor, as it not only causes great suffering to the poor animal, but renders recovery almost an impossibility.

\section{FOUL, IN TIIE FOOT}

Is a disease of horned cattle, and consists of inflammation and suppuration of the inter-digital substance, caused by overgrowth of the hoof, which when elongated gives rise to strain and inflammation of the internal structures, the irritation of dirt confined between the digits, and in some instances tuberculosis. It extends by neglect or other causes into the various articulations and bones of the foot, producing great lameness, with much fever, loss of condition, and even death. It is most commonly seen in the hind feet, but it is not unusual to meet with it in the fore feet.

In the majority of cases, if not caused by tubercular inflammation (see page 208), the disease, if attended to in time, is not so difficult to manage; the treatment being the careful removal of all loose horn under which pus is seen to burrow, mild astringents, poultices, and a purgative. In the more severe cases, where the inter-digital substance undergoes sloughing, the process must be assisted by warm poultices and fomentations. Afterwards the wound must be treated with astringents, great cleanliness, and protected by nice pledgets of tow steeped in a weak solution of carbolic acid, kept in their position by carefully adjusted bandages. There are other cases where the phalangeal bones are involved as high as the fetlock, with hard swelling of all the tissues, separation of the digits by enlargement of the 
inter-digital substance, dislocation of the inter-phalangeal articulations, caries of the articular extremities of the bones, synovial discharge, necrosis of the canons, great suffering, and lameness. Cattle, when so sorely troubled, will lie down, groan with pain, and refuse to feed, the flesh quickly wasting; great sores appear upon the various parts of the body on which they lie; and many will give up the battle altogether, and die right away as if their hearts were broken. Now, in such cases, whether the cause be tubercular deposition or not, the only method of cure is by amputation or disarticulation. I have performed these operations with the greatest.success, and I am thus in a position to suggest the best method.

If the disease has not yet extended above the metacarpophalangeal articulation, the foot and pastern may be removed by disarticulation through the fetlock-joint; but if this joint be involved to any considerable extent, it will be necessary to amputate through the canon bone. The operation is as follows: - Before the animal is cast, the diseased limb must be protected by a bandage, and when the animal is down removed from the casting rope, fastened by a cord tied around the pastern, and lield in position by two assistants; a tourniquet is then to be applied above the seat of the intended section. The knife must be strong, with a narrow blade; and care is to be taken that sufficient skin be left to form a good flap, so that the exposed end of the bone, as well as the soft parts, be completely covered when the edges of the wound are brought together. When the bone has been removed, the arteries are to be carefully taken up and secured by ligatures, and the whole extremity of the limb afterwards enveloped in carbolized lint or fine tow, firmly, but not too tightly, bound with a bandage. If the discharge is not very profuse, little after treatment is required, beyond cleanliness and occasional readjustment of the dressings. The animal must be well supported with nutritious food, and prepared for the butcher as quickly as possible.

Tumours between the claws of Cattle.-Hard nodules sometimes grow from the skin between the digits, causing a varying degree of lameness. They should be removed with tlie scalpel as early as possible. 


\section{FOOT-ROT IN SHEEP.}

There are few diseases upon the nature and causes of which so much difference of opinion exists as upon this troublesome and vexatious source of loss.

Continental, as well as some English and American veterinary writers and observers, divide foot-rot into two varieties, namely, contagious and non-contagious.

The first or contagious form is called by a variety of names, as Paronchyia ungularis ovium maligna or contagiosa (Latin); Pietin, Pietin contagieux, Mal de pied, \&c. (French); Bosartige Klauescuche der Schafe, \&c. (German); and the latter, Paronchyia inter-digitalis, or gravelling.

"Orf."-Carbuncle of the Coronet in Sheep.-This disease is one occurring more particularly in young sheep, but occasionally affects old ones. It occurs in wet seasons, more particularly in spring and autumn, and seems to be prevalent in pastures which have rough and damp grass: it also occurs amongst sheep fed on turnips, if the land happens to be damp and slushy. It is characterised by lameness, then swelling of the coronet or interdigital space; the swelling points and bursts, leaving an angry-looking ulcer, which speedily becomes filled with granulations, that readily bleed. The swelling extends up the limb, and the ulcer may attain a large size. Similar sores may appear on the face and head, and are probably due to contact. It is not a fatal disease, but is very troublesome, as when well established is slow to heal. The principal treatment is to remove to dry short pasturages, and treat the sores with astringents and antiseptics.

In his lectures Professor Dick said as follows:- " Foot-rot is the name given to a disease in sheep similar to "Foul in the foot' of horned cattle. Its consequences are disastrous and ruinous, attacking, if neglected, the whole flock, so that in feeding they actually crawl on their knees; hence it is regarded in the last degree contagious. After a good deal of investigation, however, I have arrived at a different conclusion; and I discover in its history nothing more than the result of that domestic state to which the sheep has been subjected. By nature not unlike the goat, it frequents the summits of the lofty mountains, where its hoofs, altogether analogous to those of the horse, are exposed to much tear and wear. When from these alpine regions we transfer the sheep into our grassy lawns, our moorish lands, or 
sandy soils, this wearing away of the crust is put an end to; it grows too long, and proves a great encumbrance. In this state it is exposed to many injuries, among others from the long grass of the pastures, and itself necessarily injures the soft parts beneath; and hence lameness, inflammation, suppuration, to the extent of casting the hoof, are the consequences. The circumstance of the disease occurring epidemically arises from the whole flock being placed in precisely similar circumstances."

The late Mr. Read of Crediton, in an essay on this subject, after very carefully weighing for and against contagiousness, says :"For the last three or four years I have made every inquiry of men accustomed to sheep. Some say, on my asking their opinion, that it is as infectious as the plague; for if they put a lot of sound ones with some that were lame, they all became so. But I have said-'Perhaps the sheep with whom yours were put were in a soil favourable to the production of foot-rot, and yours were taken from a healthy soil.' Such was generally the case, and such is the proof which farmers and others pretend to give of its infectious nature. Little do they imagine what is the operating cause on a soil disposed to it. That which gives it to one will give it to a liundred if there is a predisposition to take it on."

"My opinion is," concludes Mr. Read, "that you may put lame sheep with sound ones on a healthy farm, and they will soon get well, and the others will not become infected."

Mr. George Fleming brings forward some very strong facts in support of its contagious nature, and under the head of "Contagium," says as follows:- " The contagium is present in the exudation from the diseased foot, and may be termed ' fixed.' It is transmissible either directly through contact of the diseased with healthy sheep, or indirectly through litter, pastures, roads, railway waggons, or cattle ships. It is also transmissible by 'inoculation' of the morbid fluid at the coronets of healthy sheep. Indeed, though numerous observations had previously demonstrated that the extension of the malady was due to the presence of a virulent element, it was not until inoculation was resorted to that this was received as a fact. Pictet was the first (in 1805) to publish observations in this direction. He received two hundred half-bred merino shecp from Piedmont, some of which were lame from the disease. These were placed with a 
hundred other home sheep on a low hill, where the pasture was dry and of good quality; but in a short time the latter commenced to have sore feet, and before long the whole three hundred were affected. The rams, which joined them afterwards, were also attacked. This occurrence having been made public, attention was directed to the contagiousness of the malady, and similar occurrences were soon made known, especially by Girard. Gohier, to decide the question, undertook a series of experiments, after having published confirmative observations. He removed shreds of the loose horn from the claws of diseased sheep, and fastened them between those which were quite healthy; in this way he produced the malady. As the question was a serious one for the agricultural interest of France, the Agricultural Society of Paris offered premiums for the best essay on the disease, and these were awarded (in 1823) to Favre and Sorillon. The experiments successfully carried out by these authorities were perfectly conclusive. Favre, for instance, in one of his trials, simply deposited the matter between the toes of thirty-two sheep, and twenty-one became affected. Then followed observations and experiments by Letzins, Giesker, Felix, Mathieu, Delafond, Charlier, and others. Reynal has made numerous observations and direct experiments, which are entirely cunfirmative of all that had been previously published; so that there is no doubt whatever as to the existence of a virulent element in the disease, and that to this it largely owes its extension.

"Vitality of the Virus.-The virus of foot-rot appears to preserve its activity for a considerable period, though nothing definite has yet been arrived at in this respect.

"Infection.-The virus does not appear to have any influence on other animals than sheep; no case of transmission, either naturally or by inoculation, having been recorded. Fine-wooled, ligh-bred sheep, as already noticed, are most readily infected, and lambs are also susceptible; those with coarse wool are not only less readily infected, but they are more easily cured.

"The power of the infection is shown in Favre's experiments, in which twenty-one out of thirty-two inoculated sheep become affected.

"Mode of Aecess.-Infection takes place through the skin around the claws. There is no evidence to show that it can be produced in any other manner. 
"Incubation.-The incubation stage is from three to six days.

"Extcnsion.-The disease is chiefly extended through traffic in sheep at the fairs and markets; by allowing diseased animals to travel in railway waggnus and ships, and introducing healthy stock into these without a thorough cleansing having been carried out. Pastures on which affected sheep have grazed a short time previously have also been known to cause the disease in flocks succeeding them. Indeed, we can scarcely realise any more certain mode of extension than that of depasturing healthy sheep with diseased, or where the latter have been a short time before. The grass imbibes the discharge from the suppurating claws, and especially from between them, where it is most abundant and virulent; and the healthy sheep, walking through the grass, must receive continuous applications of the virus from every blade, and on the very part where experiments have proveu the skin to be most prompt and certain in absorbing it -between the toes.

" Contact between, or mixing of, the sick and healthy, even for a brief period, on roads, at fairs, or on pastures, is also a prolific cause of extension. Litter, fodder, sheds, and stables must also be included among the media which harbour and convey the contagion. As has been remarked, the extension is facilitated by certain external influences."

I have inserted these quotations in order that the reader may have both sides of the question.

I think, however, that the direct inoculation, by means of shreds of loose horn or matter from diseased feet applied between the claws of feet that were healthy, proves nothing further than that discharge, in virtue of its irritating properties, induced inflammation of the inter-digital tissues in a manner similar to that which would result from the application of any common irritant, or even a foreign body, applied to and retained in the same place, and that the other facts are incomplete, as it is not stated whether the season was damp or dry. Professor Brown's opinion upon this question is well worth quoting. He says:- " The third position which we have undertaken to discuss will require but little consideration. It refers to the 'virus' of ' foot-rot,' - the animal poison supposed to be capable of inducing the diserse by contact.

"The question of the existence of such poison among the pro- 
ducts of foot-rot must be determined by experiment; and the only foundation for the assumption of 'virus' lies in the fact that the introduction of the matter from a diseased foot to the healthy foot by puncture through the horn has prodtced foot-rot in some cases, although it has failed to do so in others.

"After all, the fact may be admitted without any question; it proves very little. We are quite prepared to learn that an injury to the horn of the foot, followed by the eontact of a mixture of moist epithelial cells and particles of dirt, will, if applied sufficiently long, produce foot-rot; we know that dirt and moisture will suffice, without any additional elements.

"Some of the experiments in inoculation failed, probably because there did not happen to be enough of the gritty particles; others because they did not remain long enough in contact with the membrane to establish any irritation; and at best there was not so much disease produced as would have occurred if the animals had been placed upon undrained land, where there happened to be a tolerably plentiful admixture of sand or grit in the soil.

"If it had ever been found that the introduction of the matter under the skin of the leg or any part of the body produced the disease, there would be fair evidence of its specific nature; but in the absence of this proof, we cannot admit an assumption which is altogether unnecessary to explain the results.

"From the present state of the argument, we may consistently deduce the conclusion that 'foot-rot' is primarily consequent upon such a derangement of the structure of the horn as permits the introduction of foreign particles, which ultimately reach the internal membrane, and occasion irritation, followed by exudation and excessive secretion."

For some time past my attention has been very much directed to this matter, and from my own observations, very carefully made, and from information gathered from many eminent flockmasters and their shepherds, I am quite satisfied that diseased sheep may, without fear of infection, be allowed to mix with sound ones on dry lands, and that eonsequently foot-rot is a non-contagious malady, and that it is manifested in two ways. First. Sheep pastured on gravelly or sharp sandy farms, wear away, especially in wet weather, the soles of their hoofs, and 
the sensitive parts become exposed; or if pastured on moorlands, or soft parks, the wall grows too long, the sole, changed by moisture, affords but an imperfect protection to the sensitive structure within, permits the penetration of dirt, and occasicnally, as stated by Professor Dick, the toe of one or both claws may bend upon the sole, and thus become a source of pain and infammation. Fungoid growtlis appear on the exposed surfaces, the l.meness increases, and there is loss of condition, as in the other form of the disease.

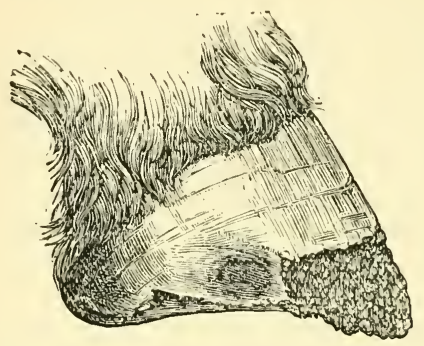

Fig. 77.-Confirmed stage of the first form of foot-rot.

The second and by far more common form of foot-rot is expressed by inflammation and swelling of the inter-digital tissues, with at first but little visible alteration in the condition of the horny

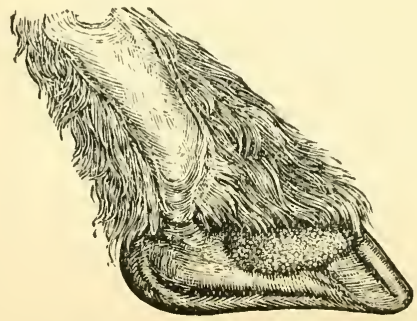

FIG. 78 shows the condition of internal wall of digit in an early stage of the disease.

sole, but the inflamed inter-digital structures soon suppurate, and discharge a fotid ichorous fluid, which burrows under the horn of 
the inner walls of the claws, and separates it from the sensitive structures within; as in the other form, the exposed structures soon sprout, forming fungoid growths, which discharge a little fotid ichor.

The lameness increases in severity as the disease advances, and in a few days the animal presents a lank appearance, and begins to lose condition.

In some cases the sensitive structures of the affected foot or feet lose their cohesion, and the horny walls hang loose around them, separated from the inflamed tissues within, except at the upper edge of the foot. The horny sole crumbles away as seen in Fig. 77, the sensitive tissues are exposed, and soon sprout as masses of fungus. The animal is now unable to put its lame feet to the ground, and will crawl on its knees, if the fore feet only be affected, and upon its abdomen, if the hind ones be the seat of the disease.

If the hoofs are not shed, it is found that the continual irritation gives rise to an augmented but intermitting secretion of horn, the hoof becomes crooked, hard, deformed, large in size, and covered with rings.

The disease-like that described by Mr. Fleming- "affects one or more feet; but ordinarily one, and passes to the others;" but sometimes only a single claw is affected.

It is important that the differential symptoms of foot-rot and foot-and-mouth disease in sheep be correctly understood.

From what has been stated, foot-rot commences either upon the solar aspect of the foot or in the inter-digital tissues. In the one case it begins below, in the other above; and by diffusion of the inflammation and burrowing of the pus extends along the inner aspect of the digits downwards to the sole, and sometimes upwards through the coronary structures, forming sinuses and ulcers above the hoof, occasionally, but very rarely, involving the tendons and bones, and causing sloughing of the whole foot, there being at all times a strong tendency to the growth of fungus from every exposed part of the foot.

Now, in foot-and-mouth disease-eczema contagiosa-the local symptoms are first manifested by redness of the skin surrounding the coronet, this redness commencing very often as a mere spot exactly above the inter-digital space, particularly at the heels, then extending all round the coro- 
net and on the pastern; the redness is succeeded by an eruption, the crop of pimples or vesicles being generally more numerous above the bulbs of the heels. The eruptive stage does not continue long, the blisters burst or dry up and form a layer of scabs, which finally fall off or dessicate, leaving the parts more or less denuded of wool for some time subsequently ; indeed it may be stated that the condition of the coronary skin is very similar to that which is induced by the action of an ordinary cantharides blister, and the changes occurring on the skin of the coronet may be classified under four heads, namely, redness, vesication, scab, and dessication.

The inflamed and vesicular condition of the skin is thought by some observers to extend into the sensitive foot, and no doubt this is a correct and proper view to take of the disease, and accounts for the very severe lameness observable in the earlier stages, the early detachment of the horny digits from their connecting laminæ, ${ }^{1}$ and for the presence of lameness in some instances before the occurrence of perceptible redness of the coronary substance. Immediately on the appearance of vesication, and some-

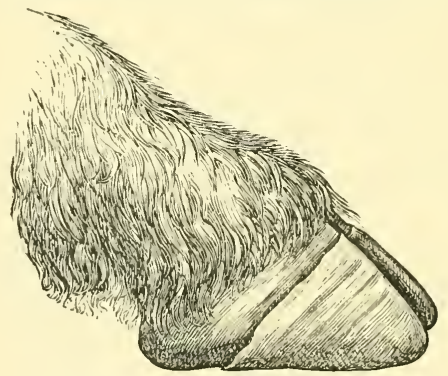

FIG. 79 shows the condition of a foot four weeks after the first or febrile stage.

times even prior to that event, the horny walls separate, more particularly at the posterior part of the feet, sometimes very slightly, occasionally a mere line of attempted separation being observable, at other times more completely, from their attach-

1 Vesication round the coronet should not necessarily produce separation of the horn therefrom; nor does it, unless, as in eczema epizootica, where, in even slight cases, the vesicles will be found to extend underneath the coronary band. 
ments to the coronary substance, or, where the attack has been violent, from the connecting laminæ also, and thus the horny digits are more or less completely separated from the sensitive and vascular structures within; and in a varying period, depending on the severity of the attack, the claw can be pulled off with ease, or is cast of spontaneously, leaving the exposed living structures smooth, red, and congested, and now of course liable to be affected by external irritants, and to sprout up in the form of fungoid granulations, and thus may be confounded with footrot by inexperienced persons. Reference to page 401 will show that even in the worst forms of foot-rot, where the horn is detached from the sensitive foot, that it still remains attached at its upper border. Now, in foot-and-mouth disease, the separation always commences at the coronary junction.

Professor Brown says- "Of the several quite distinct local diseases of the foot of the sheep, none is distinguished by the presence of a blister or vesicle in any part of the foot, while in the foot-and-mouth disease there are always vesicles present, or distinct evidence of their previous existence, and there is also a general absence of that condition of hoof which is usual in foot-rot. In exceptional cases the hoofs are elongated, much broken, and sometimes ragged and rotten; but this condition has nothing to do with the aphthous disease, which is indicated by the presence of vesicles between the claws, in the posterior part of the foot immediately above the hoof, and sometimes exactly on the portion of skin between the digits which covers the transverse ligament connecting the two sides of the foot together, and which is rendered tense, and therefore distinct when the digits are pulled apart. When the posterior part of the hoof is separated from the secreting membrane, as it often is in foot-and-mouth disease, the vascular surface is seen to be congested, but there are no signs of the so-called fungoid growths which distinguish foot-rot. In short, no two diseases can be more distinct from each other in the local appearances; but independently of the evidence afforded by the diseased parts, there is in cases of foot-and-mouth disease clear evidence of febrile action in the system. The animal's appetite may not be much affected, nor is it necessary that the demeanour should be suggestive of much suffering, but the application of the thermometer will show a rise of internal temperature when the 
animal is suffering from the aphthous affection. Making allowances for the variations of the temperature in sheep, which in health will range from $101^{\circ}$ to $104^{\circ}$, the increase is quite marked, as the temperature in the diseased sheep will range from $104^{\circ}$ to $107^{\circ}$. It will not, however, in the majority of cases, be necessary to use the thermometer to decide the question of the nature of the disease, as the local evidence will be sufficient.

"Vesicles in the mouth of the sheep, although not so generally present as in cattle, are very commonly found when they are looked for, but as the idea has long been prevalent that sheep are not affected in the mouth, it naturally has happened that the part has escaped notice, and besides, the lesion is not so prominent as in the mouths of cattle, and therefore not so readily recognised by the unpractised eye. In lambs of a few weeks old vesicles not larger than a hemp seed have been detected on the tongue. Abrasions on the lips and palate are frequently seen in sheep identical in character with those which are observed in cattle; and altogether the evidence of the identity of the disease in cattle and sheep is perfectly conclusive, even irrespective of the admitted fact that the affection is intercommunicable in the two classes of animals."

Professor Brown states that vesicles in the mouth are very commonly found when they are looked for. My experience leads me to a different conclusion, namely, that during some outbreaks they are as often absent as present, even in the earliest stage of the disease.

In addition to the fever expressed by increased temperature, acceleration of the respiratory movements, sinking of the flank and belly- "clapping," as it is called in Scotland-and loss of appetite, which precedes and accompanies the earliest local manifestation of foot-and-mouth disease, another important difference exists, namely, the lameness of foot-rot very commonly commences in one or two of the feet, and may or may not pass to the others; whereas in foot-and-mouth disease all the feet may be simultaneously affected, in which case the animal creeps in a very characteristic manner when first made to rise from the recumbent posture. In some instances all the feet may not be affected, or some may suffer more severely than others; in whichever it is manifested, the lameness does not affect the feet consecutively as in foot-rot. 
Causes of Foot-Rot.-Although it is ascertained that the tendency to foot-rot may be hereditary, and the progeny of some sheep of all breeds are more liable to it than others on the same farm, it must be confessed that the great cause of foot-rot is wet, causing softening of the horn. Where the grass is long and insinuates itself between the claws, the part of the upper and thin portion of the inner walls of the digits is the first to give way, and the disease may be said to be inter-digital, and is called the scald in some districts; but where the grasses are short, mossy, and the soil sandy, the solar aspect of the foot is the first to suffer. Whichever way it is first manifested it is entirely due to wet soil, and in its prevention and cure this fact must be borne in mind.

On hill farms it is found that this disease appears almost invariably about the end of July, and reaches its height towards the end of August and beginning of September. During this period the dews are heavy, and the grasses consequently saturated with moisture.

Prevention.- "The prevention of foot-rot," says Mr. Armatage, "is a subject which should engage more attention than it has up to the present time."

Of course it will be understood that removal from a wet to dry sound pasture is of pre-eminent importance, and from luxuriant to a shorter kind of grass. In highly cultivated farms the removal of sheep from luxuriant pasturage is often a matter of impossibility; when such is the case, experiments have been tried to harden the hoofs of the sheep, that they might resist the action of moisture, and some of these have been to some extent successful.

The Messrs. Archibald of Overshiels, Glengelt, Duddingstone, \&c., the celebrated breeders of blackface and Cheviots, inform me that they have found the following process superiorto all others:-

A wooden trough of the following dimensions is to be made. Length nine feet, breadth two feet, and depth one foot and a laalf, with lid and lock, in order that it may be locked after it has been used. Into this trough a solution of arsenious acid of the following strength is to be poured, sufficiently deep to cover the feet of sheep as they are driven through it, namely, one pound of the arsenic to five gallons of water.

Arsenic of itself is but slightly soluble in water, and in order to dissolve it readily, it is necessary to combine it with an equal quantity of an alkali, such as the common washing soda. The arsenic 
and the alkali should be put in boiling water together, in a boiler large in proportion to the quantity of water it is to contain, and for the reason that the liquid is apt to rise suddenly; the carbonic acid, combined with the alkali, is disengaged by the process of boiling, and during its escape causes rapid ebullition. For thesame reason the water, after the addition of the powder, must be slowly boiled for some time, or until the arsenic is entirely dissolved.

The sheep are to be driven through the trough containing the arsenical solution once a week ; if it is thought necessary to repeat it oftener, the liquid is to be weakened in strength by the addition of water.

To prevent the sheep jumping out of the trough as they are being driven through, it it will be necessary to place hurdles at the sides; and it is important that it be borne in mind that the sheep be folded for a short time in a place where neither they nor any other animal can have a chance of grazing, or the consequences might prove disastrous.

The Messrs. Archibald state that this, if applied about the middle of July, is not only the best preventative but the best cure of the disease, provided always that all loose and detached horn is carefully removed.

There are a great many so-called specifics for foot-rot; if looked into, however, they resolve themselves into caustics of various kinds. I have found the terchloride of antimony (butyr of antimony) as effectual, and much less painful to the animal, than any other caustic. If the fungoid growths be very prominent, it may be necessary to employ a strong acid ; in such a case nitric acid, carefully and sparingly applied by means of a teather to the surface of the growths, may be used with advantage; where there are no fungoid growths, but merely a scalding between the claws, the tincture of the terchloride of iron diluted with an equal quantity of water is a very good application. In addition, I would suggest, that after the feet are dressed with any of the above-named remedies the exposed surfaces might be brushed over with a quick-drying varnish, or a solution of gutta-percha, in order to afford an artificial covering to the inflamed and irritable tissues.

When the fungoid growths are not very large, a solution of sulphate of copper may be used, and the sheep driven slowly through it, or kept in it for a few minutes.

If necrosis of the bones occurs, amputation, if confined to ono foot, might be resorted to, in order to save a valuable ram or ewe. 

2

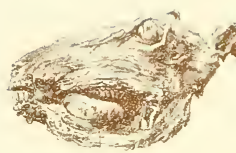

2.

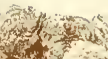

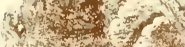

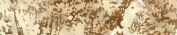
(v) A $1-1004$

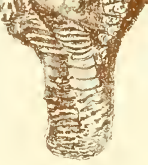

4
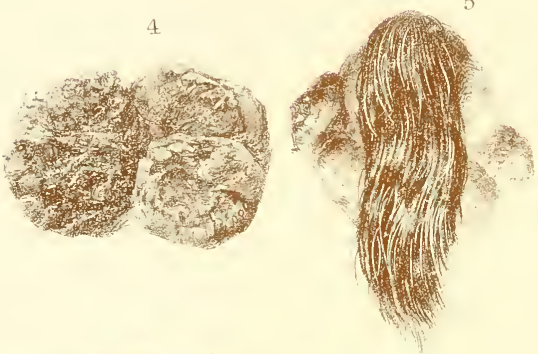

7

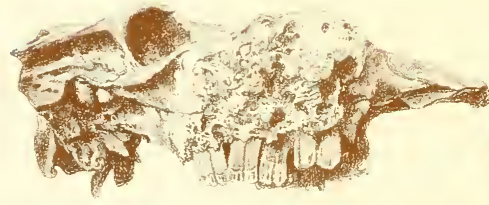

9

2

2

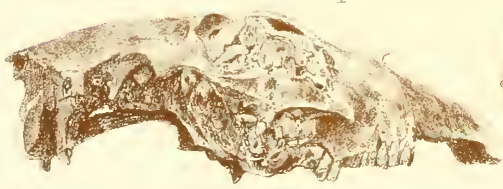

PLATE IV

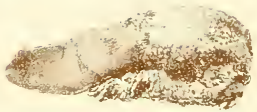

6

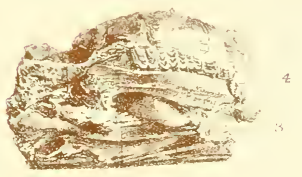

8

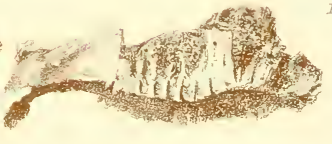

10

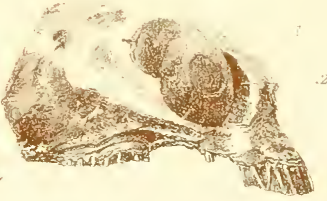




\section{TUMOURS.}

\section{EXPLANATION OF PHOTO-LITHOGRAPH, PLATE IV.}

1. Malignant Epdlis.-Mouth of ox. The microscopic characters of this tumour are given at page 419. The cells, as will be seen by the microscopic drawing, Fig. 99, partake of the nature of epithelial cancer.

2. Benign Epdlis.-Mouth of sheep. Microscopically, the structure of this tumour is fibrous.

3. Epithelioma.-Tongue of ox. The true nature of the growths are not definitely made out.

4. Medullary Cancer.-The tumour was originally roundish in form; distinctly separated from surrounding structures, and invested in a capsule of a semi-fibrous nature. The section shows various degenerative changes which have taken place within its substance. The tumour was removed by me from near the stifle-joint of a mare at Berwick-on-Tweed. It weighed four pounds, and, along with other masses of a similar character removed from the mammary gland and submaxillary space, made up an aggregate mass weighing $31 \mathrm{lbs}$. The mare died some time after the operation, and a post mortem examination revealed many other cancerous tumours in various internal organs. The microscopic characters of this tumour are represented in woodcuts, Figs. 95, 96, and 97, page 418.

5. Medullary Cancer of the Tail (not melanotic), showing the arrangement of its several masses; on the left the tumour has ulcerated. Removed by me from a cab-horse; no return of the disease.

6. Medullary Cancer of the Bones of the Face of the Ox.(1.) Large tumour. (2.) Cystic formation within the superior maxillary sinus. (3.) (4.) and (5.) Smaller medullary tumours.

7. Osteo-Sarcoma of Ox.-The specimen having been boiled, the sarcous matter is removed, the bony trabeculæ alone remaining.

8. Crstio Growth in superior maxillary sinus of a year-old colt. (1.) The cyst. (2.) Fifth and sixth molar teeth uncut. (3.) Fourth molar on the point of "cutting." (4.) Worm-eaten fangs of temporary molars undergoing absorption.

9. Dentigerous or Tоотн Tumour, containing several hundred rudimentary teeth, filling the antrum (2.), and extending from it into the cavity of the mouth $(1,1)$.

10. Large Nasal Polypus.-(1.) The tumour. (2.) Anterior naris. 


\section{CHAPTER XXII.}

\section{TUMOURS.}

\section{CLASSIFICATION.}

Tumours are included in that class of diseases named hypertrophies or overgrowths; and all their varieties consist in additions to the organised materials of the body, arising from an excess of formative force; but in the case of each kind of tumour the mode is peculiar in which this excess is manifested. A tumour differs from an inflammatory exudate in-1st. That its increase is of itself; $2 d$. That it grows as a part of the body by its own inherent force, depending on the surrounding parts for little more than a supply of blood, from which it appropriates its nourishment; $3 d$. As a general rule a tumour increases constantly, whereas an inflammatory exudation depends upon a morbid state of the parts at or contiguous to it; and increases in size only so long as the morbid action in the adjacent parts continues. Many tumours are solitary, but it very often happens that many tumours appear almost simultaneously, as in the case of verrucæ or warts, in the same animal; but such multiplicity of primary growths must be distinguished from metastatic formations, or those secondary tumours resulting from the transmission of the elements of an original tumour to other parts of the body characteristic of malignancy.

Some tumours closely resemble the tissue on and in which they grow, and are consequently called homologous tumours; whilst others differ very materially from the surrounding tissue, and are called heterologous tumours. For example, a cartilaginous tumour growing from cartilage is homologous, but growing from any other tissue, as from a muscule or a gland, it is heterologous ; thus the same variety of tumour may be in one case homologous and in another heterologous. But heterology is not limited to 
the production of a tumour dissimilar in its structure from that in which it originated, but is applied to a growth dissimilar to that in which it is situated. Thus an excessive proliferation of epithelial cells within the ducts of the mammary gland is homologous, but when this becomes excessive, and extends beyond its normal limits, as seen in cancer, it becomes heterologous.

A tumour rarely actually disappears, and thus differs from an inflammatory growth; as a rule its permanence is in accordance with the tardiness of its growth. Green says that the more rapid the growth and the more lowly the organisation of the tissues formed, the sooner do retrogressive changes occur. The carcinomata and sarcomata generally develop rapidly, and consist for the most part of cells, which quickly degenerate.

This cannot, however, be said of rapidly growing tumours in the lower animals. In melanotic sarcoma the growth is sometimes very rapid, and shows but little or no tendency to degenerative changes ; but, particularly in lymphadenoma, it will be seen that a most rapid growth is associated with the entire absence of degenerative changes.

Tumours usually develop from small beginnings. Some grow rapidly, others slowly and intermittently, and there is no limit to their growth, some reaching enormous dimensions. Their formation is always attended with more or less inconvenience and danger. The least injurious are those which grow slowly, and merely compress the surrounding tissues; but a growing tumour requires to be fed, and the organs on which it may be placed in the system generally are deprived of some nutriment: if the growth be slow this may be unimportant, but if very rapid, the results may be very grave. The gravity and importance of a tumour, benign in itself, are seriously increased by its situation. Thus a very small tumour, particularly if of rapid growth, in the brain or spinal canal may prove fatal to life; and a tumour pressing upon the æsophagus or trachea, obstructing the acts of deglutition and respiration, is a very different matter to a much larger, invading the skin, and interfering with no important function.

A tumour is said to be malignant when it has a tendency to destroy and infiltrate into surrounding tissue, when it tends to recur after removal, and when it is metastatic and heterologous; 
and benign, when its structure is not widely different from the natural tissue, or when it is not an infiltration displacing or overwhelming the original tissue of its seat; neither does a benign tumour show a natural proneness to ulceration, or if it does ulcerate, the ulcer has a tendency to heal, which is not the case in a malignant tumour, where softening precedes an ulceration which has no tendency to heal-a morbid substance like the original forming the walls and boundaries of the ulcer-this substance passing through the same process of ulceration as the primary growth, and so the ulcer spreads and makes it way through all kinds of tissue.

Tumours are now generally classified upon their histological characters, and are divided into two great groups, namely-1st, those composed of structures resembling the adult, and $2 d$, those composed of structures resembling the embryonic connective tissue types. The first group is made up of mesoblastic elements, and is arranged as follows:-

1.- Histioid or Mesoblastic Tumours.

(A.) Tumours typical of the fully developed connective tissues :-

\begin{tabular}{|c|c|c|c|}
\hline \\
\hline Туре & f fibrous tissue, & & Fibroma. \\
\hline$"$ & adipose tissue, & & Lipoma. \\
\hline$"$ & cous tissue, & & xoma. \\
\hline$"$ & $\begin{array}{l}\text { lympnatic tissue } \\
\text { cartilage. }\end{array}$ & & $\begin{array}{l}\text { Lympnoma. } \\
\text { Euchondroma. }\end{array}$ \\
\hline$"$ & bone, . & & Osteoma. \\
\hline
\end{tabular}

(B.) Tumours typical of higher tissues :-

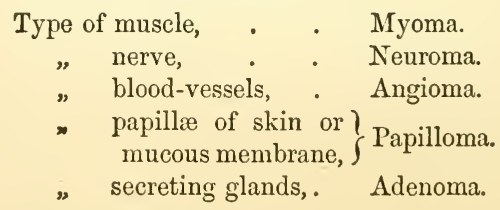

(C.) Sarcomatous tumours, composed more or less of embryonic 
or indifferent tissues, but in which there may be-always abortive-some attempts at higher development:-

Spindle-celled sarcoma, . $\left\{\begin{array}{l}\text { Small spindle-celled sarcoma. } \\ \text { Large " } \\ \text { Osteoid sarcoma. } \\ \text { Myeloid " }\end{array}\right.$

Round-celled sarcoma,.$\left\{\begin{array}{l}\text { Small round-celled sarcoma. } \\ \text { Alveolar sarcoma. } \\ \text { Melanotic sarcoma. }\end{array}\right.$

Parasitic sarcoma, . . Actino-mycosis.

2.-Epithelial Tumours, consisting not only of Mesoblastic, but of Epiblastic and Hypoblastic elements in addition.

On section these tumours are seen to consist of a dense basis membrane-cancer stroma-built of reticulated bands and trabeculæ, and containing within its areolæ a substance of a softer and different consistence, often in the form of a milky juicecancer juice. They are classified as the carcinomata, which include the four following varieties, namely-1. Scirrhous; 2. Medullary or Encephaloid; 3. Colloid; and 4. Epithelioma.

In addition to these two great groups we have cystic tumours, found in various parts of the body, due to the dilatation of obstructed ducts, the embryonic forms of parasites, and to accidental causes, as seen in the eudothelial cysts, found around tendons, serous abscesses, \&c. 


\title{
CHAPTER XXIII.
}

\author{
SIMPLE TUMOURS.
}

FIBROMA OR FIBROUS TUMOUR-PAPILLOMA-IIPOMA-NEUROMAENCHONDROMA - CRUSTA-PETROSA TUMOURS-PSAMMOMA-OSSEOUS TUMOURS-OSTEOMA-PARASITIC CYSTS-CYSTIC TUMOURSTEETH TUMOURS - SARCOMATA - MELANOSIS - ACTINO-MYCOSIS CARCINOMATA - HARD CANCER - SOFT CANCER - EPITHELIAL CANCER.

I.-SIMPLE OR HISTIOID TUMOURS.

The most common forms of tumour met with in veterinary practice are fibrous tissue tumours-Fibromata, and ordinary warts - Papilloma, fatty tumours - Lipoma, and cartilage tumours-Enchondroma.

The fibroma, a fibrous tumour usually found in parts which contain much fibrous tissue, is slow in its growth, and is not accompanied by pain or tenderness, unless accidentally inflamed; is of a hard, rounded form, with its surface smooth or dividerl into lobes; generally moveable, and contained in a wall of areolar tissue.

The most familiar example is the subcutaneous wart. These are of various sizes; some as small as a pea, others as large as a goose's egg, lodged in the subcutaneous areolar tissue, singly or in clusters. They differ much in their degree of connection with surrounding parts, being sometimes firmly attached by continuity of tissue, at other times loosely imbedded in them, and easily moveable beneath the skin. Although many groups of several tumours in each may exist in the same structure or organ, it is very rare to see them co-existent in separate organs. They have very few vessels; indeed, some seem to have no blood-vessels, and no immediate communication with the surrounding parts, but are lodged in a sac, and derive their nourishment by imbibing nutrient fluid from its walls. 
The tumours are composed of white fibrous tissue, blended with some yellow elastic fibres, closely resembling those of areolar tissue. These fibres vary in their different stages of development, and are interlaced together, forming very compact and solid masses. At first the tumours are quite unattached to the skin, but as they grow they burst through and become attached to it; and from their points of attachment prolongations, in the form of fungoid granulations, rapidly grow from the surface. Their favourite seat is the subcutaneous areolar tissue of the inferior surface of the abdomen, where they are generally found associated with the epidermic wart. When the skin is cut through, it is often discovered that a large number of them, in a nest of condensed fibrous tissue, on which numerous blood-vessels ramify, are lodged in the areolar tissue of this part. They are then easily pressed out by the hand, and this is the best method of removing them, the sac being afterwards dressed with some astringent, such as a solution of sulphate of copper. They are also found in the uterus, more especially of the bitch, where they sometimes contain a fluid in their centre.

Polypi belong to the fibrous tumours, consisting of tissue similar to that of the last-named form. The tumour sketched in Photo-lithograph, Plate IV., is an example of a very large nasal polypus.

A polypus may be defined to be a tumour attached by means of a narrow pedicle, and the most familiar example is the nasal polypus attached to the superior part of the nostril; of a softish consistence; bleeding when injured; often containing a thin limpid fluid in its centre; growing downwards, filling the cavity of the nostril, causing much uneasiness to the animal, and interfering very materially with the respiratory function.

There is a discharge from the affected nostril, often tinged with blood, especially during exercise or work. The animal makes a snuffling sound in its breathing, and frequently sneezes. The tumour cannot always be seen, but by growth becomes visible to the examiner. Sometimes it grows in the contrary direction, falls into the isthmus faucis, and is apt to become temporarily lodged in the larynx, causing the animal to breathe with the greatest difficulty, with a loud roaring sound, and often to fall down from exhaustion and want of breath. By 
great effort the animal coughs the obstructing tumour from the larynx into the fauces again, and all at once the roaring sound and difficulty of breathing disappear. Such cases are termed "Bellones" by horse-coupers.

The only treatment for polypus is removal; and the best method is evulsion by the forceps. The instrument must be passed up alongside of the polypus to its roots, where they must be fixed by strong pressure on the handles; and then the connections of the tumour must be torn by a compound movement of turning and pulling. I have removed them very successfully in this way. The nostril ought to be afterwards frequently syringed with an astringent wash, such as a solution of the tincture of the perchloride of iron.

When the polypus grows into the faures, it is quite possible to remove it-if it can be discovered by the hand-by enclosing its pedicle in a noose of strong wire, and tearing it away by strong and continuous traction. If it were possible to apply the "Ecraseur," the removal of this polypus would be rendered a very easy matter. Fibrous tumours, similar to polypi, are found in the ventricles of the brain.

Fibrous tumours, especially in the horse, are often due to the imprisonment of pus in the deep-seated inter-muscular structures. For example, an apparently fibrous turnour, with well-defined borders, and no appearance of inflammation, is often found in the inferior cervical region, beneath the levator humeri muscle. It arises from the pressure of the collar, and when cut into, will be found to consist of the enormously thickened walls of an old abscess-small in itself, and containing a little pus. Again, these tumours may be found upon those portions of the horse's sides that are liable to be bruised by the shaft of the cart. Indeed, with the exception of the subcutaneous tumours already described, the majority of the so-called fibrous tumours of the horse are due to the presence of pus too deeply seated to find its way to the surface.

These old abscesses may be removed either by excision of the whole mass, or by destruction with caustics. If not very large, perhaps the latter is the quicker method; but if very large, the knife must have the preference. It is quite useless to attempt their reduction by the use of external stimulants, such as iodine or blisters. 


\section{II.-PAPILLOMATOUS TUMOURS}

Form a class of new formations of much interest, and are of frequent occurrence. Warts (verrucæ) on the skin and on the mucous membranes are instances of them. Warts consist of a

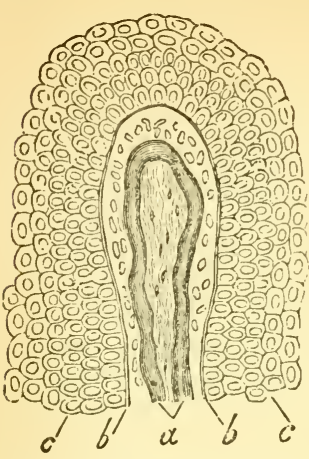

Fıg. 80.-Perpendicular section of a papilla, from an acuminated condyloma, after the addition of acetic acid. a. Vascular loop, internal to which is fibrous tissue, forming the axis of the papilla. Outside are nuclei. $\quad b, b$. Basement membrane. c. c. Epidermic cells. 250 diam.-(WEDL.)

thickening of the epidermis, produced by accumulation of its scales, with hypertrophy of the papillæ of the true skin.

These growths are found most commonly in young animals, their favourite seat being the under surface of the abdomen, the genitals, mammary glands, lips and eyeliris. In the dog they are often seen in large numbers within the mouth, appearing in a very short space of time, and disappearing as quickly. When they form on mucous mem. branes, or when found about the anus, they are called condylomata. They are of the same nature, but are softer in consistence than the wart.

They may be removed by excision or torsion; twisting or pulling by the hand being very often sufficient. If they are found within the sheath of the penis, or on the prepuce surrounding the orifice of the urethra, the patient has to be cast, and the whole mass of them removed by the cautery or knife, and their seat cauterised. If this be not done, they are apt to grow again. Warts sometimes grow to an immense size, and hang like bunches of onions below the animal's belly; when cut into, they are found not only to be warts, but also steatoma, or the subcutaneous fibrous tumours already described. Indeed, when this kind of tumour breaks through the skin, it very closely resembles a common wart. They are found in horses and cattle, and most commonly in young animals.

When in the mouth of the dog, they may be snipped off with a pair of scissors; their removal in this way is not succeeded by 
much hæmorrhage, or the mouth may be washed twice or thrice a day with vinegar, which seems to have a wonderful effect in destroying them. When on the eyelid, their removal must be performed with great caution, or the animal may be damaged for life. Gentle and repeated touches with an acid, so as to destroy a thin pellicle at a time, is the best method for their removal when so situated.

The "grapes" of chronic grease are similar to warts, consisting of thickened epidermis and of enlarged papillæ and sebaceous follicles; they are best removed with the actual cautery; shaved off, in fact, by a sharp-edged piece of iron leated to a red heat.

Another kind of epithelial tumour is found in the vagina, especially of the bitch, larger than a wart, with a lobu-

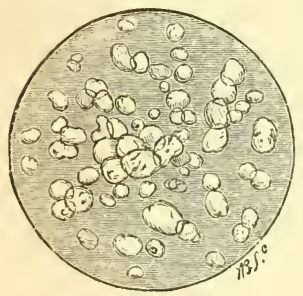

FIG 81. - Fat cells, from tumour removed from vagina of a cow by Mr. Cartwright of Whitchurch. Case described in Veterinarian for March 1872.200 diam. lated surface, and much vascularity, having a tendency to bleed. Several of these vegetations by confluence form a large tumour; they are to be removed by being enclosed in a clam, and cut off by the cautery. They may be named as vascular epithelial tumours.

\section{III.-FATTY TUMOURS OR LIPOMATA,}

Consist of normal fat cells packed together. They occasionally attain a very large size. When situated externally, they must be removed by excision, and the sac destroyed by a slight touch of the actual cautery or a caustic wash. Vaginal tumours sometimes consist of fat cells. The tumour from which the microscopic view is taken weighed $2 \mathrm{lbs} .5 \mathrm{oz}$, and was removed from the vagina of a cow by Mr. Cartwright of Whitchurch.

\section{IV.-NEUROMATOUS TUMOURS.}

The term neuroma is applied to a fibrous tumour connected with the nerves. In the human being they are of frequent occurrence, several hundreds of them being found in one sub- 
ject; but in the lower animals I know of only one set of nerves upon which they appear, and as the result of an operation, namely, upon the plantar nerves, after their division for foot lameness.

These tumours appear within an indefinite period after the operation (neurotomy); in one case four years had elapsed before they began to form. They are of a solid firm consistence, composed of a fibrous stroma, the fibres of which have a wavy outline, running parallel to or interlacing with one another, and having scattered throughout them numerous groups of cells more or less closely packed together. Rokitansky says"These tumours lie between the fasciculi of the nerve, and are interwoven with their neurilemmatous sheath; and it is a remarkable and no less important general rule, because of the symptoms which may result from its presence, or which may be set up by operations performed on it, that neuroma is never deposited in the centre of a nerve, but at its side, so that only a small part of its fasciculi is displaced. The displaced fasciculi are spread abroad and stretched over the tumour, while the greater mass of the nerve remains on the other side uninjured, and with its fibres in connection with one another."

These tumours are found as rounded or oval bodies, with their long diameter along the course of the nerve; varying in size, but never very large; moveable in the transverse but not in the long direction. They are always on the superior division of the cut nerve. They sometimes cause great pain, manifested more particularly when the horse is standing still. He will then often lift his foot from the ground, as if the pain were lancinating; but the lameness is not so evident when he is made to move. When the tumour is pressed upon, or handled in any way, the patient evinces acute agony. Sometimes, however, these tumours, although of some magnitude, cause no inconvenience; but they are apt to be struck by the opposite foot, and so cause the animal almost to fall to the ground. They sometimes make their appearance in a short time after the operation of neurotomy, and are the result of a badly performed operation; the operator having divided the nerve below the upper angle of the wound in the skin, thus leaving a portion of it in the wound, which, becoming embraced in the reparative material, forms a nucleus for the growth of the neuroma.

The only treatment is excision by the knife. 


\section{V.-CARTILAGINOUS TUMOURS, OR ENCHONDROMATA.}

These may take place in the soft parts, or in bone; their favourite seat being the region of the sternum, or upon the ribs. They are frequently caused by external injury. There are two forms of them; one being round or oval, with well-defined borders, and the other having no well-defined limits, but resembling an infiltration into the surrounding structures. In the latter case it will generally be found that the tumour arises from the development and growth of cartilage in an inflammatory exudate; and wounds involving the sternum are very apt to be accompanied by this complication.

To the touch these tumours are hard and dense, but present a slight elasticity. They gradually increase in size, and when on the sternum become a source of hindrance to the animal's movements, causing him to move with the fore legs wide apart, and with apparent stiffness in the gait. The skin over the enlargement soon becomes raw by the friction of the elbow on its surface.

The rounded form of tumour generally appears on the cariniform cartilage as a globular enlargement, hard and firm to the touch, causing little or no inconvenience to the animal, unless interfered with by the collar.

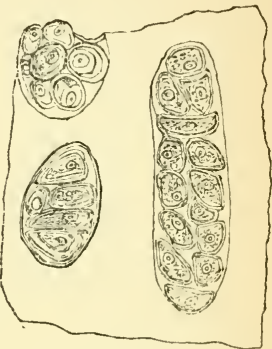

Fig. 82. - Proliferation of diseased cartilage. Large groups of cartilage cells within a common envelope (wrongly called parent cells), produced from single cells by successive subdivisions. At the edge one of these groups has been cut through, and in it is seen a cartilage cell invested by a number of capsular layers (external secreted masses). 300 diam.-(VIRchow.)

I am not aware that these tumours grow within the shell of a bone, as in the human being, but on the outside, and generally fastened to the bony wall and invested by the periosteum, which is greatly thickened and overgrown. When cut with the knife, they present a bright, greyish, translucent, or pinky-white appearance, and sometimes coarsely granular, or opaque, with gritty points, as if gradually ossifying. When examined microscopically, they are found to consist of cartilage cells, mixed with fibres of white fibrous tissue. The fibres are very delicate and tufted, and contain the cartilage cells, either singly or in groups. 
The only change that occurs in these tumours is their ossification into a cancellous structure enclosed in a thin compact shell of bone. I have never seen the fatty degeneration or cystic transformation spoken of by human pathologists; but it is quite possible that such a change may occur.

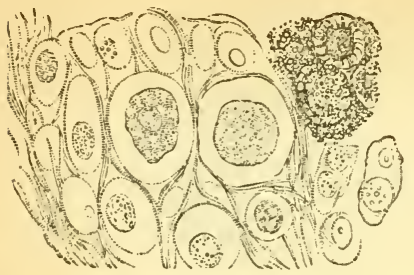

Fig. 83.

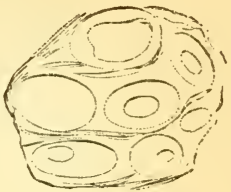

Fig. 84 .

Figs. 83 and 84.-Enchondroma. Fig. 83. Structure of a firm enchondroma. The right of the figure represents, above, mineral deposit in and around the cells; and, below, some isolated cartilage corpuscles. Fig. 84. The same, after the addition of acetic acid, rendering the whole-and especially the nucleus-more transparent. 250 diam.-(BENNETT.)

Treatment.-The only method is their removal by excision, and that as early as possible.

\section{VI.-CRUSTA-PETROSA TUMOURS.}

The following drawing represents a section of a tumour now in the Veterinary College Museum, composed of crusta-petrosa, attached to one of the molars of the ox. I have no history of the case; but upon examination find that the attached tooth is the last molar of the upper jaw.

At $A$. the tooth is represented with its table running obliquely downwards towards $C$., and its crown lost in the adventitious substance. $B$. The shining surface of the section, and $C$. the crown surface of the tumour, slightly worn upon its inferior aspect by contact with the teeth of the opposing jaw. The tumour weighs $1 \mathrm{lb} .3 \mathrm{oz}$.

Tomes describes a similar condition of the crusta-petrosa under the term dental exostosis or hypertrophy of the cementum, and he says-" It will not be forgotten that the surfaces of the fangs of teeth are coated with a thin layer of cementum. Under certain circumstances this layer becomes increased in thickness by additions on the external surface. The newly added cemen- 
tum is in every way similar in structure to that previously forming part of the tooth." He again says-"In dental exostosis

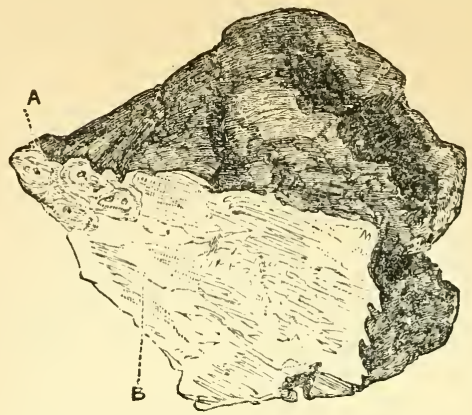

FIG. 85 .

the amount of new cementum may be very slight, or may be considerable in quantity. The affected fang may be but little enlarged, or it may be increased to twice its natural size. Near about the end of the fang is the most common situation to find the greatest amount of cementum; but you seldom find an increase on one side of the fang only, unless the opposite side has been exposed by the absorption of the gum, or deprived of its periosteal covering. In some cases nodules of cementum are found in various parts of the fang, even near the neck of the tooth." And he concludes that "dental exostosis is caused by that condition of the periosteum which is called irritation-a state usually induced by pre-existing disease in other dental tissues, and in a great majority of cases by caries-not always, however."-(Dental Physiology and Surgery, by John Tomes, Surgeon-Dentist to the Middlesex Hospital.)

\section{VII.-CALCAREOUS TUMOURS-PSAMMOMA.}

These consist of a deposit of calcareous salts in various parts of the body -in the testicle, parenchyma of organs, and upon nerves.

Psammoma often constitutes those tumours found within the folds of the lateral ventricles of the brain of the horse, and is important when sufticiently large to induce symptoms of brain pressure. 


\section{VIII.-OSSEOUS TUMOURS AND OSTEOPHYTES}

Are very irregular, of a warty stalactic shape, and are common in the neighbourhood of joints where the articular surface is affected with caries, and sometimes surround the tendons of a limb when no joint disease is present.-(See Photo-lithograph, Plate I., Fig. 4.)

They result from the ossification of an exudate which has been formed in consequence of some cause of inflammation. Osseous tumours are of a more compact structure than osteophytes, and grow more from the compact structure of the bone itself. Cases of great lameness sometimes occur from these new formations becoming inflamed from accidental causes, and I have seen necrosis of them produce sloughing of the skin, and the formation of sinuses.

\section{IX.-OSTEOID TUMOURS-OSTEOMA.}

(See Photo-lithograph, Plate I., Fig. 9.)

These are tumours of irregularly protuberant surface, affecting both the upper and lower jaws of horned cattle. They are generally of a slow, but sometimes of a rapid growth, and when so, they possess some malignity (see Ostea-Sarcoma.) They consist of a cancellous bony tissue, forming trabeculæ, which surround spaces filled with a greyish white, vascular, fibrous material, in which a varying quantity of cells and nuclei are imbedded.

These tumours may vary in their density, from the presence of more or less bony structure in their formation.

If not depending upon the presence of the actinomyces, or upon the irritation of the tubercular bacillus, when these tumours would be more properly classed amongst "inflammatory growths," the osteoma, benign in their nature, are very rarely met with in practice. The tumours of the maxillæ of cattle are generally evidences of actinomycosis, now said to be curable by iodide of potassium given internally.

The only treatment that can be recommended is the removal of the tumour at its very earliest stage, and before it has attained any size. If it be of any magnitude no treatment should be attempted, but the animal ought to be fed for slaughter, if not already fit. These tumours do not seem to cause much pain or inconvenience for a long time, and hence the animal will feed well enough. 


\section{X. - PARASITIC Cysts.}

The compound cysts include the hydatid larval conditions of many parasites, such as-

Cysticercus bovis-in the muscles of horned cattle.

" cellulose-muscles of the pig.

" pisiformis-entrails of hares, \&c.

" cucumerinus-body of dog louse.

" tonuicollis - liver, walls of mesentery, pleura, pericardium, diaphragm, \&c. of sheep and pigs.

$" \quad$ fasciolaris-liver of rat and mouse.

Ecchinococcus veterinorum - large bladder-worms in liver, heart, lungs, \&c.

Coenurus cerebralis-in brain of herbivora, particularly sheep.

Of these, however, the Ccenurus cerebralis and the Ecchincocci are the only ones which attain any size.

\section{XI.-CYSTIC TUMOURS OR CYSTS}

Are not now regarded as tumours in the strict sense of the term. They are cavities containing a liquid or pultaceous matter, enclosed in a more or less distinct capsule, which may be a new formation or a pre-existing structure, distinctly extravasated with or by its own secretion. Paget divided them into simple or barren, and compound or proliferous.

The barren cysts contain a fluid-like serum, such as that found in serous abscesses (capped elbow and capped hock), or synovia-like fluid, as in the enlarged bursæ (wind-galls); whilst others contain a more highly organised fluid, as in ranula.

These cysts, according to the same authority, have at least three modes of origin. 1st. Some are formed by the enlargement and fusion of the spaces or areolæ of the connective or other tissues. In these spaces fluids accumulate; the tissues become rarefied, and gradually the boundaries of the spaces are levelled down and walled in, till a perfect sac or cyst is formed, the walls of which continue to secrete.

$2 d$. Some cysts are formed by dilatation and growth of natural ducts or sacculi, as are those sebaceous or epidermal cysts, which, formed by hair follicles, have permanent openings. Such 
are also certain cysts containing secretions formed of enlarged tubes, such as the ovarian cysts, formed by the overgrown Graafian vesicles; and such appear to be certain cysts formed of dilated portions of blood-vessels shut off from the main streams, whilst others are degenerate, malignant growths, usually of a sarcomatous nature.

$3 d$. Many, and perhaps the great majority of cysts, such as those of the kidney, the choroid plexuses, the chorion, and the thyroid gland, are formed by the enormous growth of new elementary structures, having the character of cells or nuclei, which pursue a morbid course from their origin, or from a very early period of their development.

Simple or barren cysts may be divided into gaseous, serous, synovial, mucous, retentive, and hæmorrhagic.

Gaseous cysts have been observed by Hunter on the intestines of pigs. I have frequently seen them on the intestines of dogs. A cyst of this kind often forms on the hind quarters of horses, in the region of the trochanter major of the femur, which does not seem to contain any fluid when cut into, and upon dissection presents a mere cavity, lined by a glistening grey membrane. It can be distinctly observed in the living animal Can the term wind-gall, as applied to distended synovial bursæ, have arisen from the fact that this cyst seemingly contained nothing but air?

Serous cysts arise in two ways:-1st. From the effects of pressure; and $2 d$., without evident cause. To the first belong capped hock, capped elbow, and other so-called serous abscesses.

Serous abscesses are soft tumours, formed by an effusion of serum into the areolæ of the connective tissue of the part, which becomes condensed, and marks out the limits of the cyst. Within the cyst the fibrous trabeculæ, or bands of connective tissue, are gradually absorbed, and give place to the effusion, until at last a cavity is formed, which is filled with serum, and lined by a more or less perfect epithelium. In some serous abscesses, more particularly if caused by great pressure or friction, inflammatory products are formed, which become consolidated; or a perfectly formed cyst may inflame, and become converted into a solid tumour. In others again, the areolar structure is not so completely removed, and the serosity will be found in various alveoli or spaces, giving to the tumour a honeycomb appearance when cut into. 
When on the elbow, the best treatment is the reduction of the tumour by puncture; the cavity afterwards injected with a solution of sulphate of zinc, or of iodine, and the orifice of puncture kept open until the cavity of the cyst has become obliterated. Another plan is to excite the suppurative action in it after it has been punctured, by injecting a stimulating mixture, as ammonia and oil, or by inserting a seton through its centre. It is useless to puncture and allow the wound to close immediately, for the walls of the sac have acquired secreting properties, and continue to pour out the serosity, which will speedily fill the eavity of the cyst. It is therefore necessary to keep the puncture open until the walls of the sac have become adherent to each other, and its cavity destroyed.

When the cyst has become consolidated by the causes already given, it will often be found that a little suppuration occurs in the centre of the tumour; but it is never very extensive, and the suppurative spot is surrounded by a thick wall of condensed fibrous tissue of a greyish appearance.

There are two ways of removing the tumour when in this condition-by excision, and by sloughing it with escharotics. I have tried both plans, and prefer the latter, provided it is carefully done. I puncture the tumour in two or three places, and insert into each puncture a very small quantity of finely powdered corrosive sublimate and arsenic, in equal parts, rolled up in a small piece of tissue paper. The effect of this is twofold:-(1st.) It destroys the vitality of the tissue which it touches; and (2d.) Excites absorption throughout the whole extent of the tumour. The absorption first excited in the living structures in immediate contact with those destroyed by the agent, whereby a line of demarcation is formed between the living and dead tissue, becomes general throughout the tumour, and in a few days it will be seen that it has diminished in volume in every direction. After the sloughs caused by the escharotics have been removed, the parts should be kept clean for a few days, at the end of which it may be necessary to reapply the caustic.

If excision with the knife be preferred, the operator must make his ineision in the perpendicular direction only, as a crucial incision leaves an ugly blemish. Two incisions parallel to each other may be required in very large tumours, and about 
an inch apart, in order to remove a portion of the skin, which, if left, would be an ugly blemish.

Capped elbow is caused by the animal lying upon the heels of his shoe, and is apt to be produced by a shoe too long in the heels. It must therefore be remembered that the application of proper shoes is the first step in the treatment, and, in addition, that a pad or bandage should be placed around the foot, in order to prevent any chance of bruising during the period of treatment, and for some time after recovery. Some horses will cap their elbows, no matter how carefully they are shod, and these, whilst in the stable, should always have a boot or pad to cover the heels of the shoe and posterior parts of the foot.

The treatment for capped hock is a more difficult matter, for unless great care be taken, a small enlargement on the point of the os calcis-of a soft pliable nature-may become converted into a hard, solid, and unsightly swelling, which will lessen the value of the animal to a very great extent.

Serous capped hoeks are most frequently seen in well-bred horses, whilst capped elbows prevail amongst those of a coarser breed. Not that there is any special predisposition in either class of animal, but from the facts that coarse horses are shod with turned-up or long heels, and that the well-bred ones are liable to accidents to the points of the hocks from kicking in harness or in the stable, being, as a rule, more restless at night than the coarser-bred horse.

Some horses have a habit of scraping all their bedding from under them, and of lying upon the bare floor of the stall. Such are very frequently seen with the points of their hocks enlarged. I always look with suspicion upon horses with this blemish, as generally it is indicative of some vice.

If the enlargement is not very unsightly, it should be left alone; if there is any heat of skin and inflammation, the application of cold to the part and the administration of a purgative will constitute the best treatment. But if the sac be large and the hock unsightly, the insertion of a seton through it, not merely between it and the skin, will occasionally reduce it. There is no danger in this, for there is no synovial cavity interfered with in any way. The seton should remain in for a fortnight, but no longer, as it is apt, if kept in too long, to 
produce considerable thickening of the subcutaneous tissues of the parts surrounding its course. The cyst sometimes becomes consolidated, either from the treatment employed for its removal, or from some other cause. The repeated application of iodine, or its combination with mercury, will often reduce it very considerably. In some cases a strong application, such as one dram of the biniodide of mercury to the ounce of lard, will answer; in others, a frequent application of a milder preparation will do best. I have found the following a very nice remedy:Hydrargyrum biniod., one drachm; water, twelve ounces; and a sufficient quantity of the iodide of potassium to dissolve the biniodide. This is to be applied once or twice a day until slight soreness is produced, and reapplied when the soreness disappears. If possible, the horse should be turned out to grass, or kept in a thickly bedded box during treatment.

Cysts upon the hock or elbow, or indeed upon any part of the body, if they do not cause lameness, are not to be looked upon as unsoundness.

A form of capped hock arising from disease of the os calcis, and an unsoundness, has been already described (page 312).

In some rare instances collections of fluid-forming cysts occur in front of the patella, the knees, and other parts of the body. They may be opened with safety.

In horned cattle immense cysts form in front of the knees, caused by the animal being compelled to lie upon a hard floor. A seton through their substance, and the application of a bandage, to prevent bruising whilst the patient is lying down, will generally remove them.

Serous cysts form in the ovaries, mammary glands of bitches, in the thyroid body of all our patients, and in the facial sinuses of the horse and ox.

The cystic growths which are found in the ovaries are either simple or unilocular, compound or multilocular; sometimes scarcely increasing the normal size of the ovary, which is found to have lost its natural structure, and its place taken up by a number of small cysts; they are supposed to result from distention of the Graafian vesicles by a morbid increase of their contents. In other cases the cystic growths attain an immense size, and hold a large quantity of fluid, which distends the cavity of the abdomen, and presents a swelling externally which 
fluctuates on pressure. This form of dropsy is common in bitches. The treatment is by tapping (paracentesis), and afterwards injecting the tincture of iodine through the canula. I have repeatedly performed this operation with success. I generally introduce the trocar as near the base of the tumour as possible, and send it upwards obliquely through the skin and subcutaneons tissues. The limits of the tumour in bitches may be very well defined by a careful external manipulation.

In the cow small ovarian cysts are sometimes a cause of nymphomania. Their presence can be detected by an examination per rectum, which must be thoroughly emptied, and the hand, being introduced into it, is passed over the uterus until it reaches the ovaries. The diseased ovary is larger than the healthy one, is irregular and lumpy on its surface, and more or less clastic. Moderate compression will cause rupture of the cysts. On the following day there may be increased excitement, with trifling fever; but with quiet and a light diet these soon disappear. Goetz avers that tubercular masses in the ovaries may be dispelled in the same manner.-(Veterinary Journal.)

Ovarian dropsy has also been seen in the cow and in the mare.

Cysts in connection with mucous membranes and glands are termed "mucous cysts," and may be witnessed occasionally in the mouths and on the lips, as well as in the vicinity of the eyes, of the lower animals. I have seen that form called ranula, which is a sac containing a glairy fluid, situated under the tongue, on either side of the frænum. It impedes the movements of the tongue, and causes the patient to froth at the mouth. It requires to be removed by a pair of scissors, and the cavity touched with caustic. Pointer and setter dogs are subject to ranula.

Cysts in the thyroid body constitute bronchocele; they are said to be caused by the water which the animals drink, and that if the water be changed they will disappear. But I have some doubt as to the truth of the statement that bronchocele is solely caused by the presence of magnesian lime in the water, as I have seen well-marked cases in foals, dogs, and in large flocks of lambs, where this could not possibly be the cause. Doubtless it is found most abundantly on the magnesian limestone formations; but it cannot be denied that low situations, 
or a damp atmosphere upon elevated situations, including weakness of the circulation, will act as causes of bronchocele.

It is detected by a soft fluctuating swelling of the thyroid body, occupying one or both sides of the larynx. Treatment: Removal of apparent causes, with good food, warm shelter, and the administration of iodine or its salts. If these are not successful, and if the swelling interfere with the usefulness or value of the patients, paracentesis must be performed, and the sacs afterwards injected with tincture of iodine.

Of the compound or proliferous cysts we have examples in cysts bearing cysts, constituting the compound serous cysts of the ovaries, thyroid body, and mammary gland; in cutaneous proliferous cysts, or cysts bearing hair and skin; and in dentigerous or teeth-bearing cysts.

Of the cutaneous cysts we have many examples from the horse, cow, and dog. Hair-bearing cysts have been found in parts far removed from the outer surface of the body, as in the brain and ovaries.

Mr. Paget says, that "it is perhaps only during the vigour of the formative forces in the fœetal or earliest extra-uterine periods of life that cysts thus highly organized and productive are ever formed. The sebaceous, epidermal, or cuticular cysts that grow in later life are imperfect, impotent imitations of these, yet clearly are the same disease, and are therefore most naturally classed with the proliferous cysts, needing only to be named according to their contents."

The truth of the above quotation must be apparent to all who have seen cases of this kind, for they are generally found in very young animals as small hard tumours, some of them having a very small aperture through which some of their contents can be pressed out, whilst the majority of them are without any aperture whatever. When cut into, they are found to contain hair, along with sebaceous matter, forming a globular and hardened mass, enclosed in a cyst with a laminated pliable wall, lined with epidermis and covered with hair.

The most important compound cystic diseases that come under the notice of the veterinarian are-1st. That where multiple cysts, or $2 d$., dentigerous cystic growths, invade the superior maxillary sinuses. (Photo-lithograph, Plate II., Fig. 8, is an illustration of the former, and Fig. 9 of the latter.) 
The first form is found in animals during the active period of dentition-from one to three years old-and is recognised by a gradual enlargement of one or both sides of the face, usually of one side only, with a discharge from the nostril of an albuminous fluid of the consistence of synovia, which gradually changes into a purulent matter streaked with blood. There is a snuffling sound in the breathing, as if the nasal cavity were diminished in calibre; and this, as well as the swelling and discharge, increases-sometimes very rapidly, at other time more slowly, until at last the animal has to be destroyed.

There is no cough, or if there be, it is only accidental, and the animal usually feeds well and thrives moderately.

On making a post-mortem examination the superior maxillary sinus will be found to contain a rounded mass (made up of numerous cells, containing a glairy fluid), pressing upon the nasal borders and turbinated bones, causing them to bulge into the nasal cavity, diminishing its calibre, producing a difficulty in breathing, and a snuffling sound during the egress and ingress of the air.

Upon trephining a case of this kind, it will be discovered that the sinus contains a glairy fluid, and a friable and highly vascular mass, which forms the walls of the cysts, having a structure similar to that of the turbinated bones. Whether it is an extraordinary development of the sinus before the bones of the face are sufficiently large to accommodate it, or whether it is a new formation-a cyst-I can scarcely determine; but whatever it may be, it can be removed by an operation if it has not been 3llowed to go on too long. The method of operation is as follows:-Make an incision through the skin about four inches long, and dissect it upwards as a flap; trephine the face, near the junction of the nasal and superior maxillary bones of the side affected, in two places, about three inches apart; and with the bone forceps remove the bone between the two apertures, making the gap about an inch in width, then with a hook and sharp scalpel dissect the whole of the sac away, taking care not to disturb the young teeth. If it be found that the turbinated bones have been pressed into the nasal cavity, a portion of them may be removed also. After washing out the débris of the sac and clots of blood, bring the skir: to its proper position, and 
secure it by metallic suture. When the wound is healed, it will be found that the discharge from the nose and the difficulty in breathing have disappeared, and that the life of a valuable young animal has been saved.

\section{DENTIGEROUS CYSTS OR TEETH TUMOURS.}

Cysts containing teeth have been found in the testicles and other parts of the body; but the teeth-bearing cysts, which are of importance to practical men, are those immense collections of imperfect teeth-forming tumours within the antrum, as shown in Photo-lithograph, Plate II., Fig. 9. I have met several cases of this kind, and have extracted teeth from even so high as the base of the ear.

During life, these tumours are distinguishable by more or less disfigurement of the face, by a bulging outwards of the superior maxillary bone, accompanied in some cases by amaurosis of one eye, succeeded by atrophy of the eye from the pressure of the growing tumour. In other cases, these complications are not present, but now and then an abscess forms in the post-orbital region, which will be found upon examination to contain a hard body-an imperfect tooth.

To understand the process by which these "teeth tumours" are formed, it will be necessary to remember that the teeth of all animals belong to and arise from the membraneous portion of the digestive canal, and that at a very early period of fœtal life a provision is made for the development of the permanent teeth, as well as for that of the temporary ones. This provision, according to Goodsir, who devoted much attention to the subject, is as follows:-As early as the sixth week of intrauteral life (in the human fœtus) a groove appears along the border of the future jaws, called the primitive dental groove, which is lined by the membrane of the mouth. At the bottom of this groove projections-papillæ-spring up, corresponding in number with the temporary teeth; these gradually increase in size, and acquire the shape of the future teeth.

Whilst the growth of the papillæ is going on, partitions are. formed across the groove, by which they become separated from each other. These partitions subsequently form the bony sockets. 
The formation of these partitions places each papilla in a separate cavity; and concurrent with this process, small growths take place upon the membrane of the mouth just as it dips into the papillary eavity or follicle, which finally, by union with other growths, form a lid which covers the papillæ in a closed sac or bag.

Before the final closing of the follicle, a slight folding inwards of its lining membrane takes place.

This folding inwards of the membrane of the primitive groove is for the purpose of forming a new cavity - the cavity of reserve -which furnishes a delicate mucous membrane for the future formation of the permanent teeth. By consulting the diagram the reader will better understand this process. Fig. 1 shows the
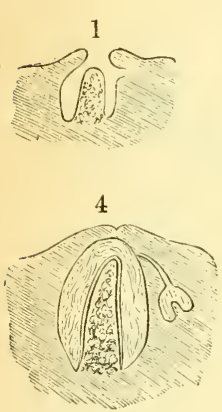
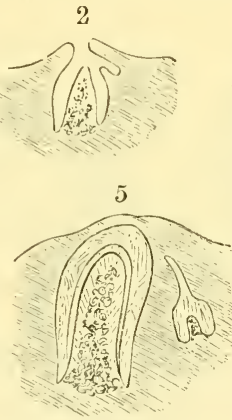

Fig. 86.
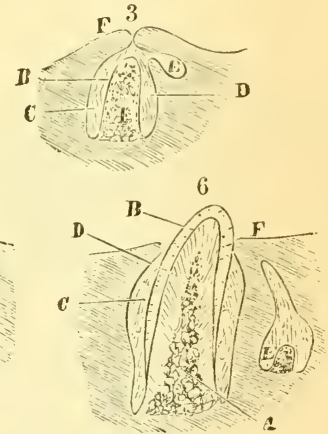

papilla standing in the groove, and a slight folding inwards of the membrane of the primitive groove near to the lid on the right side. Fig. 2 makes this more apparent; and in the other Figs. we find the folded membrane considerably altered in form, and increased in size, having a projection from its bottom part, - the papilla destined for the production of the permanent tooth detached from its follicle.-(From Simonds On the Age of the Ox, \&c., 1854.)

From a study of this, it will be found that the eavity in which the permanent tooth is developed is a mere detachment from the lining of the primitive groove, by the formation of a 
fold in the lining membrane, and that in this cavity a papilla is formed exactly in the same way as in that of a temporary tooth. Now, I look upon the formation of these dental tumours as being due to some eccentricity in this folding of the lining membrane, by which the cavity of reserve is made up of several folds; that these folds become eventually separated from each other, forming separate cavities of reserve; and that a papilla becomes developed in each cavity thus formed in a manner similar to those constituting the papillæ of the natural teeth. These irregular papillæ become finally converted into irregular teeth, which, for want of space in the mouth, are forced upwards into the antrum, and-as in the photo-lithograph-may completely block it up, as well as the posterior nasal opening. I have classified them as cystic tumours, as, in the first instance

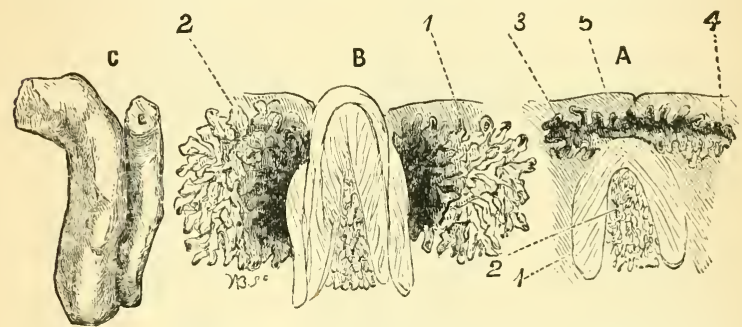

Fig. 87 and Diagram, showing supposed development of dentigerous tumour $A$, Eccentric folding of "cavity of reserve," 3 and 4 showing its two extremities 2 , the gum. B, Further development of tooth, and of the eccentric folding of the reserve cavity, the folds becoming separated from each other, each fold forming a separate cavity. $C$, shows two real teeth removed from a tumour imperfectly united, each tooth having a pulp cavity.

they are enclosed in sacs or cysts; they, however, soon burst through their investing membrane, and form a large tumour, composed entirely of teeth, having a great variety of shapes, and running in different directions. The teeth vary in size, some being very small, others nearly as large as a permanent molar; each tooth has a pulp cavity, and is composed of the same structures as the natural teeth.

Should their removal be determined upon, it will be necessary to trephine the superior maxillary sinus, and detach them with the forceps. 


\section{THE SARCOMATA.}

As already stated, the sarcoma are tumours built up on the type of embryonic connective tissue, and in which, as in embryonic life, the cellular elements predominate over the intercellular substance. The sarcoma are by far the most common forms of malignant tumours met with in veterinary practice, particularly in the horse, although all the ordinary patients of the veterinarian are subject to their inroads.

They vary in consistence from hardish nodulated masses, as in lympho-sarcoma, to those of a soft colloid character, resembling embryonic tissue in its most immature-gelatinous-stage, and when it consists almost entirely of small round cells and a soft amorphous intercellular substance. There is generally, however, an attempt at the formation of some higher kind of connective tissue, and consequently there are varieties of these tumours, but that most commonly-nay, almost invariablyseen in horses and cattle is the small round-celled sarcoma, which are very soft and rapidly growing tumours, and chiefly

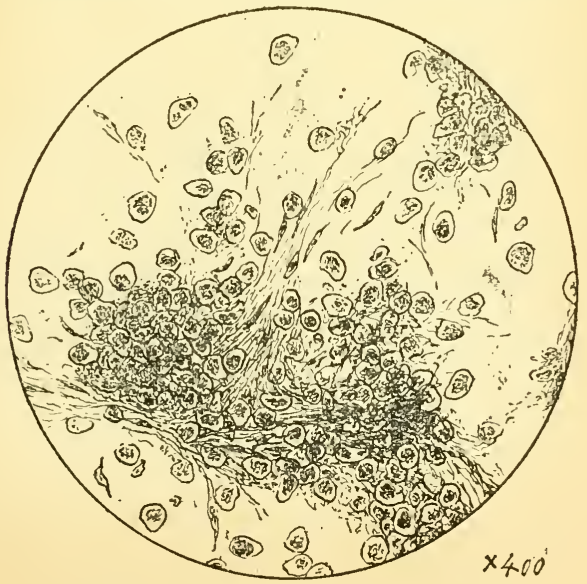

Fig. 88.-Sarcomatous cells, with a few filbres.

From the dog.

occur primarily in fasciæ, loose subcutaneous tissues, in the connective tissue of the nerve centres, frequently in the retina, 
constituting fungus hæmatodes; from the periosteum; and in man from the medullary tissue of bones; and, secondarily, these growths invade the lungs, and afterwards affect the more vascular organs, in which there is a complex capillary network.

When cut into, they are of a uniform soft, brain-like consistence, of a reddish white or grey colour, somewhat translucent or opaque, very vascular, the blood-vessels being often dilated, varicose, and liable to rupture, which gives rise to ecchymoses, the formation of blood cysts, and, if the skin be broken, to hæmorrhage. On scraping the cut surface, a thickish juice, rich in cells, is obtained, and on this account, as well as their other clinical characters, they have been confounded with medullary cancer, from which they are distinguished by the absence of alveolæ and stroma, and by the uniformity of the character of their cells.

The other tumours belonging to this group, glioma, growing from the connective tissue of the brain, spinal cord, and nerves, are large round-celled sareoma, which specially affect the mucous tissue of the pharynx and posterior nerves, where it forms a firm, almost fibrous, pale polypoid growth.

Spindle-celled Sarcoma.-In this tumour there is an attempt at the formation of a higher type of connective tissue than in the round-celled varieties. They approach the fibroma to some extent, but are slightly malignant; the intercellular substance is sometimes very scanty, or almost imperceptible, whilst in other cases it is more abundant; the cells, however, are elongated, and throw out spindles of varying length. Both large and small spindle-celled sarcoma rarely give rise to secondary growths, and are generally surrounded by a more or less definite capsule; are firm, solid, or elastic, and present a pale fleshylooking surface on section.

The Mycloid Sarcoma.-This is a spindle-celled sarcoma, consisting of small spindle cells with the addition of the large many nucleated cells, termed myeloid cells, and the tumour generally grows in comnection with bone, particularly with the jaws, constituting one of the terms of what is familiarly known as osteo-sarcoma, commonly seen in the ox. They generally calcify, and are considered the least malignant of all the sarcomata.

It may here be stated that, with the exception of the srnall 
round-celled sarcoma, the other forms already mentioned present very little interest to the practical veterinarian, as they are rarely met with amongst his patients. The only case of pure spindle-celled sarcoma that I ever had the opportunity of examining was in a well-bred cow, whose cutaneous tissues were covered with innumerable ulcerating tumours, having the histological character of this form of sarcoma.

\section{MELANOSIS, OR MELANOTIC SARCOMA.}

Melanosis, described in the first edition of this work as black cancer, is found, upon further investigation, to belong to the sarcomata, or, as already stated, tumours consisting of embryonic connective tissue.

In structure the sarcoma consists of connective tissue, which retains its embryonic characters and cells, and constitutes nearly the whole of the growth. The round cells are those generally, I may state, always found in the melanosis of the lower animals, and are similar in form to white blood corpuscles, but containing granules of dark pigmentary matter, which give the tumours their characteristic black appearance.

The dark granules of pigment not only fill the cells, but lie free in the surrounding fibres, as shown in fig. 107 (c).

Melanosis has hitherto been described as a benign disease in the lower animals, both by human and veterinary pathologists. Professor Spence says-"The melanotic cancer has this peculiarity, that a form of it occurs in the lower animals which cannot be distinguished from the black cancer in man. But in the former case it is not malignant, and has no tendency to return after removal, while in the human subject it is plainly and entirely a malignant disease-a melanotic form of cerebriform cancer." Paget says- "In the horse and dog, I believe, black tumours occur which have no cancerous character; but none such are recorded in human pathology."

I have seen a great many cases of melanosis, but I never saw one in the horse, the history of which I could afterwards trace, where the disease did not return; and if recurrence only were the special characteristic of malignancy, this would prove the true nature of the growth, without a shadow of doubt. But a 
malignant is not the only growth that is liable to recur, hence the recurrent character of melanosis can only be taken as one fact to prove its malignity.

I have also seen melanosis in horned cattle of various colours, but more particularly in those of a dark brown or black colour, and as a rule early removal has been effectual, and there has been no recurrence.

A melanotic tumour has all the specialities of malignancy. 1st. Its minute structure is not like any of the fully developed natural parts of the body. $2 d$. It is usually an infiltration; and this characteristic is much more manifest than in medullary cancer; although it may appear as separate masses, it will be found, on close examination, that the structures surrounding the tumour are deeply tinged wilh the pigmentary matter, and that they gradually disappear either by absorption or by being appropriated as materials for the growth of the malignant disease. $3 d$. It has a tendency to enlarge, not only by growth, but apparently by multiplying itself in the formation of other tumours around it, or in more remote parts of the body. $4 t h$. Ulceration in melanosis is as constant as in the other malignant tumours. And, lastly, there is scarcely a tissue or an organ that melanosis may not invade. It may, therefore, be safely concluded that "melanotic or melanoid tumours are, with very rare exceptions, sarcomatous tumours, modified by the formation of black pigment in their elemental structures." It may also be mentioned that melanotic tumours in the human being have their favourite seats in or beneath pigmentary moles.

Melanosis is, with very rare exceptions, confined to grey horses, and becomes developed as they whiten with age. Its favourite seat is on the under surface of the tail, around the anus or vagina, or the perinæum; more rarely the scrotum, mammary gland, inner surface of thigh, and on the nose or lips. These are merely its outward manifestations; for on dissection it may be found, either in large masses or as small multiple tumours, in the mesenteric, bronchial, deep inguinal, and lumbar glands, and in the spinal canal. I do not think that there is any part of the body that may not be affected by these tumours; but I wish to confine myself strictly to those cases that have fallen under my own notice. 
In some cases there are no external tumours. The dissection of one case-death occurring from dropsy - revealed a melanotic mass, weighing six pounds, situated in the bronchial lymphatic glands. In another case a tumour was found in the inguinal plexus of glands weighing above eight pounds, which, previous to death, pressed upon the crural nerves, and caused paralysis. In the majority of cases, however, there has been some outward manifestation of the disease; but in occult disease occurring in grey or very light chestnut horses, the practitioner ought to consider whether such disease may not be due to melanotic growths.

The development of melanosis is looked upon as a new excretory function, set up for the purpose of eliminating from the system the pigmentary matter which is no longer required for the purpose of tinging the hair. If it were true that the pigmentary matter was already formed in the blood, and only required to be appropriated by the structures to which it gave colour, the supposition would be justified; but the fact is, pigment is not conveyed to a part, but is formed by the cells of the tissues in which it is found, out of materials supplied by the blood. We must, therefore, look upon melanosis as being due to an exalted formative power in the cells of the tissue in which it is found. When formed in the skin of grey horses, we can easily understand that its pigmentary cells may be stimulated to increased activity by the blood, which one may suppose is highly charged with the ingredients from which pigment is formed. This would be merely an exalted natural action of the cells; but when the pigmentary matter is formed by the cells of glands, or other structures than skin, we must look upon the process as a perverted formative action.

Melanosis appears usually as a rounded tumour, small at first, but gradually enlarging in every direction within and upon those portions of the body which do not lose their black colour by age: or several of these small tumours may manifest themselves simultaneously, enlarging by growth and by coalescence, and thus forming one large tumour. Other tumours form in the neighbourhood, and usually extend along the direction of the hairless skin of the tail, anus, and perinæum, until at last there is a most unsightly collection of them, like bunches of large onions. After a time they wither in the centre; the skin 
ulcerates by a slow, degenerate, and non-suppurative processbeing seemingly converted into melanotic matter-and allows more or less of the contents of the tumour to escape. As the tumours grow the natural tissue of the part withers, its place being taken up by the melanotic matter.

The animal does not seem to suffer much inconvenience, except from the effects of friction upon the surface of the tumour when it comes in contact with contiguous parts. Thus a tumour on the tail will become sore if it presses upon another tumour on the vagina, or if by its weight it presses heavily upon the opposing healthy skin, or when the tumour or tumours are so heavy as to prevent the animal from elevating its tail during progression.

Without entering further into the details of the symptoms, it may be concluded that a black tumour in any part of the body may be called melanosis.

Melanotic growths are neither very hard nor very soft, especially the external ones. Those found in the internal parts of the body are much softer. The colour varies in shade from a dark brown to the deepest black.

Melanosis is generally confined to aged animals, but I have seen it manifested in a five-year-old grey horse, and become rapidly developed in various parts of the body.

The treatment of melanosis is early removal, when it, as well as other malignant growths, may be checked for a time; but, as already stated, its recurrent nature is so great as to preclude the hope of permanent eradication.

Lymphadenoma or Lympiho-sarcoma.-This is another form of a malignant and fatal growth occurring in all the patients of the veterinary surgeon, as well as in man, arising very often from no appreciable cause, being sometimes of very slow, sometimes of very rapid growth; differing from other malignant tumours in one particular only, namely, that it seldom invades but one kind of tissue-that of the glandular system, but as certainly proving fatal as a tumour presenting all the characteristics of the most potent malignancy.

When of a very slow growth, and situated in some external glands-(I have seen it in the lymphatic glands of the head and neck remain passive as it were for years, ultimately develop in the internal organs, and prove fatal)-it is termed a lymphoma, 
consisting of ordinary lymphoid tissue, resembling in fact a hypertrophy of gland. In this form there is a well developed reticulum and endothelial plates, and a normal number of lymphoid cells; whereas, when it becomes malignant, the cells increase to an enormous extent, the reticulum being at first scanty, but later on the reticulum becomes largely developed, with increase of the endothelial plates, and corresponding diminution of the number of lymphoid cells. But, notwithstanding its cellular character, lympho-sarcoma has little or no tendency to undergo any degenerative changes; there is no softening, caseation, or calcification as in others, more particularly scrofulous glandular growths.

Symptoms and Clinical Characters.-In cattle these tumours originate most frequently in the lymphatic glands of the head and throat, sometimes commencing in the submaxillary, from thence to the parotidean region, often following the lymphatic tracks, and extending along the course of the trachea, involving the thyroid body, and invading the surrounding tissues, from which, however, it remains distinctly separated; that is to say, it does seem to infiltrate into, but merely displaces, the surrounding tissues.

I have seen these growths in cattle extending along the intermuscular spaces in an upward as well as downward direction, and involve the face and upper cervical, as well as the inferior and lateral aspect of the throat, pressing upon the larynx, pharynx, and large veins, preventing free deglutition, respiration, and return of the blood from the head, which, in consequence, has soon presented a swollen cedematous condition, the animal being in consequence a miserable object.

The effects of these tumours are similar to those induced by scrofula; both are incurable, but lymphadenoma has no tendency to suppurate or ulcerate, whereas scrofulous or tubercular growths undergo degeneration, soften, and, to some extent, suppurate, such suppuration giving relief, for a time at least, to distressing symptoms.

I have not seen any external manifestations of lymphadenoma in the dog, but have met with masses in the spleen, mesentery liver, and kidneys. In one instance, the weight of an agglomeration of these tumours, taken from a fox terrier, was as much as seven pounds-the symptoms prior to death 
being hard enlargement of the belly, and some extent of dropsy.

In the horse lymphadenoma is generally found invading the spleen and other abdominal organs-(see frontispiece, Veterinary Medicine)-and is then difficult of diagnosis; the more salient signs being a wasting disease arising from no ostensible cause, or perhaps succeeding some trifling ailment. There is capriciousness of appetite, stiffness of the back and loins, pallidity of the mucous membranes, and loss of flesh. If there be no symptoms beyond these, the practitioner is under a great disadvantage; but if, in addition to the above, there be an appreciable increase of the colourless blood corpuscles, the diagnosis becomes less difficult, as lencocythemia-(not that it does not occur without chronic spleen disease)-is, when associated with the above symptoms, characteristic of this condition.

Other cases in the horse, which have fallen under my notice, have had well developed glandular growths about the throat and subscapular glands, which, increasing in size, press upon contiguous organs, and thus become a source of inconvenience to the animal.

In two horses that came under my notice, the glands of the throat became so enlarged as to necessitate tracheotomy to relieve the distressed breathing. In another the subscapular glands were enormously enlarged-the growth being very rapid in this instance. All of these became subject to intermittent colicky pains, finally becoming emaciated, and, after the death, had enormous growth in the spleen, clusters of smaller tumours in the liver, kidneys, in the mesentery, and in the lungs.

In some cases of farcy, lympho-sarcomatous growths are found in the spleen and other organs. I think, however, that they are mere coincidences, as the majority of farcied horses present none of the growths, whilst the tumours exist independent of equina poison.

In some instances the development of the splenic tumours is associated with an appreciable increase of the white blood corpuscles, anæmia, pallor of the mucous membranes, capriciousness of the appetite, more or less rapid emaciation, stiffness of the back and loins, hanging back the full length of the halter or head-collar (in one case a continual curling and twitching of the upper lips, and resting the teeth upon the edge of the manger), 

TWO VIEWS OF THE RAY FUNGUS (ACTINOMYCES) UNDER DIFFERENT MICROSCOPIC POWERS.

Stained with Heneage Gibbes's Tubercle Bacillos Stain, and Mounted in Canada Balsam.

Fig. 91.

Mycelium.

Conidia.

Sarcomatous Cells.

Fibrous Tissue.

$\times 500$.

FIG. 92.

W. o. W.

$\times 250$. 
scraping with the fore feet, and occasionally well-marked colicky symptoms.

In the majority of cases the spleen becomes enormously enlarged, being 70,80 , or $100 \mathrm{lbs}$. in weight; in one case over $200 \mathrm{lbs}$. When of such enormous size, there will be enlargement of the abdomen, and a possibility of diagnosing by external manipulation and examination per rectum.

Microscopically examined, these tumours are found to consist of a reticular stroma, made up partly of anastomosing ramifying cells, containing a multitude of round cells in its meshes, as in figure 89 .

If a thin fresh section of a tumour is shaken with water so that the lymphoid cells are washed out, the structure can be

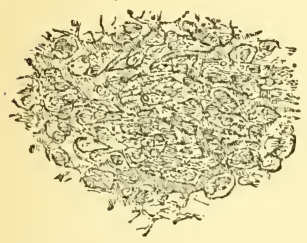

TIG. 89.-Mass of cells in meshes of reticulum.

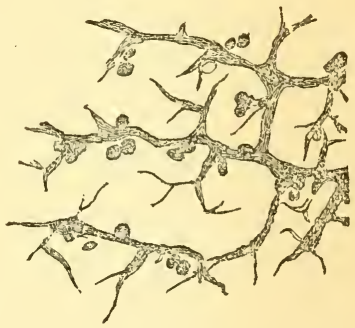

Fic. 90.-Thin section from which many of the cells have been washed and pencilled out.

more easily made ont under the microscope, and the appearance presented in fig. 90 will be seen.

\section{ACTINO-MYCOSIS.}

This malignant growth may be classified as a parasitic tumour, consisting of the development of cellular sarcomatous elements from an irritation caused by the presence of a vegetable parasite -the actinomyces or ray fungus-a radiating or star-like vegetable parasitic growth, as shown in fig. 91.

The botanical position of this fungus is not yet determined, 
some pathologists going so far as to question its vegetable origin, as it differs in many respects from its congeners.

Actino-mycosis is described by Ziegler as a progressive inflammatory affection set up by the fungus-actinomyces--and that it results in the formation of fibrous tissue granulations, and in suppuration. It attacks human beings, cattle, swine, and sheep; it has also been seen in the dog, and is communicable by inoculation, but the horse seems to resist even this test.

In cattle, the masses or tumours seldom suppurate, and sometimes attain the size of a goose's egg, affecting the tongue, the lower and upper jaws, and it has been discovered in other parts of the alimentary tracks, the lungs, skin, \&c.

The disease was described by Rivolta in 1875, by Bollinger in 1877 , and since then has been subjected to investigation by several observers. Previous to this period it was known in this country as scirrhus of the tongue, malignant epulis, tubercular stomatitis, and osteo-sarcoma. It consists of a gradual development in the tissues of the tongue, jaw, or other parts of a fungus, which, on reaching its full development, is a peculiar gland-like body, outwardly resembling a mulberry, produced by the aggregation of the club-shaped conidia of the parasite.

When affecting the tongue, it causes that organ to swell, and sometimes to attain a weight of 10 to $12 \mathrm{lbs}$, , and it is soon covered by numerous irregular shaped masses resembling warts, varying in size from a pin's head to that of an egg or larger. They are of a pale yellow colour, and are more or less soft in consistence, and the larger ones having generally broken through the buccal membrane.

The swelling and the tumours cause the tongue to lose its molvility; hence the patient eats with difficulty, and rapidly loses flesh, and as the disease is incurable, the animal should be sent to the butcher.

On post mortem examination, it will be found that the tissues of the tongue are difficult to cut with the knife, and that the cut surface presents numerous yellowish white or greyish nodules imbedded in its tissues.

\section{THE CARCJNOMATA.}

Cancerous tumours are growths characterised by the development of irregularly shaped cells of an epithelial type, each 
containing one or more nuclei and distinct nucleoli, grouped in rounded or variously shaped connective spaces termed alveoli, and which are said to be in direct communication with the lymphatics at the margins of the tumour, each alveolus having a boundary or wall of fibrous tissue, called the stroma. They are divided according to the amount and nature of the stroma, and the number and character of the cells, into hard, epithelial, and soft cancers.

\section{IIARD CANCER, OR SCIRRHUS,}

Is dense and white in its structure, arranged in masses, with projections passing from its centre to various parts of the organ which it attacks. It cuts up almost like cartilage, and after being cut the surfaces both become concave, and the hæmorrhage from them is uniform, without jet, and more excessive than from the surrounding tissues. It differs from a simple tumour by being more rapid in its growth, by its tendency to involve the lymphatic glands, to break through the organ in which it is developed, and to involve neighbouring textures. When developed in the mouth and face, it interferes with the process of mastication, and causes the animal rapidly to lose flesh; but when it is so situated as not to interfere with any of the animal or vital functions, it does not seem to affect the constitution for a long period, or until ulceration has taken place. The ulcer then involves the adjacent parts, and a fungus is thrown out, hæmorrhage occurs, and the patient suffers from irritation and exhaustion.

The only treatment is early removal, if the tumour is situated in a part where an operation can be performed. The application of escharotics is not to be recommended, excision being by far the better method. In the mammæ of dogs the operation nearly always affords relief, if the brachial or inguinal glands be not affected. I have operated on many cases, and always with success; but I have taken care not to do so when the glands are much enlarged; but if the external gland be enlarged, and when it can be ascertained that internal ones are not involved, the formal as well as the original tumour are to be excised.

Professor Bennett has suggested a method of destroying 
cancer, by injecting weak acetic acid into the tumour. The suggestion has arisen from the fact that acetic acid destroys the cells of cancer, transforming them into amorphous matter.

\section{SOFT CANCER.}

This is met with in two forms, namely, medullary sarcoma or cerebriform tumour, and the colloid, alveolar, or gelatiniform, as it is variously named. The term colloid seems to be preferred by modern writers.

(1.) Colloid Caneer.-I have met with specimens of colloid cancer, since the publication of the first edition of this work, in the ovaries, kidneys, and in the brachial lymphatics. The specimens, as well as one met with in the duodenum of the horse by Dr. Young, were found to correspond with those seen in human beings, in whom it is found as a primary disease, principally in the digestive organs, uterus, mammary gland, and peritoneum: and, as a secondary disease, in the lungs and lymphatic glands. It consists of fibres so arranged as to form alveoli or spaces, varying in size, and containing a soft, viscous, and neariy liquid matter, grey or amber-like in colour, but sometimes opaque, and of a greenish yellow hue. This is the true colloid, or glue-like substance. The density of the tumour will depend upon the proportions of its two constituent materials. If the colloid matter predominates over the fibrous material, the tumour will have a soft, fluctuating feel, and will be made up of large masses of colloid substance, intersected by white fibrous cords or thin partitions, arranged as in areolar tissue. But when the fibrous texture is predominant, the tumour will appear as a tough, white, fascia-like mass, containing small separate cavities or cysts filled with the colloid substance.

(2.) Medullary cancer is developed in the form of circumscribed tumours or infiltrations. The case from which the specimen seen in Photo-lithograph, Plate IV., Fig. 4, was taken, presented both varieties, the circumscribed, as well as the infiltrated form, in the mammary region; infiltrations only in the submaxillary space, and one well-defined circumscribed tumour (that represented in the figure), weighing four pounds, upon the outer side of the thigh. 
Many names have been given to this variety of cancer, such as fungus-hæmatodes, cephaloma, encephaloma, encephaloid, carcinoma medullare, medullary fungus, medullary sarcoma, \&c. It is found in the glands, in the inter-muscular structures, in the penis, scrotum, the orbit, submaxillary space, and in the bones and periosteum.-(See Photo-lithograph, Plate IV., Fig. 6.)

Medullary cancer may present itself as one tumour; but when cut into, it will be found, as a rule, to be made up of several snaller ones, enclosed in a distinct wall or boundary; or its multiple character may be defined by external examination. To the touch it presents a peculiar soft elastic feel of the slow fluctuation of some thick liquid, which may be taken for pus or serum by an inexperienced examiner. The veins over the tumour are congested; the skin retains its natural appearance for a long time; but eventually becomes tense and painful, the pain being referable to the tension of the nerves and surrounding tissues-the tumour itself not being sensitive-and then ulcerates and bleeds. Ulceration does not take place very readily; but when it occurs, a fungous growth soon appears, and much of the brain-like matter of which the tumour is composed, along with much blood, is discharged. A separable medullary cancer may, as a whole, present a very irregular surface, having a tendency to extend in the direction of the intermuscular spaces, as in one case, where I found it in the walls of the abdomen. In this instance the tumour was almost of a triangular shape, with its base turned backwards, extending for about fourteen inches under the panniculus carnosus, and adapting itself to the form of the spaces existing between the pectoralis magnus and serratus magnus muscles. But though the mass may be irregular in the aggregate, its component lobes are round or oval, and readily adapt themselves to surrounding parts. They grow deeply in loose areolar spaces, but their boundaries are more superficial where the surrounding structures are firm. The parts around the lobes are not usually infiltrated, as each lobe is surrounded by a more or less distinct capsule, which seems not only to enclose each individual lobe, but to extend over and involve the whole tumour. This is easily separated from the surrounding structures, and when cut into allows its contents to protrude, or, when very soft, to ooze 
out. The blood-vessels, which are numerous in the capsule, are tortuous in their course, peculiarly friable in their texture, and usually surrounded by the medullary matter. This friability of the vessels renders it very difficult to apply ligatures, as the pressure required to arrest the hæmorrhage usually breaks through the easily lacerable vessels.

When cut into, the lobes are seen to be composed of a peculiar soft substance (the medullary matter), which is easily broken and spread out with the fingers. It resembles reddish-coloured brain matter, and is sometimes softer than brain. I have never seen it white, as described by human pathologists; but the tint is usually clear, that is to say, there is no purulent or fibrinous opacity. Masses of a peculiar looking substance are seen in it. These are yellowish, rounded bodies, similar to very small cysts, and in mass resembling Indian meal or coarse porridge. This matter is often found between the lobes, as well as within them. In their centres the contents of the lobes are seen to be undergoing fatty degeneration.

When the cancer is pressed or scraped, it yields a turbicl material, "cancer juice," and leaves a small quantity of fibrous tissue, with numerous blood-vessels. This, as well as the cancer juice, is formed during the growth of the cancer, and therefore differs from the "stroma of scirrhus" by being part of the malignant growth, and not of the tissue in which it grows.

It was supposed at one time that the vascular system of these cancers was either exclusively venous or arterial ; but it is now proved, by the experiments of Lebert and others, that they contain arteries, capillaries, and veins, arranged in networks of varying closeness; and it is also probable that the difficulty of injecting veins in some of them is due to their being filled with cancerous matter, which stop the injection, after it has traversed the capillaries. The vessels are very abundant, and are not only friable in structure, but defective in muscular tonicity, hence their liability to bleed when the tumour ulcerates, or when wounded accidentally; and it is due to this that the name fungus-hæmatodes has been applied.

Medullary cancer may arise from an accidental injury, such as a blow or wound. In a case of cancer of the side of thorax and abdomen, the tumour arose in the cicatrix of an cld wound; the tumour had been present for several years, and 


\section{MICROSCOPIC ANATOMY OF CANCER.}

Fig, 94.

(A)

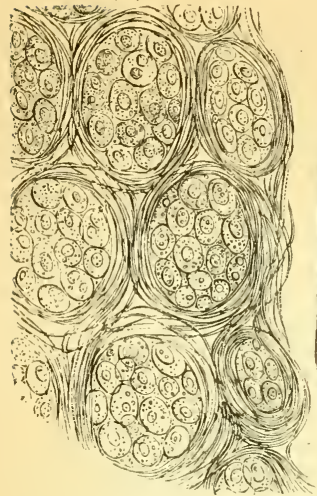

(D)

$(C)$

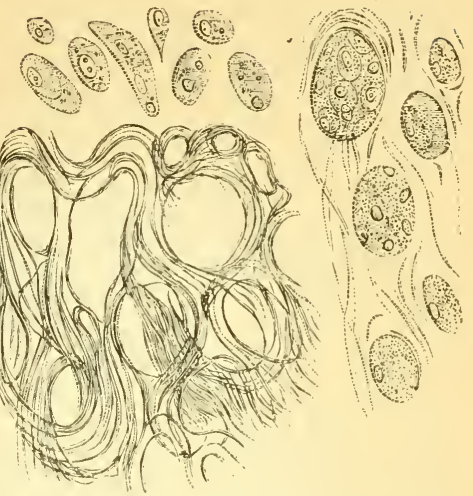

(B)

(A.) Colloid cancer. Appearance of the fibrous areolæe filled with cancer cells.

(B.) Fibrous stroma, deprived of the cells by pressure and washing.

(C.) Stroma and cells, after the addition of acetic acid.

(D.) Some of the cells isolated. 250 diam.-(BEnnetr.)

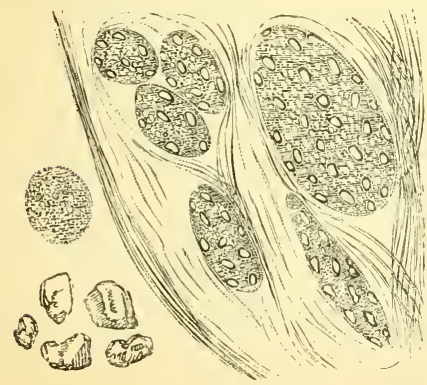

FIG. 95.

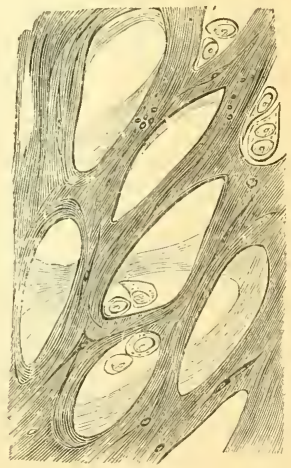

FIG. 96.

FIG. 95.-Colloid tissue, with the loculi filled with molecular matter, in which cells are commencing to form. On the left of the figure one of the molecular masses has been squeezed out of the fibrous matrix; below are masses of mineral matter. 250 diam.-(BENNETT.)

FIG. 96.-Alveolar stroma of cancer, obtained by pencilling out, under water, a very thin section of a scirrhous tumour of the breast. 220 diam.-(ARNOTT). 


\section{MICROSCOPIC ANATOMY OF CANCER.}

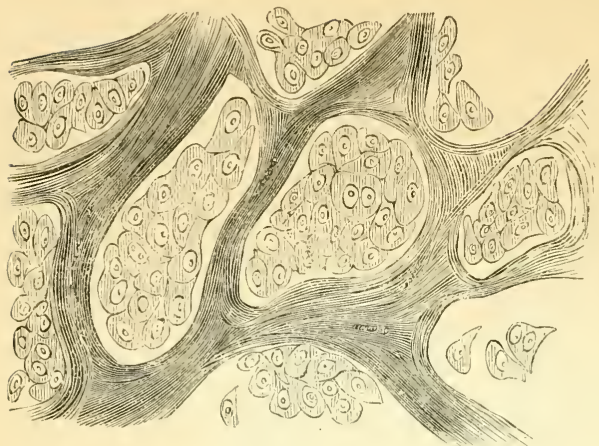

Fig. 99.
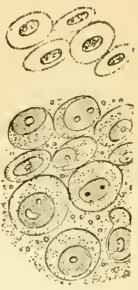

Fig. 98.

FIg. 97.

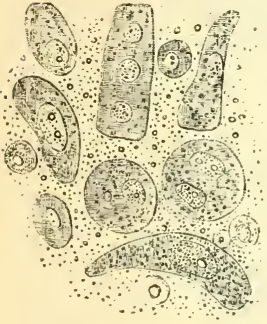

Fig, 100.

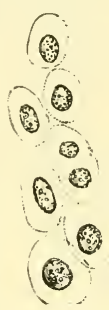

FIg, 101 .

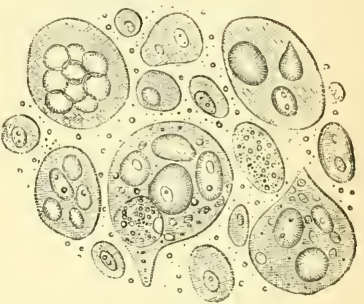

Fig. 102.

Fig. 97.-Typical mature carcinoma from a scirrhous breast. Probably by the action of the chromic acid solution employed to harden the specimen, the cells have shrunk away from the alveolar walls to some extent. 220 diam.-(ARNo'Tr.)

FIG. 98.-Young cancer cells from testicle.

Fig. 99. - The same, after addition of acetic acid.

FIG, 100.- Older cells, from a tumour in duodenum.

Fig. 101.- The same, after addition of acetic acid.

Fig. 102.-Highest development of cancer cells, including secondary cells, frum a tumour of the toe. 250 diam.-(BENNETT.)

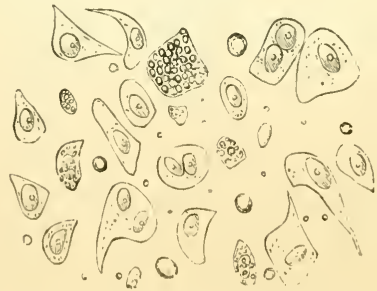

FIg. 103.-Various cells, from a carcinomatus tumour. 220 diaro.-(ARNotT.) 


\section{MICROSCOPIC ANATOMY OF CANCER.}

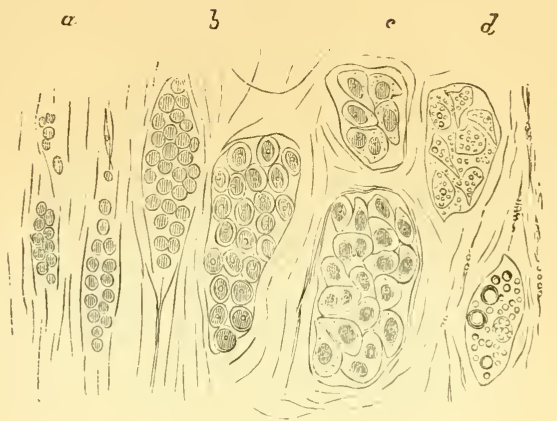

FIG. 104.

FIG. 104.-Diagram representing the several stages of carcinoma. a. Granular corpuscles collected into groups (connective-tissue proliferation?) b. Young oval nucleated cells clustered together. $c$. Typical mature structure. $d$. Gradual withering by fatty degeneration of cell elements and shrinking of the alveoli, (ARNOTt.)

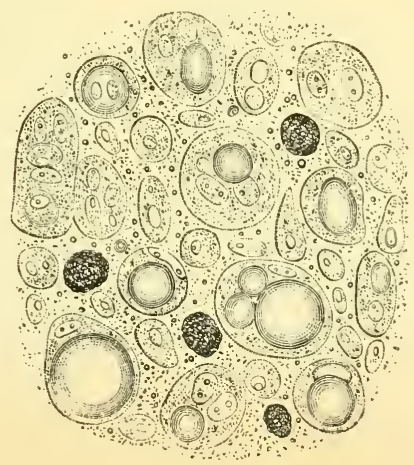

FIG. 105 .

Fig. 105.- Simple and compound cancer cells from duodenum. Several contain fluid by endosmose, which strongly refracts light. 250 diam.-(BENNETr.) 


\section{MICROSCOPIC ANATOMY OF CANCER IN HORSE AND OX.}

Fig, 106.

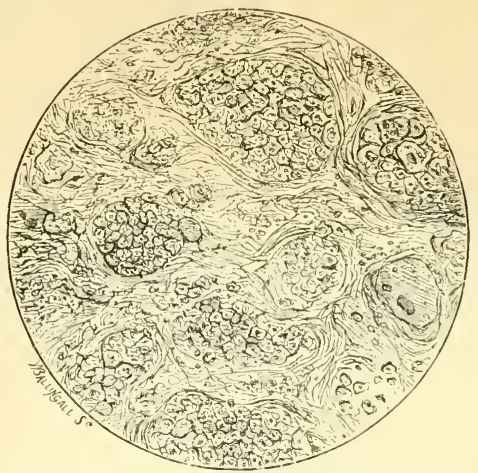

Fig. 106.-Medullary cancer of horse (specimen obtained from Plate IV., Fig. 4), showing groups of cancer cells imbedded in fibrous stroma. 200 diam.

(B)<smiles>C=C</smiles><smiles>C1CCCC1</smiles><smiles>c1cc[se]c1</smiles><smiles>[SiH3][SiH3]</smiles><smiles>C1=CC2=NC=NC2=N1</smiles>
क $\frac{1}{6}+(y)$

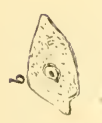

(3)

(⿹)

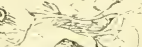

$\circ$ (3)
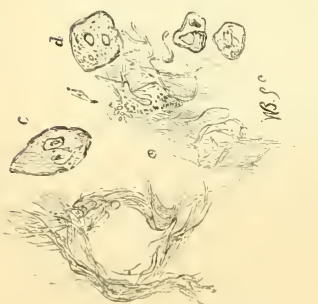

Fir. 107 .

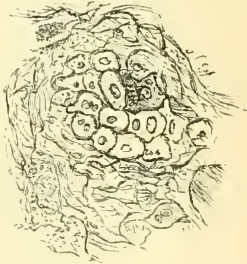

$(C)$

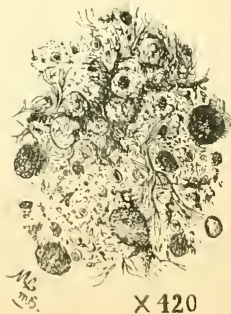

(A.) Portion of above tumour magnified 300 diam.

(B.) a. b. c. d. c. f.g. Detached cells, and fibrous stroma with empty loculi, from same tumour. 420 diam.

(C.) Melanotic sarcoma from tail of a grey horse, showing pigment matter enclosed in cells, and lying free in fibrous stroma. 420 diam. 
gradually increased in size, until it became unsightly, and interfered with the application of the saddle-girths.

\section{EPITHELIAL CANCER.}

Epithelial cancerous tumours, also known as epithelioma or cancroid, consist of a fibrous stroma, in which papillæ and epithelium are found greatly multiplied and enlarged. The cells, when microscopically examined, are found to be numerous, flat, round, oval, or elongated, containing a simple nucleus; and other cells containing large nuclei, which appear as if in process of development into cells. They differ but little from the natural epithelial cells of the part; and as the minute structure of this form deviates least of all the cancers from the natural structures upon which it grows, so its course and history exhibit but little malignancy.

Its chief site is the skin and mucous membranes, but particularly at the junction of the two; as the mouth of mucous orifices, the vulva, anus, eyelids, and edges of the mouth. It is a very rare form in the lower animals; arises from some previous local disease or injury; and is seen more frequently in dogs than in the other domesticated animals.-(Fig. 93.)

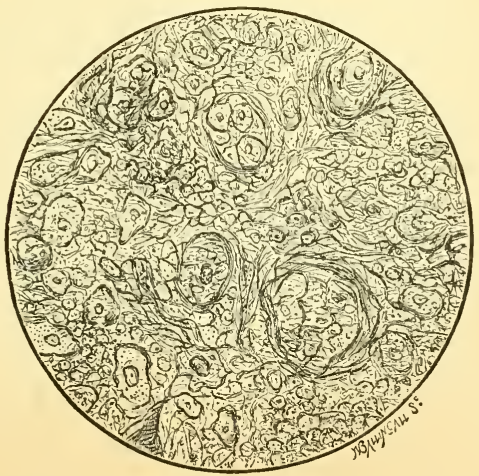

FIG. 93.-Section of epithelial cancer from palate of the ox, showing large flattened cells, with numerous smaller ones enclosed in a fibrius matrix. 250 diam. 


\title{
CHAPTER XXIV.
}

\author{
WOUNDS.
}

\begin{abstract}
DEFINITION-CLASSIFICATION-INCISED-PUNCTURED - LACERATTDCONTUSED - GUNSHOT - POISONED - TREATMENT OF WOUNDSSUTURES - COLLODION - STYPTIC-COLLOID - SHELLAC PASTE NON-INTERFEKENCE.
\end{abstract}

THE term wound signifies a recent solution of continuity of the living tissues induced by some mechanical cause.

Wounds are classified under the following heads:-Incised, punctured, lacerated, contused, gunshot, and poisoned.

The various modes of healing are considered in the following order, namely:-1. By immediate union; 2. By primary adhesion; 3. By graulation; 4. By secondary adhesion, or the union of granulations; 5. By healing under a scab. These five modes are sometimes called:-1. By the first intention; 2. By the adhesive inflammation; 3. By the second intention; 4. By the third intention; 5. Subcrustaceous cicatrisation.

1. Incised wounds.-An incised wound is that made with a clean-cutting instrument. The textures are divided evenly and smoothly; there is no tearing or bruising of parts, hence the hæmorrhage is at first much greater than in most other wounds. If the wound has been parallel to the course of the muscular fibres of the part, there is no gaping of the edges so long as the part is kept in position; but if the cut be across the direction of the muscular fibres, or transverse to the axis of a limb, the wound will be drawn apart, the deep parts more so than the superficial, owing to the retraction of the divided muscular fibres, and a cavity formed in which blood and pus are apt to collect and retard the progress of repair.

The treatment of this sort of wound is very simple, but it comprehends four important indications, namely-1. To arrest 
hæmorrhage; 2. To remove foreign bodies; 3. To effect and maintain co-adaptation; and 4. To guard against excessive inflammation. (1.) Hæmorrhage, whether arterial or venous, is to be arrested, and this is the first thing that must be attended to. If it arises from a small artery partially cut, blood of a bright red colour flows or spurts out in jets; but if it be completely cut across, the ends contract, and the hæmorrhage ceases. In some cases the bleeding will continue although the artery be divided completely across, or will take place from time to time, and prove serious. In such instances the end of the artery must be searched for, drawn out by the forceps, and tied by a ligature; occasionally it will be found necessary to enlarge the wound to do this effectually.

Venous bleeding is generally easily arrested by moderate pressure, or by an astringent application, such as a solution of the tincture of terchloride of iron. As a rule, even these slight applications are unnecessary, venous bleeding stopping spontaneously if the wound is exposed to cold air ; but if a large vein be wounded, it is often necessary to tie it with a ligature.

When the bleeding is arrested, all clots of blood, dirt, and foreign bodies are to be removed from the wound by careful sponging with tepid water. In sponging a wound, eare must be taken not to injure its surface by any undue pressure. It is quite sufficient to squeeze the water out of the sponge on to the wound, without the sponge being brought into contact with it. This is easily done by placing the sponge at a little distance above the wound, and allowing the water to flow gently over the wounded surface. If any materials are firmly imbedded in the tissues, they must be removed by the forceps or the point of the finger. Cleaning wounds with a coarse brush cannot be too highly condemned.

In wounds where muscular fibres are deeply cut, it is recommended, more especially by Mr. Syme, that the wound should remain open for about eight hours, for the purpose of allowing the discharges of blood and serum to escape; or if sutures are immediately employed, they should be applied in such a loose unanner as to allow the blood and serosity to escape, and all clots which may afterwards form washed out. This is good practice, and ensures healing by adhesion in many cases that would otherwise run on to the more tardy process of granulation. 
In many large wounds sufficient room for the escape of the discharges, which are always profuse for the first few hours, may be obtained by omitting one or even two sutures at the most dependent part of the wound, which, if it be parallel to the axis of the limb and the direction of the muscular fibres, is easily brought together and maintained so by a few sutures, placed about an inch apart. If the wound be transverse to the direction of the limb or muscular fibres, with a cavity formed by the retraction of the divided muscular fibres, it is difficult to bring its lips into apposition; and even when they are coarlapted by firm and strong sutures, the skin into which they are inserted is almost sure to slough, the process of healing being at the same time retarded by the irritation set up, and the blemish increased to a considerable extent. In such cases the best plan is to bring the lips together at their extremities, using the quilled suture, and leave a gap at the central portion of the wound; or, if the eavity be very deep and in a downward direction, it may be necessary to make a counter-opening at its inferior part, to allow the discharges to escape. If such be the case, the lips of the original wound are to be brought together, and it is possible they may heal by the adhesive process.

The edges of wounds are kept in apposition by means of sutures, pins, plasters, and bandages. Plasters are rarely used in veterinary practice, but their employment, especially in wounds in the lower portions of the extremities, may with advantage be preferred to that of sutures, as they cause no blemish. A longitudinal or even transverse wound situated on a leg is easily brought together by plasters, over which a moderately firm bandage can be applied; and a wound so treated will heal in less time, and leave a smaller blemish, than when sutures are employed.

Various forms of sutures are employed for keeping the edges of wounds together; the intermupted suture is, however, the form mostly in use, and the material "the metallic suture wire." The wire causes less irritation than thread, and is to be always used in preference. It can be had in thickness and strength adaptable to almost all wounds, with needles grooved for the purpose, from the instrument makers.

The tivisted suture, or the pinning of wounds, is performed as follows:- Having brought the lips of the wound nearly into 
contact, a "pin-director," with the pin in its concavity, is to be introduced from the outside inwards, and carried out through the opposite side to the same distance from the edge that it entered on the former side; the director is then withdrawn, and the pin left in and secured in its position by a firm wax ligature passed around it, making the figure of 8 , by which the wounded parts are drawn gently into contact. The number of pins is to be determined by the size of the wound; threequarters of an inch to one inch is a proper distance between two pins.

The Quilled Suture.-When pressure is required on the deep as well as the superficial parts, this form of suture is employed.

This suture is best applied with a strong curved needle, fixed in a handle, and having an eye near the point. This can be threaded with the loop of double twine, strong thread, or catgut. The needles must pierce the skin not closer than an inch from the edge, deeply inserted, and brought out at the same distance beyond the opposite margin of the wound. This will leave a double thread through the wound, with a loop on one side and two free ends on the other. Through each loop so formed a piece of whalebone or cane should be passed, and the threads firmly drawn over it, while the free ends are firmly tied over a similar piece on the opposite side of the wound. To maintain a more perfect apposition in the more superficial parts, a few interrupted sutures may be inserted to the margins of the wound.

When an injury is superficial, sutures are not required; and in parts where plasters cannot be applied they can be kept in apposition by collodion, styptic-colloid, or, what is more economical, a thick solution of shellac in methylated spirit, which may be prepared and kept ready for use in a wide-necked bottle with a tight-fitting cork.

As to local applications to incised wounds, fortunately the days are past when greasy ointments, friars'-balsam, black oils, and other irritating abominations were made use of, and now- - days wounds are left alone. If anything be done at all, the best application to the surface of the skin around the wound is the ordinary white lotion, gently squeezed out of a sponge, and allowed to trickle over the surrounding skin; care being taken that the sutures are not disturbed, nor the dried discharge which covers the surface of the fissure removed. Fomentations 
with hot or cold water are also objectionable, because they increase the discharge from the wounded surfaces, disturb the sutures, and invariably bring on the suppurative action.

If all goes on well, the sutures are to be carefully removed at the end of the fifth or sixth day, when it will be found generally that the wound has fairly united; but should the lips begin to gape, the surrounding parts to inflame, pus be discharged, the sutures to drag, and cut the skin, some of the sutures at least must be removed, the wound gently bathed with tepid water, and its surface dressed with very dilute carbolic acid, a solution of iodine, or the latest antiseptic dilute boracic acid. In warm weather, and in cases that begin to inflame, tow or lint dipped in cold water may be gently laid over the wound, and retained in position by a light bandage placed at a little distance from the wound. This should be occasionally wetted with cold water in which carbolic acid has been dissolved. It is, however, better not to interfere in any way, except when absolutely necessary.

The Constitutional Treatment.-Prescribe a cooling diet; administer a gentle purgative, and order quietude.

The application of oiled silk, thin gutta-percha, or any other waterproof covering to the wet dressings, is objectionable in veterinary practice, more especially in horse practice, as the parts so confined are apt to become highly inflamed, and the skin blistered.

2. Punctured wounds are produced by the penetration of a sharp or blunt pointed instrument into the tissue usually to a depth disproportionate to the aperture of entrance. Punctured wounds are the most dangerous of all wounds, and for the reasons that, from their depth, they are liable to implicate arteries, nerves, veins, viscera, and deep-seated vital parts; that the parts which they traverse are stretched and torn, and consequently are disposed to inflame and suppurate; and pus, when formed, has no free exit, and is liable to burrow extensively; that foreign bodies may be carried to great depths without being suspected, and create long-continued irritation; and finally, punctured wounds are most liable to be followed by tetanus.

The treatment of punctured wounds must be conducted in accordance with the gravity and depth of the puncture, and the 
amount of laceration and contusion. If the wound be shallow, attended with little or no bruising, and free from foreign matters, adhesion may be possible, and ought to be promoted; and so long as oozing continues from the orifice, cold water bathing is to be practised. When no more blood or serosity issues, a pledget of tow dipped in collodion, or the shellac solution, is to be placed over the opening so as to cover it entirely, or with the exception of its most depending part.

The animal is to be kept quiet; a purgative given, and a mash diet prescribed.

In more serious cases, or in the simpler ones, when inflammatory swelling supervenes, the wound must be dilated freely.

In deep punctured wounds, with hæmorrhage, it may be necessary to dilate in order to secure an artery, or to remove a foreign body; and in the minor punctures, often seen in the neighbourhood of the elbow, it is important to bear in mind that there is great risk of sub-fascial suppuration if dilatation is not practised early. If there be much tension the skin and subcutaneous fascia are to be divided longitudinally and to the same extent. Then the fascia is to be divided transversely, so as to make a crucial incision in it; this will relieve all tension. The same rule applies to partially divided muscular fibres; they must be divided thoroughly and completely, both by a longitudinal and a transverse incision, so as to give free vent to any discharges from the deeper parts of the wound.

When this is done, warm fomentations are to be frequently and freely applied; and in situations where poultices can be adjusted, they should be employed-(boiled turnips form, I think, the best poultice; they are very soft, and retain their moisture for a longer time than bran or linseed meal)-and the surface of the wound dressed with white lotion or opium, and water.

The constitutional treatment must be directed to relieve pain by opium, and the accompanying fever by a purgative, aconite, and antiphlogistic diet. If the puncture be in a foot or limb, with excessive lameness, and inability to lie down, the slings must be early employed.

Fungous protrusions or excessive granulations on the surface of the wound are not to be irritated by caustic applications; they are the tissues of the part, swollen and congested, and will 
disappear when the inflammation subsides. If, however, after the inflammation has been subdued, the exuberant granulations continue in the wound, they are to be destroyed, and the tissues stimulated to healthy action by being touched with the nitrate of silver. In some instances, owing to debility of constitution, these granulations continue as flabby, pale-looking excrescences, notwithstanding efforts to repress them, discharging a sero-purulent matter. Their repression will then be best effected by attention to the constitution of the animal, and by repeated applications of astringents.

Fungous granulations are very often caused by injudicious bandaging, and in many instances where bandages have been applied to a wounded part they will entirely disappear upon the discontinuance of the bandages, and if a wound from which foreign bodies have been removed is not irritated by bandages, undue interference, or what are termed digestive ointments, no sprouting of proud-flesh will occur. There are, however, exceptional cases, and it may be necessary in these to support the vessels of the part, and repress the granulations, by properly adjusted bandages. Their effects are to be closely watched, for generally they irritate the wound and excite fungous growths.

Sulphate of copper, either in solution or as a fine powder, is very generally applied to these granulations. I think it is a very irritating application, and not so serviceable as the nitrate of silver. In cases where the cause of exuberant granulations can be traced to the constitution, tonics, good food, and pure air are more to be depended upon than any mere local application. When the constitution of the animal is good, and the treatment proper, the cause of the continuance of unhealthy granulations generally depends upon the presence of some foreign body in the wound; and of this a wound in the foot, pressed by a small portion of horn, may be taken as a familiar example. Here we find that the strongest escharotics are powerless in suppressing the growth of "proud-flesh" so long as the horn is allowed to irritate by pressing unduly upon any part of the wound; but if the obnoxious piece of horn be removed, the granulations will cease to grow; and if the part be exposed to the air, will wither, scab over, and heal in a very short time. 


\section{CONTUSED AND LACERATED WOUNDS.}

The division into contused and lacerated wounds may be classified as one of two sections, namely-1. Contusion with ecchymosis; and 2. Contused and lacerated wounds.

A contusion is an injury inflicted by some blunt object, without perforation of the skin, and its consequences are-(1.) A degree of concussion or benumbing which may be severe without further mischief, as, for example, when a horse strikes his leg with the opposite foot, goes lame for a time, but very shortly is all right again. This is called brushing or interfering, and a repetition of it will cause some structural change in the part contused. Horses that brush require to be shod with a preventive shoe, or to wear a boot made for the purpose. This infirmity is commonly seen in the hind legs, where it is of less consequence than in the fore ones. Horses that cut or brush with their fore legs are generally bad-legged ones, with round fetlockjoints and turned-out toes; and they should be looked upon as unsound, for they may be brought to the ground at any time if the limb be severely struck. Indeed, it is a rare exception to find an animal of this conformation without broken knees, if it has done any work at all.

The second effect of contusion is a structural injury, varying in degree. 1st. There may be rupture of the smaller bloodvessels, and infiltration of the blood into the surrounding tissues, constituting ecchymosis. $2 d$. A large blood-vessel may be ruptured, and the blood extravasated in considerable quantity, tearing up the areolar tissue in which it coagulates; or if an artery be cut, a false or diffused aneurism may be the result.

The third effect may be the formation of a serous abscess.

The fourth effect may be the pulpification, disorganization, and subsequent mortification of the part contused, not merely of its surface, but of structures deeply imbedded beneath the skin. Repeated contusions of the coronet and pastern, by "brushing" or "interfering," are succeeded in the colder months of the year by violent inflammation of the skin and subcutaneous tissues, and the formation of abscesses in the parts, which sometimes endanger the life of the animal by the severity of the accompanying fever; whilst in other cases the inflammation extends into the joints, rendering the case almost hopeless. The treat- 
ment of bruises or contusions is that calculated to suppress inflammation and prevent sloughing. If the bruising be very great, there may not be much pain at the outset, and this is calculated to deceive the practitioner; he must therefore take into consideration the character of the accident, if it be severe or otherwise, and the general condition of the animal; if there be rigors, debility, or collapse immediately after the accident, he may expect the reaction to be proportionally severe.

The local treatment of contusions must be directed to soothe and prevent undue inflammatory reaction. For these purposes warm fomentations or poultices are to be employed. The constitutional treatment during the stage of collapse must be that calculated to stimulate and support, but when reaction has set in, the antiphlogistic plan must be adopted.

If much blood is imprisoned in the tissues, it will be necessary to remove it; and in those graver instances, where pulpification and sloughing are present, the process of separation of the dead tissue is to be assisted by warm poultices or fomentations, and putrefaction confined to its lowest limits by antiseptics.

It need scarcely be stated that excessive hæmorrhage must be arrested by pressure, or it may be found necessary to cut down upon the vessel, if it be an artery, and secure by ligature. During the process of sloughing, the animal strength must be supported by good food, beer, wine, quinine, and other tonics.

Lacerated Contused Wounds.-When the skin is divided, lacerated, and torn by the contusion, the lesion is denominated a lacerated wound. The edges of such wounds, whether depending upon tearing and dragging, or upon contusion and bruising, are ragged and uneven; the parts being torn rather than cut, and accompanied by much straining of the surrounding tissues. This dragging and bruising weaken the vitality of the parts; this loss of vitality and the depression of the nervous system may prevent the manifestation of much pain until reaction has been established. There is always less hæmorrhage than from an incised wound, because the vessels are irregularly divided, torn, or even twisted.

The treatment of wounds in which laceration is the chief characteristic, or when the contusion is slight, is that by which adhesion is best promoted. Sutures are not generally applicalle, 
co-adaptation being best promoted by collodion, shellac, or styptic colloid, with absolute rest and cooling applications, in addition to purgative medicine and a light diet. If inflammation and suppuration supervene, the treatment must be changed to that calculated to promote the separation of all sloughs, and the growth of healthy granulations. Irrigation with warm water, containing carbolic acid or other antiseptic, as already recommended, is that best adapted for this purpose. 


\section{CHAPTER XXV.}

wouNDS-continued.

GUN-SHOT-CANNON BALLS - TIND CONTUSIONS - MUSKET-SHOT GRAPE-SHOT - SMALL-SHOT-CONSEQUENCES OF GUN-SHOT WOUNDS -TOUNDS AND BRUISES HAVING SPECIAL NAMES-SPEEDY-CUT - TREAD - CUTTING OR BRUSHING - WOUNDS OF ABDOMINAL PARIETIES-BURNS, SCALDS, AND THE EFFECTS OF COLD-POISONED WOUNDS.

\section{GUN-SHOT WOUNDS.}

UXDER this term are included all the injuries caused by the discharge or bursting of fire-arms. They consist of severe contusions, with or without solution of continuity.

\section{CANNON BALLS.}

The cannon ball, for the distance in which its impetus and velocity are greatest, destroys everything that opposes its course. If it strikes a limb, it knocks it off, leaving a stump covered with a disintegrated mass of pulpified tissues, and bone ground down to powder. But if the shot has travelled until its impetus is somewhat lost, the injury it inflicts is ten times greater. It tears its way more deliberately, lacerates the skin, cuts the inuscles into longer and looser flaps, and splits the bones to a considerable distance above the wound. Should the shot strike the limb slantingly, it may inflict a severe laceration, with or without injury of the bone. Of such injuries, those which tear across the great vessels and nerves are the most dangerous; whilst lacerations in the length of the limb, such as the ploughing up of the outside of the thigh from hock to stifle, or from stifle to hip, may be recovered from if the great vessels be untouched. 
SPENT BALLS.

However near to the end of its career a cannon ball may be, it is still a most dangerous thing, and many a leg has been knocked off by a ball whose progress seemed as slow as that of a cricket ball. But the most important series of injuries caused by spent balls are the contusions which they inflict by striking against and rolling over parts after they have lost the velocity required for penetrating or carrying them away. Such injuries were formerly called wind contusions, being supposed to depend on the commotion of the air caused by the passage of a ball close to the part injured without striking it; but now it is known that the wind of a ball, though startling enough, has no bad consequences. In many instances, although the skin may be intact or but trivially grazed, still the parts beneath have been irretrievably disorganized, the muscles pulpified, the bones comminuted, and large vessels and nerves torn across. In less severe cases there may be enormous extravasations, with or without fracture of bone, followed by profuse and unhealthy suppuration and sloughing of the injured parts.

\section{MUSKET-SHOT.}

When a musket or pistol ball has penetrated the body, there is seen a hole, perhaps rather smaller than the ball itself, with its edge inverted; and if the ball has passed completely through, there will be a larger and more ragged orifice, with its edge everted. The oscillations of a musket ball are shown to be in the inverse ratio of its velocity, by its effects on bones. Thus, when a ball propelled with great velocity strikes against a bone of compact tissue, such as the body of the femur, it produces a comminuted fracture of the worst kind, shivering the bone into splinters, and often splitting it up to a great distance. But when the velocity of the ball is very slight, it may be flattened and rebound; or may, if it strike a sharp edge, such as the spine of the tibia, be itself split into pieces. If it strike the cancellated tissue, it will probably bore a canal through it, of which the exit may possibly be twice as large as the entrance. If the propelling force be nearly exhausted, the ball may lodge in the cancellous tissue, forming for itself a kind of chamber, from 
which it is with great difficulty extracted in consequence of the small size of the entrance. If the ball penetrate the cancellous tissue near a joint, it will most probably shiver the bone between its course and the joint.

If it strike a bone obliquely, it may dig out a longitudinal groove without fracture.

A remarkable circumstance connected with gun-shot wounds is the facility with which the ball may be diverted from its course by the slightest obstacle. Any trifling obliquity of surface, or difference of density in the parts which it traverses, may cause it to take a circuitous route. Thus, a ball may enter on one side of the head, chest, or abdomen, and may pass out at a point exactly opposite, just as if it had gone entirely through the cavity, whereas it may be found to have traversed the whole way beneath the skin.

It is always important to ascertain whether the shot has passed out of the body or whether it has lodged; and supposing there are two holes, it must be considered whether they are produced by the entrance and exit of one, or by the entrance of two distinct balls. Sometimes it will happen that a ball splits, either from defect in the casting, or from its striking against some sharp bony ridge; and although one portion may pass out, another may be lodged within the body.

It frequently happens that large masses of metal are impacted in the substance of a part, without much external indication of their presence.

Gun-shot wounds may be complicated by the presence of other foreign bodies besides the ball. 1st. Pieces of saddlery, or of the accoutrements of the horse; $2 d$. Pieces of bone or muscle which have become virtually extraneous in consequence of being dead and detached. It must be recollected that although there may be no ball in a gun or pistol, yet that the wadding may act as a ball, if the piece is discharged close to the animal.

\section{GRAPE-SHOT,}

Striking en masse, produce the effect of cannon balls; singly, of musket balls. Exploding shells cause fearful lacerations and contusions; and all of these gun-shot injuries may be complicated with severe burns from the explosion of gunpowder, with unburnt gunpowder, and other foreign bodies. 


\section{SMALL-SHOT,}

Discharged from a fowling-piece or pistol, produce different effects, according to the distance at which they strike. If the distance is great, they will, in all probability, be scattered and fall singly, peppering the animal smartly, but not penetrating beyond the subcutaneous tissue, nor doing much harm, unless the eyes be wounded. But if the distance is small, so that they strike en masse, their effects are far more destructive than those of a bullet, for they spread in the flesh, and so cause great laceration, besides the mischief arising from their presence in the tissues.

\section{PROGRESS AND CONSEQUENCES OF GUN-SHOT WOUNDS IN FAVOURABLE CASES.}

Inflammation generally comes on in from twelve to twentyfour hours after a gun-shot wound of some common part. The wound becomes swelled, stiff, painful, and exudes a reddish serum. On the second or third day pus begins to be formed, but the suppuration is limited by exudation of lymph around the wound. About the fifth day the parts in the immediate track of the ball which have been killed by the violence of the contusion begin to separate, and change from a blackish-red to a brownish-yellow colour; and from the ninth to the fifteenth day (sooner or later) the slough is thrown off. In the meantime granulations form, the wound contracts and becomes impervious at the centre, and heals up with a depressed cicatrix.

In some cases, through unfavourable accompanying circumstances, as want of timely attention, improper applications, \&c., the local and constitutional symptoms are much more formidable. The pain, redness, and swelling are more severe, the wound dry, and fever violent. When suppuration is established, instead of being confined to the track of the ball, it is diffused amongst the neighbouring muscles and under the fascix, forming numerous and irregular sinuses, so that the case may be in hand for many months. If the ball or any other foreign body remain lodged, the present inflammation and constitutional disturbance will be proportionately severe, and the resulting suppuration more profuse and accompanied by more pain, till the exciting cause is 
got rid of. But if the constitution or the parts do not possess much irritability, if the ball be small and polished, and if it press against no nerves, vessels, or other sensitive parts, it may, and often does, remain for years without creating any disturbance; a eyst being formed for it in the belly of a muscle, or the interstitial areolar tissue.

Mortification supervening on gun-shot wounds may occur under the following conditions:-(1.) When the injured parts are irrecoverably disorganized, so that they immediately cease to live: this sometimes happens to the tissues in the immediate track of the ball, or to a whole limb struck by a spent ball. (2.) From excess of inflammation following a wound. (3.) From division of the great arterial or venous trunks. This is indicated by mortification in the extremity of the limb; the foot becomes cold and insensible; this state spreads up the limb, and the parts immediately above those that are actually dead becorne slightly tumefied and discoloured; and if the animal is allowed to live, the living parts become, in the course of two or three days, hot, painful, and swollen to a great extent. The constitution becomes affected; there is restlessness, anxiety of face, the expression of the eye is haggard; fever runs high, and the poor animal sinks, not having sufficient power to throw off the gangrened part.

Treatment.-If the ball has left the body, the treatment already described for contused and punctured wounds will be applicable; namely, for the stage of collapse, stimulants, as spirits of nitre or carbonate of ammonia, and opium to relieve pain. If the foreign body is still in the wound it should be removed by incision, forceps, or otherwise ; but if it cannot be removed withont a very large incision, it had better be left alone, as it will be brought within reach by the contraction and granulation of the parts, and by the flow of pus; or it may become encysted, and give rise to no further trouble. If a ball has lodged in the substance of a bone, it should be removed by a chisel or trephine, or necrosis will follow.

Some wounds have peculiar names, very suggestive sometimes, and very ridiculous at other times. Over-reach, treads, speedycuts, are bruises and contused wounds.

An over-reach is a tread upon the coronet of the fore foot from 
the shoe of the hind foot. This accident may occur from either the inner or outer side of the toe of the shoe striking the coronet. A tread is a wound on the coronet produced by the shoe of either hind or fore foot upon the coronet of the opposite leg.

A speedy-cut is a contusion on the fore leg, either above or below the knee-joint. Horses subject to this fault should be shod very carefully, the shoe smoothly filed on the inner side, and the clinchers carefully attended to. I have sometimes seen horses that required to have their shoes removed, and their feet shortened, at least every three weeks, on this account. Speedy-cut is liable to cause a dangerous accident, the horse suddenly falling from the violence of the pain, endangering the life of the rider, and breaking his own knees. On this account speedy-cut, if it cannot be prevented by shoeing, may with propriety be considered an unsoundness.

Brushing or cutting is caused by the shoe of one foot striking against the fetlock. Generally it is the hind limbs which suffer, but not always. Young horses out of condition often do this when they are tired and exhausted. They must be shod with preventive shoes. In some cases it is necessary to apply shoes thicker on the inner than on the outside, nailed round the toe and outside only. But shoes thick on the inside are to be used as seldom as possible, and a three-quarter shoe, or one thin on the inside, without a heel on the outside, is to be tried in preference, and for the reason that when a horse strikes the fetlock with a thick shoe the blow is given with such force as to induce inflammation and permanent thickening of the joint, with a greater liability to cutting than before. When horses have been sharpened during a frost they are very apt to tread themselves, and sometimes very severely, causing extreme lameness.

\section{WOUNDS OF THE ABDOMINAL PARTETES,}

On account of the structures which they involve, and the danger of intestinal protrusion, require a speciality of management, more particularly when situated in the inferior portion of the abdominal walls.

1st. Shallow punctures, involving the muscles, but not penetrating through the whole thickness of the floor of the belly, are very apt to cause multiple abscesses. The pus formed in the 
wound, being unable to find exit on account of the smallness of the opening, burrows between the abdominal fascia and muscles, separates them from each other, causing pain and swelling; at the same time small abscesses form in various parts, which are difficult to heal.

$2 d$. Deeper punctures, penetrating almost or completely through the walls, the peritoneum being divided or not as the case may be, are apt to become enlarged by the pressure of the contained viscera, and to cause death by allowing the protrusion and escape of the intestines.

The treatment for the first form must be conducted with the view to allow the free escape of pus and other discharges, and for this it may be necessary to dilate the external opening. This, however, must be done very carefully, or the second danger may be induced. Very often the burrowing of the discharges is promoted by the drying of the surface of the wound. This is more apt to prove injurious when the hair is long, as the hair and dry discharge become matted together over the opening, which is thus effectually plugged up. Care must be taken that this does not occur, and for its prevention the hair must be clipped round the orifice, and the parts carefully washed and kept clean. If it is necessary to dilate the opening, the incision should be shallow, and along the long axis of the belly. When abscesses form, it is always necessary that they should be opened early, as the fascia, being very tough and elastic, allows extensive infiltrations or burrowing of the pus.

The deeper punctures must be treated with a view to prevent the escape of the intestines. The discharges from them must be allowed free exit; but whilst doing this, their extension by the weight of the viscera must be prevented, and this may be done very effectually by enclosing the body of the patient in a common bed-sheet, sewn firmly round him. This will form a suitable support to the weakened walls, and at the same time allow the escape of the discharges. Should it, however, become clogged up by the coagulation of these, a small hole may be made in it immediately below the wound.

When the peritoneal cavity is actually penetrated, or even when it is only nearly so, the medical treatment should be conducted with a view to lessen the danger of peritonitis. Purgatives are to be withheld, and should the pulse be at all disturbed, 
opium must be given, and the large bowels unloaded by enemas; fomentations to the belly are to be persevered in until the danger of inflammation is past.

Incised wounds upon the belly, such as those inflicted by the tusks of a boar, an accident of frequent occurrence, are to be treated by strong sutures, the collodion or shellac paste, and the broad-sheet bandage; but fomentations are not to be applied as in punctured wounds, as they would interfere with the adhesive process, unless, indeed, the danger of peritonitis be imminent.

I am clearly of opinion that purgatives should never be administered when the abdomen is wounded, whether the wound be deep or shallow, as they may excite the most dangerous complications.

Wounds penetrating the walls may heal up externally, but generally a hernia of greater or lesser magnitude remains, forming a permanent blemish.

BURNS, SCALDS, AND TIIE EFFECT OF COLD.

The first effect of cold is to diminish the vital action of the part to which it is applied. This state of depression, when not continued too long, is succeeded by a more than usual activity, or what is called reaction; and if this alternation be oft repeated, the part becomes permanently weakened, being slightly swelled, of a purple colour (as is well shown in horses with white heels), not so warm as usual, and afterwards become inflamed. The skin will now crack, and a discharge of sanguineous matter take place.

More intense cold not only weakens, but entirely suspends vital action. The part becomes pale, insensible, and shrivelled, and is said to be frost-bitten. The skin, particularly the heel, will often slough across from side to side, forming a strip of dead skin, underneath which is a deep chasm, called a cracked heel.

In other instances, and particularly during long-continued snowy weather, with partial thaws, succeeded by sharp frosts, the deeper-seated tissues of the coronet lose their vitality, and deep and extensive sloughs are thrown off, leaving the tendons, ligaments, and even the articulations exposed. The animal now suffers from all the agonizing pain, fever, emaciation, \&c., which 
characterise open joint, and too often succumbs, or has to bo destroyed.

I had a case in the winter 1874-5 where the sensitive frog was frost or rather snow bitten. A piece about the size of a florin sloughed off, leaving the navicular bursa opened; the lameness was excessive; synovia flowed abundantly for some weeks; a large sinus formed in the heel. The horse, however, fed moderately well, lay down and took plenty of rest, and the injury being in a fore foot-which makes a most important difference in all cases of severe injuries-eventually recovered. The treatment consisted in the application of poultices, succeeded by cold water and mild astringents, and finally blisters to the coronet, a high-heeled shoe having been put on the foot as soon as the poultices were discontinued, and which afforded marked relief. In the treatment of frost-bites poultices should not be applied for too long a period, for after the slough is thrown off the parts recover quicker without than with poultices; and, as a general rule, frost-bites are at first best treated with cold applications; but if sloughing has commenced, poultices, irrigations, or fomertations are to be employed, succeeded by cold mild astringents, antiseptics, or deodorizers, such as charcoal.

Burns and Scalds.-These are common at iron works, and result from horses falling on hot dross, the bursting of steampipes, \&c.

The division of burns is, from time immemorial, into three classes-(1.) Burns producing mere redness; (2.) Those causing vesication; and (3.) Those causing death of the part burnt.

The first class is attended with mere superficial inflammation, terminating with or without-more generally with-desquamation of the cuticle and temporary loss of the hair.

The second class is attended by a higher degree of inflammation, causing the cutis to exude serum, and to form vesicles, followed in most instances by suppuration and the formation of obstinate ulcers. The formation and increase of these vesicles may be often prevented by proper treatment.

The third class of burns is attended with mortification from disorganization of structure; the skin and subcutaneous tissues being literally roasted or boiled, as the case may be, the blood cuagulated in its vessels, and the circulation of the part com. pletely stopped. 
In all cases of severe scalds or burns there is more or less supervening fever, manifested by shiverings, coldness of the skin and extremities, prostration of strength, restlessness, quick and feeble pulse, and heavy or sighing respiration; the surface of the burnt part, if destroyed, will become pale and leathery, the hair coming off in patches, leaving a denuded surface, from which issues a thin serous discharge. Swelling of the part now appears, and in about four or five days a line of demarcation surrounds the dead part; the chasm widens, the burnt portion contracts and dries, leaving the exposed granulating surface full in view; the granulations are white, spongy, and moist; there is no discharge of laudable pus, but of a thinnish ichorous matter, generally of a dirty-white colour. The slough now falls off, leaving a wound of more or less magnitude, according to the severity of the burn, which takes many months to heal; it then leaves a cicatrix of a hard, dense, cartilaginous nature, which contracts more or less, pulling the surrounding skin into puckered folds, which ever afterwards constitute an unsightly blemish.

The most intractable cases are those where the regions of the elbow and shoulder are burnt or scalded. Here, in consequence of the continual action of the parts, and the attrition caused by the motion of the chest against the muscles of the inner part of the arm, the wound never heals. Very often the whole limb is burnt, exposing tendons, muscles, and destroying the tissues protecting the articular cavities; such cases should always be destroyed at once. But if the gluteal region or thick part of the thigh be burnt, involving none of the joints, recovery may ensue, although the injury be excessive.

Horses sometimes die from the first shock; the animal sinks from collapse; the rigors become severe, with great restlessness, feebleness of the pulse, sighing respiration, rapid prostration, and death.

Treatment.-In all curable cases, the Carron oil, namely, linseed oil and lime water, in equal parts, is to be applied frequently. A good plan is to dredge this over with flour, or if the oil and lime water is not to be easily got, dredging with flour is a good plan. The Carron oil and flour prevent pain, by excluding the atmosphere; and should neither remedy be at hand, the parts may be protected by cotton wool or anything 
that will exclude the air. Mr. Howell of Rochdale recommends a solution of nitrate of silver, five grains to the ounce of water, to be applied continually, or as often as each application dries, for several hours. Opium and stimulants are to be administered, and the bowels acted upon by a purgative. In about four days pus will form. The wound has now to be treated like any other suppurating sore. If the burn is slight, and the structure of the part merely inflamed, the white lotion is a good application.

\section{POISONED WOUNDS.}

Stinging insects belong almost exclusively to the orler hymenoptera, in which the sting, in the sterile females, represents the modified ovipositor, and consists essentially of two exceedingly fine sharp darts, inclosed in a tubular sheath, at the base of which is placed a special venom sac or gland whose contents are injected into the puncture by the usually barbed darts. The most familiar examples are the stings of wasps or bees. When large numbers of hymenoptera are disturbed, their attack may induce severe or even fatal consequences. The bites should be treated by dilute ammonia.

The bites or stings of insects cause a good deal of irritation and pain to animals, but are not of much importance, and seldom come under notice. Sometimes the eyes become inflamed, and the eyelids swollen and painful from this cause. The best preventive is laurel water, applied to the parts twice a week.

Spiders and Venomous Insects.-The bites of several spiders (Araneida) are said, by various writers, to be venomous. Amongst these the Tarentula (Lycosa tarentula), abounding in southern Europe, has acquired an extraordinary reputation. Direct experiments have, however, proved that its bite is attended with no more ill consequences than a slight local irritation. In the island of Elba another species of spider (Aranea B. guttata) is reputed to be dangerous to men and animals, but these reports are doubted by various writers. Amongst the insects whose bites are more than locally irritating, the tsetse (Glossina morsitans), a dipterous fly abounding in South Africa, and described by Dr. Livingstone in his travels. This fly affords a truly poisonous matter: of a septic and diffusive nature, by 
which, independently of any local irritation, the whole system becomes fatally affected.

The tsetse is a harmless looking insect, very little larger than a house fly; its bite is innocuous to man, goat, antelope, pig, ass, and all wild animals, and even to the calf whilst sucking; but fatal to the horse, domestic cattle, sheep, and dog. The symptoms do not arise immediately after the infliction of the bite, but after an interval sometimes of several weeks. There is then an appearance of general disorder, with weakness and emaciation ; running at the eyes and nose, and glandular swellings under the jaws; continual wasting of the body, and finally death.

The districts infested by the tsetse are very distinctly defined, although separated from a healthy one by a narrow river, or even an inperceptible boundary, beyond which an animal is never affected, unless it has been into a district infested by the fly. It appears that nothing has been ascertained respecting the nature of the plants or vegetation, or whether anything peculiar grows in such districts; but travellers seem satisfied that the tsetse Hy is never found except where the elephant and rhinoceros abound, and that in proportion as those animals are destroyed (lo the ravages of the tsetse diminish.

The Poison of Snakes and Vipers.-All the truly venomous vertebrata belong to the order of ophidian reptiles, which is subdivided into two large groups or sub-orders, distinguished froin each other by clearly defined peculiarities of organization. In one of these sub-orders, the viperina, the species of which it is composed are all more or less venomous; whilst in the others, the colubrina, of which the harmless ringed snake of this country is an example, the majority are innocuous as regards their lite, but formidable otherwise: for example, the boa constrictor.

Characteristics of the venomous Viperina.-Body comparatively thick and clumsy; the general aspect sombre and lurid; and the usual movements sluggish and dull. The tail is thick, and very short; the head broad, depressed, and triangular, or cordiform, joined to the trunk by a constricted neck, and covered on the summit with small scales, not plates or scutes, and having the skin usually loose and wrinkled; the eyes are small, wholly lateral, deeply lodged in the sides of the head, and shaded above 
by prominent overhanging rugose plates; the pupil is elongated and vertical; the upper lip descends on either side, like that of a mastiff, so as to conceal the poison-fangs; the scales are in almost every instance keel-shaped (carinated), or sharp-pointed (muricated). Closer examination of the internal structures of the head and oral organs will still further disclose the more decisive characters of the viperina. The maxillary bones are exceedingly short and mobile, and each supports a single longpointed and recurved tooth, termed the poison tooth or fang, perforated throughout its entire length by a slender canal.

By a peculiar arrangement of the maxillary bones and muscles, this tooth, when not in action, can be reclined into a groove in the gum, where it lies completely concealed; but it can be surdenly and forcibly erected when the viper is preparing to strike its deadly blow. The rest of the bones connected with the jaws, face, and gullet are also very loosely articulated, and so disposecl as to admit of enormous dilatations of the mouth and entrance into the pharynx. Besides the fangs, the upper jaw contains no teeth; but a series of pointed recurved solid teeth are arranged along the palate. In the lower jaw similar teeth are found at the extremity.

The fang is traversed by a narrow canal, continuous above, with a pouch or sacculus surrounding the base of the tooth, into which opens the long curved duct of the poison gland. This organ, which, with various modifications, may be described as composed of tubular follicles communicating with a common canal, has a thick aponeurotic wall surrounded by muscular fibres, in most cases apparently connected with the temporalis muscle, and which are supposed to act as compressors of the gland and ejaculators of its contents. The poison gland is usually below and a little behind the orbit; but in one remarkable instance, causus rhombeatus, it is of an enormous size, and situated on the back, extending from the nape of the neck through nearly one-sixth of the entire length of the body, lying immediately beneath the integument, and superficial to the rilus and costal muscles.

The venom of serpents when fresh is a transparent yellowish or greenish viscous neutral fluid, very much resembling saliva; insipid and almost inodorous; heavier than water, and not very readily mixable with it; the mixture when shaken becoming 
turbid, but exhibiting no obvious characters denoting its virulent properties.

It contains besides albuminous or mucous and a small amount of fatty matters and the usual salts, a peculiar principle, to which Prince Lucien Bonaparte has given the name of "echidnine" or viperine. It strongly resembles ptyaline, but possesses active poisonous properties which are retained even after it has been dried for a considerable time, if not exposed to the air.

When the poison is introduced into the stomach, it seems to produce no effect beyond a temporary local irritation; nor does it appear to induce any deleterious effect when applied to the surface of the skin, even when it has been slightly abraded; and, according to Fontana's experiments, it seems innocuous when applied to the exposed surfaces of muscular tissue, cartilage, periosteum, pericranium, the dura mater, the medullary canal of bones, cornea, tongue, lips, palate, exposed nerves; and all experiments tend to show that in order to produce its specific effects it must be directly introduced into the subcutaneous areolar tissue, and even such introduction is more certain to succeed when the poison is introduced through the fang itself than after inoculation with a cutting instrument.

When introduced, the poison appears to cause death in two ways; when very strong, by directly destroying the irritability of the nervous system, like some of the most powerful narcotic poisons; when less powerful, by diffuse inflammation of the areolar tissue, abscesses, and gangrene. In the first-named instances the symptoms are extreme depression, and a sinking, feeble, flickering, intermittent pulse, coldness of the extremities, dilated pupils, speedy insensibility, stupor, and death. In the second form, the symptoms are of the most alarming asthenic character, from the moment of the infliction of the bite, and are succeeded, if the patient live sufficiently long, by diffuse suppuration and gangrene. The post mortem examinations of such cases reveal a dark, alkaline, and fluid state of the blood, which emits a peculiarly sickly odour, intense congestion of the lungs and spleen, with other appearances indicative of "death of the blood" (necrœmia).

The local treatment consists in preventing absorption into the circulation by tying a ligature round the bitten limb upon the cardiac side of the wound; the immediate excision of the part, 
followed by the application of cups exhausted of air (cupping); followed by the free use of the actual cautery.

The constitutional treatment must be directed to combat depression by stimulants-wine, brandy, whisky, or, according to the latest method practised in India, the injection of ammonia into the veins. Other methods are recommended, such as the free use of arsenic; and Professor Bribon's antidote finds favour with some. It consists of bromine, five drachms; bichloride of mercury, two grains; and iodide of potassium, four grains. This is given to the human being in doses of ten drops, repeated, if necessary, every twenty minutes. Should it ever be deemed advisable to give this to the horse, it must be used in larger doses than these; care, however, being taken that the bichloride is not pushed too far. There are some snakes and vipers whose bites are harmless.

Animal poisons, and the poison of rabies that act peculiarly upon the horse, will be considered upon another occasion.

Sometimes the skin and subcutaneous tissues are destroyed by the action of some poisonous substance, such as the mineral or other acids. I have met with no cases except those caused by mineral acids, and the caustic alkalies, which have a similar effect when accidentally or otherwise applied to the skin. These substances, when used intentionally to remove morbid structures, or when applied to unhealthy wounds, are called caustics. When spilled upon or applied to a large surface, their effects are similar to those of burns; they inflame and afterwards destroy the part. If concentrated, they chemically destroy the vitality of the tissues, and results like those supervening on burns are the consequence. If an acid has been the cause, it is well to wash the parts in some alkaline solution, to neutralise its effect as much as possible, and to destroy any that may be left on the surface. If a caustic alkali has been the cause, a weak acidulated solution is to be used, such as vinegar and water, and the after treatment must be according to the general principles laid down for the treatment of burns. 


\title{
CHAPTER XXVI.
}

\section{WOUNDS-continued.}

\begin{abstract}
METIIODS OF REPAIR - IMMEDIATE UNION - PRIMARY ADHESION GRANULATION-SECONDARY ADHESION-HEALING UNDER A SCAB -FORMATION OF THE CICATRIX, AND COMPLETION OF THE REPARATIVE PROCESS.
\end{abstract}

REPAIR OF WOUNDS, AND THE METHODS OF HEALING.

PAGET, in his admirable Lectures on Surgical Pathology, very profoundly considers the question of the reproduction of injured and lost parts; and he says - " The ability to repair the damages sustained by injury, and to produce lost parts, appears to belong in some measure to all bodies that have definite form and construction. It is not an exclusive property of living beings, for even crystals will repair themselves, when, after pieces have been broken from them, they are placed in the same conditions in which they were first formed. The power of reproduction is exemplified in a most remarkable manner in the lower form of animals, some having the power of reproducing themselves from a fragment into a whole and perfect body; in others, the reproduction of a lost limb has been observed; but in the animals we have to deal with this power is limited to the reproduction of tissues of three classes.

" 1 st. To those which are formed entirely by nutritive repetition, such as the blood and epithelia.

" $2 d$. To those which are of lowest organization, and (which seem of more importance) of lowest chemical characters-the gelatinous tissues, the connective, and the bones.

" $3 d$. To those which are inserted in other tissues, not as essential to their structure, but as accessories, as connecting or incorporating them with the other structures of vegetative or animal life, such as nerve-tibres and blood-vessels. 
"With these exceptions, injuries or losses are capable of no more than repair, in its most limited sense; that is, in place of what is lost, some lowly organized tissue is formed which fills up the breach, and suffices for the maintenance of a less perfect life of the part."

\section{DIFFERENCES BETWEEN THE HEALING OF SUBCUTANEOUS AND OPEN WOUNDS.}

"John Hunter has long ago shown that there is a wide difference between the healing process in injuries which are subcutaneous and those that open to the air. He says-'The injuries done to sound parts I shall divide into two sorts, according to the effects of the accident. The first kind consists of those in which the injured parts do not communicate externally, as concussion of the whole body, or of particular parts-strains, bruises, and simple fractures. The second consists of those which have an external opening, comprehending wounds of all kinds and compound fractures.' He then says"The injuries of the first division, in which the parts do not communicate externally, seldom inflame; while those of the second commonly both inflame and suppurate.' It is hardly possible to exaggerate the importance of the principle here laid down, as on it is embodied the whole practice of subcutaneous surgery. Of the two injuries inflicted in a wound-namely, contusion and exposure to the air-exposure is the worse. Both are apt to excite inflammation, but the exposure excites it most certainly, and in the worst form-that is, in the form which delays the process of repair. Abundant instances are shown of this in simple and compound fractures, and in the injuries of articulations. A simple fracture may have been eaused by much greater violence than the compound one, yet the phenomena resulting therefrom are not so tedious nor so dangerous to life; or a simple fracture extending into a joint, even if caused by great violence, is a very different thing from a wound made into one, though ever so gently made. We lhave other instances in the rarity of suppurations, even after extensive ecchymoses, and the general occurrence of them when wounds are left open.

"The healing of open wounds, as already stated, may be accomplished by five different modes. 
"By what is termed immediate union is meant that process whereby the divided parts are rejoined without the production or the interposition of any new material. Mr. Hunter maintained that union by the first intention is effected by means of the fibrine of the blood extravasated between the surfaces of the injured part, which fibrine, there coagulating, adheres to both the surfaces, becomes organized, and forms a vascular bond of union between them. But it is now admitted that he was in error, and that this form of repair is simply the restoration of the parts; the apposition of their surfaces restoring the vitality and circulation.

"But blood extravasated in wounds is not without its influence on their repair, and there are evidences to prove that inasses of effused, or stagnant, or coagulated blood may be organized. These evidences include cases of blood effused in serous sacs, especially in the arachnoid, or clots in veins organizing into fibrous cords [as I have seen in farcy], or clots organizing into tumours in the heart and arteries, and the clots so organized above ligatures on arteries as to form part of the fibrous cord by which the obliterated artery is replaced. But there is also evidence quite sufficient to show that extravasated blood is not at all necessary for union by the first intention, or for any other mode of repair; and the fact is that the repair is best, and the material for it most ample, where no blood is extravasated. But though this be the usual case, it becomes a question-When blood is effused and coagulated between wounded surfaces, how are the clots disposed of? For often, though not generally, such clots are found in wounds, or between the ends of a broken bone, or a divided tendon, when an artery by its side is cut; and in most operationwounds one sees blood left on them, or flowing on their surface, when they are done up. How, then, is this blood disposed of ? If effused in large quantity, so as to form a large clot, and especially if so effused in a wound which is not perfectly excluded from the air, or if effused even in a subcutaneous injury, this blood is most likely to excite inflammation; and the swelling of the wounded parts, or their commencing suppuration, will push it out of the wounds; or, in moro favourable cases, the blood may be absorbed, and this may happen when it has formed separate clots, or more readily 
when it is infiltrated into the tissues. The absorption of blood is a very slow process, and takes as much time as the healing of a fracture; it is therefore the best plan always in cases of punctures with small openings to enlarge the orifices and remove the clot, if not very deep seated. The best time for doing this is about the third day after the infliction of the injury, as by that time there is not much danger of secondary hæmorrhage, the mouths of the wounded vessels being sealed by exudation. But if the blood is left in a wound, its absorption seems to be thus-that it is enclosed within the reparative material, and absorbed by the vessels of that material as its organization proceeds. In conclusion, extravasated blood is-1st. Neither necessary nor advantageous to any mode of healing, 2d. A large clot at all exposed to the air irritates and is ejectea ; $3 d$. In more favourable conditions the effused blood becomes enclosed in the accumulated reparative material, and while this is organizing, the blood is absorbed; and lastly, it is probable that the blood may be organized and form part of the reparative material ; but even in this case it probably retards the healing of the injury."-(PAgeT's Surgical Pathology.)

\section{IMMEDIATE UNION.}

The observations of Paget and other modern surgical investigators being conclusive that the fibrine of extravasated blood plays no essential part in the process of healing, it will now be necessary to follow the same observers into the various modes by which repair is effected.

1st. Immediate union is effected in some cases of incised wounds that admit of being with safety and propriety closely and immediately bound together; the blood, if any be shed, is thus pressed out, and the divided blood-vessels and nerves are brought into perfect contact, and union may take place in a few hours; and as no intermediate substance exists in a wound so healed, no mark or cicatrix is left behind.-(MACARTNEY's Treatise on Inflammation.)

In order that this process may be fulfilled, it is necessary that the parts be in perfect contact, and in complete repose, and that means be taken to prevent the occurrence of inflammation. 
The sccond mode of repair is that by primary adhesion, or as it used formerly to be called, by the "adhesive inflammation," and is thus accomplished:- When the divided parts are allowed to remain till the mouths of the divided vessels are entirely shut, inflammation inevitably follows, and will furnish the materials for union by throwing out coagulable lymph; this is called the adhesive inflammation. The lymph is simply laid on the cut surfaces, and scarcely any is infiltrated into the tissues; becoming organized and vascular, it connects the two cut edges, aud finally forms between them a thin layer of connective tissue, on the surface of which, if it be exposed, a very delicate layer of cuticle is developed. The smooth shining surface of this cuticle gives the peculiar character to the scar.

It was thought until very recently that the lymph exuded during the earlier stages of this process developed itself into the tissue by which the repair was effected, but this view is incompatible with the opinions held at the present time; and it will be seen by reference to the chapter on Inflammation that the formation of the new connective tissue is effected by the cells of the tissues of the part in which the inflammatory process occurs, and not by the organization of exuded lympl, which plays a passive rather than an active part in the process of repair.

Union by primary adhesion may be accomplished in a very short time. Sir James Paget mentions several instances where the repair has been thus effected in as short a time as seventeen hours.

\section{HEALING BY GRANULATIONS.}

When a wound fails to heal by either of the two processes already described, a series of changes take place in it which are termed healing by granulations; and the simplest case for illustration is that of an open, gaping wound, which from the time of its infliction is only covered with water-dressing. The process is as follows:-Blood gradually ceases to flow from the surface of such a wound; one may, however, still see some blood-tinged, serous-looking fluid oozing from it. Slowly, as this becomes paler, some of it collects, like a whitish film or 
glazing, on the surface; and this, if examined with the microscope, will be found to contain an abundance of corpuscles, having the appearance of the white corpuscles of the blood imbedded in a fibrinous film. The collection of these corpuscles on the surface of a wound, especially on wounded muscles and fasciæ, appears to depend on the peculiar adhesiveness which they exhibit as soon as they are removed from the canal of the healthy blood-vessel. The film increases slowly, and makes the surface of the wound look as if covered with a thin greyish or yellowish-white layer of buffy coat. This increase of glazing is the prelude to the formation of granulations; but while it is going on, and often for some days later, there is in and about the wound an appearance of inaction-a calm in which scarcely anything appears except a slight oozing of serous fluid from the wound. These periods of repose after severe injury may be the brooding time of either good or evil; whilst it lasts, the mode of union of the wound will in many cases be determined; the healing may be perfected, or a slow, uncertain process of repair may be just begun. Immediately after the infliction of an injury, and during this period of calm, the blood in the adjacent parts remains stagnant. During this stagnation materials may ooze from the vessels, enough to form the glazing of the wounded surfaces of certain parts; but before granulations can be formed the flow of blood must again begin, and its supply must be increased. So it may be stated generally that the first visible change which ensues after the period of calm - the period of incubation, as it is called-is an increased supply of blood to the parts in which repair is to ensue.

That which next follows, after the increased afflux of blood, is the production of the material to be organized into granulations. This is added to, or perhaps displaces, the glazing already existing upon some surfaces; and where none exists, as on fat or bone, the new material is accumulated on the bare surface of the wound. Upon this process it has been remarked by John Hunter that a white substance is one day seen, exactly similar in every respect to coagulable lymph; upon the next day this substance has been found to contain blood-vessels (to be vascular). This is granulation without suppuration, but it is very rarely observed.

The further development of the reparative material is very 
interesting, and a recently formed granulation, when examined with the microscope, will be seen to be composed of numerous cells heaped together without apparent arrangement, and connected by very little intermediate substance (protoplasm). Some are round, others caudate, spindle-shaped, elongated, or splitting into fibres. Singly, they are colourless, but in clusters they are ruddy-even independent of the blood-vessels. As the cells become developed into fibres in the deep layers of the exudation, the superficial ones, arrested in their development, become converted into pus cells, which, after having served to protect the deeper seated and more permanent ones, are thrown off in the discharge.

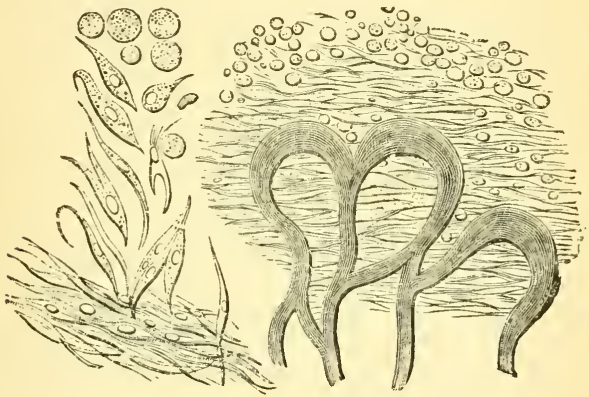

FIG. 108.- Vertical section of a granulating sore. Externally, pus corpuscles; deeper, fibre cells in various stages of development into fibres. The looped blood-vessels are seen enlarged at their extremities. Magnified 100 diameters linear. On the left the cells are magnified 200 diameters linear.-(BENNETT. )

The connective tissue thus constructed by the development of the cells gradually assumes the characters of that tissue, and as it becomes more consistent and dense, the discharge of pus diminishes; a new surface is produced, which after a time contracts, and a permanent cicatrix is formed. After a time yellow elastic tissue is developed, and becomes mingled with the white fibres of the scar.

Granulations are sometimes arrested in their development from some unknown causes, as in indolent wounds and ulcers. In these cases, as is sometimes seen on the legs and backs of horses, months may pass and the cells will not develop them- 
selves beyond one or other of their lower forms. In other cases, the cells not only do not develop themselves, but they degenerate, becoming merely granular, losing the well-marked character of their nuclei, and acquiring all the structure of the pus cell; thus they are found in the walls of fistulæ and sinuses. Or, more than this; the granulation cells may lose ali structure, and degenerate into a mere mass of débris and molecular substance. They are so found on the surface of a wound a day or two before death from exhaustion, as in fatal cases of punctured foot; and in this state they are found, and are commonly ejected when a granulating wound ulcerates or sloughs. With more active disease they become tinged with blood, or œdematous; such are the spongy masses that protrude under the name of proud-flesh or fungus in wounds, and in that disease of the foot termed canker. "All these are lindrances to healing; these are the dangers to which the healing by granulations is obnoxious; it is the proneness to these things that makes it even slower and more insecure than in its proper course it might be."-(PAGET.)

The treatment for granulation is that calculated to repress undue inflammation, in fulfilment of which all sources of irritation must be removed. Sutures, if present, should be removed, warm water dressings or irrigations applied, cleanliness enjoined, and to prevent putrescence and fotor, weak carbolic acid or other antiseptics. If the discharge be excessive, weak astringents, as the white lotion, are useful adjuncts; and lastly, it nust be remembered that all pieces of pulpified tissues, lacerated ligaments, thecæ, or skin, ought to be clipped off, and partially divided nerves cut across, and the torn portions taken away. Without these precautions sloughing ensues, and the process of repair will be greatly retarded.

\section{HEALING BY SECONDARY ADHESION, OR UNION OF GRANULATIONS,}

Occurs when even surfaces of granulations, well developed, but not covered with cuticle, are brought into contact, and so retained at rest. As often as this happens, the cells of which the surfaces are composed adhere together; vessels are developed which pass through them, forming mutual communications, aud 
the surfaces before separate are united. Out of the two layers of granulations one is formed which pursues the normal develnpment of connective tissue. There are several circumstances in which healing by secondary adhesion should be attempted; for example, in a wound presenting two separate surfaces, with a gap between them, where pressure can we applied and the surfaces brought into immediate contact or apposition, by bandaging or otherwise: this may be done in wounds of the legs, of the lips, and of the flank. In this way wounds will heal up in a very short time, whereas, if they are left to "fill up" with granulations, the process will occupy a much longer period. In applying means to produce this method of healing, certain conditions are essential to success. 1st. That the granulations are healthy, not inflamed, profusely suppurating or degenerated, as those in sinuses commonly are. $2 d$. The contact between them should be firmly but gently maintained.

\section{IIEALING UNDER A SCAB.}

This method of healing wounds is the natural one, and as such requires no art. It is the method in which nearly all wounds in animals, when not interfered with, heal. The scab is formed of the fluids that ooze from their surfaces, dust and other foreign bodies are entangled in this fluid, and under such a scab the scar or cicatrix is securely formed. The edges of this scab adhere over those of the wound, so as to form for it a sort of air-tight covering, under which it heals without suppuration, with the formation of a scar, which is more nearly like the natural parts than any scar formed in a wound that remains exposed to the air, and which does not, like it, contract so as to produce deformity of the parts about it. The scab may be formed of either dried blood, dried lymph and serum, or dried purulent matter.

The healing of a wound under a scab has always been considered a desirable process; but to its universal adoption there is some hindrance. For example, when the scab is once formed and the wound covered, it is necessary that no morbid secretion take place. Whenever, therefore, inflammation ensues in a wound covered with a scab, the exuded fluid collecting under the scab produces pain, compresses the wounded surface, or forces off the 
scab. To avoid these unfavourable concomitants, perfect rest must be enjoined, non-interference, and a cooling diet. Collodion makes a good artificial scab.

Such, then, are the several methods of healing observed after wounds of the soft parts, and in connection with them we have the perfecting of scars. In the perfection of scars two things may be observed, namely, their contraction, and the gradual perfecting of their tissues. A process of contraction is always associated with the development of granulations, and this is illustrated by the smallness of the scar in comparison with the original wound. This contraction of both the granulation and the scars is regarded as some vital power of contraction, and of a necessary mechanical effect of the changes of form and construction that the parts undergo. The same change ensues in the organization of inflammatory products, as in false membranes, indurations, and thickening of parts. In all these cases the form of the cell, while elongating, as before described, into a fusiform body, is so changed that it will occupy less space. The whole mass of the developing cells becomes more closely packed, and the tissue they form becomes much drier; with this, also, there is a diminution of vascularity. Thus, there results a considerable decrease of bulk in the new tissue as it develops itself; and this decrease, beginning with the development of the granulation cells, continues in the scar. The improvement and perfecting of the tissue of the scar is again a very slow process. The principal changes by which it is accomplished include the removal of rudimental textures, the formation of elastic tissue, the improvement of the fibrous or fibro-cellular tissue, and of the new cuticle, till they are almost like those of the natural formation-excepting always that the true skin is not reproduced with its hair follicles, \&c.-and the gradual loosening of the scar, so that it may move easily on the adjacent parts. The tissue of the scar extends down deep into the wound, fastening itself immoveably upon its surface; but after a time it becomes more elastic and looser, and the morbid adhesions are freed. Thus we see injuries to the joints followed by much stiffness; this stiffness-depending upon the adhesion of the scar to the deeper-seated partsgradually disappears, and the scar itself becomes more and more pliant, but it never assumes the exact characters of the original tissue, and a scar remains a scar throughout the animal's life. 
All the changes taking place in the various methods of healing of wounds occur in, and are visible illustrations of, the changes that take place in recovery from disease. In all there is a gradual approach of the new particles-which are successively produced-to a nearer conformity with the specific character of the parts they should replace, till repair becomes almost reproduction.

For an explanation of the process by which blood-vessels are formed in reparative material, the reader is referred to the chapter on Inflammation. 


\title{
CHAPTER XXVII.
}

\author{
RESULTS OF WOUNDS.
}

\section{ERYSIPELAS, SIMPLE AND PHLEGMONOUS-SYMPTOMS AND TREATMENT -TETANUS-IDIOPATHIC AND TRAUMATIC-VARIOUS FORMS OF -SYMPTOMS-PATHOLOGY-TREATMENT.}

THE various forms of wounds having been described, the diseases which occasionally follow them may here with propriety be considered. These are Erysipelas and Tetanus.

\section{ERYSIPELAS.}

Although the redness of skin, which is one of the characteristics of this disease in man, so that it is popularly known as " the rose," and "St. Anthony's fire," is absent, or at least cannot be perceived, in the lower animals, owing to the thickness of the epidermis and colour of the hair, yet it is essentially the same, arises from similar causes, and requires a corresponding treatment. The disease in man is divided into simple, phlegmonous, bilious, œdematous, erratic, and periodic; but in the horse the œdematous and phlegmonous are the only forms originating traumatically, and a bilious, periodic form, simulating what has been already described as lymphangitis or inflammatory œdema.

Erysipelas may be defined to be inflammation of the skin and subcutaneous areolar tissue, characterised by a diffused swelling of the parts affected, which has a remarkable tendency to spread, and is dependent upon some alteration in the blood, induced by the Streptococcus erysipelatosus.

CEDEMATOUS ERYSIPELAS.

This is the most common form of traumatic erysipelas met 
with, and generally succeeds wounds of the extremities in horses debilitated by hard work, bad keep in young plethoric animals, or in those tainted by glanders, animal malaria, or septic infection.

Symptoms.-In an indefinite period, but generally about the third or fourth day after the infliction of an injury-more particularly if such be on a depending part of the body-the skin in the immediate neighbourhood of the wound is found swollen, smooth, shining, hot, tender, and painful; the swelling gradually extends from the wound, embracing in some instances the whole superficies of a limb in the course of a few hours. The swollen surface pits on pressure where much areolar tissue is found, but where the subcutaneous tissues are hard and firm, the impression of the finger is not so well defined.

Vesication sometimes occurs, but this is not a constant symptom, and is succeeded by some amount of desquamation, more particularly at the flexures of the joints. The local manifestation of the erysipelatous inflammation is accompanied by some degree of constitutional disturbance; the pulse becomes quick, rigors are present; the animal is "fevered," as it is commonly expressed, loses its appetite, and pain is manifested by lameness if the disease be in a limb.

\section{PHLEGMONOUS ERYSIPELAS.}

This is a much more violent form than the œdematous, and is expressed by a great amount of constitutional disturbance, partaking of a typhoid character; the tendons and ligamentous structures, the fascia of adjacent muscles, as well as the skin and subcutaneous areolar tissue, become involved in the inflammation; the pain is excessive, the swelling is hard, tense, and occupies a large extent of surface. In a variable period, purulent collections form in the subcutaneous areolar tissue, or more deeply between the tendons, ligaments, and fasciæ, which on being opened discharge a watery pus, which may be mixed with shreds, or, in more aggravated cases, with masses of gangrenous tissue. The systemic disturbance is severe; rigors are frequent; pain is acutely felt; the pulse becomes small, quick, and feeble; the respirations hurried; the bowels generally constipated, the fæces covered with mucus, and the urm 
scanty and high-coloured. The desire for food is lost, but the thirst is sometimes excessive. Occasionally the inflammation extends into the articulation nearest the original injury, and the case becomes complicated with open-joint, or abscesses form along the course of the alosorbents, the animal becoming eventually farcied or glandered.

Treatment.-In the œedematous form, the bowels are to be opened by a brisk cathartic, the swollen parts frequently fomented, bandaged, and dressed antiseptically. When the cathartic has operated, saline diuretics, and liberal doses of the tincture of terchloride of iron, are to be administered, and the food is to be of the best kind.

In the phlegmonous form the treatment must be more energetic. A purgative is to be administered; the excitement combated with aconite, which has a most marked effect in allaying the irritation and fever; and when the bowels are freely moved by the purgative, the tincture of the terchloride of iron, in doses varying from two drachms to half an ounce, is to be given every four hours.

Locally, the parts are to be fomented with warm water, and smeared with oil or extract of belladonna.

If abscesses form and give distinct indications of pointing, they must be opened, but it is advisable to abstain from the use of the bistoury as long as possible, inasmuch as the admission of the atmosphere into an erysipelatous wound is apt to be followed by sloughing of the tissues.

Cases of erysipelas originating in very trivial wounds, and succeeding to surgical operations, have fallen under my notice.

\section{TETANUS.}

Tetanus is usually described as a powerful and painful spasm of the voluntary muscles, which is long-continued and uncontrollable. The spasm of the muscles is that of rigid contraction, and from its constancy and non-intermitting character, it has been termed tonic. It is frequently a result of injury, although it also occurs without obvious canse; hence it is called traumatic and idiopathic tetanus. Of all the domestic animals, the horse is most liable to tetanus. It is but rarely seen in the ox tribe, and when it does occur in the bovine animal, it is apparently of the idiopathic form. 
Traumatic tetanus may result from a very trivial injury, although it is most likely to do so after a severe laceration or puncture, more especially when nerves are injured. Wounds of the feet and joints, although giving rise to a high degree of irritative fever, seldom cause tetanus, and in my experience wounds in the region of the quarters, thighs, and fore arm, more especially if the great nerves of those parts are injured, are those most liable to cause iti.

The operations which are most commonly succeeded by tetanus are docking, castration, the insertion of setons, and in one instance which fell under my notice, a moderate blister to a fore leg proved a cause of tetauus.

Tetanus, whether traumatic or idiopathic, is rarely seen in certain districts. Mr. Cartwright of Whitchurch informs me that he has never seen a case of tetanus in his district, although he has practised there for forty-five years; and during the ten years I practised in Bradford I saw but two cases, both of which were idiopathic. In other districts of the country, tetanus, of both kinds, is exceedingly prevalent.

Tetanus is occasionally seen as an enzootic disease, simultaneously attacking several animals in the same district. During the summer of 1858 I witnessed ten cases in a fortnight. Some of these were traumatic, whilst the exciting cause of the others could not be traced. Some writers on veterinary surgery state that tetanus is more apt to prevail in cold than in hot weather. My experience is contrary to this, and that it is mostly during warm weather that the disease jrevails to any extent, although isolated cases of it occur at all times of the year.

There are several varieties of the disease, and the word tetanus is made use of to denote it generally. As a generic term, it comprehends all the varieties, but when not used in this sense it implies that the disease involves all classes of muscles equally. When the muscles of mastication are alone involved, it is called trismus. When it chiefly affects the superior cervical and dorsal muscles, causing the head to be elevated and the spine curved downwards, it is called opisthotonos. When the muscles of one side are affected, it is called tetanus lateralis, or pleurosthotonos; and in other casesrare even in the human being-the inferior muscles are chiefly' 
affected, the chin drawn towards the breast, the spine curved backwards, the disease is named emprosthotonos. In the lower animals, trismus, with opisthotonos, is generally met with. I have seen a modified form of tetanus lateralis, but the other form-namely, emprosthotonos-is, I think, unknown in either horse or ox.

Tetanus, whatever be the variety, may be acute, subacute, or even chronic. The acute is that which is most common, and most fatal; it lias a tendency to involve the whole frame, and to destroy life by arresting the respiratory movements. Amongst the variety of causes which may produce tetanus, in addition to the irritation of wounds, I have observed worms in the stomach and intestinal canal, collections of sand in the large intestines, and uterine irritation after abortion.

Traumatic tetanus follows injuries, whether inflicted surgically or otherwise, in an indefinite but limited period of time,--in some instances within an hour after the infliction of the injury, but usually the occurrence of the spasm is not observed until the wound is nearly or quite healed. Neglect in the treatment of, the presence of a foreign body in, or the application of irritating medicaments to, a wound, is apt to cause tetanus.

The Symptoms of Tetanus.-In the earliest stage there will be a stiffness of the muscles near the seat of the injury; if a limb is wounded, the animal will move it with difficulty; the stiffness spreads over the whole body; the animal will begin to champ his jaws, and grind his teeth. There is often a flow of saliva from the mouth, and a collection of froth upon the lips. The breathing now becomes accelerated, the nostrils dilated, the nose protruded, the membrana nictitans pushed more or less over the eyes, which are withdrawn within their sockets. If the animal be suddenly disturbed, the superficial muscles will be seen to twitch or tremble; the eyeballs convulsively withdrawn within the orbits, causing the patient to show the white of the eye at every convulsive retraction; the tail is suddenly elevated, and is maintained in that position by an irregular clonic spasmodic action of the levator muscles so long as the excitement continues.

At first the pulse is not much affected, and in all but the most severe attacks it continues undisturbed for two or three days; it has a hard, incompressible character, however, and as 
the disease advances it becomes accelerated, harder, and more incompressible. When the spasm becomes general, the position of the various parts of the body is regulated by the action of the more powerful muscles. The limbs are extended, flexion of them is performed with difficulty, and the patient stands with outstretched limbs. The course of the levator humeri can be easily traced, and the contraction of this, and other muscles which act upon the superior part of the cervical region, cause the neck to assume the appearance of what is termed "ewe neck." The peristaltic motion of the bowels is stopped; the urinary bladder firmly contracted; a dry, husky cough comes on when the animal attempts to swallow, and the act of deglutition is performed with a difficulty which increases from day to day. The muscles of the abdomen are rigid; the belly looks small and hard; the intercostals act imperfectly; and when the diaphragm becomes involved, the breathing is performed with very great difficulty.

Although the spasm of tetanus is of the tonic or persistent kind, there are exacerbations of a clonic intermittent character; and the whole course of the disease is marked by paroxysms of great severity if the animal be subjected to meddling attendance, strong light, or rustling noises. In a modified light, and when the animal is kept quiet, the spasms are usually diminished, and the exacerbations much milder.

But little is known about the general pathology of tetanus. Some writers are of opinion that it is due to an exalted polarity of the nervous centres, excited by the injury in the traumatic form, or resulting from a mal-condition of the blood, or the effects of cold acting upon the nerves of sensibility in the idiopathic form of the disease.

For many years, however, it was suspected by many English veterinarians that tetanus was both a contagious and infectious disease. The contagiousness of tetanus has now been almost conclusively proved, the disease having been transmitted by inoculation, particularly with material obtained from the spinal cord of tetanic subjects.

The contagiousness of the disease is due to a micro-organism - the Bacillus tetani-described by Nicolayer, 1884, and by Rosenbach, which gives rise to the formation of a ptomaine termed Tetanine. This organism is longer but narrower than 
that of anthrax, having one enlarged spore-bearing end. Nocard has demonstrated that dried blood and pus seraped from instruments employed in eastrating horses, all of which had died from tetanus, caused the disease when inoculated into rabbits. The Bacillus tetani is also found in garden mould, and it is possible to explain the non-existence or rarity of the disease in some districts, and its prevalence in others, upon the assumption that the organism common to the soil gains entrance to the animal economy.

Pearing these conclusions in mind, the practitioner will not fail to see the probable and beneficial effects of germicide treatment, both locally and generally. Weak sublimate dressings to the wound in traumatic treatment, carbolates, salicylates, and biborates internally.

In post-mortem examinations of traumatic tetanus I have invariably found the nerves leading from the injured parts to present some signs of inflammation; the neurilemma more vascular than natural, the vessels of the spinal cord engorged, and the sub-arachnoid space to contain some effusion.

Treatment of Tetanus.-Suppose the disease to be caused by docking or neurotomy, the first question to be considered is whether a portion of the stump of the tail or the end of the nerve should be removed or not. Some are of opinion that the nervous irritation is due to the nervous fibrillæ of the part being pressed upon by the contraction and cicatrisation of the reparative material of the wound, and that this explains the curious fact that tetanus generally occurs when a wound is nearly or completely healed. If this view be correct, then further amputation of the tail or excision of the nerve would afford some relief. Experience, however, does not warrant me in recommending this to be done. The wounds, or the seats of them, if healed, are in all cases to be fomented, and if practicable poulticed, the fomentations or poultices to be medicated with a solution of belladonna. Should tetanus occur soon after an injury is inflicted, the wound should be examined, and any lacerated or partially divided nerve, foreign body, or dead tissue removed.

There are some cases of tetanus so acute from their commencement that it is quite hopeless to expect any but a fatal termination; and in every case where all the symptoms are firmly established before the fourth day of attack, death may be expected. But in cases where the symptoms are slowly 
developed, some movement of the jaws still remaining, the exacerbations not very severe-more especially if the animal possess a calm quiet temper, and lives over the ninth day,--a recovery may be expected.

The wound having been properly attended to, the next thing to be done is to place the animal loosely in slings; this ought to be done early, and before the nervous excitability becomes too great. If the patient is comfortably slung, he will get used to the slings before the malady has attained its height. I recommend the slings because many horses which are in a fair way of recovery lie or fall down when the muscles begin to relax, and, when down, struggle and fight to such an extent that they seldom recover from the excitement and renewed severity of the disease thus brought on. The surroundings of the patient are of the utmost importance; the stable must be darkened; should contain no other horses; be situated in a quiet spot, removed from noises, and the door must have a lock, a key of which is to be kept by one individual (the veterinary surgeon, if possible), who is to visit the patient at most twice a day, and great care must be taken that the animal is not tormented by flies.

As a rule, the desire for food continues for several days; the thirst is considerable, and large quantities of mutritious fluids will be drunk with avidity.

The most important particulars in the successful treatment of tetanus are quietude and nourishment, medicinal agents playing but a subordinate part.

Quietude having been secured, nourishment is to be given by allowing the patient milk and thick gruel to drink; along with these, eggs may be mixed with advantage. A little hay or grass placed in the rack will often keep the animal quiet, although the attempts to swallow sometimes cause a paroxysm.

The medicinal agents that have been used in the treatment of tetanus are numerous; purgatives, opium, tobacco, Calabar bean, woorara, prussic acid, calomel, chloroform, belladonna, hyoscyamus, cannabis indicus, arsenic, chloral-hydrate, \&c. \&c. I have treated tetanus in various ways, and am satisfied that administration of a does of aloes, if it can be given without exciting the horse, followed by belladonna-which is only to be given when the patient shows symptoms of great excitement-is the best method of treatment. The Calabar bean, given in doses of two to four 
onnces of the tincture, has a most wonderful effect upon the spasms, the pulse, and the breathing; but this effect is very transient, and is succeeded by a return of the spasms with great severity. The seat of the wound is from time to time to be smeared with the extract of belladonna; and when the belladonna is administered internally, it should be either dissolved in the animal's mash or drink, or else placed between his teeth, allowance being made for the probable waste.

The prussic acid treatment, so highly recommended by the late Mr. Lawson of Manchester, has with me proved to have no special superiority; and doubtless the success of Mr. Lawson in the treatment of tetanus was due more to the tact and skill of the man than to any virtue contained in the remedy.

Those cases of tetanus which terminate favourably take usually about six weeks before the spasmodic contractions entirely subside. As soon as they can eat good food, they are to have it liberally. Corn, roots, and hay in the winter; corn and grass in the summer, and a few doses of tonic medicine, such as the sulphate of iron, will materially assist convalescence.

In the fatal cases of tetanus, the breath very frequently becomes fotid prior to death, and if the mouth be examined, a quantity of slate-coloured epithelium will be found on the inner surfaces of the lips, gums, and tongue.

The treatment of idiopathic tetanus requires no special notice. It is to be conducted upon the principles already laid down, the only difference being that no local applications are necessary. It is generally supposed that the idiopathic is more amenable to treatment than the traumatic; but, so far as I can judge from my own experience, it is even the more fatal form; and I find that I am supported in this conclusion by the veterinary surgeons of the neighbourhood of Edinburgh. 


\section{CHAPTER XXVIII.}

\section{DISEASES AND INJURIES OF THE FACIAL REGION.}

WOUNDS OF THE LIPS-TUMOURS-BRUISES OF THE MOUTH-AFFECTIONS OF THE BUCCAL MEMBRANE-SPORADIC APHTHE-PARALYSIS OF THE LIPS-OPEN PAROTID DUCT-SALIVARY CALCULIPTYALISM-RANULA-EPULIS-GLOSSITIS-ULCER AND INDURATION OF THE TONGUE IN HORNED CATTLE-PARALYSIS OF THE TONGUE.

\section{DISEASES AND INJURIES OF THE MOUTH, TONGUE, ESOPHAGUS, ETC.}

Wounds of the lips call for no special notice, further than that they are to be treated upon the conservative method; that is to say, an endeavour must always be made to bring about the union of the divided parts. It is a very common practice, when a lip is partly cut, with the divided portions hanging loosely, to remove them with a sharp pair of scissors or knife, instead of bringing them together with sutures, and retaining them in apposition till they are united. I want to impress upon the young practitioner the importance of never removing any portion of injured lips until he sees that their reunion is an impossibility; and rather than cut them off, he should allow them to be separated by the process of sloughing; for a lip mutilated by meddling surgery remains a permanent blemish, leaving the teeth exposed and the powers of prehension greatly interfered with.

TUMOUR OF THE LIP.

A tumour sometimes forms on the lips; at first firm and solid to the touch, of a variable size, occurring either spontaneously or as the result of a sting, and which in a day or two suppurates and bursts. It requires little treatment except fomentations. 


\section{BRUISES OF TIIE MOUTII.}

Various parts of the mouth, tongue, and the rami of the jaw are bruised and injured by the teeth, and by severe bits. These have already been alluded to under Diseases of TIIE Bones, and I need say but little here; but I would recommend that all "pulling horses" should be ridden or driven in easy bits-the ring-snaffle bit in preference to all others. I have often been called to horses whose mouths have been hot, swollen, and the mucous membrane torn by the bit, the lips bleeding, or the whole of the parts which are situated beneath the bit black from extravasated blood; and I have heard the rider exclaim, "The brute nearly pulled my arms off."

Many horses are ruined, as well as tortured, by severe bits and heavy hands; and it may be often seen that a horse is so tender in the mouth that he will scarcely face the bit, until he is urged, perhaps by a whip he cannot endure, and the spurs which he abominates. However, forward he must go; his pride is insulted, his dignity tonched, his courage raised, and off he goes, harder than was bargained for, pulling his rider or driver's arms off-and serve him right; but at the expense of his own delicate mouth, from which he will suffer for many a day. I have a horse at the present time, a highly-bred one, with courage, spirit, action, docility, and a most beautiful mouth, provided he is driven in a snaffle-bit; but if a curb-bit is put on, he will scarcely face it for the first few miles; but when warmed to his work, no man could hold him. He pulls and pulls, perhaps suddenly stops, shakes his head, or leans to one side of the road. Indeed, he was so dreadfully bad in his behaviour when I first bought him, that I thought he was useless; but when a proper bit was tried, I had no further trouble with him. I am satisfied that many hundreds of horses are the same as my own.

When a horse is injured by the bit or curb, time should be allowed for the mouth and jaw to regain their natural condition before the animal is bitted, and then the bit should be of the lightest and easiest description.

\section{AFFECTIONS OF THE BUCCAL MEMBRANE.}

Lampas, barbs, paps, \&c., are terms applied by the ignorant tu fancied diseases; the first to the prominent palatine bars 
of the young horse, the latter to the swollen papillæe of the tongue and cheeks. The lampas is supposed to interfere with the masticatory powers of the animal, and the veterinary surgeon is frequently requested to burn or otherwise remove it, and if he declines, some barbarous fellow very soon undertakes the task. If the veterinarian can convince the owner or driver of the horse that an operation is unnecessary, that the bars of the young horse are always prominent, that they are reddened during the process of dentition, and that those of the old may be swollen from some internal ailment, as indigestion, which a little medicine will remove, well and good; but if it be an impossibility by force of argument to impress this conviction upon the client, it is better for the veterinary surgeon to operate, although it is against his conviction, than allow the horse to be tortured by some ignorant and barbarous pretender.

Barbs and paps are swollen papillæ, or perhaps the orifices of labial, sublingual, or submaxillary glands, mistaken for growths, and cut off. I should scarcely have mentioned this in a book of this kind, except that one continually meets with men who believe in anything that is sufficiently absurd. The papillæ, or even the orifices of the gland ducts, may be swollen during dentition, catarrhal affections, and indigestion; but they are never to be mutilated by being cut off. Remove the source of irritation, and they will soon disappear. If the mouth seem painful, and if there is a dribbling of saliva, a mild astringent wash of borax or alum will tend to remove the pain, and be a source of comfort to the patient. If the stomach or bowels are at fault, gentle aperients, antacids, and stomachics will have to be administered, the quality of the food being at the same time looked into.

\section{SPORADIC APHTHE, OR THRUSH.}

A crop of small vesicıes, or even pustules, occasionally appears in the mouth of horses, particularly during the process of dentition, the eruption being called aphthæ, or thrush. In cattle, sheep, and pigs these eruptions are not at all uncommon, the buccal membrane peeling off in patches, leaving the tongue, gums, and mouth raw and painful, and rendering the animal unable to take food without great difficulty. 
Treatment.-Removal of cause, cool astringent washes to the mouth, and if ulcers form which have no disposition to heal, the nitrate of silver is to be applied.

\section{PARALYSIS OF THE LIPS,}

Generally met with in horses which are compelled to wear heavy bridles, as those used for hearses, mourning coaches, \&c., or in any class of harness-horse whose harness-bridle fits him badly. It is called in Yorkshire dropped-lip; and the term is very expressive, for the lips are pendulous, the lower one semieverted, and the horse's face seemingly lengthened by this pendulosity. They hang elongated, flaccid, and powerless; the saliva flows from the mouth, in consequence of the animal being unable to approximate his lips. When attempting to drink he pushes his head into the water up to his eyes; and whilst feeding he is compelled to gather his food with his teeth only. He therefore feeds from the bottom of the manger, pushing his nose deeply into his corn. He champs whilst eating, often drops his mouthful, and generally quids his hay.

This affection is due to an injury to the portio dura, or seventh pair of nerves. If both the nerves are injured, both sides of the lips will be paralysed, causing the pendulosity already mentioned; but if the injury is linited to one nerve, as is more usually the case, the lips will he drawn from the affected side, and the horse's mouth will appear crooked.

The seventh pair of nervis convey the motor power to a variety of muscles, but more particularly, as bearing upon the subject now under consideration, to those of the lips, nose, and lower part of the face. These nerves pass out of the cranium by the stylo-mastoid foramen of the petrous temporal bone; at first deeply buried under the parotid gland, they afterwards pass between the glands and guttural pouches, to gain the posterior border of the lower jaw, round the neck of which they turn, and, mounting to the external surface of the masseter muscle, run downwards on the cheek quite subcutaneously. In well-bred fine-coated horses they can be seen very plainly on the sides of the cheeks, and being thus superficially situated, are liable to be injured by the pressure of a heavy ill-fitting bridle. The injury causes inflammation of the nerve (neuritis) 
and its neurilemma, with swelling and exudation. The exudate pressing upon the substance of the nerve, adds to the already existing loss of function. If the cheeks are carefully manipulated, the swollen nerves can be distinctly felt.

Some writers attribute this paralysis to indigestion or other obscure causes. But I cannot conceive how it can arise except from that already stated-and this is the only cause I have yet met with in my practice-or from disease of the petrous temporal bone, through which the portio dura passes, and disease of the brain.

Treatment.-Removal of all pressure from the head and face. If the animal is tied in the stall by a head-collar, this must be removed and replaced by the neck-strap; or what is better, it should be turned loose into a box. The pressure of the head collar may seem trivial, but it is sufficient to retard the progress of recovery for an indefinite period. The food should be soft, and placed in a convenient position; a deepish manger is the best, as the horse is apt to toss it about and waste much, owing to the absence of prehension. A purgative is useful, assisting to remove the inflammation of the nerves. Fomentations and rubefacients are to be applied to the masseter region; and if these prove ineffective, the absorption of the exudate must be excited by repeated applications of the biniodide of mercury ointment. It is quite unnecessary to administer nervine tonics, as nux vomica, for the paralysis depends upon inflammation of the nerve, and its continuance upon the pressure of the exudate, the removal of which, if organic change in the nerve tissue has not been induced, will restore the power to the paralysed muscles. The biniodide may be assisted in its action by the internal administration of iodine or its salts. Commonly, this treatment will suffice, but should the loss of power still continue, setons over the cheeks, or the actual cautery, are to be tried. I have seen a great number of cases, but only one which was incurable.

AFFECTIONS OF THE DUCTS AND GLANDS OF THE MOUTH.

OPEN PAROTID DUCT.

Steno's duct winds round the inferior maxillary bone, in com- 
pany with the submaxillary artery and vein, and enters the mouth between the second and third upper molar teeth. Its course across the jaw is superficial, and hence it is liable to be opened by direct violence, as kicks or heavy blows, or by ulceration of its coats when involved in the abscess of strangles. From whatever cause it is opened, saliva is discharged from the wound, instead of flowing into the mouth. When the animal is not feeding the discharge is very slight indeed; but during mastication, more especially if the food be dry, the flow is most abundant, as the parotid gland always secretes in direct ratio to the dryness of the food.

Treatment.-Various means have been resorted to for the purpose of re-establishing the communication between the gland and mouth. If the injury is not recent, the mere closing of the external wound is insufficient, for the reason that the duct between the wound and the mouth is no longer pervious, being obliterated by the inflammatory swelling. The first step in the treatment of open parotid duct is to make an opening between the wound and mouth, along the course of the original canal, if possible; if not, an artificial channel will have to be formed, by introducing a seton, from the ulcer into the mouth, which should remain for four or five days, or until it has made a suppurating channel. It is then to be withdrawn, the external wound brought together by suture and collodion, or styptic-colloid thickly applied. When the adhesive dressing is quite firm, a little food is to be given, in order to excite the secretion of saliva, for if the gland is quiescent for any length of time, the artificial duct is apt to close by adhesion of its sides. Great care must be taken that the dressings are in no way disturbed for several days after they are applied; and in order to support the animal without exciting the secretion of any great quantity of saliva, such food as eggs, milk, and thick gruels must be allowed; for if dry food be given the secretion will be calculated to loosen and disturb the adhesive applications, to force the lips of the wound asunder, and to reduce the fistula to its original condition.

If the treatment by seton is unsuccessful, the gland should be destroyed by injecting into its substance, through the opening already existing in the duct, the following solution:-Nitrate of silver, half a drachm; nitric acid, one drachm ; water, one ounce. 
A powerful syringe will be necessary to force the injection into all the ramifications of the gland. It arrests the discharge, by causing such an amount of inflammation in the substance of the gland as to destroy its secerning functions; the tubules and ducts become filled with adhesive lymph, causing the adhesion of their sides, the destruction of gland cells, and such an alteration in its whole.structure, that it finally becomes converted into a solid indurated mass or tumour, which is gradually removed by absorption.

It must be distinctly understood that neither the insertion of a seton nor the destruction of the gland are to be attempted in recent cases; nor should caustics or the actual cautery be at any time applied. In a recently opened duct the wound should be treated by suture and collodion, and the animal forced to abstain from all solid food for at least three days after the accident. He must have gruel, milk, \&c., to drink, and be muzzled, to prevent him eating his bedding. Nauseating with small doses of aloes is very useful, destroying the desire for food, and assisting materially in the compulsory quietude of the jaws. When the saliva is partly discharged into the mouth, and partly through a small ulcer out of the duct, a smart blister will generally bring about the closure of the opening. The removal of the gland by excision, as recommended by the continental veterinarians, is never required.

\section{SALIVARY CALCULI.}

These concretions form chiefly in the parotid, sublingual, and submaxillary ducts. They are caused by an accidental nucleus, such as a small piece of hay or corn penetrating the canal-to which the salts of the saliva adhere, forming roundish or mulberry concretions blocking up the duct, which becomes enlarged and distended with saliva. The treatment is-removal, by manipulation into the mouth, if possible, or through an opening made by the knife, and treating the wound so made upon the plan recommended for recently opened duct.

Sometimes an oat insinuates itself into the orifice of the parotid duct, producing distension of it by saliva, causing it to appear as a pendulous sac on the borders of the jaw. The foreign body must be removed through the mouth. 
According to Percivall, salivary calculi are mainly composed of the carbonates and phosphates of lime. I have only one specimen in my possession, presented by Mr. Gloag, and it is composed of organic matters, namely, albumen, mucus, and cholesterine with a mere trace of salts.

\section{EXCESSIVE SECRETION OF SALIVA-PTYALISM.}

This results from disease of the teeth; some peculiar foods, as green food charged with mustard; mercurialism; any source of irritation in the mouth ; from the poison of epizootic aphthæ; and rinderpest.

Mercurial ptyalism is easily induced in horned cattle by small doses of calomel, and by mercurial dressings to the skin. In the horse, larger doses are required; although in one case which came under my notice two drachms of calomel were sufficient to cause alarming salivation and other signs of mercurial poisoning.

The treatment in all cases is the removal of the cause, repeated washings of the mouth with cold water, and mild astringents, such as vinegar, borate of soda, or alum.

\section{RANULA}

Consists in the formation of a cyst in one of the ducts of the sublingual gland, varying in size from a walnut to a hen's egg, containing a ropy fluid. (See chapter on Tusours.)

\section{EPULIS.}

(See Photo-lithograph, Plate IV., Fig. 1.)

A tumour of the gum, often of a simple growth of the same consistence as the structure from which it grows, and not likely to be reproduced when the exciting cause has been removed, and the disease extirpated. Its frequent cause is caries of some parts of the molar teeth. The lower jaw is its most common seat; it commences at the root of the molars, or in the alveolar ridges. The size and extent of epulis are various; it may be confined to the gum between two teeth, or it may involve several, if neglected, and partake of a malignant character, as the specimen portrayed in Photo-lithograph, Plate IV., Fig. 2. 
The smaller tumour (Fig. 1) is in the mouth of the sheep; the larger (Fig. 2), in the cow ; the first is benign, and the latter malignant.

The two figures in the photo-lithograph show the difference between the benign and malignant forms very distinctly; the first was smooth on the surface, of slow growth, and seemed to be unattended with pain, but interfered with the process of mastication; the other ragged, of rapid growth, frequently bleeding, and seemingly giving the animal much pain, and was accompanied by infiltration into the surrounding bones and hard palate of the characteristic cancer matter.

The benign tumour may be removed with advantage; the operation being modified according to its size and situation. One tooth or more must always be extracted, in order that the proceeding be effectual. After these have been removed, the tumour must be surrounded by an incision made with a strong knife, and if need be, part of the alveolar process is to be taken away by the bone forceps. If the tumour is far back in the mouth, it will be advantageous to divide the cheek, in order to expose the parts fully to view, and to obtain ready access to them. The wound so made will heal very well if properly secured. I have met with epulis in cattle and sheep, but never in the horse. Generally, those in the upper jaw are of rapid and unlimited growth, those in the lower of slow growth and benign nature; but if not completely removed, they are apt to grow again.

AFFECTIONS OF THE TONGUE.

GLOSSITIS.

Glossitis, or inflammation of the tongue, when occurring as a primary disorder, is consequent on injuries, scalds, or chemical irritants. The tongue is injured and inflamed by being included in the halter when the "cavil is put in the mouth ;" I have seen it cut in two by such carelessness. The proper way to put the halter in the mouth is to let the tongue go free, but a careless man will include the tongue in the loop, and thus inflict a violent injury. The tongue may be bitten severely by the animal himself, or wounded by sharp and irregular teeth. Again 
it has been cut and severely wounded by a cruel attendant out of spite; and scalded by hot drink, or irritated by such remedies as ammonia or turpentine given insufficiently diluted. The irritation from ammonia, caustic potash, or soda, may be easily recognised, the buccal membrane being dissolved by the irritant. The tongue hangs out of the mouth inflamed, swollen, having a soft saponaceous feel; its covering peeling off as a serni-transparent bluish-white material; the tongue substance very red, painful, and injected; the cheeks in the same condition. Treat with cold astringent lotions, vinegar and water answering two purposes-(1), neutralising any remaining alkali, and (2) forming a grateful application to the inflamed part.

Wounds of the tongue have to be treated on conservative principles. Nothing must be destroyed, cut off, or removed; but all torn edges brought into apposition, and held so by proper sutures. A horse with half a tongue will feed moderately well, and keep his condition, but he always requires extra care and attention, and he is less valuable in the market. It therefore behoves the veterinary surgeon to save as much as he can of this organ when at all mutilated; but if a portion be really gangrenous, it must be excised.

\section{ULCERS OF THE TONGOE,}

Are often caused by diseased and irregular teeth; dirty, foul, rusty bits; some kinds of food; or they may appear as a secondary affection, depending upon indigestion. The causes must be removed in all cases, and the ulcers treated with the nitrate of silver, alum, or borax; chlorate of potash makes a nice wash to the parts; it may also be given internally, when the ulcers depend upon internal causes.

Horned cattle are subject to induration of the tip of the tongue. It arises from no ostensible cause, and is generally incurable; and as it interferes with the powers of prehension, the animal rapidly loses flesh, and should be slaughtered at an early stage of the disease.

PARALYSIS OF THE TONGUE.

A paralysed coudition is generally present when the brain is 
extensively diseased, or suffering from the pressure of tumours, serum, pus, or extravasated blood. It may also be induced by forcible traction being applied to the tongue by the attendant when giving balls or other medicines; this not only produces paralysis, but otherwise seriously injures the tongue. The symptoms of paralysis of the tongue are-the tongue hanging out of the mouth, the animal unable to draw it in, and a dribbling of saliva from the mouth.

It is good practice in all cases of disease or injury of the tongue, when it protrudes from the mouth, to force it into its proper position; retaining it there by a bandage placed round it and the lower jaw. When the tongue hangs out of the mouth for any length of time, it is dried by the evaporation of its moisture, cracks, and ulcerates upon its surface, and becomes exceedingly painful, swollen, and inflamed.

Abscesses sometimes appear at the root of the tongue and isthmus faucis, interfering with deglutition, and giving rise to cough. The mouth is to be examined, and the abscesses onened by puncture or by laceration with the finger. 


\section{CHAPTER XXIX.}

\section{DISEASES AND INJURIES OF THE FACIAL REGION-continued.}

PARROT-MOUTH-IRREGULARITIES OF THE TEETH-DISEASES OF THE ALVEOLAR PROCESSES - CARIES OF THE TEETH - DISEASES OF DENTITION-DENTAL IRRITATION IN YOUNG CATTLE-DENTAL COUGH IN YOUNG HORSES-DENTITION FEVER-SUPERNUMERARY TEETH-DISEASES OF THE FACIAL SINUSES-OZGENA, OR NASAL GLEET.

\section{DEFORMITIES OF THE MOUTH AND IRREGULARITIES OF THE TEETH.}

Parrot-Mouth.-This is a well-known deformity, and consists in the upper incisor teeth projecting in front and overhanging the lower ones, to an extent, in some instances, of as much as two inches; in such cases the lower incisors meet the palatine ridges or "bars" when the mouth is closed.

The teeth of both jaws become much elongated, not being worn off by the attrition which keeps them at a proper length when they meet naturally.

A horse with a parrot-mouth will feed from the manger very well, but if turned to grass he experiences a difficulty in collecting his food. On this account a horse with a parrot-mouth is not so valuable as he otherwise would be.

If the lower incisors become so long as to bruise and otherwise injure the "bars" and roof of the mouth, they will require to be shortened with a rasp; the upper teeth may at the same time be shortened also.

The upper jaw is naturally broader from side to side than the lower. If this natural formation is carried beyond a certain extent, the molar teetn do not approximate during mastication, and consequently a ridge of unworn tooth material is left on the external aspect of the upper, and on the inner sides of the 
lower teeth, wounding the tongue and cheeks, and causing what is vulgarly termed "quidding."

This malformation may be due to the rami of the lower jaw being unusually close together, or to what more rarely occurs, the increased breadth of the upper maxilla; in either case, the result is the same, namely, the prevention of the natural grinding motion of the jaws by the overlapping of the unworn surfaces.

The treatment in such cases can only be palliative, and consists in the periodical rasping of the edges of the teeth, and feeding the animal on soft food.

The teeth, both incisors and molars, are sometimes irregularly placed in the mouth, the irregularities of the incisors, both as to position and number, being more common than those of the molars; and they seldom cause any disease or inconvenience, unless when, projecting beyond a moderate length, they bruise or otherwise injure the opposing jaw. They must either be removed or rasped down to a moderate length. Their removal is a very difficult matter, indeed scarcely possible without fracture of the jaw ; but they may be reduced by the rasp very easily.

The irregularities of the molars are of much greater importance, and, independently of those already described as depending upon the want of uniformity in the width of the jaws, the two opposing rows may be of unequal length. Thus the upper row may be longer than the opposing lower row ; in this case, either the first or last molar in the upper row, as the case may beand it is sometimes the one, sometimes the other-not being worn down by attrition, becomes elongated to such an extent as to cause extensive disease in the lower jaw. Again, the rows in the lower jaw may be longer than those of the upper; in this case, the unopposed teeth will grow to such an extent as to cause disease of the superior maxillary bone, and even to perforate into the superior maxillary sinus. In such cases it is necessary that the sharp projection be removed. When situated anteriorly, this is not difficult to accomplish, a good strong chisel and haminer being all that are required, in addition to the balling-iron to open the horse's mouth. It may not be necessary to cast him, if he is a very quiet beast, but all operations on the teeth and mouth are much more easily performed when the horse is cast. After the projection has been chiselled off, all remaining 
asperities and irregularities are to be smoothed down with a fine file.

When the projecting piece of tooth is situated at the back of the mouth, it is to be cut to a level with the other teeth with a concave-edged chisel, and separate guard, which may be made in a few minutes by any smith, as it consists only of a piece of iron bent at its extremity so as to grasp the back of the tooth, and of sufficient length in the handle to protrude from the mouth. The bent end is to be inserted by the operator behind the tooth; the handle is to be held by an assistant, who is to pull towards himself, exercising firm but not inordinate pressure; this will prevent the tooth from splitting when the cutting chisel is struck with the hammer. In all the other teeth but the last, the operation can be performed with "Gowing's sliding chisel," which would be the best instrument for the last tooth also, if it could be applied.

\section{DISEASES OF THE ALVEOLAR PROCESSES.}

These are extensively diseased, in some cases with enlargement of the gums, which are soft, spongy, and bleed easily. The teeth may or may not be diseased.

The causes are various, and not easily demonstrated. Mr. Varnell, whose observations on diseases of the teeth and cranial and facial regions are entitled to great respect, says:- "The causes which give rise to this condition of the maxillary bones are not easy to define. That a horse so affected is from certain peculiarities predisposed to it, there can be no doubt. For example, the teeth being placed at a distance from each other, allowing thereby the food to accumulate between them, must be looked upon as a predisposing cause. A strumous diathesis, which I believe to be more common in the horse than is usually supposed, must also, when it exists, be regarded as a predisposing cause of this affection. The particles of food which become impacted in these unusually wide interdental spaces after a time decompose, and give rise to fotid compounds, which act preju dicially upon the parts they are in contact with. The membrane which covers the gums, and also that which lines the alveoli, and is reflected on to the fangs of the teeth, become inflamed. The inflammation will extend to the bone, the blood-vessels of 
which will become enlarged, as will also the Haversian canals in which they ramify. The osseous laminæ surrounding these canals will be partially absorbed, and to some extent separated from each other, and the enlarged spaces thus produced will be filled with inflammatory exudation. Hence the soft, spongy state of the gums, their tendency to bleed from slight causes, and as their periodontal membrane is separated from the teeth; hence also their looseness in the alveoli."

Symptoms.-Imperfect mastication of food, "quidding," with loss of condition; the fæces containing much ill-digested aliment. Examination of the mouth will show to the practitioner that there are spaces between the teeth filled with hay, corn, \&c., which, from long detention, may have become foetid.

Treatment.-The treatment recommended by Professor Varnell in those cases where it is considered advisable not to dostroy the animal, is the removal of the impacted matters from between the teeth with water and a brush constructed for the purpose; the parts to be afterwards washed out with a solution of chloride of lime.

Very old horses are subject to this disease of the gums and alveolar process, as well as to looseness of the teeth in their alveoli from natural decay. In such cases no good can be done by any operation or treatment.

In addition to the treatment of Professor Varnell, the filling of the interdental spaces with gutta-percha might prove of great service.

\section{CARIES OF THE TEETH.}

Caries, dental gangrene, or decay, is almost exclusively confined to the molar teeth, although I have seen the incisors in that condition.

Caries of the molars may commence primarily in the fang, neck, or crown of the tooth.

Caries of the fang arises from inflammation of the pulp, and may be caused by a constitutional predisposition or external injury. Inflammation of the pulp does not always cause caries. I have several cases on record where the fangs were enlarged from periodontal deposit, with alscesses surrounding the fangs, without caries. Caries commencing at the fang may be due to obliteration of the pulp cavity, at an age when the vitality of the tooth depends upon the integrity of the pulp. 
I need scarcely remind the professional reader that the integrity of the teeth depends upon a due supply, both as to quantity and quality, of nutritive materials. On the fangs of the recently cut tooth but little crusta-petrosa is met with compared with that which exists in old teeth. As age advances the crusta increases, and the tooth grows from the outside.

In man, it is generally agreed that after a given time the dentine ceases to be produced, and the pulp becomes converted into osteodentine. In the horse, the pulp cavity becomes obliterated gradually by the pulp continuing to form dentine, the pulp simply giving way to its own product, which ultimately occupies its place and fills up its cavity.

In proportion as the pulp diminishes, so is the supply of nutriment to the tooth lessened, until at length it is entirely cut off from the interior; and to provide for the vitality of the tooth under these circumstances the crusta-petrosa increases in quantity on the fang, and at the expense of the perfectly formed dentine lying in immediate contact with its inner surface. That is to say, this layer of dentine is converted into crusta-petrosa by the dentinal lacunæ undergoing dilatation, and becoming identical. with the hollow spaces or cells of the crusta. The tooth now draws its nourishment from the blood-vessels of the socket, and thus it continues long after the obliteration of its pulp cavity to serve all purposes as a part of the living organism. This is the natural condition of old teeth; but when the pulp cavity is obliterated at an early age, by a too rapid formation of dentine, and consequent obliteration of the pulp when the crusta is not yet sufficiently developed to supply nourishment to the whole tooth, caries must be the result.

Many cases of caries of the teeth that have come under my observation have resulted from the above cause, and very often the disease is confined to that part of the crusta-petrosa that dips with the enamel into the interior of the tooth, splitting up the tooth into several perpendicular fragments, from crown to fang.

Caries of the neck (cervix) or the tooth is seen in those horses whose teeth are wide apart, and is caused by the food remaining in the interspaces, and by decomposition exciting inflammation in the periodontal membrane.

Caries of the neck is very commonly met with in dogs, sometimes causing abscesses in the cheeks. 
Caries commencing at the crown or table is due to a portion of the dentine losing its vitality, and the power of resisting the chemical action of the fluids of the mouth; a portion of the enamel of the crown may be fractured by the animal accidentally biting a piece of hard stone or metal contained in his food. Mere fracture of the enamel is insufficient of itself to lead to caries of the teeth of the lower animals, for it is a substance that is gradually worn off by the attrition of the teeth continually going on during mastication; but the pressure which has been sufficiently great to cause fracture of the enamel, may at the same time have caused such an amount of injury to the subjacent dentine that it dies, and progressively becomes decomposed.

In man it seems there should be death of the dentine and acidity of the oral fluids before caries can take place, test-paper applied to a carious tooth invariably showing the presence of free acid; and a very small perforation in the enamel may coexist with a considerable amount of disease in the dentine.

Mr. Tomes thus describes the process of destruction in the teeth, as well as that conservative action which, as we have already seen, takes place in caries of the bones.

He says - "When a portion of dentine has become dead, it is circumscribed by the consolidation of the adjacent living tissue. The tubes become filled up, they are rendered solid, and the circulation is cut off from the dead mass by the consolidation of the tubes. This consolidation does not go on gradually from without inwards, keeping in advance of the decay, but occurs at intervals. It would seem successive portions of dentine lose their vitality, and that the contiguous living tissue becomes consolidated." 1

We thus see that nature retards the progress of the disease by throwing barrier after barrier of defence against its inroads.

The act of consolidation is a vital one; but, according to Mr. Tomes, it is probable that the dentine thus consolidated loses its vitality. "This may be inferred from the place that it occupies, its external protective position, its decreased capabilities for capillary circulation, and its increased density."

Another interesting exhibition of vital action is the production

1 Dental Physiology and Surgery, by JoHs Tomes, Surgeon-Dentist to the Middlesex Hospital. 
of secondary dentine, by the surface of the pulp under the excitement of caries in the contiguous tissue.

Symptoms of caries of the teeth are those of pain. The horse so suffering will, whilst eating, suddenly stop masticating his food, perhaps drop it from his mouth, will rest his cheek on the manger, holding his head to one side, and after a time, when the pain subsides, will begin to feed again; or he may "quid" his food, throwing out large boluses mixed with much saliva. I have seen instances where the manger would be half filled in the morning with semi-masticated food and sour salivary secretions.

Caries of the fang will usually be denoted by an enlargement on the bone which contains it, corresponding to its position; this enlargement of the bone may be due to the formation of an alveolar abscess, or to increased deposition of crusta-petrosa (see Tumours). Thus in the lower jaw the submaxillary bone will present a prominence external to the offending tooth. This bulging portion of the bone, when caused by an alveolar abscess, as well as the soft structures external to it, will ulcerate, thus giving exit to the pus.

Caries of the superior molars, when commencing at the fang, is usually associated with a discharge of fœid purulent matter from the nostril of the side affected; this symptom may be present before those portions of the tooth outside the gums present any signs of decay. When caries of the fang attacks a first or second superior molar, the superior maxillary bone will present an enlargement similar to that already noticed in the inferior maxilla, with sometimes an ulcer of communication.

The fang may be absorbed without suppuration; if such be the case, the tooth will sink below the level of its fellows; but there will be no external enlargement, no alveolar abscess, nor ulceration of the bone.

Mr. Varnell says that the irritation of the fangs may give rise to fibro-osseous tumour, which may either project into the antrum, and partially or wholly fill that cavity, or into the mouth, interfering thereby with the process of mastication.

Again, caries of the fangs may extend outwards to the maxillary bones, causing immense gaps of ulceration in them, as shown in Photo-lithograph, Plate I., Fig. 7 ; or the ulcera- 
tion may extend into the mouth, the pus discharging itself there, leaving an unhealthy sinus.

The special symptoms of caries of the teeth may be summed up, according to M. Bouley, in the following order:-

1st. Remarkable fotor, which is peculiar to the disease, and pervading the mouth and the secretions within it.

$2 d$. Flow of saliva from the mouth.

3d. Appearance of a black spot on the carious tooth, or of a cavity varying in extent, according to the duration of the disease.

4th. Sharp pain, indicated when a tooth is struck by any instrument.

5th. Swelling of the gums, redness and pain around the diseased tooth.

$6 t h$. The accumulation of food about the diseased tooth, and which, undergoing putrefaction, produces the inost repulsive smell.

$7 \mathrm{th}$. In some cases the only symptom to be observed is that the gum has receded from the diseased tooth slightly.

By a careful examination of the mouth, the diseased tooth can be detected by the fœtor, and by an accumulation of decomposing food around it, and by an alteration in its general appearance. The "speculum oris," of which a woodcut is here

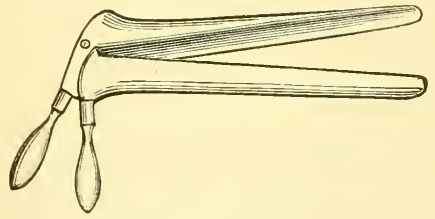

Fig. 139.

inserted, is a very useful instrument for examining the mouth of the horse.

The treatment for diseased tooth is its removal, and this is best effected by trephining the alveolus and punching the tooth out.

All the molars, except the last, may be thus removed. There may be some difficulty with the fifth, but the last is so far separated from the outer wall of the maxillary sinus, with its fang extending obliquely upwards and backwards towards the 
inner plate of the orbit, that punching is rendered an impossibility. I find, however, that the fourth molar is more liable to caries than any of its fellows. This tooth is shorter and thicker in its fang than the others. Its fang is also more deeply imbedded in the sinus than the third and fifth; more towards the cavity of the nose; and were it as long as they, it would protrude into the nasal chamber. It has therefore a short thick fang, and its pulp cavities are sooner obliterated by a deposition of dentine. In some five-year-old mouths, I find its pulp cavities quite obliterated.

The first, second, and even third molars may be withdrawn with the forceps, but they are much more easily removed by punching. The fourth, owing to the thickness of its fang, and the difficulty experienced in fixing the forceps to a worn-down tooth, is only removeable by punching from above.

I have operated in this manner both upon the upper and lower molars. The operation is very simple. Cast the animal, and trephine, so as to make the opening to correspond to the fang of the diseased tooth, allowing sufficient space above or below the root of the fang-above, if the operation be in the upper, and bclow, if in the lower jaw-for the introduction of the punch. The punch should be at least an inch in circumference at its point, that it may not cut or split the tooth.

Two or three smart, but not heavy, blows with the hammer are sufficient to dislocate the tooth ; having the balling iron in the mouth, and when this is effected, it may be removed with the smaller molar forceps, or even by the hand.

The after-treatment is very simple, and consists in frequently washing the cavity with water containing a little carbolic acid or chloride of lime. The elastic syphon is a very useful instrument for this purpose, the nozzle being introduced into the wound, and the water forced into the cavity. In this manner foreign matters, impacted food, \&c. are forced out of the sinus.

It might be supposed that an opening thus made would remain as a sinus of communication, but in all my cases, where the bones have been healthy, the alveolar cavity has become obliterated; in the course of about five weeks its sides have approached each other, and the gum over them has become quite hard. 
Some practitioners speak about stuffing the cavity with guttapercha. It is rarely necessary; indeed, it is calculated to do harm, by preventing the healing process, and producing caries of the bones.

If caries attacks a tooth early in the horse's life, the opposing tooth demands surgical attention. The carious tooth will sink below the level of the others in the row, and the opposing tooth, not being worn down by the attrition of mastication, will become elongated; it will grow to its full length, and project into the hollow of the carious tooth, and eventually become sufficiently long to wear through the gum and into the jaw itself. The same applies when the carious tooth has been removed either by disease or operation; and a horse which has been subject to the attention of the animal dentist for the removal of his teeth requires periodical examination, the unopposed tooth requiring to be cut to a level with its fellows in the same jaw, and occasionally filed upon its surface, or it will continue to increase in length, and be a source of serious injury and annoyance.

Quidding of the food may exist unassociated with disease of the teeth. I am of opinion that such cases are due to partial paralysis of the pharyngeal muscles.

Instruments for the treatment of the irregularities of the teeth can be purchased from any veterinary instrument maker. They are various; some of them very useful, many of them of no service at all.

\section{DISEASES OCCURRING DURING DENTITION.}

The three first molars are temporary teeth, and are replaced by permanent ones in the horse when he is between three and four years old, and in the ox at two years and two months and two years and nine months. In young cattle, ranging from two years old and upwards, the cutting of the permanent molar teeth is occasionally a matter of some difficulty, owing to the unshed crowns of the temporary ones becoming entangled in the new teeth, proving a source of irritation, and preventing the animal from feeding. In some parts of the country such animals are called "rotten," from their emaciated condition, and perhaps from the foetor emanating from the mouth, arising from the imprisoned and decomposing food. 
When an animal of this age is seen to stop feeding, to lose condition, or to drivel from the mouth, it is necessary that the mouth be duly examined, and if the unshed teeth are the source of irritation, they ought to be at once removed. The smaller tooth forceps will be found sufficiently strong. Many hundreds of young cattle have been sacrificed from this cause; when a proper examination has not been made, they have actually died of starvation in the midst of plenty. In the horse the same condition of the molars may occur, but it is very unusual; the corner incisors, however, may present the same anomalous condition; the loosened crown of the temporary tooth grasping the crown of the permanent one, which seems to fit its irregularities sufficiently tight to prevent its being shed in the natural manner. It must be removed, as it proves a source of irritation. Horses from four years to four years and six months should have their mouths occasionally examined to see if all is going on well; and all young teething animals-horses, cattle, sheep, and dogs -if at any time seen to fall off in condition without apparent cause, should undergo an inspection of their moutlıs in order to ascertain the condition of the teeth.

Horses at four years old are very subject to a distressing cough. At this age the third temporary molar is replaced by its permanent substitute, and at the same time the sixth molar, or that standing last in the row, is being cut. Some amount of irritation is seen to exist in the gums during the eruption of all teeth, but in some instances the irritation is excessive, and extends from the gum into the fauces and larynx. This is particularly the case with the sixth molar; and, as a result of the extension of the irritation, congh is excited, more particularly in the morning, when the animal begins to feed. The cough is loud, sonorous, and prolonged: the horse may cough twenty, thirty, or even forty times without ceasing. It is a throat cough, originating in laryngeal irritation, which the animal endeavours to be rid of by coughing.

In some instances the cough is associated with a tendency to diarrhoa, more particularly if the animal is being fed on uncrushed corn and bran. The corn, swallowed without being properly masticated, acts upon the irritable condition of the mucous membrane, and easily sets up the diarrhœea.

The cough is alarming, as it is supposed to terminate in 
roaring. There is, however, no danger of that, if the animal be not predisposed by hereditary taint; but if he is of a stock of roarers, even the irritation of teething may develop the infirmity.

The treatment for this, which may be truly called a toothcough, is careful dieting on crushed food; hay, not much bran; grass, if in season, or roots when grass is not obtainable; alkaline medicines, more particularly the bicarbonate of soda; gentle aperients occasionally, if the bowels be irregular, and if the frees are foetid, the fotor will be much diminished by a few doses of the hyposulphite of soda; the mouth to be gargled with some cooling mixture, such as the borate of soda or alum.

DENTITION FEVER (PERCIVALL).

During the active stages of the process of dentition some liorses suffer from a degree of constitutional disturbance, accompanied by loss of appetite, debility, unthriftiness, a tendency to diarrhœea, and excited pulse, but without cough or any other symptom indicating that the fever is due to disease of any internal organ. On examination of the mouth being made, the gums are found red, swollen, and tender, with the secretion of saliva much increased. Hutrel D'Arboval says :- " A sort of local fever originates in the alveolar cavities, running high or low according to the resistance the teeth encounter from the hardness of the jaws or their own disproportionate size and solidity. The gums become stretched from the pressure of the teeth against them; they dilate, sometimes split; at the same time they are red, painful, and hot even to a sense of burning. Internally, the roots of the teeth, from shooting downwards, compress the dental nerves, and painfully drag the periosteal linings of the alveolar cavities. These combined causes will sufficiently account for the local irritation and suffering accompanying teething, and enable us to explain many morbid phenomena we find appearing in horses about this, from various circumstances, the most critical period of their lives."

Horses from three to four years old are more subject to this species of dental irritation than those of a more tender age, and it is well known amongst horsemen that horses will stand more fatigue at a more tender age than they will at this; and the reason is to be found in the fact that dentition is now at the 
height of its activity. When the animal is three years old,eight permanent molars are being cut, and four permanent incisors are in active growth within the gums. At four years of age the same number of molars are cut, and the same number of incisors are at a more advanced stage of growth within the gums, in addition to the canine teeth, which make their appearance about this time. No wonder, then, that the eruption of so many teeth is a source of irritation and fever.

The best treatment is to throw the animal off work, turn him to grass if the weather permits, or into a loose box in a wellventilated spot, and give him rest until the process of dentition is completed. If the gums are red and swollen, lancing them will prove a source of great relief. This operation is easily performed in the anterior portion of the mouth, but is rather difficult at the posterior part; however, it may be doue with a proper instrument, the gum-lancet of the dentist on an extended scale being the most suitable. If the temporary teeth seem to hinder the eruption of their successors they are to be removed.

\section{SUPERNUMERARY TEETH.}

Small supernumerary teeth are often met with in the front of the molars called "wolf's teeth." They have been supposed to be a cause of ophthalmia, but this can scarcely be. They can produce no inconvenience; but if requested to extract them the practitioner can hardly refuse. The best method is to remove them with the tooth-forceps.

The question as to the influence of the teeth upon diseases of the eye might perhaps be deemed worthy of discussion, inasinuch as the dental nerve is a branch of the same nerve as that supplying the eyes with common sensibility, namely, the fifth. The older writers maintain that moon-blindness was due to the wolf's tooth, and the first procedure in the treatment was its removal.

Now-a-days, however, the supposition is not carried quite so far, and the utmost that can be said is that the irritation of teething may be an exciting cause of ophthalmia in animals whose constitutions are hereditarily or otherwise predisposed to the disease; and the removal of supernumerary teeth, lancing the gums, or the withdrawal of offending teeth, may very possibly be fullowed by some remission of the ophthalmic symptoms. 


\section{DISEASES OF THE FACIAL SINUSES.}

The facio-cranial sinuses may very reasonably be looked upon as one cavity, divided into frontal, maxillary, and sphenoidal compartments. They form air-cavities, communicating with the nasal passages by a small passage guarded by an imperfect valve. In addition to the above divisions, each compartment is divided into smaller cavities by thin osseous plates, the whole being lined by a continuation of the Schneiderian membrane, which is neither so vascular nor so thick as in the nasal passages. These cavities differ in size in different breeds, as well as in individual horses of the same breed. They are small and ill-developed in all young animals, but rapidly increase in size till full growth is attained. In the ox and sheep they communicate with the canals and foramina of the "horn processes" of the frontal bones; hence, in the malignant catarrhal fever of the ox the horns are loosenerl by the extensive suppurative inflammation extending to the cavities of the "horn flint."

In catarrhal affections, the lining membrane of these sinuses, by extension of the inflammation of the Schneiderian membrane, becomes diseased, and pours out a quantity of pus, which, lodging in the various compartments of the sinuses, becomes a source of irritation, constituting what is known as "nasal gleet" or ozœna. A collection of pus or tumours in the sinuses, if exit be not allowed, cause absorption of the bony plates by pressure.

Nasal gleet may arise from other causes than catarrhal inflammation (but this is the most common cause), such as external injuries, caries of the upper molars, disease of the superior maxilla from elongated inferior molar teeth, alveolar abscesses, hypertrophy of the fangs of the teeth, disease of the facial bones, collections of inspissated pus in the convolutions of the ethmoidal and turbinated bones, formation of cystic tumours and of calcareous concretions in the superior maxillary sinuses, and from clots of blood in a state of decomposition; and in horned cattle and sheep, from the lodgment of the larvæ of the CEstrus bovis.

General Symptoms. - "In the majority of instances there will be an irregular discharge from one nostril, indicating that the sinuses of that side of the head only are affected. This discharge will also vary very much in its quality, according to the nature of the malady, the duration of the disease, and the struc- 
tures that may be involved. The submaxillary glands will be tumefied, but loose, in the cellular bed; or, on the other hand, hard and adherent to the periosteum of the jaw. The horse may appear dull, but this symptom is not always present; the eye on the side of the head aftected will look dim, the upper lid will often droop a little, and there may be a rough appearance of the hair over the region of the part diseased. The breath from the nostril of the side affected may be offensive, indicating thereby disease of the bone or decomposition of the contained matter. Mastication may be imperfect, - a defect which points to the teeth. If the malady is of long standing, and the sinuses full of matter, or the disease peculiar in its nature, there may be a slight enlargement over the affected sinus; or if the disease be of an inflammatory character, there will be pain, and perhaps pitting of the part upon pressure; and the submaxillary or temprral arteries of the side affected may also throb more or less.

"These symptoms are diagnostic of disease of the sinuses of the head, and are, I think, sufficient to induce the practitioner to seek for the precise cause which produced them."-(VARneLL.)

When the sinuses, or a division of them, are filled with pus, percussion, applied to the outer walls, will cause a dull sound to be emitted, and by comparing the sound of the side affected with that of the healthy side, a distinction can be drawn that will be of material assistance to correct diagnosis. In some instances there will be a bulging outwards of the bones over the the spot containing the pus.

In very doubtful cases I have been in the habit of carefully exploring the sinuses by introducing a small gimlet through the outer wall; if pus be contained therein, the hollow of the gimlet will be filled with it. This will satisfy the practitioner as to whether there is pus or not, but it does not indicate the line of treatment, as some cases have done well in my practice after trephining, where no pus was contained in the sinuses.

I quite agree with Professor Varnell that the proper place for trephining the sinuses, in order to remove anything abnormal that may be contained in them, is at their most dependent part.

The spot I always select is situated about an inch superior and posterior to the termination of the zygomatic spine, varying the situation anteriorly, according to the age of the patient. If lie is young, it must be considerably nearer to the cavity of tho 
nose, as the fangs of the molar teeth are then long, and their alveoli fill up the sinus, whereas in the old horse the sinus will

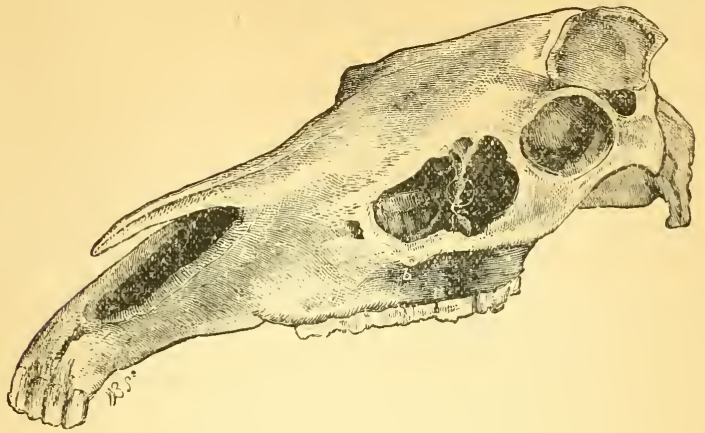

Fra. 110.-Skull cut open to show the situation of bony partition of superior maxillary sinns. The white circle is the spot selected for trephining both cavities by one opening. $a$, Space between fourth and fifth molar teeth; $b$, Termination of zygomatic spine.

extend as low as the zygomatic crest, where the opening can be made without danger of coming in contact with the teeth.

In some instances the frontal sinus has to be opened, especially when the contained pus is inspissated, and lodged in the convolutions of the ethmoidal and turbinated bones; but in the majority of instances one opening, at the most depending part of the cavity, is sufficient.

The external portion of the maxillary sinus is divided into two compartments by a transverse plate, marked in the drawing, which in many cases is perfect throughout life. The superior portion contains the alveoli of the two last molar teeth, the inferior portion the alveoli of the third and fourth, while the first and second molars are quite superficially situated; very often, indeed, their fangs actually protrude through the maxillary bone, or they may be so thinly covered by it that they can be both seen and felt through the skin of a well-bred horse. From this short description it will be seen that the guide to the operator in making the opening through the bone, so as to emmrace both cavities, will be the space between the fourth and fifth molar teeth. Of this he must judge by an inspection of the 
mouth, as the teeth are not always relatively in the same position as regards the termination of the zygomatic ridge.

To trephine successfully, it is necessary to cast the animal. I have operated without casting, but the operation is better performed when the horse is down.

It is advisable to remove a round piece of the skin and subcutaneous tissue, rather larger than the size of the trephine, and to dissect down to the bone cleanly, removing all shreds of the soft structures, or the trephine will become clogged with débris; and, to render the operation less difficult, it is a good plan to bore a small hole in the centre of the piece of bone with a small gimlet, and to introduce the centre-bit of the trephine into the hole so bored.

The best kind of trephine is that with a moveable centre-bit; but the latest improved trephines made by Mr. Mackenzie being very superior instruments, need no description.

When the piece of bone is sawn through, it will, in the majority of instances, come out of the aperture along with the saw, or it may fall into the sinus; and when it is attached to the dividing ridge of the two sinuses, it may be necessary to give the trephine a slight twist to one side, to disconnect the trephined portion from the bony plate.

The additional instruments are a strong scalpel, a pair of forceps, and an elevator, to raise the piece of bone when this is not accomplished with the trephine. This elevator, made of steel, should be six or seven inches long, from half to three quarters of an inch wide at one extremity, which is to be circular, to correspond to the trephine, and sufficiently thin to pass into the groove made by the saw.

Mr. Varnell recommends that the skin should be divided by a crucial incision, and the sinuses explored by the probe. As to the crucial incision, I find that the flaps so made are a disadvantage, interfering with the necessary daily washing and dressing of the sinuses; that they become inflamed, painful, and swollen, and cause the animal to be restive when an attempt is made to dress the part; and the wound is also apt to close too quickly if its margins are not destroyed by caustic-a bad practice. In making the circular incision, and removing the whole of the tissues contained within the circle, the operation is more easily performed, the edges of the wound do not interfere 
with the necessary ablutions, and the aperture does not close up so soon.

I see no advantage in using probes; they are calculated to wound and irritate the mucous membrane, and the contained material can be removed without them.

With Professor Varnell, I condemn the use of setons; they are calculated to do harm by keeping up irritation in a part where it is almost sure to lead to organic changes in the bones, and thus render what may be a curable into an incurable disease. Their insertion must originally have arisen from a defective knowlege of anatomy and physiology.

The opening into the cavity having been made, the practitioner will be able to judge as to the nature of its contents. If it contains pus, washing with water will remove the greater part of it. Inspissated pus, however, is very difficult to remove, and it is not advisable to exercise too great a force in washing out the sinuses, as the inspissated contents will be floated away in a few days by the formation of new pus. Large flakes will thus be carried off both by the nostril and the opening. In washing out the sinuses, if the horse be cast and the nose elevated, there is great danger of suffocating him, as the fluid will, in all probability, find its way into the larynx, and finally into the lungs. Care must therefore be exercised, the water must be injected slowly-the elastic syphon is the best instrument for this purpose-and the nose kept depressed. There is but little danger of this accident if the patient be standing.

If the sinus contain anything abnormal it must be removed. In one case that I operated upon, there was a large clot of blood, in a very fœetid condition, filling up the whole cavity. The horse had been discharging from the nostril for months; removal of the clot, however, was followed by a most rapid recovery. In other cases calcareous deposits will be found studding the mucous membrane, as if originally the tumours had been tubercular, and most probably these concretions are calcareous tubercular degenerations.

The treatment of the case after the operation must depend in a great measure upon the cause of the disease, and the state of the animal's health. If the cause be local, such as a diseased tooth, blood-clot, or imprisonment of pus, and the general health good, but little need be done, except washing out the 
sinuses daily, first with tepid, then with cold water containing some decdorizer, as the chloride of lime or zinc, carbolic acid, or Condy's fluid, afterwards with astringents, as weak solutions of the perchloride of iron, sulphate of copper, or tannic acid. The aperture must be kept open so long as the foetor and the discharge continue. The discharge at first is thick, curdled, and foetid, but by degrees it assumes a less unhealthy character, becomes thinner daily, until at last it has the same appearance as the nasal mucus. This change is always significant, and diagnostic that the case will do well; but if the discharge continue to have a fœid odour, to come away intermittingly, clotted, and sometimes sanguineous, the termination will generally be unfavourable. In several instances I have found some portion of the inner plate of the sinus, or one or more of its osseous irregularities, divested of their lining membrane, and presenting spots of caries. In such instances the injection of very dilute hydro-chloride acid, say one part to a hundred, has been of signal benefit.

I also find benefit to follow sprinkling the animal's box with chloride of lime; the continual inhalation of the chlorine having apparently a beneficial effect upon the diseased mucous membrane.

When the disease is accompanied by constitutional ill health, the local must be supplemented by general treatment. I do not agree with those who recommend that the general treatment should precede the operation, unless the animal be in a state of extreme debility. To improve the tone of the system, the patient must be removed from all depressing influences, and placed in a well-ventilated and comfortable box or stable; the food to be nutritious, but easy of digestion; the hay and corn must be of the best quality, and given liberally. Now and then a mash may be allowed,-particularly if there is any tendency to torpidity of the bowels, - with carrots or grass, if in season. The horse is to be well groomed; damp wisping is very beneficial by keeping up the action of the skin; the excretory organs must not be allowed to become dormant, or the system will become loaded with effete materials.

Medicinally, the mineral tonics have been found serviceable, particularly the sulphates of iron and copper, given in the food or in a ball. 
It is especially requisite to vary the tonics. Give the following for one week:-

Cupri Sulp. 3ii.,

Canth. pulv. gr. v., m., fiat bolus,

and administer twice a day after a full meal. The ball may be continued if the case seems to improve, but should it be thought that amendment has discontinued, the salt of iron is to be substituted for the copper. A very favourite medicine of mine is the colchicum powder, in addition to the tonics; it acts unmistakeably on the kidneys, and encourages the excretion of the effete hippurates and other urinary salts. Regular exercise, in addition to the grooning, should be insisted upon, and it is good practice to order the food to be placed near the ground, as movement of the jaws and a depending position of the head favour the discharge of the imprisoned matters.

There are many diseases of the sinuses that result in no permanent improvement after operation and treatment. Upon this point the observations of Professor Varnell are most valuable. He says, in the Veterinarian for May 1867, "Diseases of the sinuses of the facial region of the horse's head, arising, as previously stated, from external violence, are seldom treated successfully, therefore it is not often that a radical cure is effected. Generally speaking, the disease advances comparatively quickly, or it may be, on the other hand, it progresses very slowly, which will depend upon the age and constitution of the horse, and the pathological nature of the malady. It is seldom, however, that he is allowed to die from the effects of the disease itself, humanity, if not economy, suggesting the advisability of having him destroyed.

"The osseous boundaries of the sinuses, together with the membraneous covering, the inner one in particular, become thickened from being infiltrated with inflammatory exudation, the blood-vessels and Haversian canals enlarge, the bone softens, and its cancelli become filled with a semi-gelatinous or oily material, the thickening of the membrane gradually increases, its surface becomes uneven, and when its structure is examined it will be found to have somewhat of a greas character. From these causes the sinuses, which gradually become diminished in capacity, will, in the process of time, also become partly or 
wholly filled with matter resembling porridge, commingled with small particles of oily matter.

"If the practitioner suspects these structures to be in the above condition, or that the sinuses contain such materials as I have described, he will hesitate before he commences his operation; for if his experience corresponds with mine as to the result in such cases, he will come to the conclusion that they are incurable. For my own part, if I felt satisfied that the pathology of the disease in any case that was presented to me corresponded with the above description, I should certainly decline to have anything to do with it in the way of treatment; but as it is difficult to diagnose such cases with any degree of certainty, a prognosis is worth but little. Our investigations, therefore, should be extended beyond a mere surface examination. I will suppose that the evidence is sufficient to convince the practitioner that the sinuses are diseased, but the precise nature of the affection he is unable to determine. What course under such circumstances should be taken? I should advise that one or more of the sinuses be opened; which can easily be done with a small trephine or even with a gimlet. Having now examined the walls of the sinuses, the lining membrane, and some of the material which they contain, he will be prepared to advise what course should be adopted. If the character of the disease should turn out to be simple in its nature, and that there is a fair chance of its being treated successfully, he will have partly accomplished the operation for the cure thereof; or if, on the contrary, he considers the affection to be incurable, he will be prepared to advise that the patient be disposed of or destroyed, as may be deemed best.

"Disease of the sinuses is sometimes complicated with one of a similar character of the nasal passages; indeed, it will be an extension of the affection from one part to the other, or it may have commenced simultaneously in both eavities.

"The symptoms indicative of the nasal passage as well as the sinuses being affected are as follows:-The discharge, which is never very copious, is nevertheless continuous; the breathing is embarrassed, particularly if the opposite nostril is closed, when a considerable impediment will often be evinced, and percussion gives a dull sound over the whole of the side of the face.

"If the mucous membrane of the nasal passages, when thus 
affected, be examined post mortem, it will be found much thickened throughout, but in some places more so than others; its structure will have a somewhat greasy appearance, and its surface covered with a greyish-yellow, sticky fluid, which, if examined by the microscope, will be found to contain large irregular cells, granular matter, oily particles, and pus cells, the latter not so abundant as from the appearance of the fluid might have been expected. The meatuses may be nearly closed by the thickened membrane, and the spaces between the folds of the turbinated bones entirely obliterated; and very often the matter will have accumulated between these folds to such an extent as to form a considerable enlargement, in fact, an abscess, which on being opened will be found to contain a muco-purulent, or a glairy, viscous fluid.

"Cases of this kind have been often mistaken for glanders, and horses destroyed in consequence. This would be an error in diagnosis, of course, but a very unimportant one ; for although the disease is not, as far as we know, contagious, nevertheless it is incurable, and if the horse's health is impaired by bad keep and exposure, there is a possibility of its degenerating into glanders.

"I have met with another disease affecting the sinuses of the face of the horse, dissimilar, however, to the one I have just described. The symptoms which I have observed to accompany this affection are dulness, the eye on the side affected is dim and watery, and there is a slight intermittent discharge of thin greyish matter from the nostril of the same side. The submaxillary glands on this side may also be enlarged; and if so they will be hard and somewhat firmly attached to the periosteum of the jaw-bone.

"If the sinuses be trephined, as recommended in the previous affection, for the purpose of ascertaining the nature of the malady, instead of the bone being soft and much thickened, it will be thicker than natural, and its density much increased. The mucous lining will also be much thickened-in some places more so than in others,-and it will have the character of a dense fibrous membrane. The sinuses will contain more or less fluid of a dark greyish colour, which, when examined, will be found to contain large, irregular, round cells, much débris of broken-up cells, and some elongated, spindle-shaped cells, but no oily particles or pus cells." 
Mr. Varnell then relates an instance of malignant growths in the sinuses, but the evidence brought forward is very unsatisfactory, the case to my mind being one of glanders, although its true nature was rather obscure.

In the chapter on Tumours the reader will find cystic growths in the sinuses described, also the symptoms and treatment of nasal polypi.

The dog is occasionally subject to ulceration of the nasal chambers, and to the formation of small growths upon the mucous membrane. I cannot assign any causes; probably catarrhal affections may be the originators. I have removed the growths, and even a portion of the septum ad nasum, with good effect.

In connection with the growth of the face and sinuses, deformities are apt to occur from no ostensible cause in the form of rounded enlargements of the maxillary bones. The enlargements may be confined to one or they may affect both cheeks, without discharge from the nostrils, and seemingly causing no inconvenience to the horse. All treatment is calculated to do harm; therefore, so long as they remain as mere deformities, the practitioner ought not to interfere. Frictions with iodine and blisters, the remedies generally employed, very often stimulate to activity what otherwise would remain latent.

\section{NASAL GLEET}

In the horse frequently depends upon no traceable cause, and has been variously named dropsy of the sinuses (VARNELL), catarrhus sinum frontalis et maxillaris (GAMGEE), and coryza. In some cases there may be a lodgment of pus in and a discharge from the sinuses, while in others the discharge is from the nasal membrane only. The character of the secretion, and the whole train of symptoms accompanying it, are very baffling. The horse may be unthrifty, with or without cough, the coat harsh, skin dry, appetite capricious or depraved, in some instances good, but the assimilative powers seem to be at fault. The discharge is intermittent, yellowish-white in colour, does not stick to the nostrils, as in glanders, and there is no ulceration, as in that disease ; sometimes containing a few shreds of coagulable matter, which is symptomatic of disease of tooth or bone, as the coagulation is caused by phosphoric acid or $\mathrm{H}_{2} \mathrm{~S}$, which are formed by the decomposing matter. The Schneiderian membrane may be excoriated, presenting patches of abrasions resembling ulcers, but not the characteristic pit-like depression of the glanderous ulcer. The 
rucous membrane is pale, perhaps thickened and infiltrated, or is of a coppery hue; and the eye of the affected side appears sunken, gummy at the lids, with a slight discharge of mucus from its inner canthus.

In every case of this kind the practitioner should be very guarded in his diagnosis, as the disease may be glanders in its latent form. Let the animal be isolated, kept in a loose box; all pails, brushes, combs, sponges, \&c. made use of for cleaning and feeding him must be kept apart until time develops the true nature of the malady.

Inoculation with the discharge is unsatisfactory, as inflammation and suppuration of the lymphatics are induced by any unhealthy ichorous discharge.

Great improvement often results from the effects of a sharp fly blister to the face, over the sinuses and nasal chambers, tonics being discriminately administered, and the best of food allowed. Injections of cold water or some slight astringent wash, as the tincture of the terchloride of iron, in the proportion of an ounce to the quart of water, or of sulphate of copper, daily, are often very beneficial. Inhalations of sulphureous acid gas, largely diluted with air, are useful. The gas is made as follows:-Burn half-an-ounce of prepared sulphur-the milk of sulphur in preference, being a pure preparation-in a moderate sized loose box or stable, and make the animal inhale it for half-an-hour every day. It lias a stimulating and tonic effect on the mucous membrane, and if sufficiently dilute, does not irritate.

A chronic nasal discharge, with a snoring and snuflling sound during breathing, may arise from some cause of obstruction in the nostrils-polypi, or a thickening of the Schneiderian membrane,-causing constriction of the anterior nares. These sources of obstruction and irritation being removed, the discharge and abnormal sounds disaypear. 


\title{
CHAPTER XXX.
}

DISEASES OF THE PHARYNX, ESOPHAGUS, ETC.

\begin{abstract}
POST-PHARYNGEAL ABSCESS - PUS IN THE GUTTURAL POUCHES PHARYNGEAL POLYPI - CHOKING, CLINICAL RECORDS OF ESOPHAGOTOMY-DILATION AND STRICTURE OF THE ESOPHAGUS -WIND-SUCKING AND CRID-BITING.
\end{abstract}

\section{POST-PHARYNGEAL AESCESS}

Is a collection of pus situated in the cartilaginous division of the guttural pouches. Being placed between the two cavities, it bulges into both, presses upon the pharynx, and interferes with the act of deglutition, and as it increases in bulk, fills up the posterior nares, draws up the vellum pendulum palati, and causes the animal to breathe through the mouth.

It may be associated with strangles, or it may appear independently of that disease; its first symptoms being discharge from the nose, sore throat, difficulty in swallowing, the food and water being returned through the mouth.

The pus being confined in the tough cartilaginous structure of the guttural pouches, prevents the abscess from bursting spontaneously; it therefore becomes a necessity to open it by operation, and for this and all operations within the mouth, the best instrument is a long straight bistoury, enveloped in tow or lint to within about half-an-inch of its point. This precaution having been taken, the horse's mouth is to be kept open by a wide ballingiron; one hand of the operator is then to be introduced into the fauces, and by firm pressure he is to find out if there be fluctuation; on this being ascertained, the bistoury is pushed along the hollow of the hand, and plunged into the fluctuating swelling, cutting neither to the right nor left, for fear of injuring the carotids, but keeping the incision in the centre of the swelling, a 
gush of pus will immediately escape into the mouth. I own there is some difficulty in the operation, but if nothing be done, the animal's life is sure to be sacrificed.

The operation may be performed through the guttural poucher, or the puncture might be made through the nostril, but I think the best way is that through the mouth.

\section{COLLECTIONS OF PUS IN THE GUTTURAL POUCHES}

Differ from post-pharyngeal abscess by the presence of more or less external swelling; a bulging outwards of the parotid glands; and by an intermitting discharge from the nostrils of thick creamy, perhaps slightly curdled pus. The difficulty in swallowing, although at first perhaps very great, is not persistent, disappearing upon the cessation of the pharyngeal inflammation.

The diagnostic signs of pus in the guttural pouches are: first, a discharge from either one or both nostrils when the head is depressed, or an occasional nasal discharge, as from an overHow through the openings of the eustachian tubes; a swelling more or less extensive, without hardness, on the side affected, with a flow of pus from the nostril when the swelling is pressed upon.

The Treatment.-Cut through the skin immediately behind the posterior border of the parotid gland, above the carotids and jugulars, the incision is to be at least two inches long; after dissecting carefully through the thin superficial layer of muscular tissue, and exposing the edge of the gland, the finger is to be introduced into the very loose areolar tissue situated beneath it, and pushed until the wall of the guttural pouch, or enveloping capsule of the pus is felt; an effort is now to be made for the purpose of overcoming it by the pressure of the finger. Sometimes it will give way, but if not, a canula is to be introduced into the space made by the finger, through which the sac is to be punctured by the stillet, and the whole cavity washed out with warm water, forced in by the elastic syphon. The wound must afterwards be kept open by plugs of tow, and the parts thoroughly washed daily with water containing carbolic acid or some astringent, using the syphon in order to do it effectually. 
Mr. Gamgee has described a very formidable operation, under a more formidable name, viz., Hyo-vertebrotomy, for the purpose of giving exit to pus in the guttural pouches. The operation is quite unnecessary ; and I am surprised that any one knowing the anatomy of the parts so well as Mr. Gamgee, should not have thought of operating underneath the parotid gland. If both pouches are filled with pus, the operation is to be performed on both sides.

\section{PIIARYNGEAL POLYPI.}

Tumours, with long pedicles, are sometimes found in the fauces, which by falling into the pharynx or larynx constitute pharyngeal or laryngeal polypi.

The symptoms which they produce are those of choking, difficulty of breathing, incessant efforts to cough, and a flow of saliva from the mouth. In some instances "intermittent roaring" is the only symptom of their presence.

They are detected by examination of the fauces, and are to be removed by the "écraseur," torsion, and when they present a broad base, they are to be destroyed, "sloughed out" by escharotics.

\section{CHOKING.}

Choking in the horse is generally caused by the lodgment of a quantity of dry food, such as corn, cut hay, or chaff, swallowed rapidly by a greedy feeder; by a piece of carrot or potato, or by a ball of large size or hard consistence, especially if wrapped in thick coarse paper, becoming lodged in the pharynx or œesophagus.

Eggs forced into the pharynx have often proved a source of obstruction.

"Horses with voracious appetites," says Mr. Percivall, "are very apt in their avidity to bolt their corn whole, and gulp it down so rapidly, that the successive portions, instead of passing into the stomach, accumulate within the gullet, and block up its canal. Only a small collection, or else a large and extended one, may in this manner ensue before the animal feels or expresses uneasiness. All at once he leaves off feeding; next, he makes every effort in his power to complete his imperfect swallow, and gulp down the cause of his distress. Should he not succeed, his 
throat and neck become, through his gulping and ineffectual exertions, spasmodically drawn up; and prooably he gives every now and then a loud shriek, no less expressive of his own anguish than excitive of the compassion of those around him. Should he attempt to swallow water, the fluid, together with the saliva abounding in his mouth, returns through his nostrils. These urgent symptoms are not, however, always present, and they depend very much on the position of the obstructing body. Thus, when it is in the pharynx, the distress, coughing, and slavering are very urgent. When the obstruction is in the cervical portion, there is a visible enlargement on the course of the esophagus; the general symptoms being great anxiety of countenance, sunken head, tremors, and partial sweats over the body, with great exhaustion shortly after the occurrence of the accident."

An obstacle lodged within the thoracic portion of the œsophagus does not give rise to the more urgent symptoms of choking; an animal may evince a desire to drink, and may swallow water until the osophagus is filled from the foreign body upwards, and which, distending the tube, causes violent attempts at vomition. In cattle and dogs the act of vomition is very perfectly performed, but in the horse the contents of the œesophagus are discharged through the nostrils, and in lesser quantities than in other animals. Occasionally, however, the discharge may be through the mouth as well as the nose, and the food may be expelled with violence, and forced to a sufficient distance to plaster walls, rack, and manger.

In the cow the obstruction is generally a piece of turnip, potato, leather, such as the sole of an old shoe-this animal heing very fond of chewing any material of the kind that may fall in her way. In the dog, pieces of meat, bones, pins, and needles, are generally the causes of choking.

Choking with dry food, in the horse, especially in those cases where the whole length of the œsophagus has been distended, is the most dangerous; but I have seen a most severe and obstinate instance caused by an egg, given with the belief that an unbroken egg was a cure for the coiic.

The symptoms in the cow are tympanitis of the rumen, involuntary action of the jaws, flow of saliva from the mouth, and a violent cough, causing forcible expulsion of the fæecs and urine. 
Tympanitis is also occasionally present in the horse. In the dog violent retching and cough, with staring, prominent eyes, are the most conspicuous signs.

In some rare cases in the horse the actual cause of disorder may be overlooked, more especially when the foreign body is within the thoracic portion of the œsophagus, and I have seen cases where loss of appetite, bloodshot eyes, constipation, and discharge of saliva from the mouth, were the only apparent symptoms, until a more minute examination proved the existence of a swelling along the course of the cosophagus, on the left side of the neck.

Mr. Gamgee arranges the causes under two heads: "1st. Those dependent on the animal itself; and $2 d$. The nature of the food." With reference to those under the first head, they are- " 1 st. Any influence which may favour the contraction of the throat or gullet on the object swallowed. $2 d$. Inflammation or ulceration of the throat and gullet, which favour choking. The ulceration which follows bad accidents of this description, and which is especially troublesome a week after an animal has been relieved (of choking), often causes a dangerous accumulation of alimentary matters low down in the œsophagus. $3 d$. Organic disease of the œsophagus, especially constrictions such as are observed in crib-biting horses. 4th. Injuries and diseases of the salivary apparatus or organs of mastication, whereby food is imperfectly chewed and moistened. If the parotid ducts in a horse are both opened, so as to allow of the escape of the secretion, the animal soon suffers from impaction of the gullet. $5 t h$. Voracious appetite and rapid deglutition of bulky or dry food."

The following cases may be interesting. They are from a paper published in the Veterinarian, by Mr. King of Stanmore:-

Mr. King observes that "choking is common among old horses whose grinders are imperfect, and whose keen appetites incite them to bolt their corn. He has seen the oesophagus in this manner distended "almost from the stomach to the throat"a case in which recovery is very rare. Mr. King's practice is to pour down fluids, and press and squeeze the œsophagus, with a view of mingling the liquid introduced among the masses of corn; and this manipulation has occasionally succeeded.

The following case shows how much a practitioner may be led astray by false or imperfect accounts :- 
Some years ago Mr. King was called to a horse belonging to a coach proprieter. The owner said his horse "had a bad sore throat, and could not swallow." Mr. King examined the throat and gullet, but, finding nothing, suspected nothing. The horse was blistered and drenched; but the liquids all returned without any effort being made to swallow them. The animal died; and on examination there was found, within the thoracic portion of the cesophagus, a ball composed of the ashes of tobacco, enveloped in double paper. At first, all knowledge concerning this discovery was stoutly denied; but afterwards it was confessed that the ball had been administered for worms. Had not such delusion been practised, the probang would have been used, and, Mr. King thinks, have proved effectual.

Mr. King also observes, "there is a notion abroad, new-laid eggs will improve the condition of horses; and the practice is to auminister them with the shell only starred in a few places: a practice that lias in some instances been the means of choking the animal."

Mr. King was once called to a horse with a reported "sore throat." The groom swore he knew no cause for it. Mr. King, however, had reasons for entertaining doubts of the man's veracity, and therefore proceeded at once to pass a probang. On the return of the instrument the bulb was found covered with fragments of egg-shell. The horse speedily recovered. Mr. King has had related to him, on good authority, two similar cases in cattle practice.

The following irremediable and fatal case of the same description occurred to Mr. T. Cooper, V.S., Coleshill.

In December 1834 Mr. Cooper was called to Dunton Hall, to a bay horse that was taken suddenly unwell. Mr. Cooper found the animal "coughing violently, and stamping with his fore feet, with saliva running from his mouth, which he occasionally attempted to swallow, but the greater part returned through his nostrils." It was evident there was obstruction. The horse had been eating Swedish turnips. Mr. Cooper passed a whalebone down the cosophagus, " and a rounded substance could be distinctly seen driven before it. The horse after this appeared to be relieved; he ate some hay and drank some water, and was left for the night." Next day he was much worse. He did not cough, but heaved very much at the flank; refused all food and 
drink; was dejected; saliva with mucus ran from his nose, and much of it he swallowed. He was bled; took an aperient with digitalis ; and his throat was blistered, from a notion that "the substance might have injured his throat." Third day, much the same. "Takes gruel from a bottle, and will not eat." Mr. C., from the first, had no hope of saving him, and early next morning he died. On dissection, a large-sized hen's egg, entirely whole, was found firmly impacted in the œsophagus, within a few inches of its cardiac termination; the parietes of the tube around the egg being "much dilated, and ulcerated nearly through." The groom confessed he had given the egg a few hours before Mr. C. was sent for, with a view of improving the horse's condition. The balls which had been given must have passed the egg in a liquid state, probably along with the grucl. - Veterinarian, 1835.

Treatinent of Choking.-In all cases where the obstruction is at the back of the mouth, or high in the throat, and within reach of the operator's hand, it is to be removed through the mouth. When the impaction is in the pharynx, and barely within reach of the fingers, its removal will be rendered less difficult by pulling the tongue well out of the mouth; but if it be impossible to remove it thus, the object may be pushed upwards by an assistant. Should the offending body be sharp-pointed, it will be necessary to remove it by the forceps; in dogs this form of choking will be most commonly met with.

Failing removal by the mouth, or when the object is too far down to be reached by the hand, it should be manipulated on the outside, and gently pushed upwards and downwards; and if it can thus be made to move either way in the slightest degree, its total spontaneous disappearance may confidently be looked for. Should this method, however, fail, the object may be removed by causing the animal to swallow liquids, more especially demulcents, such as gruel, or what is better, an emulsion made by mixing equal quantities of oil and water, blencled together by the addition of a small proportion of the carbonate of potash.

In choking with dry food, the emulsion must always be used, as the probang is inadmissible, on the ground that it merely acts as a ramrod, and converts a loose impaction into a hard, almost impermeable, mass. In addition to causing the animal to swal- 
low the demulcent liquid, the mass within the œesophagus is to be broken up by gently rubbing the neck, and by pushing it upwards and downwards by gentle manipulation. The practice of having the throat rubbed by a strong broomstick, worked upwards and downwards by two powerful fellows with might and main, as if a horse were made of iron, and not of flesh and blood, cannot be too highly censured. The manipulation must be directly over the impaction, and ought to be performed by the veterinary surgeon's own hands.

In one case of choking with locust beans, bran, and chaff, in which the symptoms of distress were very severe, and called for immediate relief, the following method proved eminently successful: - Water was administered until the œsophagus became quite full above the seat of the impaction. This induced a violent fit of coughing; the whole of the fluid was thus forcibly ejected, along with some of the impacted mass. The process was repeated after short intervals of rest. Each fit of coughing brought up more and more of the solid materials with the water until the whole mass was thus got rid of. It may be mentioned that the horse became very restive, and fought against the water being introduced through his mouth. I was consequently forced to give it by the nostrils.

I recommend this method where the symptoms demand immediate relief as being safe and effectual. The water ought to be given by the mouth if possible, but if this be impossible, through the nostrils, as there is no danger if carefully performed.

When the offending body is a turnip, carrot, egg, potato, a ball, or other object that may be removed with safety by pressure from within the gullet, and when removal by manipulation and by swallowing liquids has failed, the probang must be brought into operation.

Probangs are of various sizes, and may be purchased from the instrument makers. I therefore need not describe them further than to say that horses require a smaller one than horned cattle, and that a stillet with a screw at the end may be occasionally required. The introduction of the probang into the œesophagus of the horse is very difficult, and it is generally necessary to " cast" the animal. In the cow this difficulty is not experienced; a gag in the mouth, and two or three strong fellows to hold the 
patient, being generally sufficient. Armatage's is the best form of gag.

In introducing the probang, it is essential-1st, that the animal's nose should be elevated, in order to make the mouth and throat as near a straight line as possible; $2 d$, that the neck should be kept straight; $3 d$, that the probang should be pushed with care over the epiglottis ; $4 t h$, that the pressure brought to bear upon the obstructing object should not be violent, but gentle and intermitting, as firm and persistent pressure is apt to cause rupture of the osophagus; for it must be remembered that generally the tube is spasmodically closed beyond the obstacle, and severe and continuous pushing increases this condition, causes the obstacle to tear the cuticular coat, and finally to penetrate the œsophagus altogether. If gentle taps with the cup-end of the probang be insufficient, the "screw stillet" is to be introduced, and an attempt made to transfix the obstacle with the cork-screw termination of the stillet, and to withdraw it through the mouth. The operation may be compared to that of drawing a cork out of a bottle; but should the operator fail in removing the obstacle by this method, it will be good practice to pierce it in several places by the screw, and thus break down its integrity, and leave the completion of the operation to its gradual softening, more particularly in those instances where the symptoms are not very urgent. In cases where the impaction is within the thoracic portion of the csophagus, nothing more call be done; but should it be within the reach of the operator, it may be deemed necessary to perform "œsophagotomy." This operation is very easily performed. It should be adopted, however, as a last resource, as wounds of the œsophagus are difficult to manage, and tend to produce a stricture of the tube.

If the foreign body can be distinctly made out by examination, the operator should, in the first place, have a twitch put on the horse's nose-in the cow, the "bull dogs;" in the second place, the head must be elevated and neck extended to throw the muscles into a state of firmness and tension; then an assistant is made to press the off side of the neck upon the obstacle, so as to cause it to bulge outwards as far as possible: these preliminaries being completed, the surgeon must make a bold longitudinal jncision on to the object, cutting through the skin, muscles, and cesephagus at one stroke of the knife, but taking care not to 
make the wound too large. If the incision be not made boldly, the animal will become restive, and render the operation very difficult.

The offending body being removed, the divided parietes of the osophagus are to be brought together by two or three sutures, using fine catgut for the purpose (the carbolised catgut now made use of by surgeons), and which, I may mention, was recommended by Professor Dick at least forty years ago. The wound in the skin must be closed with strong metallic suture wire, previously dipped in diluted carbolic acid. In fact, everything must be done to insure union by the adhesive process. Pads, bandages, and all other remedies recommended by veterinary writers, do much harm, by irritating the wound, and setting up the suppurative process.

When the wounds have been carefully closed by the sutures, and when the surrounding parts have become dry, the approximation of the external lips may be further strengthened by collodion or styptic colloid, after the application of which the parts must not be touched for several days-until, in fact, it is seen that adhesion has taken place. During this time the animal is to be allowed a gallon, or as much milk as he will drink at a time, three times a day; his head to be tied to the rack; and should the milk escape through the wound-and this need not occur if the application of the sutures has been properly performed-the oesophagus is to be supported by the attendant applying a clean cloth to the wound with a gentle pressure, so as to prevent the separation of its lips; it is, however, better not to do this unless it is imperatively called for.

Mr. Gamgee recommends that œsophagotomy should be performed early in cases of choking from impaction throughont the whole length of the gullet, or, when the obstruction is situated in the horse, in its thoracic portion. I am of opinion that he is too hasty in his conclusions, and that everything should be done to remove the offending body in the other ways described. We are, however, indebted to Mr. Gamgee for his suggestion, that when choking is caused by an egg in the cervical portion of the œsophagus, the egg should first be pierced with an exploring needle, and afterwards crushed by a blow.

In concluding this subject, I may remind the reader that the persistence of "choking" depends, first, on closing of the 
a'sophagus, immediately behind the seat of the obstruction, and, secondly, on tumefaction, caused by the irritation set up by the foreign body. These conditions should be borne in mind in the treatment of a difficult case; and instead of ruthlessly endeavouring to push the object down with the probang, an attempt should be made to withdraw it with the screw; and failing this, the spasm of the osophagus should be modified by the administration or subcutaneous injection of morphia or aconite-the latter in preference, as it has a direct influence on the cardiac extremity of the osophagus, producing antiperistaltic action and attempts at vomition.

\section{DILATATION AND STRICTURE OF THE CESOPIIAGUS.}

These two conditions are very frequently associated: for example, if a stricture is situated at any part of the tube, all the portions above it become dilated from the constant accumulation of aliment.

I have met with cases of stricture caused by scalding, by tumours pressing apon the œsophagus, and in one instance stricture of the whole tube was found to depend upon inflammation and thickening of its walls, the symptoms of which prior to death were-inability to swallow, attempts at vomition, and the presence of a diffuse swelling along the course of the œsophagus. The subject of it was a cow, and it seemed to have arisen from no traceable cause. The following case, published in the Veterinarian for 1830 , by Mr. Cheetham of Glasgow, throws light upon several points connected with this subject:-

"Mr. Cheetham was called to attend a mare belonging to an officer of the 4th Dragoon Guards. She discharged masticated food from the nose; and on the near side of the neck there was a swelling in the situation of the œesophagus as large as a person's arm, commencing about six inches from the pharynx, and gradually increasing to opposite the sixth cervical vertebra, and there terminating abruptly. There had existed a partial obstruction for many months, which had so increased of late, that the animal had been obliged to be drenched with water to wash down the contents of the sac; on other occasicns a probang had been used. After such palliations as these, a blister was applied crer the tumour, and she was turned to grass. While there it 
was observed that food lodged in the sac of the œsophagus was frequently returned into the mouth, and afterwards re-swallowed, and then passed into the stomach. On being taken from grass, a quantity of corn was given to her, in order to ascertain if the stoppage still existed. The corn accumulated the same as before. Mr. C. determined on an operation. He made an incision four inches long in the nesophagus, opposite the sixth cervical vertebra. The tube seemed divested of its muscular fibres, and was composed of cuticular coat alone. The contents of the sac were removed, and she was drenched with warm water to wash out the osophagus. The sac appeared three or four inches in diameter; but the opening leading from it below was so contracted that it only admitted a probang half-an-inch in diameter. After the operation the mare drank freely of warm water, which, by applying pressure upon the wound, passed unintermuptedly into the stomach, though without the pressure the greater part escaped. She was bled, and had an aperient. The wound was fomented, and poulticed, and dressed; and the mare partook freely of gruel. Some sloughing followed, which brought away part of the œsophagus; after which the wound became healthy. Mr. C. now introduced a probang, of the dimensions of the first he used, through the stricture; which operation he repeated twice or thrice a day for ten successive days, with probangs of larger size. Thus was the stricture-which appeared to have been seated at the place where the tube enters the chest-overcome; and since then the probang has been occasionally introduced by the owner himself. To assist the mare in swallowing, the sac was aided in its action by pressure, accomplished by a broad breast-plate furnished with a pad. The sac gradually grew less; and the mare at length became enabled to consume her rations, and soon after recovered all her life and gaiety."

\section{STRICTURE OF CARDIAC EXTREMITY OF EESOPHAGUS.}

"The appearance of the cardiac stricture is this-the osophageal orifice at the stomach is contracted to the utmost degree: in one of my cases, a sharp-pointed instrument was with difficulty introduced. The muscular fibres surrounding the strictured part are prodigiously augmented in volume, and, in addition, there is a morbid thickening of their lining, arising from deposition into 
the cellular substance interposed between the muscular and cuticular coats. The cuticular membrane is thrown into rugæ unusually large, and is evidently increased in substance. The stomach itself is not affected.

"Treatment.-The two cases that came under my notice were not unmasked until death had afforded the opportunity of investigating their rature, and consequently were not submitted to any specific or appropriate treatment. Were I to encounter another, I should endeavour to pass a bougie of proper size, as far as, or even into, the stomach, with a view of ascertaining the seat and nature of the obstruction; which, being ascertained to be stricture, might possibly admit of dilatation, or of the conveyance of caustic to it. Should, however, so long a passage for the bougie render it unavailable against the stricture, we must do as Mr. Cheetham has already done, make an incision through the neck into the cesophagus, and pass the bougie or probang from there. A cardiac stricture would of necessity prove a very troublesome, perhaps an intractable, affair ; one within the neck, or even the chest, might admit of being overcome."-(Percivall's IIippopathology.

Professor Dick reports an interesting case of dilatation of the cesophagus.-(See Veterinary Papers by Professor Dick.) He compares the affection to globus hystericus in the human being. This, however, is a purely nervous affection; whereas the case in question was due to some lesion of the osophagus.

\section{CRIB-BITERS AND WIND-SUCKERS.}

Horses which are crib-biters or wind-suckers are to be considered as unscund, as the vices generally arise from or cause indigestion, induce colic, tend to lower condition, and to depress the vital powers.

A crib-biter seizes the manger, or some other fixture-the collar-shank, when nothing else can be taken hold of-with his teeth, arches his neck, and makes a belching noise. After a time the abdomen becomes evidently enlarged.

Many crib-biters thrive moderately well, whilst others are always unthrifty, dry in the coat, and hide-bound. Some practitioners maintain that air is swallowed during the act, whilst others state that gases are expelled from the stomach, and that the continued 
belching or eructation causes further derangement oi the stomach, the increased formation of gases, and the consequent tympanitis. I was at first disposed to think that the first opinion was the correct one, as it is quite possible for air mixed with saliva to be swallowed; but further experience inelines me to the latter, namely, that gases are expelled, and that increased disorder of the stomach is indueed by the habit. A chronic crib-biter may be easily recognised by the appearance of the ineisor teeth, which are worn and rounded at their anterior borders, and by an enlarged or hypertrophied condition of the muscles which depress the jaw, the sterno-maxillaris, stylo-maxillaris, \&c., and generally by the mark of a strap on the neck.

A wind-sucker smacks his lips, gathers air into his mouth, extends his head, or presses it against some solid body, arehes his neek, gathers his feet together, and undoubtedly swallows air, blowing himself out, sometimes to a tremendous extent. Of the two vices this is the worst, a wind-sucker being more subject to colic, indigestion, and polyuria than a erib-biter.

To prevent crib-biting, a muzzle or a neck-strap made for the purpose is sold by saddlers; and for wind-sucking a strap studded with sharp points of iron opposite the lower part of the jaw, is the best preventive; whilst the indigestion from which these animals seem to suffer is best combated with purgatives, alkalies, roek salt in the manger, and regular work. WVant of work, indigestion, and the irritation of teething are generally the causes of these vices. 


\section{CHAPTER XXXI.}

\section{DISEASES OF THE EYES.}

CONJUNCTIVITIS - NEBULE-ALBUGO-STAPHYLONA - CLCERS - GLAUCOMA - AMAUROSIS - STRABISMUS - ECTROPIUM - ENTROPIUM TRICHIASIS-DISTICHIASIS-WARTS-WOUNDS-HAWS-LACHRYMAL FISTULA - STRICTURE-PARASITES-FUNGUS HIMATODESPERIODIC OPHTHALMIA-CATARACT-REMOVAL OF EYEBALLDISLOCATION OF EYEBALL-MELANOSIS OF IUUIOURS-EXAMINATION OF EYE BY CATOPTRIC TEST AND BY THE OPHTHALMOSCOPE.

\section{TRAUHATIC OPITHALMIA - SIMPLE OPHTHALMIA- CONJUNCTIVITIS,}

CAUSED by a blow, as the stroke of a whip, bites of insects, common cold, or the lodgment of a foreign body.

Inflammation of the superficial structures of the eye is manifested by closure of the eyelids, swelling of them, and increased secretion of tears, which flow down the cheeks, scalding the skin to such an extent that it soon becomes divested of hair at every part over which the tears flow, and the eye is retracted and partly covered by the memlrana nictitans. If the eyelids be turned up, the conjunctiva will be found in a state of extreme congestion, and covered by a number of red streaks. The progress of inflammation, as seen in the eye, when conjunctivitis proceeds perhaps from a simple catarrhal affection, is very instructive. First of all, there is a slight weeping, and if one may judge from actual personal feeling and experience, there is a sense of irritation, as if a foreign body were in the eye. The surface of the cornea is dim and blue-looking, and vascularity is seen only at its margin and the parts external to it, because it possesses no vessels in its intimate structure, and the gradually increasing opacity is due to exudation (as already explained) within its ultimate cellular structure. 
If the inflaumation be due to external injury, the mark of its seat will generally be distinguishable by the greater intensity of the opacity immediately surrounding it. If it be due to contusion of the eyelids, there may be no mark on the eye itself, nor upon the eyelids; but generally these may be seen to present a spot of ecchymosis or congestion, if a careful examination be made.

The question of origin sometimes becomes a matter of importance, as a man's character might be at stake, the master perhaps blaming his servant for striking the animal's eye and causing the disease. In such instances the veterinarian is often appealed to by both master and servant.

Treatment.-If a foreign body is present in the eye, it must be removed. Should it be a hay-seed or corn-chaff (a very frequent cause of ophthalmia, more especially in cattle), and adherent to the coats of the eye, the removal is often a matter of some difficulty, requiring the use of the forceps or a strong feather. The removal of the foreign body must be performed quickly, as the membrana nictitans is brought into powerful play by the rapid retractions of the eye-ball into the socket. In such instances it will be found necessary to transfix the membrana nictitans with a needle and thread, or a strong ienaculum, before the irritant can be removed. After the cause is removed, but little treatment is required beyond fomentations and exclusion of light. In the more severe cases it may be necessary to reduce the congestion of the vessels by local bleeding from the angular vein, situated on the face, immediately below the eye, or by scarifying the inner surface of the congested eyelids. For my own part, I think the latter a bad practice, and apt to increase the irritation, and I always bleed from the "angular vein." In addition to the bleeding, the eye should be bathed with tepid water, or a decoction of poppy-heads, if the pain seem urgent, and covered over with a light piece of calico, properly adjusted over the ears, and fastened below the chin. This is to be kept wet with a decoction of poppies, or some lotion containing cocaine, and the parts bathed repeatedly every day.

Belladonna, applied in the form of a soft extract externally, is very useful in all forms of ophthalmia. It keeps the pupil dilated, and prevents adhesion of the iris to the lens. Bella- 
donna and hyoscyamus have the same effect, but the belladonna is the stronger, and is best applied, particularly if there be much irritation, to the eyebrows and outside of the eyelids, in the form of a paste of the consistency of honey. Should it, however, be deemed desirable to apply the active principle, atrophine, the following solution may be dropped into the eye :-

\section{R Atrophiæ sulphat., gr. iv.; aqua distil. $\xi^{\text {i. }}$}

A purgative is to be administered, and followed by febrifuges and diuretics.

The film that spreads over the anterior aspect of the eye consists of an exudate, which continues so long as the irritation lasts, but afterwards gradually disappears by absorption. Many think that it is necessary to destroy it by causties, as if it were an outer skin which had grown over the eye. This is a mistake; the deposit is within the structures of the cornea and conjunctiva, and until the inflammation has subsided, all irritating applications are caiculated to do harm.

In simple ophthalmia, resulting from an injury to the cornea, the opacity radiates from the seat of injury, whilst in catarrhal inflammation of the eyes the opacity converges inwards from the margin of the cornea. This form of ophthalmia is most frequently met with in connection with an epizootic disease which has been termed "pink-eye" by horsey individuals. It is an epizootic inflammation of the areolar tissue, and I have named it Epizootic Cellulitis, partaking of the nature of rleumatism, and the ophthalmic complication is in all probability due to the presence of the rheumatic poison.

The ox, sheep, and dog are subject to superficial conjunctivitis from cold or other causes, the dog especially, as a complication with " distemper."

In all these animals, as well as in the horse, the treatment is first to subdue inflammatory action by soothing remedies; and, secondly, to restore the tone of the vessels by applying very mild astringent washes, as the sulphate of zinc, alum, \&c., in water, and to avoid exposing the eye to strong light until it is thoroughly restored.

NEBULA-ALBUGO.

A partial opacity remains after the remoral of the general 
dimness or film. The opacity of the cornea caused by an injury often remains as a permanent blemish, being due to the presence of reparative material in the wound, and to a deposition of lymph within the layers of the cornea immediately surrounding it. An opacity of this nature is at first of a bluish tinge, and is then called a nebula; as it becomes older it turns to a pearly-white colour, and is then called an albugo. These terms are quite conventional, and perhaps objectionable. The opacities are in fact nothing more than cicatrices, and they undergo the same changes as those of other wounds, namely, they contract with age, become firmer in their structure, but never entirely disappear.

Occasional touches with the solid nitrate of silver, or a solution of it applied with a camel-hair pencil, will hasten the absorption of the surrounding lymph. The practice of blowing irritating matters through a quill into the eye is calculated to irritate the whole surface of the conjunctiva, entail suffering upon the animal, and do harm.

These opacities are, only when sufficiently large, or when so situated as to interfere with sight, to be regarded as causes of unsoundness.

The true Albugo, or Leucoma, is often seen in dogs, and seems to arise as spontaneous spots of opacity on the cornea, without previous inflammation, and generally as the result of malnutrition consequent on debility or disease, such as the distemper. Very young dogs are most prone to them, especially if improperly fed, or taken away from the mother too soon. They are best treated locally by the solid nitrate of silver, carefully anplied, and generally by good diet and cod-liver oil.

Since Percivall, Coleman, and others have passed away, writers upon veterinary surgery have described simple ophthalmia as if of every-day occurrence; but the fact is, it is a very rare thing to meet with instances of it as an idiopathic disease. Mr. I'ercivall says, writing in 1826-"First, as it attacks the human eye, it is considered as a spontaneous or idiopathic affection, and has been so named-ophthalmitis idiopathica; but the affection in horses has, I believe I may say unexceptionally, some apparent or assignable cause." Again the same authority writes, "In regard to the causes of common ophthalmia, I have already averred that they are local, mostly apparent, and not constitu- 
tional. Professor Coleman and our best modern vetcrinary writers all concur in this etiology; and I may add, that the disease would never probably have appeared in print in any other form had the subject not fallen into hands that have shown themselves unqualified by experience to pen any very correct or useful information about the matter." Evidently the truth of Percivall's observations seems to have been overlooked, facts ignored, and theories worked out, to suit each man's fancy, and idiopathic conjunctivitis described as a common affection.

\section{STAPIIYLOMA,}

A disease of the eye, so named from its being thought to resemble a grape. In this disease the cornea loses its transparency, rises above the level of the eye, and even projects beyond the eyelids in the form of a whitish-coloured tumour, which is sometimes smooth and sometimes rough on its surface.

Staphyloma is not a rare disease amongst dogs ; is occasionally seen in horned cattle; but I have not seen it in the horse in its true form, although a spurious staphyloma may sometimes be witnessed as a result of an incision through the cornea propria, allowing the bulging outwards of the eornea elastica.

Staphyloma, as occurring in the $\log$, seems to arise from two distinct pathological conditions-1st, a growth of a tumour of a compact, solid nature upon the cornea; $2 d$, a bulging of the cornea, caused by distension of the anterior chamber by an increased secretion of its natural contents (dropsy of the aqueous chambers). In this form the cornea yields to the distension produced by the turgescence of the humours of the eye, as the various serous sacs yield to an accumulation within them.

In the first form it will very often be found that a small ulcerous excavation exists in the centre of the tumour, and that the tendency of this ulcer is to eat its way through the cornea, and destroy the eye by allowing the escape of its contents. This form of staphyloma admits of considerable amelioration. If an ulcer be present, it should be touched slightly with the point of the nitrate of silver: this arrests the process of ulceration; afterwards the thickening can be removed by excision or by caustic. In dogs I have often transfixed the tumour with 
a needle and thread, and have cut it out with a scalpel; but should there be any difficulty in doing this, the nitrate of silver or caustic potash may be applied with freelom. The eschar of one cauterisation should be allowed to fall off, and the effects to subside, before the application of the caustic is repeated.

The staphyloma due to dropsy of the eye admits of but one remedy, and that may prove to be only palliative. The cornea is to be punctured, to allow the escape of the contained fluids. If this operation is not performed, the cornea will in time be absorbed; the contents of the eyeball will then escape, and the eye itself become entirely destroyed. This form of staphyloma is generally met with in pet dogs, especially the King Charles spaniel, and is a source of great grief to their lady owners. After the operation of puncturing the eyeball, purgatives and "short commons" are to be prescribed.

Ulcers on the cornea are best treated with the nitrate of silver, either in solution or in its solid form. If there is constitutional disease or debility, the general system must be attended to.

\section{DISEASES OF THE HUMOURS OF THE EYE.}

Very severe injuries to the eye may cause the character of the aqueous humour to be entirely changed. Instead of being a clear, colourless liquid, it becomes reddened with extravasated blood, milky-white by effusion of serum, or replaced by a solid fibrinous mass, which, after undergoing various transformations in colour, from bluish-white, amber, or brown, is finally converted into a pearly-white material, partially or wholly filling up the anterior chamber, causing total blindness of the eye affected, or interfering with vision to a very great extent.

\section{GLAUCOMA,}

A disease in which the vitreous humour loses its transparency, and assumes a blue colour. It is a very uncommon disease, and is usually associated with cataract or with amaurosis. It has been said that in glaucoma the hyaloid membrane covering the vitreous humour is absorbed, and that the humour is in an unconfined fluid condition. In some dissections that I have 
made, I have fourd that the vitreous humour has been converted into a semi-cartilaginous or even calcareous mass, and that instead of fluidity there was solidification of the humour and its membrane. The glaucoma of old age may occur without previous inflammation, and in dogs it most commonly occurs in this way; but in horses I have always found it associated with some traces of previous ophthalmia. The term blue can scarcely be applied to the lower animals, green or bluish-green being more significant. Melanosis of the humours has been observed.

\section{DISEASES OF TIIE NERVES OF TIIE ETE.}

Amaurosis, as a disease of the eye sui generis, is a condition of the optic nerve and its expansion-the retina-whereby they lose the power of receiving and transmitting the impressions of objects to the great nervous centre, the brain. Amaurosis as a disease of the optic nerve is incurable, but the condition often exists as a symptom of other diseases, more particularly of organic and other lesions of the brain, the effects of various vegetable and animal poisons, and of excessive hæmorrhage; but it is not my purpose to discuss these at the present time.

Amaurosis, gutta serena, or glass eye, in the horse presents the following symptoms:-A preternaturally dilated, round, and motionless pupil; the eye lucid or glassy in aspect; the eyelids opened more than natural-in fact, the animal may be said to stare. The gait and the motion of the ears are indicative of blindness.

It is possible to have amaurosis in one eye, but this is very rare in the lower animals, and is generally indicative of brain disease. Amaurosis with cataract is a common termination of constitutional ophthalmia. Excessive hæmorrhage has produced sudden but permanent amaurosis, both in the horse and cow. Can it be that, in such instances, the paralysis is due to the failure of the circulation in the arteria centralis retinæ? In the human subject, "amaurosis is sometimes due to exhaustion of the optic nervous apparatus, and is often a mere accompaniment of general nervous exhaustion, arising from great loss of blood, or excessive discharge of secretions," \&c.-(Wharton JoNES.) 
To detect the paralysed condition of the iris consequent upon the loss of power in the optic nerve, all that the practitioner has to do is to place the animal in a strong light. If the pupil remains round and large, although the eye presents no sign of organic change, amaurosis is present.

Percivall, Gamgee, and others recommend various remedies for maurosis. It is, however, quite useless to expect any good from them, the affection being incurable, when not symptomatic of some other disease. When it is so, the treatment must be directed to the removal of its cause. In horned cattle amaurosis is present in parturient apoplexy, hydatids in the brain, impactions of the stomach; in some rare instances, during the latter stage of hæmato-albuminuria, in syncope from heart disease or concussion of the brain, and during the fits of megrims.

\section{STRABISMUS-SQUINTING.}

Irregular action of the muscles of the eye never occurs in the lower animals except as a sign of another disease, as indigestion, tetanus, cerebral meningitis, acute uræmia or uræmic poisoning in the horse, and in lead-poisoning both in the horse and ox.

\section{ABYORMAL POSITION AND DISEASES OF THE EYELIDS.}

ECTROPIUM, OR EVERSION OF THE EYELIDS.

The eyelid is drawn away from the eyeball, its conjunctival surface turned out, and its ciliary margin displaced, downwards or upwards, according as the lower or upper lid is the seat of the ectropium. The eyeball, being thus deprived of the protection of the eyelid, is exposed to constant irritation, by which a chronic conjunctivitis is kept up, weakening the eye, and giving rise to specks and vascularity of the cornea.

This is a very rare condition of the eyelids. I have seen only one case of it in the horse: it was in the lower eyelid, and associated with the opposite condition-entropium-of the upper lid.

In the dog it is also rare compared to entropium, and always, in my experience, affects the lower lid.

Treatment.-If not very extensive, the conjunctiva moderately thickened, it may be reduced by the nitrate of silver, 
applied as follows:-The eyelid being everted to a greater extent, by traction on the neighbouring skin, the caustic is to be pencilled on the conjunctiva in a direction from one angle of the eye to the other, parallel to, but at a little distance from, the margin of the eyelid. After the application of the caustic the part is to be wiped with a bit of lint, and then pencilled with sweet oil. It is often advantageous to scarify the conjunctiva before applying the caustic. The cauterisation may be repeated in the course of three or four days; and when it is found insufficient to effect the desired object, it is better to have recourse to the excision of an elliptical-shaped piece of the thickened and sarcomatous conjunctiva parallel to the margin of the eyelid.

In the case of the horse referred to, the ectropium being large, excision was performed at once; and in order to effect this it became necessary to draw away the lid from the eyeball by traction on the neighbouring skin; the diseased conjunctiva was then pinched-up with strong forceps, and the piece cut off with a sharp scalpel. The piece removed must always be of such a breadth as appears sufficient, in order that when the cicatrisation is complete, the contraction of the conjunctiva may neither be so great as to invert the eyelid, nor so little as still to leave some degree of eversion. Further directions for the operation, when it appears necessary to modify the foregoing, may be obtained by referring to Joves' Ophthalmic Medicine.

\section{ENTROPIUM, OR INVERSION OF THE EYELIDS.}

This is the converse of ectropium; the free margins of the eyelid and the eyelashes are turned in against the eyeball, which they keep in a state of great irritation by the friction they exert upon it.

The margin of the eyelid may be inverted in part of its extent, constituting partial entropium, but more commonly the entropium is total. One eyelid only may be affected, or both lids of one or both eyes.

Pointer and setter dogs are very frequently thus affected; the horse very rarely. The distress occasioned by the friction of the margin of the lid and the eyelashes against the eyeball when an attempt is made to use the eye, must be very great. There is intolerance to light, which forces the animal to keep 
the eye closed to a greater or lesser extent. The conjunctivie become vascular, opaque, thickened, and ultimately dry and cuticular. There is redness of the lids, with increased secretion of tears, and other signs of conjunctivitis.

Very often entropium is congenital, but it may occur at any time of the animal's existence, from relaxation of the integuments of the eyelid and spasmodic contraction of the orbicularis palpebrarum muscle. Wharton Jones says, "In consequence of the firmness and breadth of its tarsal cartilage, and the existence of the levator palpebræ muscle, simple relaxation of the integuments of the upper eyelid seldom produces any great degree of entropium; it merely hinders the eyelid from being freely raised, constituting one form of ptosis (falling down of the upper eyelid). It is the lower eyelid which is most generally the seat of entropium from relaxation."

In this form of entropium the eyelid is simply rolled back upon itself, sometimes so much that the margin, with the cilia, lies in the inferior palpebral sinus of the conjunctiva. If the finger be applied to the outside of the eyelid, and the skin pressed down a little, the margin of the lid starts into its place, and will continue so of itself, so long as the eye is quiescent, but when the animal winks, it will fall back with a jerk into its former state of inversion.

Treatment consists in the excision of a portion of the relaxed integuments. The excised portion should be of an elliptical shape, and of such a breadth, that when the edges of the wound are brought together, the eyelid will be retained in its proper position. Though the piece of integument ought to be removed from as near as possible to the margin of the eyelid, a sufficient breadth of skin must be left at the margin for the insertion of stitches.

After the excision of the fold, the edges of the wound are to be brought together by metallic sutures, and left without further interference to heal by the first intention.

I have operated, and always with success, upon a great number of dogs and a few horses.

TRICHIASIS AND DISTICHIASIS.

Trichiasis is a growing-in of the eyelashes against the eye- 
ball, the border of the eyelid remaining in its proper position, which circumstance constitutes the distinction between it and entropium. Distichiasis is the converse of trichiasis.

Trichiasis is of frequent occurrence in dogs and cattle, but seldom seen in the horse, and it admits of being relieved only by the operation for entropium.

\section{WARTS ON THE EYELIDS.}

Warts are not uncommon on the cutaneous surface of the eyelids, or on their border. They are very often of a diffused encrusted nature, and difficult to remove.

If pedunculated, it is best to remove them by excision or ligature; if broad in the base, strong acetic acid is the best application.

\section{WOUNDS OF THE EVELIDS.}

Wounds of the eyelids are to be treated upon conservative principles; nothing must be destroyed; the edges are to be securely kept in apposition by fine silver suture wire.

The membrana nictitans is occasionally thickened by repeated attacks of inflammation; but little can be done, as it must on no account be excised; serving as it does instead of hands to the horse in the removal of foreign bodies. Warts sometimes grow on the membrana nictitans; these are to be carefully excised with the scalpel, being first transfixed by means of a suture, or the tenaculum.

\section{LACHRYMAL FISTULA.}

Iychrymal fistula is caused by a blow fracturing the bones through which the lachrymal duct runs.

\section{STRICTURE OF THE LACHRYMAL DUCT.}

This is caused by thickening of its lining mucous membrane; from catarrh of the nose extending into the duct, or from the specific inflammation of glanders. The tears flow over the side of the face, constituting what is termed "watery eye." 
Watery eye may also result from tumefaction of the eyelids, occasioning not only an increased secretion of tears, but a diminution in the size of the puncta lachrymalis, increased secretion of tears from the lodgment of an irritant in the eye, and from a catarrhal or other inflammation of it.

"The common seat of stricture of lachrymal duct appears to be at its superior part, and it is best treated by syringing with cold water from the puncta lachrymalis downwards, or it may be necessary to dilate it with a suitable bougie."-(Percivalu.)

\section{PARASITES.}

Worm in the eye is unknown in this country, but is met with in India, and sometimes in Canada. The best account of this parasitic disease that I have seen is contained in a letter from Mr. Charles Percivall, dated June 24th, 1825. ${ }^{1}$ He says- "In low humid situations in India, where fogs are prevalent, or where there is stagnant water, especialiy after an unusually wet season, worm in the eye is a very common occurrence. It is also seen in other parts during the cold months, from the begimning of October to the latter end of February, and particularly during the continuance of an easterly wind. The symptoms seem to be conjunctivitis, the cornea obscured by 'nebulous effusion,' the eyelids closed, and intolerance to light. On close inspection a small white worm can be discerned floating in the aqueous humour, at one time rising to the superior, at another sinking to the bottom of the chamber."

The method of treatment is by "puncturing the cornea at its upper and outer margin, and allowing the parasite to escape with the aqueous humour. This spot is selected for the operation because the cornea is here least dense; and the upper instead of the lower margin, because the aqueous humour, which gradually re-forms, will be less likely to again escape whilst the wound is healing, than if the incision had been made at the lower part."

The best instrument is a sharp-pointed scalpel, which should be pushed flatwise through the cornea, as near to its junction with the sclerotica as possible, making an oblique opening under the cornea; the sides of the wound will then fall into close contact with each other, and be in a favourable position for uniting 
by the first intention; whereas if the puncture be made directly through the substance of the cornea, whether its direction be perpendicular or horizontal, the sides of the wound will be pushed apart when the chamber begins to fill, and the healing process retarded.

I have not seen special directions for the operation by any Indian veterinary surgeon; but having performed the operation of puncturing the cornea for dropsy of the humours in the dog, I feel I am in a position to give preference to the method just described. Two kinds of worms have been found in the eye-the filaria oculi or filaria equi, and the strongylus (strongylus equinus) - and the same kinds of worms are also found in the intestines, the areolar tissue of the loins-supposed to produce the disease called kummivree-and in the blood-vessels. The filarixe are small white parasites, about an inch in length, of an attenuated and cylindrical form, having a mouth and anus, also an intestinal canal suspended in a cavity of the body, and, like other round worms, the sexes are distinct.

These worms find their way into the animal's body along with the water he drinks, either as fully developed parasites, or as ova (eggs). Both the parasites and their eggs are abundantly found in the stagnant waters of India.

\section{FUNGUS II AEMATODES.}

Fungus hæmatodes consists of a dark-coloured vascular tumour, growing within the cavity of the orbit, appearing at first as a small red spot at the posterior part of the eye; becoming larger, it involves the eye and the surrounding orbital bones. The tumour is malignant in its nature, being a very vascular medullary cancer; so vascular, or so infiltrated with blood, that it looks like a blood-clot; is of rapid growth, and if not entirely removed by excision at a very early stage, admits of no cure.

This form of cancer is most commonly met with in horned cattle; but I have repeatedly seen it in the horse. To remove it with any probability of success, the operation must be performed early ; everything within the orbit must be included in the operation, and the surface cauterised with the hot iron, not only to restrain the hæmorrhage, but to destroy any chance remains of the malignant growth. If the neighbouring glands are at all enlarged, 
they also must be removed. If the patient be a cow, ox or sheep, and if in fair condition, it should be slaughtered, as the tumour tends to return even if removed early, and death is apt to occur from marasmus, pain, and hæmorrhage.

\section{PERIODIC OPITHALMIA.}

Periodic ophthalmia is now rarely met with. Fifty years ago thousands of horses became annually blind from ophthalmia ; now-a-days one seldom sees a case of blindness from this cause. This happy result is due to the enlightened writings of Coleman on ventilation, and to the advance of veterinary science-facts which the public seem to ignore.

It has been variously termed "specific ophthalmia," " periodic ophthalmia," or "moon-blindness."

The term "periodic ophthalmia," is here retained, as it implies the recurrent or intermittent character of the disease; but it throws no light upon the pathology.

Periodic ophthalmia is a constitutional affection-cachectic or diathetic, probably rheumatic-arising from some calsse operating primarily on the constitution, and secondarily on the organ of vision, terminating in an opacity of the crystalline lens, termed cataract.

Symptoms.-The attack generally comes on suddenly, without ostensible cause, and in the night. The eye presents sigus of weakness, the upper lid droops, the eye seems smaller than its fellow, it is drawn into the orbit by the retractor muscle; removed, as it were, as far as possible from the light, which, from its sensitiveness, it cannot bear: intolerance to light is also manifested by repeated rapid nictitations, and by recoil of the globe within the orbit. This is especially evinced when a strong light is suddenly brought to bear on the eye-and it is further protected by the drooping of the lids, its natural curtain; the membrana nictitans projects; the tears flow over the lower lid and bedew the face. When the eye is more closely inspected, the cornea is dull; the dulness, at first most intense at its margin, but soon spreading over its surface, rendering a view of the interior structure of the eye an impossibility. The dulness is inclined to an amber colour; and it was maintained by Professor Dick that this was due to the fulness and pressure 
of the parts belind the cornea, and, says the Professor, "Were that once relieved, the cornea would immediately regain its transparency."-(Dick's Manual of Veterinary Science.) I think, however, that an impartial investigation of the matter will prove that the opacity is due to the cornea being involved in the inflammation.

Percivall, describing the opacity of the cornea and anterior chamber, says - "At the beginning, the anterior chamber preserves its pellucidity, so that the iris and pupil can be seen, the latter contracted, the former unchanged in colour; in the course of two or three days, sometimes earlier, the chamber becomes obscured by a dingy-white or amber-coloured deposit floating within it, through which the pupil is hardly discernible, contracted, and looking much like the black eye of a garden bean." The opacity of the cornea proceeds from its circumference to its centre, until at last the whole of its surface becomes of a dull greyish hue, and in some cases blood-vessels are seen ramifying over it. When the dulness is great, the iris is invisible, but when it can be seen, it will be found that the pupil is narrow and contracted, the eye altogether presenting evidence of intolerance to light. In some instances direct evidence of iritis can be distinguished early in the disease, the iris being of a dead amber colour from a deposit of lymph on its surface, as well as upon the corpora nigra.

Some writers are inclined to give preference to some one particular structure of the eye as the seat of the disease. I am of opinion that it may be considered as panophthalmitis, or inflammation of the whole eye, commencing primarily as "ophthalmia interna posterior."

The remaining symptoms are turbidity of the aqueous humour; the corpora nigra lose their jetty blackness; the pupil becomes more and more contracted; the conjunctiva intensely reddened; and in some cases the vessels crowd around the margins of the cornea, across which numbers of them shoot in irregular lines; occasionally pus forms in the anterior chamber (hyopyon); and in rare instances the inflammation may terminate in suppuration of the entire coats, and consequent disruption of their contents, but the common termination is cataract.

The inflammation is apt to move from one eye to the other, and for this reason, and on account of its recurrent nature, the disease has been described as "gouty ophthalmia." 
The subsidence of the attack is marked by a diminution of the inflammatory symptoms, the haziness slowly disappears from the cornea, the pupil becomes larger, less contracted, rounder, disclosing the lens altered to a hazy grey colour, the result of change in its substance, or a deposit on its surface, the prelude to cataract; the pupil in many instances does not regain its former dimensions, even after a first attack, the iris having become fixed by adhesion; the cornea may regain its natural hue, but the iris is more or less permanently altered in aspect and colour, and the eye seems smaller than natural: this is caused by its continuing in an irritable condition, and its being drawn backwards by the retractor muscle, even after the apparent subsidence of the disease. As time advances, the opacity of the various structures becomes less apparent; but sometimes the cornea is clouded by an interlaminal deposit, and generally the eyebrow remains in a peculiarly wrinkled condition. This wrinkled appearance of the eyebrow after the acute symptoms have passed off, is characteristic of periodic ophthalmia. The eye, having regained more or less of its natural appearance, may remain free from active disease for an indefinite period. In some instances the disappearance of the acute symptoms may go on satisfactorily for three or four days, when, without any appreciable cause, the eye is again found highly inflamed, the eyelids closed, and the animal seemingly suffering intense pain; whilst in other instances the active sigus of the disease totally disappear for several weeks or months, and then recur again and again, until cataract is fully developed; other cases are marked by complete subsidence of the disease in one eye, and its appearance in the other; its mode of procedure in such cases is to attack one eye, then the other, until both are permanently altered in structure, and the animal is left totally blind.

Ordinarily, blindness and complete disorganization are followed by a cessation of the paroxysms; but there are exceptions to this, and I have met with cases where acute inflammation has recurred periodically for years after a cataract has been fully developed.

It is quite possible that one attack may disorganize the organ to such an extent as to cause it to assume the appearance of a bulbous mass, the iris driven into the aqueous chamher, adherent to the cornea; the lens dislocated; and all the structures, 
including even the vitreous humour, so cemented together that scarcely a vestige of the original appearance of the eye is discernible. These instances are, however, rare, and mark the severity of the inflammation; the majority of cases being of a much milder type, recurring and finally ending in cataract, ossification of the choroid, retina, and the whole contents of the chambers.

The late Professor Coleman, from very extensive observations made at a time when this disease was exceedingly prevalent, arrived at the conclusion that the affection resulted from the same conditions as those which engendered glanders and farcy, namely, contaminated atmosphere, resulting from ill-paved, illdrained, unventilated stables; and Mr. Percivall, in discussing the subject, after adducing evidence that mules and asses were quite as subject as horses to ophthalmia during the Peninsular war, says - " Shall we say, by way of a summary rationale, that the effluvia arising from the breath, perspiration, urine, dung, \&c., generate an animal poison, and that this, being in a gaseous form, is diffused through the atmosphere of the stable, and with it carried into the system through the medium of the air-passages, skin, or alimentary canal-most likely by the first, but. possibly by one of them-wherein it breeds disease in the circulating fluids, which breaks out, under a local form, in the lungs, skin, nose, eyes, \&c.? Or shall we say that these parts are affected locally in the first instance, and subsequently contaminate the system? Let us postpone the consideration of an answer until we come to include glanders in the same family of poisonous influences." Professor Coleman adduces as proofs that it is not a local affection:- " 1 st. The constitutional derangement, which he says may often be traced to the digestive organs and skin, and is in some respects unlike simple irritative disorder; $2 d$. The character of the inflammation, which is neither so acute nor so rapidly progressive as simple ophthalmia often is, and yet the one readily yields to proper treatment, whilst the other obstinately continues its course; $3 d$. The eye may be locally exposed to volatile ammonia, or any animal effluvium we can obtain by artificial means, and no such effect will be produced."-(Percivall's Lectures on the Veterinary Art.)

I have nothing to suggest with regard to successful treatment. The disease is incurable, and has baftled the efforts of all who 
have endeavoured to combat it. It is very true that the inflammatory stage is seemingly modified and cut short by varions remedies; but the disease recurs, and terminates only when the sight is completely destroyed. Some writers affirm that when it attacks both eyes alternately it is better to destroy the one and save the other-that when one is destroyed it occurs no more. Surely this is a mistake. I have over and over again seen it attack one eye repeatedly until cataract was completely developed, then attack the other until the same result was produced.

An acute attack is always accompanied by febrile disturbance; to relieve this a purgative is useful, to be succeeded by febrifuges, such as the nitrate of potash, aconite; and, acting upon the knowledge that effete materials are circulating in the system, colchicum may be given with advantage. The local pain is to be soothed by fomentations, anodyne collyria and belladonua, but no irritating materials should be made use of.

Prevention is of greater importance than attempts to cure what has already proved itself beyond the reach of any and every known remedy.

Horses subject to this disease continually change owners; and, as a legal question, the ability to discriminate between the healthy and unsound eye hecomes a matter of great importance to the veterinarian; and Mr. Percivall says, very correctly"Any single one of the following appearances may, unless its origin can be satisfactorily traced to simple ophthalmia, excite our suspicions; but a combination will warrant an unfavourable prognostic:-A sunken or gloomy aspect of the eye altogether, compared with the other; prominence of the membrana nictitans; vascularity or pinkness of the conjunctiva; a watery state of the eye; dimness or cloudiness of the cornea, particularly around its margin; dulness or discoloration of the iris ; corpora. nigra yellowish, whitish, or spotty; pupil smaller than the other; perhaps hazy or milky, or containing a minute white speck in its centre, which is an incipient cataract."

Another symptom, and one of great importance, which Mr. Percivall does not notice, is the wrinkled or furrowed appearance of the upper lid and eyebrow.

The common termination of periodic ophthalmia, viz., eataract, is a very important subject to consider in its medicolegal aspect. 
A cataract is usually developed as follows:- " A grey or white speck is seen, after the subsidence of the acute inflammatory stage of periodic ophthalmia, in the centre of the pupil; this grows at each successive attack until it quite fills up the aperture; vision grows less and less distinct during its formation. The growth of the incipient speck during the time that the organ is suffering from ophthalmia is commonly rapid; but as soon as the inflammatory action has subsided, its progress becomes comparatively slow, and now and then it appears to remain quite stationary; for as soon as cataract has formed, the inflammation generally begins to decline, and the eye seldom receives another attack; there are, however, some exceptions to this. And it is also, I believe, an established fact, that not only does the inflammation permanently leave the cataractous eye, but, should the other have remained free from the disease, it is in less danger of being attacked. Under such circumstances, therefore, a cataract is hailed as a favourable omen; this is explicable, seemingly, on the principle of sympathy, which is known to have great influence in ophthalmic pathology, and the circumstance has been considered weighty enough to recommend the artificial destruction of one eye in disease to preserve the other in health."-(PercivalL.)

Although cataract is generally a sequel to periodic ophthalmia, it by no means follows that this is the only cause. Many instances of fully developed eataracts in both eyes have come under my immediate observation without any preceding inflammation. At the present time I have a carriage-horse under my care. Four years ago I examined him as to his soundness, and passed him. I have seen him continually during this period, and am positive he has not suffered from any acute disease of the eyes. Some two months ago he began to exhibit symptoms of defective vision, by shying, \&c. Upon examination, I found a star-like cataract in each eye; these have rapidly increased in size, and they now embrace nearly the whole structure of the lens, but there is not a trace of inflammation in either eye, nor has there been any since I first examined him.

Writers upon human ophthalmic surgery ascribe cataract to a great variety of causes.

1st. In some cases a blow in the eye, without any penetration of its tunics, ruptures the capsule; while in others, cataract, 
generally attended by amaurosis, follows a blow on the eye, or a blow or fall on the edge of the orbit, without any apparent rupture or dislocation. This effect may not show itself for several years after the injury."-(Mackenzie's Practical Treatise on Eye, 1854.)

"The lens may become opaque in consequence of a blow or concussion of the eye without solution of continuity. I have seen many such instances.

"In a patient who had received a blow in the eye from the fist, seen by Beer in twenty-four hours after the accident, the capsule was torn, the lens split in two and quite opaque, there was slight effusion of blood into the anterior chamber, and considerable ecchymosis of the conjunctiva."-(LAURENCE on the Eye, 1844.)

$2 d$. "Lenticular cataract consists in a marasmus and opacity of the proper substance of the lens, and not in any opaque deposit; but nothing is known of the exact nature of the change. It may be looked upon in some degree as a natural effect of old age."-(Wharton Jones.)

The late Sir David Brewster was of opinion that at least one cause of cataract was an inordinate saline condition of the aqueous humour, and that, owing to this extreme salinity of the humour (upon the principle of exosmosis and endosmosis), the fluid contained in the lens became diminished, its concentric laminæ being thus separated from each other, and that the proper treatment for cataract was abstraction of the aqueous humour by puncturing the cornea, after which the patient was to abstain from partaking of salt with food.

All cataracts have been classified under two heads, namely, the true and the spurious.

\section{TRUE CATARACTS.}

The opacity may be seated in the lens itself, or in its capsule, or in both lens and capsule at the same time; different kinds of true cataracts are accordingly described, namely, lenticular, capsular, and capsulo-lenticular. The distinction of these different kinds is not of such great importance to the veterinarian as to the human oculist, as operation for the removal of 
cataract is inadmissible in the lower animals; total blindness being preferable to imperfect vision.

Again, cataracts are described by the terms hard and soft. Hard cataract is that commonly seen in the horse; in the dog soft cataract is generally met with; it is a very common disease in this animal, and is not preceded by any signs of inflammation. It presents in its earlier stages a glistening tendinous aspect, and a stellate appearance, resembling a healthy lens made opaque by the action of re-agents. The lens seems larger than natural, and the pupil is generally dilated.

Pet dogs that are over-fed, especially those which are fond of sugar, frequently become blind from cataract. I have known some cases apparently recover under a more healthy regimen, and when compelled to take exercise, but ordinarily the lens assumes the appearance of broken spermaceti.

Spurious or false cataracts, so called in contra-distinction to opacities of the lens, consist in opaque deposits of lymph, blood, or pus on the anterior capsule, obstructing the pupil. They may originate in severe traumatic ophthalmia, or they may co-exist with true cataract, and arise from the same cause. As a medico-legal question, the time necessary for the formation of cataract becomes a subject of great importance. The case of Lord Randolph Churchill versus Day has an important bearing upon it, as well as upon the liability of veterinary surgeons.See Veterinarian, 1871.

Usually cataract is only completely developed after repeated attacks of periodic ophthalmia, and in a period of time varying from two to twelve months; but there are cases where one attack is sufficiently severe to destroy not only the transparency of the lens, but the integrity of the whole organ of vision; and, to state what has happened in my own experience, a fully formed cataract has been developed in ten days from the commencement of an attack of ophthalmia; the opacity of the anterior portion of the eye being removed to such an extent as to allow the cataract to be plainly visible. Such an instance as this is rare.

I have already stated that cataract in the horse may proceed from other causes than inflammation, and it will be well to bear this in mind, shonld an action at law, similar to the one already quoted, be raised at some future time. 


\section{DISLOCATION OF THE EYEBALI.}

This is not at all an uncommon occurrence in dogs, particularly in those beautiful-eyed ones, the King Charles spaniel. The cause is accidental, a fight with another dog, or more commonly with a cat. The eye is actually "scratched out," and hangs pendulous on the cheek. In all such cases the eye must be returned, and, wonderful to relate, the after usefulness of the organ is not always impaired.

The method of returning the eye is as follows :-After washing away all extraneous matters, dirt, blood, \&c., with tepid water, let an assistant, who is to stand behind the dog, open the eyelids as far apart as possible, then the operator is to press gently, but firmly, upon each side of the anterior aspect of the dislocated globe with the balls of his thumbs until the globe is replaced within the orbit. But should such pressure prove ineffectual, the outer angle of the eyelid is to be divided with a pair of scissors or bistoury-a small snip is sufficient-when the eye can be replaced without difficulty; the incision in the lids being afterwards drawn together by a single suture. It must be borne in mind that in reducing dislocation of the eye, extreme pressure must be avoided, or the eye will be irretrievably damaged, and that pressure is to be exerted more upon the sides than upon the front of the globe, for pressure on the front will flatten it, and cause it to bulge laterally.

\section{REMOVAL OF THE EYEBALL.}

Except in malignant disease, it is not necessary to remove the whole of the eye; if the cornea is removed, the humours, lens, \&c., escape, the eye will collapse, and its various coats become adherent. Removal of the eye is sometimes necessary in dogs suffering from irritating staphyloma or incurable ulcers on the cornea, and in the horse after severe injuries to the eye, resulting in permanent tumefaction and enlargement of the whole organ, preventing closure of the eyelids, and subjecting the animal to much pain from the irritation of dust or other foreign bodies.

\section{EXAMINATION OF THE EYE.}

An ordinary examination of the eye is made by turning the 
horse's head away from a strong light, shading the eye with a black hat, and obscuring any white article of clothing that the examiner may be wearing at the time. But in doubtful cases of soundness of the eyes, a more searching test is required, and the mode of examination is by reflection of light, callerl the catoptric test. For the application of this test, the horse must be put in a dark place, and should the pupil be sensitive to light, it must be dilated with belladonna.

When a lighted candle is moved before the healthy eye, three images of it may be observed. First, the erect image, that moves in the same direction as the candle, is produced by reflection from the surface of the cornea; secondly, another erect image, produced from the anterior surface of the crystalline lens, which also moves in the same direction; and thirdly, a small inverted image, situated between the other two, is reflected from the posterior surface of the lens, and moves in the contrary direction to the others and to the movements of the candle

In cataract the inverted image is rendered indistinct or abolished, and the second erect one sometimes indistinct also. In glaucoma, the deep (or second) erect image is rendered more evident than in a sound eye, and it is only in a very advanced stage that the inverted image is obliterated. In amaurosis all the images are as distinct as in the healthy eye, but there is no contraction or dilatation of the pupils as in health.

\section{EXAMINATION BY THE OPITHALMOSCOPE.}

Very small cataracts are discoverable only with the aid of vis instrument; the catoptric test not being always sufficiently satisfactory.

In order to examine with the ophthalmoscope, much practice with the instrument is required, and the veterinarian should become an adept in its use before resorting to it in an important case.

A dark place is necessary, and the examination is much facilitated by first dilating the pupil with belladonna.

The examiner is to stand in front of the horse, with the instrument in his hand. An assistant stands near the horse's shoulder, holding a lighted candle (this is better than a stronger light). The examiner is to manipulate his instrument until the light of the candle is reflected into the horse's eye; in many 
cases this is sufficient to bring an opacity of the lens distinctly into view, a cataract looking like a dark or black spot in the illuminated eye; but should this not prove sufficient, the eye may be magnified by the lens which accompanies the ophthalmoscope, and the examiner can limit his vision to the eye by looking through the small aperture at the back of the instrument.

It may here be mentioned that the small instruments made use of by oculists are the best; the larger ones made by veterinary instrument makers are not nearly so good, and are apt to deceive. 


\section{CHAPTER XXXII.}

DISEASES OF THE HEAD, NECK, VEINS, ETC.

OTORRHEA-EXTERNAL CANKER OF THE EAR-POLL-EVIL-FISTULOCS WITIERS-CIRCUMSCRIBED AND DIFFUSE PHLEBITIS-THROMBUS -VARICOSE VEINS-PHLEBOLITES-ENTRANCE OF AIR INTO THE VEINS.

\section{DISEASES OF THE EAR.}

WITH the exception of liability to accidental injuries-and gangrene of the concha sometimes seen in scarlatina-the ear of the horse seems exempt from disease; the dog, however, is subject to two well-known forms of disease of the ear, namely, internal and external canker.

Internal Canker, Otorrhoca, is an inflammation of the lining membrane of the meatus auditorius externus, with a discharge of purulent matter, sometimes mixed with blood, which may occasionally coagulate, block up the tube, and cause deafness. When the inflammation extends to the internal ear it constitutes "otitis," a most painful affection, causing constitutional disturbance, in some instances delirium, coma, and death. The inflammation of the mucous lining of the outer meatus is very often aggravated, and otitis produced by irrational treatment.

The disease occurs chiefly in spaniels and retrievers, but no breed is exempt; the practice of "cropping the ears" does not seem to predispose to it; indeed, it is mostly met with in dogs with pendulous ears. The origin of the disease is local or constitutional ; the local, caused by the lodgment of water, dirt, or any foreign matter in the meatus; and the constitutional, arising from improper and over-feeding, want of exercise, catarrhal affections, and the extension of skin diseases.

Treatment.-When of local origin, the treatment must be directed to the subjugation of the inflammation by local soothing 
applications, such as fomentations to the part, weak solutions of acetate of lead and opium applied warm (poured, not injected) to the ears. A purgative should be administered in the first stages, and the animal kept on a cooling diet for a few days.

This method of treatment will generally afford relief; but should the disease continue, a stronger astringent is to be applied, or it may be necessary to stimulate the part to healthy action by the nitrate of silver, of the strength of five grains to the ounce of water. As an astringent, a solution of the terchloride of iron is a very effectual one, and it may be necessary to inject it with a syringe: however, great caution must be exercised, and violent remedies avoided, if possible, in the treatment of what is already a painful affection. If this caution is not exercised, much suffering is inflicted upon a dumb beast, and a tractable disease rendered incurable. If the dog be in a debilitated condition, either from the long continuance of the disease, or want of proper diet and attention, he must be supported with good food, and stimulated with tonics, the sulphate of quinine more particularly.

In those painful forms where acute otitis exists with febrile disturbance, great restlessness; and the animal often howling with pain, rubbing his ears and head with his paws, and evidencing signs of much irritability, the general and local treatment must be of an active kind; continual fomentations with warm water, or a decoction of poppies must be prescribed; a seton inserted behind the ear, and, in addition to a brisk cathartic, small and repeated doses of the tincture of aconite, or a solution of morphia, are to be administered.

When the cause is constitutional, the general health of the dog must be attended to, and in some cases I have found it necessary to prescribe a continuance of tonic alteratives, such as the liquor arsenicalis, with an occasional aperient.

\section{EXTERNAL CANKER.}

This is an abraded or wounded condition of the tip of the ear, arising from accidental injury, or from the dog, especially a water dog, violently shaking his head and ears. Rarely is this affection found in any but long-eared dogs, and in them, owing to the habit of shaking the head, it is almost an incurable disease. 
The abrasion or wound may at first be exceedingly small, a mere bruise on the border of the ear; but after a time the tip of the ear commences to split, the cleavage being increased by the repeated shakings of the head and violent flapping of the ears. The treatment must first be directed to the prevention of this shaking, and for this purpose a net-cap must be fastened around the dog's head and ears. A French authority recommends the application of a blister over the external surface of the ear. This allays the intolerable itching, and substitutes a smart pain, which prevents the shaking of the ear. This precaution against further injury, with cleanliness, a purgative, regular food and exercise, a gentle touch of the nitrate of silver, and, as in internal canker, preventing the dog from "taking the water," will generally effect a cure. If the ear be split to any depth, and if, after a recovery and removal of the cap, the wound again begins to spread, the edges of the cleft must be pared and brought together by fine-metallic-sutures, and at the same time all diseased portions upon the tip removed with the knife. If the edges do not completely unite after one operation, it must be repeated until they do so; and failing to cure by these means, the dog must be "cropped."

\section{POLL-EVIL.}

This is a fistulous ulcer situated on the supero-posterior portion of the cranium immediately behind the ears of the horse, and is caused by accidental violence, or by the habitual use of a tight bearing-rein.

At the first stage it may be recognised as a soft fluctuating tumour surrounded by inflammatory swelling, enlargement of the superior cervical lymphaties, and stiffness of the neck; or the inflammation of the surrounding tissue may have subsided, leaving a prominent swelling-a serous abscess.

Treatment proper before pus is formed.-Reduce the inflammation, if present, by the application of cold water to the part, and by the administration of purgatives internally. When the inflammation is overcome, reduce the swelling by friction with iodine, but do not puncture; but if suppuration is established, the abscess cannot be opened too soon, and it is best to make the incision at its base, to allow the pus to escape from 
its most inferior part. For this purpose, a free incision is to be made, and it must not be allowed to close too soon; fomentaticns are to be repeatedly applied, but no special application is required, the ordinary treatment for abscess being all that is necessary.

If the abscess has already burst, is discharging a fotid unhealthy pus, and the opening is surrounded with fungous granulations, the disease will be found to have assumed a most formidable aspect; for not only will the subcutaneous areolar and muscular tissues be involved, but the ligamentum nuchoe also; which, being tough and elastic, imprisons the pus, causing it to burrow in various directions, and, having a low organization, its healing powers are exceedingly torpid. The treatment of a case of this description is a matter of some difficulty, and to be complete, a thorough examination must be made with the probe of all the fistulous ulcers and sinuses. These must be freely laid open to their very base, and the whole dressed with a solution of the bichloride of mercury, the chloride of zinc, or the muriate of antimony; a thin layer of the parts laid open will thus be destroyed, and the whole converted into a common wound. The repeated applications of caustics and escharotics are not required, indeed they do much harm; when once the part is converted into a healthy-looking wound, all that is necessary is to keep it clean, dress with mild astringents, and prevent the opening from closing too quickly.

In many cases, setons inserted from the original opening along the direction of the fistulæ, and brought out upon the opposite side of the poll, are very successful; but if there be many sinuses, the seton treatment is not to be depended upon, and it is better to use the knife freely.

When poll-evil is caused by the bearing-rein, it is deepseated, perhaps involving the synovial membrane of the occipitoatloidean articulation, and some cases have terminated in anchylosis of this joint, causing the animal to be permanently stiff-necked. In some instances the ulcerative process has penetrated the capsules of the first or second cervical articulations, causing sudden death by pressure upon the medulla spinalis; in others, pieces of the bones become detached, keeping up the irritation and formation of pus; these must bo searched for and carefully removed. 
FISTULOUS WITHERS.

Fistulous withers resembles poll-evil in all particulars except its seat. It is caused by bruises from ill-fitting saddles; but some horses, from the conformation of the withers, are more prone to be thus injured than nthers. Horses with high withers are particularly liable to the injury. Sometimes the spines of the dorsal vertebræ become affected with chronic inflammation from repeated bruisings; their extremities spread out, or some portions of them become necrosed. These are complications which must be provided for in the treatment, which is to be conducted upon the same principles as that for poll-evil, namely, to make a depending orifice for the discharge of purulent matter, either by seton, incision, or by a counter-opening made at a point below the bottom of the sinus.

\section{INFLAMMATION OF THE JUGULAR VEIN.}

Inflammation of the jugular vein occurs as a sequel to bleeding (phlebotomy), and is due to a constitutional predisposition to local inflammation - as in that condition co-existent with acute laminitis-accidental disturbance of the wound after the operation, or to carelessness on the part of the operator.

In order to secure the healing of the puncture made by the lancet or fleam, by immediate union, it is necessary to bring the lips of the wound immediately and closely together. The blood, if any, on its surface is thus pressed out, and the divided bloodvessels and nerves are brought into perfect contact. Union may thus be effected in a few hours. If this method of union is not secured, the wound may heal by the adhesive inflammation; but if suppuration occur, the inflammation will in all probability extend to the coats of the vein.

The more immediate causes of phlebitis are said to be, using a rusty fleam, bungling in performing the operation, or from the animal rubbing the pin against the stall or manger.

Pathology.-Inflammation of a vein is characterised by a reddening of all its coats, owing to congestion of its vasa vasorum; an exudation is poured out from the coats of the vein, which, along with its contents, forms a solid coaguium. The experiments of Gendrin go to prove that the clot is not 
wholly due to coagulation of the blood contained in the inflamed portion of the vein; for he found, when a portion of a vein was insulated by securing it between two ligatures, emptied of its blood, and inflammation excited by an irritant injection, that a plastic solid substance was formed, which filled up the whole calibre of the vessel. In some instances the plug is formed of concentric layers of straw-coloured lymph, with a dark sanguineous clot in its centre. At first the clot is but loosely attached to the interior of the vessel, but subsequently it becomes more strongly adherent, the surrounding areolar tissue infiltrated with serum, and that in contact with the vein adherent to it by a fibrinous exudate. In this manner the vein and surrounding textures are firmly united.

Traumatic inflammation of the jugular vein is always circumscribed-sometimes "suppurative," sometimes "fibrinous." The thrombus or coagulum extends in an upward direction (from the wound) considerably beyond the primary seat of the inflammation, and terminates in a conical point. The inflammation may terminate by this clot becoming organized, and the vein converted into an impervious cord, without the advent of the suppurative process ; but in the majority of cases one or two circumscribed swellings appear along the course of the coagulum, in which fluctuation and the other characters of abscesses may be detected. The pus in these abscesses is confined by a coagulum, above and below, which forms a barrier to its admission into the general circulation.

Whilst these changes are going on in the inflamed vein, the lips of the wound are separated, everted, are redder than natural, and discharge a sanious material, mingled with blood; the surrounding parts also are in a state of tumefaction and tension, whilst the vein in its course towards the head conveys the sensation to the fingers of a hard cord of considerable thickness.

It seems peculiar that the formation of the thrombus or clot should extend towards the head and from the heart in inflammation of the jugulars, and towards the heart when other veins, such as the brachials or saphenæ, are accidentally inflamed. We are not to suppose that the inflammation does not extend below as well as above the puncture in phlebitis of the jugular; indeed, a thickening of the coats of the vessel can be distinctly felt through the skin, but the obliterating clot is not found 
below the seat of puncture; and Mr. Percivall, in treating on this subject, has been at much pains to account for this peculiar disposition in the inflammation to extend so generally towards the head when the jugular is punctured, and towards the heart when it takes place in any other vein; being in the one instance against the course of the circulation, and in the other with it, as is invariably seen in the human being. To reconcile these seeming discrepancies, Mr. Percivall observes, "that although the $o b$ structed state of the vessel is not the exciting cause of the inflammation, it invariably directs the course of it. It therefore remained to inquire in what manner the obstruction was prevented in the previous course of other veins similarly affected, as the saphena and plate vein of the horse, and the basilic and cephalic of the human." Now these veins, Mr. Percivall ingeniously argues, freely anastomosing with contiguous trunks, preserve a continual flow of blood up to the obliterated part; but above this, such anastomosis does not exist, the communicating branches being few and small; consequently, the blood remains to coagulate and to continue the disease. The jugular is similarly situated upwards, for it has no anastomosis to carry off the obstructed blood above the puncture, in which direction, therefore, the inflammation proceeds, the obstruction being prevented downwards towards the heart; for, having once emptied itself, the inflammation and tumefaction will prevent its receiving more blood, by which means no offending coagulum remains. Thus Mr. Percivall argues that the deviation from what is considered as a fixed law in human pathology-that this inflammation always occasions obliteration in the vein towards the heart-is thus reconciled, and that " the same cause is operating under different circumstances." -Lectures, vol. i., p. 103.

Speaking to Professor Spence of this peculiar tendency in the clot to extend upwards in inflammation of the jugulars, he kindly showed me a specimen of inflammation of these veins in man, where the thrombi extended upwards even to the cranial sinuses. From this circumstance I think we may safely conclude that the same law applies in man as in the horse.

The best treatment for circumscribed phlebitis is the application of a smart canthariules blister along the course of the inflamed part, and a cure will be effected in a very short time. The blister is to be applied whether suppuration be present or 
otherwise. If abscesses have already formed, they must be opened before the blister is applied, for if this precaution be not taken, the pus may break down the barrier which surrounds it, thus entering the circulation, and leading to very serious or even fatal consequences.

I find that writers upon human surgery recommend leeches, fomentations, poultices, and other methods of treatment which -in veterinary practice, at least-have been found to retard the healing process, and I would suggest very respectfully that the method by blistering is worthy of their consideration.

Inflammation of the jugular always terminates in the conversion of the vessel into an impervious cord, by the organization of the clot; and for this reason a horse having suffered from it should not be turned out to grass, as the collateral circulation seems to be insufficient to convey the blood from the head whilst it is kept in the depending position during grazing; and as a consequence much serum is effused into the areolar tissue of the face, cheeks, lips, and nostrils, interfering with the process of mastication; and by diminishing the calibre of the nostrils, preventing the due admission of air into the lungs.

\section{DIFFUSE PHLEBITIS.}

This originates from irritation of a vein, as from punctured wounds in their vicinity, or it may supervene on the circumscribed form, the barrier to the admission of the pus-already described-giving way from some cause. The symptoms differ from those of the circumscribed form by extension of the swelling along the course of the vein, swelling of the lymphatics, acute œdema, and tension of the part resembling an erysipelatous inflammation.

In acute diffuse phlebitis the inflammation extends along the lining membrane; fibrinous deposits are formed in various parts, and even in the right side of the heart. In other cases, where the progress of the disease is slower, the joints become affected by purulent deposits.

Wherever there is extensive venous circulation, as in the lungs, liver, joints, and intermuscular areolar tissue, there venous congestion and effusion take place; inflammation and abscesses follow. The following case may advantageously be 
here noted:-A mare, whilst being fastened to the carriage, ran away; the carriage was caught by a protruding wall, and she was thrown with violence, the elbow and arm being lacerated extensively. The wounds were treated with care. On the third day the patient commenced to shiver; the swelling increased to a great extent, the leg down to the foot having the appearance of a tense odematous infiltration, the shoulder and neck rapidly assuming the same condition; the breathing became hurried, the pulse rapid and feeble; the appetite entirely left her; and from this time she rapidly sank, and died in two days. The post mortem revealed the lungs in a state of excessive congestion, and permeated almost throughout with commencing suppuration; the cavæ, jugular, axillary, radial, brachial, humeral, metacarpal, and even plantar veins of that side were found inflamed throughout, their coats congested, thickened, and surrounded by a semi-purulent fibrinous deposit, whilst fibrinous clots were found within them. The elbow, knee, and fetlock joints were highly inflamed, and contained much unhealthy synovia. The onset of phlebitis is characterised by a fit of rigor, succeeded by great constitutional disturbance and the symptoms already mentioned.

\section{THROMBUS.}

Occasionally after the operation of bleeding there is extravasation of blood into the areolar tissue surrounding the puncture, including considerable swelling of the parts; this is called a thrombus, and is caused by the operator drawing the skin too far out whilst introducing the pin; by the opening in the skin being too small to allow the free escape of the blood, or by the vein being loosely attached to the surrounding parts, and rolling during the operation. However, no bad consequences follow if the opening be closed, a cold wet sponge held to the part for a short time, and the animal's head tied to the rack.

\section{VARICOSE VEINS}

Are not frequently seen in the lower animals. Occasionally the jugular presents a varicose dilatation after it has been bled from, as if its coats had been weakened at the part by the 
operation. The saphena is sometimes dilated by pressure of a " bone-spavin," or by very prominent cuneiform bones. A case of a very large varix on the course of the saphena was published in the Edinburgh Veterinary Review, by Mr. Hunt, Birmingham.

The veins of the extremities of horned cattle present varicose dilatations along their course in the form of sacculated or knotty protuberances on various parts of the vessels; the contained blood is at first in a fluid state; but an alteration not unfrequently occurs, the blood coagulates, and the vessel becomes obstructed.

The formation of these coagula is an effect of inflammation in the coats of the vein; this inflammation may be slight, or it may run on to suppuration, giving rise to small abscesses. I have repeatedly met with this form of phlebitis in cattle underfed and kept in wet, cold situations. It seems to arise from debility of the circulation, and relaxation or want of tone in the coats of the vessels.

The treatment for dilatations without inflammation :Better food, warmth, and comfort; tonics, and pressure by bandages; and, in addition, when suppuration is established, the abscesses are to be opened, and blisters applied, but no pressure.

If a large varix, without any tendency to inflammation and formation of a clot, should be met with by the veterinary practitioner, the vein is to be obliterated; and the method at present adopted is to introduce two needles, one above and one below the dilatation, kept in these positions by a thread tied around them so as to cause mediate pressure on the venous coats. The needles are not removed, but allowed to ulcerate their way through. Care must be taken not to introduce the needles into the vein, but underneath it, so as to ensure that the circulation is absolutely cut off.

\section{PHLEBOLITES.}

Phlebolites or Vein-Stones.-Concretions have been found in dilated veins in the neck, and other parts. They are formed by the calcareous degeneration of coagula, and are composed of the phosphates of lime and magnesia. An interesting case is reported in MorTox's work, On Calculous Concretions. 


\section{ENTRANCE OF AIR INTO VEINS.}

Air rapidly injected into a vein causes immediate death, but if injected slowly it only causes great distress. The accidental admission of air into the veins has often resulted in a fatal termination, and advantage has been taken of the knowledge so gained, for the purpose of destroying old or otherwise worthless horses by this easy mode of death. An accidental admission of air into a vein, causing death, may occur after bleeding; for example, if the pressure upon the jugular which is maintained by the finger or vessel containing the blood be suddenly withdrawn, a vacuum is formed, into which the air rushes with a hissing, gurgling, or sucking sound. The animal is suddenly seized with symptoms of faintness and convulsive breathing, falls, and perhaps immediately dies. In other instances the fatal termination is not so rapid, but a great faintness seems to overcome the animal. He may fall and breathe with difficulty; a churning noise is said to be heard at the heart, the action of which is extremely feeble. If the quantity of air which has entered is but small, the animal may rally; but more copious entrance causes death by syncope; in sorne cases there are convulsions, whilst in others death seems to be due to mere exhaustion.

The mode of death is as follows:-The blood becoming mingled with air, assumes a frothy character in the right ventricle; it is sent through the pulmonary artery, but becomes more or less arrested in the pulmonic capillaries, in consequence of the right ventricle being unable to overcome the mechanical. obstacle presented by air-bubbles in these vessels. The quantity of blood transmitted through the lungs for the systemic circulation grows less and less, according to theincrease of obstruction and arrest of the blood in the capillaries of the lungs. The supply to the head is inadequate to afford due stimulus to the nervous centres, and syncope results. If circulation be not restored, this continues; the respiratory movements then cease, and life becomes extinct; the heart last failing in its action from want of its necessary stimulus-the blood.

From some experiments made by me and one of my pupils (Mr. Hutcheon), it was found that some of the air was eliminated 
into the areolar tissue of the lungs, constituting interlobular emphysema, and that the inspiratory movement was performed with greater difficulty than the expiratory.

The Cause.- "Owing to the tendency to the formation of a vacuum in the pericardium during inspiration, there is, during that process, a suction action, called by some 'venous inspiration,' in the veins within and near the thorax, extending to a limited extent only, and ceasing where the coats of the veins collapse. The space in which this suction action exists is that in which venous flux and reflux are perceptible. Accordingly, it is found that the accident has always occurred when veins have been opened in the cervical and brachial regions."(SPENCE.)

To prevent the occurrence of this accident, after the operation of phlebotomy, it is only necessary to remove the pressure from the vein slowly and carefully; but should it occur, and not prove immediately fatal, the treatment should be directed to maintain an adequate supply of blood to the head. For this purpose stimulants are to be administered; and if the animal falls, he is to be kept in the recumbent position; frictions are to be applied to the extremities; and should the urgent symptoms pass away, the treatment is to be conducted with the view of preventing the occurrence of inflammation of the lungs, a sequel which, experience has shown, is apt to ensue.

The subject has been carefully investigated by a commission appointed by the French Academy, and by the lahours of Magendie, Amussat, Wattman, and Cormack. 


\title{
CHAPTER XXXIII.
}

\author{
DISEASES OF THE ARTERIES AND LYMPHATICS.
}

\begin{abstract}
DISEASES OF TIE ARTERIES-ARTERITIS-EMBOLISM - ANEURISM TRUE ANEURISM - THE DISSECTING ANEURISM - FALSE ANEURISM - ANEURISMAL VARIX - VARICOSE ANEURISM-DEGENERATION OF THE ARTERIAL COATS-CALCAREOUS, CARTILAGINOUS, AND FATTY DEGENERATION - INJURIES TO ARTERIES-INFLAMMATION OF THE ABSORBENTS.
\end{abstract}

\section{ARTERITIS.}

INFLAMMATION of the arteries is a rare affection in the lower animals. Cases of inflammation of the iliac arteries are, however, recorded, and I have seen instances of it. There is a fine specimen in the College Museum, and the history of the case from which it was obtained is contained in the following letter:-

\section{Letter from the late Professor Barlow to Dr. Gairdner.}

“1 Pilrig Street, 12th December 1855.

“My Dear Sir-I have recently met with a few instances in which arteries of considerable size have been almost entirely plugged up with fibrinous clots, firmly adherent to their walls. In these cases, during life, there was sometimes visible, but unexplained, atrophy of certain muscles in regions specially supplied by such vessels; and sometimes, when a main trunk, such as the aorta posterior, became thus plugged, there was palsy of the hind parts (of course, I speak of the horse). At first I fancied these things to possess no material interest, and did not preserve the vessels. However, this day week, a pony greatly disabled behind, but not completely paralytic, was brought for dissection. I found a large plug of adherent fibrine in the aorta post., just where this vessel divides into two iliacs on each side. (In the horse, you will remember, there is no 
common iliac, but the aorta post. divides into internal and external iliacs). This plug was firmly adherent to the roof of the artery; that is, to that part lying in contact with the vertebræ. It was not sufficiently large to obstruct the stream of blood completely, but it must have caused a material lessening of the stream. The internal iliacs, however, were completely plugged up, and the outside of the fibrinous clot was adherent to their walls-in many places all the way round. In one place especially, a calcifying process is taking place in the coagulum. If such things are not already too familiar to you, I wish you would look in at Clyde Street any day before two P.M., for the condition is to me somewhat new.-Yours very truly,

JoHN BARLOW."

In all the instances recorded of arteritis in the horse, the disease was of a chronic and limited character, invading a portion, more or less in extent, of one, or at most two, large arteries and their branches. The diffuse suppurative arteritis described by writers upon human surgery, so far as my knowledge extends, is quite unknown. Arteritis is generally caused by an injury, though such may not generally be traceable. The iliac arteries, and more especially their branches, are imbedded in very powerful muscles, and during the violent contractions of those muscles are liable to be injured, the result of the injury being inflammation; exudation from the walls of the vessel, forming the nucleus of a clot; the clot being, as in phlebitis, composed of lymph exuded from the walls of the vessel, and of coagulated blood. The formation of the coagulum always begins at some definite fixed spot, and points to some source of local irritation; from this it extends until the artery is plugged up to its origin from the parent trunk.

The symptoms of plugging of the external iliacs are coldness of the extremity, with muscular debility, which increases with exercise, and atrophy of them; and should the arteries of both sides be plugged, the symptoms simulate those of paraplegia. The diagnostic sigu, however, is absence of pulsation in the artery, detectable by examination per rectum. The loss of blood supply to the extremity furnished by these arteries is not sufficient to cause gangrene; a modified circulation being maintained by branches of other arteries, which auastomose 
with those of the occluded vessels. The want of circulation is scarcely shown during repose; there seems to be but a slight degree of muscular debility; but when the animal is compelled to move, he immediately exhibits signs of inability.

Virchow, in his work on Cellular Pathology, describes the process of the coagulation of the blood in the vessels under the term "thrombosis." A clot so formed he calls a thrombus, and the impulsion or projection onward of a clot or thrombus, detached from the walls or valves of the vascular systenı, is described by him under the term "embolism."

Thrombi may be propelled from the heart to the arterial peripheric vessels, or they may form in veins and travel to the heart.

He says:- "As long, however, as the thrombus is confined to a branch vessel, so long the body is not exposed to any particular danger; the worst that can happen is, that, in consequence of a peri or meso phlebitis, an abscess may form and open externally. Only, the greater number of thrombi in the small branches do not content themselves with advancing up to the level of the main trunk, but pretty constantly new masses of coagulum deposit themselves in the blood upon the end of the thrombus, layer after layer; the thrombus is prolonger beyond the mouth of the branch into the trunk in the direction of the current of the blood, shoots out in the form of a thick cylinder farther and farther, and becomes continually larger and larger. Soon this prolonged thrombus no longer bears any proportion to the original thrombus from which it proceeds. From a lumbar vein, for example, a plug may extend into the vena cava as thick as the last phalanx of the thumb.

"It is these prolonged plugs that constitute the source of real danger, as the stream of blood may detach minute portions, hurry them away with it, and wedge them tightly into the nearest system of arteries or capillaries.

"Many cases of sudden death that would otherwise be inexplicable, are thus accounted for." The question is one of importance to the veterinarian, and is well worthy of further study.

In the human subject clots occasionally originate from very trivial local causes, chillains even being the starting-point. 
May not causes as trivial as this operate in producing fatal results in the lower animals?

I think that in no case of swelled legs, enlarged glands, subacute inflammation, or hardening over the course of the lymphatics or superficial veins, should the possibility of a fatai termination by embolism to the right side of the heart be overJoked, or the possible supervention of suppurative phlebitis. Professor Gamgee, in his Domestic Animals in Health and Disease, mentions a case of embolism supervening upon the insertion of a seton for the cure of bone-spavin.

In farcy-a disease where the lymphatics are inflamed-1 have invariably discovered coagula in the splenic vessels, these liaving been originally formed in the veins closely connected with the inflamed lymphatics, conveyed to the heart in small detached particles by the returning stream of blood, propelled from thence into the arterial system, and deposited in the capillaries of the spleen and other organs. In epizootic cellulitis-a disease characterised by diffuse inflammation of areolar tissue-death frequently occurs, when the animal is to all appearance recovering, from embolism to the right side of the heart; whilst in other instances of the same disease the thrombi have been propelled from the heart to the cerebral arteries, and death has resulted from coma, or from a slow degenerative disease of the brain, ensuing from deficient blood supply.

\section{ANEURISM,}

A pulsating tumour containing blood, and communicating with the interior of an artery.

Aneurisms are divided into true and false. "A true aneurism is that which is due to disease of the arterial texture, whether the diseased condition be a degeneration and dilatation of all the coats of the vessel, or an ulceration of one or more of them, leading to dilatation of the external fibrous coat.

"A false aneurism is nothing more or less than a wounded artery, the blood, prevented from escaping externally, becoming coagulated in the areolar tissue, which becomes condensed, and forms a sort of cyst, though generally a very imperfect one. as in some cases the force of the blood-current dissects the textures widely. The wound in the artery remains open, and 
enlarges by degrees, so that pulsation is in this case also a marked feature in the tumour."-(SPENCE.)

Many cases of aneurism in the horse are recorded, and most commonly aneurisms of the anterior mesenteric artery and posterior aorta have been met with. The accompanying drawing, which is taken from a specimen in the possession of Professor Walley, will illustrate the common situation of aneurism.

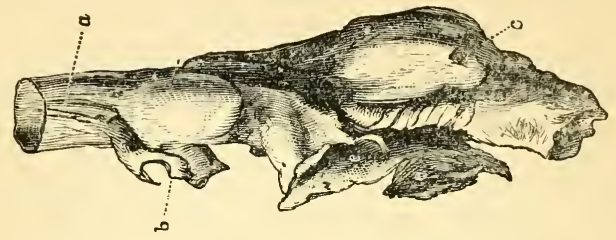

Fig. 111.-Aneurism of the posterior aorta, with calcareous degeneration of the walls of the sac.

$a$, Aorta, natural size.

$b$, Small dilatation, with the cut ends of the anterior mesenteric and coeliac axis.

$c$, Large aneurismal sac, laid open.

There are records of scattered cases of aneurism in large numbers, but the most interesting memoir bearing on this subject appeared in the Vienna Quarterly of Veterinary Scicnce for 1852 , by Dr. Bruckmiiller. It refers to aneurism of the anterior mesenteric artery. Hering was the first to indicate the frequency of this lesion, but Gurlt doubted the correctness of Hering's observations. Bruckmiiller examined carefully, from the 22d May to the 30th September 1851, 65 horses, with a view to determine the frequency of this aneurism, and found only six animals perfectly free from it; 59, or 91 per cent., had dilatation of the mesenteric artery to a greater or less extent. In 19 cases there was simple thickening of the arterial coats, without or with very slight dilatation; 8 small aneurisms from the size of a pea to a hazel nut; 29 larger, varying from the size of a walnut to that of a hen's egg; and 3 with hardening of the coats. The ages of the animals varied from six to twenty, chiefly from six to eleven. Aneurism is therefore not a very rare, but a very common disease in the horse; and in a number of cases of mesenteric aneurism parasites are found occupying recesses in the thickened coats. The 
parasite is a variety of the Strongylus-Strongylus armatus, varietatis minoris-which is often found in the textures of solipeds.

With reference to the presence of parasites in the anterior mesenteric artery, I may state that they are scarcely ever absent in the ass, and that the coats of the vessel, as well as those of the cœliac axis, are thickened-calcareous-and present aneurismal dilatations.

In the investigation of obscure causes of disease in the horse, it may be of advantage to bear in mind the frequency of aneurism.

In the dog I have witnessed cases of aneurism of the posterior aorta, and the symptoms are much better developed than in the horse. In the $\operatorname{dog}$ suffering from this form of aneurism, the pulsations of the vessel can be felt by laying the hand upon the "walls of the abdomen; in fact, the expansion and contraction of the artery (pulsations) can be both seen and felt; a thrill is also communicated to the pulsations of the femorals. In addition to this diagnostic sign, the animal suffers from marasmus, debility of the muscles of the hind limbs, loss of appetite, and a tendency to vomition.

Treatment.-Aneurism of the aorta, mesenteric, iliac, or other deep-seated artery, is beyond the reach of surgical interference; in the $\operatorname{dog} I$ have, however, seen beneficial results follow the persistent administration of the iodide of potassium. But in the more external aneurisms, should the veterinarian meet with such an affection, the treatment is by continuous pressure, or by ligature, and in veterinary practice the operation of Hunter is the best. The Hunterian operation is performed by tying the artery on the cardiac side of the tumour, at some distance from the tumour where the artery is most accessible, and where its coats are most free from disease. After the operation, the aneurismal tumour is gradually diminished by absorption; and with regard to the main trunk on which the aneurism is seated, it has been found in human surgical practice that it has become obliterated from the first branch above the ligature to the first below the aneurism.

The method of operation by acupressure is now generally practised by surgeons. The employment of the catgut suture is also highly spoken of. The catgut is dissolved after a certain 
time, and removed by absorption, without causing the ulceration which follows the common thread suture.

The changes that occur in an artery after it has been tied are as follows:-Plastic exudation takes place from the extremities of the middle and inner coats at the line of deliquiation, with exudation into the areolar tissue surrounding the artery. Its different coats can no longer be distinguished; and sooner or later, by means of a thin exudation of lymph exterior to the portion of coagulum formed by the coagulation of the blood, the walls of the artery and the surrounding areolar tissue become condensed into one mass, in which original parts can no longer be distinguished; and by the organization of this exudation into fibrous tissue the artery becomes permanently obliterated.

\section{THE “DISSECTING ANEURISM,"}

Is fortunately a rare variety even in the human being. This form of aneurism is due to rupture of the internal and middle coats of the artery, which allows the blood to escape outwards and to burrow under the external coat, separating it from the middle coat, so as to form a cavity, extending in some cases for a considerable distance in the course of the vessel, and involving more or less of its circumference. It is generally met with in the aorta, and is a cause of much agony.

Aneurism of a large artery, such as the aorta or mesenteric, may cause sudden death by rupture of the arterial coats; the symptoms being those common to all excessive hæmorrhages, namely, rapid sinking of the vital powers, pallor of the mucous membranes, coldness of the surface of the body, extremities, and mouth,-the animal generally, before dying, emitting a peculiar shriek of agony.

\section{FALSE ANEURISMS.}

Traumatic or false aneurisms may be diffuse or circumscribed. In the diffuse the blood becomes extravasated in the tissues, forming a sort of flattened mass over the vessel, which communicates to it a feeling of pulsation.

In the circumscribed, the areolar tissue surrounding the opening in the artery becomes condensed, so as to form a sac which limits the eflused blood. 


\section{ANEURISMAL VARIX.}

When a direct communication is accidentally or otherwise made between an arterial and venous trunk, a disease may he formed which is called "aneurismal varix." It may occur on any part where an artery and vein in proximity to each other are both wounded. Three wounds are made before the disease takes place; for example, an aneurismal varix may occur from venesection, especially when the blood is drawn from the jugular; a large fleam being used, or a lancet plunged too deeply, the vein is wounded on each side, and the carotid artery is penetrated; the wound upon the outer side of the vein may heal, but that on its inner side and that in the carotid may remain open, and through these openings a communication is established between the two vessels. The effects of this direct ingress of the arterial blood into the vein causes the latter to become widened, and to present sac-like dilatations; the artery on the distal side of the disease becomes smaller, and its coats thinner, whilst on the proximal side of the wound it is usually widened.

The symptoms of aneurismal varix are the swelled condition of the vein, the feeble pulsation of the artery beyond the seat of the disease, and a thrill or bruit being perceptible when the part is auscultated.

This form of aneurism calls for no special remarks as to treatment. Horses were very often affected with it in the days of excessive and repeated bleedings, and they seemed to suffer no inconvenience.

\section{VARICOSE ANEURISM}

Differs from the last form of false aneurism by the communication between the two vessels being indirect; thus, the blood escaping from a wound in the artery passes into the surrounding areolar tissue, which it distends into a sac, and from this sac it is discharged into a vein.

In the treatment of false aneurism, especially the diffuse form, the flow of blood into the tissue must be arrested by cutting down on the tumour, cleaning out the coagula, and tying the artery upon both sides of the wounded point. In small wounds of 
arteries, and where the tumour is circumscribed, a bandage, in situations where it can be firmly applied, may suffice to arrest the hæmorrhage and produce the closure of the wound in the vessel.

I have repeatedly seen cases of diffuse aneurism from a wound of the external thoracic artery, accidentally inflicted by the point of the scissors whilst the horse was being "clipped," the areolar tissue at the lower part of the chest being loose, and admitting a large amount of blood, a huge tumour has been thus formed in a short time, requiring active interference. In such cases the practitioner must "cast" the animal, and operate on the artery in the manner already described.

\section{DEGENERATIVE DISEASES OF THE ARTERIAL COATS.}

There are three forms of degeneration of the arterial coats frequently met with in the horse. Rarely, indeed, is an old subject dissected at the College without some change being detected in the coats of some of the large arteries.

The most common form is "calcareous degeneration." In the figure shown at page 582 this change had taken place to some extent. The seat of the calcareous change is the transverse fibres of the middle coat, in which it appears in the form of spicula or plates, involving a greater or less extent of vessel, and in some specimens it is so complete as to convert the affected part into a mere passive tube. In consequence of this change in the middle coat, the external surface of the artery presents an uneven appearance, unequally dilated at some parts, and slightly contracted at others. The internal coat may be unchanged at the commencement, but it soon becomes shrivelled, irregular, and ruptured at the margins of the deposit.

In old horses this change is very common in the posterior aorta, anterior mesenteric, the arteries of the extremities, the coronary (cardiac), and arteries of the brain. Indeed, the so-called "staggers" of old horses is mostly due to this degenerative change in the cerebral arteries, or death from apoplexy may be due to rupture of a cerebral artery which has been thus rendered friable and easily ruptured.

Calcareous deposition differs from true bone in important particulars; it is without fibrous structure and vascularity; it 
presents an irregular granular appearance, and is destitute of obvious arrangement.

Chemically, it is composed of the phosphate and carbonate of lime, and animal matter (LASSAIGNE), and it constitutes one of the various examples of that calcareous degeneration common to old age, which is displayed in the increasing proportions of earthy matters in various parts of the animal body.

\section{CARTILAGINOUS DEGENERATION.}

Cartilaginous deposits are chiefly found in the small arteries, at a considerable distance from the heart, and present themselves in various forms of transparency, consistency, and connection with the inner membrane.

They are deposits upon the inner membrane, and in some instances are easily peeled off, leaving the membrane entire; at other times they are firmly adherent to it. "The only changes which they seem to undergo, after assuming a cartilaginous consistency, is increase of thickness."-(PIRRIE.) "They originate in the exudation of arteritis, which is at first of a gelatinous consistence, but gradually becomes firmer, and eventually supplants the inner membrane, on the free surface of which it was originally effused."-(Вizoт.)

\section{FATTY OR ATHEROMATOUS DEGENERATION.}

Fatty metamorphosis of the arterial coats is rarely met with in comparison to the calcareous form, but it may be witnessed in the coronary (cardiac) and in the cerebral arteries of ol ${ }^{3}$ horses, such as old favourite carriage-horses, which have led a life of ease and good living; and very lately I met with this condition, along with atrophy and fatty degeneration of the ventricular walls, in the heart of a brewer's horse, which for some years had done little or no work. This form of degenerative disease consists of a deposition of fatty granules of a pale yellowish colour, situated between the middle and inner coats of the artery. Later on, the coats of the vessel have a thickened leathery appearance, due to degenerative changes having taken place in all its tissues, by which its power of contractility is more or less destroyed, and if it be cut open, well-marked 
patches of a yellowish colour will be found scattered on its inmer surface, whilst deposits of earthy matter alternate with the fat.

I have seen gangrene of the tail in cows, particularly old cows. May it not have been due to this condition of the arterial coats? The subject is well worthy of further consideration.

\section{IXJURIES TO ARTERIES.}

In the lower animals wounds of arteries are fortunately rare. When an artery is wounded the hæmorrhage is much more rapid than from a vein; the blood is of a bright red colour, and discharged in jets, synchronous with the contraction of the heart. At first the stream is full, and is pushed from the wound with considerable force; but as the animal grows faint the jerks are more perceptible, and the flow diminishes. When an artery is torn it is unequally lacerated; its external coat is twisted and drawn out, whilst the divided internal and middle ones retract, and are thrown towards the centre of the canal. The elasticity and vitality of all the coats are impaired for some little distance from the wound, and the current of blood in the injured part is diminished or altogether arrested for some distance, and the bleeding is thus prevented.

If an artery be split in a longitudinal direction and across the course of the circular fibres, the bleeding is most profuse when the part is relaxed; but if it be kept on the stretch, the bleeding becomes less profuse. If an artery has been cut partially in a transverse direction the bleeding is very profuse, for the transverse fibres separate from each other, and the opening gapes. Cutting an artery completely across is attended with its retraction within the sheath and surrounding tissue, and the bleeding is arrested if the vessel is not large. Thus, when the palatine artery has been partially divided, the hæmorrhage continues; but if the incision be made deeper, and the artery completely cut across, the bleeding gradually ceases.

In the case of a large artery, however, this proceeding is not sufficient, although the bleeding may be arrested as the animal grows faint, the pressure of the surrounding clot being sufficient at the time to arrest the bleeding; but no sooner does the faintness pass away, and the circulation become strong again (unless 
a clot has formed within the artery, from effusion of lymph and coagulation of the blood), than secondary hæmorrhage occurs. A large artery, when divided, ought to be secured by ligature, or acripressure upon both sides of the wound; for if it be secured on the cardiac side only, the bleeding will continue by the flow of the blood being re-established through the medium of the collateral and anastomosing branches.

The methods of arresting hæmorrhage, particularly if the wounded vessel or vessels be small, by the application of styptics, or the actual or potential cautery, are very successful; and they act by producing a clot, which may be temporary only, but before it is cast off the natural lımostatic process has time to be completed.

"The actual cautery should be applied at a red heat only, not at a white heat, lest the slough should separate too soon. The cautery slightly impairs the vital functions of the coats of the vessel at the injured point, a clot forms, and the circulation is arrested."-(SPExcE.)

\section{LYMIPIANGITIS, OR INFLAMMATION OF THE LYMIPHATICS.}

This affection resembles bilious erysipelas in the human being, and is known among horsemen by a variety of terms. In Scotland it is called a "weed;" in England a "shot of grease," "shake," and "Monday morning disease."

Inflammation of the absorbents is usually confined to one hind leg; occasionally both hind legs are simultaneously or successively affected, and more rarely the fore limb, generally the right-off-is the seat of the disease.

The local inflammation is commonly preceded by a shivering fit-rigor-which may continue for some hours; and as a rule the severity of the attack is denoted by the intensity and duration of the rigor. The rigor is accompanied by more or less restlessness; and lameness in the affected limb is manifested at an early stage. To the rigor a hot stage succeeds. The animal now blows, sometimes with great intensity, whilst sweats bedew the body; the pulse is hard, full, and strong, the accompanying fever being of the "sthenic type;" the visible mucous membranes injected, bowels constipated, the secretion of urine scanty, the urine high-coloured, and loaded with hippurates and urea. 
The local inflammation is manifested by swelling of the inguinal glands when it is in a posterior extrenity, and of the brachial glands when a fore limb is affected; the swollen glands are very painful when pressed upon, and a swelling extends downwards from them, first as a narrow elevation upon the inner side of the thigh or arm, but soon extending in every direction; it involves the whole circumference of the limb, from the glands first inflamed down to the foot. As the swelling increases, the pain and lameness subside.

Mr. Haycock, who was the first to give this disease a scientific name, very graphically describes a case of it which occurred in his practice. He says:- "The horse is standing in the stable on three legs, the left hind limb being held with the foot from the ground. Great anxiety is depicted on the animal's countenance, and he frequently looks round at the limb held up. The respirations are forty-eight per minute; the pulse ninety-six, and hard and cord-like to the touch. The nostrils are dilated to their full extent, and the perspiration rolls in drops from the sides of the abdomen, the shoulders, and the thighs. The affected limb is greatly distended upon its inner surface, from its junc. tion with the body to the very foot. The lymphatic glands are swollen into large lumps or masses, and towards them, in all directions, run a great number of lymphatic vessels, enlarged to the size of a thick quill. These enlarged vessels exist on the outer as well as the inner side of the limb. The surface of the swelling is covered with a serous exudation; the mouth is dry and clammy; and great desire is evinced for cold water." The horse lived for two years after the attack, the limb remaining thicker than natural. At the end of that time Mr. Haycock had the opportunity of dissecting the affected limb, and he describes its condition as follows:- "On the removal of the skin from the hind extremities, I at once observed a difference in the colour of the exposed structures. The fascia of the diseased limb was covered with a yellow-coloured fluid, not unlike as though the tissue had been coated over with a quantity of bile, or very yellow serum. On cutting deeper, I perceived the areolar tissue to partake of the same colour, and a yellow liquor exuded from it; the tissue itself was also changed; it was dense, thicker, and partook more of a fibrous character than natural. This change was observable in all the areolar tissue 
of the linib; at least, all that I examined. The lymphatic glands were also greatly enlarged; and on cutting into their substance, the knife met with considerable resistance; a scirrhous structure was exposed; the yellow fluid flowed forth in abundance; and on carefully exposing the vessels which terminated in the glands, and slitting them up, the same kind of liquor was liberated. The internal surface of the vessels presented here and there patches of a light red colour; and on comparing the vessels with those of the other limb, they were without doubt larger; many of them were double the size in calibre." -(Haycock's Contributions to Veterinary Pathology.) One remarkable symptom was observable in the foregoing case that is rarely witnessed, namely, the visible enlargement of the lymphatics after the accession of the swelling of the limb. In all the cases that have fallen under my notice, the enlargement of the absorbents, though undoubtedly present, has been hidden or masked when the areolar tissue has become swollen.

Horses that have once been attacked by lymphangitis are liable to a recurrence of the malady, and generally one attack succeeds another periodically, until the limb assumes an enlarged or distended condition termed elephantiasis,- - a state in which it is found that the areolar tissue of the limb has become hypertrophied by the organization of some portion of the exudate formed at each successive attack.

Inflammation of the lymphatics, unless when excited by a specific poison, such as the morbid material of glanders and farcy, seldom runs on to the formation of pus. Occasionally, however, the lymphatic glands, more particularly the inguinal, suppurate and discharge the contained pus. Suppuration of the glands may be suspected when lymphangitis continues longer than usual, and when the swelling extends along the abdomen, sheath, or mammary glands. After a time, the swelling in the groin becomes more prominent, but it never has very well defined limits, appearing more as a purulent infiltration than as a true abscess. As soon as it can be ascertained that pus is formed, the parts must be freely laid open, a good depending orifice being essential, for the pus is apt to burrow into the surrounding fascia and areolar tissue.

The causes of lymphangitis are irritation of the lymphatic glands by chyle rich in nutritive products, and, in some rare 
instances, blood in a highly fibrinous condition owing to metamorphosis of tissues. The first is produced by over-feeding, more particularly when the animal is not called upon to perform his ordinary labour; and the irritation commences in the mesenteric glands, for it has been shown that every meal which is eaten produces a certain irritation in these glands; the constituents of the chyle conveyed to them acting as physiological stimuli to the gland substance, whereby they become enlarged. This irritation extends from the mesentery, along the chain of lymphatic ganglia imbedded in the sub-lumbar and deep inguinal regions which connect the thoracic duct with the first visible lymphatic glands-the superior inguinal ganglion. The course of the disease supports this view of its pathology ; its peculiarity being the way in which it extends from above downwards; the extension downwards along the limb affected being merely a continuation of the process which has already taken place in the glands within the cavity of the abdomen. In some instances lymphangitis is preceded by symptoms of intestinal irritation, colicky pains, and constipation of the bowels. This latter condition is generally constant, unless in those instances, common in Scotland, where the disease is produced by over-feeding on green food, particularly upon rye-grass, when, instead of constipation, a condition approaching to diarrhœa is often witnessed.

When the lymphatics are inflamed in consequence of the blood being in a highly fibrinous condition, brought about by metamorphosis of tissue, it will be found that the animal has not been eating for some days: in one instance within my recollection, sixteen days elapsed before any manifestation of lymphangitis. An explanation is difficult, and can only be arrived at by assuming that the vitality of the animal was supported by the pabulum derived from its own tissues; and that such nutrient material, being highly loaded with fibrine, was calculated to act as an irritant to the glands and ducts of the lymphatic system.

Treatment.-In the first stage-that of rigor-fomentations to the affected extremity, warm clothing, purgatives, and at the same time allowing the animal to drink a moderate quantity of water. The administration of stimulants, unless the rigor be very continuous, is not called for; indeed, stimulants are apt to be productive of harm, by causing a powerful reaction; but 
should the shivering persist, notwithstanding the application of warmth, friction, clothing, and bandages, moderate doses of spirit of nitre may be administered. As a powerful stimulant to the vessels of the skin, inducing a general warmth over the surface of the body, I may mention that tincture of arnica, in doses varying from one to two ounces, ean be given with advantage.

In the second or hot stage, bleeding from the jugular is generally recommended. I have experimented upon this matter, and have arrived at the conclusion that, although moderate bleeding does no harm, it does no good; it neither modifies nor cuts short the disease; and in cases where the animal is debilitated previous to the attack, the withdrawal of blood not only increases the severity of the local inflammation, but prolongs its duration as well. Fomentations to the affected limb, to induce an early swelling, and the administration of a cathartic, constitute nearly all the treatment that is required. After the eathartic has operated, diuretic remedies may be given with advantage; and should the fever be very high, the pulse harl, and the animal showing signs of much pain and restlessness, tincture of aconite is to be repeatedly administered. Some practitioners are in the habit of giving a small cathartic, and sending the horse to ordinary work whilst suffering from this malady. Such practice is irrational, and defeats its own object; for exercise, though carefully regulated, if given early in the disease, causes the swelling to assume a permanency of character which it is difficult to remove. Exercise certainly dissipates the swelling at the time; but after the patient has stood for a few hours, the swelling returns, and it is no less curious than true, that each fresh exudation tends more and more to become crganized.

The condition called elephantiasis, or a chronic thick leg, is difficult to treat. A large quantity of fibrous tissue formed in the limb assumes the character and functions of one of the proper elements of the part, and remains as a permanency. Drastic cathartics, powerful diuretics, iodine, and its salts, have been given, but with little or no advantage; whilst blisters, setons, and the actual cautery to the limb itself have been tried with like effect. I think much can be done for the prevention of this condition by carefully feeding the animal; and when it 
is actually present, by avoiding exercise or work, until at least the inflammatory condition of the part has subsided.

In chronic swelled legs, large doses of veratrum are recommended by some veterinarians. When the swelling is due to a mere dropsical effusion, this remedy is a most effectual one ; but when the adventitious tissue is organized, it is useless to punish the animal for no possible good result.

The lymphatic vessels may be inflamed from causes that may be traceable to some local lesion, as wounds, and particularly bruises; the inflammation of the lymphatics being denoted by swelling along their course, their coats becoming thickened and infiltrated with exudation. This condition is notably produced by saddle or harness galls, when the bruise may be seen surrounded by swollen lymphatic vessels, running in various directions, and to the extent of a foot or two, in some instances. Again, a pricked or punctured foot may give rise to inflanmation of the lymphatics, with swelling of them both above and below the knee or hock joints, giving rise to a difficulty in diagnosis without a thorough examination of the foot.

Sympathetic inflammation, and even suppuration of the lymphatic glands, may also be occasionally witnessed; but they call for no special comment, nor any guidance for treatment beyond what has already been laid down.

Suppurative inflammation of the lymphatics gives rise to small isolated abscesses along their course, forming, as it were, loci of the disease, each of which appears to serve as a fresh focus of morbid action; this may be witnessed in farcy, a disease with which I do not intend at present to deal, and occasionally in sympathetic inflammation connected with violent diseases of the foot or leg.

During the early stages of inflammation of the lymphatics a restricted and cooling diet is to be prescribed; for the reasons already laid down, that highly nutritious food is a direct stimulus to the glandular system. After the more acute symptoms have passed away, and when debility supervenes, the animal's strength must be maintained, and the absorption of the cxudate encouraged by a well regulated nutritious diet; whilst the swollen limb is to be strengthened and supported by moderately firm bandages; the absorption of the exudate, stimulated by frictions with the hand, and some gentle stimu- 
lating embrocation. Iodine is not specially called for unless tho exudate assumes a callous or hard condition. If it be remembered that the exudation and swelling are owing to the irritation, and that they will disappear like the swelling of any ordinary inflammation when the irritation is removed, much difficulty in the due application of suitable treatment will be overcome, and the animal saved much needless pain and suffering from the effects of the so-called "absorbent stimulants." 


\title{
CHAPTER XXXIV.
}

\author{
HERNIÆ, ETC.
}

REDUCIBLE, IRREDUCIBLE, AND STRANGULATED HERNIE-CMBILICAL OR EXOMPHALOS-VENTRAL, INGUINAL, AND SCROTAL IIERNIEGUT-TIE IN CATTLE.

\section{HERNLE.}

Abdominal herniæ, or ruptures, are divided into reducible, irreducible, and strangulated, according to their condition; and into inguinal, scrotal, ventral, umbilical, and diaphragmatic, according to their situation.

A hernia is the protrusion of a piece of intestine, omentum, or both, through one of the natural openings: a rupture, the protrusion of intestine, omentum, or both, through the muscular walls, but not through the skin, although the terms are made use of synonymously.

\section{REDUCIBLE IIERNIA.}

A hernia is reducible when it can be easily returned into the abdomen. It consists of a soft fluctuating swelling, unattended with heat, pain, or even uneasiness. It is generally large after a full meal, and decreases in size when the bowels are empty. When the animal coughs, it becomes tense, larger, and communicates a sudden impulse to the hand of the examiner. Writers upon human surgery lay it down as a rule, that when the swelling is elastic, uniform, and compressible, and if its return be sudden, and attended with a peculiar gurgling noise, there can be no doubt that the hernia is formed of intestine. If the swelling be more solid and uneven, feeling doughy to the touch, receives an impression from the fingers of the examiner, and if its return be gradual and unattended with any peculiar sound, there can be no doubt of its being an omental hernia only; if a portion of the swelling be elastic, and returns suddenly with a gurgling noise, and if the remaining part be 
doughy and its return be more gradual, the hernia is in all probability formed of intestine and omentum.

\section{IRREDUCIBLE HERNIA.}

A hernia is said to be irreducible when it is not strangulated, but yet cannot be returned into the abdomen. The causes that prevent reduction are: -1 st. The bulk of the protruded parts being out of proportion to the opening through which they would have to return. $2 d$. Adhesions of the protruded parts to the hernial sac through the medium of organizable lymph, which assumes the form of filamentous bands, admitting of some degree of movement between the intestine and sac, or a more firm union, constituting what has been called "the fleshy union." In this form the union is close, firm, and deep, so that the sac and its contents cannot be separated from each other, but form a solid mass, the circulation of which is common to both sac and intestines.

\section{STRANGULATED HERNIA.}

A hernia is said to be strangulated when the contents of the sac experience such a degree of pressure as not only to cause them to be irreducible, but also, by compressing their blood-vessels, to disturb, impede, or suspend the circulation within them. This condition speedily causes inflammation in the protruded parts, which extends to others within the abdomen.

\section{HERNIE, AS NAMED ACCORDING TO THEIR SITUATION.}

ExomphaLos-A protrusion of any portion of bowel or omentum through the navel, forming a tumour at that part, is called umbilical hernia or exomphalos. It is very frequently congenital, and is more commonly met with in calves than in foals. If not congenital, it generally takes place very shortly after the birth of the foal or calf, and arises from the yielding of the umbilical opening; the closure of which, although it may be complete, is still lax and weak. In some cases this laxity and weakness remain until the animal is two or even three 
years of age, and a rupture may happen from any violent effort. This hernia, more especially in foals, is occasionally spontaneously reduced. As the animal grows, the omentum and mesentery, being, in proportion, shorter in the adult than in the young animal, are drawn into the abdomen, and the opening closes.

Treatment.-The animal, having been kept short of food for a few hours, is to be cast, and the contents of the sac carefully returned. The reduction effected, the skin is to be drawn from the belly, pinched up, and confined in its folded state by the plain wooden clams, placed upon the sides of the fold, or by two skewers run through it at each extremity of the opening in the abdominal walls, and as close as possible to the surface of the abdominal fascia. The skewers are to be connected, and the doubled skin made into a firm fold by a piece of strong twine wrapped round them, firmly but not too tightly, embracing the folded skin. In applying sutures, clams, or skewers, for the reduction of hernia, wherever situated, the objects to be attained are, the union of the internal surfaces of the folded skin, and the production of an exudate to block up the hernial opening. To do these successfully, the external pressure is not to be so great as to cause rapid sloughing of the skin, but to induce adhesive inflammation of the opposing surfaces. If there be occasion to tighten the ligature or apply a fresh one, there is no necessity to cast the animal. I recommend this method, as I have known of cases where very tight ligatures have been applied, and where the skin has rapidly sloughed, result in protrusion of the bowels, and death.

In calves the hernial sac frequently suppurates, the skin and surrounding structures inflame, the animal goes off its food, and calves with such navels are looked upon as bad thrivers. When means are employed for the reduction of the hernia in cases of this description, the process of consolidation, after the clam or sutures have been removed, is not very satisfactory, and what appears to be a successful result in many instances proves delusive; the sac filling again, and perhaps to a greater extent than before surgical interference. Animals of this kind are often of a tuberculous constitution, and a repetition of the operation is dangerous; the want of proper tone and vitality in the parts rendering them liable to excessive sloughing, which, by exten- 
sion through the walls of the abdomen, allows the escape of the intestines, and causes the death of the animal.

In those cases where the tumour is of a globular form, a ligature round its neck is often effectual. Ligatures, clams, sutures, and skewers must be allowed to drop off spontaneously, or the operation may prove incomplete.

\section{FISTULOUS UMBILICAL HERNIA.}

Of this form of hernia, with artificial anus, the following interesting case is recorded in the Veterinarian for 1833 by Professor Dick:

The horse, four years old, was bought at Kinross Market, with a considerable enlargement of the umbilicus upon him. He did his work for some time, but it was with stiffness and unwillingness. Shortly afterwards he was seized with a "ting," and a farrier was sent for, under whom he got better. He was put to work again, but proved still stiff and unwilling. Shortly after, "an opening formed at the navel, by which the whole of the fæces were passed off." Mr. Thomson, V.S., Redstone, was sent for, who "stitched up the opening, which was large enough to admit a person's fist, and applied a roller with a pad of tow over it, also occasionally adhesive plaister." "Adhesion took place, and granulations formed very rapidly for some time; but a very small opening remained, which seemed extremely obstinate, yet was making a little progress, and to all appearance would have been entirely closed in a short time "-when he died. The intestines proved in many places perforated by ulceration, apparently the effects of lumbrici. There seemed to be no disease about the umbilical opening, except adhesion between the intestines and the parietes of the abdomen. I found it to be a part of the ileum that had fallen into the opening, about a foot from the cæcum. Nature had formed a tube of about two inches long, quite distinct from the ileum, by way of a rectum; but when it was a good deal healed up, air seemed to be sucked in by it, and passed off per anum. In drinking, the water passed off by it without any of the aliment being mixed with it; it seemed almost as clear when passed as when drunk. If he got the benefit of his meat, he received no benefit of his drink ; for it passed off as fast as he drank it; and from this cause, apparently, 
he became very much emaciated. During the time Mr. Thomson attended him he showed no symptoms of disease, save a slight attack of gripes. Before death the wound became reduced to the size of a quill, discharging white mucus, but no fæees. He was allowed only two pints of water a day, with some peameal, with a small quantity of hay. When Mr Thomson first saw the horse, he gave a clyster of water-gruel, which occasioned much inconvenience, "seemingly by emptying the posterior bowels too much, as flatus passed, seemingly from the orifice of the anus, and he worked like a pair of bellows for four hours." Mr. Thomson then injected a solution of sulph. zinc at the orifice, which caused griping pains for a short time; but they did not recur, although this was afterwards frequently repeated.

Analogons to the above operation in principle, but simpler in application, is one that has been practised with great success by Mr. Pattie, Yoker, Glasgow. His account, in the Veterinarian for 1836 , of his mode of operating is - " The colt is not cast, nor submitted to any restraint beyond that of having his head held. The hernial tumour is emptied by forcing its contents into the the belly; the loose integument forming the pouch is gathered into the left hand, while the right surrounds it by a ligature placed as closely as possible to the abdominal parietes, and drawn sufficiently tight to interrupt the circulation. On the second day there is considerable tumefaction around the incarcerated integument, which also in a slight degree partakes of the engorgement, feels cold, and often clammy and moist. When the ligature has not been sufficiently tight, or the pouch so large as to require strong compression for arresting the circulation, it is hot and tender. In all cases more than one ligature is necessary. Generally on the third day the first cord is loose. The circle it embraces has been reduced, partly by absorption and partly by incision, and there is no longer any compression. If neglected after this, the tum our rapidly increases in size, and is attached by a neck whose diameter is limited by the ligature. It is necessary, therefore, to see the patient twice or thrice a week to renew the ligature. The second, third, or fourth, should so many be required, must be placed above that which preceded, and close to the abdomen. They relax in from one to three days, and are then useless, save for the parpose of supportiug 
those which follow. The whole drop off, along with the tumour, in from ten to twelve days. The place from which the pouch is detached is neither raised nor excavated. It is a flat, granulating surface, as large as a halfpenny, and seldom broader than a half-crown. No further treatment is required, save, perhaps, a little astringent lotion to hasten cicatrisation, or an ointment to exclude flies."

\section{VENTRAL HERNIA.}

Ventral hernia differs from the umbilical and inguinal forms, from the circumstance of the protrusion occurring through an artificial opening in the abdominal walls, produced by violence, and not through natural apertures, as in the other instances.

A ventral hernia may be situated on any part of the abdominal parietes, except the navel, and is caused by laceration of some of the muscular or tendinous fibres composing the abdominal walls. The size of the tumour varies greatly in different cases; and strangulation of this hernia rarely occurs. Sometimes a partial or incomplete ventral hernia is met with, namely, rupture of the outer layer of the abdominal muscles, the internal layer being intact.

Large ventral herniæ are rarely curable, but should the muscular gap be small, the methods recommended for umbilical hernia may be employed with success. In the generality of cases there is, however, no necessity for adopting any kind of treatment.

A case of ventral artificial anus is recorded in the Veterinarian for 1837, by Mr. Karkeek, V.S., Truro, which is highly interesting, both on account of its rarity and the successful issue of the treatment adopted. Mr. Karkeek says :-

"Two years since a pony mare received an injury from the horn of a bullock on that portion of the abdomen situated between the cartilages of the false ribs, inclining a little to the left side, producing a ventral hernia about the size of a cricket ball. From a kick received upon the place from the toe of the shoe of a boy, very serious injury resulted, which terminated in an opening through the lacerated muscles into the colon itself; being that portion of its second flexure which forms the upper and anterior arch, and the liquid and pulpy contents soon issued freely from the aperture. The nare continued for 
three weeks in this state, when I was requested to examine her -about the 6th of January. I found the opening nearly large enough to admit my four fingers, and it had a very unhealthy appearance. The discharge of pulpy and watery food was great, and the smell very offensive; so much so, that it was with difficulty any person could be found to attend her. The pulse was between 50 and 60 , and the appetite tolerably good."-The mare being with foal, and an old favourite besides, her owner was very desirous to have something done. There were two favourable circumstances - the length of time since the injury, and the absence of inflammation._- "Having cleaned the wound," says Mr. Karkeek, "I closed the opening with a strong suture of pack-thread, with a common packing needle, taking in as much of the integuments and abdominal muscles as possible. I then applied a pledget of tow soaked in a solution of chloride of lime, and supported the whole by means of a thick woollen bandage laced along the spine. I ordered the wound to be cleansed and the solution to be applied every morning, and her head to be tied to the rack to prevent her lying down. About five weeks afterwards, being in the neighbourhood, I called to inquire after my patient, when to my surprise $I$ found her alive and well, the wound having completely healed. Had this case happened nearer my residence, I should have endeavoured to have instituted some experiments with regard to the process of digestion on different kinds of food; and this, I believe, might have been done without endangering the life of the animal, as there was a copious discharge of food for three weeks previous to my attending the mare."

Professor Simonds relates a case of extensive ventral hernia in the Veterinarian for 1839 , to which the reader may refer. The result was most successful; but as a rule it is safer not to treat this form of hernia otherwise than by a compress and suspensory bandage, that can be tightened at pleasure, as there is great danger of sloughing of the skin, and consequent protrusion of the intestine.

INGUINAL HERNIA.

Inguinal hernia, or bubonocele, almost always makes its appearance suddenly, and hastens to become strangulated. The 
contents of this hernia consist almost in all cases of the small intestines. From the looseness of their attachment, their volume, their general emptiness, and their energetic contractility, they most readily enter the inguinal canal. The duplicatures and flexures of the colon are the parts next most liable to protrusion. In respect to the omentum-which is so short that one would conceive it impossible it could ever reach the conal, withont laceration at least-its protrusion is uniformly the effect of some violent intestinal commotion, and is never the occasion of much mischief. When the contents are intestines solely, the hermia is denominated an Enterocele; when nothing but omentum, Epiploeele; when both combined, Entero-Epiplocele." - (GIRard on Inguinal Hernia. Paris, 1S27).

The lower animals are much less subject to this form of hernia than man, and this is not less due to the position of their bodies than to the arrangement of the muscular and fibrous envelopes forming the floor of the abdomen. M. Girard says :-

"In man, the intestinal mass is bearing downwards, and particularly upon the inguinal regions, where the openings-the abdominal ring and crural arch-are situated. In quadrupeds, on the contrary, in consequence of the oblique inclination, forwards and downwards, of the floor of the belly, from the flank to the brisket,

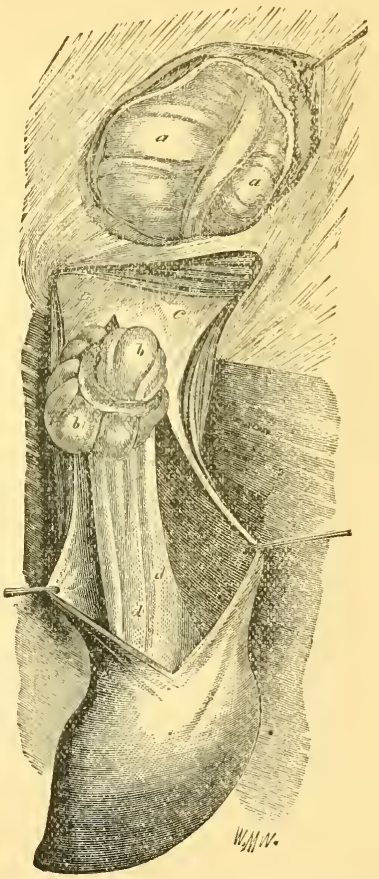

Fig. 112.-Inguinal Hernia (Girard). $a, a$, Portion of the colon, continuous with $b, b$, small intestine, which is fixed in the inguinal canal; $c, c$, is the neck of the peritoneal sheath, which is enlarged from the passage of the intestine into it ; $d, d$, tumefied portion of spermatic cord. (From GAMGEE's Domestic Animals.) 
the intestinal mass gravitates against the diaphragm, pushing it forward and occasionally rupturing it. The resistance afforded by the parietes of the belly is likewise greater, owing to the increased density and peculiar disposition of the coverings of the abdomen, the fascia superficialis being thicker, more elastic, and more developed than in man, and particularly towards the pubes, and being supported by the panniculus carnosus, an envelope that does not exist in man; added to which-not to mention the advantages arising from the oblique and straight muscles, which latter are much broader than in man-the fascia transversalis is considerably stronger and more expanded. Connect with these facts the practice of castration at an early age, one consequence of which is the contraction of the inguinal canal, and there will appear sufficient to account for the comparative exemption of the horse from inguinal rupture; and at the same time, for the unheard-of occurrence of the species denominated femoral." 1

Inguinal hernia is most frequently met with in stallions and young animals, and essentially consists of a passage of a fold of intestine into the inguinal canal through the internal abdominal ring. It is very frequently seen in India and on the Continent, but it is uncommon in this country, from the fact that stallions are not often used for working purposes.

It is but rarely seen in the gelding, and scarcely ever in the mare, although it is possible that it may occur in both.

It occasionally presents peculiarities:-1st. "It may occur with or without visible tumour; and it may EITHER be acute, chronic, simple, strangulated, continued, or intermittent. In some cases there exists a thickening of the membranes, adhesions of

1 "This was written in 1827, but since then an 'unheard-of' case has happened to M. Seon, V.S. to the Garde Royale. He was called, while on the march, to a mare with a swelling as large as his fist in the upper and fore part of the inside of the thigh. The existence of hernia was evident beyond dispute. By compressing and pushing its contents backward and upward, he caused the whole of them to re-enter the canal, but they speedily re-appeared. Bandages and compresses of tow kept the hernia reduced, but their tightness caused alarming tumefactions, which required their removal; and the consequence was, on the sixth day the hernia returned. The mare was now cast, the hernia reduced, and pledgets of tow, dipped in melted pitch, plastered upon the situation of the tumour, and over them, one, twelve inches in diameter, of pitched strong canvas. As soon as the pitch had set, the mare was let up. In ten days afterwards the plasters had fallen off, leaving some ulcerations, which readily healed. The place opposite the termination of the femoral canal subsequently exhibited a species of callus."(Percivall's Hippopathology.) 
the coverings of the hernia to one another, and occasionally to the intestine within them. In other instances hernia is complicated with hydrocele, the tumour assuming another shape, and acquiring considerable magnitude. Besides these differences, the hernia may be imperceptible, at least to the view, in consequence of having protruded no further than the inguinal canal, in which state it is called a bubonocele; when it pervades the canal and descends into the scrotum it takes the appellation of oscheocele. Either of these forms may be recent or chronic, reducible or irreducible. Hernia very rarely exists on both sides. It occurs oftenest on the right, a circumstance which is not easily explained."-(Girard on Inguinal Hernia.)

The Symptoms.-Whenever a stallion, or even a gelding, is affected with symptoms of colic, more especially if the symptoms of abdominal pain are apparently relieved when the animal is laid upon his back, a position he will maintain for half-an-hour or more at a time, it is the duty of the veterinary surgeon to examine for hernia. This is done by first emptying the rectum of its contents by an enema, and then exploring the internal abdominal ring, by passing one hand into the rectum, and manipulating the scrotum with the other hand. The strangulated intestine can be easily felt should the hernia exist. If the hernia is not reduced, the symptoms increase in severity, the pain becomes continuous, cold sweats bedew the body, more especially the scrotum and thighs; the animal sighs, the pulse becomes thready, the eyes injected, and the pupils dilated.

The indications of the presence of an inguinal hernia before actual strangulation has taken place are, according to Mr. Percivall, as follows :- “Indisposition to work, erected head, appetite impaired, pain succeeding; the animal breathes deeply, paws, and puts himself into various postures to obtain relief. There are cases in which the horse appears as if he were languishing from over-fatigue. Now and then the gut returns of itself, and the patient becomes suddenly restored to ease. A second descent, however, commonly takes place, and that-should it likewise return-becomes followed by a third, and so on, until, from the volume it acquires, the hernia becomes permanent. The testicle on the hernial side, though felt drawn up, irregularly descends and ascends. This symptom is highly pathognomonic, and one demanding that the practitioner should, 
without loss of time, examine into the state of the inguinal canal."

The following account of scrotal hernia I have taken the liberty of transcribing from Percivall's Hippopathology:-

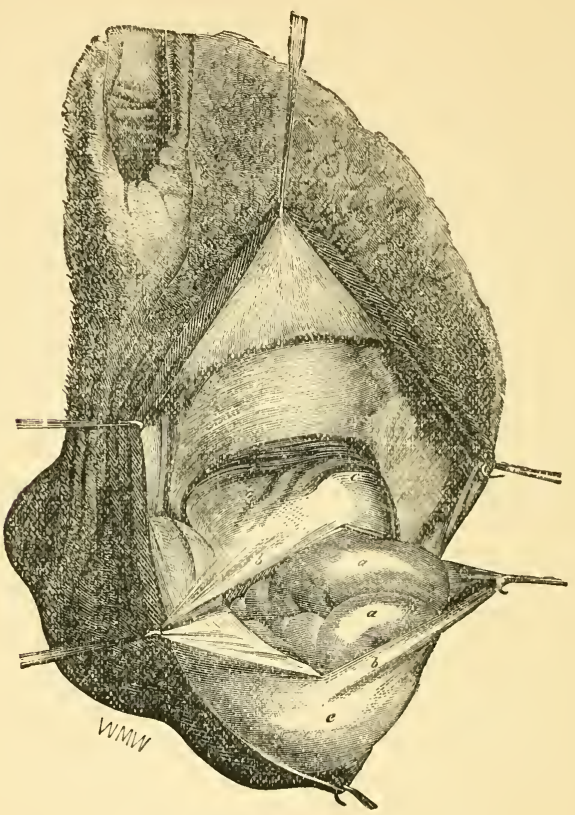

FIG. 113. - Scrotal hernia, showing at $\alpha a$ the fold of intestine in the scrotum; $c c$ is the wall of the hernial sac; $e$ represents the elevation of the tunica vaginalis produced by the testicle.-(GIRARD.)

\section{SCROTAL HERNIA, OR OSCHEOCELE,}

" Owes its production to dilatation of the vaginal sheath of the testicle, combined with relaxation of the fibrous tissue surrounding the ring, and is at first mostly intermittent; that is, it disappears during repose, and returns under exercise or exertion ; which variable condition continues until such a descent takes 
place as renders the tumour, from its weight, incapable of yielding to the retraction of the surrounding parts; in this condition its augmentation goes on, until the matters accumulated within the gut produce obstruction, and that becomes followed by strangulation. These changes, so far from being sudden, proceed rather slowly; and accumulation and obstruction always precede strangulation. While the accumulation is going on, we may observe loathing of food, dulness, indisposition to move; also, as the engorgement proceeds, loss of appetite, constipation, borborygma, colic. Strangulation adds virulence to these symptoms, occasioning, as in recent hernia, the greatest distress, until gangrene takes place, and then all pain suddenly ceases, and cold sweats; shiverings and convulsions close the scene.

"Strangulation.-Practical observations show us that old hernire become strangulated from engorgement, and not from stricture around the neck of the sac at the ring; that can be considered but as a secondary cause. The circumstance of stricture following, however, accounts for the symptoms of strangulation being in these and the afore-mentioned cases essentially alike; being found to vary only in their succession and rapidity of progress. It may be observed, however, that many horses having scrotal hernix not only escape strangulation, but continue to do their work with a large tumour swinging between their thighs; Gibson mentions a case in which " the gut extended the scrotum down to the hock," apparently without any inconvenience from it, beyond what may arise from its bulk and weight. This is a fact which argues most strongly against meddling with such tumours unless we be peremptorily called on to interfere.

"Diagnosis.-It is not always easy to distinguish scrotal enterocele from other swellings of the genitals, and particularly when the hernia is complicated with sarcocele or variocele, or thickening of the cord, or a combination of these affections. The tumour of an enterocele does not preserve a general uniformity; it is commonly most bulky next the abdomen, increasing from below upwards; indeed there are cases in which its volume below little, if any, exceeds that of the scrotum. The swelling yields to pressure, and returns to its form after being compressed. If it be raised up with the hand, it sensibly diminishes in volume, from part of its contents being withdrawn into the abdomen; 
which retraction sometimes is attended with a gurgling noise. Should it be deemed advisable to examine into the state of the inguinal canal, its openings will be found to be more or less dilated and encumbered; and this is an infallible proof of the existence of hernia.

"One diagnostic more I would add, which seems to have escaped the observation of our learned author; and that is, the selfexpansion of the swelling under the effort of coughing. Grasp the tumour with one or both hands, softly but closely, and then let another person cough the horse, and the swelling will be found suddenly to expand under the effort, and as quickly to receut again. Might not this criterion supersede the troublesome business of exploration per rectum et vaginam penis?

"Morbid Consequences.-In almost all chronic herniæ we meet with serous effusion, either into the cavity of the tunica vaginalis, or into the cellular tissue uniting the hernial coverings. Morbid thickening of the tunics is a much rarer occurrence, and one of which M. Girard has seen but few examples. The comparative rarity of cases of adhesion between the gut and sac in horses, ( irard thinks, may be ascribed to the non-employment of artificial pressure, by trusses and bandages, as in man. Mr. Charles Percivall, however, informs me that the occurrence is by no means so uncommon in India, where castration is much practised at a late period of life."

\section{CONGENITAL SCROTAL HERNIA.}

This, the most frequent, but the least dangerous species of hernia, is an attendant on birth, augmenting up to the third or sixth month, after that diminishing, and ultimately disappearing. Should it continue without lessening in volume for a year or eighteen months, it may be considered as, and is in fact become, a chronic or permanent scrotal hernia. In case the swelling, however, instead of being always the same, at intervals diminishes, and continues so to do, more sensibly as time advances, it will in the end recede altogether; and though it return again at times, still, the relapses growing less marked or frequent, at last the gut will enter the ring no more.

In the fetus in utero inguinal hernia is found. M. Lingmenard, V.S., who has practised for twenty years in Normandy, a great 
breeding country, has ascertained, by a vast number of observations, that inguinal hernia is invariably present at birth, even in abortions and in subjects still-born.

After birth.-Herniæ making their appearance a few days after birth are also to be included in the class of "congenital." In these cases the gut becomes hernial in the same manner in which it does in adult age ; it slips through the peritoneal aperture at the ring, and either drags down the testicle along with it, or else follows that organ in its descent; the testicles in ordinary cases not descending prior to the sixth or seventh month. The experienced practitioner above named, M. Linguenard, calculates that about one-fourth of the Norman colts are foaled with scrotal hernia; but that in the majority of them it disappears in the course of growth. In the Recueil de Médicine Vétérinaire for July 1828 appears the following:-These swellings (scrotal hernix) occasionally make their appearance in the scrotum of the colt a few days after birth. Sometimes they occupy one side only of the bag; occasionally both are distended. In a few instances the scrotum becomes as large as a child's head: these are true scrotal hernix. A portion of intestine has descended into the scrotum. Bandages and topical applications are perfectly useless, or worse-producing irritation and pain. At an uncertain period the swelling begins spontaneously to diminish, and at length entirely disappears. When it occupies both sides of the scrotum, it goes back more tardily; and the retraction of one side seems to be quite independent of that of the other.

Causes.-It is worth while to inquire if the herniæ, prior to birth, originate from causes similar to those that occasion it in after age. Certain movements of the full-grown fotus appear very likely to produce hernia, especially at a time when the inguinal apertures and passes are so lax as almost to invite entry; indeed, both the ring and inguinal canal in the fotus appear proportionably larger than in the adult, and evidently possess more extensibility. The parietal parts-the fibrous aponeurosis of the abdomen, the borders of the external ring, the dartos, and the cremaster-being all as yet but imperfectly developed, possess little power to oppose hernia. No sooner has the foetus left the womb, however, than these several parts by degrees acquire strength, until they attain energy sufficient to 
react upon an incarcerated hernia, raise it upwards, and ultimately force it back again into the abdominal cavity and retain it there.

\section{EPIPLOCELE}

Is a frequent companion of enterocele, without adding anything to the importance of the case; indeed, epiplocele of itself is so far from being dangerous that it has occurred without symptoms either of pain or disordered function. Protruded omentum, without intestine, gives rise simply to a soft indolent tumour in the groin, unvarying in volume, unless it receive additional contents-a circumstance that serves at once to distinguish it from enterocele. I think I may add to this coughing, as a corroborating diagnostic. M. Roupp assured M. Girard that in the course of the practice of castration on cart-horses, he had on several occasions met with hernial omentum, and had invariably amputated the protrusion without the slightest ill consequences.

Thickening of the membranes will render the dissection of the dartos from the sac both tedious and difficult; and this may exist to such a degree-in one case they were found an inch in thickness-that for the clams we shall be compelled to substitute a strong waxed ligature for the compression of the cord, which should be fastened by a running knot.

In the case of sarcoccle the operator must be guided by circumstances. Should the tumour consist of intestine principally, the operation is to be conducted the same as for thickened membranes. Sarcocele may render the tumour so firm and compact as to deprive it of every sign or feeling of containing intestine; and should the operator neglect to explore the ring, this concealment may lead him into fatal error, in case he might determine on the removal of the sarcocele. Whenever intestine is detected, he must take care to make himself sure about its return before he ventures to apply either clams or ligature to the sarcocelatous swelling.

Adhesions between the hernial gut and its sac are so rare that M. Girard has seen but one instance of their occurrence; though it would appear, from what has been already stated, on the authority of Mr. Charles Percivall, that between the testicle and its vaginal covering they are by no means uncommun. 
When adhesions of the first kind do occur, it becomes nevessary to open the sac, in order to destroy them, before the reduction can be accomplished, a case wherein the clams will have to be applied upon the bare cord.

In the cases of stoppage and strangulation, herniotomy becomes necessary, and must be practised without delay; otherwise, scrotal hernia in general admits of time for deciding on the operation, and for preparation for it by dieting, blood-letting, \&c.

After the operation, the veterinarian will, besides enjoining a low and appropriate diet, bleed and purge and administer injections, according as the case may seem to require. The animal had better stand with his croup elevated, and be tied up so that he cannot lie down.

Hernia in geldings is a disease of the rarest kind, the veterinary annals of our own country affording ample proof of this; but the fact of there being cases on record is sufficient to show that a case may occur, perhaps at a moment least of all expected; and that we may not be taken unprepared, it behoves us to possess every information requisite for the treatment of such an accident.

In geldings inguinal hernia takes the same course, and is susceptible of the same terminations, as in stallions. Trusses and bandages are all ineffectual; a surgical operation is the only means of causing contraction and closure of the inguinal canal. And this consists simply in returning the bowel, and the application of clams - no cutting being required-upon the outside of the skin, the same as is practised for umbilical hernia.

Treatment of Strangulated, Scrotal, and Inguinal Hernioe in the Stallion.-If the hernia be recent, and not very firmly strangulated, the intestine is returnable by manipulation, even without casting the animal; but should any difficulty be experienced, the horse must be cast, turned upon his back, and maintained in that position, with his croup raised by bundles of straw properly adjusted. The operator will now introduce his hand into the rectum, which has been already emptied by enemas, and explore the internal ring, where he will feel the imprisoned gut. He is then to endeavour to disengage the hernial portion by gently withdrawing it into the abdominal cavity, at the same time aiding its retraction by pushing it with the other hand. Should there be much difficulty in effecting the return 
in this way, the practitioner is to desist, violence being too often the forerunner of strangulation and gangrene. This operation is called the Taxis ; it is recommended by M. Girard, Mr. Percivall, and others. I am, however, of opinion that it is very often an objectionable method of procedure, endangering the animal's life, by causing gangrene of the imprisoned intestine; and from the excessively lacerable condition of the rectum, generally associated with hernia, there is great danger of its walls becoming luptured, no matter how careful the veterinary surgeon may be. It must also be borne in mind that although reduction may be effected by the Taxis, without the occurrence of rupture of the rectum, or the infliction of so much injury to the strangulated intestine as to cause gangrene, notwithstanding the opinion of some practitioners, I maintain that the operation is incomplete unless it be followed by castration; for, without this, the protrusion is likely to recur, and may even do so immediately after the animal has risen. It is, therefore, better to castrate the animal, being careful not to wound the intestine; and the covered operation, namely, that method by which the tunica vaginalis reflexa is left uncut, is that recommended by Percivall and others. My own opinion on the subject will be found in the chapter on Castration.

The covered operation is performed as follows:-The skin and dartos muscle are to be carefully separated from the tunica reflexa until the hernial sac is fully exposed to view; an incision, sufficiently large to introduce the finger, is then to be carefully made into it. When this is done, the operator is to pass his finger into the opening, and discover the stricture; then he is to introduce a strong "bistouri cache" along his finger into the stricture, and divide it, taking care to keep the back of the bistoury towards the imprisoned bowel. In many cases when the canal is thus dilated the bowel will slip into the abdominal cavity with very little trouble; but should a large quantity of intestine be imprisoned, it will be necessary to enlarge the opening in the tunica reflexa to an extent sufficient to allow the bowel to be pulled out and gently unravelled before it can be returned.

The return being effected, the scrotum, including the skin, cord, and its tunics, are to be enclosed in a plain clam, which is to be left on until it sloughs off. Without this precaution 
the operation is unsafe; for the inguinal canal, which has by the operation been made sufficiently large for the incarcerated intestine to return to its proper position, is by the same operation so dilated as to present no impediment to a recurrence of the protrusion.

The constitutional treatment of the animal, before the operation, must be conducted with a view to allay pain and relax the parts; for this purpose large doses of opium are to be given, and the operation may be performed under chloroform.

After the operation, fomentations to the abdomen, repetition of the opium until all symptoms of pain have subsided; and to prevent displacement of the clam and injury to the scrotum, it is advisable to tie the horse's head to the rack, giving him very gentle walking exercise.

Purgatives are to be avoided both before and after the operation, because excitation of the peristaltic action of the bowels increases the contents of the hernial sac, thus rendering the return of the gut more difficult, and by stimulating the bowels generally, converts the irritation of the part into a general inflammation of the intestines. A continuance of the constipation produced by the opium is not to be feared, indeed it is to be encouraged for a reasonable period, say three or four days, for it gives time for the injured portions of the bowels to become restored to their healthy condition. Should the constipation extend beyond this time, and if the practitioner is convinced that the patient suffers from it, a very mild aperient is to be administered-say, one pint of castor or linseed oil ; the large bowels emptied by enemas, and irritation of them prevented by allowing none but the simplest food, bran and linseed mashes being very suitable, and demulcents, such as linseed tea, to drink.

The hernia which sometimes immediately follows ordinary castration, produced either by the violent struggles of the animal while under the operation or in the act of rising, the escape of the bowels being facilitated by a natural lax condition of the abdominal rings and canal, is to be treated like the foregoing, the gut being first returned, when this is possible, and the scrotum and its contents included in a plain (not caustic) clam.

Mr. Stanley of Warwck, late of the 5th Lancers, has furnished 
me with the following account of herniæ as they occur in India.

Inguinal and scrotal hernia are frequently met with in our Indian cavalry, many of the horses being stallions. No age, breeding, or size is exempt or specially predisposed; but countrybred coarse horses are more often afflicted with chronic hernia, probably because there are more of them entire. Stud-bred English-crossed are always castrated.

These forms of hernia are frequently met with on the Continent of Europe, and occasionally in our home breeding establishments.

There are two forms: 1 st. The acute; and $2 d$. The chronic.

Causes.-Fatigue and heat of climate, by relaxing the vascular and muscular fibres, cause the testicles to descend, so that the scrotum appears remarkably large and dependent; the weight of the glands thus gradually dilates the internal ring, and the bowel during its vermicular action glides along the cord into the inguinal canal, and on to the scrotum, constituting the affection under consideration. Hydrocele, violent kicking, severe exertion, getting cast, are all exciting causes.

This affection in our patients is incorrectly termed rupture, because there is no laceration of tissue; all entire horses being liable to it at any time, as the lining membrane of the scrotal sac is simply a continuation of the peritoneal membrane lining the abdomen, and reflected over the viscera; so that there is an opening from the former to the scrotum, connected by a passage termed the inguinal canal, the upper border of which canal (formed of peritoneal membrane) constitutes the internal abdominal ring, which is supported by the fascia of the abdominal muscles, through which it passes by a slit-like aperture. It is through this opening, or mouth of the inguinal canal, that the herniated bowel first passes from the abdomen towards the scrotum ; and if this opening is small, a few inches of the bowel become fixed, the ring acting as a ligature. The vessels of the imprisoned bowel, now in the inguinal canal, become engorged, gaseous distension aggravates the mischief, and we have a case of acute inguinal hernia. The symptoms resemble those of colic or spasm of the bowels, with these additions ; intense, uninterrupted abdominal pain; the testicle on the affected side, drawn close to the abdomen, is not let down on walking, and the 
opposite gland is raised and lowered uneasily; perspiration drops from the scrotum, the loins, and flanks; the leg on the affected side sometimes halts in walking; in some cases it is held up, and the sufferer will bite at the part. The pulse at first is little disturbed, but it very soon beats rapidly, and gets hard, small, and wiry, indicating intense pain ; the countenance is extremely anxious, breathing, panting; the patient is not still an instant, but up and down, rolling, and even screaming with agony, and, later on, straining violently, as if to void fæeces. Examination per rectum, which should be made in every case of doubt (with a little patient experience), will decide at once whether it be inguinal hernia or not, simply feeling with the fingers the cord passing into the ring or mouth of the inguinal canal. If it is clear, the ends of one, two, or even three fingers may go into the ring; if the bowel is there, the ring is found choked and the bowel fixed, so that it may be taken hold of and pulled (of course, to a limited extent), and the patient will instantly strain with all his might. Comparison with the opposite ring will always decide the case.

This examination is easily made while the horse is standing. Nothing (unless there is a considerable length of bowel in the canal-then its inferior border may be defined) can be seen or felt externally in a purely inguinal case. Unless relieved by operative surgery, death results in from nine to twelve hours.

Post Mortem.-Having removed the hind leg on the affected side, on opening the scrotum bloody serum escapes, and the testicle is inflamed. By laying open the inguinal canal, and following the course of the cord, we find it much swollen, highly inflamed, and infiltrated with a sero-fibrinous effusion; and at the top of the canal, close to and just outside the abdominal wall, is a small knuckle of purplish-black intestine, filled with gas, as tight and hard as a ball. The strangulating ring is simply peritoneum inclosing the cord with this bit of bowel. Inguinal hernia terminates more rapidly and fatally than scrotal, owing to the aperture of the ring being so small.

Scrotal hernia may be acute or chronic. The acute is when the herniated bowel has travelled down the inguinal canal into the scrotum, and strangulation has quickly followed. The symptoms are less acute in the early stage, slower in progress, 
and prove fatal a few hours later. The remarks as to diagnosis in the inguinal form apply here, excepting that the scrotum is full of bowel and the canal distended by it. Nevertheless, examination per rectum is absolutely necessary to confirmation.

Chronic scrotal hernia is undoubtedly more ccmmon than suspected, and where the ring has gradually become very much dilated, is accompanied by very little inconvenience to the animal; simply rolling on his back being sufficient to reduce the hernia, as it slips back by gravitation into the abdomen. There was a harness-horse which, after trotting a few miles, always stopped, and could not be prevented lying down, but after having a roll would get up and go on with his work for a time, and then repeat the act, -all explained by a scrotal hernia. Animals that escape pain escape also observation and detection of the affection. There is always danger of ingesta passing with the bowel into the scrotum, producing distension and strangulation; but the more usual consequence is gradual enlargement of the scrotum, a succession of attacks of abdominal pain, gradual vital depression, with loss of condition, emaciation, and inability to work. Then operative intervention is imperative.

Treatment of the acute and strangulated form must be early and decisive, and the operation is one of our greatest triumphs in veterinary surgery, as the patient not only recovers, but is positively saved from a most painful death.

To proceed, give internally a powerful sedative, then secure the horse on his back, his feet being raised by a rope thrown over a beam; draw the hind-leg on the affected side away from the body, and forwards, so as to relax the ring and muscles of the groin; hold the testicle, and manipulate along the cord, so as to force the bowel back, if possible, into the abdomen. Valuable assistance may be afforded by dragging on the bowel per rectum; if the case be chronic, and the canal and ring large, reduction is readily effected in this way. If, on the contrary, the hernia is fixed immoveably, opening the scrotum and amputating the testicle will, in some cases, assist reduction, but is not recommended, as the bleeding makes the operation a very dirty one. The simplest and most effectual method is to cut down on to the inguinal canal close to the internal ring, making a small opening through its peritoneal tunic just to almit a finger (beware of wounding the bowel immediately beneath). The 
pressure of the finger may suffice to return the bowel, but more often, on attempting to pass the tip of the finger through the ring to the abdomen, it will be found so tight that this is impossible ; keep the tip of the left fore-finger against the ring as a guide; slide a narrow guarded bisioury along the guide (with the right hand) through the ring, with the cutting edge outwards and forwards; compress the handles as to bring the sharp edge against the ring; and if properly done, its giving way is instantly felt; remove the bistoury, and press the bowel from the canal towards the belly. It goes readily enough, and as the last portion falls in (the finger should feel round inside the ring to make sure all is clear), the poor sufferer gives a deep sigh of relief, and from that moment the pain ceases. No suture or compress is necessary; the parts being already inflamed and swollen, the wound will keep the animal quiet, and set up sufficient inflammatory swelling and adhesions to prevent effectually the recurrence of the affection. By these means the testicles are spared, and a very speedy cure effected. Cases have returned to work perfectly healed in three to five days after the operation; others have had scrotal abscesses form, a few weeks later, ultimately making good recoveries. The subsequent treatment consists of sedatives, purgatives, enemas of tobacco smoke, fomentations, \&c., \&c., as for inflammation of the bowels: in a case ultimately successful, the bowels were not moved for sixty hours.

It is almost impossible, and indeed would require an instrument a foot long, to cut through the ring by passing a bistoury up the canal from the scrotum, besides giving no certainty as to effecting our object; and by cutting the canal immediately below the ring (readily done, owing to the difficulty of passing the bistoury through the tight ring), the bowel, on pressure, readily disappears, but the patient dies. The post mortem shows us the bowel protruding through the ring, and forced through the slit in the canal, outside the peritoneal wall, and lying between it and the internal abdominal muscle. To prevent such grave issue, a flat hook on the point of the guard of the bistoury, projecting up so as to protect the point of the blade, is necessary; this hook being passed through the ring into the abdomen prevents the ring from slipping off the end, and also protects the bowels in the abdomen from injury, and greatly facilitates the success of 
the operation. The reduction of chronic hernia has been already described, and the prevention of its recurrence suggests itself. Castration by the covered operation is often most effectual; but if the internal ring and canal are large, the canal may burst above the clam, and the intestines consequently escape voluininously. To meet this difficulty successfully, pass a seton needle, six or eight inches long, through the skin posterior to the canal, and high up close to the ring; carry it under the canal, in contact with the external abdominal muscle, and bring the point out again through the skin in front of the canal; twist tape in the form of a figure 8 over the two ends of the needle, moderately tight, thus applying acupressure to the canal close to its superior orifice, so that hernia even of the smallest portion of the bowels is impossible; then castrate by the covered operation; remove the needle on the second or third day (to avoid sloughing), and adhesive inflammation completes the cure.

Complications occur from previous injury leaving the testicle adherent to the scrotum, large hydroceles, hypertrophy of scrotal walls, \&c.; a severe blow on the scrotum, injuring the testicle, as from a kick, or when struck by the butt of a lance or sword scabbard. The symptoms of suffering are scarcely distinguishable from hernia, examination of the ring per rectum being necessary to a correct diagnosis.

"In the Recueil for this year (1875), MI. Barry, of La FertéMilon, describes his method of operating for strangulated inguinal hernia. After stating that the reduction of this hernia is one of the most serious operations the veterinary surgeon has to perform, and acknowledging that he had been very unsuccessful with the recognised modes of procedure; he asserts that the one to which he is now about to refer is that which has constantly yielded favourable results, and is as easy as it is simple.

"The following is the procedure:-After having placed in readiness a quantity of pure oil, a convex bistoury, a suture needle, and a piece of strong twine one to two yards in length disposed as a large bleeding cord, the patient is thrown and fixed on its back as for castration; and the envelopes of the testicle are carefully incised, so as to lay bare the hermial sac. When the knuckle of intestine has been exposed it is dressed with the oil all over, and even gently withdrawn a little in 
order that the inflamed part may likewise be oiled. Then the testicular envelopes are formed into a kind of funnel, into which a quantity of the oil is poured, with the object of more thoroughly lubricating the intestine, as well as the spermatic cord and inguinal ring (into which the index finger is introduced), and ensure the contact of the oil with every part of the strangulation. This done, one hand inserted in the rectum attempts to reduce the hernia by gentle internal traction, while the other assists the reduction of the loop by taxis, in moving the intestine from side to side and up and down, and in doing this to allow the oil to penetrate the canal the viscus has to traverse; this movement powerfully assists in the accomplishment of the operation.

"The oil has a triple advantage:-1. It prevents the irritating action of the external air; 2 . It greatly facilitates the gliding of the intestine, which has become dry from the inflammation; 3. It soothes the latter to a certain extent. There is nothing to be apprehended from the ingress of a little into the abdomen.

"If, notwithstanding what has been recommended, the hernia resists attempts at reduction, this resistance can be overcome in combining the action of the oil with puncture of the intestine, which was resorted to in human medicine a few years ago.

"Barry mentions a case which fully illustrates his mode of reduction of inguinal hernia, and which he was called upon to attend. The animal was lying on the right side, the hernia was on the left. The disordered movements which it had exhibited were now succeeded by a suspicious calmness, cold perspiration, small thready pulse, torpor, and other indications which betrayed the approach of death. Obtaining the consent of the owner to operate, he had the horse put in position, and on incising the tunics of the testicle he found the duplicature of intestine of a deep-red colour, and even livid in three patches-always a very grave feature. The loop was well oiled by the finger, and some oil was poured into the cavity in the manner above described; all the parts were thoroughly lubricated, and the index finger was introduced around the walls of the inguinal canal, which rermitted the oil to descend more readily, and assisted in bringing the strangulated intestine a little farther out. This organ was greatly distended and very voluminous, and puncture suggesteci itself; this was done by means of a suture needle in three places 
for two-thirds of its length; at the third puncture a slight noise was heard, and little air-bubbles appeared around the opening. Barry's right hand in the rectum, in concert with the left, which moved the flexure about two or three times, then promptly reduced the hernia.

Castration was afterwards performed by ligature, which Barry prefers to the clams; these irritating the wound by the great pressure they exercise on the tissues already inflamed. In the covered, as well as in the uncovered operation, the author has always been successful with the ligature. The horse was in a short time afterwards at work.

"Barry asserts, in commenting on this procedure, that the abdominal organs are not always so extremely sensitive to contact with the air as is generally believed; and he incidentally alludes, in support of this statement, to the case of a foal which he castrated, and which the following morning had the omentum hanging as low as its hocks-hernia of the peritoneum. This extruded membrane was red and very inflamed, and Barry was rather puzzled what to do; however, he had the foal thrown; with the right hand in the rectum lie seized the omentum, while with the left he pulled it out until the white non-irritated peritoneum appeared; then with his two hands pulling in opposite directions to stretch it, he caused the owner to amputate the inflamed parts with a convex bistoury; this done, internal traction completed the business.

"The animal recovered without manifesting the slightest derangement."-(FLEMING in Veterinarian, March 1875.)

\section{SCROTAL HERNIA IN PIGS.}

Scrotal hernia is very common in young pigs, and such should be castrated by the covered operation. The general practice amongst pig-gelders is to castrate in the ordinary way, and stitch up the divided scrotum; sometimes they include the intestine in the suture, and cause the death of the animal. I have cut pigs with hernia by merely enclosing the scrotum in a loop of twine, first returning the intestine, and allowing the parts to be removed by sloughing, and they lave done well. Pigs are, during some seasons, peculiarly liable to suffer from tetanis after castration. 


\section{GUT-TIE IN CATTLE.}

Gut-tie occurs in certain districts in young oxen, more especially in the counties of Northampton, Buckingham, and Lincoln, and results from a peculiar method of castration by which the spermatic cord is left too long. It occurs only in oxen that have been castrated at an early age, and generally shows itself when the animal is about two or three years old.

The spermatic cord, when divided, recedes into the abdomen, but adheres partly to the portion of intestine in immediate contact with it; or, according to Mr. Armatage and other writers, the spermatic cord becomes fixed by adhesions to the abdominal ring, and is sometimes separated some distance from the pelvic bones, but united to them by a layer of peritoneum. In consequence of pressure against this membrance, rupture takes place, and a small knuckle of intestine passes through, and shortly becomes strangulated. Sometimes the peritoneum passes before the intestine, forming a pouch or sac. This lesion occurs in oxen used for draught. As the animal grows, the portion of the cord attached to the intestine, remaining short in proportion to the size of the growing animal, draws the adherent gut towards the margin of the pelvis (the course of the vas deferens); the fold of peritoneum, separating the pelvic from the abdominal cavity, is pressed upon and ruptured by the gut; the sac so formed incarcerating it, and causing symptoms similar to those of strangulated hernia. It is found in the right side, because the left side is mainly occupied by the rumen.

The symptoms are very characteristic: no passage of fæces, but a discharge of blood-covered mucus from the rectum; continual and increasing abdominal pain, the patient constantly crossing his legs, and backing himself; he loathes his food, and occasionally lies down. The animal may bear up against it for three or four days, particularly if death is not hastened by the injudicious administration of purgatives.

Treatment.-Some cases are relieved by passing the hand into the rectum, and reduction effected by pushing the impediment upwards and forwards, so as to lift the imprisoned fold through the opening in the peritoneum. Should this not succeed, an incision is to be made into the right side of the flank, and the intestine liberated by cutting the rudimentary spermatic 
cord, and lifting the imprisoned gut from the sac. The wound in the skin is to be closed by suture.

The lesion and the methods for its relief are well known to the veterinarians practising in the districts where it is commonly met with, and they say that the animal should not be cast, but the operation performed whilst he is standing.

Diaphragmatic and Mesenteric Hernice will be considered hereafter, and in conjunction with internal diseases of the digestive organs. 


\section{CHAPTER XXXT.}

\section{DISEASES OF THE BLADDER, EXTERNAL ORGANS OF URINATION, ETC.}

DISEASES OF THE BLADDER, VAGINA, VESICULE SEMINALIS, MAMMARY GLAND, ETC.-CYSTIC CALCULI IN THE HORSE, OX, SHEEP, AND DOG-LITHOTOMY - LITHOTRIPSY-LITHOTRITY - URETHRAL AND PREPUTIAL CALCULI-SABULOUS MATTER - URETHRITIS-PHYMOSIS-PARAPHYMOSIS-AMPUTATION OF THE PENIS-VAGINITIS -LEUCORRHGE-MAMMITIS.

A condition of the urine in which there is a tendency to a deposit of its solid elements is not unfrequently witnessed in the horse, ox, and sheep; occurring either in the form of an amorphous impalpable powder, sediment, or sabulous deposit; in a crystalline form, gravel, or as a hard concretion, calculus, or stone.

In my own experience, the sabulous deposit is most frequently met with in mares, the hard calculus in the stallion and gelding. The gravelly form in oxen and sheep, as incrustations on the mucous membrane of the bladder and urethra, giving rise to suppression of urine, violent irritation of the bladder, and frequently causing the death of the animal.

In the human being it is found that the formation of calculous concretions depends upon various conditions of the body, or diathesis, the most remarkable of which are-1st. The lithic or uric acid diathesis; $2 d$. The phosphatic diathesis; and $3 d$. The oxalic diathesis. In the lower animals, the dog excepted, the conditions of urine leading to the deposition of solids in the bladder are limited to two, namely, the presence of the carbonate of lime, or of the ammonio-phosphate of magnesia.

In the horse and ox, the carbonate of lime calculus is that generally found; in the sheep and pig, the ammonio-magnesia plosphate; and in the dog, the uric acid calculus. 
The following may be taken as an average analysis of the various calculi :-

Carbonate of lime, . . $\quad 85.03$

Carbonate of magnesia, . $\quad$. $\quad 3.62$

Phosphate of lime, . . . . 5.81

Organic matter, . $\quad . \quad$. $\quad 4.21$

Water and loss, . $\quad . \quad$. $\quad 1.33$

$\overline{100 \cdot 00}$

In addition to these constituents, the oxalate of lime, silicic acid, and iron are occasionally found.

Ox (Fürstenberg).

Carbonate of lime,

Carbonate of magnesia, . . . . 10.0

Carbonate of iron,

Organic matter, .

Water and loss, .

$3 \cdot 0$

Silicic acid, inconstant.

Carbonate of lime,

Sheep (Fürstenterg).

$100 \cdot 0$

Phosphate of lime,

Ammonio-phosphate of magnesia,

Uric acid,

Organic matter, .

Iron, water, and loss,

Renal Calculi.

Dog (Lassaigne).

Uric acid,

Ammonia,

Phosphate of lime,

$30 \cdot 8$

Oxalate of lime, .

The foregoing analyses of Fürstenberg have very clearly shown that the calculi of sheep differ from those of the horse and $o x$, in containing the ammonio-magnesian phosphate. It would be interesting-would space permit-to inquire into this 
question. It may, however, be taken for granted that the cause lies in the quality of the food. Very probably the sheep, from which the specimens were obtained, had been fed on turnips and cake, both articles of food being rich in the constituents of the ammonio-magnesia phosphate.

In the horse, as well as in the domestic animals, the carbonate of lime is a constant component part of the urine; and when, from some cause, it is in excess, it becomes deposited within the bladder, either as a sediment or calculus. It is not, however, necessary that it be present in excess ; for it is found that horses which, from the nature of their work, are compelled to retain their urine for many hours at a time, are most subject to calculous deposits. For this reason, hunters are more commonly affected than other horses.

In the mare, cystic calculi form around nuclei, such as pieces of straw, stones, or even iron. I have a specimen in my possession removed from a mare, in which the calcareous matter is laminated around a piece of iron. It can readily be understood that any foreign body accidentally or maliciously introduced into the bladder will cause a precipitation of the urine salts.

Causes.-In addition to those enumerated, the causes of urinary deposits are to be looked for in the nature of the animal's food and water. Some clovers, for example, cause the formation of large quantities of urine salts (often seen on the floor of the stables in the form of a reddish-yellow sand); and should any irritation of the bladder be induced, an increased secretion of mucus will be the consequence, and a nucleus is thus formed, around which the salts may be deposited.

Symptoms in the Horse.- Some stiffness in the hind limbs; repeated motions of the tail; a frequent desire to urinate, exhibited by the animal stretching himself out, drawing his penis, but with little or no result. At other times the excretion of the urine is performed with apparent ease; but it will be often seen that the flow suddenly stops, and the further attempts of the animal to empty the bladder prove in vain. He will continue stretched out for a short time; he may then kick at his belly, groan, sigh, and perhaps lie down in pain. These results are produced by the calculus being forced into the neck of the bladder, and mechanically arresting the further passage of 
the urine. In other cases, and more especially in those where the bladder contains much sabulous matter, there is incontinence of urine, which, flowing over the thighs and legs, excoriates the skin, and produces an erythematous condition of it, causing the animal much annoyance, and giving rise to much foetor. In mares thus affected, incontinence is almost a constant symptom; and in the horse a dribbling of urine generally succeeds the more perfect act of urination.

The urine is sometimes of a high colour, sometimes whitish or yellowish white, and deposits a thick sediment when allowed to cool in a vessel, and a pungent odour of ammonia is emitted by some specimens.

Examination of the Paticnt for Cystic Calculus.-Examination per rectum- " a mode of inquiry even known to Vegetius," savs Mr. Percivall, "is the veterinarian's grand confirming test of the presence of calculus. It may be said to constitute his diagnosis, for it will assuredly resolve all his doubts and apprehensions, and, moreover, can be easily and readily practised without the risk of any injury to the patient."

In this method of examination it is usual to empty the rectum by an enema of warm water, and to choose, as the most favourable time for examination, that immediately succeeding the act of urination, it being easier to detect the stone in an empty than in a full bladder.

If the bladder be full at the time of examination, it should be emptied either by pressure brought to bear upon it by the hand of the operator through the walls of the rectum, or by the introduction of the catheter.

It is recommended by Mr. Percivall that, if the stone is not. found by examination per rectum, the horse should be cast, and the bladder examined whilst he is laid upon his back. It may certainly be necessary to do this, but in the few cases that I have seen the diagnosis has always been easy without casting.

Treatment.-The calculus having been detected, it becomes the duty of the veterinarian to remove it; and to do this an operation is necessary.

Removal of the Calculus in the Mare.-The calculus may be removed without cutting the urethra, and the method is as follows :- 
The hard of the operator, being well oiled, is to be introduced into the vagina, on the floor of which, at a distance of four inches from the external opening, the meatus urinarius may easily be felt. The operator, after carefully lifting up the fold of mucous membrane, which here acts as a valve, is to introduce the fore-finger into the urethra; retaining the finger in the urethra, he must pass the smaller spoon-bill forceps with the other hand. It is necessary that the passage of the forceps be guided by the finger which is already in the urethra, as it is almost an impossibility to pass it beyond the valvular fold without this. When the forceps is fairly in the urethra, its entrance into the bladder is to be effected slowly, its blades being at the same time slightly opened and closed, for the purpose of dilating the passage.

The forceps having reached its destination, the operator is to introduce his right hand along the vaginal canal, or if he cannot get it sufficiently forward, into the rectum, and guide the stone into the blades.

Firm hold of the stone having been thus obtained, the forceps is to be withdrawn with a gentle rotatory movement; but should its blades be too small to grasp the calculus, a larger pair must be introduced, or it may be necessary to crush the stone before its removal can be effected. The urethra of the mare is much larger than that of the horse, and, by patience and care, it can be dilated sufficiently to allow the passage of a very large calculus.

\section{LITHOTOMY IN THE HORSE OR GELDING.}

Cast the animal upon the off side; and when anæsthesia is fully induced by chloroform, an assistant is to draw out the penis and introduce the male catheter. The operator, having placed his right hand in the rectum, is to direct the catheter into the bladder, as it is apt to enter the ejaculatory ducts, and mislead him during the rest of the operation. The liability of the catheter to enter one of the ejaculatory ducts rather than the bladder is a matter that seems to have escaped attention; but it is liable to occur, especially in aged animals: the operator must therefore feel that the instrument passes along the median line into the bladder.

The catheters made by the instrument makers answer the 
purpose very well; they should, however, be provided with a whalebone stillet, the wire ones usually provided being apt to bend upon themselves, and remain in the bent position when the point of the catheter reaches the ischiatic arch. When the instrument has reached the symphysis pubis, the stillet must be withdrawn, in order to allow the catheter to pass easily along the pelvic floor.

The catheter having reached its destination, an incision is to be made upon it, at the perinæum, so as to freely expose the interior of the urethra, and to bring the catheter fairly and clearly into view. The catheter being withdrawn, the smaller forceps is to be introduced into the urethra, and by a gliding motion pushed into the bladder. Some little difficulty is generally experienced in doing this, from the fact that the sphincter vesica contracts pretty firmly upon the instrument; the operator must therefore exercise a little patience, or the parts may be torn by a forcible introduction.

Many writers describe lithotomy as a very complicated operation, and that it must be performed by various cuttings with long-bladed bistouries, guided by “directors," \&c., and Professor Dick used to say that it was necessary to take up the pudic artery; but none of these are requisite, one free incision, sufficiently large to admit the forceps, is all that is required. I have operated in this manner, both for the stone, and experimentally, before my class repeatedly, and always successfully. It is advisable that the forceps be warmed and dipped in oil before it is introduced, as the sensation of cold causes the sphincter of the bladder to contract with some force, and its introduction to be a matter of difficulty.

The operation of cutting for the stone is called lithotomy; that of crushing the stone without cutting, lithotripsy ; and that of boring or rubbing the calculus, in order to reduce it to powder, lithotrity. These two latter methods are inapplicable, except in the mare; but it may be necessary to crush the stone in the horse before removal, and, in such a case, the operation would be a combination of lithotomy and lithotripsy.

Provided the stone cannot be grasped whilst the horse is upon his side, he must be placed upon his back, care being always taken that the hand in the rectum sliall guide the stone into the forceps. 
The after treatment of the case consists in washing out the bladder with tepid water; the approximation of the lips of the wound by sutures; allaying irritation and pain by opium; and keeping the bowels regular by gentle aperients.

In some experiments which I conducted, the skin was drawn to one side before the incision was made; this caused the wound in the urethra to be subcutaneous. I found, however, that although the urine did not escape through it very profusely, there was a collection of it in the areolar tissue, which led to a high degree of inflammation and purulent infiltration.

\section{LTHOTOMY IN THE OX.}

The introduction of the catheter is rendered impossible in this animal, owing to the curve of the penis; and hence the removal of a calculus can only be accomplished by cutting on to the urethra. Calculi are, however, most commonly found in the urethra in these animals, and by following its course externally it will be found distended and fluctuating until the obstruction is reached. This has to be cut upon and removed, and, as Professor Dick used to say, "speedy relief will be given." In some cases in the ox it has been recommended to tap the bladder through the rectum; the necessity of this is very doubtful, as it can always be reached by a perineal incision and the introduction of a catheter through the opening thus made.

\section{URETHRAL CALCLLI}

Occur in sheep as well as in oxen, and give rise to symptoms of great distress. The affected animals repeatedly attempt to urinate, and like the ox, pant, grunt, are restless, alternately lie down and rise, become listless, and if not relieved, die with symptoms of abdominal pains and irritative fever. They should be examined, and the calculi removed, as in the ox.

\section{PREPUTIAL CALCULI.}

When these calculi attain a sufficient size to produce strangury, they cause inconvenience. They are sometimes true calculi 
in the horse, but more commonly are composed of dirt mixed with the secretion of the sebaceous follicles of the prepuce and sheath. In order to prevent inconvenience from these concretions, the sheath must be examined and washed. Horses with large pendulous sheaths are very subject to them.

In the ox and sheep, when fed on turnips or other phosphatic food, long stalactite-like deposits of the phosphates form around the preputial opening, causing obstruction to the emission of urine and much inconvenience; they, and the hair or wool they are attached to, are to be cut off.

\section{REMOVAL OF SABULOUS MATTER.}

In the case of a gelding, an operation as for lithotomy has to be performed, and the contents of the bladder scooped out by the forceps or a spoon, and the bladder thoroughly washea by a stream of water forced in by "Reade's pump." In the mare this can be effected without cutting.

Animals that have much sediment in the urine should be allowed small doses of hydrochloric acid in their water every now and then; the acid passing out of the body through the kidneys and bladder will dissolve the earthy carbonates, which, thus rendered soluble, are ejected with the fluid constituents of the urine.

\section{DISEASES OF THE EXTERNAL ORGANS OF GENERATION.}

It may be accepted that the lower animals are free from specific diseases of the generative organs, and that those affections characterised by a discharge are due to simple inflammation of the mucous membrane, catarrh, debility, or tumours.

\section{URETHRITIS-INFLAMMATION OF THE URETHRA.}

This may occur in the gelding as well as in the entire horse; in the former from a general catarrhal condition of the mucous membrane, and from the irritation of some medicines, such as cantharides or croton; in the latter-in addition to the above causes-from copulation too frequently performed, the general system being at the same time rendered susceptible of inflammation by stimulating food or medicines. Hutrel D'Arboval 
says that a stallion may be thus affected from covering a mare with a small vagina. In bulls, this affection is not at all uncommon, but $I$ am of opinion that it is more a result of injury due to rashness in the act than to any other cause.

Symptoms.-Frequent desire to urinate, the act performed with difficulty; frequent erection of the penis; and a discharge from the urethra. The genitals may afterwards swell, and ulcerations may form upon the prepuce.

Treatment.-The parts to be bathed with warm water, dressed with mild astringents, which may be injected into the urethra, and the animal to have mild aperients and the bicarbonate of soda. If the case be chronic, the animal may have to be cast, the parts examined, and all ulcerated surfaces dressed with the nitrate of silver.

Hutrel D'Arboval describes a disease in the horse simulating syphilis, characterised by inflammatory irritation of the glans penis, extending to the sheath, presenting a tense, shining appearance, and giving rise to phymosis or paraphymosis.

I have seen a condition similar to this originating in cancer of the penis, as well as in those causes described by D'Arboval in his dictionary.

Causes.-According to D'Arboval, this form of irritation is found in horses whose genitals are habitually dirty; or arisin $:$ from accidents, or the introduction of foreign substances into the sheath.

Treatment.-Emollient fomentations to the parts; cleanliness; if abscesses form, they must be laid open, dressed with weak carbolic acid lotions, and the general system modified by mild cathartics, bicarbonate of soda, and demulcent drinks, such as linseed tea. In the more chronic cases, it may be necessary to apply the nitrate of silver, or even to amputate the penis.

The Vesicul e Seminalis are but very rarely the seat of disease; but $I$ have met with instances in young bulls, where they have been distended by an accumulation of epithelium, imperfect semen, and débris. The symptoms are, pain and straining in defæcation, and difficult micturition. On exploring the rectum to discover the cause of these symptoms, a fluctuating tumour will be discovered upon one, perhaps both, sides of the pelvis, pressing upon the urethra and neck of the bladder. 
The accumulation within the vesicle seems to be due to some cause of obstruction in the ejaculatory duct, for by firm yet gentle pressure the contents can be forced out, and the tumour reduced in a few minutes, the contents being discharged through the urethra. If the symptoms recur, it will be necessary to repeat the $\epsilon \mathrm{x}$ amination, and again press out the accumulation.

\section{PHYMOSIS,}

" $A$ morbid condition of the prepuce or sheath, which, from contraction of the orifice, prevents the drawing or exit of the penis." -(Percivall.)

Phymosis is the result of inflammation or engorgement of the sheath round about the orifice, or of enlargement of the glans penis, or of co-existence of these states. Blows, kicks, contasions, wounds, abscesses within the sheath, the presence of warts or excrescences of any kind, polypi even, may all be set down as occasional causes. In geldings the penis becomes diminished in volume and length, so much so in some horses as not to appear protruded in the act of urination; in which case the sebaceous secretion furnished by the interior of the prepuce accumulates within the folds of the integument, and acquires, by detention, irritating properties, which cause the glans penis to inflame and swell to that degree that the animal can no longer pass his urine. In addition to these causes phymosis is occasionally seen when the sheath is much swollen from œdema, produced by want of exercise, disease, the stings of insects, or castration.

The treatment must depend upon the cause. If that be inflammatory, antiphlogistics, fomentations, and perhaps scarifications ; if œdematous, scarifications, frictions with the hand, exercise, diuretics, or purgatives, as the case may be; and when associated with debilitating diseases, tonics ani good food. Cold fomentations are generally of great service.

\section{PARAPHYMOSIS.}

The penis is protruded in paraphymosis, and cannot be withdrawn within the sheath. It may arise from a weakened condition of the penis, sometimes associated with debilitating 
discases, or a paralysis sui generis, or from swelling of the glans penis, with protrusion and enlargement of it, arising from an accident or castration; the sheath forming a tight constriction round it, and preventing its retraction. "The penis paraphymosed" (says D'Arboval) " appears protruded out of its sheath to the extent of half a foot, swollen to the size perhaps of a man's thigh, evidently in consequence of effusion into the cellular tissue of its envelopes, and is curved in the form of an arc, and knotted from partial circular contractions, which, when excessive, are productive of coldness of the organ. Its extremity, the part most tumefied, turns of a red brown; violent inflammation accompanies all this, and the consequent pain is extreme. For all there is so much swelling, however, in general the urine gets a passage, though, should the inflammation run very high, and spread to the body of the penis, gangrene may be the result."

In this country paraphymosis arises from œdema or inflammation of an already weakened or paralysed penis, and is usually met with in old geldings, or in young ones when suffering from debility or disease. In the one case the paralysis is usually a permanent condition; whilst in the other it is temporary, depending upon muscular debility, and usually disappearing when the general strength of the animal is restored. Instances are recorded where the pendulous penis has been returned into its sheath, and maintained there by firm stitches, and the animal sold as a sound one, the trick not being discovered until the new owner has had possession for a few hours, or even a day or two, when, the sutures giving way, the penis has returned to its former pendulous position.

Treatment.-For paraphymosis resulting from paralysis, amputation is the only remedy; but for the same condition, when arising from accidental causes, or from a state of general debility brought about by disease or other weakening influences, the symptoms of urgency only, namely, the pain, swelling, febrile disturbance, and other associated conditions, demand the practitioner's attention. In many instances the swelling will depend upon an œdematous condition of the parts generally; the sheath will be swollen, forming a constricted neck around the pendulous penis, arresting its circulation to some extent, and finally producing a tense inflammatory swelling. In cases of general 
debility, or of any disease accompanied by pendulosity of the penis, and œdema of the sheath, legs, and under parts of the abdomen, the local treatment must be directed to prevent partial strangulation of the penis, by puncturing the sheath, fomenting with cold water, and applying friction to the part; the general system being at the same time treated by diuretics to stimulate and promote the absorption of the effusion; tonics, and more especially a combination of nux vomica and iron, to excite the appetite, to promote the assimilation of the food, and act as a nervine tonic upon the structures of the weakened muscles of the system generally, and upon those of the penis more particularly; and, in addition, the horse must have good food, regular exercise, and careful grooming.

Should actual inflammation of the penis exist, with great engorgement of its vessels, and a tense, shining swelling of its whole substance, it may be necessary to suspend it; at the same time making use of the suspensory bandages for the application of soft poultices, as boiled turnips, to as much as can be covered of the inflamed structures.

For the rapid reduction of the swelling-and this is a point of much importance-cold applications are preferable to warm; cold water, cold astringent lotions, and cold poultices. In addition to scarifications, in all cases that admit it, exercise is useful, by removing venous engorgement and exciting the absorption of fluids. In cases resulting from an accident or an operation, it may be necessary to give a purgative, or, perhaps, anodynes and sedatives; but in those arising from debility, except, indeed, the local symptoms are very severe, depletion of any kind is to be aroided.

Mr Broad of Bath recommended the following treatment:-

Put the penis into a tight elastic stocking by squeezing it with the hands, and as it reduces, lessen the size of the stocking, and suspend the penis as much as possible. If there be much effusion into the surrounding tissues, apply a wide canvas band, with long straps and buckles, round the body, so as to support and cause pressure to the swollen tissues. A few hours' compression from the stocking will often reduce it sufficiently to enable the liorse to retract it. 


\section{AMPUTATION OF THE PENIS.}

Iustances occur when it becomes necessary to remove the penis by amputation. "A state of disease" (says Mr. Percivall), "either of the penis or of its preputial covering, such as has resisted, or seems likely to resist, all treatment; or, indeed, such as would probably occupy any unreasonable length of time to cure, might, perhaps, warrant a recourse to amputation."

I have performed this operation upon four horses, and in tro of them with permanent success ; in one with temporary success, whilst one case terminated fatally in a few hours after the operation, the animal being very aged and feeble. Three of the animals were geldings, and one a stallion. Two of them had cancer of the penis; the others were suffering from paralysis and ulceration of that organ. The operation was performed as follows :- The animal having been cast and chloroform administered, a catheter was introduced into the urethra, and retained there by the passage of a ligature round the penis, an inch or two above the seat of the intended incision. The ends of the ligature were left long-the ligature itself being of strong twine-so that the penis could be fully controlled during the operation. When a large portion of the penis had to be removed, the ligature was passed through the healthy portion of the penis with a strong packing-needle, in order to prevent withdrawal into the sheath before the arterial branches were secured. Everything being now fully at command, the diseased structures were removed by a bold circular incision, the arteries searched for, and secured by ligature. But little hæmorrhage occurred in any of the cases operated upon until some hours had elapsed; but at this time some discharge of blood took place, and continued occasionally for a few days, and arose from the corpus carernosum becoming filled with blood, in consequence of slight erections of the penis, resulting from the irritation of the parts, more especially during the act of urination. In one case-that of the stallion-these erections were frequent, and the hæmorrhage-which was of a dark venous character-alarming, but it could always be controlled by the application of cold water. In order to prevent this venous hæmorrhage, I-in one case-allowed the ligature and catheter to remain in situ for some time, fastening the catheter to the belly by means of a cord tied round it, and carried over the 
aninul's back. I cannot say that it did mucil good, as it caused some uneasiness, and had to be withdrawn.

In the case which ended fatally the penis was so swollen and indurated that it was impossible to draw it from the sheath, and a long incision had to be made through the integuments, immediately below the ischial arch, and the parts amputated through the opening thus made.

In some cases, the flow of urine becomes arrested after the parts have healed up, the process of cicatrisation corrugating the urethral opening; and in such it has been found necessary to divide the condensed cicatrix.

This condition only arose in one of my patients, and he, being old, was not submitted to this procedure. M. Barthelmy, in a paper upon this subject, read before the Royal French Academy of Medicine, thus concludes :- " 1 st. That amputation of the penis may be performed on the gelding without any apprehension from hæmorrhage; $2 d$. That to avoid any obstruction of the urethra, a pipe should be placed in the canal, and, by rings affixed to it, sustained therein for at least two months."

HYDROCELE, OR DROPSY OF THE SCROTUM,

Is a very rare disease in this country. Occasionally a gush of serum issues from the scrotum during castration, but it is of no consequence.

\section{DISEASES OF THE VAGINA AND MAMMARY GLAND.}

Vaginitis, or inflammation of the vagina, except as a complication of catarrhal influenza, or a result of difficult parturition, is almost an unknown disease. This arises from the fact that the female organs of generation remain in a state of inactivity during the greater part of the year, and is in accordance with the general law which exempts from disease those parts whose functions are rarely called into action.

Vaginitis, unless it result from the violence employed in delivering the mare of her young, or inflicted during the act of coition, or injury otherwise done, is merely characterised by a discharge from the vulva of a greyish-white mucus, which soon changes to a yellowish-white purulent-louking material. The 
discharge of this material is inconstant; it collects in the fossa navicularis, and comes away in gushes at uncertain periods, particularly when the lips of the vulva are opened. The mucous membrane is reddened at the earlier stages of the disease, but becomes blanched when the affection has existed for some time. There is but little constitutional disturbance. Very probably the animal is suffering from a common cold, contracted during the period of œstrum; and generally but little treatment is required.

When vaginitis has been caused by violence of any kind, symptoms of a much graver character than the above manifest themselves. The mucous membrane becomes highly injected, at first dry and hot; but a discharge soon takes place, of a thin sanious material, very acrid in its nature, irritating all parts with which it may come in contact, and increasing the inflammation already existing, causing such an amount of straining as to threaten inversion of the uterus, and calling for the free administration of opium. I have seen this form in the cow and bitch, as well as in the mare. When succeeding parturition, it is accompanied by much systemic disturbance, and may prove fatal by extending into the uterus.

Treatment.-This must be directed to lessen the local irritation, and to prevent the straining which it excites. This straining becomes in some instances an alarming complication, and it may be so severe as to cause inversion of the uterus. Warm fomentations to the loins and perinæum are very soothing, and assist in promoting a discharge of a less irritating material from the inflamed surface. The vagina may be injected with warm water containing opium, and should there be any foetor, Condy's fluid or the hyposulphite of soda can be added with advantage. The constitutional treatment must be that calculated to soothe without debilitating; for it must be remembered that the inflammation and the accompanying fever are of a low type-the inflammation partaking of the diffuse nature of erysipelas, and the fever of the typhoid character. Opium, belladonna, or hyoscyamus, in combination with the bicarbonate of soda, are best calculated to allay the pain; and should the bowels be constipated, enemas are to be employed in preference to purgatives.

No active steps should be taken at first to suppress the 
discharge by the injection of any astringent remedy. The inflammation must be first reduced, and the cessation of the discharge will generally follow. Should the discharge become chronic, continuing for a long time after the more urgent symptoms liave passed away, the disease is then termed leucorrhœa, fluor-albus, or the whites, and must be combated by local astringent injections, and the administration of tonics, more especially the salts of iron or copper, combined with small doses of cantharides.

Many cases of vaginal discharges depend on tumours of a cancerous, fatty, or epithelial character, or upon bruises or ulcers caused by the wilful introduction of hard substances, such as fork-handles, by mischievous boys. In all cases the parts are to be carefully examined, and the offending cause removed. I remember one case of a young mare being injured by a large stallion, where the vaginitis so produced was succeeded by the formation of multiple abscesses in various parts of the body. She eventually recovered under a tonic treatment.

Tumours in the vagina are but rarely seen in the mare, but they are not uncommon in some breeds of cattle; they vary in size from a marble to a goose's egg, are round or oval in form, and the mucous membrane covering them is traversed by enlarged and congested veins. These seldom require to be interfered with; but if they cause irritation, discharge, pain, or obstruct the emission of urine, they are to be removed, by torsion if pedunculated, or by clam and cautery if broad in the base.

Vaginal hæmorrhoids are sometimes met with in bitches; either single or multiple, pedunculated or on broad bases. They are prone to bleed, and cause a good deal of irritation and pain, manifested by some degree of straining, difficult micturition, and general restlessness. The best method of removing them is with the cautery or some strong caustic, as their bases or roots must be destroyed, or they are almost sure to recur. If caused by high feeding and want of exercise, the patient should be purged and the causes avoided.

\section{MAMMITIS, OR INFLAMMATION OF THE MAMMARY GLAND.}

Acute inflammation of the udder is of rare occurrence in the mare, but is frequently met with in the cow, and is sometimes highly contagious. I have seen it in mares during the active 
period of lactation, also when the gland has been inactive-in mares with foals at their feet, and in others which had never been pregnant. The exciting causes may be general or local. To the former belong irregularities of diet, a tendency to the disease termed lymphangitis or weed, frequent recurrence of the periods of œstrum; and to the latter, contusions, the direct application of cold, and external injury. Cows are subject to it, when in a plethoric condition at the time of parturition, or if the animal be turned to a rich pasturage, when the organ is in high activity. The last cause is a very common one, and acts by stimulating an organ in an already high state of activity; and it is an example of a healthy congestion being transformed into an inflammation by over-stimulation.

Inflammation of the mammary gland is also very frequently caused by a cruel practice called "hefting," or "over-stocking." This consists in the preparation of the cow for show or market, by allowing her to go unmilked until the mammary gland becomes fearfully distended with its secretion, in order that the milk vessel may have what is considered a fine appearance. Not contented with allowing the gland to become filled until it can no longer contain the milk, the natural provision for relief, namiely "spontaneous flow of milk," is prevented by plugging the teats with cobbler's wax or gutta-percha. ${ }^{1}$ Some scoundrels go even farther than this. They have a mould made to resemble a most symmetrical udder, with the teats in a perfect position, which is fastened below the udder, the animal being put in a frame made for the purpose, and as the gland becomes distended with milk it is thus moulded into the required shape. This is a species of cruelty that the law of the land ought to suppress. It is often followed by violent inflammation, resulting in the obliteration of one or more quarters of the gland, or even by gangrene.

Cows giving no milk, when being fattened for the butcher, frequently suffer from a modified form of mammitis, which retards the process of fattening very materially. This is caused by the retention of some milk in the gland, where it becomes curdled, and acts as an irritant. There is a very cruel but very effective method for the prevention and cure of this in some

1 Latterly a more artistic method has been discovered, and is extensively practised. The process consists in covering the teat, after it is first manipulated into proper shape, by a solution of white gutta-percha or collodion, which rapidly dry and reniler the How of the milk impossible. 
parts of the country, namely, excision of the teat of the inflamed quarter of the gland. This allows the milk to escape; but it can be equally effected by an incision at the superior part of the teat, or in some cases by frequent frictions with belladonna.

Acute mammitis seldom attacks the whole gland, but is confined to one or more quarters, and is expressed by swelling, heat, pain, and redness of the part inflamed, with an alteration in the physical properties of the milk, which is curdled, whey-like, and nixed with blood. There is generally much accompanying systemic disturbance; rigors, succeeded by increased heat of skin. The bowels are generally disordered, being either constipated or unnaturally loose.

The exudation formed in the substance of the gland very frequently destroys its secreting properties, blocking up the acini, lactiferous ducts and sinuses, and leading to the conversion of the glandular structure into a mass of fibrous tissue. This alteration of structure is followed by wasting of the previously swollen part, which becomes a hard, almost cartilaginous mass, smaller than the healthy portions of the gland, and constitutes an unsoundness. A cow with only three-quarters of the udder secreting milk is of less value than when the whole is infact. In some instances the secreting properties may return after the next calving, but in others this desirable result is not obtained. The other terminations are-(1.) Resolution of the inflammation, and restoration of the lactiferous structures to their natural condition; (2.) Suppuration, the gland becoming hard in parts, which afterwards suppurate and burst externally, or the pus may be discharged through the teat; and (3.) Gangrene of the inflamed quarter. This result is apt to occur in cows that are narrow between the thighs-Alderneys and Guernsey, and other narrow cows-and is caused by the pressure of the thighs upon the swollen udder; or it may result from the scverity of the disease in cows that are well formed in this respect.

Treatment.-Removal of the exciting cause, attention to the state of the digestive organs, and for the purpose of removing any source of irritation which may be contained in them, gentle cathartics and alkalies are useful. The accompanying fever is to be moderated by aconite and the nitrate of potash; and should the cow be inclined to eat, she must be fed upon food devoid of much milk-producing constituents. This is essential to success, as it gives rest to the inflamed structures. Soft food of any kind 
is to be prohibited, and water must be allowed sparingly. Sorne practitioners recommend stimulants; but unless there be gangrene of the gland, and much general debility, this method is calculated to do harm.

The local treatment is of great importance. In the first place, the milk ought to be frequently drawn; and if this cannot be done with the hand, the teat-syphon must be used. The withdrawal of the milk, which is curdled and often mixed with blood, and fomentations with warm water, are generally sufficient for the milder cases; but when the udder is much swollen, it becomes a duty to relieve the animal of the increase of weight - which of itself is a source of much suffering-by the application of a broad bandage, made so as to envelope the whole gland, and fastened over the back. This bandage can be made to contain a poultice; and if holes be cut in it for the passage of the teats, there will be no necessity to remove it when it is desirable to milk the cow. The best material for a poultice in cases of this kind is spent hops. They are very light, contain much moisture, and can be easily changed when necessary; they are also very soothing, and do not irritate when they become dry. The udder is to be rubbed with the extract of belladonna, which also may be dissolved and mixed with the poultice. The belladonna serves a twofold purpose; it allays the pain and irritation, and, by relaxing the sphincter of the teat, allows the spontaneous escape of the milk. If suppuration occur, the abscesses are to be opened, the pus allowed to escape, and the wounds dressed with mild astringent antiseptics.

Gangrene of the gland may necessitate the removal of the dead portion by excision; but before using the knife the practitioner must ponder over the probabilities of the case. The late Mr. Barlow recommended early excision. I am, however, of opinion that this should not be done, for the dead parts, if time be allowed, will separate from the living, and are then easily removed by moderate traction; the foetor of the discharges being in the meantime modified by deodorising agents, as Condy's fluid, carbolic acid, chloride of zinc, or the hyposulphite of soda, and the animal's strength supported by good food, milk, eggs, as well as ale or stout, and the appetite stimulated by stomachics. 


\title{
CHAPTER XXXVI.
}

\author{
CASTRATION.
}

CASTRATION-PRECAUTIONS TO BE OBSERVEU BEFORE THE OPERATION IS PERFORMED-METHODS OF OPERATING-CONSEQUENCES OF CASTRATION - SWELLING - HERNIA-SCIRRHOUS CORD-FISTULA OF THE SCROTUM-PERITONITIS-GANGRENE-TETANUS-PARALYSIS-AMAUROSIS-GLANDERS AND FARCY.

THE operation by which the horse is emasculated is generally performed upon him when he is about one year old, and at a season of the year when the weather is neither too hot nor too cold; the latter end of April or the beginning of May is the period generally fixed upon. This time of the year and the age of the animal are sanctioned by universal custom, and for the reasons that the colt is sufficiently strong to bear the operation without much danger, and that the young grass is becoming ready for him when he has recovered. Colts thin and low in the neck should form exceptions to the general rule, and ought to be allowed a few months more time "to furnish" before being operated upon.

Precautions to be observed before operating.-1st. To examine the scrotum, in order to ascertain if hernia be present, and whether the testicles have descended, for in some instances, either from narrowness of the inguinal canal, deficiency of force in the contraction of the gabernaculum testes, or some other cause, the testicles, or one of them, may be retained in the abdominal cavity.

$2 d$. Not to operate upon a thin, weakly colt, nor upon one suffering from any disease.

$3 d$. Not to operate during very cold weather, when an easterly wind is blowing, nor in sultry weather, when flies prevail. 
4th. Not to operate upon an animal, of any age, which is confined in an ill-ventilated, ill-drained, or otherwise unhealthy shed or stable; nor upon one stabled along with a great number of horses.

$5 t h$. To recommend that all colts intended for the operation should have a daily allowance of corn for a few weeks prior to being cut.

$6 t h$. To be very careful that all the instruments required for the operation be scrupulously clean, and the scalpels sharp.

$7 t h$. If the practitioner has conducted a post-mortem examination of any animal, or has attended a case of parturition, or has performed any operation calculated to produce a taint that may cling to his clothes, hands, or instruments, he is to delay performing the operation of castration for at least one day thereafter.

This last precaution is very important, and I may add to it, that colts should not be cut near the dissecting-room of any veterinary college.

Preparation for the Operation.-If the colt be in good condition, and fit for the operation, he is merely to be kept short of bulky food, and to be stinted in his water for a few hours prior to the operation. It is a mistake to starve the animal for a day or two, as is the custom in some districts; and he should have a small feed of corn on the morning prior to being cut. If the colt be not in condition for the operation, it must not be performed for some weeks, the animal to be well fed in the meantime.

In the case of an older animal, which has covered or has been otherwise worked before being cut, no preparation is necessary, provided he is in working condition; but if he is fat, has been standing idle for some time, or from other causes is out of working condition, he should be prepared by one or two purgative doses, regular exercise, and good hard food. It is an error to suppose that the strength and vital powers of a horse should be reduced before he is operated upon. He cannot possess too much health and strength. In fact, a horse, to stand an operation well, ought to be in the condition of a prizefighter when prepared for battle.

The animal-whatever his age may be-being deemed in fit condition for the operation, the practitioner will proceed to 
cast him for the operation, and administer chloroform if thought expedient. As most operators have a way of their own, I neel not describe, in a book of this kind, how it ought to be done, except indeed that I cast animals of all ages for this operation with the ropes, that I throw them upon the near side, and that I sometimes operate without turning upon the back, and without including the fore feet in the ropes. In cases, however, where the testicles are very small and difficult to get at, I find it necessary to turn the animal upon his back.

The animal, being now prepared for the final act of the operation may be deprived of his testicles in a variety of ways, which I need not describe, as I consider that the most humane way is the best of all ways, and that is the operation by torsion, performed as follows:-The operator is to be provided with two clams, one made of two pieces of smooth rounded iron, jointed, the other of flattened steel, as represented in the drawing.

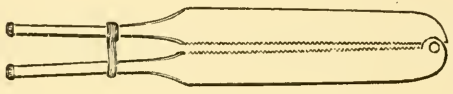

Fig. 114.

The operator having grasped the testicle intended to be first removed-and it is good practice to remove the smaller one first, when the colt is young, or the one farthest away if they are both of a good size-with his left hand, is to secure it in position by passing the smooth elam round the scrotum, so as to press the skin upon the spermatic cord, and thus prevent the withdrawal of the testicle by the cremaster muscle when the incision is being made. When the testicle is firmly included in the clam, the scrotal skin is to be pulled tight over it, and the incision made close to the raphe, and low down towards the sheath, in order to allow the escape of all subsequent discharges, and prevent swelling. The incision is to be nade with a bold sweep of the scalpel, and is to be at least three inches long, when the testicle will generally pop out. The smooth clam is now to be removed, and the flat clam-serrated upon its edges-applied to the spermatic cord, above the epididymus, and securely 
fastened by pulling its handles together and fastening them with the slide. The testicle being now firmly secured, the operator is to divide the non-vascular posterior part of the cord, close to the surface of the clam and above the epididymus, by running a sharp knife flatwise upon the clam, taking care, however, not to cut beyond what is seen to be white and bloodless, and leaving the testicle and vascular cord untouched with the knife. The vascular portion of the cord is now to be firmly embraced with the torsion forceps as close as possible to

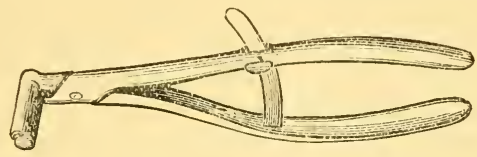

Fig. 115.-Torsion forceps.

the surface of the clam, and the testicle cut off within about an inch from the forceps. The forceps-the grasp of which having been fixed by the screw-is now to be turned slowly round with the right hand of the operator, the left being engaged in keeping the clam steady, when it will be seen that the tissues of the cord quickly give way, until the spermatic artery alone remains ; this gives way more slowly, and very frequently will remain unbroken till it is drawn out as small as a piece of thread. In some instances it will be found necessary to reapply the torsion forceps after a few turns have been made, owing to the cord commencing to give way at a distance from the clam, which-if not prevented by the reapplication of the forceps-would leave a quantity of bruised tissue attached to its extremity.

It is important that but as little as possible of the cord, bruised by the forceps, should be left attached, and that the cord should be made to break across as near to the clam as possible. It is also important to bear in mind that the forceps should be turned slowly and steadily, rapid jerking twists being calculated to rupture the artery before its coats are sufficiently bruised. When the division of the artery is complete, the clam is to be slowly removed, the cord allowed to escape into the scrotum, and the other testicle operated upon. I have operated 
by this method for the last fourteen years, and always with satisfactory results.

The operation by ligature of the artery seems the most surgical and humane, but experience has proved that it is the most unsuccessful of all methods. The late Professor Dick recommended the ligature for a number of years; but towards the end of his life he was forced to acknowledge, and frankly did so, that it was attended with frequent fatal results, the very presence of the ligature seemingly inducing a prejudicial effect, irritating the cord, and causing peritonitis or abscesses.

When hernia is present, operation by torsion is inadmissible, that by the clam being the only method which can be performed with safety; and what is termed the "covered operation," namely, that in which the tunica vaginalis is left undivided with the knife, and dissected from the scrotum, has been recommended. I consider this method a very undesirable one, and calculated to be succeeded by unsatisfactory results; and even if it were always successful, I fail to see the advantage of dissecting the skin and dartos muscle from the tunica vaginalis, and making a wound that is sure to suppurate profusely, when a much more simple method, namely, that of including the scrotum in a plain clam, can be easily performed. I therefore strongly recommend that when a hernia is present, the intestine should be returned into the abdomen, and the scrotum and its contents upon the side of the hernia included in a strong wooden clam (not a caustic calm), placed as close to the external ring as possible, and the whole mass allowed to slough. In this way no incisions are made, no painful dissections; and the barrier to the descent and escape of the intestines, by the skin being included in the adhesive process, is much stronger than when it consists of the serous tunics only.

In castrating aged horses particularly, it is often found that the testicle has become firmly adherent to the scrotum, generally at the inferior and the posterior part of the testicle.

If the adhesion be slight it can be broken down easily; but in some cases it is so extensive as to render this an impossibility, and the operator is compelled to dissect the adhesion until the cord is clearly reached.

Mr. Collins recommends that the covered operation be performed when the tunics are much thickened. Why not 
include the scrotum, and thus do away with the necessity for dissection?

In some cases one or both testicles will be imperfectly developed, small, and almost beyond the operator's reach; but by careful traction they may be included in the clam and removed.

\section{THE CONSEQUENCES OF CASTRATION.}

These are either favourable or unfavourable, normal or abnormal. The normal or natural results are, a varying but slight degree of inflammation, some swelling, and a discharge of serum or pus.

The swelling which succeeds the operation varies very much in different subjects; in some limited to the sheath, and consisting of a mere serosity; whilst in others it extends along the floor of the abdominal walls, even as far forward as the sternum. This, however, need cause no alarm, so long as the animal eats well, and usually a few punctures with the lancet will, by allowing the serosity to drain away, cause its disappearance in a day or two; but when the cord becomes tumefied to any great extent, alarming consequences may be apprehended, and endeavours must be made to suppress the inflammation by fomentations with warm water to the part, assisted by the administration of sedatives and febrifuges.

Some operators are in the habit of applying irritant ointments to the divided end of the cord immediately after the operation, in order, as they say, to produce a discharge of matter. This is a very reprehensible practice, and calculated to inflict much pain. There is no necessity for the establishment of any suppurative action; indeed, the very fact of there being any suppuration at all, indicates that an amount of inflammation, calculated to retard the healing process, has been excited by the operation, and instead of irritating the parts with abominable mixtures, such as sulphuric acid, olive oil and turpentine, it is the duty of the practitioner to perform the operation in such a manner as to cause but the slightest possible degree of pain, and to do nothing having a tendency to retard the process of repair by the adhesive inflammation.

It will be found occasionally that the edges of the wounds unite in a very short time after the operation, and that a consi- 
derable effusion into the scrotal sac subsequently occurs. In such, the rule of non-interference must be slightly departed from, and it becomes necessary to apply warm fomentations to the part, in order to soften and separate the united edges, and allow the escape of the incarcerated serosity; but even in such instances the fomentations need not be repeated.

The inauspicious consequences of castration are, hernia, scirrhous cord or champignon, peritonitis, gangrene, tetanus, amaurosis, glanders, and farcy.

Homorrhage need not occur if the operation be properly performed; a slight degree of bleeding from the vessels of the scrotum is of no consequence, except when it occurs in lambs. In these animals even a very slight amount of bleeding into the scrotum is followed by very serious consequences; indeed, this sometimes causes a great fatality amongst lambs castrated in the months of September and October. It sets up a very high degree of erysipelatous inflammation in the cord, extending to the peritoneum, which, along with the cord and surrounding structures, speedily becomes gangrenous. The blood imprisoned in the scrotum of the lamb, decomposing very rapidly, seems to exert a toxic effect upon the surrounding tissues, therefore great care must always be taken that the operation is performed without hæmorrhage; and for this reason I am of opinion that the scrotum of lambs five or six months old should be opened with the actual cautery, and all bleeding thus prevented.

The admission of air into the peritoneal sac, which often occurs when the horse rises after the operation, and is demonstrable by a rushing or gurgling sound, never does any harm. Indeed, air must always be admitted into the peritoneal sac, and the instances mentioned where the sound often causes much alarm differ from others merely in such admission being accompanied by a rushing noise.

Farey and glanders occasionally result from castration; but they are merely accidental, and depend upon the animal being predisposed prior to the operation, or that he has been subjected to some taint immediately subsequent to its performance.

Paralysis is also recorded as a result; and in all probability it is due to some injury to the vertebræ or muscles of the back or loins, occurriug whilst the animal is secured for operation. 
Tetarius may result from castration, although the operation has been carefully performed. It happens here as it does when caused by any wound or injury, and the operator cannot be held responsible, although attempts have been made to recover damages when death has so occurred.

Amaurosis.-This complication has been observed by several writers, but in every instance the horse has been subjected to improper treatment shortly after the operation, or the hæmorrhage has been excessive. In some instances sight has been restored in a few days, whilst in others the animal has remained permanently blind.

Hernia after castration has been already noticed.

Champignon, scirrhus of the cord, or sareocele, arises from castration with the caustic clam, when the operator has neglected, while removing the clam, to separate the adhesions which always take place between the cord and the lips of the wound; from castration with the actual cautery when the cord has been left too long, or when the cremaster muscle has suffered from some debility, and the extremity of the cord has remained in contact with the wounded scrotum, or has slightly protruded beyond the opening. Mr. Percivall, translating D'Arboval, gives an extended account of this affection; but it is evident that neither he nor his French authority had ever considered the above-mentioned causes. In order to prevent scirrhus of the cord, it is necessary when the operation is performed by the clam, that the operator should introduce his finger into the wound, and gently separate the cord from the scrotum, by tearing the adhesions asunder, and pushing the cord upwards towards the abdominal ring. There is no difficulty in effecting this at the time the clam is removed, the material constituting the adhesions being of a non-vascular, easily lacerable nature; whereas, if the separation be not effected, the end of the cord, imprisoned in the scrotal wound, and subjected to sources of irritation, becomes inflamed, and finally constitutes a fibro-vascular mass, sometimes attaining the size of a man's head, forming a fungous tumour several pounds in weight. Mr. Percivall states that the tumour varies in size and form; sometimes its largest part is below, sometimes above. In my own experience, the tumour has been largest below, appearing as a cauliflower excrescence on the cut end of the cord, the cord 
itself being at the same time much swollen, indurated, and firmly adhering to the surrounding structures. The presence of the tumour, and the pain from the inflammation of the part, cause the animal to become stiff during progression, and to draw up the leg of the affected side whilst standing; to be "tucked-up in his flank," to lose his appetite, and present other signs of constitutional disturbance. Occasionally the limb becomes swollen from an erysipelatous inflammation, with much lameness, and a tendency to suppuration of the inguinal glands. Should both cords be diseased, all the above symptoms are aggravated; the animal no longer lies down, but, as $\mathrm{Mr}$. Percivall states, " continues to be preyed upon by an exhausting suppuration and fever, which bring on marasmus and death. The cord on dissection appears solid, thick, and scirrhous, and as large as a man's arm, all the way from the wound to the loins, with vessels in it the size of one's finger, and divers abscesses, full of black, sanious, fœtid matter; the kidney, haunch, and thigh of the same side containing fistulous ulcerations; the bladder having the appearance of a scirrhus; in fine, all the surrounding parts participating more or less in the disorganization."

I have made the above quotation from Percivall for the reason that I have never had an opportunity of making a post mortem examination of a case of sirrhus of the cord.

Treatment.-Acting upon the conclusion that the fungous growth proceeds from peripheral irritation, and not from intrinsic causes, as previous disease of the cord, or the tubercular or cancerous diathesis, the practitioner will lose no time in removing as much of the mass as he possibly can reach with the knife. I have operated repeatedly and successfully, and have proceeded as follows:- The animal being cast, I have dissected the tumour from the surrounding scrotum, as high up as possible, sometimes removing a portion of skin about half-an-inch in breadth along with the diseased mass; then having fairly separated it from the healthy structures, I have placed the flat clam, Fig. 114, upon the cord, as near the abdominal ring as possible, and have removed the tumour by very slow torsion, twisting it slowly round with the hand, until it has completely given way. There has been no hæmorrhage, although I have removed a champignon between six and seven pounds in weight 
by this method. Mr. Percivall, quoting from D'Arboval, recommends the ligature, and when the scirrhus has extended along the cord too high up to be included in the ligature-a fact, he says, "that may be ascertained by manual examination, per rectum, and that the cord itself is indurated, and increases in volume upwards, and has contracted adhesions with the surrounding parts, and is likely to degenerate into a cancerous mass, reaching from the scrotum to the loins, other means must be sought. One resource is left us, that of piercing or boring the cord with a red-hot iron of sufficient length, and straight, and about the diameter of the finger. The scirrhous cord is to be drawn forth to the extent that it will bear by an assistant, who at the same time diverges the lips of the wound, while the operator plunges the cautery into the scirrhus, and thrusts it longitudinally through it; he may likewise cauterise its sides both within and without the scrotum; the object being to induce a suppurative process which may consume it." With every due deference to Mr. Percivall, and to the authorities from whom he quotes, I think this a very barbarous method, irrational in principle, and cruel in application; I look upon the growth of the fungus to be due to-except in the instances above mentioned, and which may be set down as incurable by any external application or operation-the irritation of the dividend extremity of the cord being kept up by its adhesion to the wound in the scrotum. When the source of irritation no longer continues, and its removal is effected by excision of the tumour, the inflammation of the cord, and all accompanying symptoms will gradually subside. In fact, the continuance and growth of the tumour depend upon a cause analogous to that which produces proud-flesh in a wound, namely, irritation.

\section{CHRONIC SUPPURATION, OR FISTULA OF THE SCROTUM.}

I have frequently met with cases of a chronic induration of the cord, seemingly arising from the same cause as champignon, namely, adhesion to the scrotal wounds, in geldings of various ages. In such, the cord is hard, and enlarged within the scrotum, and from time to time suppuration occurs within its substance; abscesses form and discharge an unhealthy purulent matter. The formation of these abscesses occurs periodically, and may 
ie induced by very trivial exciting causes, such as a common cold, or a hard day's work. The cord then inflames, the animal becomes lame, stiff, feverish, and unfit for work, and will occasionally remain in this condition for several weeks after the abscesses have discharged their contents. Geldings subject to this affection are generally unthrifty, go wide behind, and with a degree of stiffness in their gait. They are unsound, and should the veterinarian be called upon to examine a case of this kind, even months after the animal has been purchased, he need not hesitate to certify as to the unsoundness, provided he can discover that the animal has had abscesses upon a previous occasion; and this can always be done by examination of the scrotum, upon the surface of which depressed cicatrices, indicating the seat of former abscesses, will be found.

The treatment is removal of the diseased cord by torsion.

A case is reported by Mr. Percivall of a tumour of the cord, from this cause, weighing twenty-nine pounds.

\section{PERITONITIS.}

Inflammation of the peritoneum may manifest itself as early as the second day after castration has been performed. It is generally due to one of the following causes:-The prevalence of easterly winds; exposure to cold; the animal being unfit for operation, by either being too fat or too lean; dividing the cord too high up; or it may originate from some constitutional tendency to inflammatory disease when all external circumstances are good.

The symptoms are those of dejection and dulness rather than of acute pain; the animal seems to suffer most excruciating agony, but is too depressed or too much afraid to express his suffering in the usual manner. If there are colicky pains, their manifestation continues but for a short period, and gives way to great depression; the animal standing almost immoveable; a tucked-up and tense abdomen; hurried breathing; quick, hard, wiry, rapid pulse; coldness of the extremities; rapid sinking, and death; occasionally the animal becomes delirious, or comatose and paralysed.

Traumatic peritonitis is an inflammation partaking of the nature of erysipelas, spreading rapidly from its point of origin 
over the whole surface of the serous membrane. The disease is accompanied by a low adynamic form of fever; changes in the blood are induced by which its various constituents become broken up, and discharged by the urinary organs, tinging the urine a dark red or coffee colour.

Upon a post mortem examination being made of an animal which has died from this form of peritonitis, the small intestines, and sometimes the stomach and large ones, will be found to contain large quantities of this altered blood, passive hæmorrhage into the canal having occurred prior to death. It has the appearance of very dark red, or reddish-brown, ill made coffee, containing the grounds.

While the intestinal canal is thus partly filled with altered blood, the surface of the peritoneum presents a variety of appearances. In some parts it is studded with dark red spots, whilst in other parts it is covered by an exudation of an aplastic nature. Surrounding the abdominal ring, a diffuse dark red blush is generally seen, extending over more or less surface, as the case may be. In some instances the exudation of lymph las been abundant, and bands of false membranes may be seen uniting the intestines to each other, and to the abdominal walls; whilst others are characterised by effusion of a turbid serosity more than by exudation of lymph. Several quarts or gallons of serum will often be found, although the animal has been ill but for a few hours. In places, the congested vessels of the peritoneum present a streaky appearance, but what is properly termed congestion is never very strongly marked; this is in all probability due to the readiness with which effusion and exudation take place; the intense dark red colour, found here and there over the surface of the membrane, having more of the characters of ecchymosis or gangrenous extravasations than of true congestive spots. When the intestinal convolutions are separated from each other, a film of exudation is seen to extend across the interspace, and it is seen that the exudation collects in the furrow between the convolutions of the various intestines more abundantly than elsewhere. The effusion is often mixed with loose flakes of coagulable lymph, and it seldom presents the straw colour of a true serous effusion, being generally tinged with blood, or containing ill-conditioned purulent matter, which gives it a dirty grey, or even greenish hue. 
Treatment.-Generally the disease terminates fatally in a few hours, and may be considered as beyond the reach of remedial measures; but if any treatment is to be adopted, it must be that calculated to soothe and support,-opium, stimulants, and the application of hot water to the abdomen, succeeded by mustard. Bleeding, purgatives, and depressants are inadmissible. The remarks of Mr. Ferguson of Dublin upon the treatment of peritonitis are very pertinent, and ought to be carefully studied by veterinary surgeons. They are to the effect that peristaltic action and movement of the bowels should be arrested by opium in peritonitis (see Veterinarian for 1871). If the above remedies are ineffectual, the probabilities of a favourable termination are very remote; but should the acute symptoms yield, great care must still be taken that the patient be subjected to no cause by which a relapse may be induced. Purgatives must be withheld; the food must be of the most easily digested nature. Boiled linseed, with bran well soaked in boiling water, answers admirably, as it is calculated to keep the fæces pultaceous without producing increase of the peristaltic action of the bowels. 



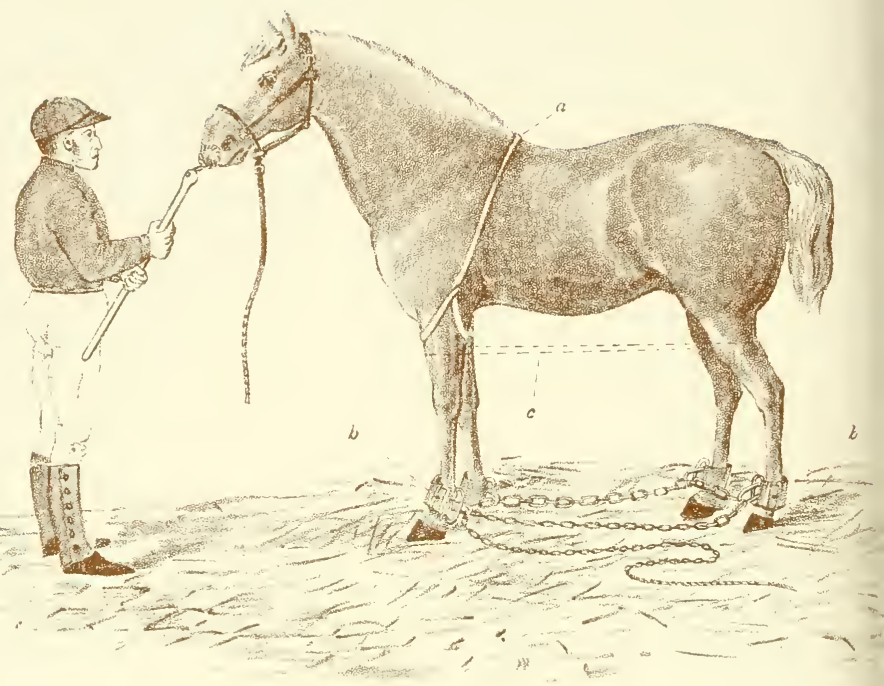

FIG, I.

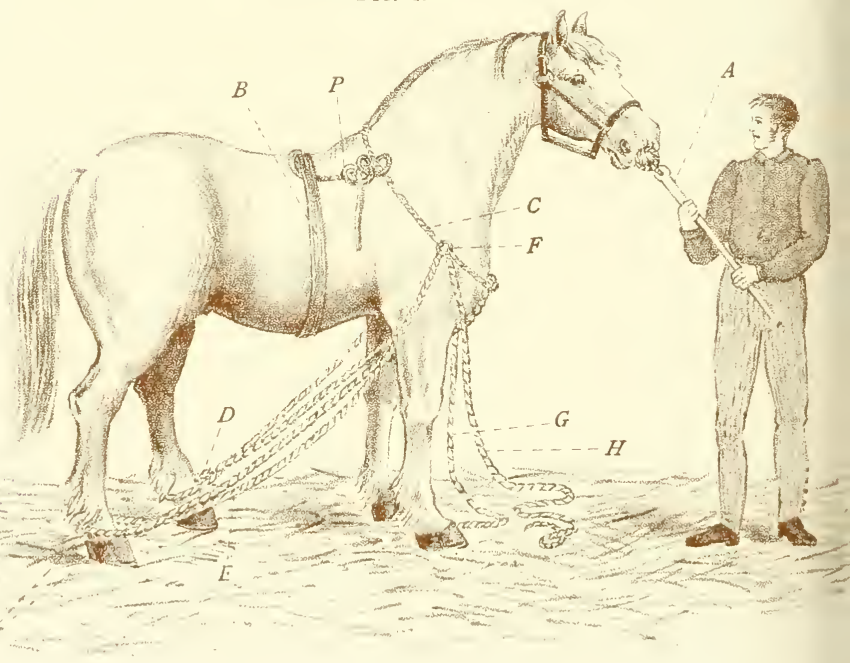

FIG. 2. 


\section{CHAP'TER XXXVII.}

\section{CASTING}

Is the mothod by which animals are secured so as to render the performance of operations safer to both the practitioner and the subject.

There are two methods in ordinary use, the one by "side lines," the other by "hobbles."

Casting by side lines is the method chiefly employed for young animals, and is proceeded with in the following manner.

A rope (which has been in use so as to render it soft) about an inch in thickness and fifty feet in length is doubled, and the doubled end tied in a firm knot, having a loop of about three feet in length.

A bed of straw, peat moss, shavings, or other soft and clean elastic material-never a manure heap-having been made, measuring about twelve feet square, the animal, having a strong head collar on, is led on to the middle of it blind-folded, and a twitch applied to its nose (Plate V., Fig. 2, $A$ ).

A surcingle or back strap (Fig. 2, $B$ ) is fastened tightly round the animal's chest. The loop of the side line (Fig. $2, C$ ) is then passed over the animal's head on to the neck, like a collar, with the knot undermost. The loop is then secured to the surcingle on either side by a strap or rope $(P)$ to prevent the loop slipping on to the animal's neck. The two ends are then passed between the fore legs, one is taken to the outside of the near hind leg, below the hock, passed round to the inside, under itself $(D)$, and up to the neck loop, and passed through it.

The other is taken to the outside of the off hind leg, passed round to the inside, under itself $\left(E^{\prime}\right)$, and up to the neck loop, and passed through it $(F)$.

Two or three men then lay hold of the free end of the near rope $(G)$, and stand by the near quarter of the animal. Other two or three men lay hold of the off rope $(H)$, and stand in front 
and to the off side of the animal, and at a given word the men all pull. The animal's hind legs are thus drawn under him, and he falls on his hind quarters, the ropes at the same time slipping down into the hollow of the heels $(K)$; the animal is pushed on to his side, and both legs are drawn tight up near the neck. The rope is then passed round the fetlock, and a hitch drawn over the foot on to the fetlock and pulled tight. The fore leg on that side is then secured, and the foot brought near, and outside of the fetlock of the hind leg, the position then being, the fore leg outside, its foot opposite the fetlock of the hind leg, and the foot of the hind leg being opposite the fetlock of the fore one. A litch or two of the rope is then cast over the fore fetlock, thus making it secure, and the remaining end of the rope wound round the two pasterns. A similar process is gone through on the legs of the opposite side.

The second method by the hobbles is as follows:-

Hobbles are obtained like those shown in Plate VI., Z. They are nothing more nor less than four leather straps (capable of being lengthened or shortened to fit different animals), three of them having an iron link at either end; the one link $(Y)$ being longer and narrower than the other $(X)$, so as to pass through it $(P)$; and a fourth one-the main lobble (Plate VI.)-having, in addition to the link $Y$, a socket for a screw-bolt, called the key, with which to secure the chain.

Also a chain capable of passing easily through the links is obtained, about ten feet long, having its first sixteen links sufficiently large to allow the insertion of " the $D$," and on its first link having attached a screwed socket $(S)$, fitted to the space $T$ To the other end of the chain is attached a strong rope about ten feet long.

The animal is led on to the bed twitched and blind folded, and, if about to be cast on the off side, a flat rope or $w c b$ (Plate V., Fig. 1, a) looped round the near fore arm, and passed to the off side, to be held by an assistant.

"The key" and " $D$ " are taken charge of by an assistant.

The main hobble is applied to the near fore pastern, and the other hobbles to the other pasterns, taking care that the buckles (Plate VI. and Plate V., $b, b$ ) are to the outside, the points of the straps of the fore buckles pointing backwards, and the points of the straps of the hind ones pointing forwarls. 

The smaller hobble links are then passed through the larger ones.

The assistant at the main hobble (Plate V.) passes through its projecting link the end of the chain, which is then passed from the inside through the link of the off fore hobble, then from the outside through the link of the off hind hobble, and from the inside through the link of the near hind hobble, and up again to the main hobble, and its screwed end $(S$, Plate V.) secured in the space $T$ by the key.

One or two men then lay hold of the web ( $a$, Fig. V.), and three or four men lay hold of the free end of the chain and rope, and at a given signal all pull. The animal's four legs are thus drawn together, and he is pulled over by the men at the web. The man holding the head should endeavour to keep the animal's neck straight, and not allow it to bend towards the chest.

The chain is drawn as tight as possible, and the $D$ inserted into the last link of the chain passed through the link of the main hobble, and then screwed up, to prevent it from slipping back. The free end of the web is then passed round the near hind leg and back again round the near fore one, and twisted round so as to prevent its slipping (Plate V., dotted line $c$ ).

If the animal is to be cast on the near side, the main hobble is to be placed on the off fore pastern, and the web on the same leg.

When the operation is over, the web must first be removed, then the key must be unscrewed, and the hobbles, thus being freed, removed from the legs; the twitch is then taken off, and the animal allowed to rise. 


\section{CHAPTER XXXVIII.}

\section{AN ESTHESIA.}

Andesthesia, or deprivation of sensibility, is of two kindsgeneral and local,-is obtained by various means, and is used to relieve suffering when animals are in pain, or when they are about to undergo some painful operation.

General anæsthesia is usually produced by causing the patient to inhale chloroform, ether, or a combination of these, also by

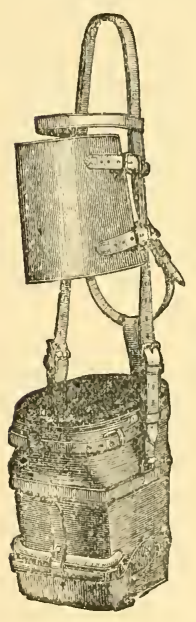

FIG. 116. the subcutaneous injection of morphia or atropia; or by the administration, as a drench, of laudanum, chloral hydrate, \&c. ; but I prefer the inhalation of chloroform, or a combination of that with sulphuric ether.

As a rule it is necessary to cast the animal before administering the anæsthetic, but in some it may be administered whilst the animals are standing; and in old animals, which have stiff backs or suspected anchylosis of the vertebræ, I strongly recommend the administration of the drug in the standing position, and as a consequence the animal sinks naturally to the ground. Care must, however, be taken that there are several assistants at hand, so as to prevent the animal from injuring itself during the excitement from the first action of the anresthetic.

If Carlisle's muzzle-an apparatus I can highly reccmmend (see Fig. 116) - is made use

Carlisle's muzzle. of, about two ounces of the anæsthetic will generally suffice.

Local anæsthesia is produced by the application to the part of cocaïne, morphia, or atropia; or by the subcutaneous injection of cocaïne, morphia, or atropia, by the long-continued 
application of cold water or ice, or by the application of ether spray.

Cocaine, in the College practice, is the most effective and eertain, and has the great advantage of not interfering with any healing process afterwards, like ice or ether spray. The parts must first of all be clipped elosely, and a 10 per cent. solution of cocaïne applied three or four times at intervals of two or three minutes. 


\section{CHAPTER XXXIX.}

\section{DISEA ES OF THE SKI Y.}

CZASSIFICATION-WILLAN ANI) BATEMAN'S, BENNETT'S, AND BUCH ANAN'S CLASSIFICATIONS-ERYTHEMATOUS INFLAMMATIONS-CLIPPING HORSES-HERPES- URTICARIA.

An essay was read a short time ago at a provincial veterinary association by a veterinary surgeon, who stated that Professor Spooner, in his lectures, instructed his class to the effect that if all the skin diseases of the horse were put in a sack and shaken up, they would turn out to be mange. With all due deference to the essayist, I must demur from his conclusions; and from much observation, I am in a position to state that true mange is a rare disease in this country.

In the human being diseases of the skin are so numerous and so important as to demand a speciality of study; but in the lower animals they are found to be much simpler in their nature, fewer in their varieties, and more amenable to treatment. At the same time, many of them bear so close a resemblance to those of man, that a classification based upon the researches of the more recent dermatologists, may with advantage be adopted by the veterinary surgeon.

There are three classifications:-1st. The artificial one of Willan, Bateman, and others; $2 d$. The natural arrangement of Alibert and others; and $3 d$. A pathological classification, founded on the supposed morbid lesions; but of these the classification of Willan and Bateman is considered the best for practical purposes. It is comprehended in the following definitions of terms in common use in the description of skin discases:- 
Order 1. Pimples. - Papulae are simple, solid acuminated elevations of the cuticle, resembling an enlarged papilla of the skin. They commonly terminate in a scurf, and sometimes, though seldom, in slight ulcerations on their summits.

Order 2. Scales.-Squamce consist of cuticle in patches, plates, or laminæ, in which the epidermic cells are morbidly adherent, hard, thickened, whitish, and opaque. These scales cover either small papillæ, red elevations, or larger deep-red and dry surfaces.

ORDER 3. Rashes.-Exanthemata are composed of variously formed, irregular sized, superficial red patches, which disappear under pressure, and terminate in desquamation.

Order 4. Blens,-Bulle. These differ from vesicles in their size, a large portion of the cuticle being detached from the skin by the interposition of a watery fluid, usually transparent. The skin is red and inflamed underneath the blister.

Order 5. Pustules.-Pustulce consist in circumscribed elevations of the cuticle, and contain pus. They have red and inflamed bases, and are succeeded by an elevated scab, which may or may not be followed by a cicatrix.

Order 6. VesicLES._Vesiculce, small acuminated or orbicular elevations of the cuticle, containing lymph, which, at first clear and colourless, may become amber-coloured, opaque, or pearl-like. They are succeeded by a scurf or a laminated scab.

Order 7. Tubercules. - Tuberculae, small, hard, indolent elevations of the skin, sometimes suppurating partially, sometimes ulcerating at their summits.

ORDER 8. SPOTS.-Maculae are permanent discolorations or stains of some portions of the skin, often with a change of structure. They may be whitish, dusky, or dark.

Dr. Bennett has expunged Bullo from these orders, and added two others, namely, those which depend upon the presence of parasitic animals and plants, and which he calls respectively Dermatozoa and Dermatophyta. His classification is as follows :- 
Order I. Exanthemata.

Erythema.

Roseola.

Urticaria.

Order II. Vesicule.

Eczema.

Herpes.

Scabies.

Pemphigus.

Order III. Pustule.

Impetigo.

Ecthyma.

Acne.

Rupia.

Order IV. Papul.e.

Lichen.

Prurigo.

Order V. Squam.e.

Psoriasis.
Pityriasis.

Ichthyosis.

Order VI. Tubercula.

Lepra Tuberculosa.

Lupus.

Molluscum.

Order VII. MaculeE.

Lentigo.

Ephelides.

Nævi.

Purpura.

Order VIII. Dermatozo..

Entozoon folliculorum.

Acarus.

Pediculus.

Order IX. Dermatophyta.

Achorion Schönleinii.

Grubii.

(Mentagra.)

Objection is taken to these forms of classification upon the gruunds that they are mainly anatomical, and that they throw no light on the causes, the pathology, or the treatment of the various diseases. "Febrile diseases are associated with the non-febrile, and local and trivial ailments are associated with those of grave import. They entirely overlook the circumstance that what may be papular to-day may be vesicular tomorrow, and pustular eventually. And, on the other hand, distempers which nature has plainly brought together, and connected by striking analogies and resemblances, are by these arrangements put widely asunder."-(IVATsoN.)

The late Dr. Buchanan, of Glasgow, in a paper "On the Theory and Classification of Inflammation of the Skin," says that skin diseases, like all other diseases, ought to be classified according to their nature or pathology; but when the cause is unknown, some other principles must be sought for, under which groups may be formed; and he finds that in the pathological processes recognised as inflammations, new formations, 
and homorrhages, skin diseases may be arranged into three or more groups.

\section{Classification of Skin Diseases.-(Dr. Buchanan.)}

\section{Class I.-Inflammations.}

Group I.-Simple inflammations (allied to simple dermatitis).

(1) Erythema-(a), simplex; (b), multiforme; (c), chronicum -comprehending papulatum, nodosum, stroplutus, squamosum, pityriasis, furfuracea, membranacea, rubra.

(2.) Herpes (simplex and zoster).

(3.) Urticaria (idiopathic, from indigestion of particular kinds of food; from uterine affections, or persistent).

(4.) Dermatitis (idiopathic, as from burns, or from frostbite ; or symptomatic, as of erysipelas ; or phlegmonodes, as furunculus, anthrax, Aleppo tubercle).

(5.) Pempligus (benign, persistent, and foliaceous).

Group II.-Eczematous inflammations (allied to eczema).

(1.) Eczema (erythematodes; E. papulosum-comprising lichen simplex, and prurigo; E. vesiculare; E.rubrum; E. pustulosum - comprising impetigo sparsa, figuratis, and pilaris; E. lichen; E. squamosum; E. pityriasis). (2.) Acne (comprising A. simplex; A. pilaris; A. rosacea).

(3.) Ecthyma-(a), simplex; (b), chronicum, rupia; (c), gangrenosum.

(4.) Psoriasis (punctata, guttata, nummularis, circinata, (lepra), gyrata, confluens).

Group III.-Ulcers.

(1.) Idiopathic.

(2.) Sympathetic.

(3.) Constitutional.

\section{Class II.-New Formations.}

Group I.-Homologous New Formations.

(1.) Epidermic (epithelial growths, comprising callosities, clavus, ichtlyyosis, cornucutaneum).

(2.) Pigmentary (lentigo, ephelis, moles, melanosis, chloasma, silver stain, leucopathia). 
(3.) Dermic (cicatrix normal, or cheloid, cutaneous tumours (wens), multiple tumours (mycosis), molluscum simplex, condylomata, verruca vulyaris, verruca mollis).

Cinoup II.-Heterologous New Formations.

(1.) Pseudoplasms (lupus-comprehending maculosus, tuberculosus, hypertrophicus, exedens, serpiginosus; and lepra-comprehending maculosa, tuberculosa, ancesthetica, exulcerans).

(2.) Neoplasms (epithelioma, carcinoma).

Class III.-Hæmorrhages, e.g., Petechio, Vibices, Ecchymosis, Purpura.

CLaSS IV.-Diseases of Accessory Organs, e.g., Hair, Nails, Sweat Glands.

Class V.-Diseases defined by Uiriform C'auses.

Group I.-Parasitic diseases.

, II.-Syphititic eruptions.

" III.-Eruptions of Specific Fevers.

"IV.-Scrofulodermata.

The classification of Dr. Buchanan, containing though it does many diseases of the skin which are never seen amongst the lower animals, is yet that best adapted for the purposes of description; and although I have given the table in its entirety, I intend to describe in the text those diseases only to which the lower animals are liable.

\section{ERYTHEMATOUS INFLAMMATIONS.}

\section{(1.) ERYTHEMA,}

Inflammation of the outer layer of the dermis, including not only the slight inflammation produced by mildly irritating agents, such as rubefacients, cold, stings, \&c., but also spontaneous inflammations depending on systemic or constitutional disturbances. Its character or expression is that of uniform redness, (but the redness can only be seen in the colourless skin, such as white heels of horses, and mammary glands of cows), with heat, swelling, and irritation. 
The causes of erythema are cold and heat operating alternately on the skin; wet, friction, dirt, pressure, and constitutional causes, such as hereditary predisposition, debilitating diseases, plethora, and poverty. There are three forms of erythema described by some writers. An examination of the divisions will at once show the reader that the causes are described as the diseases. Thus, erythema intertrigo is a superficial inflammation of the skin, produced by a chafe, gall, or fret, induced by friction of one part of the skin against the other, by the harness, or by the irritation of urinary discharges flowing over the skin, as when an animal is long confined in the slings, when suffering from cystic calculus, or any cause of non-ability to urinate properly. Again, erythema paratrimma means erythema from pressure, such as saddle-galls; and erythema chronicum includes cracked heels, and chapped teats of newly-calved cows and ewes. I refer to this, in order to explain to the reader the various methods adopted in describing skin diseases.

Erythema, as seen in the horse, may be divided into acute and chronic; the latter form being that commonly witnessed in long-standing "cracked heels" of horses, where the skin presents upon its surface bran-like, scaly crusts, the limbs swelling more or less at night, and the animal evincing perhaps a slight stiffness in gait when first moved in the morning, but no suppuration.

An acute form of erythema is often witnessed in prolonged wet weather, involving the limbs to a considerable extent; sometimes all the four legs, arms, thighs, and surface of the abdomen are covered over by patches of superficial inflammation. This disease is generally known under the elegant and euphonious term of "mud-fever." It is caused by the irritation of wet dirt, and, I was going to say, negligent grooming, but a circumstance lias just lately come to my knowledge that compels me to hesitate. It will be generally known that the winter of 1871 was a very wet one, and consequently mud-fever a very prevalent disease. Speaking one day to a large cab-proprietor and jobmaster in this city, and casually referring to the prevalence of sore legs from this disease, he informed me that none of his cab-horses were so affected, whilst his job-horses were all more or less so. The reasons he gave were that the cab-horses were never groomed at night: that they came in at all times, dirty 
and wet, were turned into their stables, but never groomed till the morning; the dirt was then dry, and was brushed off; whilst his best horses (out on job in gentlemen's carriages), which came in early and had their legs washed, dressed, bandaged, and otherwise made comfortable, were all affected with sore legs.

Mud-fever is occasionally attended by a considerable degree of systemic disturbance (hence the term, I suppose), excessive lameness from irritation and pain, and desquamation of large patches of the skin; occasionally it is several weeks before recovery takes place. Even after the febrile symptoms have disappeared, an amount of unthriftiness continues; the hair and cuticle come off in patches on various parts of the body; the appetite remains capricious, and any condition which the horse may have previously possessed soon disappears. Now and then limited suppurations occur in the flexures of the knees, hocks, and pasterns.

Mr. Broad, of Bath, writes to me as follows:- "Nud-fever.The plan of clipping the hair with the clipping machine, from off the legs especially, is the predisposing cause, as it leaves the skin so bare that it cannot so readily resist the effects of irritants of any kind as when protected by its natural covering. The hot water washing opens the pores of the skin, and a free secretion sets in, which, however, becomes suddenly checked from the clothing and bandages not being put on before the vessels of the skin become congested, and subsequently inflamed; hence the disease. The horse is often, after washing, immediately led from the hot steaming stall or wash-house into the open air to his box, the temperature of which is very low; and in other cases he is exposed to currents of cold air from the opening of doors during the process. There is also another very common and bad practice-that of thoroughly wetting all the legs at the commencement of the washing, so that the water may run on to the feet to moisten the dirt and render them more easily cleaned. Mud-fever may occur after washing with cold water, but not with proper and ordinary attention to clothing and bandaging. I have never known it to occur when the mud has been allowed to become dry and then well brushed off without the application of water; not even when the horses have been hunted in districts supposed to cause it. My opinion of the mud of the supposed blistering districts is that it is there 
more plentiful, and of a more sticky character, consequently requiring a longer and more extensive application of the hot washing process. As preventives, allow the hair to remain on the legs from the upper part of the hocks and knees downwards, wash with cold water, clothe, and bandage.

"I strongly condemn the practice of washing hunter's legs before they return to their stables, as I also do that of carriage and hack horses when left at hotel stables for an hour or two, as in such cases they are never rubbed dry or bandaged; the result is often either cracked heels or mud-fever.

"Treatment.-Mix eight parts of glycerine and one of plumbi dia. sol., and apply once or twice daily; give walking exercise, and seldom will there be any other medical treatment required."

Treatment of Erythema.-In limited erythema, aperients, rest, poultices to the parts, succeeded by diuretics, tonics, and the local application of zinc ointment, or, what is very useful, a thick liniment made by adding two ounces of liquor plumbi to six or eight ounces of olive oil. The cause, in the acute as well as in the more chronic form, must be inquired into, and, if possible, removed. Chronic erythema requires to be treated by astringents, avoidance of moisture, diuretics, tonics; and, should these fail, the part is to be pencilled over with a solution of nitrate of silver (one drachm to the ounce of water); or a mild blister is to be applied, and the system generally altered by the administration of the liquor arsenicalis for several days or weeks.

In the more diffuse form the whole of the parts affected are to be fomented with warm water, and dressed over with the zinc or lead preparation: bandages do harm. The constitutional treatment:-Purgatives, febrifuges, diuretics, succeeded by tonics; and should the case be obstinate, arsenic or quinine.

Horses, when clipped very closely, and since machine clipping has become general, suffer very frequently from the disease, which manifests itself as a diffuse form of erythema a few days after the operation. The inflammation extends over the greater part of the surface of the body, and terminates in a few days in small patches of desquamation of the cuticle and hair. Before clipping machines were used, a form of erythema was found, resulting from the effects of the singeing which generally completed the operation imperfectly performed by the scissors. 
Tlis was even much more formidable than that met with nowa-days. If there be much pain and irritation of the skin, and if the animal be afraid of his harness, and easily galled by the collar or saddle, a few days' rest is to be prescribed, together with fomentations to the parts affected; and the following lotion applied :- $\mathrm{R}$. Plumbi acet. $\xi \mathrm{i}$, aqua $\mathrm{Oj}$; ; or the ordinary white lotion may be used.

With reference to the clipping of horses, I am of opinion that it is a great advantage; they work better after being clipped; thrive on less food; are less liable to disease; are stronger, healthier, and more cheerful ; and, when sick, recover in a much shorter time. It is not my intention to discuss the question ; I merely wish to counteract a ridiculous idea propounded by $\mathrm{Mr}$. Gamgee, that clipping is injurious to the horse.

I strongly recommend the Irish method of clipping, namely, clipping all parts of the body except the legs. The hair that is left on the legs protects them from the irritation of wet and dirt; and, when horses are used for hunting purposes, from the penetration of thorns, \&c.

\section{ERYTHEMA MAMMILARUM.}

This is not a rare variety of erythema, and is seen in the cow, affecting the cutaneous covering of the mammary gland; sometimes associated with inflammation and swelling of the gland substance; at other times uncomplicated. It presents itself as a diffuse redness, with heat and pain of the skin. It is generally met with about the time of parturition, and is best treated by a mild cathartic, and the local application of a cooling lotion.

\section{(2.) HERPES.}

" This disease is expressed by patches of irregular form and variable size, upon each of which there arises a group, cluster, or crop of vesicles. It is often preceded by considerable local irritation, cutaneous pain, or neuralgia, which sometimes remains after the eruption has died away. The vesicles ultimately rupture, and a gum-like scab forms over the group, which shrivels and contracts upon itself."-(ArTKEN.) The clusters vary much 
in size, and the vesicles are larger than those of eczema; they do not burst so quickly, and their contents rapidly become milky. A common seat of herpes is the upper lip, constituting what Professor Dick called "crusta labialis ;" occasionally it is seen on the skin surrounding the hoof, and it is then called herpes coronalis, or crown scab. It begins with some heat, and redness, should the skin be white, on a small patch, upon the surface of which the vesicles appear.

Herpes is found in the mouth, on the mucous membrane of the rectum, vagina, and in the interdigital space of horned cattle, sheep, and pigs; in the so-called epizootic aphtha, eczema epizootica, and murrain, or foot-and-mouth disease.

During the prevalence of some epizootic diseases amongst, horses, groups of large vesicles form on the face, lips, even within the mouth, and on the mucous membranes. The vesicles are only seen at their outset, because the epithelium retains the fluid exnded under it but for a very short time, so that a white spot is left, produced by maceration of the epithelium, or, if this be removed, a shallow excoriation. No crust is formed, owing to the constant soaking of the parts in saliva and mucus.

HERPES CIRCINATUS, OR VESICULAR RING-WORM.

There are two forms of ring-worm in the horse- a non-contagious form, the herpes circinatus; and a contagious ring-worm, tinea tondens, or furfaceous herpes, which will be described hereafter.

Herpes circinatus is an eruption of vesicles upon inflamed patches of skin, which assume a circular form. The circles are of varying size, and sometimes enclose an area of healthy skin. They increase in size by their circumference.

In very slight attacks the vesicles are very small, and their contents are disposed of by absorption, the eruption terminating by desquamation.

Causes.-Some disturbance of the digestive functions, or irritation of the respiratory mucous membrane; the eruption being an effort of the system to eliminate some disposition to internal disease. Horses which have been lately imported from Ireland are very subject to this form of herpes; it seems to depend upon a general disturbance of the system, brought about by the sea voyage. 
Treatment.-If very slight, little or no treatment is necessary. The parts may be covered over with the lead liniment, and a diuretic given; but when the vesicles are large, the base inflamed, and the animal disposed to rub himself, a smart cathartic is to be administered, the parts bathed with the acetate of lead lotion, and should there be excoriations, an ointment of nitrate of silver, ten grains to the ounce of lard, should be used. If the eruption be associated with debility, as is commonly the case in young horses which have passed through an attack of a debilitating disease, tonics and good food are to be prescribed. Mr. Erasmus Wilson recommends that when herpes has become chronic, a blister should be applied.

\section{(3.) URTICARIA-NETTLE-RASH-SURFEIT.}

This is a frequent form of skin disease in the horse, and consists of an eruption of elastic eminences, roundish or oblong in shape, and attended with itching.

The lumps rise quickly, and upon the greater part of the body, generally beginning upon the neck, and frequently disappearing as suddenly as they come. They are unequal in size; some like hemp-seed, others as large as beans, and flattened upon their surface.

The peculiarity of this form of eruption is its suddenness. I have often seen the lumps appear upon all parts of the body in the course of a few minutes. It is caused by some disorder of the digestive apparatus, and is sometimes preceded by colic and diarrhœa.

A sudden change of diet will frequently produce it, and it is not at all uncommon when horses are first fed on grass. It is supposed that poisonous herbs, a draught of cold water when the animal is heated, sudden exposure to cold and damp, and calculi or worms in the intestines, are causes of it.

Treatment.-As a rule, this is a very simple matter, a mild purgative being all that is necessary. Should there be much itchiness-the animal rubbing itself-the skin must be bathed with warm water, dressed with the acetate of lead lotion, and low diet prescribed for a few days.

Dr. Maclagan reports the analysis of the urine in a case of uticaria, in which there was deficiency of urea and uric acid, 
"We venture to propose, as the pathological view of the case. that the defect was a deficiency of the products of the transformation of the tissues, and that the retention in this way in the system of matters which ought to be eliminated from it might be the cause of the cutaneous eruption."-(Edinburgh Monthly Journal.) If this be the correct view of the pathology of urticaria, good results may be expected from the administration of colchicum in obstinate or recurring nettle-rash.

In some cases of nettle-rash desquamation of the cuticle and hair occurs; and, what is very peculiar, the hair never assumes its original colour, but remains white on the seat of the rash, the animal being ever afterwards spotted upon those parts where the rash was situated. 


\section{CHAPTER XL.}

\section{DISEASES OF THE SKIN-continued.}

DEFINITION-DIVISION-VARIOUS FORMS OF ECZEMA - SIMPLER-RUBRUM-LICHEN-IMPETIGINODES-ACUTE AND CHRONIC GREASEELEPHANTIASIS-CRUSTA LABIALIS-TREATMENT OF ECZEMATOUS ERUPTIONS-EFFECTS OF CARBOLIC ACID ON THE DOG.

\section{ECZEMATOUS INFLAMMATIONS.}

THE majority of skin diseases, particularly in the lorse, are due to the expression of some form of eczema; indeed, we may look upon it as the commonest skin disease.

Eczema, as defined by Willan and Bateman, is " an eruption of minute vesicles, not contagious, crowded together, and which, from the absorption of the fluid they contain, form into thin flakes." There is first an erythematous state of the skin, with heat, tingling, and itching, soon followed by the formation of vesicles; and the elementary lesions of the skin in eczema may be divided as follows:-(1), An erythematous condition of the skin ; (2), vesicles; (3), pustules; (4), papules ; (5), fissures . (6), a mixture of several or of all these lesions.

The vesicles, when they occur, are usually developed at the orifices of the cutaneous follicles, are easily ruptured, and discharge an albuminous alkaline fluid, which is glutinous and irritating to the skin. When the vesicles are not ruptured, they terminate by absorption of the fluid; but the disease does not often come to an end with the drying up of one crop of vesicles. More commonly, the eruption is successive, and crop after crop of vesicles is formed; or the surface, on which they first appeared, remains red and raw, and continues to discharge a 
fluid resembling that contained in the vesicles. After the disease has existed some time, there is a thickening of the derma, which becomes corrugated, more especially in those parts of the body where the skin is naturally loose and moveable.

The individual vesicles of eczema do not last long, and in some cases no vesicles are found; but the skin is inflamed, and fissures make their appearance in the epidermis. These fissures are at first superficial, but become deeper if the disease continues. This form has been called by Dr. Buchanan eczema rimosum; and in the horse it affects the flexures of the knees and hocks, termed respectively mallenders and sallenders, by horsemen and farriers. Eczema characterised by fissures is grenerally a chronic form of the disease, and when affecting the limbs it has been designated by Greve, Psoriasis carpi ct tarsi.

VARIOUS FORMS OF ECZEMA.

\section{(1.) ECZEMA SIMPLEX, HUMid TETTER,}

A non-contagious disease, usually commencing about the neck, shoulders, back, and thighs. It usually comes on suddenly, and is manifested by itchiness, which causes the animal to rub and bite itself until the hair and cuticle are brought off, leaving the skin red, raw, and inflamed. Successive crops of vesicles develop themselves, dry on the sore skin, or discharge a fluid which seems to cause an extension of the disorder. The parts of the body most usually affected are those which are covered by the saddle or harness; but it mav affect any part, such as the head, neck, quarters, thighs, and fore-arms. It is generally called mange, but it differs from true scabies in two essential particulars, namely, it is not contagious, and does not depend upon the presence of a parasite.

Eczema simplex is sometimes very troublesome and difficult to treat. In some horses it occurs periodically, or when an alteration is made in the diet. As a rule, it is a summer disease, prevailing in the warmer months; but in a few cases which have come under my notice, it has been developed in the winter only, and when the horses were clipped. These are very exceptional and peculiar cases, clipping in other instances favuring the clisappearance of the disease. Eczema simpley. 
is very common in Elinburgh, and most usually ittacks dray and farm horses. In the winter months it attacks horses fed upon boiled food, meal-seeds (liusks of oats), or coarse straw. In the summer, horses well cared for are subject to it when first put on green food, and almost invariably an animal which has suffered from one attack is liable to a recurrence.

In such instances eczema occurs symptomatically as a consequence of some constitutional disturbance. In other cases, the disease is induced by the direct application of irritants, heat, cold, strong ointments of sulphur, tar, and blisters.

It may attack several animals at the same establishment, and this may lead one to suppose that it is true scabies; but it is not so, and the reason why several animals are thus attacked is explainable by the fact that they are all partaking of the same kind of food, and subjected to the same treatment in other ways.

This form of eczema is generally considered to be true contagious mange; but I am satisfied, from extensive opportunities of carefully noting the disorder, that it is not so; and although it may, and often does, attack a number of animals simultaneously, it is generally confined to a few in the same stud, and these will suffer for months without communicating the disease to others. If the history of an outbreak amongst a number be inquired into, it will usually be found that there has been a sudden change of diet.

\section{(2.) ECZEMIA RUBRUM,}

The red inange of dogs, in which there is an eruption (due to a parasite, see Scabies) of vesicles on a very red inflamed skin; the redness is best seen in white dogs. It is usually confined to the thighs, belly, and chest. In chronic cases the skin becomes tumefied and corrugated at the parts affected.

MI. Percivall, in his work on the effects of medicines, says that "a form of eczema similar to the red mange is induced by the incautious application of mercurial ointment to the skin, accompanied by loss of appetite, salivation, closure oi the eyelids, great dulness, fœetid exhalations from the skin and mouth, and sometimes death."

A considerable number of cases of this form of eczema has 
been recorded as occurring in cattle and dogs; and in books on skin diseases it is described as eczema mercuriale. I have seen it result from the irrational administration of hydrargum cum creta.

\section{(3.) LICHEN}

Is a papular form of eczema; the eruption, commencing as small papules either isolated or confluent, and becoming excoriated, discharges a serous fluid, which ultimately concretes into a crust. This forrii is confined to the legs of horses, and situated along the course of the flexor tendons. Sometimes all four legs are affected, frequently one or two; and from the peculiarity of appearance it gives to the legs the disease is known as "Rat tails." This appearance is due to an exudation around the hair follicles; the hairs remain unchanged, except from friction, and emerge from an elevated papulæ, giving to the leg an appearance of being covered by the tails of rats.

\section{(4.) ECZEMA IMPETIGINODEs, or PUSTULOSUM.}

"In eczema impetiginodes the skin is highly inflamed and swollen; the vesicles, which in many places are aggregated into confluent clusters, often communicate with each other, and form a continuous vesicle of some extent. Their contents, at first limpid, speedily become turbid and puriform, and in a short space of time are effused upon the surface by rupture of the epidermis. The purulent secretion, after its effusion, concretes upon the broken surface, and produces yellowish laminated crusts, often of considerable extent. When the crusts are rubbed off or removed, the exposed surface of the derma presents a vivid crimson colour, partly concealed here and there by films of whitish lymph, and secreting an abundant ichorous fluid having a reddish tinge. This secretion hardens, if the inflamed surface be exposed to the influence of the atmosphere, into a thin dark-coloured scab, which remains, unless disturbed by accident or design, until the excoriated surface is healed.

"The eruption of eczema impetiginodes, as of the milder forms of eczema, is successive; fresh crops of pustular vesicles are produced as the first decline, and in this way the disease is prolonged, especially if irritated by the employment of injudicious remedies. In the latter case the affection often lapses into the chronic form of eczema."-(WILson.) 
This form of eczema is frequently seen in the horse, under the well-known appellation of " grease," which may be defined to be an inflammation of the skin at the back of the heels and fetlocks, where vesicles and pustules form, yielding a fotid discharge. The disease is sometimes associated with febrile disturbance. In some instances, swelling of the limb or limbs affected may precede the eruption ; whilst in others, the eruption precedes the swelling. The hind limbs are more frequently affected than the fore ones. The discharge is sometimes very profuse, and it is said that it is capable of inducing an eruption in cows and human beings similar to that of variola; on this account it has been termed equine lymph.

I am of opinion that the evidence as to the specific character of this equine lymph is not sufficient to warrant a conclusion, and that the subject is deserving of further investigation.

The discharge from the pustules and vesicles of grease irritates the surface over which it flows; and the skin of the heels -which in health is peculiarly soft and pliable-becomes rigid; the natural sebaceous secretion of its follicles is arrested, and, as a consequence, the movements of the limb cause the skin to crack, and to become a mass of soreness, ulceration, and fungus, accompanied by heat, pain, and lameness. When the disease is of this type, it is very apt to assume a chronic character. The febrile symptoms, along with the heat, pain, and lameness, diminish; but the swelling still continues, and the skin is constantly moist and greasy from the discharge, which is thick, fœetid, and mats the hairs together. Masses of fungoid granulations now appear, springing from the unhealthy sores, consisting of hypertrophied papillæ, covered over by abnormal horny scales of epithelium, loosely attached to their surfaces, easily rubbed off, and exposing a highly-vascular sensitive surface beneath, which bleeds at the slightest touch. These excrescences are commonly called "grapes," and they belong to a class of skin diseases described by dermatologists as "acne," or chronic inflammation of the sebiparous glands, characterised by the eruption of hard, conical, and isolated elevations, which sometimes suppurate on their summits, or pour forth an inordinate quantity of secretion; whilst in other cases their action is torpid, the sebaceous matter is concreted into a solid form, and distends the excretory duct and hair follicle even to the 
orifice. Professor Hering has found in chronic grease large numbers of acari, called sarcoptes hippopodus, of which the accompanying woodcut (Fig. 117) is an illustration.

Hering says of it that its body is twice as long as broad, beset all over with hairs like satin; head retractile; proboscis consisting of two valves moving laterally; mouth directed rather downwards ; close to it two small palpi ; eight feet, five-jointed,

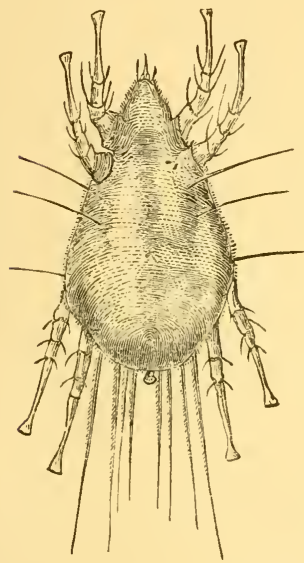

FIG. 117. - Sarcoptes hippopodus.-(Hering.) the last joint as long as the four preceding, with a small sucking disc at the end, and two small hairs on each joint. Two pairs of feet originate near the head, and two posteriorly on the belly. On the abdomen a small prominence, and four long, straight, plumose bristles; their length $0 \cdot 16$, their breadth $0.08-0.085^{\prime \prime \prime}$. The three pairs of bristles on the back and those at the abdomen can be raised like the tail of a peacock. The large bristles are plumose; the hairs on the joints of the feet diminish in length towards the extremity of the foot. Only the third joint of the first pair of feet has a longer hair.

The presence of this parasite in chronic grease is accidental; and other diseases, such as canker, mallenders, and sallenders, are apt to hecome complicated with a mange- caused by this parasite. Gerlach designates this epizoon symbiotes equi, and says that the disease induced by it may be called foot-mange; but it has nothing in common with canker, or other known cutaneous eruption. Attention is at first drawn to a horse with this disease by his rubbing his fore legs, or striking constantly with the hind ones during the night. The seat of the disease, and the ready detection of numerous parasites in clusters where the crusts or scabs form, about the horse's heels, \&c., suffice to enable us to diagnose the malady.

The swelling of the legs affected with grease, at first consisting of material capable of reabsorption, becomes transformed, in 
neglected cases, into a low form of fibrous tissue, constituting what is termed elephantiasis; in some cases the affected limbs become enormously enlarged from this cause.

The causes of grease are both intrinsic and extrinsic. Of the intrinsic and predisposing, "breed" is the most common, grease being generally met with in hairy-legged cart-horses with big broad feet and light "washy" bodies. Some are of opinion that white legs are peculiarly liable to this disease; but a careful and impartial investigation of the matter has led me to conclude that this idea involves a popular fallacy.

The exciting causes of grease are, improper food, especially moist, inferior, and cooked food, clipping the hair of the heels and legs, filth, and neglect.

In some instances the best bred and best cared for horses are liable to grease; but in them it scarcely ever assumes a chronic character, but partakes more of an erythematous than of an eczematous nature. In race-horses, the process of sweating induces cracked heels; the sweat, running down the hollow of the heel, dries, and leaves the part impregnated with its salts, which act as irritants to the skin.

\section{IMPETIGO LABIALIS ET FACIALIS.}

The disease occurs in the lower animals as crusta labialis, from eating some kinds of food, and is chiefly seen affecting animals with white faces and legs. Some veterinarians state that it is unknown to attack any but the white portion of the skin, and that animals without white hairs are exempt from the disease. Professor Roll, of Vienna, refers to it in the following terms:-There occurs amongst horses with white faces, and only at pasture, an eruption which consists in small pustules over which yellow crusts form, and which adhere to the rough and thickened skin. An ointment is required to favour the separation of the scabs, and no further treatment is called for.

Mr. Gamgee mentions several other authorities, who describe the disease as occurring upon white parts of the body, and that its origin is due to the eating of vetches which had become subject to honey-dew; buckwheat in flower and seed; and hot and wet weather, after animals had eaten of a variety of wild polygonum; and that in some instances the white portions of 
the skin inflamed and sloughed, while the dark parts were unchanged. This peculiarity can scarcely be said to apply to the disease as it manifests itself in this country, where colour or the want of it does not affect its course and progress. I have repeatedly seen it in sheep, as well as in cattle and horses, fed on certain clovers, which seemed to excite the disease more by being brought into contact with the skin than by any effect upon the general constitution; the eruption being confined to those parts of the body which were continually touched by the herbage, namely, the lips, nostrils, coronets, and pasterns.

Treatment.-The removal of the affected animals from the field containing the cause will generally be sufficient; but should surgical interference be required, the parts are to be rubbed over with the zinc or lead ointment, and purgatives administered. If there be much pruritis, the irritable parts are to be bathed with the following lotion:-

Re Acid Hydrocyanic, . . . $\quad$ కss. Aqua, and afterwards covered over with lard.

Mr. Robertson of Ellon recommends the ointment of the nitrate of mercury.

\section{TREATMENT OF ECZEMATOUS ERUPTIONS.}

The different forms of eczema present varieties of character peculiar to themselves, and these must be borne in mind when they become subject to the veterinarian's care. In most instances, when not induced by direct local irritation, the eruption may be looked upon as a safety-valve to the system, and, consequently, the discharge by which it is accompanied must be suppressed very gradually, and not before a counter one, from the intestinal mucous membrane, has been medicinally or otherwise established.

In all cases the treatment is to be'regulated by the severity of the symptoms, and by the particular cause and locality of the disease. When it is of constitutional origin, purgatives are to be employed, which are to be succeeded by diuretics, antacid stomachics, and when the disease is chronic, specific alteratives.

Different purgatives are used, according to the animal treated: for the horse, aloes; for horned cattle, sulphate of soda or mag- 
nesia ; and for dogrs, jalap and calomel, caster oil, or the syrup of buckthorn. The diuretics should be those that excite the excretion of the solid constituents of the urine, such as colchicum, with the nitrate of potash, or iodide of potassium. When the eczema is associated with disorder of the digestive apparatus, loss of appetite, and irregularity of the bowels, the bicarbonate of potass or soda, and bitters, as gentian, quassia, or cinchona bark, are to be administered. In most cases of irregularity of the bowels, where the fæces emit a fœetid and peculiar odour, I have found two-ounce doses of the hypo-sulphite of soda very useful. Of specific alteratives, arsenic, in the form of arsenite of potass, is the most valuable. For the lower animals it is to be prepared in the simplest manner possible, and its preparation is as follows:-Acid arsen., $3 i$.; potass carb., $3 i$.; aqua, 3 xii.; mix, and boil slowly, until the arsenic is dissolved, and strain when cold. Prepared in this way, it is almost tasteless, and quite odourless. Each ounce of the solution contains five grains of arsenic; the dose is from half an ounce to an ounce of the liquor, two or three times a day for the horse, mixed with his food or water; horned cattle will take rather larger doses; and the dog, thirty to sixty drops, according to size.

For animals in low condition, the arsenic may be combined with vegetable tonics, and given in the form of a ball, nux vomica making a valuable combination with it.

The diet is to be regulated according to each particular case. Some animals are so constituted as to be affected by what in others proves to be the simplest of food, namely, green fool. In such instances, there must be a change to dry food, and in all, during the earlier stages of the disease, there must be some alteration and restriction both as to the quantity and quality. It must also be borne in mind that all rough articles of diet, such as meal-seeds, coarse straw, and other inferior and damager stuffs, are very prone to cause skin diseases. Locally, the parts affected are to be bathed with warm water, or poulticed, if possible. The irritation and itchiness (pruritis) are relieved by the application of a diluted solution of the diacetate of lead, the white lotion, or a solution of the carbonate of potash; in cases where the itching is very extreme, by hydrocyanic acid largely diluted. When in this state of itchiness, the animal should be fastened, so as to prevent his biting or otherwise damaging him- 
self. To the parts that have already been made raw, a mixture of acetate of lead and olive oil may be applied with advantage.

The local applications to the impetiginous form of eczema differ somewhat from the above, and consist of fomentations and poultices, succeeded by mild, and afterwards by strong, astringents.

A very useful application to greasy and cracked heels is found in the oxide of zinc ointment. The benzoated oxide of zinc ointment is recommended by Erasmus Wilson as the most perfect local application for all chronic inflammations of the skin.

Professor Dick, who was very successful in the treatment of grease, recommends that the legs should, in the early stages, be washed twice a day with soap and water, and a solution of sugar of lead and sulphate of zinc applied. "This," says the Professor, "may not be chemically scientific, but I have found it superior to anything else. When grapes abound, powdered sulphate of zinc should be introduced among them, which is found often to supersede the application of the actual or other cauteries; and, in like manner, strong washes with dilutel sulphuric and nitric acids prove not less efficient."

I have found it necessary in many cases to remove the grapes with the actual cautery, more especially when very numerous; and for this purpose two blacksmiths' fire-shovels are the best instruments; one to be made sharp at its edge, and heaterl to a red heat, to remove the excrescences; the other kept cold, and placed between the skin and hot shovel, to prevent undue burning. In this manner I have removed many scores of these excrescences in a few minutes; whereas, if they had been destroyed by caustics, such as the sulphate of zinc, corrosive sublimate, or the strong acids, the destruction must have been effected by slow degrees, or deep and extensive sloughings of the skin and subcutaneous tissues would have occurred. When, however, the grapes are few in number, their points may be carefully dressed with the sulphate of zinc or corrosive sublimate, eare being taken that the applications be not too extensive.

The fotor of the discharge of grease, either in its acute or chronic form, is much modified by carbolic acid. In the acute stage, a little of the pure acid is to be added to the lead or zinc 
lotion ; while in the chronic, the crude acid may be applied, for one dressing, undiluted. In this form it stimulates the skin to healthy action; but when applied after the first time, it must be diluted with oil or glycerine, one to twenty parts, or inflammation and sloughing will be induced. As primary applications, even in the treatment of chronic grease, poultices are very useful; they clean the parts, soften and remove scabs, and allay the inflammation of the skin; boiled turnips are best, and they may contain yeast, charcoal, carbolic acid, or the hypo-sulphite of soda, as deodorisers.

In chronic grease, after the bowels have been well opened by a cathartic, arsenic and diuretics are to be prescribed until the anasarcous swellings disappear; and should there be a disposition to a return of the disease, the arsenic is to be continued, and combined with tonics.

When it is deemed necessary to change the arsenic for another remedy, the sulphates of iron and copper may be substituted.

Chronic eczema, when expressed by an eruption other than that of grease, requires a speciality of treatment applicable to itself.

1st. The crusts and scabs, after being soaked with oil for a few lours, are to be removed by washing; if the hair is long it must be clipped, and this applies to the greasy as well as to the other forms of eczema. When the hair is removed and the parts thoroughly cleaned, a sufficiency of the following may be applier, not only to the diseased, but to a good deal of healthy skin; remembering that whatever the remedy may be, much depends upon its effectual application :-

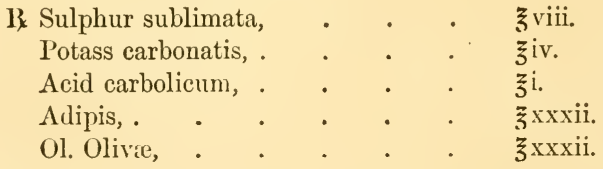

This is to be left on the skin for two or thrce days, and then washed off with soft soap and warm water. It acts as a cutaneous stimulant.

The other remedies available in chronic eczema are lime water, bichloride of mercury in weak solution, tar ointment, 
and mercurial ointment; and to relieve the itching (pruritis), when it is very great, hydrocyanic acid lotion.

Canine skin diseases require to be treated with very great care; remedies that cause a great amount of constitutional disturbance in the dog have no such effect when applied to other animals : for example, carbolic acid, although carefully prepared and diluted, and as carefully applied, produces in some instances so much depression as to cause death in a few hours, by a gradual failure of the heart's action (asthenia); in others, where the first depressing effects of the remedy have been overcome by stimulants, warmth, or electricity, the animal has fallen into a state of marasmus, with sunken eyes, fœtor of the breath, formation of sordes on the teeth, "tarry" fæces, total loss of appetite, and death in six to twelve days. For these reasons I have discontinued the carbolic acid in $\operatorname{dog}$ cases, although it is an excellent local remedy. It might be supposed that these toxic effects result from the dog licking himself, thus introducing the acid into the stomach. I held this opinion at first, but further observation of cases where such precautions were taken as to render the licking of the poison an impossibility, has convinced me that it is absorbed into the system through the skin; that it has a peculiar effect upon the dog, and, consequently, is a dangerous remedy. For similar reasons the mercurial ointment is unsafe.

For the dog the following is the safest and best cutaneous stimulant:-

R. Unguentum sulpho-alkalinum.

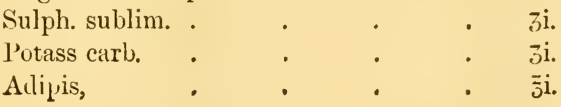




\section{CHA P TER XLI.}

\section{DISEASES OF THE SKIN-continued.}

URINOUS ECZEMA.

UNDER the above term I have to describe a disease manifested by a peculiar condition of the skin, which $I$ have seen a few

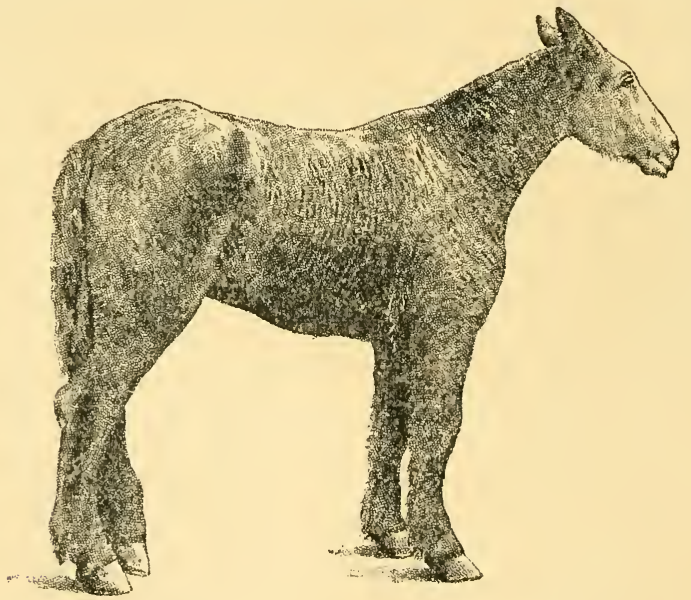

FIG. 118.-Appearance of the mare-mane, body, and legs clipped.

times during my experience, and which is shown in the photograph.

The first case occurred many years ago, and was seen in consultation with Mr. Aitken, V.S., Dalkeith. A young cart horse 
was said not to have urinated for three months, and, in addition to the symptoms about to be described, there was a constant flow, from the lower part of the mane, of a urinary fluid, which flowed over the shoulder and down the limb. The excretion had a strong urinary and ammoniacal odour. The horse either died or was destroyed, and unfortunately no post mortem examination was made.

The second case was that of an old circus horse belonging

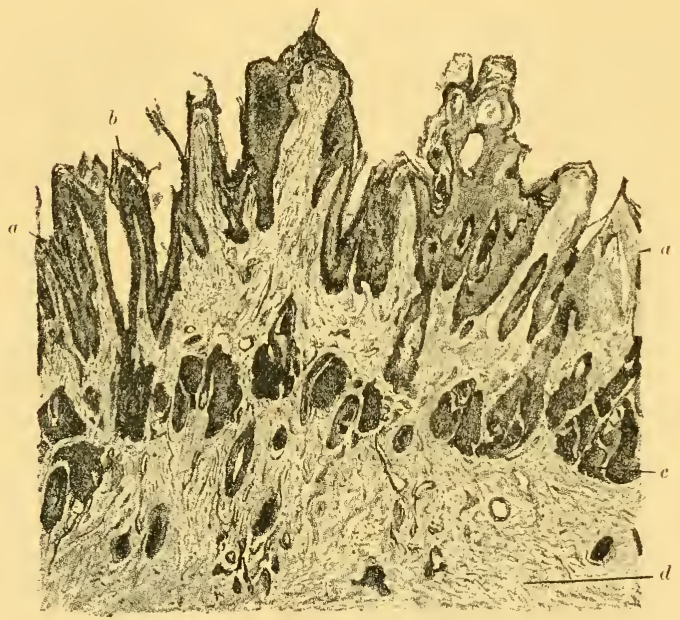

FIG. 119.-SKIN. Vertico-transverse section of skin. $(\times 20$.

a. Rete mucosum.

$b$. Remainder of desquamated superficial layers of epidermis.

c. Hypertrophied sebaceous glands.

d. Cutis vera distended and softened.

to Mr. Newsome. This horse continually sweated an ammoniacal fluid from both flanks, and the skin of his legs was very much thickened; he passed small quantities of urine, having a thick, mucilaginous character, ate fairly well, but gradually became emaciated. The horse leaving Edinburgh alive, I had no chance of making a post mortem examination, but I know that he died shortly after I had seen him. 
I next had an opportunity of examining the kidneys of an animal which had presented the symptoms of the disease, but which I did not see in life. The kidneys presented all the appearances shown in the photographs.

The animal from which the photographic illustrations were taken was treated by my son and myself from the commencement of her illness-from 11th December 1890 to 6th March 1891-until she was destroyed at the College.

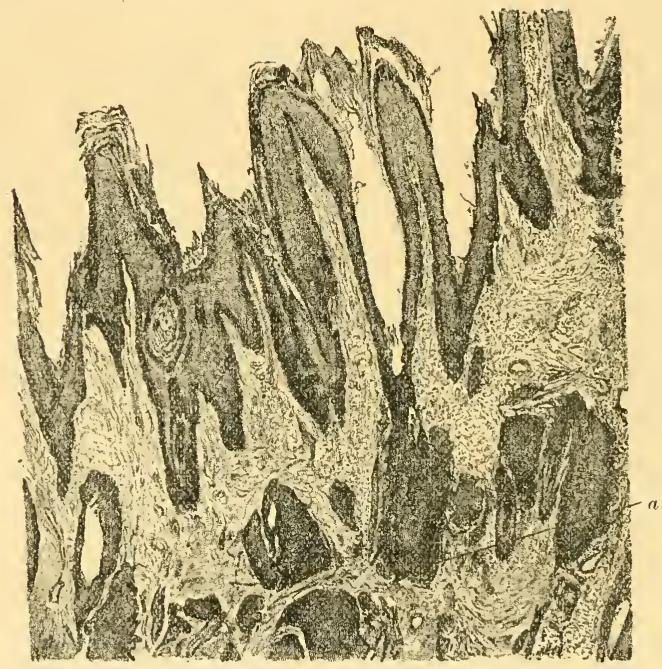

Fig. 120.-Skiv. Under a higher power. $(\times 40$.

a. Supposed altered duct of sudoriferous gland.

At first she presented the symptoms of scarlatina, from which she apparently recovered; but before being put to work the appearance of the body and extremities, shown in Fig. 118, began to manifest itself, although she fed well and kept up her condition,--in fact at one time improved in condition and became almost fat. About a year before this final illness she had an attack of azoturia.

The symptoms may be described as follows:-1st. Swelling 
of the extremities, from the surface of which a fluid having a fotid and urinary smell constantly exuded; the skin became excoriated, particularly at the flexures of the joints, upon the inner surface of the thighs, and between the forearms and chest; an eruption then appeared on the whole surface of the body, including head and tail, from which issued a fluid which concreted into thick squamæ or crusts.

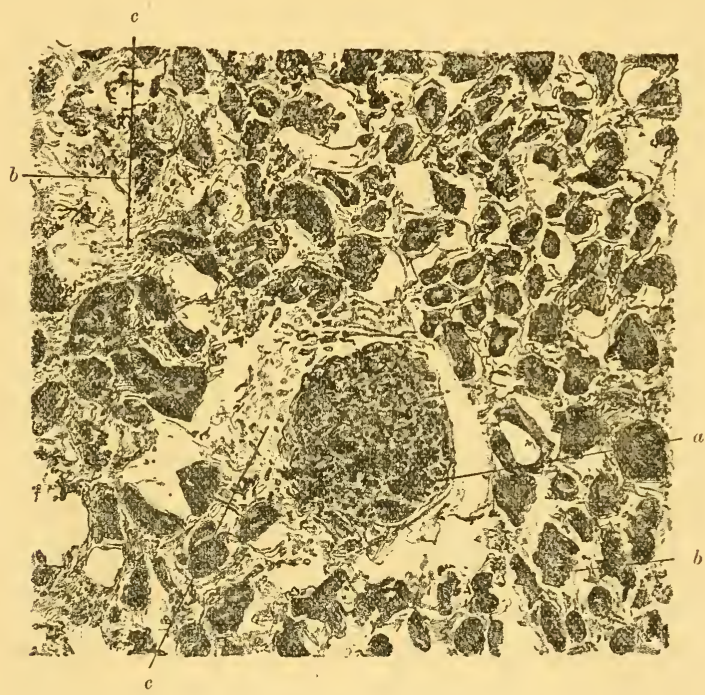

Fig. 121.-KInNer. Cortical part. $(\times 350$.

a. Malpighian body.

b. Transverse section of secreting tubnles with altered epithelium.

c. Connective tissue slightly increased; rich in cell elements.

These adhered to the hair, and were almost irremoveable with comb or brush,-in fact they could scarcely be removed even by washing with alkaline fluids. No amount of grooming could keep the animal clean, for as soon as one set of crusts were removed others rapidly formed; the whole surface of the skin became slightly swollen, and tender to the touch. There was a disinclination to movement, and when the animal was 
made to move, it did so very stiffly, and gave evidence of pain, arising from friction of the excoriated surfaces and movement of the rigid swollen and tender skin; the limbs became more pronouncedly swollen, but at no time was the swelling very great.

Throughout the course of the disease, and in all cases seen by me, the bowels were rather constipated, the freces being hard, dry, and pellety, and covered over with a slight mucosity.

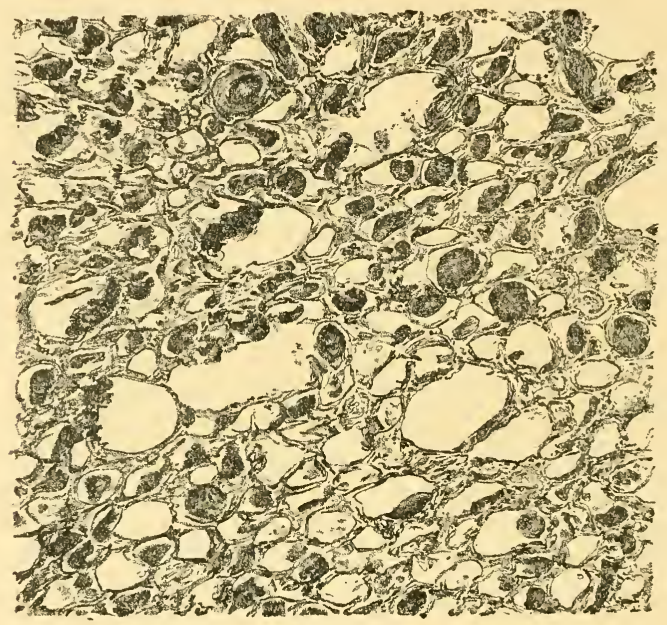

Fig. 122.-Kinner. Boundary layer transverse to tubules, which are partly denuded of epithelium, some of which may be accident.l, but remaining cells are similarly changed to above. $(\times 350$.

On examination of the liver, kidneys, and skin-the only organs which gave evidence of disease on post mortem examination-the liver had a soft and degenerated appearance, the kidneys were slightly enlarged, soft, and pale, the skin thickened.

The Skin.-The surface layers of the epidermis were largely desquamated, and the papillæ of the derma hypertrophied. There was enlargement of the sebaceous follicles, which were 
blocked with cells; a few of the sudoriferous glands were also enlarged, but the vascularity of the skin was only slightly increased. There were a few enlarged lymphatics here and there, and the subcutaneous tissue was open, loose, and contained much serum.

On analysis of the cutaneous excretion it was found that it contained large quantities of nitrogen in the form of ammonia,

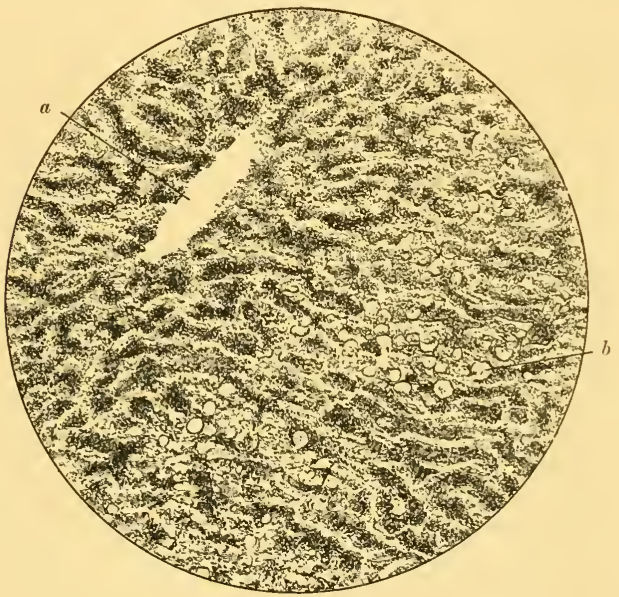

1 Fig. 123.-Liver. Cells increased in granularity, and showing fatty degeneration; destruction greatest in periphery of lobules. $(\times 300$.

a. Hepatic vein.

b. Periphery of lobule where degeneration is greatest.

a small proportion as urea, a proportion as albuminoids, about 5 per cent. of fat, and also salines in the form of phosphate, sulphate, and chloride of sodium, with a little chloride of potassium, and the proportion of water as low as 10 per cent. It was noticed that there was a large proportion of volatile butyric, and a small amount of lactic acid. It will be seen from the photo-micrographs that the papillæ of the skin are hypertrophied

1 The photo micrographs of Skin, Kidneys, and Liver are by Dr. James Hunter, Professor of Physiology, New Veterinary College, from sections by Prufessur W. O. Williams. 
and covered only by the deep layer of the epidermis, the superficial ones having been almost completely desquamated. This points to the fact that there was an exudation from the whole skin surface, irrespective of gland openings.

The Kidneys. - The changes in the kidneys were those of a chronic character, the degeneration universal, but most advanced in the secretory portion. The comnective tissue throughout the kidneys was but slightiy increased, and that was of

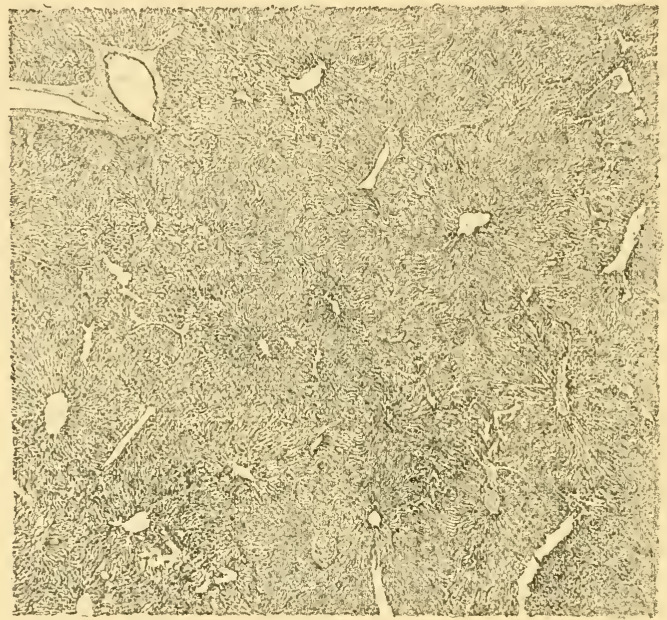

Fig. 124.-Liver. Showing several lobules undergoing the same changes, as shown in Fig. $123 .(\times 65$.

an embryonic type. No marked changes were observed in the nervous tissue.

The Liver.-The change in the cells, which is universal throughout the liver, is much more pronounced towards and at the periphery of the lobules. This leads to the inference that the morbid material enters the liver by the interlobular vessels; that is to say, the degeneration is due to something conveyed to the liver by the blood-vessels rather than from any initial change in the organ itself. 
ANALYSIS OF SWEAT, BY PROFESSOR IVISON MACADAM. Fat, ${ }^{1}$

Butyric acid,

Lactic acid,

Ammonia (volatile), ${ }^{3}$

14.93 per cent.

Substances soluble in alcohol and soluble in water, ${ }^{4} 8.01$,

Substances soluble in alcohol, but insoluble in water, ${ }^{5}$

Substances soluble in water, but not in alcohol, ${ }^{\circ}$

Substances insoluble in water and alcohol, ${ }^{7}$.

26.97,

$1.7 \cdot 2$

1.72

$3 \cdot 55$

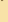

Water, .

$1 \cdot 12$,

7.59

$29 \cdot 28$

$6 \cdot 79$,

$99 \cdot 96$

1 Consisting mostly of glycerides of oleic and palmitic acid.

2 Partly in combination with ammonia.

3 In combination as butyrate of ammonia.

4 Saline matter,

Organic and volatile matter,

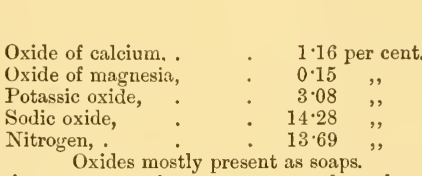

5 Contains no nitrogen; neutral to test papers and purely organic in character, mostly sulphur (highly sinelling compounds).

6 Saline matter,

Organic matter, .

Phosphoric anhydride, .

Calcic oxide, .

Magnesic oxide,

Potassic oxide,

Sodic oxide, .

Chlorine,

Sulphuric anhydride, .

Nitrogen,

$25 \cdot 14$ per cent.

21.41 per cent

$100 \cdot 00$

78.59 , ,

Calcium, evidently combined with organic acids, chlorine with sodium, potassium and magnesium as phosphates, nitrogen as soluble albuminates, \&c.

7 Saline matter,

Organic matter,

$4 \cdot 23$ per cent.

$95 \cdot 77$,

$100 \cdot 00$

Nitrogen, $14 \cdot 87$ per cent.

Sand,

Oxide of iron, .

$1 \cdot 01$

Magnesic oxide, 0.14
$2 \cdot 11$ per cent.

0.12,

83,

$0 \cdot 14$,

Phosphoric acid present as calcium and magnesium phosphates, nitrogen mostly as albumen. 
ANALYSIS OF TIIE URINE, BY PROFESSOR IVISON MACADAM.

The urine, except under the action of strong diuretics, was scanty; had the appearance of linseed oil, and on analysis gave the following result:-

$\left.\begin{array}{c}\text { Specific gravity at } 60^{\circ}= \\ \text { Water } 1000\end{array}\right\}$

Per 1000 parts :-

Total solid matter

Organic constituents,

Saline matters,

Chlorine, .

Potassic oxide,

Sodic oxide,

Calcic oxide,

Magnesic oxide,

Phosphoric anhyd.

Sulphuric anhyd.

Carbonic anhyd.

Undetermined and loss,

$67 \cdot 750$

38.060

Urea,

Albumen (coagulated by heat),

Mucus,

Fixed acid $=$ Lactic acid,

Hippuric acid,

It will be noticed that the urea is much diminished in quantity (about 31.0 being the normal), and that the quantity of mucus is enormous. This condition of the urine was permanent throughout the disease. Potassium, it will be seen, is very abundant.

\section{TREATMENT.}

Various remedies have been tried in this disease, but so far the results have not been satisfactory. The preparations of arsenic, as contained in Fowler's and Donovan's solutions, have been given extensively without beneficial results; the salts of potash, nitrate, iodide, and chlorate; tonics, both vegetable and mineral, have proved non-effective. Symptoms, however, have been alleviated, and in one case a permanent cure has been established, by the repeated administration of purgative medi- 
cine. At the first the bowels should be freely moved with aloes, and the aperient action maintained by the daily administration of sulphate of magnesia in about four ounce doses per day, or in sufficient quantity to keep the fæees in a pultaceous condition. By this method of treatment the congestion of the kidneys and liver is relieved, and the chances of at least further organic changes in these glands prevented.

With regard to local treatment, it can be palliative only: the diseased condition of the skin being secondary, and due to the irritation of the products of its vicarious function, the parts should be kept clean, and the irritation resulting from the exposure of the raw surfaces kept at a minimum by suitable dressing.

Once a week, or oftener, if necessary, the whole surface of the body should be dressed with an emulsion of olive oil, potash and water, and containing creosote or carbolic acid; this dressing is to be washed off with soap and warm water in about twenty-four hours after application, and the skin thoroughly cleansed. Then dry dressings of carbolised chalk, iodoform and chalk, or some other absorbent dressing, should be sprinkled freely over the whole surface, but more particularly over the flexures of the limbs and other excoriated spots. The diet should be generous but not stimulating. If in season, green food only should be given. 


\section{CHAPTER XLII.}

\section{DISEASES OF THE SKIN-continued.}

SQUAMOUS INFLAMMATION OF THE SKIN-PSORIASIS-PITYRIASIS DISORDERED SENSIBILITY OF THE DERMA - PRURIGO-INFLAMMATION OF THE SEBACEOUS FOLLICLES-ACNE-SITFASTS-TREATMENT.

\section{SQUAMOUS INFLAMMATION OF THE SKIN,}

DisEasEs characterised by inflammation and hypertrophy of the derma, and the production on the diseased skin of laminæ or scales. Of this disease we have two examples, namely, psoriasis or dry tetter, and pityriasis.

\section{PSORIASIS,}

"A chronic and non-contagious inflammation of the derma, characterised by the development of patches, which are irregular in form and size, and covered by irregular scales of epithelium. The patches are raised abcve the level of the surrounding skin; they are flat upon the surface, or somewhat more elevated in the centre than at the circumference, and are frequently intersected by deep fissures and chaps, particularly where the disease occupies a surface of large extent."-(WILsON.)

In the horse we have familiar examples of the above in what have been already mentioned, namely, psoriasis carpi et tarsi, or mallenders and sallenders. At first mallenders and sallenders are generally due to an eczematous eruption, but as they become chronic, the discharge with which they are attended in the earlier stages ceases to flow, and the disease is characterised by the formation of scales.

l'soriasis is usually met with in horses otherwise quite 
healthy, and it is essentially a chronic and often an intractable disease; but it never spreads over any great extent of surface, being generally confined to the flexures of the limbs.

I have reason to think that, like lymphangitis, grease, and anasarcous swellings of the legs, it is often of hereditary origin.

The scaly eruptions of psoriasis are unsightly, lower the value of an animal, and are occasionally troublesome, crack, inflame, and discharge a bloody serosity.

Treatment.-Generally remedies seem to have no effect, and beyond modifying the condition of the cracks when inflamed, the efforts of the practitioner are productive of no positive results.

To prevent the formation of the cracks and chasms, the scales should be dressed occasionally with some oily material; and when they become much thickened by accumulation, they are to be well soaked in an alkaline solution, carefully washed off, and the raw skin, thus exposed, touched with the nitrate of silver. An occasional application of the unguentum hydrargyrum is also useful.

In the case of a valuable animal, where the eradication of the disease is a question of importance, the treatment must be both local and general.

The general treatment comprises a purgative, followed by arsenic and cantharides, either separately or in combinationthe iodide of arsenic, or by a triple compound of arsenic, iodine, and mercury, which may also with advantage be employed as a local remedy; it is made as follows :-

1x Liquor hydriodatis arsenici et hydrargyri : triturate of finely levigated metallic arsenic, 6.08 grains; mercury, 15.38 grains; and iodine, 50 grains, with 1 drachm of alcohol, until the mass be dry, and changed in its colour from a deep brown to a pale red. Next triturate the mass for a few moments with eight ounces of distilled water; transfer the solution to a bottle, arld to it half a drachm of hydriodic acid, and filter, making up to eight ounces by means of distilled water if there is any deficiency. The solution is of a golden yellow colour, and each drachm contains - water, 1 drachm; protoxide of arsenic, $\frac{1}{8}$ grain; protoxide of mercury, $\frac{1}{4}$ grain; iodine converted into hydriodic acid, $\frac{4}{5}$ grain. The dose of this for the horse is from two to three ounces twice a day, care being taken that its effects 
are not pushed too far. When arsenic or its combinations can no longer be tolerated, the tincture of cantharides may be tried; and lastly, should these prove ineffectual, sulphuric acid may be internally administered. Of all local applications tar ointment is the best.

\section{PITYRIASIS,}

A name derived from the Greek word $\pi i \tau v \rho a, b r a n$, and given to a morbid condition of the skin, characterised by the rapid production of numerous fine white scales, with no exudation into the derma, and no albuminous secretion on the surface.

This is a very common disease among horned cattle, particularly young stock, and affects various parts of the body, the neck and shoulders, the rump, sides, \&c. It is accompanied by some degree of itchiness, and the scurf often furnishes a habitat for the production and development of vermin.

The development of pityriasis seems to be due to an ill-furnished condition of the animal body, arising from poor food, and it generally prevails in the winter and early spring months, and disappears spontaneously when the cattle are put into good pastures.

The treatment must consist of good food, the alleviation of the itchiness, and the destruction of the vermin which become associated with it, by sulphur ointment or an ointment of stavesacre. If the disease attack an extensive surface, the sulphur is the safer remedy, as the animals lick their skins, and may take more stavesacre into their stomachs than is good for them.

Pityriasis in the horse is due to a condition of the system associated with the presence of the oxalate of lime in the urine.

The pathological relations of the oxalates, and the conditions conducive to their formation, are not intended for discussion at present; but I may point out that the bran-like scurf, found on the coats of horses in every way carefully attended to and groomed, is associated with a degree of indigestion, frequent urination, more or less loss of condition, debility, harshness and dryness of the skin, and "hide-bound ;" and that most frequently the disease is found to affect animals which are irregularly worked but otherwise well treated.

Some kinds of food, such as carrots and turnips, favour the 
development of oxalic acid in the circulation; and on this account they must not be given in cases of pityriasis.

In some instances this bran-like scurf seems to be due to a deficient secretion of the sebaceous follicles of the legs and heels, induced by the use of soft soap or very hard water; however, it is not correctly pityriasis, but erythema, accompanied with fine branny scales.

Scales also form on various parts of the body, caused by contact with ill-fitting harness; but this is not properly pityriasis, but desquamation of cuticle owing to irritation.

Pityriasis is distinguished from psoriasis by the absence of thickening of the derma, by the scales being finer, separating more easily, by their very rapid production, and by the disease not attacking the flexures of the limbs.

Treatment.-The treatment is to be general, and to consist in the prohibition of all articles of diet calculated to furnish much saccharine matter. A gentle purgative is to be administered, succeeded by the persistent use of pitch internally. The pitch seems to have some influence in preventing the conversion of the starchy and saccharine constituents of the food into oxalic acid.

Liquid pitch made into a ball of sufficient size, with linseed meal, answers the purpose very well, the dose being regulated by the size of the horse.

Alkaline lotions may be applied to the skin if the scurf is at all thick; but, as a rule, the scales speedily separate from the skin, collect on the surface of the hair, and are easily removed with the brush.

\section{DISORDERED SENSIBILITY OF THE DERMA.-PRURIGO.}

Prurigo or Pruritis.-The most striking feature of this condition is severe itching, and it very often consists of what Mr. Percivall calls " a sort of cutaneous furor, to which horses, pampered and little worked, are liable, and which appears constitutional in its origin."

There is insufferable itching, and the habit, once contracted, is exceedingly difficult to get rid of.

Cases of this kind must be treated by purgatives, alkalies, and alkaline washes, such as the following:- 


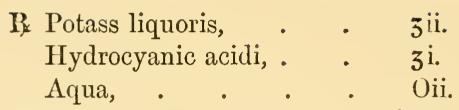

Mix, and apply to all the irritated parts of the skin.

Pruritis of the tail is a very common form of the disease, and seems to become a habit with some horses. Sometimes, however, it depends upon the presence of ascarides in the rectum. The treatment consists in giving enemas of a decoction of quassia to destroy the parasites, dressing the tail with mercurial ointment, or with equal parts of paraffin and fish oil, and if the disease affects the skin generally, give twice daily, flour sulph. 3ij., arsenicum gr. ij., mixed with the food; and when the prurigo is a habit, the root of the tail must be protected by a strong leather band, securely fastened whilst the animal is in the stable. 1

\section{ACNE}

Affects the sebaceous follicles of the skin generally, and appears as pimples, commonly known as warbles or grubs in the skin. The parts most frequently affected are the withers, back, and neck, and more particularly the " root of the mane." The eruption of acne begins as an elevation of variable size, the summit of which suppurates, and remains for several days as a pustule with an indurated base, leaving a swelling, which slowly disappears. The disease is very prevalent in the spring, sometimes affecting the tail as well as the mane and neck. When situated in parts which are liable to be chafed by the harness, these pustules are very troublesome, often rendering it necessary to throw the animal off work until they are quite healed.

Harness lined with thick, coarse woollen cloth, is a cause of this, as well as other skin ciiseases; and it may be mentioned here that all harness ought to be lined with light smooth leather.

The Treatment.-If the pimples are hard, and suppurate slowly, poultices nicely applied are very useful; but when inapplicable, fomentations are to be employed, and the parts clressed with the lead or zinc lotion. The general treatment is to be directed to the removal of all constitutional causes of derangement, and

1 For circumscribed itchings, the mercurial ointment is perhaps the best remedy that can be applied; but when the disordered surface is extensive, it is dangerous to use it, anı it must give way to a more harmless remedy. 
this is best effected by purgatives, diuretics, and stomachics. Sulphur, internally administered, is also very useful, stimulating the cutaneous glands, and assisting the process of " casting the coat;" a process with which these eruptions seem to have a close affinity.

If the tumours become indolent, they require stimulation with iodine or the iodide of mercury.

Occasionally the pressure of the harness upon these elevations produces a circumscribed form of gangrene of the skin, surrounded by a red circle of inflammatory congestion and suppuration. After a time, the gangrenous skin assumes a hard, horny, or leathery appearance, adheres firmly to the subcutaneous tissue, is surrounded by an angry-looking suppurating wounc, and from the difficulty experienced in its removal, the white gangrenous patch has been called a " sitfast."

These sitfasts are also seen to arise independently of any previous pimple, and are caused by the pressure of badly fitting harness.

The centre of the sitfast seems to retain some portion of its vitality, and from its connection with the more perfectly living tissue being to a degree retained, it is not thrown off spontaneously, as in the case of a more completely gangrenous patch. The circumference of the patch dries, shrivels up, and irritates the surrounding living tissues, which suppurate moro or less abundantly.

Treatment.-Removal of the leathery patch by carefully dissecting it out is the only expeditious method of treatment, the wound, so made, to be afterwards treated in the usual way. Some writers recommend blisters and caustics, but they are quite inapplicable, and the only circumstance that admits of the use of a caustic, is where a thin pellicle of the degraded material is not removed with the knife. In such a case the nitrate of silver, carefully applied, is a useful remedy.

For an account of cutaneous new formations, the reader is referred to the chapters on Tumours.

Purpura hocmorrhagica, searlatina, variola, and other affections which have hitherto been described as diseases of the skin, are mere symptoms of grave pathological conditions of the body, dependent on an alteration of the fluids belonging to a class of blood diseases, which I hope to describe at some future period. 


\section{CHAPTER XLIII.}

\section{DISEASES OF THE SKIN-continued.}

SUABIES - DIFFERENT VARIETIES OF ACARI - SARCOPTES-DERMATOJ)ECTES - SYMBIOTES-CLASSIFICATION-EXPERIMENTS OF HERING, HERTWIG, AND OTIIERS AS TO THE TRANSMISSIBILITY OF THE MITES TO MAN-MANGE IN THE HORSE AND OX-SCAB IN SHEEP - MANGE IN THE DOG AND CAT-TREATMENT OF MANGEDISEASES DUE TO EPIZOA-POULTRY LOUSINESS-FLEAS-TICKS -BOTS-MAGGOTS.

PARASITIC DISEASES OF THE SKIN.

THIs order of skin diseases may be considered under two heads:-(1.) Diseases due to the presence of parasites from the animal kingdom (dermatozoa); and (2.) those due to parasites from the vegetable kingdom (dermatophyta).

\section{DISEASES DUE TO ANIMAL PARASITES.}

\section{SCABIES.}

Scabies, mange, itch, or scab, is a contagious skin disease, due to the presence of animal parasites belonging to the class arachnida, the order acarida, or mites; sub-order, crawling mites; family, sarcoptes, of which there are, according to Gerlach, three genera :- $a$, sarcoptes, that burrow in the skin; $b$, dermatodectes, that simply bite and hold on to the skin; and $c$, symbiotes, living together in large numbers, and piercing no farther than the epidermis in search of food.

All these parasites live on serosity, the effusion of which is caused by the irritation which they exeite. 
Mr. Gamgee, translating from Gerlach, gives the following arrangement of the species belonging to each genus :-

A. Sarcoptes.
1. S. hominis.
2. S. equi.
3. S. suis.
4. S. canis.
5. S. catis.
6. S. caniculi.

$B$. Dermatodectes.
1. D. equi.
2. D. bovis.
3. D. ovis.

C. Symbiotes.

To the genus sarcoptes belong also:-
S. rupicapræ.-(Herivg.)
S. dromedarii.-(Gervais.)

The experiments of Hering, Hertwig, and others have proved that the mange insect may be transferred from the lower animals to man; that they bore the human skin, erect galleries in it, and cause itch-like eruptions. In general, however, the eruptions last only as long as the individual life of the mites transferred. Upon this point observations vary, some extending the continuance of the eruption to a period of six weeks, whilst others limit it to two or three. All are, however, agreed that they do not procreate in the human skin; that, although those in attendance on mangy horses may suffer from the disease, they do so only very slightly, and but for a limited period.

\section{SARCOPTES HOMINIS (ACARUS SCABIE).}

"The male is about one-third smaller than the female. $\mathrm{He}$ has suckers on two hind feet, and possesses on the abdominal surface genital organs, all of which characters are absent in the female. She, on the other hand, in addition to her size, and the negative marks alluded to, is characterised by three kinds of horny spines, which are scattered over her back. The suckers, or ambulacria, are organs of locomotion; the mandibles enable it to cut the epidermis, and extract fluid from the tissues, which passes through a delicate œesophagus, the termination of which is undermined; the body of the animal being filled with an unorganized, very fine molecular pulp. A short, delicate tube may also sometimes be observed at the anus-a supposed rectum. No respiratory apparatus can be discovered, although 
the creature may be seen to swallow minute bubbles of air, which pass down the osophagus, and, like the nutritive juices, diffuse themselves through the interior. At all events, aninal juice and air are both necessary to the life of the acarus."(BOURGUIGNON.)

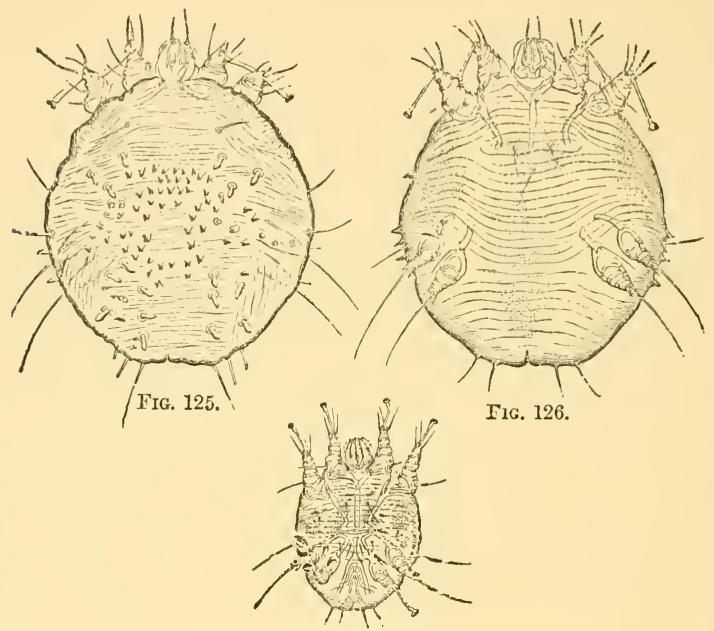

Fig. 127.

Fig. 125. - Dorsal surface of the female acarus scabie.

FIG. 126.-Ventral

Fig. 127. - Ventral surface of the male acarus. - (Bourgurgnon.) 100 diam.

It is the female only which burrows in the epidermis of the human skin. All the male acari go free on the surface of the epidermis, where sexual intercourse between male and female is said to take place. When an impregnated female is placed on the surface of the skin, it seeks a suitable spot to penetrate, and raising its head at right angles to the surface, it burrows, digs, or eats its way between the scales into the deeper layers of the epidermis, where it imbeds itself, derives nourishment, and goes through the process of parturition, till it dies. Having found a suitable place, an egor is laid, and each day another, the animal 
penetrating a little farther each time, leaving its deposited eggs to occupy the space previously inhabited by itself. The direction of the canal is oblique, the portion first formed being, of course, nearest the surface. As the old epidermis is thrown off, new layers of euticle being formed from the deeper strata, the first-laid eggs are gradually thrust upwards to the surface, where they are finally extruded; while the recently deposited ova remain in the canal, close to the parent female, whose instincts lead her to make the canal in such a way that her eggs reach the surface about the time the young ones are ready to come out of the shell. The newly hatched acari, male and female, crawl about the skin, and are said to moult their skins three times before they reach maturity; this occupies nearly five weeks. They then enter into sexual intercourse; the female burrows the skin, as before described, and dies at the blind end of the canal or caniculus, after depositing her ova; whilst the male rambles about on the surface, entering into sexual intercourse with many females, a proof of the necessity for fewer males than females.

Authors differ as to the time required for incubation; some say that egghatching occurs in eight or ten days; whilst Gerlach brings forward experiments to prove that three days are sufficient for this purpose.

\section{SARCOPTES EQUI.}

The males of this pretty large species, which is visible to the naked eye, are about $\frac{1}{15}$ "' in length and breadth, resembling a square notched at its four angles; arched on its back and belly, and

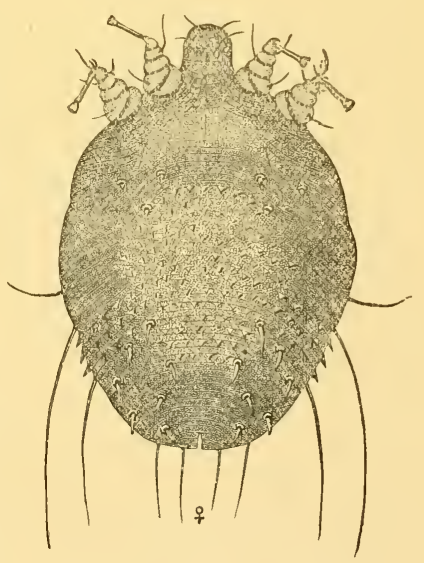

Fig. 128. - Sarcoptes equi.-(GerLach.) tolerably thick; the body covered with alternate furrows and varied lines, running transversely on the back and abdomen in a semicircular form. On the back there are small tibercles, 
and towards the front, long stiff hairs. The females, which are far more abundant, only difler from the males in their size, being about one-third longer, and in the structure of the two posterior pairs of feet, which are attached to the belly, close together, of equal length and strength, but shorter and weaker than in the male. The external (third) pair of feet has two long terminal hairs, but no sucking disc. The internal (fourth) pair has the rudiments of a claw, a sucking disc, and a fine terminal hair at the base of the stalk of the sucking disc. The colour of the parasite is whitish; the horny skeleton reddish-brown-ferruginous. The eggs are very large, coated with a sticky mass, and are often carried about by the female between her legs. The eggs are deposited in small galleries under the epidermis. The young are very small, but grow very quickly during the first four days; and, according to Hertwig, have their eight feet at once, but only six according to Hering. Their movements are effected quickly, and by means of the feet provided with sucking discs. They may be kept alive for three weeks without nourishment. Wherever they penetrate the skin, a small knotted elevation is produced, with a small passage, at the extremity of which the acarus sits. The epidermis becomes soft, separates from the true skin, and an exudate is thrown out, which, along with the epidermis, dries into scaly scurfs, that become detached.

The transference of this acarus from the horse to man has been proved by many observers-for example, E. Viborg, Sydow, Osiander, Greve, Groguier, and Hertwig-as well as the transference of the disease so caused from one man to another; but the mites do not propagate on the human skin, and the symptoms of their presence spontaneously disappear about the twelfth day.

The sarcoptes equi is transmissible to horned cattle; but it is as yet doubtful if the dog, cat, and pig are susceptible of the mange inluced by it. Gerlach failed to communicate the disorder to the sheep, although the mites were by him placed on the skin.

THE DERMATODECTES EQUI.

The dermatodectes equi, which has hitherto been looked upon as the veritable sarcoptes or acarus equi, does not live, 
like the latter, on the skin of other creatures besides the horse. Its existence is short, if conveyed to the skin of man, or of an ox, sheep, dog, \&c., and the form of horse mange which it induces is, therefore, essentially confined to the equine species; and that form of mange with which we are most conversant is connected with the existence of this acarus.

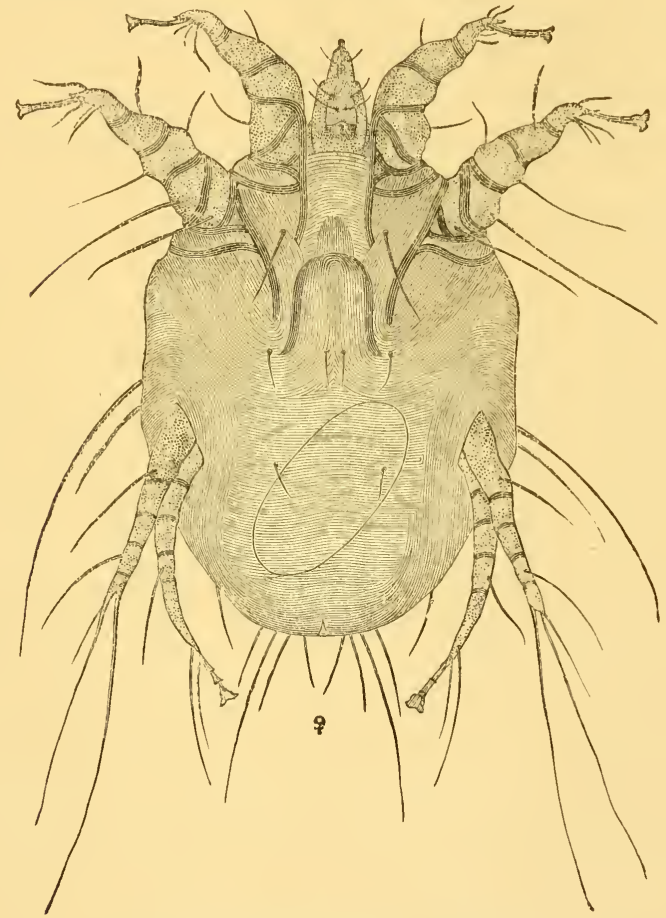

F1G. 129,--Dermatodectes equi.-(GERLACH.)

\section{SYMBIOTES EQUI.}

" This parasite, like the dermatodectes equi, seems to live only on the horse; and it is readily obtained from beneath the scales, 
by laying the latter on a paper in the sun, where next day they are found in small clusters."-(GAMGEE.)

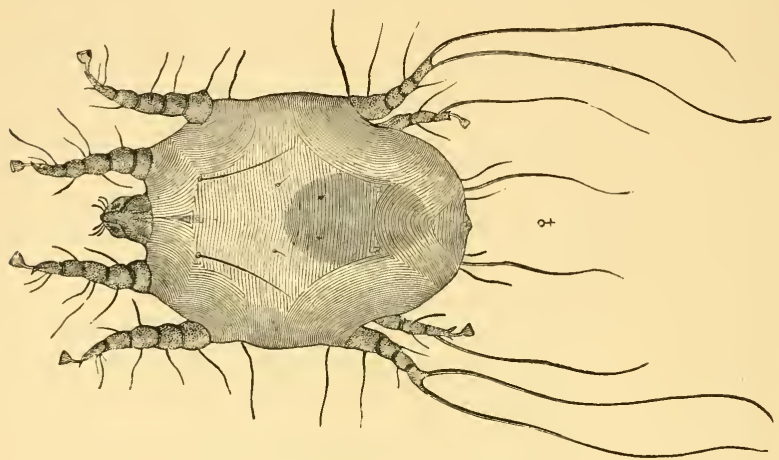

Fig. 130.-Symbiotes equi.-(GERLACH.)

According to Mr. Gamgee, who is translating from Gerlach, the finding of the various mites which constitute so essential a feature in mange, "has as yet been attended with great difficulty," and the various procedures for their detection are as follows :-

(1.) "Animals placed in the sun, and carefully examined; the acari (sarcoptes) do not get on the surface of the epidermic scales and on the hairs, as dermatodectes do ; and these can be seen with the naked eye.

(2.) "The sarcoptes are found below the scales or scabs, which may be so adherent to the skin that, on being removed, the latter may bleed. The dermatodectes are observed, after the animals have been exposed to the sun, on the outer part of these scales; but the sarcoptes are only seen on the inner. The scales examined should always be fresh, and they may be laid on black paper in a warm place in the sun, and then examined by a weak lens embracing a wide field of view. The small sarcoptes are only discovered when the cuticle has been removed with the scab.

(3.) "The sarcoptes are most certainly and easily found when the scales are laid with their under surface uppermost on the arm. In the course of twelve hours they pass from the 
scales on the arm, and burrow into the skin at the part covered by the scab. On removing the latter, the parasite is seen as a white spot on a somewhat reddened skin, or upon a small red papula. With the point of a needle the white summit of the epidermic vesicle is punctured, and the sarcoptes obtained. If the inflammatory nodule be allowed to pass to the state of a bladder, then the mite is rarely found. If there be only a few acari on the scales applied to the arm, the red points or nodules are only seen next day, and before the appearance of this eruption the insects are not, as a rule, to be found on the skin; if many of the parasites exist on the scales, then in the course of a few hours they may we obtained on the arm.

"The dermatodectes, especially of horses and cattle, are readily found in a similar way. Sometimes the scales need only be on the human skin one hour.

"When sarcoptes are sought by placing scabs on the skin of the arm, lest any should remain bare behind, the skin should be rubbed with a little oil of turpentine or some oatmeal. With the other forms of parasites mentioned this precaution is unnecessary."

Symptoms of Mange in the Horse.-As already indicated, there are three varieties of mange, each being due to a particular parasite, which seems to induce symptoms differing in some particulars. The first variety (sarcoptes) causes a vesicular eruption and intense itching, which increases towards night. This pruritis is supposed to be due to an acrid fluid or saliva which the parasite deposits in the gallery in which it is lodged.

The favourite seats of this form of mange are the sides of the neck and withers, whence they may extend over the surface of the body, except, according to Mr. Fleming, those parts covered by long hair, as the root of the mane and the tail.

If the invaded parts be examined by the hand, small hard pimples may be felt, which, if looked at closely, will be found to consist of a small scab easily removed, exposing round small moist surfaces about an eighth of an inch in diameter. The character of this form of scabies is eczematous, and at a more advanced period large surfaces become destitute of hair, and are covered with dry powdery crusts of variable thicknesses. At a later period the skin becomes thickened, wrinkled, and fissured, assuming the appearance of the skin of the rhinoceros. 
This constitutes the chronic or inveterate stage, and may (as Mèquin asserts) persist for a long time after all traces of the parasite have disappeared-become, in fact, constitutional, where the temperament or diathesis, such as herpatism, is allied with it. The disease may then be designated chronic lichen, and demands different treatment.-(FLEMING.)

The course of the disease is slow in horses well fed and cared for; but in those under less favourable circumstances it spreads with great rapidity, and may even cause death from irritation and exhaustion. Mr. Fleming, who has seen the disease in the Crimea, says:- " It has been remarked that after the disease had been cured, the hair on the affected parts grows very rapidly, and longer than elsewhere; and also that it is darker coloured, as if the poison of the sarcoptes had exercised a specific influence on the hair bulbs."

Symptoms of Dermatodectic Scabies.-This is the variety of mange usually recognised in this country; indeed, I am not aware of having seen the sarcoptic form, except during an outbreak which occurred in 1872-73, and which was conveyed from Edinburgh to Melrose, proving fatal to several horses. It is characterised by great itchiness, associated with the formation of papules and pustules. The papules are distinct elevations about an eighth of an inch in height, and a third of an inch in diameter, soon having upon their surface a vesicle, that is quickly ruptured, allowing the serous contents to escape: these serous contents dry and form a crust. The pimples continue to dis. charge, and the crust increases and continues moist, giving an appearance different to the dry powdery sarcoptic crust.

These parasites invade the upper border of the neck and root of the tail; they deposit a secretion more acrid and irritating, and, having long mandibles, they thus cause more serious alteration in the skin than the sarcoptic; but notwithstanding, this form of scabies is not so serious, is less contagious, spreads more slowly, and is more easily cured than the other form.

Symptoms of Symbiotie Scabies.-These parasites, like the dermatodectic, live in colonies, and thus differ from the sarcoptic, which lead an isolated existence. They invade the limbs of horses, and do not burrow into, but merely bite the skin, causing the production of serum, which, along with the débris of the epidermis, concretes into numerous crusts that break in large flakes. 
The itching which they cause is not so severe as in the other two forms, and they are much more stationary than the dermatodectes, the bites of which cause an exudation and a thickening of the epidermis, which defies the further action of their mandibles, and compels them to attack the surrounding healthy skin.

Two parasites seem to infest the legs of horses, viz., the one above described and the sarcoptes hippodus, described at page 677, Fig. 117.

To complete the diagnosis, the presence of the parasites must be determined, see Figs. 128, 129, and 130, it being remembered that the sarcoptic variety, owing to the fact that the female parasites preponderate very largely, are unsociable in their habits, are fond of wandering, are more contagious than the dermatodectic, and much more so than the symbiotic.

\section{MANGE IN THE OX.}

Two kinds of mites have been discovered in the mange of horned cattle:-(1.) The Dermatodectes; and (2.) Symbiotes bovis, which was first observed by Gohier, upon Hungarian oxen.

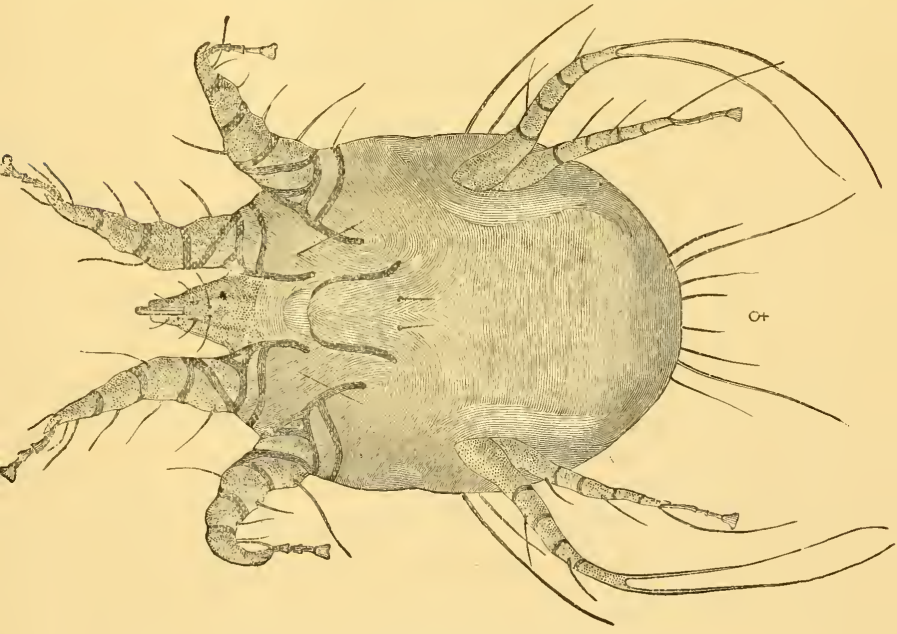

Fig. 131.-Dermatodectes bovis.--(GerLAcH.) 
SYMPTOMS IN THE OX.

The symptoms are similar to those in the horse. The dermatodectes are generally observed about the withers and root of the tail, and soon extend to the whole body, except the limbs, causing severe itching, a falling off of the hair, thickening, hardening, and corrugation of the skin, along with cracks or fissures. The animal rubs and licks itself, causing wounds and abrasions, from which an exudate is poured out, which, along with the débris of the epidermis, forms crusts.

The symbiotic parasites locate themselves about the base of the tail and vicinity of the anus, where, unless there is absolute neglect of cleanliness, they remain, causing irritation and formation of scabs.-(FLEMING.)

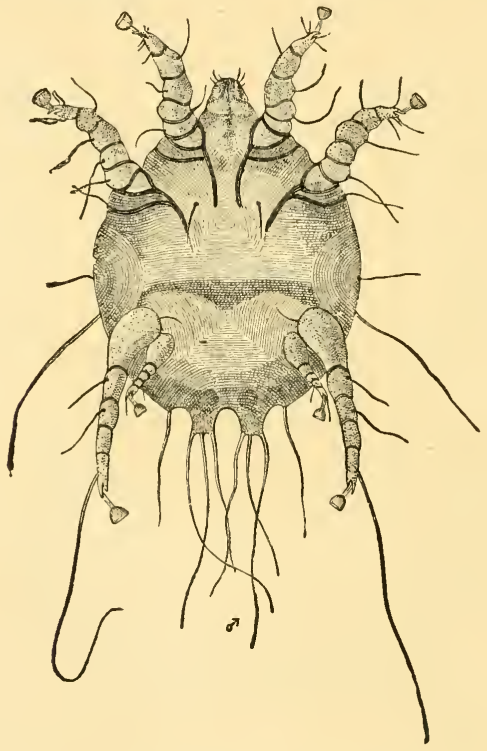

FIG. 132.-Symbiotes bovis.-(GerLacr.) 


\section{SCAB IN SHEEP.}

Three forms of scab are described, viz., the sarcoptic, dermatodectic, and symbiotic. The dermatodectic or psoroptic is the common form known in this country ; it is intensely contagious,

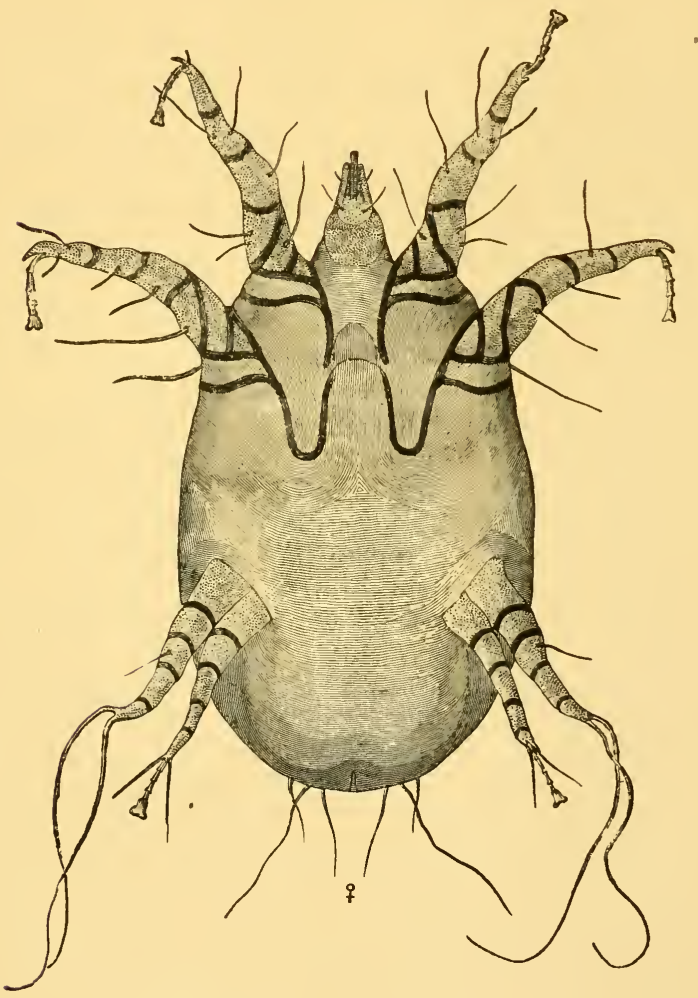

Fig. 133.-Dermatodectes ovis,-(GE.RLACH.)

and spreads with great rapidity, the parasite which causes it 
propagating most rapidly, as the following table furnished by Gerlach will show :-

\begin{tabular}{|c|c|c|c|c|c|c|c|c|c|}
\hline Generati & & & Days. & & & Females Born. & & & Males Born. \\
\hline 1 st. & • & . & . 15 & . & . & 10 & & & . $\quad 5$ \\
\hline $2 d$. & . & . & 30 & . & . & 100 & & & 50 \\
\hline $3 d$. & . & . & 45 & . & . & 1,000 & & & 500 \\
\hline $4 t h$. & . & . & 60 & . & . & 10,000 & & & 5,000 \\
\hline 5 th. & . & . & 75 & . & . & 100,000 & & & . 50,000 \\
\hline 6th. & . & . & . 90 & . & . & $1,000,000$ & & & . 500,000 \\
\hline
\end{tabular}

Its first symptom is itchiness, attempts to allay which soon give the animal a ragged appearance, tufts of wool being pulled out, leaving white patches. If the animal be caught and the bare parts examined, a slightly prominent pimple of a yellowish red colour will be observed. Upon the surface of this pimple a small blister or vesicle soon forms, its contained serosity, being discharged, forms the crust or scab, and at a little distance the parasite may be discovered amongst the wool. If the bare patches be gently rubbed or scratched, the sheep will express its pleasure by rapid movement of the lips or smacking.

The parts first attacked are the back, sides, and shoulders, and but very rarely the lower parts of the body and limbs.

The vesicles soon become confluent and sometimes pustular, the wool is easily detached along with the crusts, and the underlying skin is thickened, wrinkled, inflamed, and scaly.

The sarcoptic variety was discovered in 1838 by Delafond, the parasite being analogous to the acarus of the human species.

According to Mr. Fleming, "its first indications are observed on the upper lip around the nostril, and rarely on the eyelids and round the ears, by its appearing as a well-defined vesicle with a red areola. Very intense pruritis causes the animal to lick the lip, rub the parts against any object it can find, or scratch them with its hind or fore feet. The vesicle is in this way ruptured, the yellowish serosity it contains escapes, and forms a round hard crust. Analogous papulæ and vesicles appear on the limbs which have been employed in rubbing the lip and nose; and these parts are soon covered with dense greyish crusts, beneath which the sarcoptes congregate. At a later period the disease invades the whole of the face, cheeks, eyelids, and the lower portion of the limbs; though, strange to say, it does not attack those parts of the body covered with wool. The diseased patches extend and become more numerous and co- 
alesce; until at length such a region as the face presents only one large crust covering the skin, which is indurated, wrinkled, cracked, ulcerated, and bleeding.

"At this advanced stage, respiration, vision, and even the prehension of food, may be obstructed by the thickness and size of the crusts, and the tumefaction existing around the natural openings. A fatal termination has not, however, according to Reynal, been noted."

I am satisfied of having seen one case that was on the point of death from starvation and inability to breathe, arising from the lips and nostrils being indurated and scabbed, and in which the parasites were discovered. The symbiotic, like that in the horse, attacks the legs, spreads but slowly, and is not very contagious.

Treatment.-The first essential is the separation of the healthy from the unhealthy stock, and, as a precautionary measure, the healthy sheep should be dipped with any of the reputed dressings. A very eflectual one is a preparation of arsenic (one pound to twenty gallons of water) rendered soluble by the addition of an equal quantity of an alkali; for example, the carbonate of potash. Sheep should be dipped in dry weather, and penned for the night after the operation, to prevent them eating the drippings from the wool along with their food. The affected stock must be dressed individually; the wool being first clipped from the margin of the scabbed part (as there the parasites are located), and the part then dressed with tobacco juice, mercurial ointment, spirit of tar, or sulphur ointment. As a dip, carbolic acid is very good, but it has this disadvantage, that it destroys the colour of the wool. The flockmaster must remember that, though he may have destroyed the parasite, the ova yet remain, and therefore careful watching is necessary, and a second or even a third application of the dressing may be required.

This disease is included in the Contagious Diseases Act, 1869. The dermatodectes ovis is moderately hard; the male is roundish, the female more oval. Each of the external posterior feet has two long bristles; the fourth pair of feet in the male is rudimentary. The horny framework of the feet is reddish brown. In order that the reader may compare the delineations of Geriach and those of Kuchenmeister, I have inserted the following cuts :- 


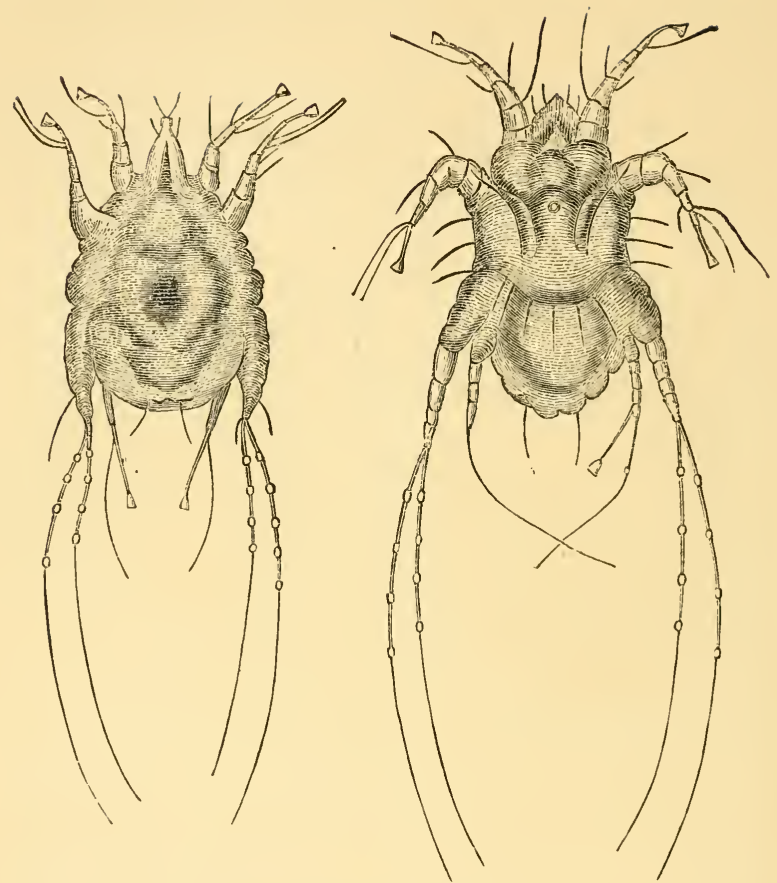

FIG 134.-Sarcoptes ovis, from above. FIG. 135. - Sarcoptes ovis, from beneath. (After KuchenMeISTER.)

\section{MANGE IN THE PIG}

Is due to the presence of the sarcoptes suis (see Fig. 136) which was discovered by Gurlt, and afterwards seen by Hertwig and Gerlach.-(GAMGEE.) It is transmissible to man.

\section{MANGE IN THE DOG.}

Dog mange is due to the presence of the sarcoptes canis. The disease is transmissible to man, but it is not yet shown whether it can be communicated to other animals. 
Its symptoms are as follows:-Itchiness, formation of red points like flea-bites, vesicles, pustules, and scabs.

Where the skin is thinly covered with hair, as upon the inside of the thighs, under the abdomen, \&c., the red points are very apparent; and in consequence of the continual itching and scratching, the skin upon these parts becomes red and inflamed.
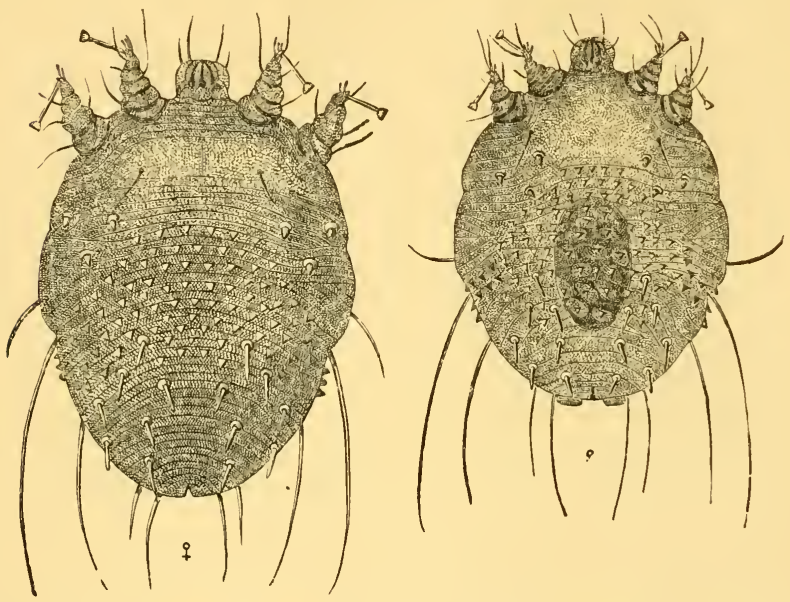

Fig. 136.-Sarcoptes suis.-(Gerlach.) Fig, 137.-Sarcoptes canis.-(Gerlach.)

Mr. Fleming is quite correct in stating that these red points are due to the presence of the parasite, although "red mange" was generally supposed to be an eczematous disease. I have satisfied myself that it is parasitic and contagious, and that what are termed scabby mange, watery mange, and dry or red mange, are one and the same disease differently manifested. The mange louse of the dog is the habitat of the cysticercus cucumerinus ; hence mangy dogs are subject to tapeworm-Tœnia cucumerinus - from swallowing the mange parasites, in the body of which is looped the germ of what becomes developed into the mature tapeworm in the dog's intestinal canal. Pups suckled by mangy dams, as a rule, fall a prey to the tapeworm when a few weeks old. On this account the dam ought to be thoroughly cured of mange before the birth of her young. 


\section{FOLLICULAR SCABIES.}

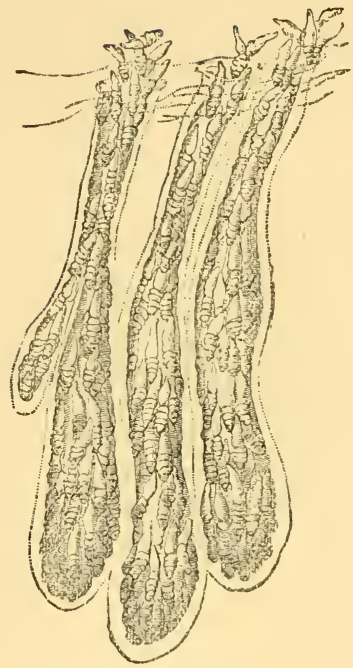

Fig. 138.

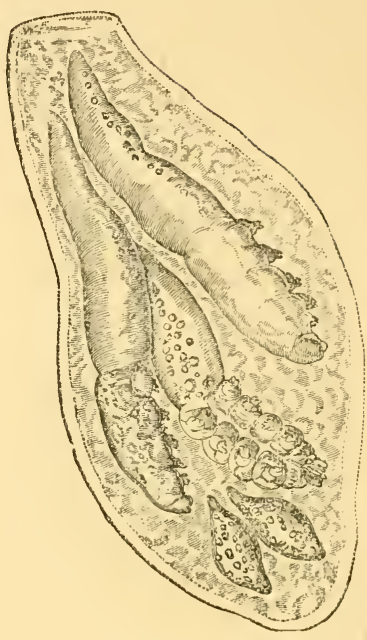

Fig. 139.

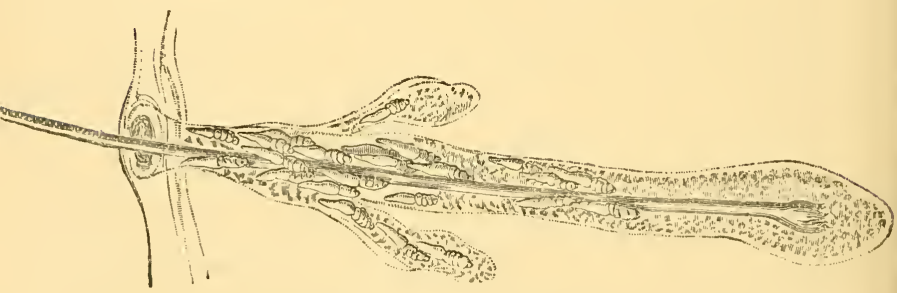

FIg. 140.-Acari folliculorum.

Fig. 138. - Three follicles of the skin of the dog, containing entozoa. 100 diam.

FIG. 139.-Cul-de-sac of a sebaceous follicle, containing three animalculæ in different positions, and two eggs. 350 diam.

FIG. 140.- Hair and its follicle, in which may be seen the animalculæ descending towards the root of the hair and cul-de-sac of the follicles.-(GrobY.) 100 diam.

This form of mange is due to the presence of the acarus demodox folliculorum.-(See Figs. 138, 139, and 140.) 
In the first edition of this work it was stated that " this entozoon is not known to cause disease." Shortly after the book was issued, I was told that this acarus not only caused disease in dogs, but the disease was considered by Continental veterinarians to be incurable; and thanks to Mr. Fleming (see Veterinary Sanitary Science and Police, vol. ii. p. 45 4 ), and Mr. W. Hunting (see Veterinary Journal, September 1875, p. 167), the English reading veterinary surgeons were made acquainted with the symptoms and nature of this form of mange.

The demodox folliculorum found in the dog is identical with that in the human being, in whom it seems to cause no disease or inconvenience. Indeeil, according to Erasmus Wilson, the difficulty seems not to be to find these creatures, but to find any individual, with the exception of newly-born children, in whom they do not exist. Gruby transferred the parasite from man to the dog, in whose skin it both lived and propagated. This dog was kept and watched for two years, and in that time all the hair follicles had been invaded, and the hair had disappeared. Mr. Fleming thinks that there may not be a specific identity between that found in man and that of the dog, and further experiments would be interesting; but the result of the experiment of Gruby is very strong evidence of their identity.

This parasite measures from $\frac{1}{64}$ to $\frac{1}{135}$ of an inch in length, and from $\frac{1}{55}$ to $\frac{1}{555}$ of an inch in breadth, and is composed of a head, thorax, abdomen, and a long obtusely pointed tail.

The head represents in form a truncated cone, flattened from above downwards, and is furnished with two maxillary palpi, which admit of extensive motion. The thorax is the broadest part of the animal, and is composed of four segments, and having four legs on each side. The abdomen varies in length and admits of certain movements; internally an alimentary canal, and a brownich mass, supposed to be the liver. It has a mouth, directed obliquely downwards from the head, and an anus at the opposite extremity of the body.

The parasites inhabit the sebaceous and hair follicles. Their heads are directed inwards towards the base of the follicles; and when very numerous, they are placed back to back, and their feet applied to the walls of the ducts.

The symptoms and treatment are so well given by Mr. Hunting, that I feel I can do no better than quote them. 
"The symptoms of the disease are seldom seen in the first stages; they consist inerely of circumscribed spots from which the hair falls, and upon which are noticeable a few small pimples. These patches extend rapidly, and fresh ones appear on other parts. Any portion of the skin may be affected, but the head, legs, belly, and sides are usually the seat of the disease. The affected places are almost hairless, and what hair remains is easily pulled out; small pimples and pustules stud the surface, the latter varying in size from a pin's head to that of a pea. The confluence of the pustules, and the discharge of their contents, give rise tu scabs; these crack and bleed, and so produce a most repulsive appearance. In white-haired dogs, the skin is red; in all it is extremely hot, and emits an unpleasant odour. The irritation does not excite much scratching, but the dog frequently shakes himself. More pain than itching seems to accompany the disease. In cases where the whole body is affected, loss of condition is most marked; and in cold weather the almost total loss of hair may cause death, if the animal be not kept in a warm place. This stage, too, is always accompanied by a ravenous appetite, due, probably, to the rapid loss of animal heat.

"Diagnosis. - In white dogs the colour of the skin may cause the disease to be mistaken for 'red mange,' or 'eczema.' The circumscribed spots in the first stages may be confounded with some forms of tinea; and the loss of hair and presence of scabs seen in the fully developed disease may easily be mistaken for ordinary scabies. The pustules, the heat of the skin, and the comparatively slight itchiness shown, are, however, nearly diagnostic. Positive diagnosis can only be made by the aid of the microscope and the detection of the parasite. If we puncture one of the pustules, and mix its contents on a slide with a little water, the acari are easily discovered. I have found as many as thirty in one pustule. Sometimes we may detect them on the root of a hair removed from an affected spot.

"The disease is contagious, but not to such an extent as scabies. My own dog had been in daily contact with an affected one for a fortnight, and showed no signs of the malady. Inoculation of one or two dogs has been unsuccessful, but that it is contagious is beyond doubt. I know a kennel in which this aisease appenred about three months after an infected dog had been allowed to live with the others for a week or two. The 
kennel contained about half a dozen $\operatorname{dog} s$, which were often changed; and cases of the disease broke out in this place, one after another, for months.

"The duration of the disease is very long. I have seen a case of two years' standing; but I do not think this length of time could be much exceeded, as the pitiable condition of the animal indicated a fatal termination, unless treatment had been adopted. I am inclined to think that the development of the disease, or I might say its period of incubation, is very slow. At any rate, a healthy dog remains a long time in contact with diseased ones before he shows symptoms of it.

"Treatment.-My first two or three cases were all unsuccessful, and it was not until I compared notes with Mr. Duguid, the veterinary surgeon to the Brown Institution, that I found this was the experience of every one else. We then worked together, and tried preparations of mercury, sulphur, iodine, carbolic acid, and other parasiticides, but still with no effect. These substances were applied either in watery solutions, or in oil, or as ointments, and were used of various strengths, so as in some cases to affect the system, or to produce a caustic action on the skin. It then occurred to Mr. Duguid, that as the parasites were situated deeply in the skin, any application to reach them must first soften and break up the cuticular covering. A mixture of creosote and oil, to which was added a strong solution of caustic potass, was tried, and its action was soon most marked. To facilitate matters, we also adopted the plan of washing the dog with warm water and soft soap, previous to applying the dressing. This is most useful in all parasitic skin diseases, but in none so conspicuously as this; as it softens the cuticle, and removes all scabs, \&c., allowing the drug every cliance of reaching the parasites.

" Carbolic acid, used with oil and caustic potass, is not effective, nor is any drug without the alkali. Creosote ointment was tried without effect by Weiss. Caustic solutions failed in Haubner's hands, but the two combined are, as our experience shows, perfectly reliable. The following is the formula:-

$\begin{array}{lll}\text { B. Creosote, } & \cdot & \text { ziv. } \\ \text { Olive oil, } & . & \text { 弓vii. } \\ \text { Sol. potassæ, } & . & \mathbf{z}^{\mathrm{i}} .\end{array}$


Mix the creosote and oil, then add the caustic solution. Dress the affected spots about twice a week, and allow longer intervals as soon as the skin becomes soft and tender. In cases where the whole body is affected, we adopt the plan of shaving the animal as soon as the skin is sufficiently smooth for the action of a razor; and in all cases it is good policy to shave about an inch of hair off the sound skin all round the diseased spots. This prevents the spread of the parasites. On a case I have now under treatment, and in which most of the skin was affected, I have tried clipping instead of shaving, and with good results. I fancy the absence of hair not only allows the dressing to act better, but injuriously affects the parasite. If this really be the case, may it not account for the difference in the symptoms caused by the parasite on man and on the dog?

"My experience as to the renewal of hair is opposed to that of Weiss, who states that it is not regenerated. I find that, except upon those spots where small ulcerations have been followed by a cicatrix, the hair is reproduced, and that it grows rapidly, but not until the parasites are totally eradicated. This is remarkable, as one would naturally expect such damage as is sustained by the follicles would have destroyed their function. When we had cured our first case, we certainly did not expect the renewal of hair, and were not a little agreeably disappointed.

"A cure requires from three to eight months, and even a longer time than this must be allowed for the growth of the hair."

\section{MANGE IN CATS}

Is due to the presence of the sarcoptes cati (GERLACH), which

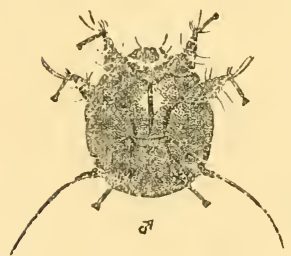

FIG. 141, --Sarcoptes cati.(GerLach.) differs from the other burrowing mites only in its relative size. It lives on the skin of the different feline animals, and is very commonly met with, inducing mange, in zoological gardens and travelling menageries. Like the mange of the other animals, it is lighly contagious, and occasionally all the cats of the district become affected with the disease. Neglected cat mange presents itself as a very loathsome and even fatal disease; the 
animal becomes one mass of scabs, underrun with purulent matter, emitting a most loathsome smell, and presenting such evident signs of distress, emaciation, and debility, as to call for the immediate destruction of the sufferer.

Symptoms.-Great itchiness of the skin, more especially when the animal is warm, or at night; a peculiar scaliness and undermined state of the epidermis; and the presence of the acarus.

\section{TREATMENT OF SCABIES.}

True mange being a contagious disease, it is essential that all animals suffering from it should be isolated, and everything with which they may have come in contact purified. Thus, with horses the clothing is to be boiled in a solution of soap and carbolic acid; and the harness, saddle, and grooming utensils washed with soap and warm water, and dressed with a solution of arsenic or corrosive sublimate, in the proportion of ten grains to the ounce of water. After being so washed and dressed, they are to be kept for several days exposed to dry air, washed again with soap and water before they are used; and before they are again put on the horse, they should be sprinkled on the side next to the horse's skin with sulphur. These may seem useless precautions, but in many cases the harness and clothing are lined with thick scabs, containing the ova of the parasites, the vitality of which is so great as almost to defy all efforts to destroy it.

Pastures in which scabby sheep have been grazing should be kept empty for some weeks ; all posts and other rubbing-places should be examined, and purified with carbolised whitewash, and all adhering portions of wool carefully removed and burnt.

Scabies being a local disease, is curable by topical remedies, and it may always be got rid of by frictions with the simple sulphur ointment. For horses, dogs, cattle, and cats, this alone is generally sufficient, provided it be properly applied, and no part affected with the disease left untouched with the remedy. The sulphuret of calcium is also highly recommended for diffuse mange, where it would be improper to cover a large extent of skin with an impermeable coating. It is made as follows:$2 \mathrm{lbs}$. of sulphur, $1 \mathrm{lb}$. of quicklime, and $16 \mathrm{lbs}$. of water. These are to be boiled together, and continually stirred until the ingredients are combined. 
In horse mange the unguentum staphysagriæ is a most effective remedy, and I should conclude that a decoction of stavesacre would prove as effectual in sheep scab; but I have no actual experience in the matter. The formula for the ointment is as follows:-

\section{1) Pulv. delphini staphisagriæ, 3 ii. Adipis, vel. ol. palmæ, $\quad$ ¿viii. Ol. olivæ. \\ zi.}

Mix, and digest at $100^{\circ}$ in a sand-bath, and strain. M. Bourguignon, with his microscope, watched with great care the effects of various remedies on the acari, and arrived at a conclusion that the most energetic remedies that could be employed for their destruction were solutions of the iodide of potassium and of the iodide of sulphur, which killed them in eight minutes. A solution of the extract of staphysagria was the next in virulence, destroying the acari in fifteen minutes. To prove the relative value of these two remedies, the following experiments were made:-The hands of an itch patient were immersed in a solution of the iodides for two hours, so as to strongly impregnate and colour the integuments. On examining the acari immediately afterwards, they were as lively as ever, but on the next day they were all dead and the eggs destroyed. The epidermis was greatly shrivelled, and in three days complete desquamation occurred, carrying with it acari, grooves, and eggs, and leaving the cutis raw and tender. The action on the skin was evidently too strong. A bath of a solution of the alcoholic extract of stavesacre was then made, and immediately after two hours' immersion of the hands all the acari were found dead, and, with one exception, the eggs destroyed. So far from irritating the integument, this application at once caused the itching to cease, and produced such a calmative effect that M. Bourguignon proposes it as a local remedy for inflammation. The eruptions also appeared to be rapidly cured by it.

Horses, cattle, pigs, dogs, and cats should be thoroughly washed with soft soap and warm water, before any remedy is applied to the skin for the destruction of parasites and the cure of mange; and no remedy should be allowed to remain on the skin for more than three days, at the end of which time the animal is again to be washed and the remedy reapplied. When the disease is due to the sarcoptes, the ova are contained in 
galleries beneath the epidermis, and it is very possible that al: are not reached by the dressing until the animal has been dressed three or four times.

Smearing sheep with greasy ointments, or dressing them extensively with oil of tar or mercurial ointment, is a very dangerous practice, and often causes many deaths. They prove injurious to the animal by clogging the wool and rendering the skin impervous, thus preventing the cutaneous exhalations, and causing the accumulation of effete materials in the blood-carbonic acid, ammonia, and other organic products-which are naturally thrown off through the pores of the skin. The symptoms of the disease so produced are hurried breathing, small, frequent pulse, blood-shot eyes, and a discharge of frothy mucus from the nose and mouth. The post mortem appearances are congestion of the lungs, the trachea and bronchii filled with mucus, and the great veins filled with a dark-coloured semicoagulated blood.

In mercurial poisoning the symptoms are those of insalivation, purging, redness of the skin, foetor of the breath, and rapid sinking.

Treatment.-The animals must be clipped and thoroughly washed with soap and water. They must be supported by stimulants, such as wine, whisky, or other spirituous liquors, afterwards treated with great care, and have good food and warm shelter.

\section{DISEASES DUE TO EPIZOA.}

The second class of parasites that infest the skin of the lower animals consists of insects which live upon the skin.

\section{LICE,}

(Pediculida, class insecta, division articulata, and order anophura), are parasites destitute of wings, hatched from eggs, undergoing no transformation in the process of their development, but shedding their skins a certain number of times. The state of lousiness is termed phthiriasis, and is seen in poor, half-starved, or very old animals. The best treatment is clipping, if the hair be long, washing the animal with a decoction of stavesacre, one ounce of the powdered seeds to a pint of water, and taking care 
that the animals do not lick themselves for some time after the remedy is applied.

Debility seems to be the predisposing cause, rendering the animal a proper habitat for the propagation and development of these parasites. It must be overcome by good food, tonics, and cleanliness.

One form of lousiness in the horse deserves special mention, namely-

\section{POULTRY LOUSINESS, OR PIITHIRIASIS EQUI.}

Attention was first drawn to this disease by Professor Bouley, whose observations are published in the Veterinarian for April 1851. He says-“ "Its commencement is instantaneous. All at once the horse is seized with violent itching. So sudden and irresistible is the desire the animal possesses to scratch himself, that he is not easy for a single moment. He rubs his skin against every resisting body near him, stamps the ground continually, strikes his belly, bites every place he can reach with his mouth, manifesting by his continual movements the burning itching by which he is devoured. At night his torments increase, so much so, that should the animal be abandoned to himself, he rubs and bites himself to that degree that he tears his skin, and carries portions away in his mouth, denuding himself extensively of his scarf-skin; nor does he relax until smarting pains succeed the insupportable torments of the itching."

The itchiness is accompanied by an eruption of very small vesicles on the skin-some solitary, others in greater number, occupying more or less extent of surface. These are succeeded by depilation of the epicermis and hair, leaving a small, perfectly circular, bare surface, of the size of a lentil or the smallest silver coin. The formation of these circular spots by consecutive desquamations goes on rapidly; in a few days a horse with the most shining coat may be spotted over with circular patches, devoid of hair, and in the course of a week the hair and epidermis will be destroyed over a large extent of surface.

The disease does not seem to interfere with the general functions, and, apart from the violent itchiness and excitement which the animal experiences, he appears in perfect health. "When, however," says M. Bouley, " the disease becomes of long duration, the subject of it will be apt to fall off in his appetite, to grow 
thin, and to lose his condition for work from the gradual wasting of his powers."

An interesting letter was published by Mr. Henderson, of London, immediately after M. Bouley's communication. Messrs. Moon, Woodger, and others have since drawn attention to the matter: all agree that the parasite causing this disease is the hen-louse, and that the horse becomes subject to the annoyance from being stabled near hen-houses.

Treatment.-Remove the cause, and wash the animal with a decoction of tobacco or staphysagria; whitewash the stables, and observe cleanliness.

\section{FLEAS-PULEX IRRITANS.}

Dogs are much troubled with the common flea; and the best remedy for it is the Persian insect powder. Its prevention should, however, be made the primary object; and this can only le attained by attention to cleanliness. Not only is the dog to le repeatedly washed, but also its kennel ; and instead of straw, sawdust, especially pine sawdust, should be used as litter.

\section{TICKS.}

Ticks belong to the order Acarida (Acarida magna), and family Ixodiadoc. They are found on the skins of horses, cattle, sheep, and doggs. Some have their mouths in the form of a sucker, by which they fasten on the skin; others are free, and have no sucker. They attach themselves to the skin so deeply and firmly, that it is impossible to remove them without tearing away the skin to which they are fixed, and they multiply so rapidly in hot climates that animals have been known to die from exhaustion. For further information on the natural history of those parasites, I beg to refer the reader to Kuchenmeister. They generally attach themselves to the jaws, belly, between the thighs, under the tail, and other parts of the animal least protected by hair.

The proper treatment for their destruction is to cut off their hodies with a pair of sharp scissors, or to kill them with oil of turpentine.

The common shecp-tick, or ked, as it is called in Scotland, 
belongs to the genus melophagus. It is of a dark reddish colour, destitute of wings, and is so tenacious of life that it will exist in a fleece twelve months after the animal is shorn. They become numerous in winter, and are best destroyed by an arsenical dip.

\section{FAMILY OF BOT-FLIES (ESTRIDEA).}

In this division the eggs are deposited in the skin of our patients, and are there developed into the larvæ of the fly.

The female cestrus has a horny ovipositor, which slides out and in, like a telescope, and bears five teeth at the end. On the one hand, it has been asserted that this ovipositor is used as a boring apparatus in burying the eggs at the moment of laying ; on the other, that it has not sufficient strength for this purpose, that the eggs are stuck upon the hairs, and that only the larvæe bore under the skin.

The bot of the ox, Estrus bovis, is best known in this country, and, according to Mr. Bracy Clark, it is the largest European species of this genus. It is not unfrequently seen in country situations, on the backs of oxen and cows, causing the formation of tumours as large as pullets' eggs on the sides and about the back and loins, which are called by the country people warbles, wornils, wormuls, and sometimes bots. Further, Mr. Clark says- "That we may continue the history of these flies with some degree of uniformity, we shall commence its operations with some remarks on the deposition of the eggs. This act appears to be attended with severe suffering, or apprehension at least, which makes the cattle run wild and furious, and gad and stray from the pastures; and hence the ancient epithet of gad-fly. When yoked to the plough, the attack of this fly is attended with real danger, since they (the cattle) become perfectly uncontrollable, and will often run directly forwards through the hedges, or whatever obstructs their way.

"When cattle are attacked by this fly, it is easily known by the extreme terror and agitation of the whole herd. The unfortunate object of the attack runs bellowing from arnong them to some distant part of the heath or nearest water; the tail, from the severity of the pain, is held with a tremulous motion straight from the body, and the head and neck stretched out to tho utmost." 
The larva, at first white, smooth, and transparent, enlarges, becomes brown in colour, and when it has attained its full growth, effects its escape from the abscess by pressing against the external opening; and finally wriggles itself out, drops to the ground, and, seeking a convenient retreat, becomes a chrysalis, and then a fly.

Bots are found under the skin of domesticated and other animals besides the ox, and also in man.

The tumours caused by the bots in cattle are sometimes of the size of a walnut, generally situated upon the back and shoulders. They are easily cured by pressing out the bot with the fingers.

\section{MAGGOTS.}

Maggots, or "the fly," are very troublesome to sheep in the sultry months of the year. They are the larvæ of the large blow-fly hatched from its ova, deposited in the fleeces of the sheep, particularly about the rump and tail of the animal when in a dirty condition; the filth forming a proper habitat for the growth of the insect. The maggots burrow into the skin, render it sore, causing suppuration and deep fissures. The sheep suffers greatly, loses its appetite, is dull, and dejected; and unless the maggots are destroyed, and the wounds kept clean, death may result. In Australia this is a great pest, and not only are sheep liable to be fly-blown, but men are often attacked by the fly, and have their noses and mouths filled with maggots.

Sores of all kinds are liable to be fly-blown in the summer time in this country, and wounds of the feet are very often found at this time of the year to contain maggots.

The best remedy for the destruction of the maggot is a combination of one part of oil of turpentine to three parts of oil. 


\section{CHAPTER XLIV.}

\section{DISEASES OF THE SKIN-continued.}

FAVUS, OR HONEYCOMB RING-WORM-EXPERIMENTS OF GRUBY-THE ACHORION SCHONLEINII-THE YELLOW CRUST DUE TO THE EXUDATION-TRANSMISSIBILITY OF THE DISEASE FROM ANIMALS TO MAN, AND VICE VERSA-TINEA CIRCINATUS-THE TRICOPHYTON DISCOVERED BY MALMSTEN-IDENTITY OF FAVUS AND TINEA CIRCINATUS - LETTER FROM MR. M'GILLIVRAY OF BANFF TREATMENT OF RING-WORM.

DISEASES DUE TO VEGETABLE PARASITES-DERMATOPHYTA.

THE vegetable parasites, or epiphytes, which cause diseases of the skin, are microscopic growths belonging to the lowest class of vegetable existence, namely, the fungi cryptogamia. Most of them are composed of simple sporules, germs, or cells, placed side by side, or end to end.

Two forms of skin diseases traceable to vegetable parasites are now pretty well understood; they are the tinea tonsurans, also called tinea tondens or ring-worm, and favus, honeycomb ring-worm, or scald-bead.

\section{FAVUS, OR HONEYCOMB RING-WORM.}

This is a disease little known in this country, and is described as a fungus parasitic disease, composed of cup-shaped scabs, sometimes distinct and separate, at other times indistinct or confluent. These fungi are capable of being implanted from one animal to another, from man to animals, and animals to man; and Gruby tried the effects of the inoculation of the parasite on vegetables, and succeeded, by inoculating the bark of an oaktree, in getting a favus-cup identical with that which grows on the heads of children. 
Pathology.-The disease depends upon a cryptogamic fungus, named the achorion Schönleinii, after Schönlein, who was the first to suggest that the yellow crusts were constituted by a vegetable parasite. The primary seat of the parasite is in the

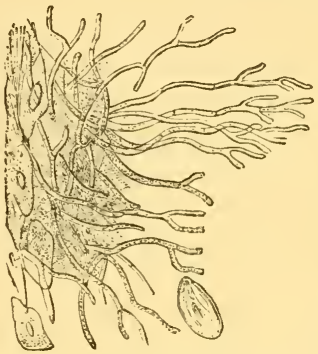

Fig. 142.

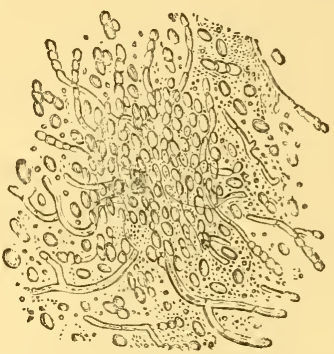

FIG, 143.

Fig. 142, - Branches of achorion Schönleinïi in an early stage of development, growing from molecular matter, and mingled with epideruic scales, from a very minute favus crust.

Fig. 143. - Fragments of the branches more highly developed, with numerous sporules and molecular matter, from the centre of an advanced favus crust. - (BENNETT.) 300 diam.

depth of the hair follicle outside the layer of the epithelium, which covers the root of the hair, and which forms the "inner root sheath of Kölliker."-(AITkEx.)

By making their sections of the favus crusts, and treating

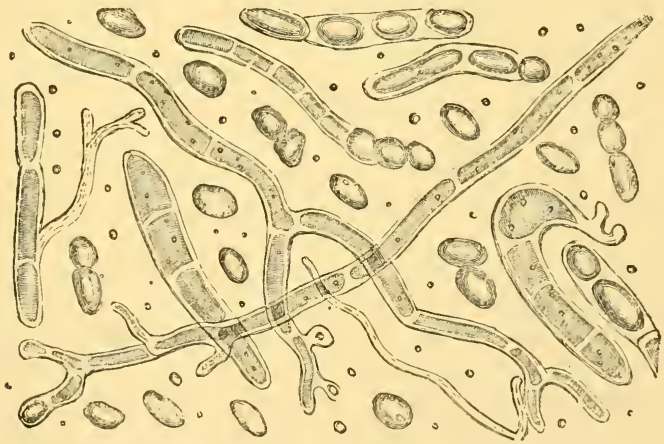

FIG. 144. - Thalli, mycelia, and sporidia of the achorion Schönieinit, showing the mode of reproduction.--(BENNETT.) 800 diam. 
them with liquor potassæ, the vegetations can be discovered microscopically-using a magnifying power of 300 diametersto consist of numerous little oval or rounded bodies; the sporules of the fungus having a diameter of about $\frac{1}{3000}$ of an inch. A number of cells united end to end form simple or jointed and branching tubes, developed from the sporules. Little granules or nuclei may be seen in the interior of the spores. The tubes vary in diameter, and hairs in the vicinity of the favus crusts are impregnated with the fungus.-(Bazin, Draper, AItken, Anderson.) Mr. Erichsen considers "that the matter of favus is a modification of tubercular disease of the skin," and this view is supported to some extent by Bennett, who is of opinion that the tubercular matter furnishes the soil from which the mycodermatous vegetations spring.

Chemically, the matter of favus has been found by Thenerd to be composed of coagulated albumen, 70 ; gelatine, 17 ; phosphate of lime, 5 ; water and loss, 8 parts in every 100 parts.

Of the transmissibility of the disease from man to animals there can be no doubt; the translations of Mr. Fleming from the Continental journals furnish ample proofs of this fact. I remember some years ago being called upon to attend a number of animals, at a farm in Yorkshire, for ring-worm, the crusts upon which presented, except on the horses, a yellow colour and a honeycomb appearance. The disease had attacked over twenty horned cattle, three horses, some dogs, and several cats. One fact in connection with this outbreak was, that the cats were very fond of sitting on the backs of the cows and horses, and, doubtless, the disease had been caught from mice by the cats, and transmitted by them to the other animals about the place.

liemak found that the sporules underwent developmental changes on the cut surface of an apple, as well as in animal fluids to which sugar had been added; but no such changes took place when they were mixed with distilled or spring water, the serum of blood, solution of albumen, pus, muscle, or any other animal tissue. In these cases, the animal tissue, as well as the favus crust, became decomposed, and infusorial formations were developed. From this we learn that the achorion grows under the same circumstances as all other moulds. Inoculation with the favus crust does not always succeed, 
and Bennett is of opinion that it is necessary to have certain exudations in peculiar states of the constitution, or disintegrated matters which have undergone particular chemical changes, probably from acid secretions of the skin, before the disease can be produced.

Be this as it may, it is very true that filth and dirt upon the skin constitute a favourable soil in which the parasite may grow, provided the animal be subjected to a source of contagion.

In all probability the secretions of the cutaneous glands, accumulated upon the surface of the body, and there undergoing the acid fermentation, become the nidi which favour the growth of the favus.

Symptoms.-The disease commences with a slight itching,

$a$

$b$

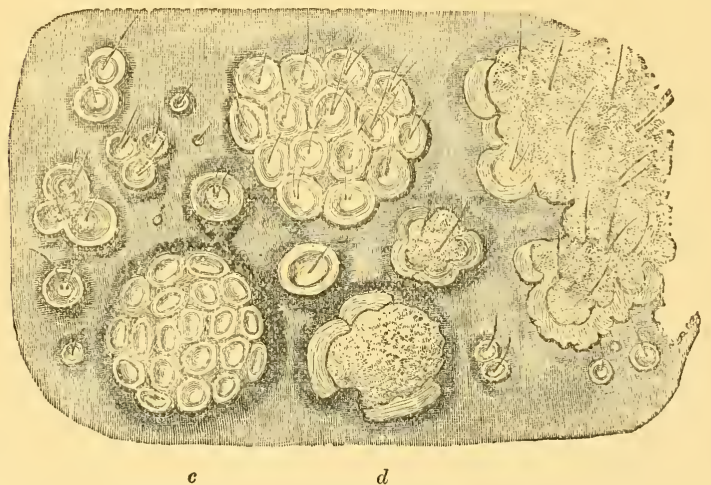

FIG. 145. - $a$, Isolated crusts of favus, presenting the lupine seed-like depression in different stages of growth (so called Porrigo lupinosa); some are arranged in groups of twos and threes. $b$, A larger group of these crusts, somewhat compressed at the sides like a honeycomb (Porrigo favosa). $\quad c$, Another group, which occurred on the shoulder of a young girl ; no hairs passed through the centre of these crusts. $d$, Large isolated crusts in an advanced state of growth ; the external ring is cracked, and the friable centre is enlarged and elevated. $e$, Numerous crusts aggregated together so as to form an irregular elevated mass ; traces of the original form may be observed in the cracked rings round the margin. Natural size.-(BENNETT.)

followed by an eruption, which soon assumes the appearance of yellow scabs of a circular form. The patches of eruption are sometimes very numerous, of a yellow colour when recent, but they become lighter as they grow older, and the crust, at first 
rather firm in consistence, becomes easily reduced to powder. The smell of the scab is peculiar, and is compared to the urine of a cat, or to a cage where mice have been kept. This is supposed by Dr. Lowe to be due to a species of alcoholic fermentation (methylamine), in connection with the vegetable growth. When a crust of recent formation is removed, a circular depression, wider and deeper than the favus, is seen, and at a more adranced stage the ulceration penetrates below the dermoid tissue.

"On examining the hairs which pass through the farus crusts, it will be often found that they present their healthy structure. At other times they evidently contain long-jointed branches, similar to those in the crust, running in the long axis of the hair, which is exceedingly brittle. There can be very little doubt that the tubes and sporules, after a time, completely fill up the hair follicle, and thence enter the hair, causing atrophy of its bulb, and the baldness which follows the disease."-(BeNnetT.)

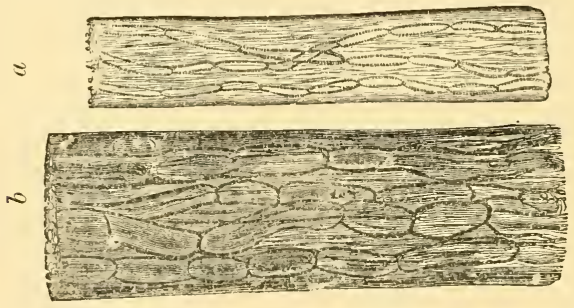

Frg. 146. - a, A light hair, containing branches of the achorion Schönleinii (magnified 300 diameters linear). The wood-cntter has made the branches too beaded. $b$, A dark-coloured hair, containing branches of the plant.-(BENNETT.)

Treatment.-The disease can only be eradicated by the destruction of the parasitic growth, and as a rule this is not so difficult to accomplish in the domestic animals as it seems to be in man. I have, however, seen some very aggravated cases in eats, but the severity of the disease was due more to neglect than to any primary virulence. Corrosive sublimate made into an ointment with lard is recommended by M. St. Cyr. The iodine ointment seems to answer very well in this country, but before it is applied, the scabs are to be removed by washing them well with soft soap and warm water. 
Many preparations are recommended by physicians, such as a solution of sulphate of copper, or of the nitrate of silver in the proportions of seven grains to ten ounces of water ; unguentum picis; an ointment of the cocculus indicus, 3i. to 3ii.; adipis, $\xi$ i. ; nitrate of merctry ointment, a solution of nitrous acid, sulphureous acid, and a variety of other remedies. The application of the stick nitrate of silver round the patches is another favourite method of treatment, and when all these fail, the hairs are removed singly with a pair of forceps (depilation.)

\section{TINEA TONSURANS.}

Ring-worm is a common skin disease in the lower animals, and, unlike favus, is not confined to those ill cared for, but is unequally seen amongst carriage-horses, hunters, and well-groomed cattle, and is defined to be an affection implicating the hairs of the skin, usually assuming a circular form. The hairs, becoming dry and brittle, have a tendency to crack or break across and fall off, leaving isolated patches of baldness, constituting what is termed alopecia circumscripta. This condition is more particularly met with in the dog ; generally, however, an erythematous eruption, accompanied by slight swelling of the skin and some itchiness, manifests itself, and a fungus ultimately appears, which had been developing between the true skin and epidermis. This fungus has a whitish appearance, and a powdery or fine bran-like aspect. It covers the epidermis between the hairs, and forms around them a complete whitish sheath. The hairs break unevenly, become ragged at their ends, and have the appearance of having been eaten through; at the same time their broken stumps are much altered, bent, and twisted, and are lighter in colour than the healthy hair.

II. Bazin states that the parasite may destroy the hair-bulb and the capsule of the hair follicle, give rise to the formation of pus, and cause perfect baldness of the part.-(ANDERSON, BAZIN, Hillier, Aitken.) This form of ring-worm differs from the vesicular form (herpes circinatus) by the absence of vesicles, and by the formation of scurf or seales around single hairs, or in patches surrounding several hairs. Again, if a hair in the vesicular form be pulled gently, the probability is that it will come up by the root, as in the case of a healthy hair, but in this form it will break off near the skin, or within its follicle. 
Pathology.- " The nature of this disease is to be studied in the botany of the cryptogamic parasite called the Tricophyton, discovered by Malmsten in 1845 . It consists of oval transparent spores or globules, about Many of these are isolated, others constitute by their juxtıposition articulated filaments. Its anatomical seat is the interior of the roots of the hair. The hairs and fungi simultaneously increase; the former seem larger than usual, are paler in colour, lose their elasticity, soften, and break off when they have risen some one or two lines above the surface."-(AITKEN.)

Ring-worm may affect any part of the animal body, but its favourite seats appear to be the hind quarters, back, neck, and face. It is a contagious disease, and depends upon the presence of the vegetable parasite already described. It differs from favus in the colour of the scabs, which are greyish-white instead of yellow. The microscopic appearance of the plant, its structure, and its powers of propagation are identical with the Achorion Schöıleinii. " There are numerous facts which justify the belief that there exists but one essential fungus, whose sporules find a soil for development and growth upon the surface, or even within more secluded portions of the body; and that varieties in the growth of that fungus are due to differences in the constitution of the individual, to the moisture, exudation, soil, or temperature under which the development of the fungus takes place. The exact nature of these differences is not yet understood; but the production of irritant acids and gases are constant accompaniments of the growth of such parasites, by the chemical action of the vegetable cell ; for it does not undergo development without exciting a chemical decomposition in the pabulum on which it feeds, and the different stages in its growth give rise to alcoholic acid and putrefactive fermentations. Thus their irritant action very soon may establish an eruption. But the ratio of eruption to parasite is not constant; for an amount of fungus which will simply produce the death of hair in one person or part of the body, may in another produce irritation, eruption, or violent inflammation. These different results may be due to two causes -(1), constitutional peculiarity in different individuals; (2), peculiarity of structure of a part as regards density, heat, moisture, and chemical and anatomical composition of the part."(AITKEN, Lowe.)

Experiments are wanting to prove the possibility of the ring- 
worm crust of the horse producing the favous crust of the dog and cat, and vice versa; but from the foregoing observations, and from the opinion of Bennett, already quoted, that acid secretions favour the development of the favous crust, and from microscopic researches into the identity of the parasites, I an almost inclined to conclude that the ring-worm of the horse may be the favous of the cat and dog, and that the yellow crust of the latter animals, and the more rapid development of the fungus, are due to the natural acid condition of their cutaneous excretions. 1

The following woodcut of the microscopic anatomy of the parasite of ring-worm is from original drawings by Professor Vaughan of this College. The scab from which the specimen is prepared was removed by me from a carriage horse, the property of David Corsar, Esq., of Arbroath. The ring-worm, a solitary patch, was situated on the off-side hind quarter, and presented the usual appearance of the affection, namely, a round, baldish patch, covered by a greyish-white crust.

Treatment.-The treatment of ring-worm, like that of favus,

1 The opinion I had ventured to express as to the identity of Tinea favosa and Tinea circinatus, and that the colour of the crust depended upon the constitution of the animal, was very fortunately confirmed by Mr. M'Gillivray of Banff, in an admirable paper on Porrigo, published in the Veterinarian for March 1872. After reading Mr. M'Gillivray's paper, I took the liberty of asking him a few queries upon the subject; and I feel I can do no better than publish the answer I received by return of post :-

“ No. 4 Bridge Street, BanfF, 6th March 1872.

" MY DEAR SIR-Your letter came to hand this forenoon, and it gives me the most sincere pleasure to answer your queries, as follows :-

" 1. As a rule, yellow crusts are not found in ring-worm in horned cattle. I have, however, seen genuine favous crusts in two such cases.

"2. Contagious materiel from the grey crust of cattle or horses undoubtedly (in so far as my experience goes) produces often, but not invariably, the characteristic yellow crust of favus in the human subject.

" 3 . The crusts or scabs of Porrigo in the dog are generally greyish in colour. In the cat I have invariably found them of a very bright yellow. In the hare I never saw them otherwise than greyish to the naked eye.

"So much for your 'queries.' My own candid opinion is that the class of the animal has a good deal to do with the colour of the superincumbent crust or scab of porrigo or tinea. This is the only satisfactory way of accounting for the fact of grey crusts in cattle producing yellow crusts in man.

"I am, my dear Sir, faithfully yours,

" WV. Williams, Esq.,

"Alex. M'Gillivray."

Principal, Veterinary College, Edinburgh." 
consists in the destruction of the cryptogamic plant. This is effected by the application to the spots-after first removing

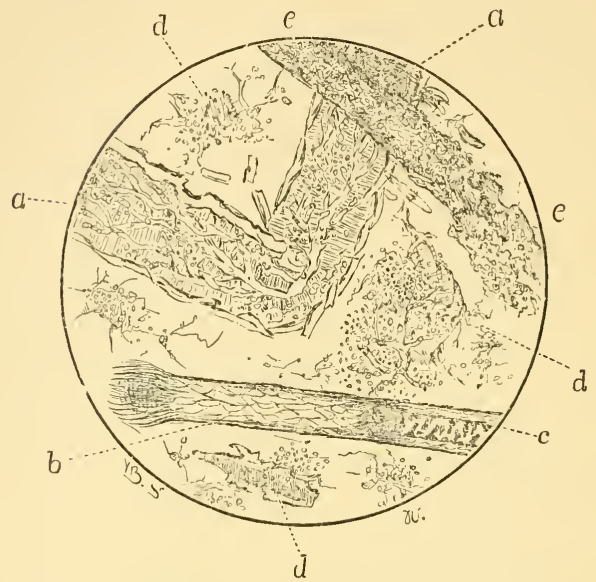

Fig. 147.-Microscopic appearance of ring-worm crust after the addition of liquor potasse. $a, a$, Large branch of the achorion, showing thalli in an advanced condition, with sporules arranging themselves end to end. $b, c$, Hair containing the plant in an early stage of development: at $b$ the fungus is distinctly seen in the centre of the hair-tube ; at $c$, the hair is represented as yet uninvaded by the parasite. $d, d$, Scattered sporules. $e, e$, Hair irregularly enlarged, with its cortical layer broken down by the growing parasite. 200 diameters.

the crusts by washing the parts with warm water, soft soap, and carbonate of potash - of the iodine ointment. To prevent a recurrence of the disease, the stable should be thoroughly cleaned and whitewashed, the harness and collars carefully washed with soap and water, then brushed over with a solution of iodine or corrosive sublimate, and the clothing of the animal boiled. 
I N D E X. 


\section{INDEX.}

Abrominal Parietes, Wounds of, Abscess,

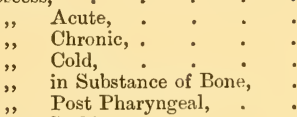

\section{Acarus Scabie,}

Arne, .

Aconite in Inflammation, :

Actual Cautery, . . .

Actino-Mycosis, . . . .

Acute Abscess, . . . .

Adhesion, . : .

After Birth, Hernia occurring, and

Causes, . . . . 574

Air into Veins, Entrance of, . $\quad 576$

Aitken and Lowe on Pathology of Ring-Worm, .

Albugo or Leucoma, $\quad 735$

Alison, Dr., on Blood-Letting in Inflammation,

Alkalies in Inflammation, . .

Alveolar Processes, Diseases of, .

Amaurosis, .

Amp after Castration, .

Anæsthesia,

Anatomy of Bones, : $\quad \cdot \quad$ : 149

Anchylosis of Vertebræ, . $\quad 203$

or Stiffening of Juints,

Anderson on Fractured Olecranon,

Aneurism, Dissecting, :
Varicose, :

• •

Aneurismal Varix, . $\quad$. $\quad 585$

Animal Parasites, . . . 700

Antero-Iliac Spine, Fracture of, . 122

Antero-Maxilla, Fracture of, . 98

Antimony in Inflammation, . 73

Aphthæ, Sporadic, . . . 495

Arterial Coats, Degenerations of, . $\quad 586$

, Calcarenus Degeneration of, 586

, Cartilaginous Degeneration of, 587

" Fatty Degeneration of, . 587

Arteries, Injuries to, . . . 588

Arteriss and Lymphatics, Diseases of, 578 Arteritis,

"Letter on, from Professor Barluw, .
Arteries and Lymphatics, Diseases of -continued.

Thrombosis, Virchow on,

Aneurism, . .

580

581

581

Treatment of, $\quad 583$

Dissecting Aneurism, - 584

False Aneurisms, . $\quad$ • 584

Aneurismal Varix, . $\quad 585$

Varicose Aneurism, . $\quad 586$

Degeneration of Arterial Coats, .

Calcareous Degreneration of Arterial Coats,

586

Cartilaginous Degeneration,

586

587

", Fatty or Atheromatous De. generation,

Injuries to Arteries, : $\quad 588$

Lymphatics, Inflammation of, Lymphangitis, 589

Mr. Haycock on, . . 590

Causes of, . . . 591

Treatment, : $: \quad 592$

592

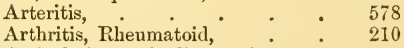

Articulations, Gliding, of Hocks,

Diseases of, . . . $30^{\circ}$

Back-Rope, Evil of, . . 120

Bandages, Kinds of, . $\quad$. 84

Barlow, Professor, on Arteritis, : $\quad 578$

Bearing-Rein a cause of Poll-Evil . $\quad 568$

Benign or Simple Tumour, . . 427

Bennett, Dr., Classification of Skin

, Diseases, . . . $6 \% 2$

, on Counter-Irritation, - 320

,, on Favus, . . 733

,, Effect of Mercury on Bone, 200

Bistouri Caché for Quittor, . . 390

Bits, Severe, . . . 494

Bladder and External Organs of Urination, Diseases of, . $\quad 623$

Urinal Deposits, . . 623

Causes and Symptoms of

Deposits,

Cystic Calculus, Examination for, . . 626

Calculus in Mare, Removal of, 626

Lithotomy in Horse or Gelding, 627

", in $\mathrm{Ox}, \quad$. 629

Calculi, Urethral, : : 629 
Blarder and External Organs of Urination, Diseases of-continucd.

Sabulous Matter, Removal of, 630

", Generation, Diseases of External Organs of,

Urethritis, Symptoms and

Treatment,

D'Arbovalon Syphilis in Horse, 631

Phymosis, Percivall and D'Arboval on,

Paraphymosis, Treatment of, 632

Penis, Amputation of,

Hydrocele or Dropsy of Scrotum,

Vagina and Mammary Gland, Diseases of,

Vaginitis, or Inflammation of Vagina,

Treatment of, .

" Mammitis, or Inflammation of Mammary Gland,

, Treatment of, . . Bleeding, Local and General, " Method of,

Blistering, Hints on, . . .

Tilisters,

Blood Effusion, : $\quad . \quad$.

, Inflammatory Appearances of,

Blood-Letting in Inflammation,

, Influence opposed to,

Plood-S̈pavin

Second,

Jog-Spavin,

Bones, Fractures and Diseases of, .

Modes of Fracture,

635

636

637

638

640

322

Symptoms of Fracture,

, Modes of Union,

Formation and Kinds of Callus, $80-81$

Stages of Repair, according to

Dupuytren,

Microscopic Characters of New,

Treatment of Fractures,

Splints and Bandages, Kinds of,

Block Tin Splints,

Compound Fractures, .

Treatment of,

Unpropitious Symptoms, .

Separation of Epipliyses, .

False Joints,

Treatment of,

Syme's Treatment, .

Causes of Non-Union,

Particular Fractures of,

Fracture of Inferior Maxilla,

Treatment of,

From Pressure of Bit, and

Treatment,

Symptoms of Fracture by Curb, 92

from Punching out Incisors,
Bones, Fractures and Diseases of-continucd.

" Professor Varnell's Treatment of, 94

", Treatment by Mr. Walker's Apparatus,

Treatment by Gutta.Percha,

Fracture of Antero-Maxilla,

Fracture of Nasal,

Professor Varnell's Treatment of, 99

Removal of Necrosed Fragments,

Fracture of Superior Maxilia, and Treatment,

Fracture of Erontal,

Treatment, . . . 102

Tendency to Tetanus, - 102

Hæmorrhage in, . . 103

Comphications of, . . 103

Fractures of Cranial Bonss, 104

Kinds of, $\quad . \quad 104$

Simple Fissure, . . . 105

,, Simple Fracture with Depression, . . . . 105

Trephining, . . . 106

Punctured Fracture, . . 107

Compound Fracture, . 108

of Crest of Occiput, common in Coal Pits, Prevention,

Concussion of the Brain, Collapse, Reaction, and Inflammation of,

Different ways in which Concussion proves fatal,

Post Mortem Appearances,

Mr. Liston's Theory, Treatment,

Hæmorrhage, . .

Syme's Method of Trephining the Cranium, .

Hernia Cerebri and Treatment, 110

Fractures of Vertebræ, Wry Neck,

Fistulous Withers, . $\quad$. 118

Broken Back, . . . 118

Fracture without Displacement, 118

Fracture during Operations, $\quad 120$

Evil of the "Back-Rope," 121

Fractures of Sacrum, and Treatment, . . . 121

Fractures of Coccyx, and Treatment,

Fractures of Pelvic, . . 122

Fracture of Anterior Iliac Spine, 122

Exact Seat of Fracture, how determined,

Fracture through Symphisis Pubis,

Fractures of Tubcrosity of Ischium,

Fractures of Femur, . . $\quad 125$

of Trochanters, $\quad . \quad 126$ 
Bones, Fracturegand PAGE

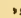

3

,
Fracture of Patella, . . 127 of Tibia, . $\quad$. $\quad$. 128 of Calcis, Treatment, $\quad 129$ of Seapula, . . . 131 of Humerus, Recovery inpossible, of Radius and Uina, of Olecranon, transversely, Mr. Anderson's Metbud of Detection, Treatment, in Bones of Knee,

Metacarpal and Suffraginis, Split Pastern, Sesamoids, Recovery impossible, 137 of Navicular, . . . 137 of Pedal, .

Treatment,

Froken Ribs, : : $\quad$ • 140

Treatment,

Luxations or Dislocations without Fracture, Case of, of Patella,

'Treatment,

Partial Dislocations of Fetlock-Joints,

Dislocation of Head of Femur impossible in Horse,

Dimpossible in Horse, $\dot{0}$

Case of Dislocation of Cervical Vertebr:e,

Classitication of,

Anatomy of,

Ostitis, or Inflammation of,

Ostitis and Periostitis of the Metacarpals, or Sore Shins,

Symptoms,

Splints,

Kinds of Splints,

Produce Lameness, when, .

Peculiarity of Lameness, .

How to distinguish it,

and Articulations, Diseases of, Splint, Treatment of,

Subcutaneous Periosteotomy,

Pyro-Puncture,

Scrofulous Ostitis,

Causes and Pathology, .

Treatment, . .

Ulceration and Caries, Syme on, 163

Treatment, . . 168

Necrosis,

Pathology of, . . .

Treatment,

Necrosis, Internal, rare, .

Subperiosteal Suppuration,

Abscess in Substance or.

Diagnosis and Treatment of,

Iiseases of, Non-Inflammatory, 178

142

143

144
153

154

155

156

157

158

158

159

159

159

160

162

163

168

172

174

175

176

176
Bones, FracturesandDiseases of - continued.

\section{" Rickets or Rachitis, . 178}

," Treatment of, . . 180

, Rokitansky on, . . 181

, Mollities Ossium, . . 182

, Cases of, $\quad . \quad$. 183

, How accounted for, . . 184

" Osteo-Porosis, . . 184

, Post Mortem Appearance, by

Professor Varnell, . • 189

, in Sheep, Mr. Robertson on, 193

,, Rokitansky on, . . 195

" Fragilitus Ossium, - • 197

, Osteo-Sarcoma, . . 199

, Treatment, . . . 200

, Effects of Mercury on, . 200

" Case by Professor Bennett, 200

Bones of Knee, Fracture of, . 135

Bone-Spavin, . . . 301

Bot Flies, . . . $\quad 726$

141 Bouley on Poultry Lousiness, . 709

Bourguignon on Sarcoptes Hominis, $686^{\circ}$

142 Brain, Collapse of, . . . 109

Bridle for preventing Fracture of Occiput,

109

Broad's, Mr., Shoe for Laminitis, and Treatment of,

Broad, Mr., on "Mud-Fever," and Treatment of,

Broken Back,

\section{Broken Knees,}

Fracture with and without Displacement,

Brok Kinds of, : :

Bronchocele, : $\quad$ : $\quad$ :

Bruises of the Mouth, . . .

Brushing or Cutting,

Bubonocele, . . . 602

Buccal Membrane, Affections of, . 494

Buchanan, Dr., Classification of Skin

Diseases,

Buffy Coat,

663

Burdon Sanderson on Inflammation, 15

Burns, Scalds, and Effect of Cold, 465

Calcareous Degeneration of Arterial Coats,

,' Tumours, '. • 420

Calcis, Fractures of, . . . 129

Calculi, Salivary, : $\quad \cdot \quad \cdot 499$ ," Urethral, : $\quad . \quad 629$

Calculus, Cystic, Examination for, $\quad 625$ 
Cancer--continued.

, Medullary, . . . 444

"Scirrhous or Hard, .

, Soft,

Canine Skin Diseases, Treatment of,

Canker,

," Internal, or Otorihos,

Capped Elbow,

Capped Hock,

Capped Knee,

Horned Cattle liable to,

Carbuncle of Coronary Band,

Cardiac Stricture of (Esophagus, .

Caries of Teeth,

Carpitis or Inflammation of the Knee,

Carpus in Dogs, Dislocation of,

Cartilage, Morbid Condition of,

" Redfern, Dr., on Wounds

$$
\text { of, . . . . }
$$

Cartilaginous Degeneration of Arterial Coats, . Tumours,

Case of Gelatinous Degeneration after

Neurotomy,

Cases of Osteo-Porosis,

Casting, Methods of, . : : 655

Castration,

, Precautions before Operation,

" Preparation for Operation,

, Torsion, Operation by,

" Operation when Hernia is present,

Consequences of,

", Inflammation after, :

" Farcy, Glanders, and Paralysis after,

"Tetanus, Amaurosis, Champignon, Scirrhus of the Cord or Sarcocele, after,

Champignon, Treatment of,

9

D'Arboval on Champignon,

Chronic Suppuration or

Fistula of Scrotum, .

Peritonitis, . .

Post Mortem of, .

Treatment of, .

Hernia following,

when Hernia is present,

Cat, Mange in, . .

Cataract,

, False or Spurious, .

True, . .

Gattle, Gut-Tie in, : .

Causes, additional, of Lameness, .

", of Anchylosis of Vertebræ,

", of Carbuncle of Coronary Band, 372

", of False Quarter, aud Caution, 374

" of False Quarter, and Caution, 374

$\rightarrow$
443

444

683

383

567

566

$24-425$

425

265

371

539

507

265

144

214

219

587

433

352

642

643

644

646

647

647

648

$64 S$

649

650

651

651

652

653

654

613

646

720

560

562

561

621

240

204

74

Causes of Inflammation,

,, of Pain in Inflammation, .

" of Scrofulous Synovitis, .

" of Sprain of Flexor Tendons,

of, . . . . 207

Causticking in Bone-Spavin, . 310

Cautery, Actual, . . . . 323

Cervical Vertebræ, Dislocation of, Case of,

Champignon or Scirrhous Cord, after Castration,

,, Treatment of, - . 650

Cherry's, Mr., Remarks on Carpitis, 265

Choking, . . . . 530

Chorea, Definition of, . . 245

ChronicInflammation, Treatment of, $\quad 76$

76
208

$\begin{array}{lll}\text {," Scrofulous Synovitis, } & \text { - } & 208 \\ \text {," } & 651\end{array}$

Clams in Treatment of Hernia, : 611

Clark, Mr. Bracy, on Estrus Bovis, $\quad 726$

Classification of Bones, . . 148

, of Joints, - : 203

" of Skin Diseases, . 660

" of Tumours, . . 408

,, of Wounds, . . 448

Clipping of Horses, - . . 668

Coarse Hocks, . . . . 307

Coccyx, Fractures of, $\quad$. $\quad 122$

Cold, Effect of, - . . . 465

Comparison between Mucus, Epi-

thelium, and Pus, . . $\quad 39$

Compound Fractures, $\quad$ - $\quad$ - 86

,. Treatment of, - . 86

Concussion of the Brain, $\quad$. 109

," Fatal Terminations of, $\quad 110$

, Hæmorrhage in, . . 115

, Post Mortem Appearances, 112

, Professor Syme on 'Tre-

$\begin{array}{ccc}\text { phining, } & \text { : } & 115 \\ \text { Congenital Hernia, } & \text { : } & 60 \mathrm{~S}\end{array}$

Contact, Mortification by, . $\quad$ - 59

Contused and Lacerated Wounds, 455

Consequences of Castration, . $\quad 647$

Corns, . . . . . . 381

Coronary Band, Carbuncle of, . $\quad 3 i 1$

Coronitis, Inflammation of Coronary

Substance, . . . . 370

Cough, Dental, . . . . 514

Counter-irritation, Theory of, - 319

Cramp in Stifle, . . . . 296

Cranial Bones, Fractures of, - 104

Crib-biters, . . . . 540

Cross Lameness, . . . . 233

Crusta Labialis, . . . . 678

, Petrosa Tumours, : 419

Cüb. Petrosa Tumourd, : : : 314 
Cystic Calculus, Examination for,

,' Tumours, various kinds,

Cysts, Compound, or Proliferous,

, Dentigerous,

" Sercus, in Horned Cattle, .

D'A rboval on Champignon, on Syphilis in Horse,

Definition and Classification of Wounds, 448 , of Lameness,

Definitions of Inflammation, .

Degeneration of Arterial Coats,

$$
\begin{gathered}
, \quad \text { Gelatinous, and Ruptured } \\
\text {, Tendon, Case of, . }
\end{gathered}
$$

Dental Cough,

Dentition, Diseases during, . . Fever,

Deposits, Urinal,

Depression, Simple Fracture with,

Jerma, Disordered Sensibility of, .

Dermatodectes Bovis, . .

Equi,
Dermatozoa,
Dermatophyta,
Diagnosis of Lameness,
" of Lameness, Rules for,
" of Shoulder-Joint Lameness
", of Scrotal Hernia,
", of Splint Lameness,
, and Treatment of Bone

$$
\text { Abscess, }
$$

Diagnostic Signs of Navicular Arthritis, 340

Diathrodial or True Joints,

Dick, Professor, on Fistulous Umbilical Hernia,

19

,

" on Foot-Rot, on Laminitis,

Diffuse Phlebitis, Treatment of Grease,

Dilatation of Esophagus, :

Diseases of Alveolar Processes, of Arteries, :
of Bladder, :
of Bones,
during Dentition, :
of the Ear,
of External Organs of Urina-
tion,
of External Organs of Genera-
tion, of Eyes, of Facial Region, . of Facial Sinuses, of Feet, . of Gliding Articulations of

Hocks, of Head, of Hock, of Horn-secreting Structures,

626

573

538

506

578

623

513
PAGE

Diseases-continued.

", of Humours of Eye, . 547

," of Joints, and Classification, 203

" of Lids of Eye, . . 549

", of Ligamentous and Ten-

dinous Structure of Hock, 311

of Lymphatics, . . 578

of Mammary Gland, : $\quad 636$

of Mouth, . . . 493

of Neck, - . $\quad$. $\quad 566$

of Nerves of Eye, . $\quad 548$

Non-Inflammatory, of Bone, 178

of Esophagus, . . 528

of Pharynx, . . 528

of Pyramidal Process of Os Pedis,

of Shoulder-Joint, • $\quad 328$

of Skin, . . . 660

Skin, due to Animal Para-

sites, . . . . 700

Skin, due to Epizoa, : 724

Skin, due to Vegetable Pars-

sites, $\quad 729$

Skin, Parasitic, : : 700

of Tongue, : $\quad$ - 501

of Vagina, : $\quad$. $\quad 636$

of Veins, - . $\quad$ - $\quad 566$

Dislocations, . . . . 142

,, of Carpus in Dogs, . 144

,, of Cervical Vertebræ,

Case of, $\quad 145$

of Eyeball, : $\quad 5 \quad 563$

," Partial, of Fetlock-Joint, 144

Disordered Sensibility of Derma, . $\quad 697$

Dissecting Aneurism, . . 584

599 Distichiasis, . . . . . 551

400 Dog, Mange in, . . . $\quad 714$

356 " Follicular Scabies in, . . 715

681 Dropsy of Joints (Hydrops Articu-

566 Ectropiuma Impetiginodes, or Pustulosum, 675 lorum), . . 227

, of Scrotum, - . . 636

Dupuytren's "Stages of Repair" of

Bones,

Ecraseur, The, . . . . 530

, Mercuriale, . . . 675

". Rubrum, . . . 674

", Simplex, : $\quad: \quad$ : 673

630 Eczema, Various Forms of, . 673

542 Eczematous Inflammation, . 672

493 Elbow Lameness, . . : 260

517 Elephantiasis, . . . . 593

325 ," from Grease, . 678

301 Tumours, . . . . 416

566 Entropium, . $\quad . \quad$. 550

299 Epidermic Tumours, • • . 415

370 Epiphyses, Separation of, . . 87 
Epiplocele,

Epithelial Tumours,

PAG

Epizoa,

Epulis-(See Actino Mycosis)

Equine Lymph,

Erysipelas after ivounds,

$\begin{array}{cl}\text {," Edematous, } & \text { Phlegmonous, : } \\ \text { Erythema, } & \text {. }\end{array}$

" Mammilarum, . .

Erythematous Inflammation, .

Examination for Cystic Calculus, Exomphalos, of Eye, . . .

Extravasation, : $: \cdot \dot{*}^{\circ}$

Exudation in Inflammation, .

Eyes, Diseases of,

"Traumatic and Simple Ophthalmia, Conjunctivitis, . Treatment,

"Nebula, Albugo, or Leucoma,

", Percivall on, . .

,, Staphyloma, . .

,Diseases of Humours of, Glaucoma,

"Diseases of Nerves of, Amaurosis, Gutta Serena or GlassEye,

Strabismus, Squinting,

"Abnormal Position and Disease of Lids of, Ectropium,

Wharton Jones' Treatmeni

", Entropium,

Trichiasis, Distichiasis,

Warts on Eyelids, Wounds of Eyelids, Lachrymal Fistula, Stricture of Lachrymal Duct,

Parasites, Mr. Charles Percivall on,

Fungous Hæmatodes,

Periodic Ophthalmia, or MoonBlindness, Symptoms,

Percivall on,

Cataract,

Percivall on $\cdot{ }^{\circ}$

True Cataracts, . $\quad$.

Spurious or False Cataracts,

Dislocation of Eyeball,

Removal of Eyeball, . .

Examination of the Eye,

by Ophthalmoscope, .

Diseases of Humours of, .

" Diseases of Lids of, . .

" Diseases of Nerves of,

" Diseases of Nerves of,

", Method of returning, :

"Parasites of

Ey'eball, Dislocation of, ${ }^{\circ} \cdot$

" Removal of,
440 Facial Region, Diseases and Injuries of, 493

675 , Diseases of Moutl, 
Facial Rugion, Diseases and Injuries of continued.

" Pus in Gutteral

Pouches, Treatment, 529

Pharyngeal Polypi, the "Ecraseur,"

Choking, Percivall on, 530

", Gamgee on Causes of, Cases of, .

Treatment of, .

Use of Probang, .

Essophagotomy, Carbolised Cat-Gut Suture,

Dilatation and Stricture of Esophagus,

Case by Mr. Cheetham, 538

$$
10
$$

Uardiac Stricture of Essophagus, Percivall on Treatment, Crib-Biters and WindSuckers,

Facial Sinuses, Diseases of, :

False Aneurisms,

False-Joints,

Fetlock-Joint, Partial Dislocation of,

False Joints, Treatment of ; Syme's 'Treatment,

False Quarter,

False or Spurious Cataract, . .

Farcy after Castration, .

Fatty or A theromatous Degeneration of Arterial Coats,

Fatty Tumours,

Favus or Honeycomb Ring-Worm,

Febra Pyogenica,

Feet, Diseases of,

, Shoeing, preliminary Remarks upon,

"Shoe used at the College,

" Disease of the Pyramidal Process of Os Pedis,

Ossification of Lateral Cartilages (or Side-Bones),

"Treatment,

", Navicular Arthritis (various Theories as to), Contractions of Foot in, :

"Sprain or Laceration of Fibres of Perforans in,

", Symptoms of,

$\rightarrow$

Diagnostic Signs of,

Pathology of,

" Pathology of, • •

" Preatment, Seton, - $\quad$ -

"Neurotomy,

"Unfavourable Results of Neurotomy,

" Case of Gelatinous Degeneration and Rupture of Tendon,

332

333

351

352
Feet, Diseases of-continued.

1PGB

,, Appearance of Navicular Bone

in the same Case,

, Pathological Anatomy of Gelatinous Degeneration in Man,

, Extract from Spence's Lectures on,

, Laminitis or Inflammation of, Acute, Subacute, and Chronic,

Professor Dick on, : $\quad$ :

356

356

", Pathology of, . . . 357

, Symptoms of, in both Fore Fect, 353

, in Hind Feet, . . 364

, Treatment, Mr. Broad's Treatment and Shoe,

" Diseases of Horn-secreting Structures,

,, Villitis, Coronitis (or Inflammation of Coronary Substance), 370

,, Treatment, . . . 370

" Carbuncle of Coronary Banc, 371

,, due to what, and Treatment, 372

, False-Quarter, . . . 373

" Causes, Treatment, and Caution, 374

", Sand-Crack, . . . 374

, Treatment, . . . 376

"Frenchand English Operationsfor, 377

, Keratoma, Treatment, . 378

, Seedy-Toe, . . . 380

," Corns, - . . . 381

, Treatment, . . . 382

, Thrush, . . . . 382

" Canker, : : : : $\quad$ : 383

, Treatment, . . $\quad 384$

, Accidental Injuries to, . 387

, Pricks in Shoeing, and Gathered Nails, . . . 387

,, Detection and Treatment, - 388

, Caution in Paring, . . 389

,, Weak Feet, • . . 389

, Quittor, . . . 389

,"Treatment, Modes of, Bistouri Caché, . . . . 390

, Foul in the Foot, : : 393

, Foot-Rot in Sheep, - . 395

, Mr. Fleming on, . . 395

" Professor Dick on, - . 395

" Difference between, and Foot- . and-Mouth Disease,

Femur, Fractures of, . : 125

340 Fetlock-Joint, Sprain of, : : 283

342 Fever, Dentition, . . . 515

" Inflammatory, • • - 60

Fistula, Lachrymal, : $\quad \cdot \quad: \quad 552$

" Salivary, • • • 497

Fistula of Scrotum, • • • 651

Fistulous Umbilical Hernia, : 599

"Withers, . 106, 118, 570 
Fleas, Pulex Irritans,

Flexor Brachii, Sprain of

, Metatarsi, Subcutaneous liaceration of,

,, Perforans Tendon liable to Injury,

" 'Fly," Tendons, Sprain of,

Follicular Scabies in Dogs,

Foot, Contraction of, in Navicular

Disease,

Foot-Rot, Professor Dick on,

Formation of Callus,

$$
\begin{gathered}
\text { of Pus, } \\
\text { of New Vessels (Virchow } \\
\text { and Paget), } \\
\text { of Sinuses, . }
\end{gathered}
$$

Foul in the foot,

Fotus in Utero, Inguinal Hernia in, Fractures, of Anterior Msxilla, . of Bones of Knee, .

Modes of, Symptoms of, of Back during Operations, of Calcis, of Coccyx, Compound, Compound, of Crest of Occiput, 108 of Cranial Bones, . . 104 of Femur, . $\quad$ • 125 of Frontal Bone, . $\quad$. 101 of Humerus, . . . 131 of Antero-Iliac Spine, . 121 of Iliac Spine, how determined,123 of Inferior Maxilla, Treatment of, by $\mathrm{Mr}$.

Walker's Apparatus, . of Nasal Bone, of Metacarpi, of Navicular, . Non-union, Causes of, . of Olecranon, tranversely, of Os Pedis, of Particular Bones, of Patella, . of Pelvis, from Pressure of Bit, . $\quad 92$ from Punching out Incisors, 93 Punctured, of Radius, of Ramus of Lower Jaw, of Sacrum, of Scapula of Sesamoids, : : of Suffraginis, of Superior Maxilla, through Symphysis Pubis, of Tibia, 'reatment of,
Fractures-continued. of Trochanters, " . 126 ", of Tuberosity of Ischium, 125 , of Ulna, . . . 134 ,, of Vertebræ, . • . 117 Fragilitus Ossinm, . . . 197 Frontal Bone, Fracture of. . . 101 Fungus of the Brain, . . . 116 Fungus Hæmatodes, . . . 554

Gamgee on Aneurism, . . . 551 Gastrocnemii, Injuries to, . . 313

Gathered Nails, . . . 387 Generation, Diseases of Exterual Organs of, Gerlach's Classifiction ${ }^{\circ} \cdot{ }^{\circ}$ Gerlach on Parasites, . . . 706 Girard on Inguinal Hernia, . . 605 Glands of Mouth, Affections of, - $\quad 497$ Glanders after Castration, . . 648 Grape and Small Shot Wounds, 460, 461 Glass-Eye, . . . . . 548 Glaucoma, . • . . $\quad 547$ Glossitis, . . . . . 501 Goodsir, Professor, on Morbid Condition of Cartilage, . 215 on Motion of Hock-Joint, 305

Granulations, Healing by, . . 477 Grease, . . . . . 671 ," Causes of, . . . 678 ," Causing Elephantiasis, . 678 , (Chronic), Sarcoptes Hippopodus in, . . . 677 ,, Treatment of, . $\quad . \quad$ : 681 Gun-Shot Wounds, . . $\quad 458$ " Progress, if favourable, 461 Gutta Serena or Glass-Eje, • - 548 Gut-Tie in Cattle, • • • 621 Guttural Pouches, Pus in, . $\quad$. 529

Haycock, Mr., on Lymphangitis, . $\quad 590$ Head, Neck, Veins, \&c., Diseases of, 566 ," Ear, Diseases of, 566 , Internal Canker, or Otorrhcea, 566 ,, Treatment, . . . 566 ," External Canker, : . $\quad 567$ ", Poll-Evil, . • . . 568 " Treatment, . . . . 568 , Caused by Bearing-Rein, $\quad 569$ ,, Fistulous Withers, . . $\quad 570$ ," Jugular Vein, Inflammation of (Phlebitis), . . . 570 , Pathology of, . . . 570 , Treatment, . . . 572 " Diffuse Phlebitis, $\quad$ • $\quad 573$ ,"Thrombus, . : : $\quad 574$ " Varicose Veins, . . . $\quad 574$ , J'hlebolites or Vein Stones, . $\quad 575$ ", Entrance of Air into Veins, $\quad 576$ ", Spence on Cause of, . . $\quad 577$ 
Mealing by Granulations,

" by Secondary Adhesions, .

" of Subcutaneons and Open

Wounds, difference (Paget), 474 under a Scab, . . • 481

"Wounds, Mode of, - 448

Healthy or Laudable Pus, . . 33

Heat in Inflammation. .

Hectic condition in Strangles,

Hereditary Tendency in Immobilité,

Shivering, \&c.

Herniæ,

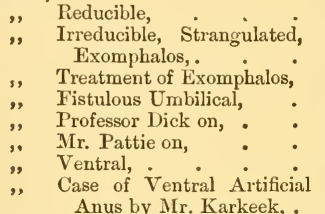

,

,

,

3)

,

,

$\rightarrow$

9

,

,

3

9 ,

9

,

5

$\div 9$

,

98

99

,

,

,
PAGE

477

480

48
33
22

22
44

248

596

596

597

598

599

599

600

601

601 Simonds, Professor, on Ventral, 602 Inguinal or Bubonocele, . 602 Girard on, Peculiarities of, . Symptoms and Treatment, following Castration, . Scrotal or Oscheocele, . Strangulation in, . Diagnosis of, . . Morbid consequences of, Congenital, Inguinal in "Fœetus in Utero," after Birth and Causes, .

Epiplocele, Scrotal, Operation for, in Stallions,

Simple, Operation for,

M. Barry's Method of Operating,

Herniotomy, . . .

Taxis and Clams in, .

Stanley, Mr., on Inguinal and Scrotal, .

Scrotal, in Pigs,

Gut-Tie in Cattle and Treatment,

IIernia Cerebri, present, Mode of Castration,

Herniotomy,

Herpes,

" Circinatus or Vesicular IingWorm,

High-Heeled Shoe in Injury to Flexor

Perforans Tendon, . • . 317

Hip-Joint Lameness, • • • 291

Hock, Capped, . . . 312, 425

, Coarse, . . . . 307

(605

606

607

607

608

608

608

609

610

616

616

615

611

612

613

620

621

116

646

611

668
Hock, Sprung,

PAGE

301

, Diseases of Gliding Articula. tions of, .

, Diseases of Ligamentous and Tendinous Structures of, .

Joint Lameness, True, .

310

299

", most susceptible to Bone-Spavin, 303

Honeycomb Ring-Worm, . $\quad 729$

Horn-Secreting Structures, Diseases of, 370

Horses, Clipping of, . . . 668

Horse or Gelding, Lithotomy in, . $\quad 627$

Humerus, Fractures of, . . 131

Humid Tetter, . . . . 673

Hydrocele or Dropsy of Scrotum, . 636

Hydrops Articnlorum, . $\quad 227$

Hæmorrhage after Castration, . 648

" in Concussion of Brain, 115

". in Wounds, . . 449

Immediate Union of Wounds, . 476

Inmobilité, . • . . . 247

Impairment of Function in Inflamma-

mation, . . . . 24

Impetigo Labialis et Facialis, : 678

Incised Wounds, Hæmorrhage, - 448

Inferior Maxilla, Fracture of, 91

Inflammation, . . • • 1

9

Definitions of,:

Pathology of, . $\quad 2$

Exudation, . . 3

,

Phenomena of, .

Stasis in, . 9

9

Parenchymatous and

Secretory, . . 12

Virchow on, . . 12

Causes of, . . 14

,

Burdon Sanderson on,

15

Varieties of, due to

Causation, . $\quad 16$

Local Symptoms of, 18

Redness,. . . 18

,

Pain, . • . 19

Cause of Pain, - $\quad 21$

,

Swelling, . . 22

$\rightarrow$

Heat, . 22

Impairment of Function, 24

Chronic, . • . 25

Terminations of, - 26

Resolution, . . 26

Adhesion, . . 27

Formation of New Vessels, Virchow and Paget on, 27

Blond Effusion or Extravasation, .

Suppuration or Formation of Pus,

Virchow on Pus, 32

Healthy or Laudable Pus, 33

Abscess, - . 35

Acute, - . $\quad 35$

Cold, - $\quad$ - 35 
Inflammation-continucd.

Chronic, .

Virchow on, .

Ulceration,

Varieties of, the Blood,

Inflammatory,

by Contact,

Types of,

Buffy Coat,

Dr. Alison on, School, Bleeding, in,

of Bone, .

Eczematous,

Erythematuus,

Diffuse Suppuration,

Superficial Suppuration,

Mucus, Epithelium, and Pus, Comparison between,

White Corpuscles, .

Febra Pyogenica, or Strangles,

Symptoms of :

Tracheotomy, .

Hectic condition,

Pyæmia, . .

Pathology of, .

Signs and Treatment of,

Reabsorption of Pus,

Formation of Sinuses,

Ulcers, Varieties and Treatment of,

Mortification, or Death of a part,

Necræmia, or Death of

Inflammatory Fever, Professor Miller on,

Inflammatory Appearances of Blood, .

Treatment of, :

Old View of, .

Blood-Letting in, :

Views of the Modern

Local and General

Method of Bleeding,

Second Blood-Letting,

Influences opposed to Blood-Letting,

Use of Purgatives in,

Opium, Aconite, Antimony, and Mercury

Alkaline Remedies in,

Nitrate of Potash, .

Chronic, Treatment of,

after Castration

of Coronary Sulsstance,

PAGE

Inf

Inflammation-continued. of Feet, . . $\quad 356$

570

265

589

638

249 $\begin{array}{ll}\text { of Muscles, or Myositis, } & 249 \\ \text { Squamous, of Skin, . } & 694\end{array}$ of Synovial Metnbranes, 206 Traumatic, of Joints, 223 of Vagina, . . $630^{\circ}$

Inflaminatory Appearances of Blood, 65 Fever, . . 608 Fever, Types of, . $\quad 64$ Mortification, . : 55

Inguinal Hernia in "Fotus in Utero," 608 or Bubonocele, - 602 Injuries, Accidental, to Feet, • 387

, to Arteries, . . . 588 ", of Facial Region, : : 493 ", to Gastrocnemii, : . 313 Internal Necrosis, rare, . . 174 Iodide of Arsenic, Preparation of, . $\quad 695$ Irreducible Hernia, . . . 597 Irrigation, Method of, for Open Joint, 225 Ischium, Fractures of Tuberosity of, 125

Joints, Diseases of, Classification of, 202 Anchylosis of Vertebræ, - 203 " Anchylosis of Vertebra, • $\quad 204$

". Symptoms obscure, · • • 205

,' Tymptoms obscure, : : : 205

", Diathrodial, or True, $\quad$ • 206

" Inflammation of Synovial Membrane, or Acute Syno-

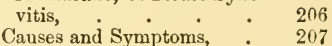

". Treatment, : : 207

", Chronic Serofulous Synovitis, 208

, Pathological Anatomy, and Causes of,
Calcification of Synovial Fringes, . . 210 $\begin{array}{ll}\text { Rheumatoid Arthritis, : } & 210 \\ \text { " Symptoms, } & 211\end{array}$ ", Anatomical Character of, : 212 ," Similar to Rheumatic Gout, 212 , Morbid Condition of Cartilage, 214 ", Goodsir on, . 215

", Dr. Redfern's Conclusions on, 218

, Healing of Wounds in Articular Cartilage, Redfern,

Traumatic Inflammation of, or Open Joint, . • 223 Condition of Part, : $\quad$ - 224

". Treatment, . . . 224

" Method of Irrigation, : 2. 225

", Dropsy of (Hydrops Articulorum) - see also Lamenrss, , Stiffening of, or Anchylosis, 
Joints, Osseous or True, Fibrous, I,igamentous, Spurious,

Jugular Vein, Inflammation of,

Karkeek, Mr., on case of Ventral Artificial Anus,

Kennel Lameness in Dogs, :

leratoma,

Kinds of Anchylosis, : $\quad$ :

Knee, Fracture of Bones of, .

Kunnmirree,

Labialis, Crusta, .

Lacerated Contnsed Wounds,

Laceration of Perforans Fibres in

Navicular Disease,

Laceration, Snbcutaneous, of Flexor Metatarsi,

Lachrymal I uct, Stricture of, . Lameness, Fistula of, :

", Diagnosis of, $\quad$ Manifestation during repose

and movement,

Cross, .

9

s)

Definition of,

Pain and Weakness are canses,

Rules for Diagnosis of,

,

$\rightarrow$

9

30

,

9

9

9

os

os

,

,

,

,

,

$\rightarrow$

Lautnesses, Particular,

Shoulder,
"Jisease of Shoulder
Joint,

PAGK

Lamenesses, Particular-continued.

PAGR

Sprain of Flexor Brachii, 255

Causes, Result, and Treatment, . . 255

Shoulder-Slip, . . $\quad 256$

Why most in Plough Horses, . . 257

Symptoms, . . 258

Treatment, . . 259

Wasted Shoulders, an

Unsonndness in Law, 259

Elbow Lameness, . 26?

Diagnostic Signs of, . 261

Olecranon wounded in Hnnting, Curious Symptoms,

Sprain of Radial or Superior Carpal Ligament,

Capped Knee, . $\quad 264$

,

s)

,

,

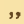

,

I)

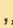

,

s,

,

,

Horned Cattle liabls to,

Carpitis, or Inflammation of Knee,

Mr. Cherry's Remarks,

Speedy Cut, • . 269

Symptoms of, . . 269

Prevention and Treatment of,

Broken Knees, : 270

Sprain of the Flexor

Tendons, . . 273

Causes, . . 274

Symptoms and Treatment, . . . 275

Tenotomy, . . 276

Sprain of Suspensury Ligament, . * 278

Percivall on the Ligament, . . 278

Special Treatment, . 283

Sprain of Fetlock-Joint, 283

Sesamoiditis, . . 254

Pathology and Symptoms, 234

Ring-Bones, Kinds of, 285

Treatment, . . 289

in Posterior Extremities, 291

Hip-Joint, . . 291

Hip. Joint, Symptoms of and Treatment,

Sprain and Atrophy of Crural Mnscles,

Stifle subject to Cramp, 296

Subcutanenus Laceration of Flexor Metatarsi Muscle, . 296

Case of it, . . 297

Diseases of Hock, True Hock-Joint, . . 299 Bog-Spavin, . . $2 \varepsilon 9$ 
I amenesses, Particular-continued.

" Blood-Spavin, $\quad$ Diseases of the Gliding Articulations of the Hocks, Bone Spavin, Definition,

, Percivall's Theory, -
" Form of Hock most susceptible,

" Goodsir on Motion of Hock-Joint, • • 305

Coarse Hocks, . . 307

Pathology of Bone-Spavin, 307

Causticking in. . 310

Diseases of the Ligamentous and Tendinous Structures of Hock, Thorough-Pin,

Treatment,

311

" Treatment, . .

, Capped Hock, . .

", Injuries to Gastrocneinii,

", Curb,

Treatment,

Tendon of Flexor Perforans liable to Injury,

Treatment of, Highheeled Shoe, .

Theory of Counter-Irritation, .

Treatinent of, Dr. Bennett on Counter-Irritation,

Rubefacients and Blisters, 321

", Hints on Blistering generally,

Actual Cautery,

Setons,

Lameness, Kennel, in Dogs,

Laminitis, or Inflammation of Feet, Kinds of, .

Laudable Pus,

Jawson's, Mr., Treatment of Tetanus,

Leucoma,

Leucocytes, ivandering in Inflammation, 545

Lice,

Lichen,

liston's, Mr., Theory on Concussion of the Brain,

Lip, Tumour of,

Lipomata, or Fatty Tumours,

Lips, Paralysis of,

Lithotomy in Horse or Gelding, . in $\mathrm{Ox}$,

Jower Taw, Fracture of Ramus of,

Luxations,

$$
\text { , of Patella, : }
$$

I.ymphangitis or Weed,

Lymphatics, Diseases of,

Inflammation of,

301

301

312

313

314

315

316

317

323

324

250

158

356

589

579
PAGE

M'Gillivray, Mr., on Ring-Worm,

Maggots, . . . . . 727

Mallenders, . $\quad . \quad . \quad . \quad . \quad 679$

Mammary Gland, Diseases of, $\quad 636$

Mammilarum, Erythema, . . $\quad 663$

Mammitis or Inflammation of Mam-

mary gland,

Mange in Cat,

638

$7 \cdot 20$

714

705

709

714

233

626

435 " causing Stringhalt, - 246 \begin{tabular}{ll} 
" causing Stringhalt, $\quad$ Development of, & 246 \\
\hline & 436
\end{tabular}

Mercury on Bone, Effects of, by Professor Bennett, . . 200

Mare, Removal of Calculus in,

Melanosis, . . . .

$$
\text { in Inflammation, - . } \quad 73
$$

in Osteo-Sarcoma, . $\quad 184$

Mercurial Poisoning in Sheep, : 708

Metacarpi, Fractures of, : . 135

Method of Bleeding, . . . 70

70
563

Microscopic Characters of new Bone, 83

Miller, Professor, on Inflammatory Fever,

Modern School Views on Blood-Letting, 69

Modes of Fracture of Bones, . $\quad 78$

, of Union of Bones, . . 79

Moroid Condition of Cartilage, . 214

," Consequences of Scrotal Hernia, 608

Mortification, . . . . 55

322 ", Varieties of, . . 56

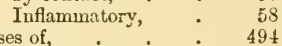

, Deformities of, - . 504

" Diseases of, . . . 493

, Glands andDuct, Affections of, 497

" Parrot, . . . 504

Moon Blindness, . . . . $\quad 555$

Mucous Cysts and Bronchocele, - 442

Mucus, Epithelium, and Pus, Comparison of,

Mud-Fever, . • . . . 660

Muscle, Subcutaneous Laceration of

Flexor Metatarsi,

296

Musket-Shot Wounds, . . . 459

Myositis or Inflammation of Muscu-

Nasal Bone, Fracture of, . 98

, Gleet, . . . . 517

, Polypus, : . . . 428

Navicular Arthritis (various Theories), 332 $\begin{array}{ll} & 137 \\ \text { Nebula, Bone, Fracture of, : } 544\end{array}$

589 Necrused Bone, Removal of, . . 97 
Neck, Diseases of,

Necrosis,

Internal, rare,

Nerves of Eye, Diseases of,

Nettle-Rash,

Neuromatous Tumours,
Neurotomy in Navicular Arthritis,

Unfavourable Results of,

New Bone, Microscopic Characters of,

Non-Inflammatory Bone 1)iseases,

Non-Union of Fracture, Causes of,

Occiput, Comnound Fracture of Crest of, 108

Old View of Treatment of Inflammation, 68

Olecranon, Fractures of,

, Wounded in Hunting,

Curious Symptoms, .

Omphalitis,

Open Joint,

Operations, French and English for Sand-Crack.

, for Serotal Hernia in Stallions,

,

for Simple Hernia, by Torsion,

Opium in Inflammation,

Ophthalmia, Periodic or Specific, .

Simple or Traumatic,

Ophthalmoscope, The, . .

Orf,

Oscheocele,

Osseous Tumours,

Ossification of Lateral Cartilages, or

Side-Bones,

Osteo-Porosis,

Osteo-Sarcoma,

Ostenid Tunours, .

Ostitis,

, and Periostitis of Metacarpi,

Otorrhoea, Serofulous,

Over-Reach,

Ox, Lithotomy in,

," Mange in,

Ozcena,

Esophagotomv,

Esophagus, Cardiac Stricture of,

$\begin{array}{ll}, & \text { Dilatation of, . } \\ , & \text { Diseases of, }\end{array}$

(Estridia,

Stricture of,

Estrus Bovis, .

Paget on Formation of New Vessels, ,, on Healing of Wounds, , on Inflammation,

Pain causing Lameness,

, in Inflammation,

Paralysis after Castration,

, of Lips,
566

.

PAGE

Paralysis of Tongue, . . . 502

Paraphymosis, and Treatment, . 632

Parasites, Animal, . . 700

700

Classified by Gerlach, . 700 of Eye, Vegetable Skin Diseases due to, 729

Parasitic Skin Diseases, . . 700

Parenchymatous and Secretory Inflammation,

Paring, Caution in,

Parrot-Mouth, . . . . 504

Patella, Fractures of, . . . 127

," Luxation of, . . . 142

Pattie, Mr., on Hernix, : $\quad 600$

Pathological Anatomy of Gelatinous Degeneration in Man,

353

Anatomy of Scrofulous Synovitis,

209

Pathology of Bone-Spavin, - . 307

730

2

357

$3+2$

169

162

570

48

284

604

Peculiarities of Inguinal Hernia, .

Pedal Bone, Diseases of Pyramidal process of, Fracture of, .

Pelvis, Fractures of,

Pharyngeal Polypi,
Pharynx, Diseases of, : $\quad$ : $\quad$ 530

Phenomena of Inflammation, . 4

Phlebitis, . . . 570

, Diffuse, . . . 573

Phlebolites, or Vein Stones, . $\quad 575$

Phthiriasis Equi, . . . . 724

Phymosis, Percivall and D'Arboval on, 632

Pig, Mange in, . . . . 714

, Scrotal Hernia in, . . 620

Pityriasis, . . . . .. 696

Plough-Horses, why most subject to

Shoulder-Slip, . . . . 257

Pointing in Navicular Arthritis, : $\quad 347$

Poisoned Wounds, . . : 468 
PAgR
Polypi,

" Nasal, : $\quad$ • $\quad$ • $\quad$ • 413

" Pharyngeal, : . $\quad$. 530

Post Mortem after Peritonitis, - 653

Posterior Extremities, Lamenesses of, 291

Potash, Nitrate of, in Inflammation, 74

Poultry Lousiness,

Pricks in Shoeing,

P obang, Use of, .

Precautions before Castration, . 642

Preparation for Castration, - • 643

Prevention of Speedy-Cut, . . 270

P'rimary Adhesion of Wounds,

Prurigo, or Pruritis,

Pruritis,

Psoriasis,

Psoæ Muscles, Situation of, :

," Strain of,

Pulex Irritans,

Punctured Fracture,

$$
\text { ,, Wound, }
$$

Purgatives in Inflammation,

Pus, Formation of,

, in Guttural Pouches.

, Reabsorption of,

Pustulosum, Eczema,

Pyro-Puncture,

Рyæmia,

$$
\text { , Patholoyy of, }
$$

Quittor,

Rachitis,

Radial Ligament, Surain of́, .

Radius, Fractures of,

Ranula,

Reabsorption of Pus,

Redfern, Dr., on Morbid Condition of Cartilage,

$$
\text { , on Wounds in Carti- }
$$

Redness in Inflammation, :

Reducible Hernia lage, . . . 219

Removal of Calculus in Mare,

$$
\text { ," of Eyeball, }
$$

," of Sabulous Matter,

Repair and Methods of Healing of Wounds,

Resolution, .

Riesults of Neurotomy, when unfavourable,

of Sprain of Flexor Brachii,

$$
\text { ", of Wounds, }
$$

Rheumatic Disease,

liheumatoid Arthritis,

$$
\text { " " Anatomical }
$$

,

Rivs, Brokes,
724

387

Rickets, Cause of,
Rokitansky on,
Treatment of, .

Rokitansky on Osteo-Porosis, : 180

," on Rickets, - . 181

Rubefacients, . . . . 321

Rules for Diagnosis of Lameness, . $\quad 239$

Sabulous Matter, Removal of, . $\quad 630$

Sacrum, Fractures of, . . . 121

Saliva, Excessive Secretion of, $\quad$ 500

Salivary Calculi, . . . . 499

"Fistula, . . . . 497

Sallenders, . . . . . 704

Sand-Crack, . . . . 374

Sarcocele after Castration, . . $\quad 649$

Sarcomatous Tumour, . . . 433

Sareoptes Canis, . . . . 715

721

703

" Equi, : Hippopodus in Cilronic

Grease,
Hominis, or Acarus Scabie, 701

" Hominis, or Acarus Scabie, 701

," Suis, . . . . 715

Scab in Sheep, . . . . 711

389 Scabies, . . . . . 700

Scapula, Fractures of, . $\quad$ - 131

179 Scirrhus, or Hard Cancer, : $\quad .443,649$

262 Scrofulous Ostitis, . . . 160

135 ," ," Causes of, . 162

500 Scrotal Hernia, Oscheocele, . . 606

51 , . in Pigs, . . 620

218 tion for, . 611

Scrotum, Fistula of, . . . 651

19 Seedy-Toe, . • • • . $38 \mathrm{C}$

18 Setons, . . . . . 324

$596 \quad$ ", in Navicular Arthritis, . $\quad 349$

626 Sesamoids, Fractures of, . . 135

563 Sesamoiditis, . . . . 284

630 Severe Bits, . . . . . . 494

Sheep Ointments dangerous, : 723

473 Sheep, Scab in, . . . . 696

26 Shivering, . • . . . 247

Shoe used at College, . . . 327

351 Shoeing, Prelimiuary Remarks upon, 325

255 , Pricks in, . . . 387

484 Shoulder-Joint, Disease of, . . $\quad 253$

235 , Lameness, . . . 253

210 " Slip, . . . . 256

Side-Bones, . $\quad . \quad$. 329

Signs, Diagnostic, of Elbow Lameness, 261

Simonds, Professor, on Ventral Hernia, 602

212 Simple Fissure, • • . . 105

110 Simple Hernia, Operation for, : 602 
PAGE

Simple or Traumatic Ophthalmia, .

Sinuses, Formation of, .

Sitfasts,

Skin, Diseases of, .

"Classification of, : $\quad$ :

" by Dr. Bennett, . . .

,, by Dr. Buchanan,

,Erythematnus Jnflammation,

,. Erythema, .

,, 'Mud-Fever,'

, Mr. Broad's Treatment of,

"Treatment of Erythema,

,, Clipping of Horses,

,, Erythema Mammilarum,

, Herpes,

", Circinatus or Vesicular Ring-

$$
\text { Worm, }
$$

Urticaria, Nettle-Rash, Surfeit,

Eczematous Inflammation,

.. Eczema, Various forms of,

, Simplex or Humid Tetter,

$$
\text { Rubrum, }
$$

Mercuriale, :

Lichen,

. Eczema Impetiginodes, or Pustulosum, .

", Sarcoptes Hippopodus in Chronic Grease,

Elephantiasis from Grease, .

, Causes of Grease, .

" Impetigo Labialis et Facialis, Crusta Labialis,

,, Treatment of Eczematous Eruption, .

Professor Dick's Treatment of Grease,

Treatment of Grease,

Treatment of Grease by actual Cautery, .

Treatment of Chronic Eczema,

Urinous Eczema, .

Squamous Inflammation, :

Psoriasis,

Mallenders and Sallenders,

Treatment of Psoriasis,

Iodide of Arsenic, Preparation of, 695

Pityriasis,

Treatment of,

. Disordered Sensibility of Derma,

Prurigo, or Pruritis,

Acne, or Warbles,

Sitfasts, Treatment of,

Parasitic,

Due to Animal Parasites or Dermatozoa, Scabits,

Species of Parasites, after Gerlach, 700

"Sarcoptes Hominis (Acarus Scabie), 701
Skin, Diseases of-continued.

," Bourguignon on, . . 701

, Sarcoptes Equi, . . . 703

Dermatodectes Equi, . . 704

, Symbiotes Equi, . . . 705

", Gerlach on Parasites, . . 706

, Mange in the $\mathrm{Ox}$, . . 709

"Symptoms in the $\mathrm{Ox}, \quad$. $\quad 710$

,. Scab in Sheep, . . . 711

, Dermatodectes Ovis, . . 711

", Sarcoptes Ovis, . . . 714

", Mange in Pig (Sarcoptes Suis), 711

$, \quad, \quad$ in Dog (Sarcoptes Canis), 714

, Follicular Scabies in Dog, . 715

,, Mr. Fleming on, . . . 716

, Mr. Hunting on, . . . 717

, Mange in Cat (Sarcoptes Cati), $\quad-21$

,, Treatment of Scabies, . . 721

, Stavesacre, Experiments with, $\quad 722$

"Sheep Ointments dangerous, 723

„ Due to Epizoa, . . . 724

" Iice, .

", Poultry Lousiness (Phthiriasis Equi),

Bouley on, . : : $\quad 724$

Fleas (Pulex Irritans), $\quad 725$

"Persian Insect Powder, - $\quad 725$

", Ticks, . . . . 725

" Other Animal Parasites, . : $: 26$

" Botflies, (Estridia), . . 726

, Estrus Bovis, . . . $\quad 726$

" Bracy Clark on Estrus Bovis, $\quad 726$

" Maggots, or the "Fly," . 727

, Due to Vegetable Parasites, $\quad 729$

, Favus or Honeycomb Ring. Worm, Pathology of,

, Tinea Tonsurans, or Ring-IVorm, 734

, Aitken and Lowe on Pathology of, 735

"Mr. M'Gillivray on Ring-WVorm, 736

"Treatment of Fing-Worm, . $\quad 736$

Snakes, Poison of, . . . 469

Sore Shins, . . . . 153

". Symptoms of, . . . 154

Spavin, Bog and Blood, . . $\quad 299$

,, Bone, . . . 301

Specific Ophthalmia, . . . 555

"Speculum Oris," The, . . 511

Speedy-Cut, . $\quad: \quad 269$

Spence, Prufessor, on Aneurism, . 581

$\begin{array}{ll}, \quad \text { on Cause of Air in Veins, } & 577 \\ , \quad \text { on Gelatinous Degeneration, } & 353\end{array}$

Spent-Balls, Wounds from, . . 456

Splints, . . . . 155

,, Block Tin, . . $\quad 85$

for Fractures, Kinds of, - 84

Kinds of, $\quad$ - 156

Lameness, Diagnosis of, . 158

when producing Lameness, $\quad 157$ 
Splints, Treatment for,

PAGE

Split Pastern,

Sprain or Strain

, of Fetlock-Joint,

," of Flexor Brachii,

", of Flexor Tendons,

" of Perforans Fibres in Navicular

Disease,

of Radial or Supero-Carpal Ligament of Suspensory Ligament,

Sprained Back,

Squamous Inflammation of Skin, .

Squinting,

Stallions, Operation for Scrotal Hernia in,

Stanley, Mr., on Inguinal and Scrotal Hernia

Staphyloma,

Stasis,

Stavesacre, Experiments with,

Stifle-Joint Lameness,

," Subject to Cramp,

Strabismus,

Strain,

, of Psoæ Muscles,

Strangles,

$\begin{array}{ll}\text {," } & \text { Hectic Condition } \\ , & \text { Tracheotoms of, } \\ \text {, } & \text { Tracheomy }\end{array}$

Strangulated Hernia,

Strangulation in Scrotal Hernia,

Stricture, Cardiac, of CEsophagus,

Stringhalt, of Lachrymal Duct,

Structure of Tumours,

Subcutaneous Periosteotemy,

Subperiosteal Suppuration,

Suffraginis, Fractures of,

Supero-Carpal Ligament, Sprain of,

Superior Maxilla, Fracture of,

Suppuration, Chronic, .

, Diffuse,

, Subperiosteal,

Surfeit, Superficial,

Suspensory Ligament, Sprain of, .

Suture, Carbolised Cat-Gut,

Varieties of,

Swelling in Inflammation,

Symbiotes Equi,

Syme, Professor, on Caries and Ulceration,

, on Trephining for Concussion of the Brain,

, Treatment of False Joints,

Symphysis Pubis, Fracture through,

Symptoms of Anchylosis of Vertebræ,

, of Favus,
158

136

241

283

255

273

262

278

242

694

549
Symptoms of Fracture of Bones, of Hip-Joint Lameness,

" of Hip-Joint Lameness,

\section{" of Inguinal Hernia,} of Kennel Lameness,

, Local, of Inflammation, of Laminitis, of Navicular Arthritis, of Rheumatoid Arthritis, Curious, of Wounded Olecranoll, . . 261 of Scabies, . . . 701 of Shoulder-Slip, . . 258 of Speedy-Cut, . . 269 of Sore Shins, . . 154 of Sprain of Flexor Tendons, 275 of Strangles, Unpropitious, in Compound Fractures,

Teeth, Caries of, . $\quad 507$

, Irregularities of, . . 504

,, Tumours, . . . . 430

Tenotomy, . . . . 276

Terminations of Inflammation, - $\quad 31$

Tetanus, . . . 486

" after Castration, . . 649 ", from Fracture of Frontal

Tetter, Humid, : $\quad{ }^{\circ} \quad: \quad 673$

Theory of Counter-Irritation, . $\quad 319$

Thorough-Pin, . . . . 310

Thrombus, . . . . . 574

Thrush, . . . . . 382

Tibia, Fractures of, . $\quad$. $\quad 116$

Ticks, . $\quad . \quad$. $\quad . \quad 725$

Tinea Tonsurans, or Ring-IVorm, . 733

Tongue, Affections of, . . . 501

Tracheotomy, - • . $\quad 46$

Traumatic Inflammation of Joints, 223

Tread, . . . . . 463

Treatment of Aneurism, . . $\quad 583$

," of Burns, : : . 467

", of Canine Skin Diseases, 693

," of Canker, . . . 384

", of Champignon, : . 650

," of Chronic Eezema, : 692

". Constitutional, of Wounds, 452

". of Eczematous Eruptions, 689 


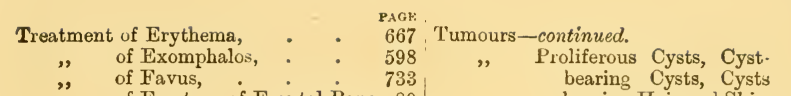

" of Fracture of Frontal Bone, 89

" of Grease, . . . 682

", of Inflammation, . . 66

,, of Inguinal Hernia, . 611

, of Laminitis-Mr Broad's

$$
\text { Shoe, }
$$

of Lymphangitis, : $\quad$. $\quad 592$

of Mammitis, . $\quad$. 640

of Open Joint, . $\quad$. 224

of Peritonitis, . . 654

of Phlebitis, . . . 572

of Pityriasis, . . $\quad 697$

of Psoriasis, . $\quad 695$

of Quittor, . . . 390

of Ring-Bones,

of Ring-Worm,

of Sand-Crack,

of Scabies,

of Side-Bones,

and Signs of Pyæmia,

of Sitfasts,

Special, of Sprain or Sus.

pensory Ligament, . of Splint,

$"$

of Tetanus,

, of Ulcers,

Trephine, Use of,

159 Unsoundness, Wasted Shoulders au,

489 Urachus, Pervious, . . . 163

53 Urethral Calculi, . . . 629

511 Urethritis, Symptoms and 'T'reatment, 631

Causes and Symptoms,

Trichiasis, for Cystis,

551 Urination, Diseases of External Or-

126 gans of,

Simple, : : : 412

,, Polypi (Class of Hibrous Tumours),

Nasal Polypus,\& Treatment, 414

Epidermic and Epithelial Warts,

Lipomata or Fatty, :

Neuromatous,

Enchondromata,

Crusta-Petrosa,

Calcareous,

Osseous,

Osteoid (Osteo-Sarcoma),

Cystic (various kinds),

Serous Cysts, Capped Elbow, 423

Capped Hock,

Serous Cysts in Horned Cattle,

Mucous Cysts and Bronchocele, eases of Alveolar Processes, 506

on Diseases of Facial Sinuses, 517

Treatment of Fracture of

Lower Jaw,

Treatment of Fracture of

Nasal Bone,

on Osteo-Porosis

189

Vagina, Diseases of, . . . 636

Tumours in, . . 638

Vaginitis, or Inflammation of Vagina, 636

Varicose Aneurism, . . 585

, Veins, . . . . 574

Varieties of Mortification, . $\quad 55$

, of Tetanus, . . . 487

Various Forms of Eczema, : . $\quad 673$

Varix, Aneurismal, . : . 585

425 Veins, Diseases of, . . 566

426 " Entrance of Air into, - $\quad 576$

Vein-Stones, . . . . 575

Veins, Varicose, . . . . 574

427 Ventral Hernia, : $\quad \cdot \quad \cdot \quad \cdot 601$ 
Vertebræ, Anchylosis of, , Fractures of,

Vesicular Seminalis, Distension of,

PAGE

203

117

631

669

Villitis,

Virchow on Formation of New Vessels, on Inflammation, .

on Pus, . on Superficial Suppuration, on Thrombosis,

Walker, Mr., Cradle for Broken Jaw, 95 Warbles,

Warts,

on Eyelids,

Wasps and Bees, Stings of, .

Wasted Shoulders an Unsoundness in Law,

Weak Feet,

Weakness causing Lameness, .

Weed or Lymphangitis,

White Corpuscles in Inflammation,

Wilson on Eczema Impetiginodes,

Wind-Suckers,

Wounds,

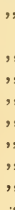

Definition, Classification of, and Modes of Healing, Incised, Hæmorrhage in, Varieties of Sutures, Constitutional Treatment, Punctured,

Contused and Lacerated,

Lacerated Contused,

Gun-Shot,

From Spent Balls, .
Wounds-continued.

Musket-Shot, $\quad$. $\quad 459$

Grape-Shot, • • . 460

Small-Shot, . . . 461

Gun-Shot, Progress and Consequences if favourable,

461

Treatment,

Burns, Scalds, and the Effect of Cold, . . . 465

Treatment, . $\quad 467$

Poisoned, Stings of Wasps and

Bees, Poisons of Snakes, 468

Over-Reach, Tread, and Speedy-Cut, . . 462

Brushing or Cutting, . 463

of Abdominal Parietes, . 463

Repair of, and Methods of Healing, . . . 473

Paget on, . . . 473

Difference between Subcutaneous and Open, from Paget, 474

Immediate Union, . . 476

Primary Adhesion, . 477

Healing by Granulations, $\quad 477$

Secondary Adhesion, . $\quad 480$

Under a Scab, . . 481

Results of, . . . 484

Erysipelas, Edematous, 484

Phlegmonous, . . 485

Treatment, . . 486

Tetanus, . . . 486

Varieties of, . . . 487

Symptoms, . . . 488

Treatment, . . . 489

Mr. Lawson's, : : 491

459 Wounds of Eyelids, . $\quad$ : $\quad 552$ 

TUFTS UNIVERSITY LIBRARIES

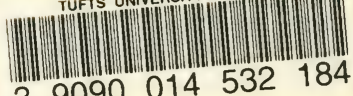

39090014532184

$$
\begin{aligned}
& \text { Wiliansiwinin } \\
& \text { iozz-1900. } \\
& \text { The prini ius avd } \\
& \text { praitice of }
\end{aligned}
$$

Seaverns

SF

753

W5

1893 
